Supporting Information

\title{
The Impact of Charge in a Ni(II) Polymerization Catalyst
}

Eva Schiebel ${ }^{\dagger a}$, Maria Voccia ${ }^{\ddagger a}$, Laura Falivene ${ }^{\ddagger}$ Lucia Caporaso ${ }^{{ }^{*}}$, Stefan Mecking ${ }^{\dagger *}$

+ Chair of Chemical Materials Science, Department of Chemistry, University of Konstanz, 78457 Konstanz, Germany

$¥$ Dipartimento di Chimica e Biologia, Università di Salerno, Via Papa Paolo Giovanni II, I-84084 Fisciano, Italy

a contributed equally

*Corresponding Authors

Email:lcaporaso@unisa.it,stefan.mecking@uni-konstanz.de 


\section{Contents}

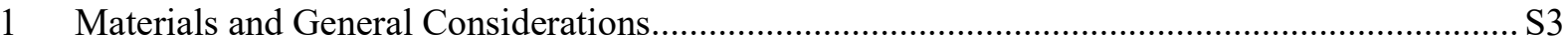

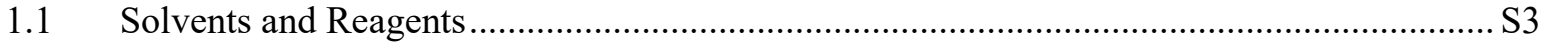

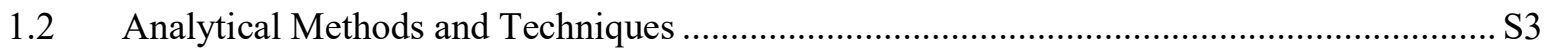

2 Synthesis and Characterization of the Complexes ……...................................................... S4

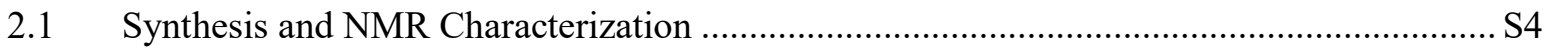

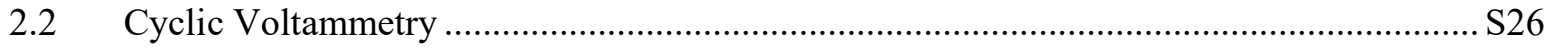

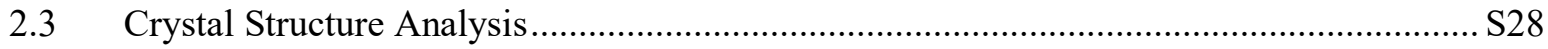

3 Ethylene Polymerizations and Oligomerizations ……………................................................. S32

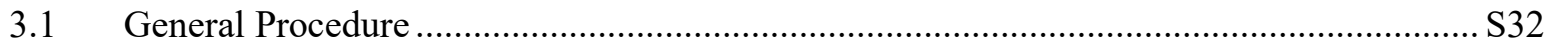

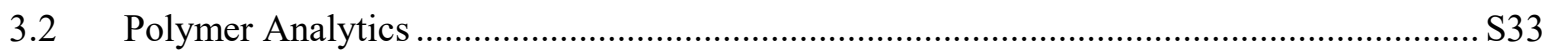

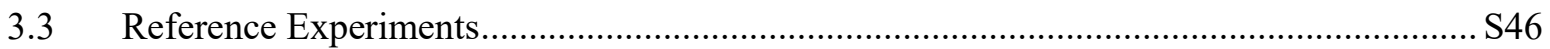

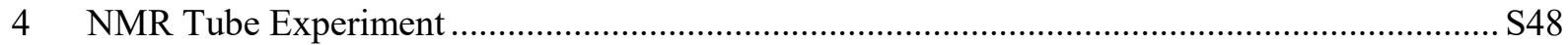

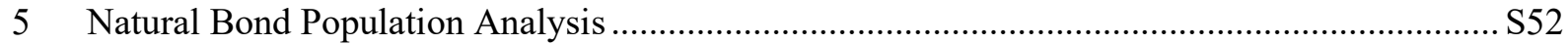

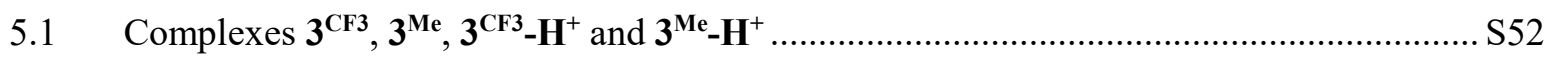

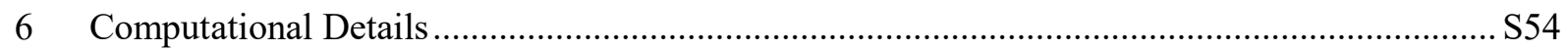

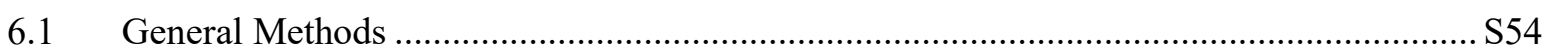

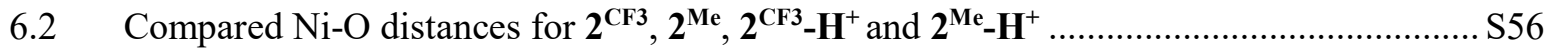

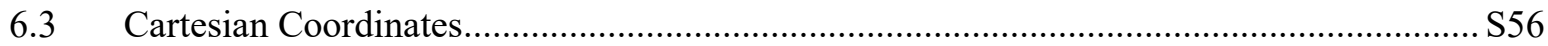

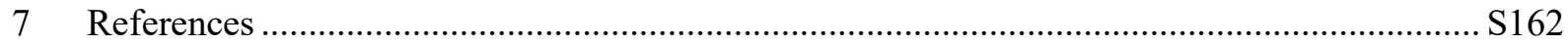




\section{Materials and General Considerations}

Unless noted otherwise, all manipulations of air and moisture sensitive materials were carried out under inert gas atmosphere using standard glovebox and Schlenk techniques.

\subsection{Solvents and Reagents}

Solvents were dried and degassed using standard laboratory techniques. THF was distilled from sodiumbenzophenone ketyl, benzene from sodium, pyridine from $\mathrm{CaH}_{2}$. Pentane, methylene chloride and toluene were dried and degassed by passing through columns, equipped with alumina and BASF R3-11 catalyst. 4-Methyl-2,6diformylphenol ${ }^{1}$, the terphenylanilines, ${ }^{2}\left[\text { (tmeda) } \mathrm{NiMe}_{2}\right]^{3}$ and Brookhart's acid $^{4}$ were synthesized according to reported procedures. 4- ${ }^{t} \mathrm{Bu}-2,6$-diformylphenol was obtained from $\mathrm{ABCR}$, nickelallyl chloride was obtained from MCAT. Ethylene (grade 3.5) and hydrogen (grade 5.0) was supplied by AIR LIQUIDE and used as received. All other commercially available reagents and starting materials were supplied by MERCK, ACROS, ABCR or ACTIVATE SCIENTIFIC. $\mathrm{CDCl}_{3}$ was supplied by MERCK, all other deuterated solvents by EURISOTOP.

\subsection{Analytical Methods and Techniques}

Nuclear magnetic resonance (NMR) spectra were recorded on a BRUKER Avance III HD 400 ( ${ }^{1} \mathrm{H}: 400.1 \mathrm{MHz}$, $\left.{ }^{13} \mathrm{C}: 100.6 \mathrm{MHz},{ }^{19} \mathrm{~F}: 76.1 \mathrm{MHz}\right)$, a BRUKER Avance III 400 or a Bruker Avance III 600 spectrometer $\left({ }^{1} \mathrm{H}: 600\right.$ $\left.\mathrm{MHz},{ }^{13} \mathrm{C}: 151 \mathrm{MHz}\right) .{ }^{1} \mathrm{H}$ chemical shifts were referenced to the solvents residual proton signals $\left(\mathrm{CDCl}_{3}: 7.26\right.$ ppm, $\left.\mathrm{C}_{2} \mathrm{D}_{2} \mathrm{Cl}_{4}: 5.91 \mathrm{ppm}, \mathrm{C}_{6} \mathrm{D}_{6}: 7.16 \mathrm{ppm}, \mathrm{THF}-d_{8}: 1.72 \mathrm{ppm}, \mathrm{CD}_{2} \mathrm{Cl}_{2}: 5.32 \mathrm{ppm}\right) .{ }^{13} \mathrm{C}$ chemical shifts were referenced to the carbon signal of the deuterated solvent $\left(\mathrm{CDCl}_{3}: 77.16 \mathrm{ppm}, \mathrm{C}_{2} \mathrm{D}_{2} \mathrm{Cl}_{4}: 74.30 \mathrm{ppm}, \mathrm{C}_{6} \mathrm{D}_{6}: 128.06\right.$ ppm, THF- $\left.d_{8}: 67.21 \mathrm{ppm}, \mathrm{CD}_{2} \mathrm{Cl}_{2}: 44.35 \mathrm{ppm}\right) .{ }^{19} \mathrm{~F}$ chemical shifts were referenced to external $\mathrm{BF}_{3} \cdot \mathrm{OEt}_{2} .{ }^{11} \mathrm{~B}$ chemical shifts are referenced to internal standard. NMR spectra of polyethylenes were recorded with addition of $5 \mathrm{mg} \mathrm{mL}^{-1}$ of $\mathrm{Cr}(\mathrm{acac})_{3}$ as paramagnetic relaxation agent. The NMR assignments were confirmed by common 2D NMR experiments $\left({ }^{1} \mathrm{H}^{1} \mathrm{H}-\mathrm{COSY}-\mathrm{QF},{ }^{1} \mathrm{H}^{13} \mathrm{C}-\mathrm{HSQC}-\mathrm{ed},{ }^{1} \mathrm{H}^{13} \mathrm{C}-\mathrm{HMBC}\right)$. MESTRENOVA software by MESTRELAB RESEARCH S.L. (V14.1.0) was used for data evaluation. X-ray diffraction was performed at $100 \mathrm{~K}$ on a STOE IPDS-II diffractometer, equipped with a graphite-monochromated radiation source $(\lambda=0.71073 \AA)$ and an image plate detection system. Crystals were placed on a fine glass fiber with grease or oil. The selection, integration and averaging procedure of the measured reflex intensities, the determination of the unit cell dimensions and a leastsquares fit of the $2 \Theta$ values as well as data reduction, LP-correction and space group determination were performed using the X-Area software package delivered with the diffractometer. Using OLEX2 ${ }^{5}$, the structure was solved with the ShelXT ${ }^{6}$ structure solution program using Intrinsic Phasing and refined with the SHELXL using Least Squares minimization. ${ }^{7}$ Graphical representation were created by the ORTEP-3 V2.02. ${ }^{8}$ for WINDOWS XP software package. Cyclic voltammetry (CV) measurements were performed in a custom-built one-compartment cell. A spiral-shaped Pt wire and an Ag wire as the counter and reference electrodes are sealed into glass capillaries that are introduced via Quickfit screws at opposite sides of the cell. APt electrode is introduced as the working electrode through the top port. It is polished with first $1 \mu \mathrm{m}$ and then $0.25 \mu \mathrm{m}$ diamond paste (BUEHLER-WIRTZ) just before measurements. The experiments were performed with approximately $5 \mathrm{~mL}$ of dichloromethane under an argon atmosphere. ${ }^{n} \mathrm{Bu}_{4} \mathrm{NPF}_{6}$ was used as supporting electrolyte. Referencing was performed with addition of $\left[\left(\mathrm{Me}_{5} \mathrm{Cp}\right)_{2} \mathrm{Fe}\right]$ as an internal standard after all data have been acquired. The scans were repeated with internal 
standard. Final referencing was performed against ferrocene/ferrocenium $\left(\mathrm{Cp}_{2} \mathrm{Fe}^{0 /+}\right)$ with $\mathrm{E}_{1 / 2}\left(\mathrm{Cp}_{2} \mathrm{Fe}^{0 /+}\right)=-550 \mathrm{mV}$ vs. $\mathrm{Cp}_{2} \mathrm{Fe}^{0 /+}$. Electrochemical data were acquired with a computer-controlled BASI potentiostat. Differential scanning calorimetry (DSC) was carried out on a NETZSCH DSC 204 F1 with a bicyclic temperature program from 20 to $160{ }^{\circ} \mathrm{C}$ with heating and cooling rates of $10 \mathrm{~K} \mathrm{~min}^{-1}$ in closed $40 \mu \mathrm{L}$ alumina pans under a nitrogen atmosphere. Gel permeation chromatographic (GPC) analysis was performed on two different apparatus: Measurements for oligomers completely soluble in THF were performed on a POLYMER LABORATORIES PL-GPC 50 instrument with two PLgel $5 \mu \mathrm{m}$ MIXED-C columns in THF at $50^{\circ} \mathrm{C}$ with RI detection against polystyrene standards. Measurements for all other oligomers/polymers were conducted in 1,2-dichlorobenzene on a POLYMER CHAR GPC-IR instrument, equipped with PSS Polefin Linear XL columns (3 x $30 \mathrm{~cm}$, additional guard column), an infrared detector (IR5 MCT, concentration signal) and a viscometer in 1,2-dichlorobenzene at $160{ }^{\circ} \mathrm{C}$ at a flow rate of $1.0 \mathrm{~mL} \mathrm{~min}^{-1}$. Molecular weights were determined against linear polyethylene from PSS Polymer Standards or by using universal calibration against polystyrene from PSS Polymer Standards (software: PSS WinGPC, version 8.32). Gas chromatographic (GC) analyses were carried out on a PERKIN ELMER CLARUS 500 GC system equipped with an Elite-5 capillary column $(30 \mathrm{~m} \times 0.25 \mathrm{~mm} \times 0.25 \mu \mathrm{m}$, phase description: $5 \%$ diphenyl $-95 \%$ dimethyl polysiloxane) using a flame-ionization detector (FID). Helium was used as the carrier gas with a gas flow of $1.5 \mathrm{~mL} \mathrm{~min}^{-1}$. An injection volume of $1.0 \mu \mathrm{L}$ was used. The oven program started with $50{ }^{\circ} \mathrm{C}$ and the column was then heated at a rate of $25^{\circ} \mathrm{C} \mathrm{min}-1$ to $230{ }^{\circ} \mathrm{C}$, to $330{ }^{\circ} \mathrm{C}$ with a rate of $10{ }^{\circ} \mathrm{C} \min ^{-1}$ and kept isothermal at this temperature for $10 \mathrm{~min}$ (injector temperature $350^{\circ} \mathrm{C}$; detector temperature $350{ }^{\circ} \mathrm{C}$ ).

\section{Synthesis and Characterization of the Complexes}

\subsection{Synthesis and NMR Characterization}

\subsubsection{Synthesis of Bis(imino)phenols}

\subsubsection{Synthesis of $\mathbf{1}^{\text {CF3 }}$}

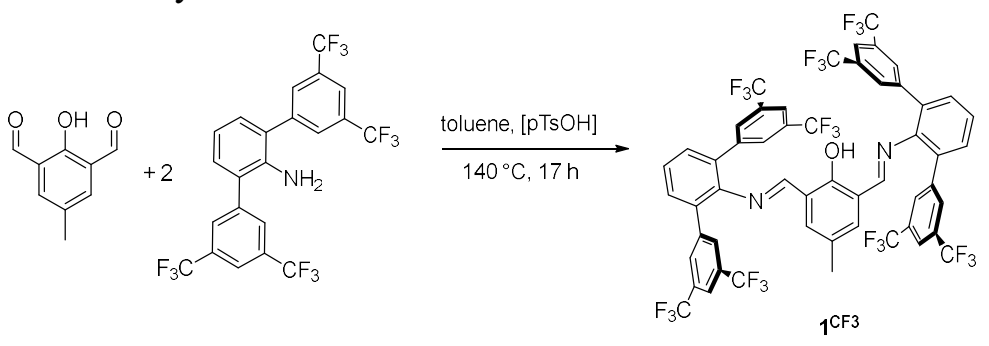

Scheme S1: Synthesis of $\mathbf{1}^{\text {CF }}$.

2,3-Di(3,5-bis(trifluoromethyl)phenyl)aniline (2.0 g, $3.86 \mathrm{mmol}, 2.0$ equiv.) and 4-methyl-2,6-diformylphenol (317 mg, $1.93 \mathrm{mmol}, 1.0$ equiv.) were dissolved in $500 \mathrm{~mL}$ of toluene. $p$-Toluenesulfonic acid (pTsOH) (5 mg) was added. The round bottom flask was connected to a soxhlet extractor loaded with molecular sieves. The reaction was refluxed at $140{ }^{\circ} \mathrm{C}$ for $17 \mathrm{~h}$. The solvent was removed, and the product obtained as yellow oil in $95 \%$ purity (2.32 g, 1.93 mmol, quantitative). 


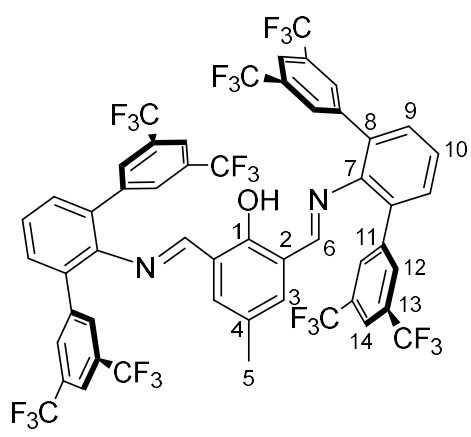

${ }^{1}$ H NMR $\left(400 \mathrm{MHz}, \mathrm{THF}-d_{8}, 320 \mathrm{~K}\right): \delta(\mathrm{ppm})=12.11(\mathrm{~s}, 1 \mathrm{H}, \mathrm{OH}), 8.16(\mathrm{~s}, 2 \mathrm{H}, \mathrm{H}-6), 8.01\left(\mathrm{~d},{ }^{4} J_{\mathrm{HH}}=1.7 \mathrm{~Hz}, 8 \mathrm{H}\right.$, $\mathrm{H}-12), 7.82\left(\mathrm{~d},{ }^{4} J_{\mathrm{HH}}=1.7 \mathrm{~Hz}, 4 \mathrm{H}, \mathrm{H}-14\right), 7.61\left(\mathrm{~d},{ }^{3} J_{\mathrm{HH}}=7.7 \mathrm{~Hz}, 4 \mathrm{H}, \mathrm{H}-9\right), 7.46\left(\mathrm{t},{ }^{3} J_{\mathrm{HH}}=7.7 \mathrm{~Hz}, 2 \mathrm{H}, \mathrm{H}-10\right), 7.29$ (s, 2H, H-3), 2.12 (s, 3H, H-5).

${ }^{13} \mathbf{C}\left\{{ }^{1} \mathbf{H}\right\}$ NMR $\left(101 \mathrm{MHz}, \mathrm{THF}-d_{8}, 320 \mathrm{~K}\right): \delta(\mathrm{ppm})=166.4$ (C-6), $160.3(\mathrm{C}-1), 148.9(\mathrm{C}-2), 142.7$ (C-7), 134.9 (C-3), $132.3\left(\mathrm{q},{ }^{2} J_{\mathrm{CF}}=33.4 \mathrm{~Hz}, \mathrm{C}-13\right), 132.3(\mathrm{C}-8), 132.1$ (C-9), $131.3\left(\mathrm{q},{ }^{4} J_{\mathrm{CF}}=3.7 \mathrm{~Hz}, \mathrm{C}-12\right), 130.0(\mathrm{C}-4), 129.3$ (C-11), $126.8(\mathrm{C}-10), 124.5$ (q, $\left.{ }^{1} J_{\mathrm{CF}}=272.5 \mathrm{~Hz}\right), 121.5$ (C-14), 19.9 (C-5).

${ }^{19} \mathbf{F}\left\{{ }^{1} \mathbf{H}\right\}$ NMR $\left(376 \mathrm{MHz}, \mathrm{THF}-d_{8}, 320 \mathrm{~K}\right): \delta(\mathrm{ppm})=-63.9\left(\mathrm{CF}_{3}\right)$.

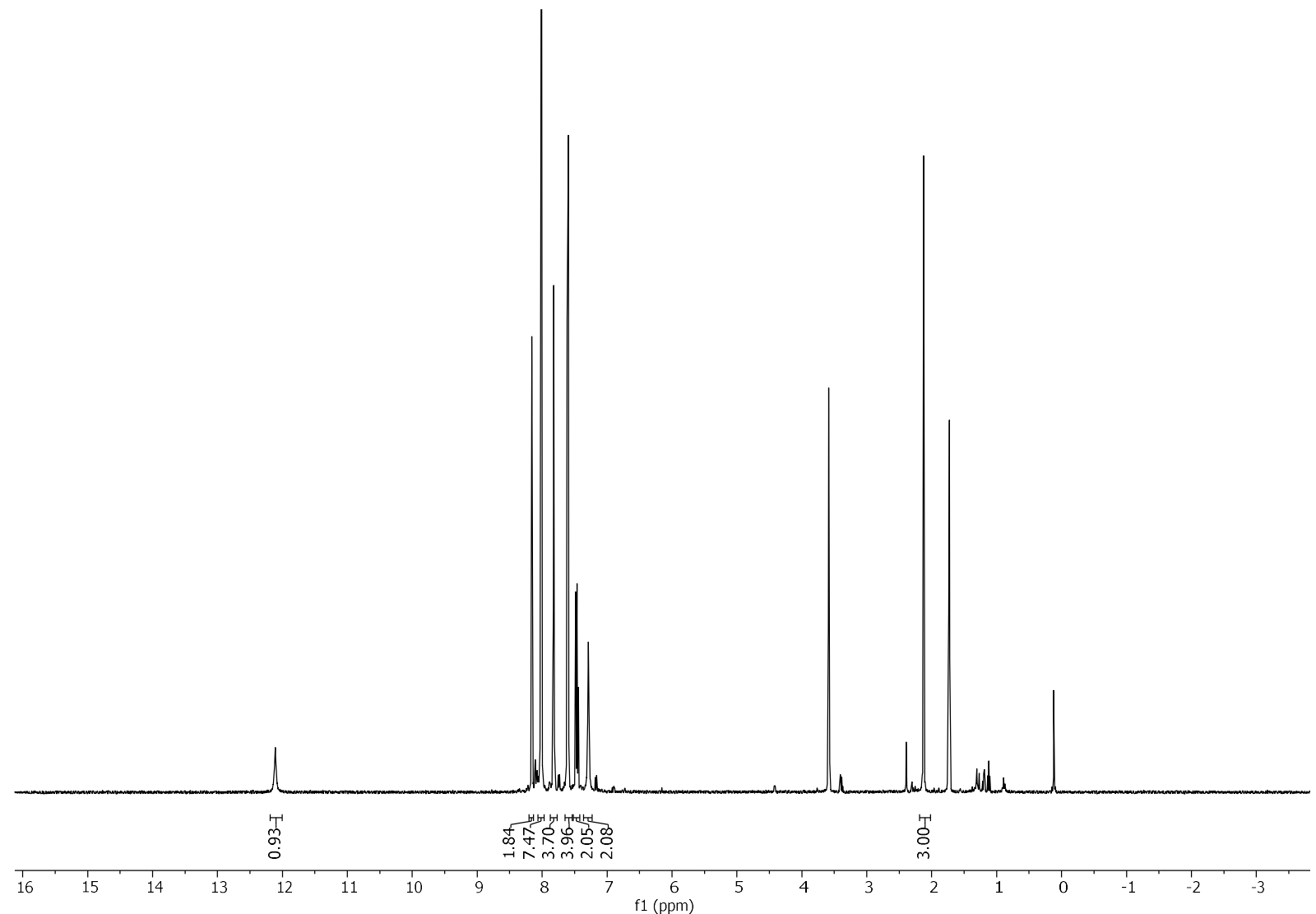

Figure S1: ${ }^{1} \mathrm{H}$ NMR spectrum of $1^{\text {CF3 }}$ in THF-ds at $320 \mathrm{~K}$. 


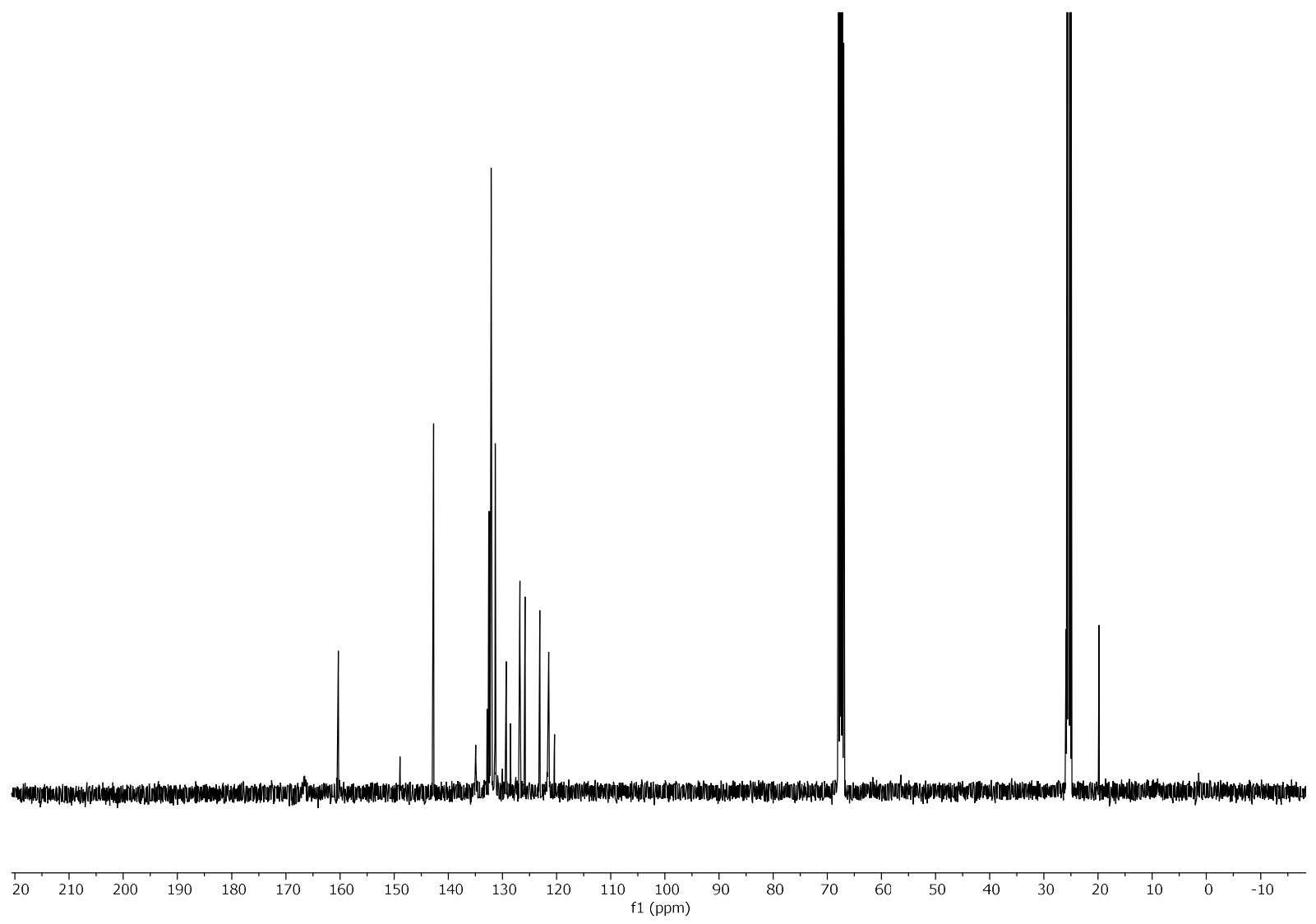

Figure S2: $\left.{ }^{13} C_{\{1}{ }^{1} H\right\}$ NMR spectrum of $\mathbf{1}^{C F 3}$ in $\mathrm{THF}-\mathrm{d}_{8}$ at $320 \mathrm{~K}$.

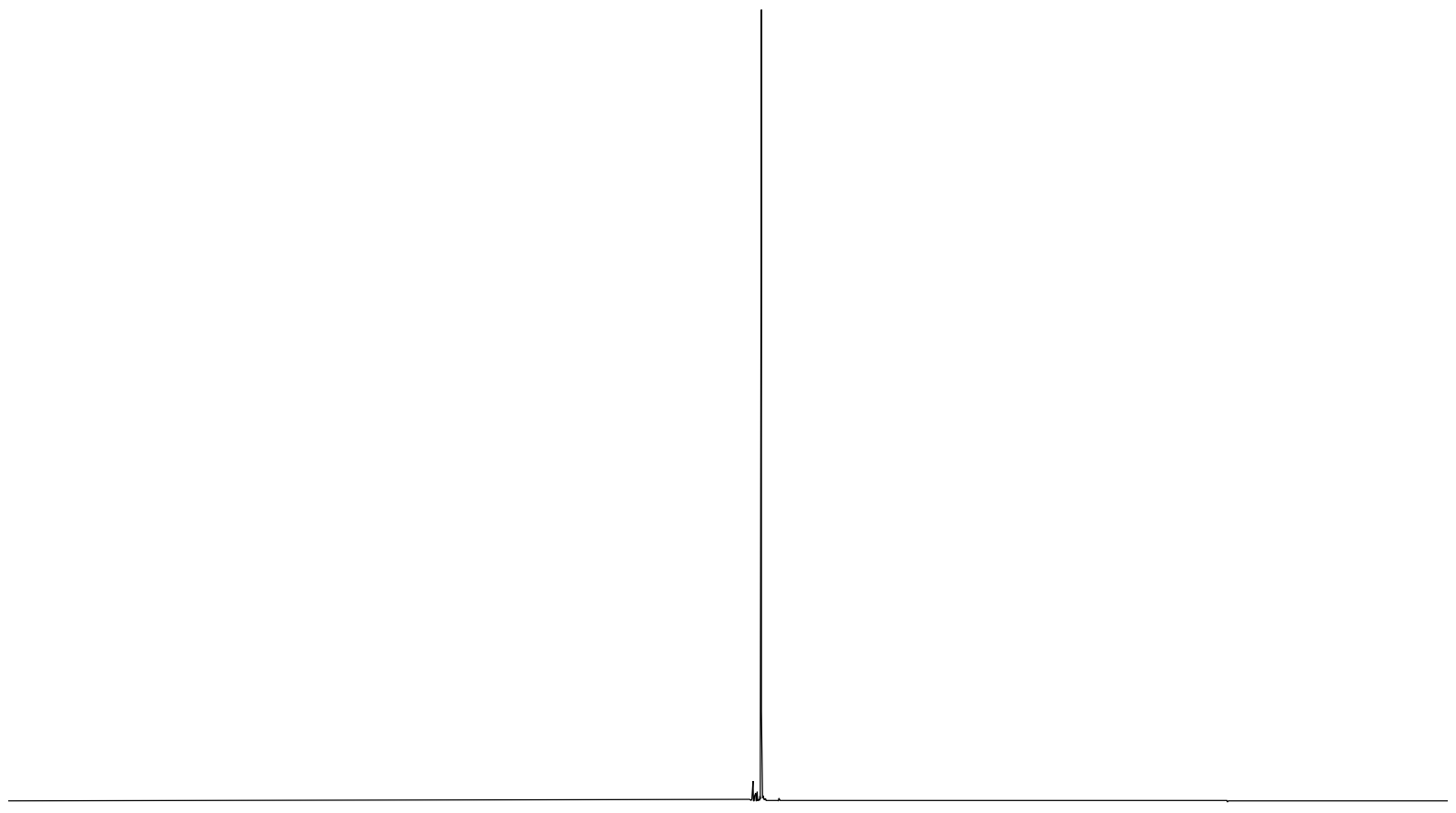

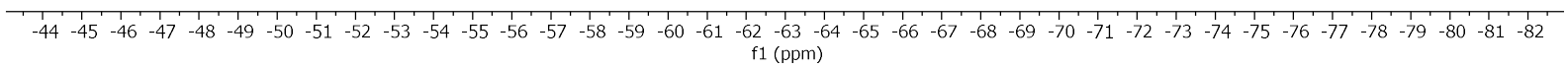

Figure S3: $\left.{ }^{19} \mathrm{~F}^{\mathrm{I}} \mathrm{H}\right\}$ NMR spectrum of $\mathbf{1}^{\mathrm{CF} 3}$ in $\mathrm{THF}$-do at $320 \mathrm{~K}$.

Sw 


\subsubsection{Synthesis of $\mathbf{1}^{\mathrm{Me}}$}
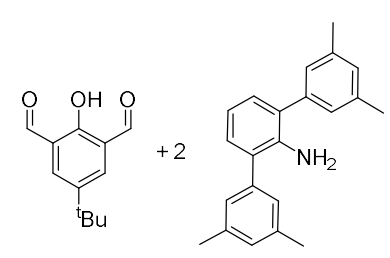

$\underset{\text { r.t. } 4 \mathrm{~h}}{\stackrel{\mathrm{MeOH}}{[\mathrm{CHCOOH}]}}$

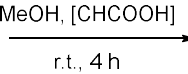

Scheme S2: Synthesis of $\mathbf{1}^{\mathbf{M e}}$.

2,3-Di(3,5-di(methyl)phenyl)aniline (200 mg, 0.66, 2.1 equiv.) and 4- ${ }^{\text {tert }}$ butyl-2,6-diformylphenol (65 mg, $0.32 \mathrm{mmol}, 1.0$ equiv.) were dissolved in $10 \mathrm{~mL}$ of dry $\mathrm{MeOH}$. Formic acid was added, and the solution stirred for $4 \mathrm{~h}$ at room temperature. The yellow precipitate was filtered off and washed with cold $\mathrm{MeOH}(2 \times 4 \mathrm{~mL})$. The product was obtained as yellow solid (145 $\mathrm{mg}, 0.19 \mathrm{mmol}, 60 \%$ ).

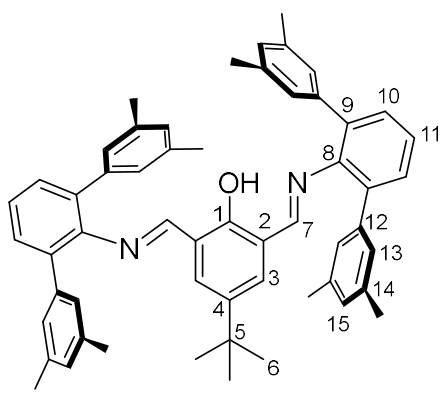

${ }^{1} \mathbf{H}$ NMR $\left(400 \mathrm{MHz}, \mathrm{CDCl}_{3}, 300 \mathrm{~K}\right): \delta(\mathrm{ppm})=12.51(\mathrm{~s}, 1 \mathrm{H}, \mathrm{OH}), 8.11(\mathrm{~s}, 2 \mathrm{H}, \mathrm{H}-7), 7.39-7.32(\mathrm{~m}, 4 \mathrm{H}, \mathrm{H}-10)$, $7.30-7.20$ (m, 2H, H-11), 7.02 (s, 8H, H-13), 6.82 (s, 4H, H-15), 2.21 (s, 24H, Ar-C $\underline{H}_{3}$ ), 1.16 (s, 9H, H-6).

H-3 is not visible due to dynamic processes of the bis(imino)phenols.

${ }^{13} \mathbf{C}\left\{{ }^{1} \mathbf{H}\right\}$ NMR (101 MHz, CDCl, $\left.300 \mathrm{~K}\right): \delta(\mathrm{ppm})=166.3(\mathrm{C}-1), 159.1$ (C-2), 140.8 (C-8), 139.8 (C-4), 137.5 (C-14), 134.4 (C-9), 132.2 (C-12), 129.9 (C-10), 128.3 (C-15), 128.1 (C-13), 124.9 (C-11), 34.0 (C-5), 31.3 (C-6), $21.4\left(\mathrm{CH}_{3}\right)$.

The atoms C-3 and C-7 are not visible due to dynamic processes of the bis(imino)phenols. 


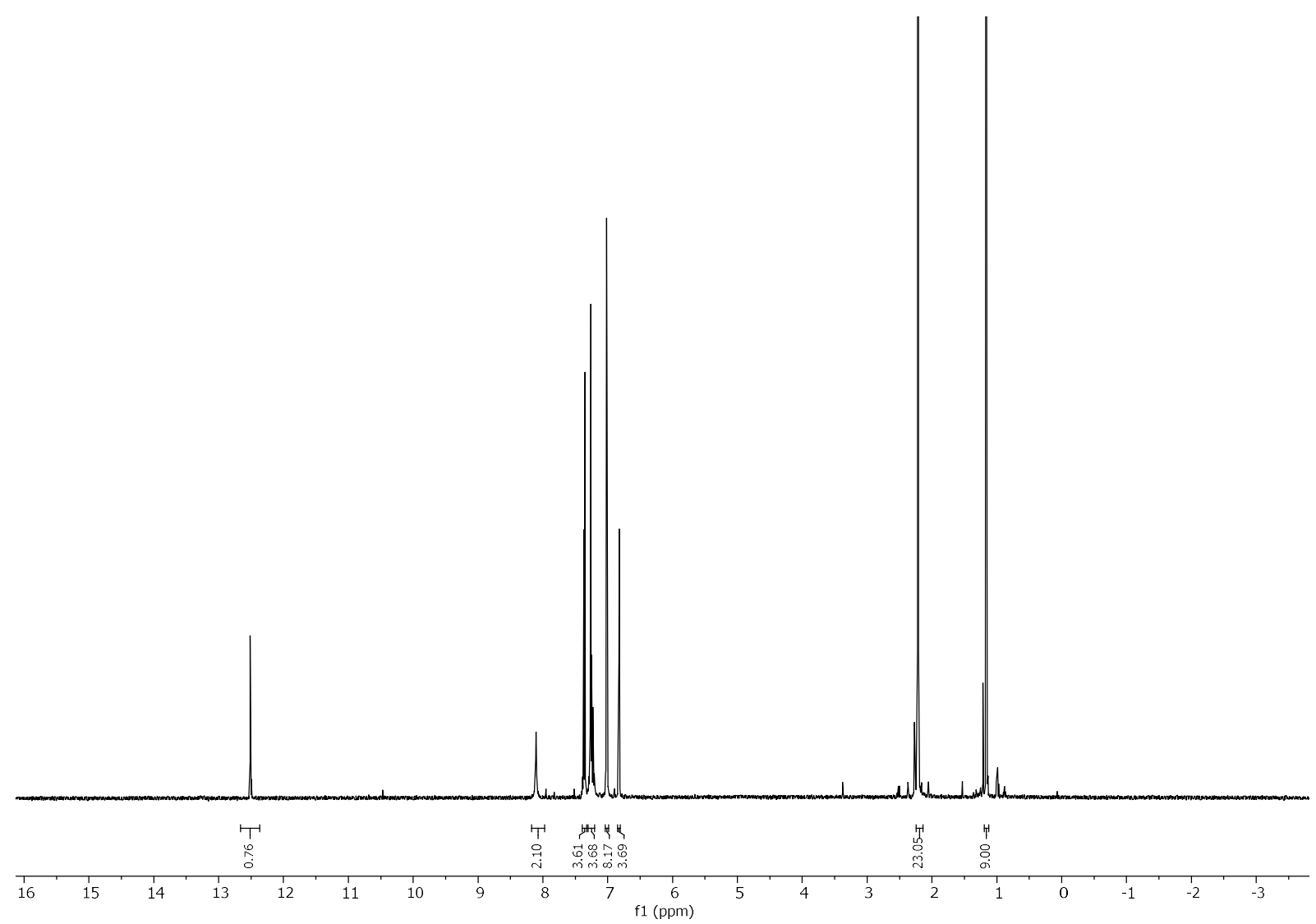

Figure S4: ${ }^{1} \mathrm{H}$ NMR spectrum of $\mathbf{1}^{\mathrm{Me}}$ in $\mathrm{CDCl}_{3}$ at $300 \mathrm{~K}$.

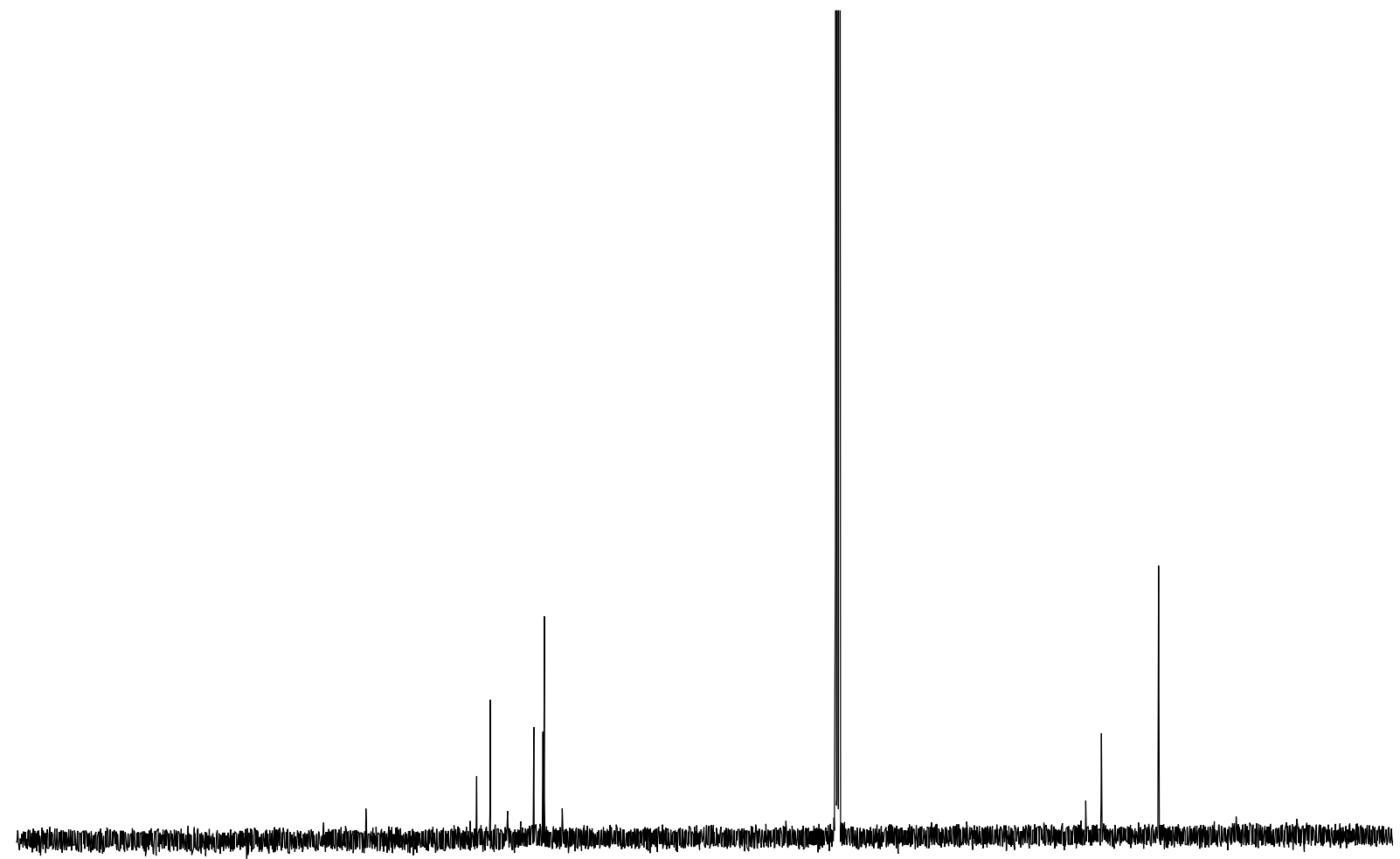

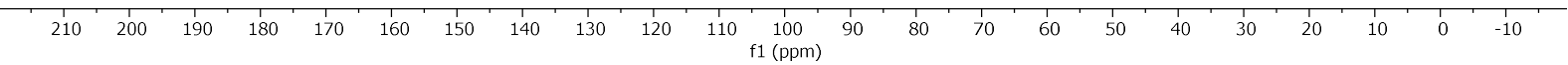

Figure $\mathrm{S} 5:{ }^{13} \mathrm{C}_{\left\{{ }^{1} \mathrm{H}\right\}} \mathrm{NMR}$ spectrum of $\mathbf{1}^{\mathrm{Me}}$ in $\mathrm{CDCl}_{3}$ at $300 \mathrm{~K}$. 


\subsubsection{Synthesis of Nickel Complexes}

\subsubsection{Synthesis $\mathbf{3}^{\mathrm{CH} 3}$}

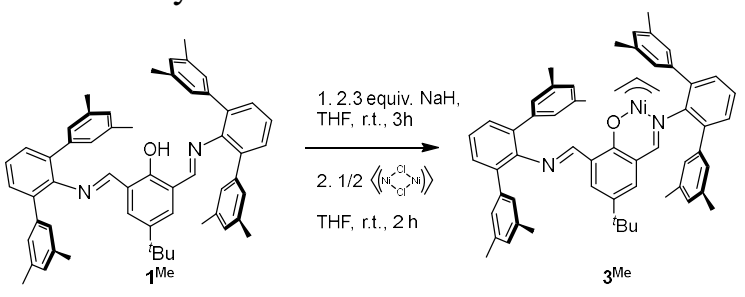

Scheme S3: Synthesis of $3^{\text {Me }}$.

A solution of ligand $\mathbf{1}^{\mathrm{Me}}(200 \mathrm{mg}, 0.27 \mathrm{mmol}, 2.0$ equiv.) in THF (10 mL) was added slowly to solid NaH (15.1 $\mathrm{mg}, 0.63 \mathrm{mmol}, 2.3$ equiv.). The solution, which slowly turned from yellow to orange, was stirred for $3 \mathrm{~h}$ at room temperature. The reaction mixture was filtered over a syringe filter and added to allylnickel chloride dimer (46.8 $\mathrm{mg}, 0.14 \mathrm{mmol}, 1.0$ equiv.) in THF ( $5 \mathrm{~mL}$ ). After stirring for $4 \mathrm{~h}$ at room temperature, the suspension was filtered over a syringe filter and the solvent was removed. The residue was dissolved in benzene, frozen by cooling in a liquid nitrogen bath, and all volatiles removed by sublimation. $3^{\mathbf{M e}}$ was obtained as a yellow-orange solid (221 $\mathrm{mg}$, $253 \mu \mathrm{mol}, 93 \%)$.

This complex was not active in ethylene polymerization experiments.

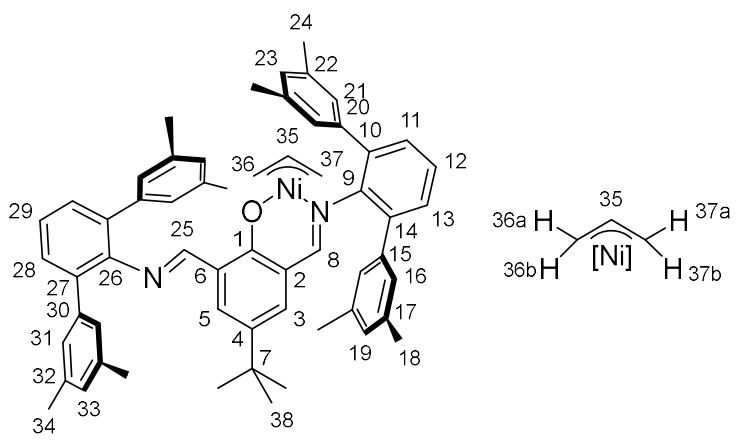

${ }^{1} \mathbf{H}$ NMR $\left(400 \mathrm{MHz}, \mathrm{C}_{6} \mathrm{D}_{6}, 300 \mathrm{~K}\right): \delta(\mathrm{ppm})=8.85(\mathrm{~s}, 1 \mathrm{H}, \mathrm{H}-25), 8.26\left(\mathrm{~d},{ }^{4} J_{\mathrm{HH}}=2.7 \mathrm{~Hz}, 1 \mathrm{H}, \mathrm{H}-3\right), 7.53(\mathrm{~s}, 1 \mathrm{H}$, $\mathrm{H}-8), 7.45\left(\mathrm{~d},{ }^{3} J_{\mathrm{HH}}=7.6 \mathrm{~Hz}, 2 \mathrm{H}, \mathrm{H}-28\right), 7.36\left(\mathrm{dd},{ }^{3} J_{\mathrm{HH}}=7.7 \mathrm{~Hz},{ }^{4} J_{\mathrm{HH}}=1.5 \mathrm{~Hz}, 1 \mathrm{H}, \mathrm{H}-13\right), 7.31\left(\mathrm{dd},{ }^{3} J_{\mathrm{HH}}=7.7 \mathrm{~Hz}\right.$, $\left.{ }^{4} J_{\mathrm{HH}}=1.5 \mathrm{~Hz}, 1 \mathrm{H}, \mathrm{H}-11\right), 7.26(\mathrm{~s}, 4 \mathrm{H}, \mathrm{H}-31), 7.24$ (s, 2H, H-16), $7.15-7.09$ (m, 2H, H-29 and 12), 6.98 (s, 2H, H-21), 6.77 (s, 2H, H-19 and 23), $6.75\left(\mathrm{~d},{ }^{4} J_{\mathrm{HH}}=2.7 \mathrm{~Hz}, 1 \mathrm{H}, \mathrm{H}-5\right), 6.74(\mathrm{~s}, 2 \mathrm{H}, \mathrm{H}-33), 5.15-5.27\left(\mathrm{tt},{ }^{3} J_{H H}=13.3\right.$, $\left.{ }^{3} J_{\mathrm{HH}}=6.8 \mathrm{~Hz}, 1 \mathrm{H}, \mathrm{H}-35\right), 2.63\left(\mathrm{dd},{ }^{3} J_{\mathrm{HH}}=6.8 \mathrm{~Hz},{ }^{4} J_{\mathrm{HH}}=2.2 \mathrm{~Hz}, 1 \mathrm{H}, \mathrm{H}-37 \mathrm{a}\right), 2.16(\mathrm{~s}, 12 \mathrm{H}, \mathrm{H}-34), 2.11(\mathrm{~s}, 6 \mathrm{H}$, H-18), 2.09 (s, 6H, H-24), 2.09* (d, $\left.{ }^{3} J_{\mathrm{HH}}=13.3 \mathrm{~Hz}, 1 \mathrm{H}, \mathrm{H}-37 \mathrm{~b}\right), 1.64\left(\mathrm{dd},{ }^{3} J_{\mathrm{HH}}=6.8 \mathrm{~Hz},{ }^{4} J_{\mathrm{HH}}=2.2 \mathrm{~Hz}, 1 \mathrm{H}, \mathrm{H}-36 \mathrm{a}\right)$, $1.35\left(\mathrm{~d},{ }^{3} J_{\mathrm{HH}}=13.3 \mathrm{~Hz} 1 \mathrm{H}, \mathrm{H}-36 \mathrm{~b}\right), 1.09$ (s, 9H, H-38).

*overlapped by methyl signals H-18 and H-24.

${ }^{13} \mathbf{C}\left\{{ }^{1} \mathbf{H}\right\}$ NMR $\left(101 \mathrm{MHz}, \mathrm{C}_{6} \mathrm{D}_{6}, 300 \mathrm{~K}\right): \delta(\mathrm{ppm})=167.5(\mathrm{C}-8), 164.9(\mathrm{C}-1), 162.0(\mathrm{C}-25), 152.3(\mathrm{C}-9), 150.9(\mathrm{C}-$ 26), 141.1 (C-30), 139.3 (C-15), 139.1 (C-20), 137.7 (C-22), 137.5 (C-17), 135.8 (C-32), 135.5 (C-14), 134.9 (C10), 134.3 (C-27), 133.1 (C-5), 130.9 (C-3), 129.9 (C-29), 129.9 (C-13), 129.5 (C-11), 128.9 (C-33), 128.6 (C-31), 128.5 (C-16), 128.4 (C-2), 128.2 (C-21), 127.9 (C-19 and 23), 127.7 (C-6), 125.7 (C-12), 123.7 (C-28), 119.3 (C4), 111.3 (C-35), 59.9 (C-37), 49.1 (C-36), 31.1 (C-7), 21.3 (C-34), 21.1 (C-18 and 24). 


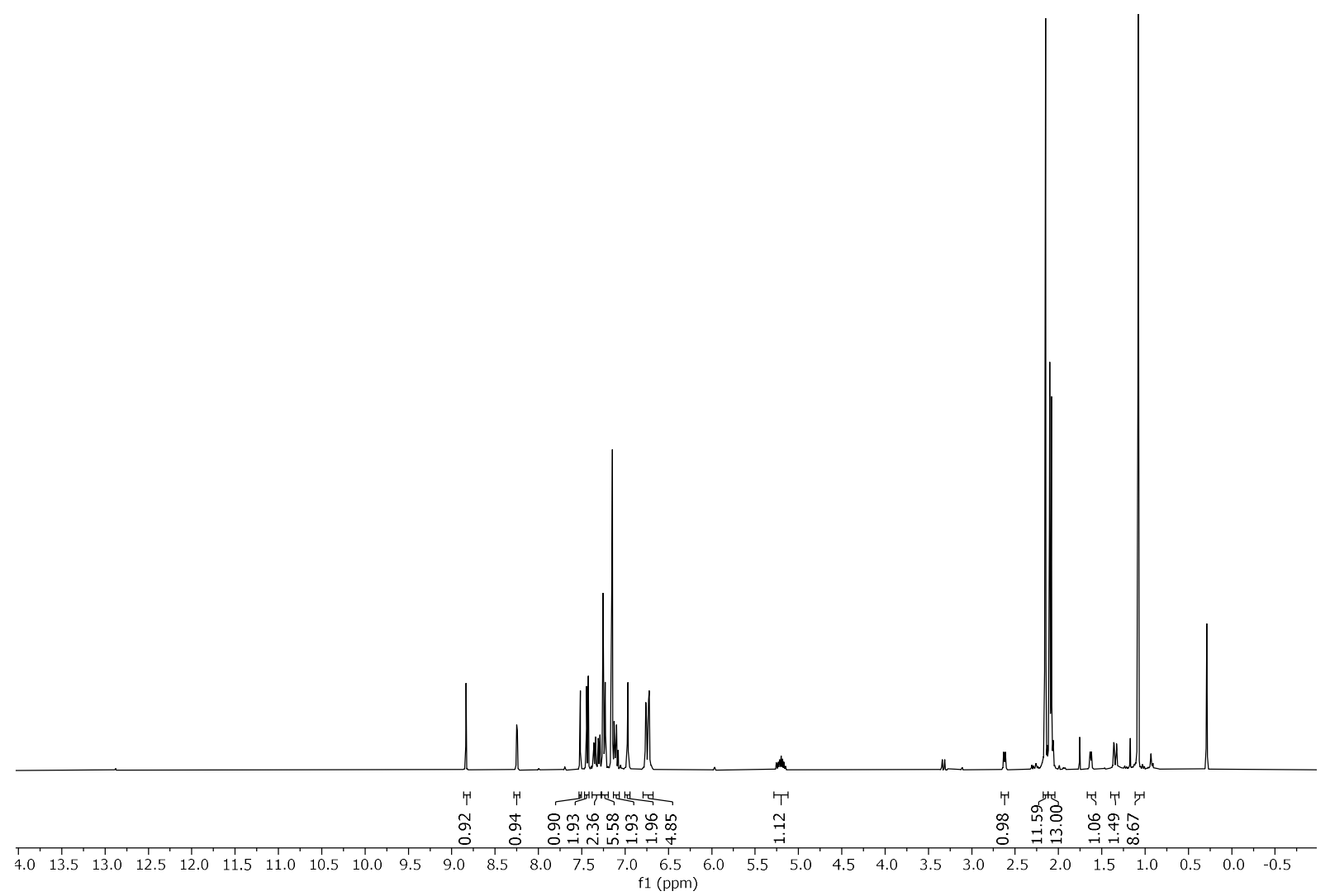

Figure S6: ${ }^{1} H$ NMR spectrum of $3^{\text {Me }}$ in $C_{6} D_{6}$ at $300 \mathrm{~K}$.
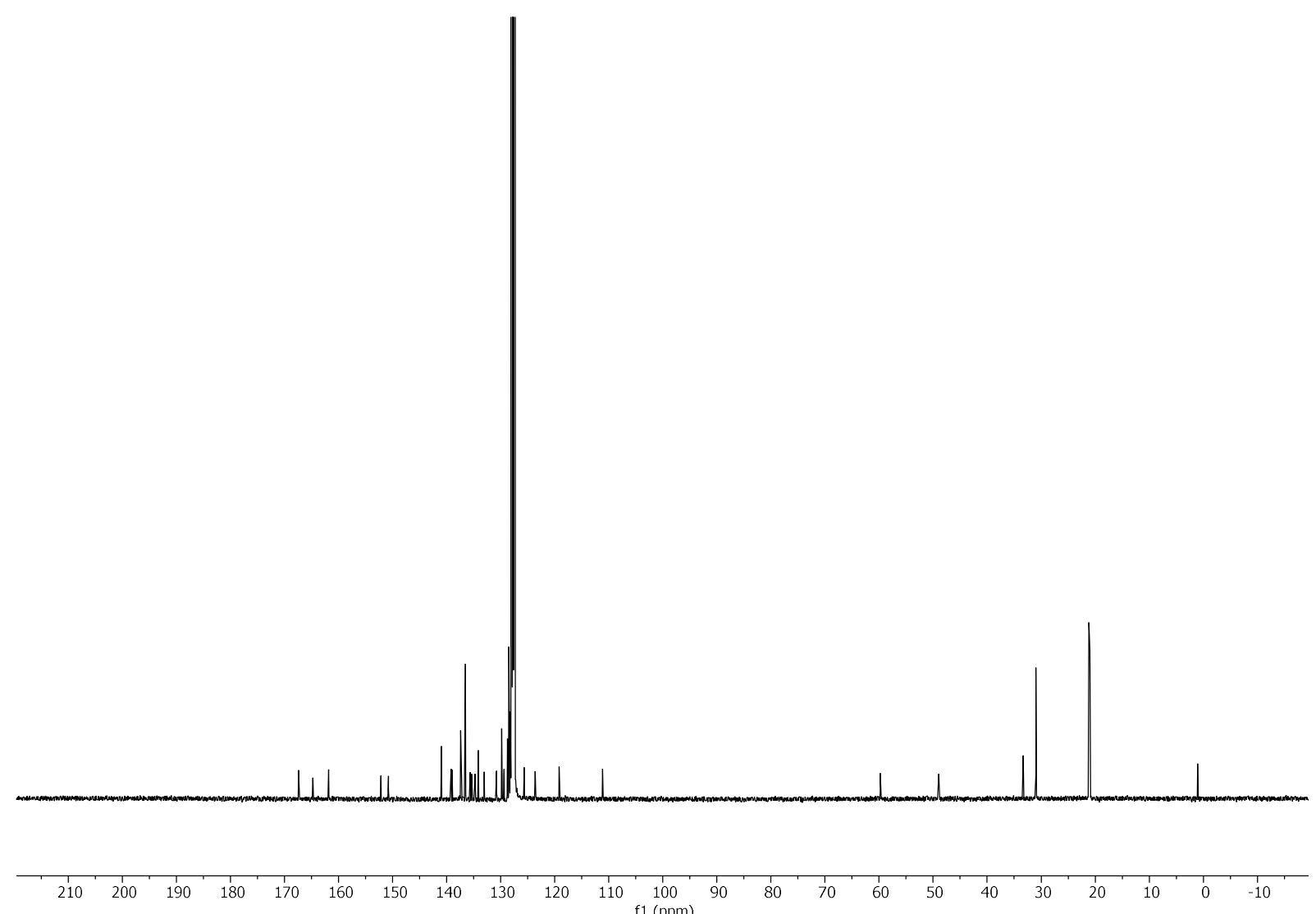

Figure $S 7:{ }^{13} C_{\left\{{ }^{1} H\right\}}$ NMR spectrum of $3^{M e}$ in $C_{6} D_{6}$ at $300 \mathrm{~K}$. 


\subsubsection{Synthesis of $\mathbf{3}^{\mathrm{CF} 3}$}
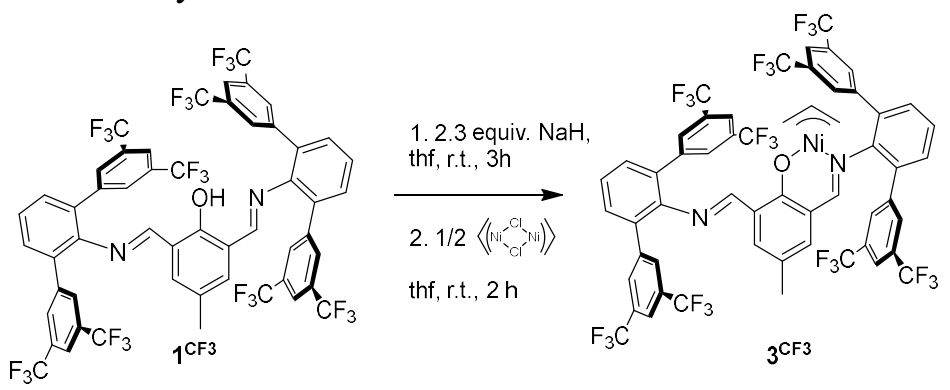

Scheme S4: Synthesis of $3^{\text {CF }}$.

A solution of $1^{\text {CF3 }}(200 \mathrm{mg}, 0.17 \mathrm{mmol})$ in THF $(10 \mathrm{~mL})$ was added slowly to $\mathrm{NaH}(9.5 \mathrm{mg}, 0.39 \mathrm{mmol}, 2.3$ equiv.). The yellow solution immediately turned orange-red and was stirred for 7 hours at room temperature. The mixture was filtered over a syringe filter and stored at $-30{ }^{\circ} \mathrm{C}$ in a glovebox. $84 \mu \mathrm{mol}$ of the crude product was added to allyl nickel chloride dimer ( $11.4 \mathrm{mg}, 42 \mu \mathrm{mol}, 1.0$ equiv.) and stirred for $5 \mathrm{~h}$ at room temperature. After filtration, the solvent was removed. The yellow solid was washed with pentane to give $\mathbf{3}^{\text {CF3 }}$ (88.9 $\mathrm{mg}, 70.4 \mu \mathrm{mol}, 83 \%$ ).

This complex was not active in ethylene polymerization experiments.

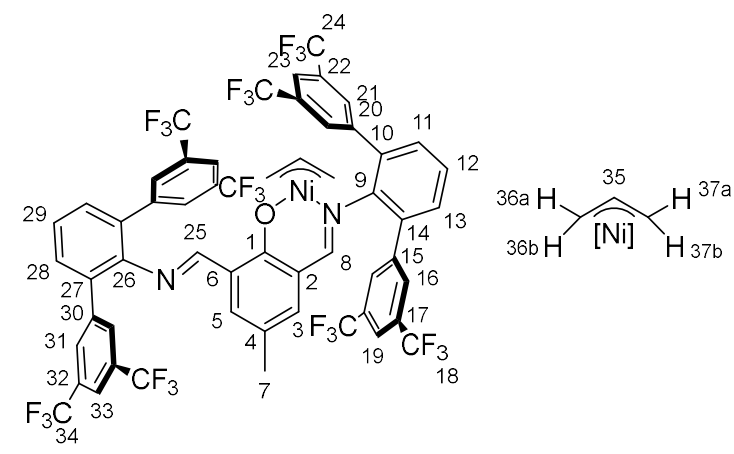

${ }^{1} \mathbf{H}$ NMR $\left(400 \mathrm{MHz}, \mathrm{C}_{6} \mathrm{D}_{6}, 300 \mathrm{~K}\right): \delta(\mathrm{ppm})=8.37(\mathrm{~s}, 1 \mathrm{H}, \mathrm{H}-25), 7.92\left(\mathrm{~d},{ }^{4} J_{\mathrm{HH}}=2.5 \mathrm{~Hz}, 1 \mathrm{H}, \mathrm{H}-5\right), 7.83(\mathrm{~s}, 4 \mathrm{H}$, H-31), 7.82 (s, 2H, H-16), 7.70 (s, 2H, H-33), 7.67 (s, 2H, H-19 and 23), 7.55 (s, 2H, H-21), 6.89 - 6.98 (m, $3 \mathrm{H}$, H-28 and H-29) $6.88\left(\mathrm{dd},{ }^{3} J_{\mathrm{HH}}=7.6 \mathrm{~Hz},{ }^{3} J_{\mathrm{HH}}=7.6 \mathrm{~Hz}, 1 \mathrm{H}, \mathrm{H}-12\right), 6.83\left(\mathrm{dd},{ }^{3} J_{\mathrm{HH}}=7.6 \mathrm{~Hz},{ }^{4} J_{\mathrm{HH}}=1.7 \mathrm{~Hz}, 1 \mathrm{H}\right.$, H-13), $6.77\left(\mathrm{dd},{ }^{3} J_{\mathrm{HH}}=7.6 \mathrm{~Hz},{ }^{4} J_{\mathrm{HH}}=1.7 \mathrm{~Hz}, 1 \mathrm{H}, \mathrm{H}-11\right), 6.77(\mathrm{~s}, 1 \mathrm{H}, \mathrm{H}-8), 6.29\left(\mathrm{~d},{ }^{4} J_{\mathrm{HH}}=2.5 \mathrm{~Hz}, 1 \mathrm{H}, \mathrm{H}-3\right)$, $5.11-5.23(\mathrm{~m}, 1 \mathrm{H}, \mathrm{H}-35), 2.55\left(\mathrm{dd},{ }^{3} J_{\mathrm{HH}}=7.9 \mathrm{~Hz},{ }^{4} J_{\mathrm{HH}}=2.5 \mathrm{~Hz}, 1 \mathrm{H}, \mathrm{H}-37 \mathrm{a}\right), 2.1\left(\mathrm{~d},{ }^{3} J_{\mathrm{HH}}=13.4 \mathrm{~Hz}, 1 \mathrm{H}, \mathrm{H}-37 \mathrm{~b}\right)$, $1.88(\mathrm{~s}, 3 \mathrm{H}, \mathrm{H}-7), 1.11\left(\mathrm{dd},{ }^{3} J_{\mathrm{HH}}=6.6 \mathrm{~Hz},{ }^{4} J_{\mathrm{HH}}=1.8 \mathrm{~Hz}, 1 \mathrm{H}, \mathrm{H}-36 \mathrm{a}\right), 0.91\left(\mathrm{~d},{ }^{3} J_{\mathrm{HH}}=12.7 \mathrm{~Hz} 1 \mathrm{H}, \mathrm{H}-36 \mathrm{~b}\right)$.

${ }^{13} \mathbf{C}\left\{{ }^{1} \mathbf{H}\right\}$ NMR $\left(101 \mathrm{MHz}, \mathrm{C}_{6} \mathrm{D}_{6}, 300 \mathrm{~K}\right): \delta(\mathrm{ppm})=166.7(\mathrm{C}-8), 165.1(\mathrm{C}-1), 163.4(\mathrm{C}-25), 151.8(\mathrm{C}-9), 150.5(\mathrm{C}-$ 26), 142.0 (C-30), 140.5 (C-15), 140.4 (C-20), 138.1 (C-3), 135.2 (C-5), 132.0 (C-10), 131.8 (q, ${ }^{2} J_{\mathrm{CF}}=33 \mathrm{~Hz}, \mathrm{C}-$ 32), 131.7 (q, $\left.{ }^{2} J_{\mathrm{CF}}=33 \mathrm{~Hz}, \mathrm{C}-17\right), 131.6(\mathrm{C}-14), 131.2$ (q, $\left.{ }^{2} J_{\mathrm{CF}}=33 \mathrm{~Hz}, \mathrm{C}-22\right), 130.8$ (C-27), 130.7 (C-28), 130.6 (C-11), 130.4 (C-31), 130.3 (C-13), 130.2 (C-16), 129.9 (C-21), 126.5 (C-2), 126.3 (C-12), 124.8 (C-29), 123.8 (C-6), $123.8\left(\mathrm{q},{ }^{1} J_{\mathrm{CF}}=273 \mathrm{~Hz}, \mathrm{C}-33\right), 123.4$ (q, ${ }^{1} J_{\mathrm{CF}}=272 \mathrm{~Hz}, \mathrm{C}-18$ and 24), 121.1 (C-19 and 23), 120.3 (C-33), 118.7 (C-4), 111.9 (C-35), 61.8 (C-37), 48.5 (C-36), 19.3 (C-7).

${ }^{19} \mathbf{F}\left\{{ }^{1} \mathbf{H}\right\}$ NMR $\left(376 \mathrm{MHz}, \mathrm{C}_{6} \mathrm{D}_{6}, 300 \mathrm{~K}\right): \delta(\mathrm{ppm})=-62.6\left(\mathrm{~s}, \mathrm{CF}_{3}, 12 \mathrm{~F}\right.$, terphenyl bound to non-coordinated $N),-62.8\left(\mathrm{~s}, \mathrm{CF}_{3}, 6 \mathrm{~F}\right.$, terphenyl on coordinated $\left.N\right),-62.9\left(\mathrm{~s}, \mathrm{CF}_{3}, 6 \mathrm{~F}\right.$, terphenyl on coordinated $\left.N\right)$. 


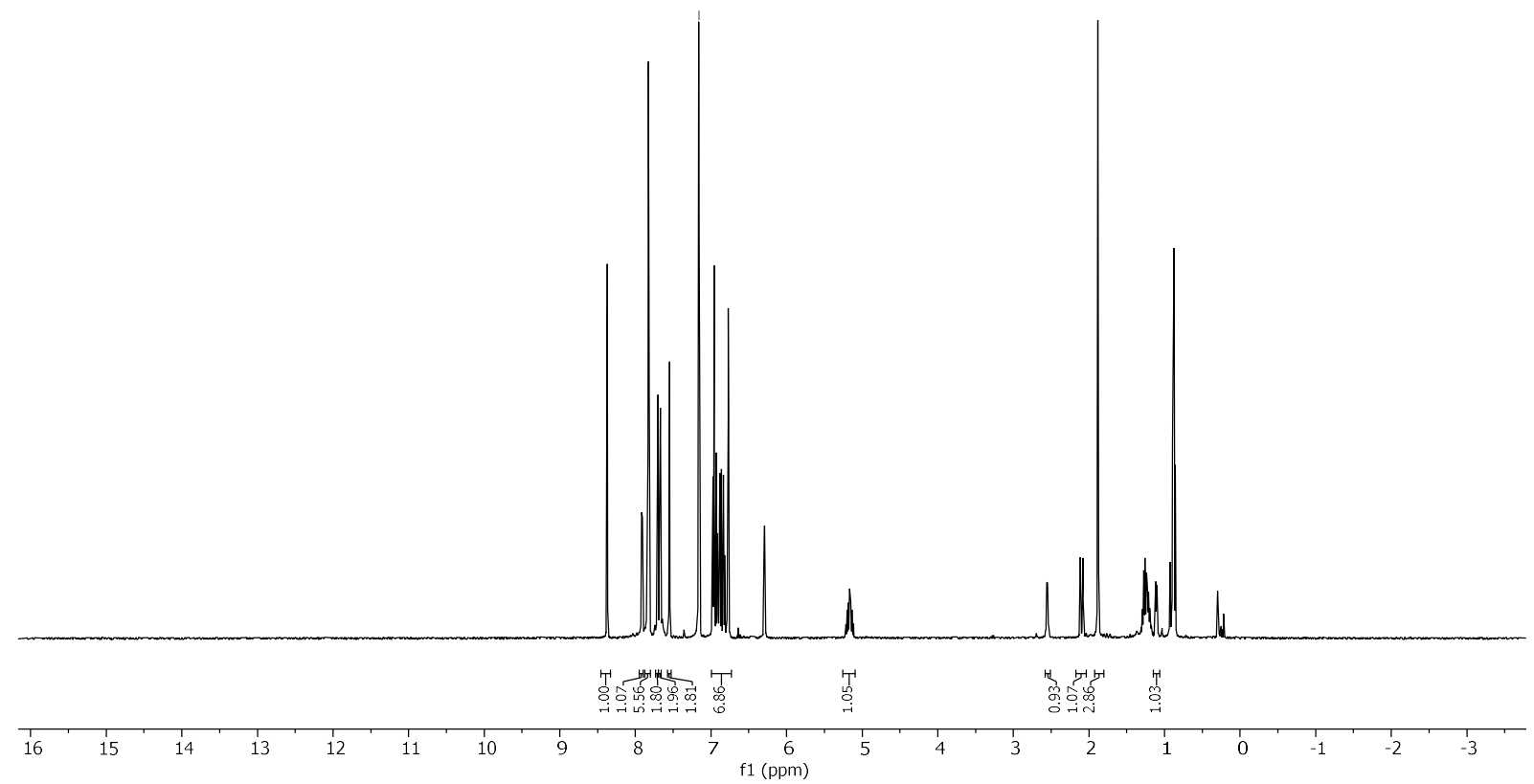

Figure S8: ${ }^{1} \mathrm{H}$ NMR spectrum of $3^{\mathrm{CF} 3}$ in $\mathrm{C}_{6} \mathrm{D}_{6}$ at $300 \mathrm{~K}$.

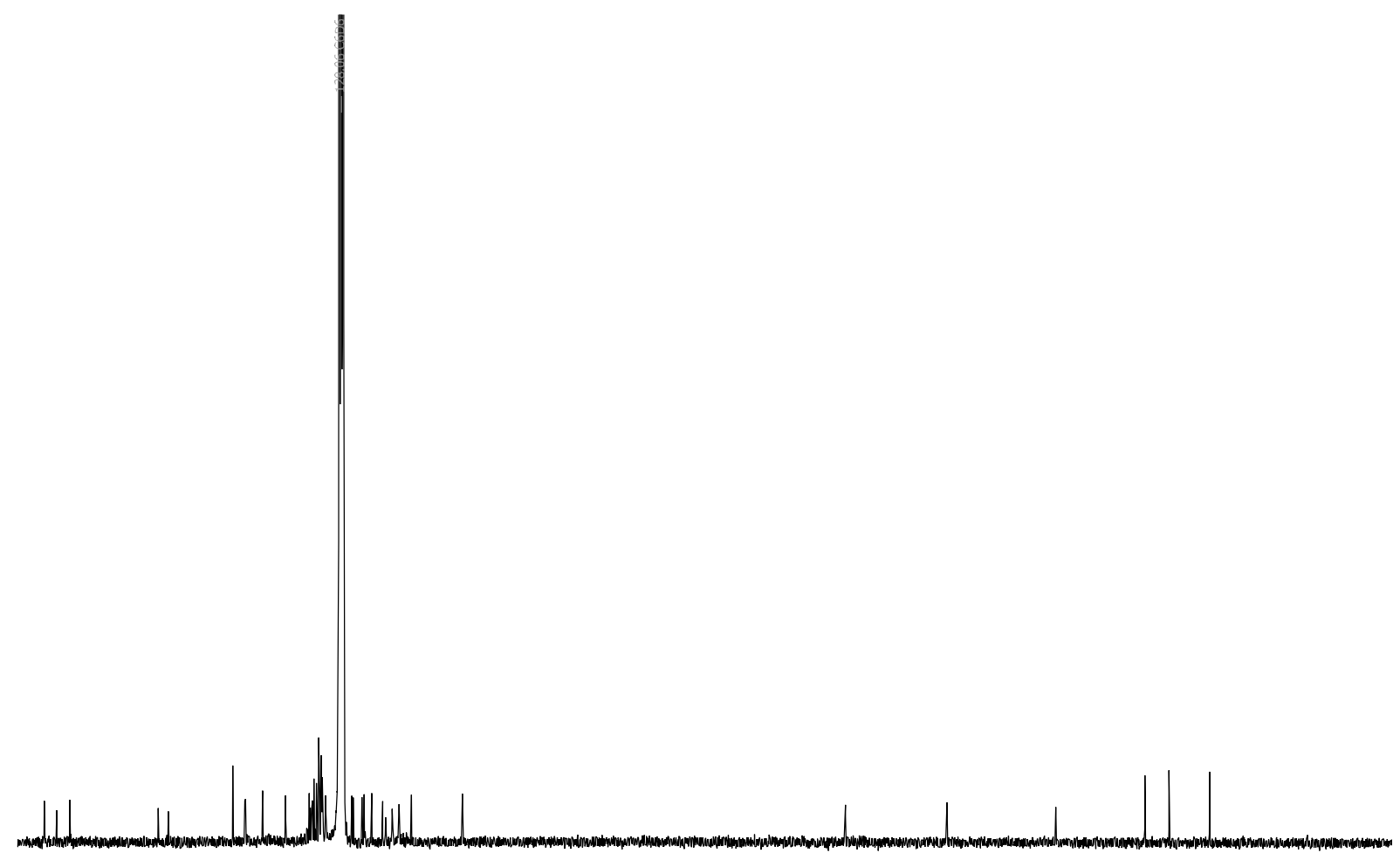

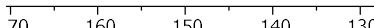

80

$70 \quad 60 \quad 50$

Figure S9: $\left.{ }^{13} C_{\{}^{\{l} H\right\}$ NMR spectrum of $3^{C F 3}$ in $C_{6} D_{6}$ at $300 \mathrm{~K}$. 


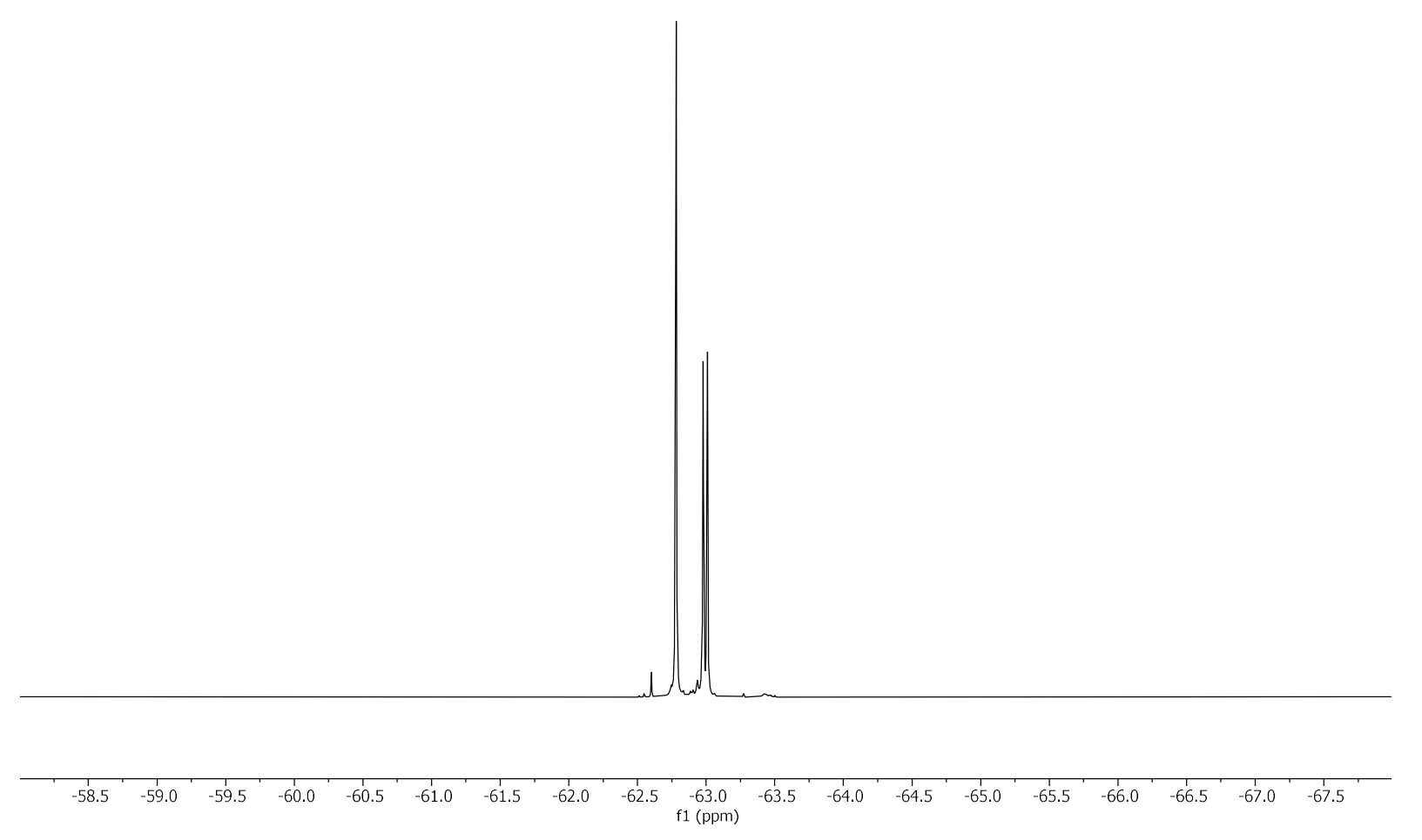

Figure S10: ${ }^{19} \mathrm{~F}\left\{{ }^{1} \mathrm{H}\right\}$ NMR spectrum of $3^{\mathrm{CF} 3}$ in $\mathrm{C}_{6} \mathrm{D}_{6}$ at $300 \mathrm{~K}$.

\subsubsection{Synthesis of $\mathbf{2}^{\text {CF3 }}$}
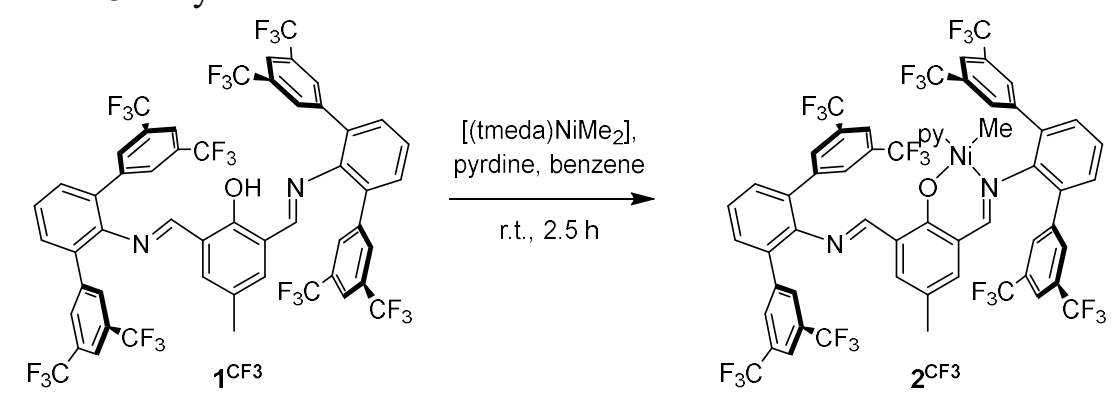

Scheme S5: Synthesis of $2^{\text {CF3 }}$.

$\mathbf{1}^{\text {CF3 }}$ (116.3 mg, $100 \mu \mathrm{mol}, 1.0$ equiv.) and [(tmeda)NiMe 2 ( $22.4 \mathrm{mg}, 110 \mu \mathrm{mol}, 1.1$. equiv.) were dissolved in $5 \mathrm{~mL}$ of benzene. Pyridine (160 mg, $2.0 \mathrm{mmol}, 20$ equiv.) was added, and the mixture was stirred at room temperature for $2.5 \mathrm{~h}$. The reaction mixture was centrifuged, the solution frozen by cooling in a liquid nitrogen bath, and all volatiles removed by sublimation. The crude product was washed with pentane $(2 \times 3 \mathrm{~mL})$. The product was obtained as a red solid (126 mg, $96 \mu \mathrm{mol}, 96 \%$ ).

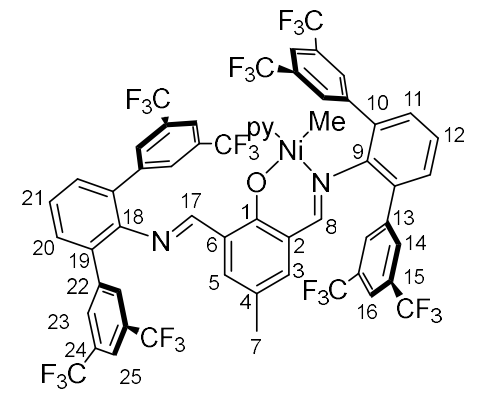


${ }^{1} \mathbf{H}$ NMR (400 MHz, $\left.\mathrm{C}_{6} \mathrm{D}_{6}, 300 \mathrm{~K}\right): \delta(\mathrm{ppm})=8.13-8.05(\mathrm{~m}, 4 \mathrm{H}, \mathrm{H}-23), 7.95\left(\mathrm{~d},{ }^{3} J_{\mathrm{HH}}=4.9 \mathrm{~Hz}, 2 \mathrm{H}, o-p y\right), 7.91$ (s, 1H, H-17), 7.89 (d, $\left.{ }^{4} J_{\mathrm{HH}}=2.5 \mathrm{~Hz}, 1 \mathrm{H}, \mathrm{H}-5\right), 7.81$ (s, 2H, H-25), 7.74 (s, 4H, H-14), 7.71 (s, 2H, H-16), $6.95-$ $6.83\left(\mathrm{~m}, 6 \mathrm{H}, \mathrm{H}-11, \mathrm{H}-12, \mathrm{H}-20\right.$ and H-21), $6.62(\mathrm{~s}, 1 \mathrm{H}, \mathrm{H}-8), 6.38\left(\mathrm{t},{ }^{3} J_{\mathrm{HH}}=7.7 \mathrm{~Hz}, 1 \mathrm{H}, p\right.$-py), $6.24\left(\mathrm{~d},{ }^{4} J_{\mathrm{HH}}=2.5\right.$ $\mathrm{Hz}, 1 \mathrm{H}, \mathrm{H}-3), 6.14$ (t, $\left.{ }^{3} J_{\mathrm{HH}}=7.1 \mathrm{~Hz}, 2 \mathrm{H}, m-\mathrm{py}\right), 1.91$ (s, 3H, H-7), -1.04 (s, 3H, Ni-C $\left.\underline{H}_{3}\right)$.

${ }^{13} \mathbf{C}\left\{{ }^{1} \mathbf{H}\right\}$ NMR (101 MHz, C6 $\left.\mathrm{D}_{6}, 300 \mathrm{~K}\right): \delta(\mathrm{ppm})=167.7$ (C-8), 165.6 (C-1), $162.3(\mathrm{C}-17), 151.2(\mathrm{C}-9), 150.7$ (C18), 150.4 (o-py), 142.0 (C-10 or -C19), 141.5 (C-10 or C-19), 138.5 (C-3), 136.2 (p-py), 135.1 (C-5), 133.3 (C22), $131.9\left(\mathrm{q},{ }^{2} J_{\mathrm{CF}}=33.3 \mathrm{~Hz}, \mathrm{C}-15\right.$ or C-24), $131.4\left(\mathrm{q},{ }^{2} J_{\mathrm{CF}}=33.0 \mathrm{~Hz}, \mathrm{C}-15\right.$ or C-24), 130.8 (C-11 of C-20), 130.7 - 130.6 (m, C-23), 130.6 (C-11 or C-20), $130.6-130.3$ (m, C-14), 126.8 (C-12), 124.7 (C-21), $124.5\left({ }^{1} J_{\mathrm{CF}}=273\right.$ $\left.\mathrm{Hz}, \underline{\mathrm{CF}}_{3}\right), 124,0\left({ }^{1} J_{\mathrm{CF}}=273 \mathrm{~Hz}, \underline{\mathrm{CF}}_{3}\right), 123.2$ (C-4 and C-6), 122.7 (m-py), $121.4-121.0$ (m, C-16), $120.9-120.5$ (m, C-25), $120.4(\mathrm{C}-2), 19.6(\mathrm{C}-7),-7.5\left(\mathrm{Ni}-\underline{\mathrm{C}} \mathrm{H}_{3}\right)$.

${ }^{19} \mathbf{F}\left\{{ }^{1} \mathbf{H}\right\}$ NMR (376 MHz, $\left.\mathrm{C}_{6} \mathrm{D}_{6}, 300 \mathrm{~K}\right): \delta(\mathrm{ppm})=-62.7\left(\mathrm{CF}_{3}\right.$, terphenyl bound to the non-coordinated $\left.N\right),-62.9$ $\left(\mathrm{CF}_{3}\right.$, terphenyl bound to the coordinated $\left.N\right)$.

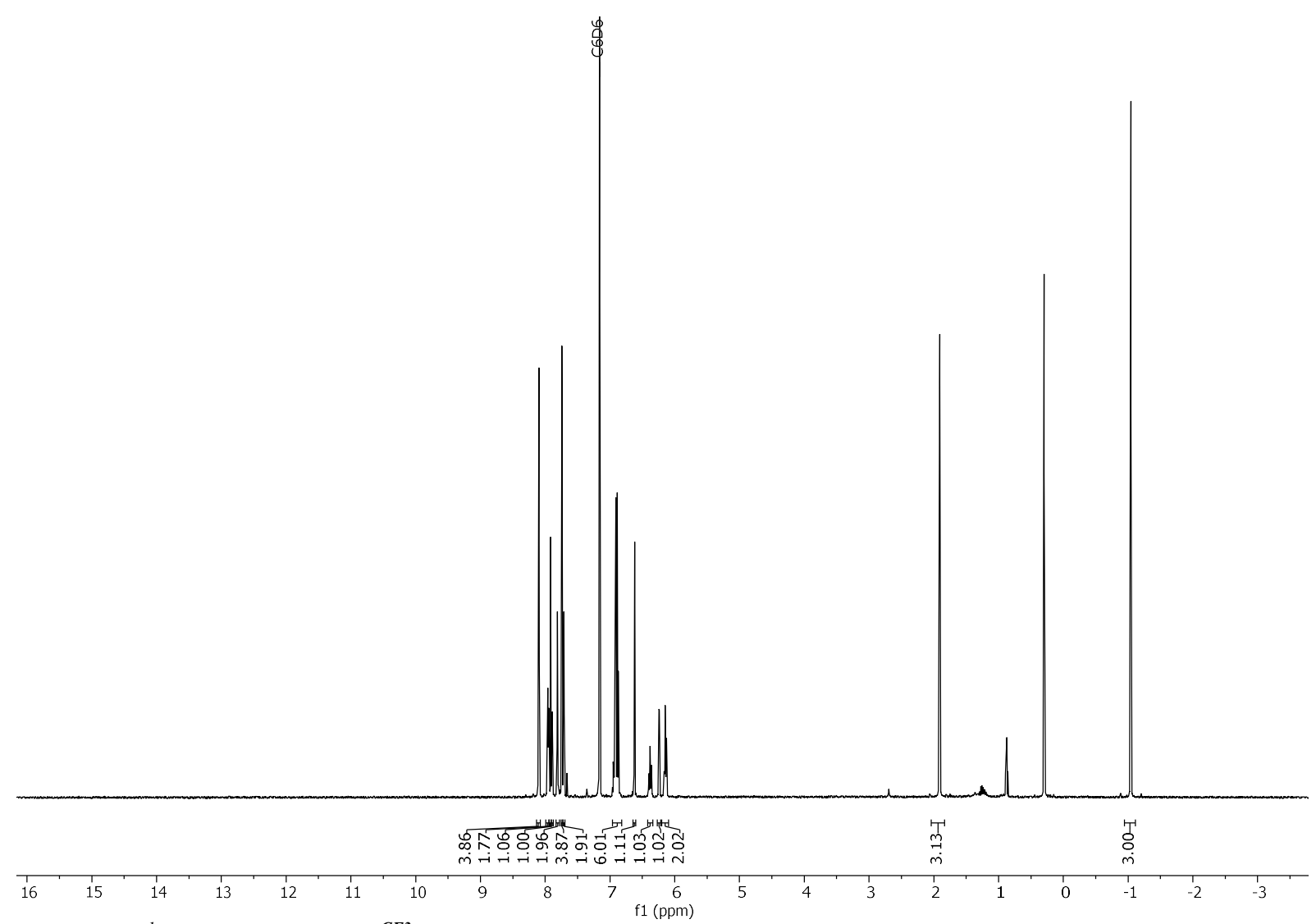

Figure S11: ${ }^{1} \mathrm{H}$ NMR spectrum of $2^{\mathrm{CF} 3}$ in $\mathrm{C}_{6} \mathrm{D}_{6}$ at $300 \mathrm{~K}$. 


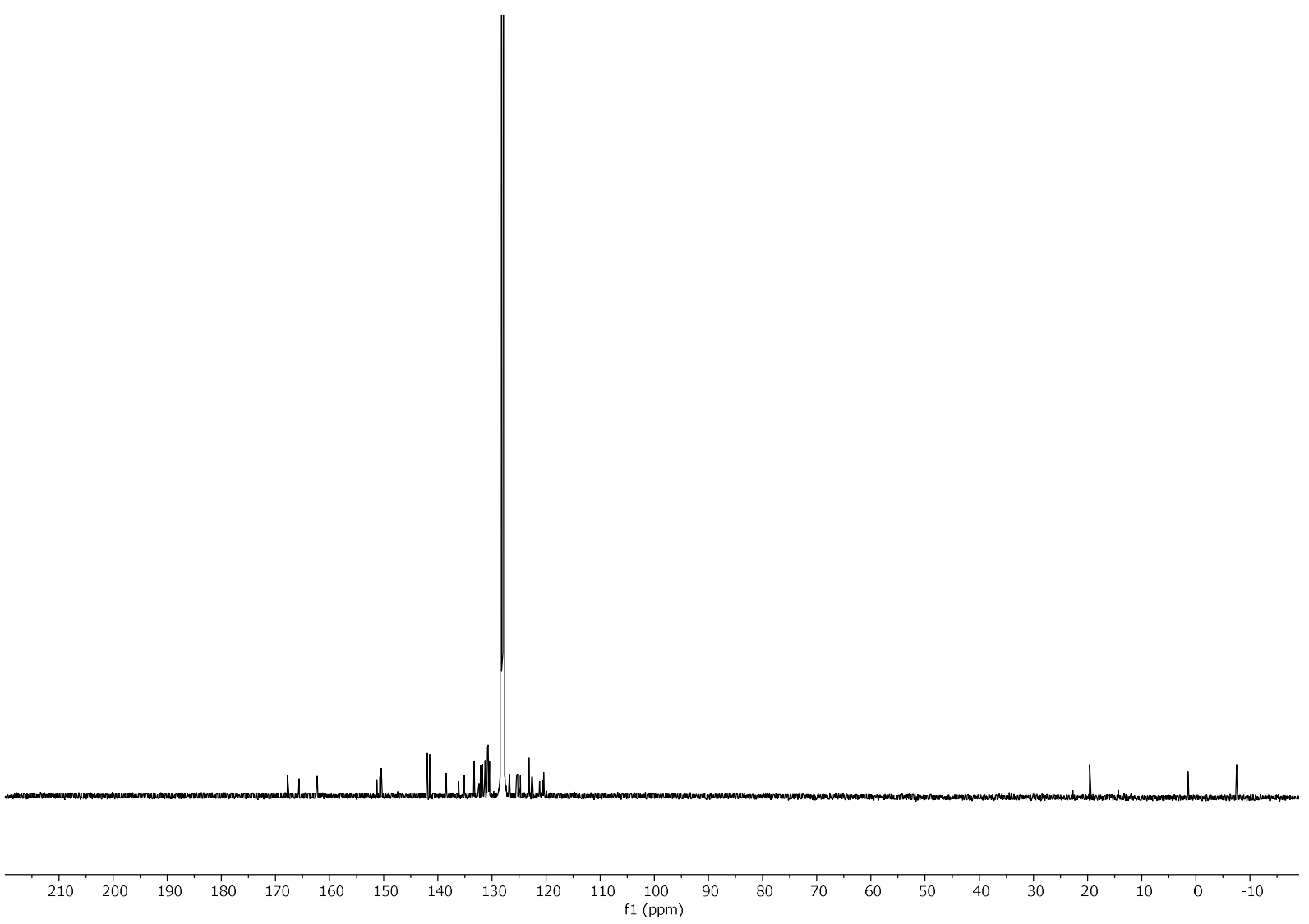

Figure S12: $\left.{ }^{13} C_{\{}{ }^{1} H\right\}$ NMR spectrum of $2^{C F 3}$ in $C_{6} D_{6}$ at $300 \mathrm{~K}$.

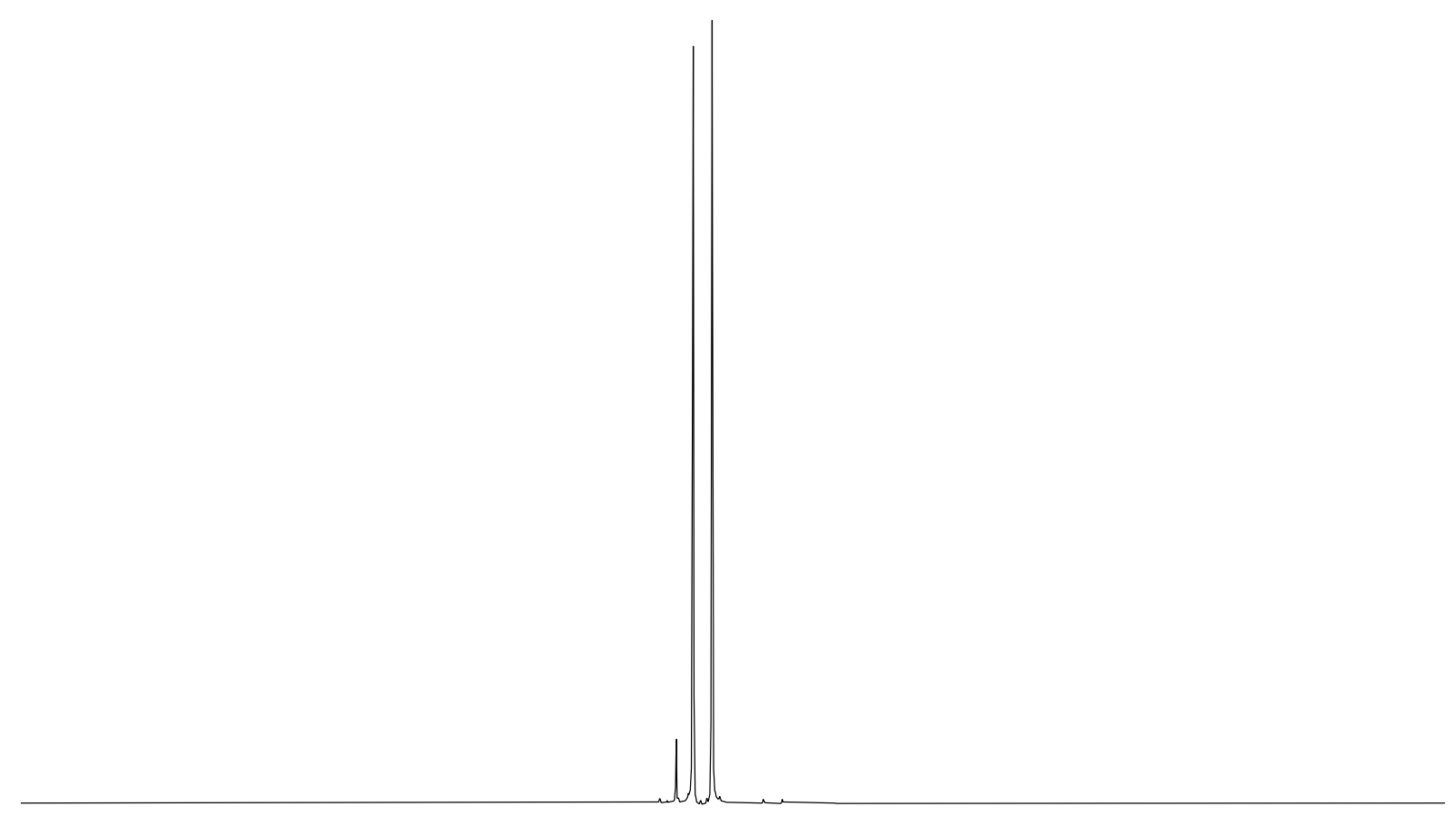

$\begin{array}{lllllllllllllllllllll}-58.5 & -59.0 & -59.5 & -60.0 & -60.5 & -61.0 & -61.5 & -62.0 & -62.5 & -63.0 & -63.5 & -64.0 & -64.5 & -65.0 & -65.5 & -66.0 & -66.5 & -67.0 & -67.5\end{array}$

Figure S13: ${ }^{19} \mathrm{~F}\left\{{ }^{1} \mathrm{H}\right\}$ NMR spectrum of $2^{\mathrm{CF3}}$ in $\mathrm{C}_{6} \mathrm{D}_{6}$ at $300 \mathrm{~K}$. 


\subsubsection{Synthesis of $\mathbf{2}^{\mathrm{Me}}$}
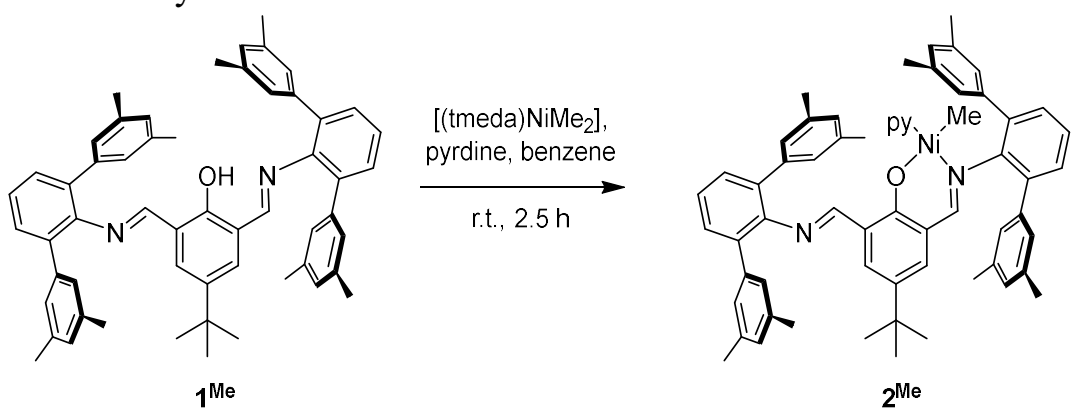

Scheme S6: Synthesis of $2^{\mathrm{Me}}$.

$\mathbf{1}^{\mathrm{Me}}$ (38.7 mg, $50 \mu \mathrm{mol}, 1.0$ equiv.) and [(tmeda)NiMe 2 ] (11.2 mg, $55 \mu \mathrm{mol}, 1.1$. equiv.) were dissolved in $4 \mathrm{~mL}$ of benzene. Pyridine ( $80 \mathrm{mg}, 1.0 \mathrm{mmol}, 20$ equiv.) was added, and the mixtures was stirred at room temperature for $1 \mathrm{~h}$. The reaction mixture was centrifuged, the solution frozen in a liquid nitrogen bath and the volatiles removed by sublimation. The product was obtained as red solid ( $42 \mathrm{mg}, 45 \mu \mathrm{mol}, 90 \%$ ).

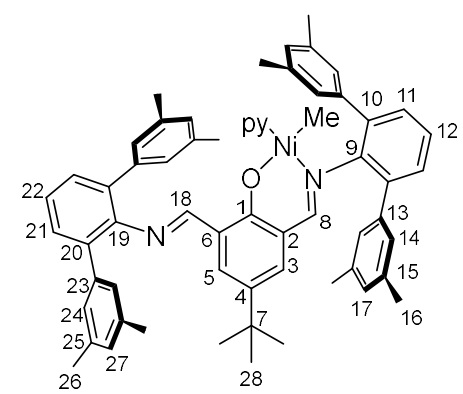

${ }^{1} \mathbf{H}$ NMR $\left(400 \mathrm{MHz}, \mathrm{C}_{6} \mathrm{D}_{6}, 300 \mathrm{~K}\right): \delta(\mathrm{ppm})=8.35(\mathrm{~s}, 1 \mathrm{H}, \mathrm{H}-8), 8.30\left(\mathrm{~d},{ }_{4}^{4} J_{\mathrm{HH}}=2.8 \mathrm{~Hz}, 1 \mathrm{H}, \mathrm{H}-5\right), 7.94\left(\mathrm{~d},{ }^{3} J_{\mathrm{HH}}=5.5\right.$ $\mathrm{Hz}, 2 \mathrm{H}, o$-py), $7.43-7.38$ (m, 8H, H-11, H-21, H-24), 7.19 (s, 4H, H-14), 7.10 (t, $\left.{ }^{3} J_{\mathrm{HH}}=7.6 \mathrm{~Hz}, 1 \mathrm{H}, \mathrm{H}-12\right), 6.88$ (s, 2H, H-27), $6.76\left(\mathrm{~d},{ }^{4} J_{\mathrm{HH}}=2.8 \mathrm{~Hz}, 1 \mathrm{H}, \mathrm{H}-3\right), 6.71(\mathrm{~s}, 2 \mathrm{H}, \mathrm{H}-17), 6.47$ (t, ${ }^{3} J_{\mathrm{HH}}=8.3 \mathrm{~Hz}, 1 \mathrm{H}, p$-py), $6.21\left(\mathrm{vt},{ }^{3} J_{\mathrm{HH}}\right.$ $=5.9 \mathrm{~Hz}, 2 \mathrm{H}, m-\mathrm{py}), 2.19(\mathrm{~s}, 12 \mathrm{H}, \mathrm{H}-16$ or H-26), $2.14(\mathrm{~s}, 12 \mathrm{H}, \mathrm{H}-16$ or H-26), 1.17 (s, 9H, H-28), -0.70 (s, 3H, $\left.\mathrm{Ni}-\underline{\mathrm{C}}_{3}\right)$.

H-22 is overlapped by the solvent residue signal.

${ }^{13} \mathbf{C}\left\{{ }^{1} \mathbf{H}\right\}$ NMR (101 MHz, C6 $\left.\mathrm{D}_{6}, 300 \mathrm{~K}\right): \delta(\mathrm{ppm})=168.5$ (C-18), 165.5 (C-1), 161.2 (C-8), 151.7 (C-9), 151.3 (o-py), 150.6 (C-19), 141.1 (C-10), 140.6 (C-20), 137.7 (C-23), 136.9 (C-13), 136.8 (C-25), 135.6 (p-py), 135.1 (C-4), 134.1 (C-15), 133.4 (C-5), 130.6 (C-3), 130.1 (C-21), 129.0 (C-11), 128.9 (C-27), 128.7 (C-24), 128.6 (C-14), 126.6 (C-2), 125.9 (C-22), 123.7 (C-12), 122.6 (m-py), 121.3 (C-6), 33.7 (C-7), 31.5 (C-29), 21.5 (C-16 or $\mathrm{C}-26), 21.4(\mathrm{C}-16$ or $\mathrm{C}-26),-8.03\left(\mathrm{Ni}^{-} \mathrm{CH}_{3}\right)$.

C-17 is overlapped by the solvent residue signal. 


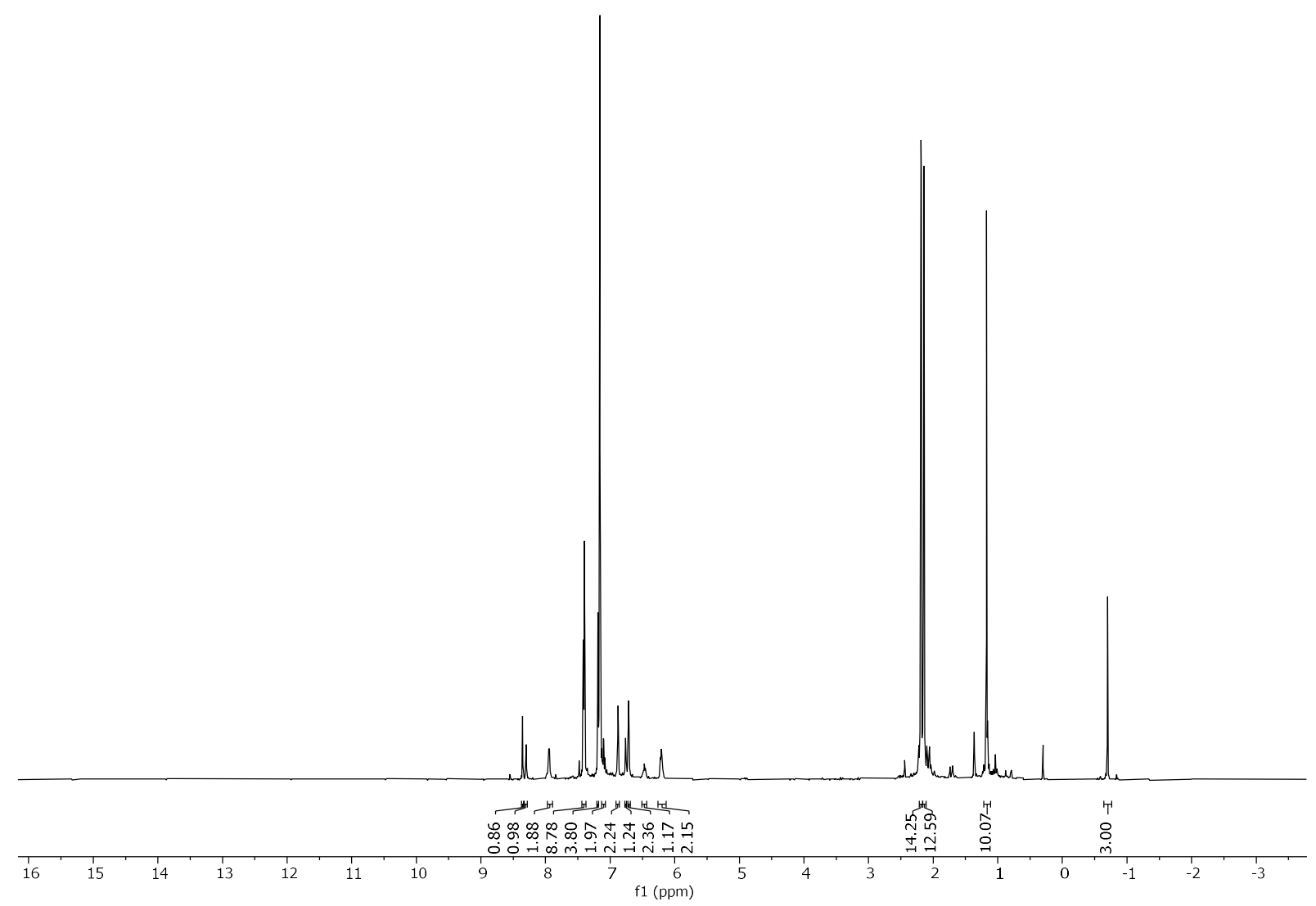

Figure S14: ${ }^{1} H$ NMR spectrum of $2^{M e}$ in $C_{6} D_{6}$ at $300 \mathrm{~K}$.

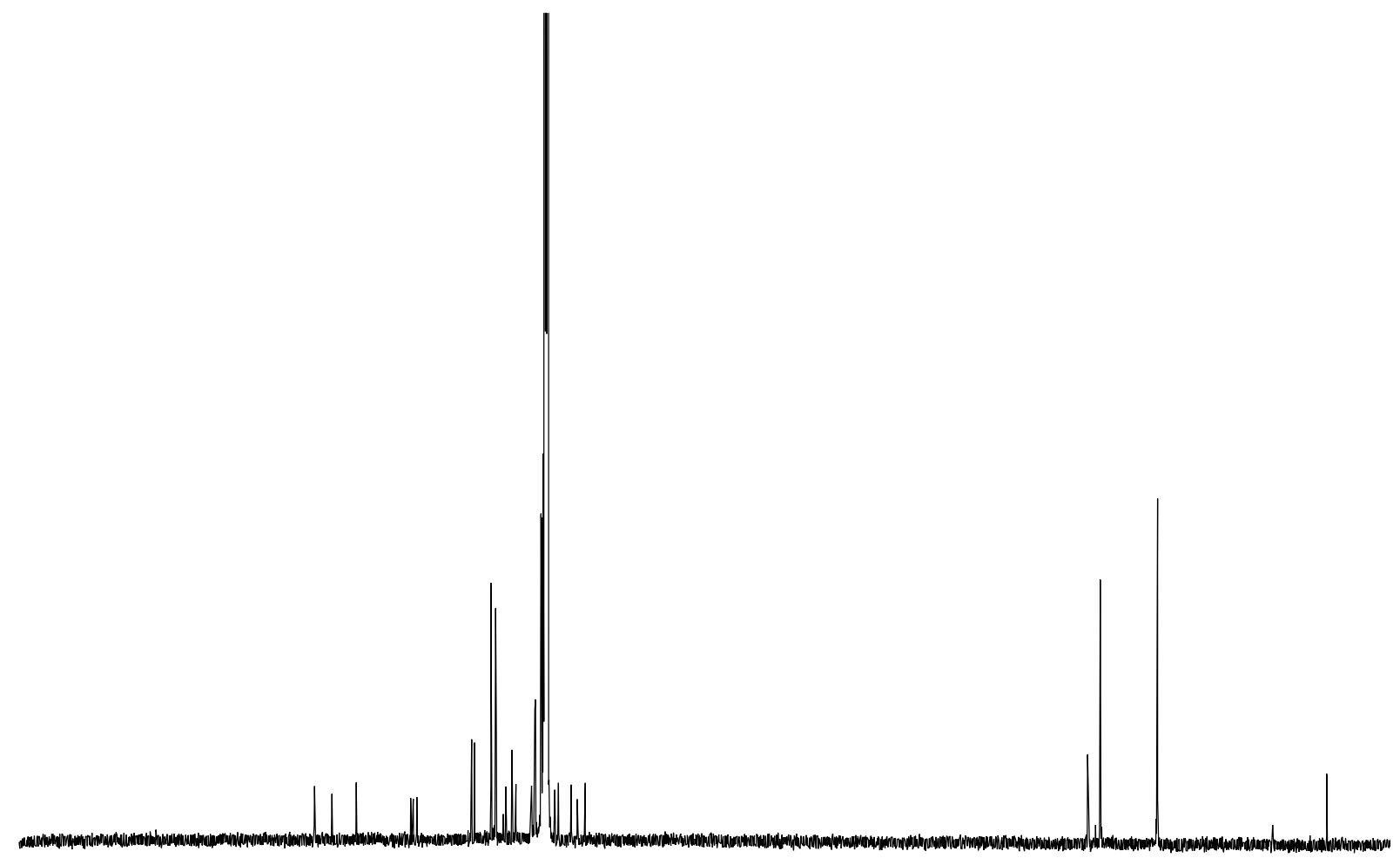

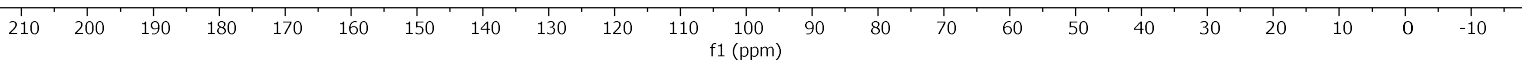

Figure S15: $\left.{ }^{13} \mathrm{C}_{\{1}{ }^{1} \mathrm{H}\right\}$ NMR spectrum of $2^{\mathrm{Me}}$ in $\mathrm{C}_{6} \mathrm{D}_{6}$ at $300 \mathrm{~K}$. 


\subsubsection{Protonation}

2.1.3.1 General Procedure for the Protonation of the Complexes

The neutral Ni(II) complex (100 $\mu$ mol, 1.0 equiv.) was dissolved in $\mathrm{Et}_{2} \mathrm{O}(10 \mathrm{~mL})$ and the red solution cooled to $-78^{\circ} \mathrm{C}$. $\left[\mathrm{H}\left(\mathrm{OEt}_{2}\right)_{2}\right]\left[\mathrm{BAr}_{4}{ }_{4}\right](100 \mu \mathrm{mol}, 1.0$ equiv. $)$ was dissolved in $\mathrm{Et}_{2} \mathrm{O}(1 \mathrm{~mL})$ and added to the complex slowly over one hour. The dark-red solution was allowed to warm up to room temperature and the solvent was removed.

2.1.3.2 Synthesis of $\mathbf{3}^{\mathrm{Me}}-\mathbf{H}^{+}$
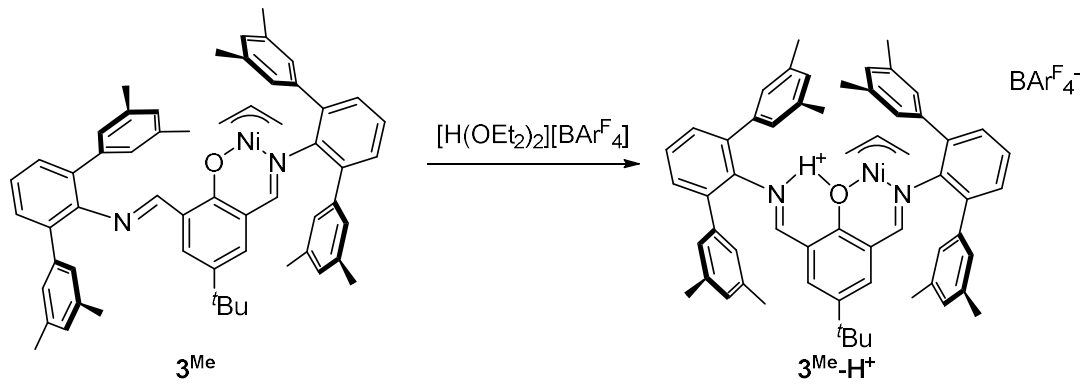

Scheme S7: Synthesis of $3^{M e}-H^{+}$.

$\mathbf{3}^{\mathrm{Me}}(8.0 \mu \mathrm{mol}, 7.0 \mathrm{mg})$ was dissolved in $5 \mathrm{~mL}$ of THF and cooled to $-78{ }^{\circ} \mathrm{C}$. $\left[\mathrm{H}\left(\mathrm{OEt}_{2}\right)_{2}\right]\left[\mathrm{BAr}_{4}^{\mathrm{F}}\right]$ was dissolved in $0.5 \mathrm{~mL}$ of $\mathrm{THF}$ and added to the complex. After warming to room temperature, the solvent was removed. As $\left[\mathrm{H}\left(\mathrm{OEt}_{2}\right)_{2}\right]\left[\mathrm{BAr}_{4}\right]$ is capable of initiating a cationic polymerization of THF, poly-THF is present in the product mixture.
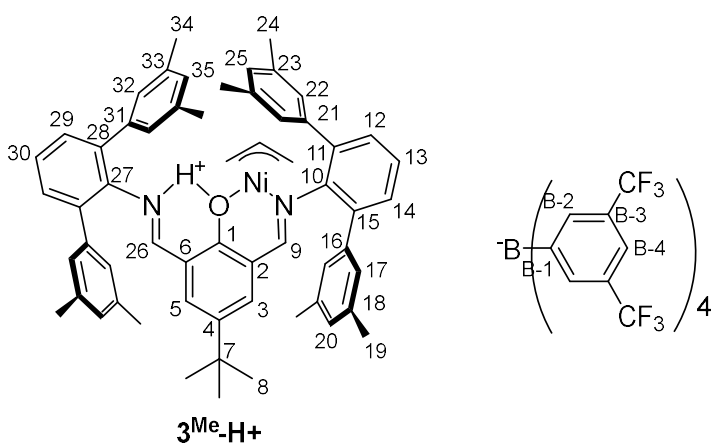

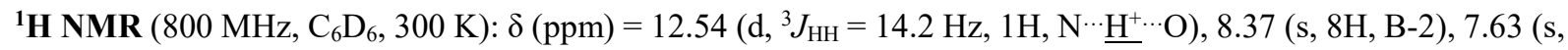
4H, B-4), $7.22\left(\mathrm{~d}, 1 \mathrm{H},{ }^{3} J_{\mathrm{HH}}=7.8 \mathrm{~Hz}, \mathrm{H}-14\right), 7.17$ (m, 1H, H-13), 7.10 (d, 2H, $\left.{ }^{3} J_{\mathrm{HH}}=7.6 \mathrm{~Hz}, \mathrm{H}-29\right), 7.7 .08(\mathrm{~d}, 1 \mathrm{H}$, $\left.{ }^{3} J_{\mathrm{HH}}=7.9 \mathrm{~Hz}, \mathrm{H}-12\right), 7.07$ (t, 1H, $\left.{ }^{3} J_{\mathrm{HH}}=7.6 \mathrm{~Hz}, \mathrm{H}-30\right), 7.16$ (s, 1H, H-9), 6.94 (s, 2H, H-17), 6.84 (s, 1H, H-5), $6.76\left(\mathrm{~d},{ }^{3} J_{\mathrm{HH}}=14.2 \mathrm{~Hz}, 1 \mathrm{H}, \mathrm{H}-26\right), 6.74(\mathrm{~s}, 1 \mathrm{H}, \mathrm{H}-25$ or H-20), 6,73 (s, $1 \mathrm{H}, \mathrm{H}-25$ or H-20) 6.70 (s, 2H, H-22),

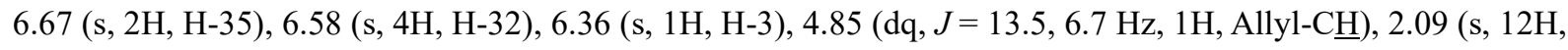
H-34), 1.97 (s, 12H, H-19 and H-24), 1.80 (m, 1H, Allyl-CH $\mathrm{CH}_{2}, 1.53\left(\mathrm{~m}, 1 \mathrm{H}\right.$, Allyl-C $\left.\underline{H}_{2}\right), 1.39\left(\mathrm{~d},{ }^{3} J_{\mathrm{HH}}=11.4 \mathrm{~Hz}\right.$ 1H, Allyl-C $\left.\underline{\mathrm{H}}_{2}\right), 1.21\left(\mathrm{~d},{ }^{3} \mathrm{~J}_{\mathrm{HH}}=13.5 \mathrm{~Hz}, 1 \mathrm{H}, \mathrm{Allyl}-\underline{\mathrm{C}}_{2}\right), 0.90(\mathrm{~s}, 9 \mathrm{H}, \mathrm{H}-8)$.

${ }^{19} \mathbf{F}\left\{{ }^{1} \mathbf{H}\right\}$ NMR $\left(376 \mathrm{MHz}, \mathrm{C}_{6} \mathrm{D}_{6}, 300 \mathrm{~K}\right): \delta(\mathrm{ppm})=-62.1 \mathrm{BAr}^{\mathrm{F}}{ }_{4}-\mathrm{CF}_{3}$.

${ }^{13} \mathbf{C}\left\{{ }^{1} \mathbf{H}\right\}$ NMR $\left(101 \mathrm{MHz}, \mathrm{C}_{6} \mathrm{D}_{6}, 300 \mathrm{~K}\right): \delta(\mathrm{ppm})=168.4(\mathrm{C}-9), 167.9(\mathrm{C}-26), 167.2(\mathrm{C}-1), 162.7$ (B-1), 162.5 (B1), 162.3 (B-1), 162.0 (B-1), 150.0 (C-10), 143.9 (C-5), 139.9 (C-4), 139.1 (C-33), 138.8 (C-11 or C-15), 138.5 (C-11 or C-15), 138.3 (C-3), 138.1 (C-16 and C-21), 137.8 (C-18 or C-23), 137.7 (C-18 or C23), 136.8 (C-28), 136.1 (C-27), 134.5 (B-2), 131.7 (C-31), 130.8 (C-29), 130.2 (C-12), 130.0 (C-14) 129.8 (C-13 and C-35), 129.1 (C-20 or C-25), 128.1 (C-17), 127.1 (C-32), 127.0 (C-20 or C-25), 126.1 (C-30), 124.3 (q, $\left.{ }^{1} J_{\mathrm{CF}}=274.0 \mathrm{~Hz}^{\mathrm{CF}} \mathrm{F}_{3}\right)$, 
121.39 (C-2), 115.7 (C-6), 117.7 (B-4) 114.1 (Allyl- $\underline{\mathrm{CH}}), 56.9$ (Allyl- $\left.\underline{\mathrm{C}} \mathrm{H}_{2}\right), 53.8$ (Allyl- $\underline{\mathrm{CH}}_{2}, 33.0$ (C-7), 29.9 (C-8), 20.8 (C-24 and C-19), 20.7 (C-34).

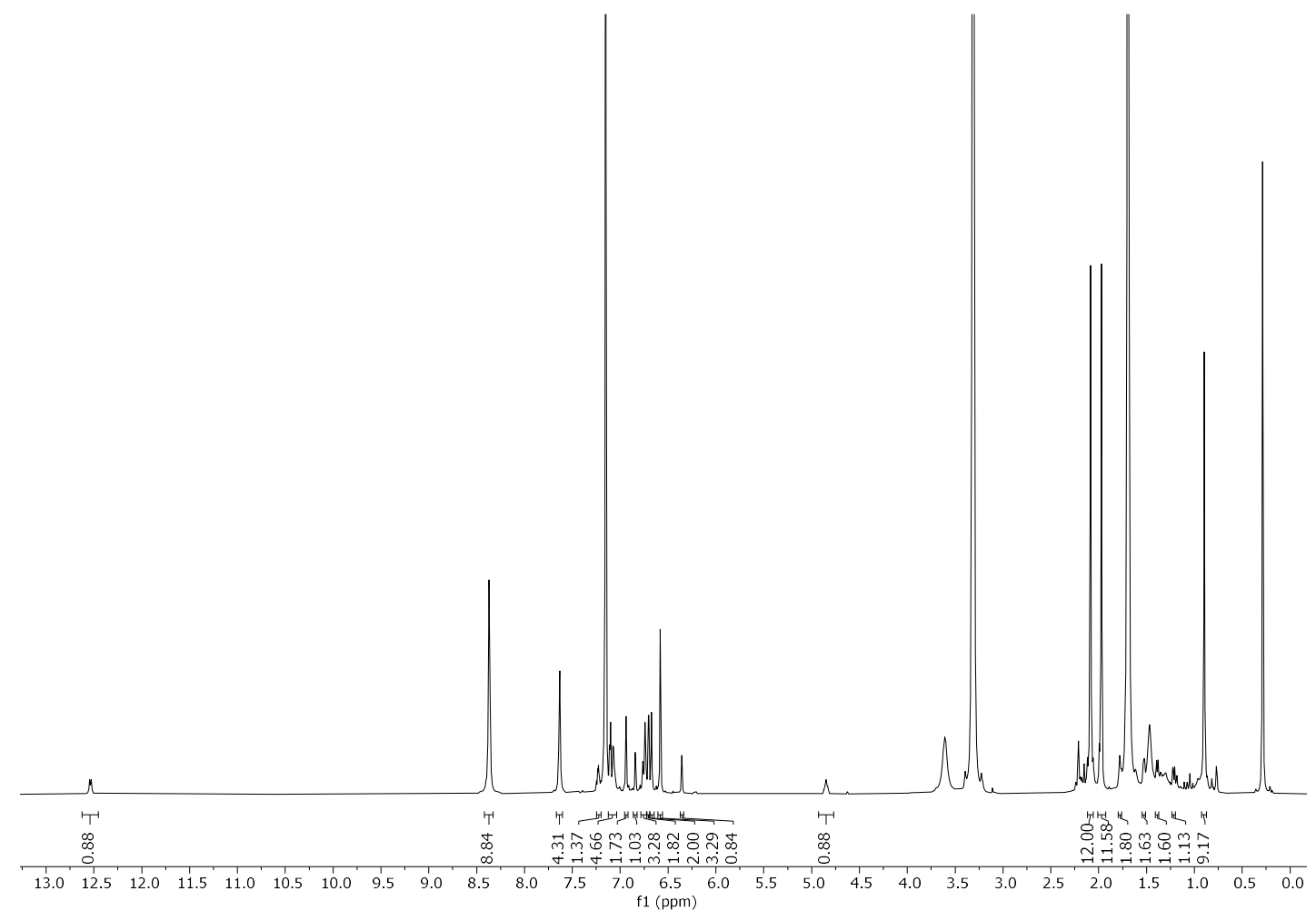

Figure S16: ${ }^{1} H$ NMR spectrum of $\mathbf{3}^{\mathrm{Me}}-\boldsymbol{H}^{+}$in $\mathrm{C}_{6} \mathrm{D}_{6}$ at $300 \mathrm{~K}$. 

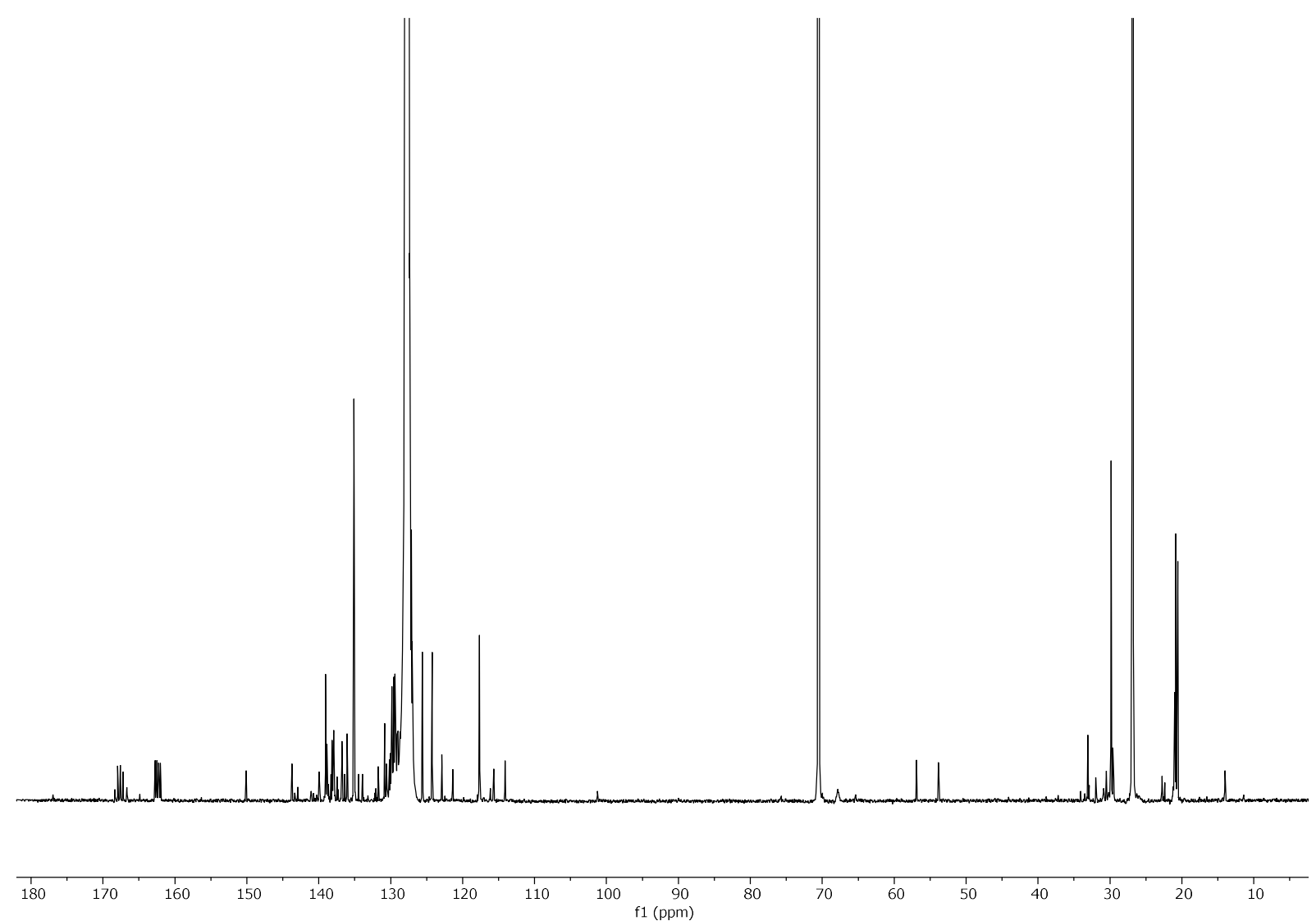

Figure S17: $\left.{ }^{13} C_{\{}^{\{1} H\right\}$ NMR spectrum of $3^{M e}{ }_{-} \boldsymbol{H}^{+}$in $C_{6} D_{6}$ at $300 \mathrm{~K}$.

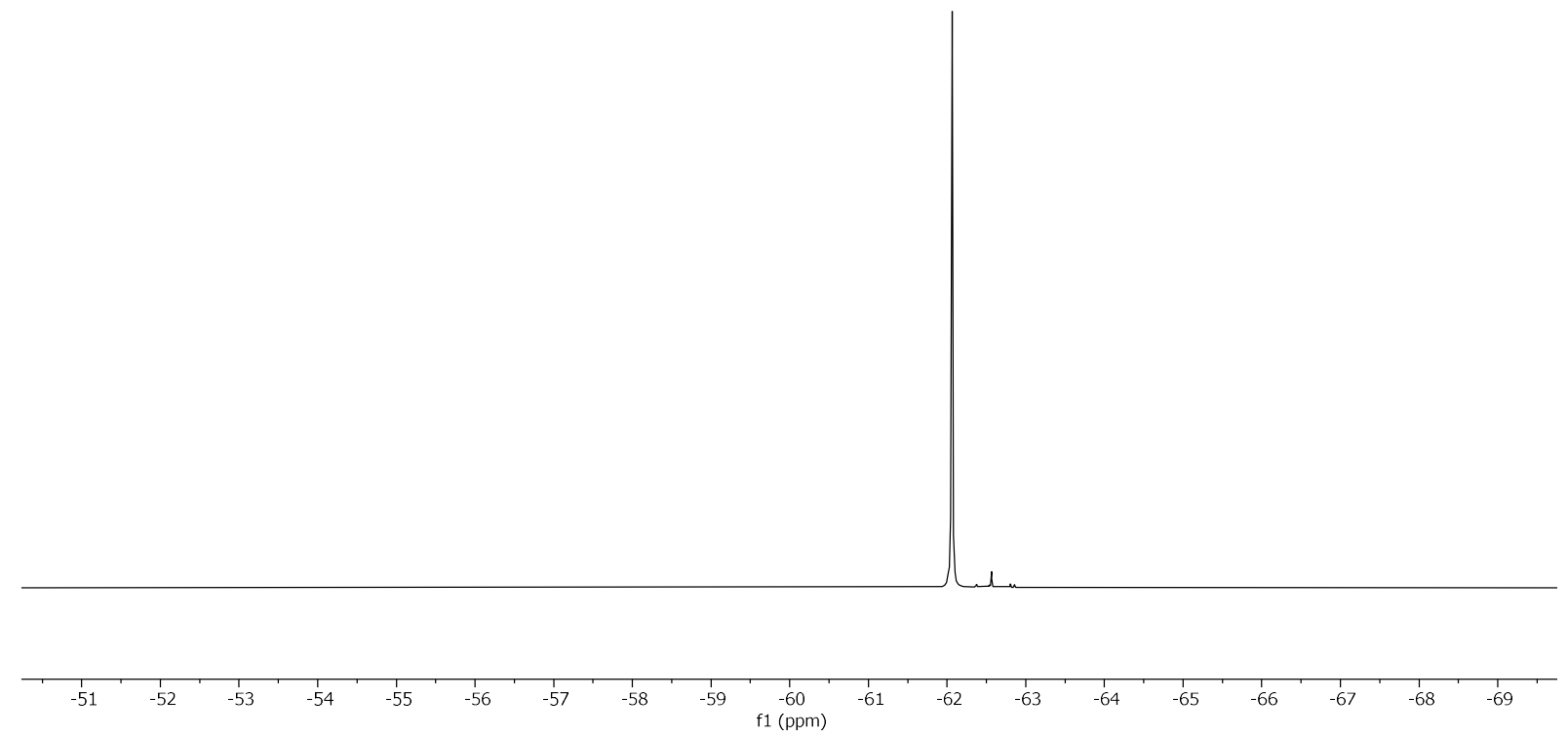

Figure S18: ${ }^{19} \mathrm{~F}\left\{{ }^{1} \mathrm{H}\right\}$ NMR spectrum of $3^{\mathrm{Me}}-\boldsymbol{H}^{+}$in $\mathrm{C}_{6} \mathrm{D}_{6}$ at $300 \mathrm{~K}$. 
Note that especially in the ${ }^{13} \mathrm{C}\left\{{ }^{1} \mathrm{H}\right\}$ NMR spectra additional impurities arose from decomposition in solution during the prolonged times required for acquisition (several days).

\subsubsection{Synthesis of $\mathbf{2}^{\mathbf{C F} 3}-\mathbf{H}^{+}$}
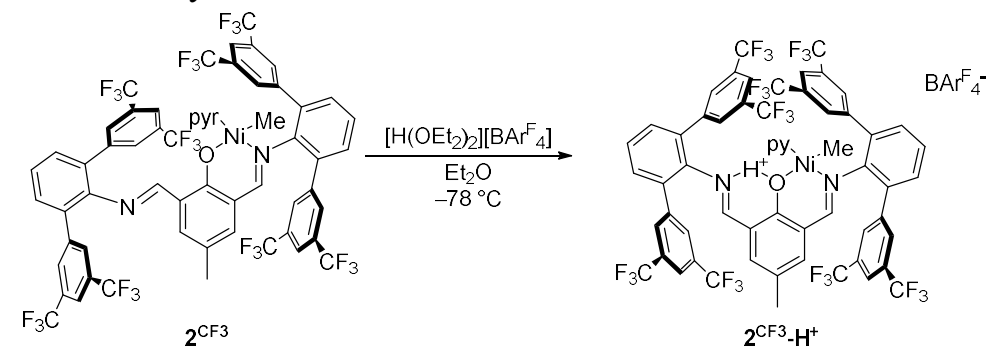

Scheme S8: Synthesis of $2^{\mathrm{CF}^{3}}-\mathrm{H}^{+}$.

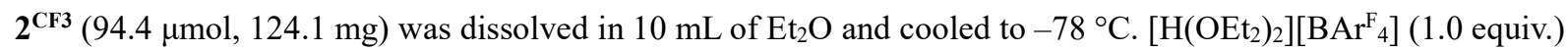
was dissolved in $1.0 \mathrm{~mL}$ of $\mathrm{Et}_{2} \mathrm{O}$ and added to the complex slowly over one hour. After warming to room temperature., the solvent was removed and the product $\mathbf{2}^{\mathbf{C F} 3}-\mathbf{H}^{+}$obtained as dark-red solid $(94.4 \mu \mathrm{mol}, 209 \mathrm{mg}$, quantitative).
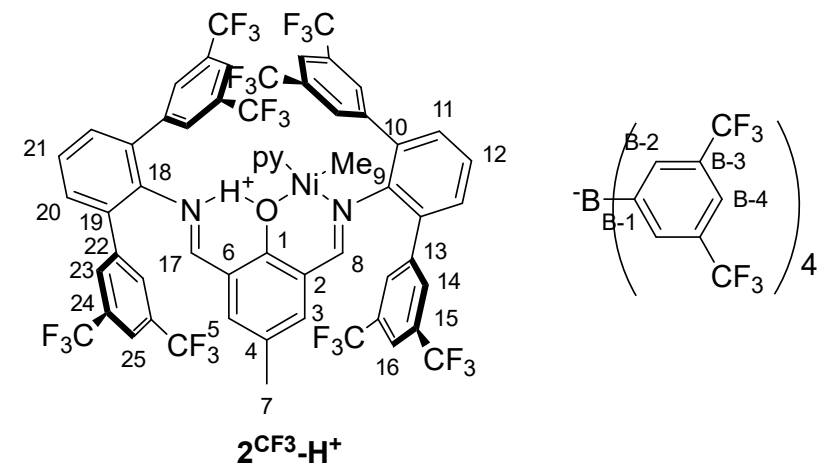

${ }^{1} \mathbf{H}$ NMR $\left(400 \mathrm{MHz}, \mathrm{CD}_{2} \mathrm{Cl}_{2}, 300 \mathrm{~K}\right): \delta(\mathrm{ppm})=12.19\left(\mathrm{~d},{ }^{3} J_{\mathrm{HH}}=13.4 \mathrm{~Hz}, 1 \mathrm{H}, \mathrm{N}^{\cdots} \underline{\mathrm{H}}^{+\cdots} \mathrm{O}\right), 8.25\left(\mathrm{dt},{ }^{3} J_{\mathrm{HH}}=5.0\right.$, ${ }^{4} J_{\mathrm{HH}}=1.6 \mathrm{~Hz}, 2 \mathrm{H}, o$-py), $8.13\left(\mathrm{~d},{ }^{4} J_{\mathrm{HH}}=1.6 \mathrm{~Hz}, 4 \mathrm{H}, \mathrm{H}-23\right), 8.09$ (s, 2H, H-16), 8.07 (s, 2H. H-25), 7.97 (m, 1H, H-8), $7.77-7.73$ (m, 9 H, B-2 and H-21)7.61 -7.57 (m, 6H, B-4, H-11 and H-20), 7.69 (d, ${ }^{3} J_{\mathrm{HH}}=13.4 \mathrm{~Hz}, 1 \mathrm{H}$, H-17), $7.58-7.55$ (m, 1H, H-21), $7.50-7.44$ (m, 5H, p-py and C-14), 7.42 (d, $\left.{ }^{4} J_{\mathrm{HH}}=2.4 \mathrm{~Hz}, 1 \mathrm{H}, \mathrm{H}-\right), 7.09-7.03$ (m, 3H, m-py and H-3) 2.19 (s, 3H, H-7), -0.66 (s, 3H, Ni-C $\left.\underline{H}_{3}\right)$.

${ }^{19} \mathbf{F}\left\{{ }^{1} \mathbf{H}\right\}$ NMR $\left(376 \mathrm{MHz}, \mathrm{CD}_{2} \mathrm{Cl}_{2}, 300 \mathrm{~K}\right): \delta(\mathrm{ppm})=-62.8\left(\mathrm{BAr}^{\mathrm{F}}{ }_{4}-\mathrm{CF}_{3}\right),-63.4($ Nickel-complex-CF 3$),-63.5$ (Nickel-complex-CF ). $^{2}$

${ }^{13} \mathbf{C}\left\{{ }^{1} \mathbf{H}\right\}$ NMR (101 MHz, $\left.\mathrm{CD}_{2} \mathrm{Cl}_{2}, 300 \mathrm{~K}\right): \delta(\mathrm{ppm})=168.9(\mathrm{C}-17), 168.4(\mathrm{C}-8), 162.5$ (B-1), 162.0 (B-1), 161.5 (B-1), 161.0 (B-1), 149.8 (o-py), 149.7 (C-5) 148.3 (C-1), 142.1 (C-3), 138.0 (p-py), 134.8 (B-2), 132.7 (C-21), $132.4(\mathrm{C}-20), 132.2(\mathrm{C}-11), 132.0\left({ }^{1} J_{\mathrm{CF}}=268.8 \mathrm{~Hz}, \underline{\mathrm{CF}}_{3}\right) 131.8$ (q, $\left.{ }^{2} J_{\mathrm{CF}}=31.2 \mathrm{~Hz}, \mathrm{C}-24\right), 131.6(\mathrm{C}-6), 131.1$ (C--12), 130.3 (C-19), 129.3 (C-14), 128.6 (q, ${ }^{2} J_{\mathrm{CF}}=30.9$ Hz, B-3), 128.5 (C-4), 125.2 (m-py), 124.5 (C-10), 124.3 $\left(\mathrm{q},{ }^{1} J_{C F}=272.4 \mathrm{~Hz}, \underline{\mathrm{CF}}_{3}\right), 124.1(\mathrm{C}-22), 122.9(\mathrm{C}-25), 122.6\left(\mathrm{q},{ }^{1} J_{C F}=271.5 \mathrm{~Hz}, \underline{\mathrm{CF}}_{3}\right), 122.3(\mathrm{C}-13), 121.9(\mathrm{C}-2)$,

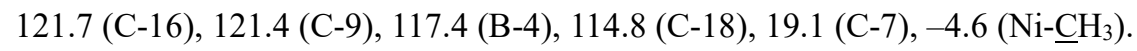




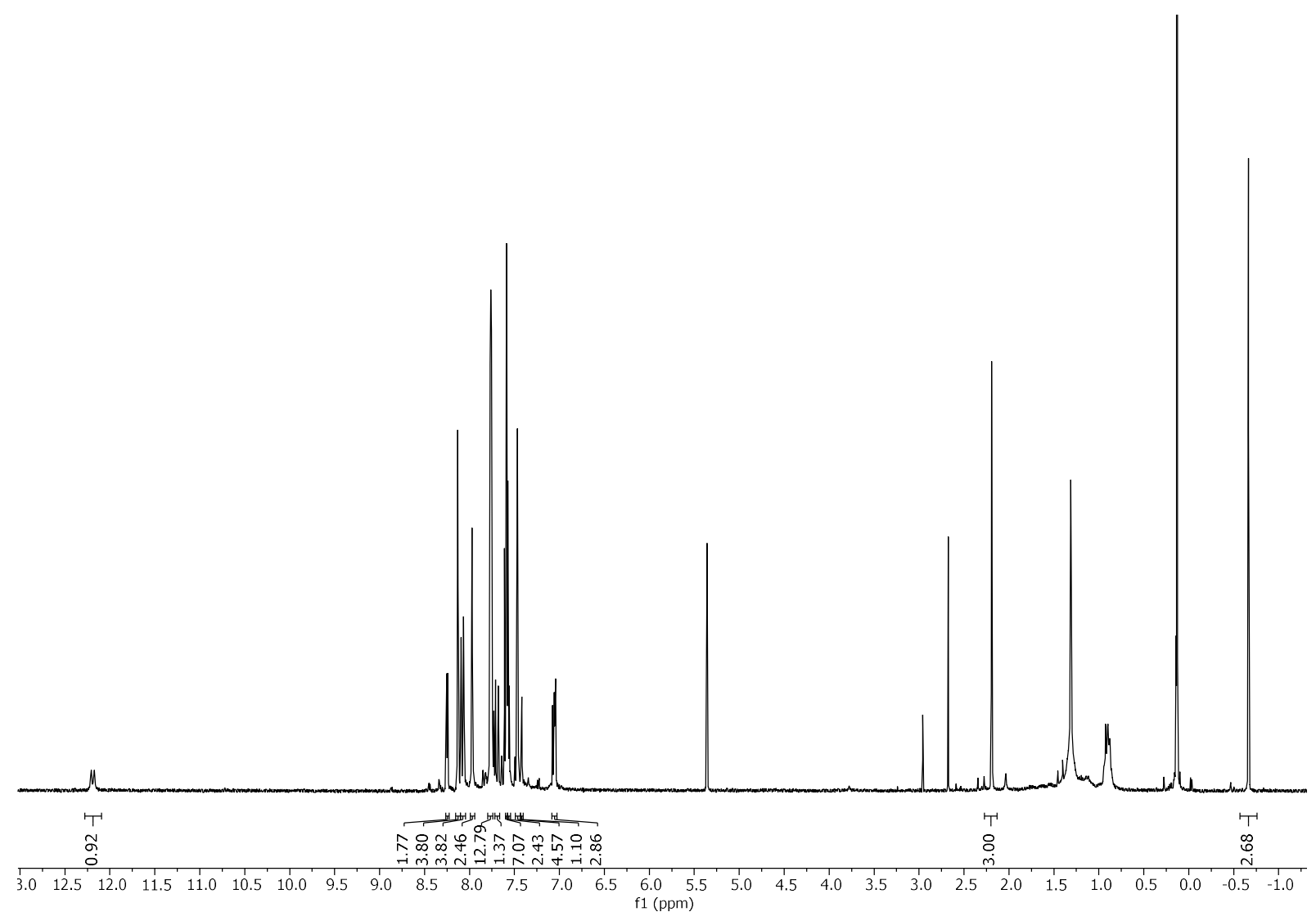

Figure S19: ${ }^{1} \mathrm{H}$ NMR spectrum of $\mathbf{2}^{\mathrm{CF}^{3}} \mathbf{H}^{+}$in $\mathrm{CD}_{2} \mathrm{Cl}_{2}$ at $300 \mathrm{~K}$.

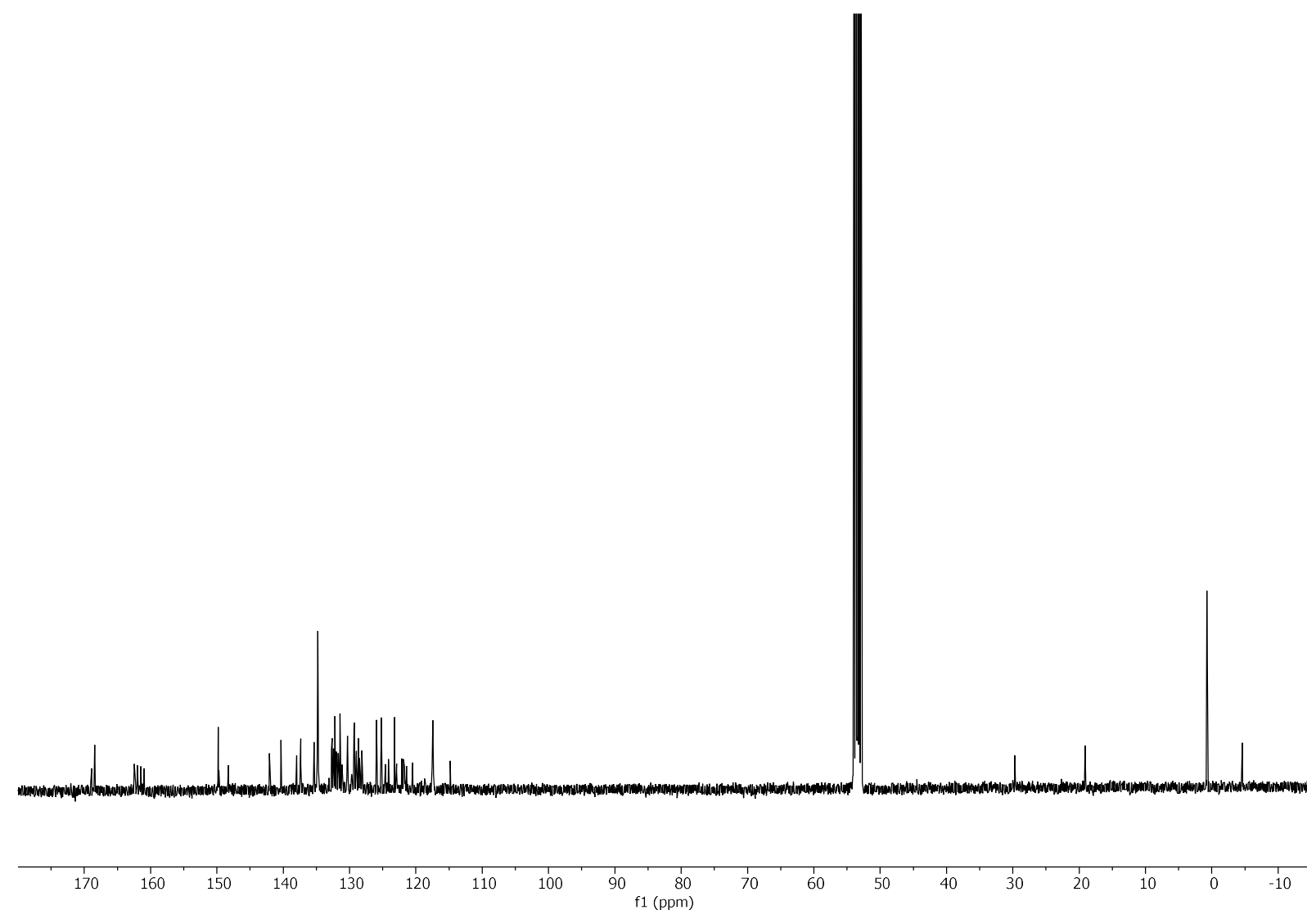

Figure S20: ${ }^{19} \mathrm{~F}\left\{{ }^{\mathrm{I}} \mathrm{H}\right\}$ NMR spectrum of $2^{\mathrm{CF}^{3}} \boldsymbol{H}^{+}$in $\mathrm{CD}_{2} \mathrm{Cl}_{2}$ at $300 \mathrm{~K}$. 


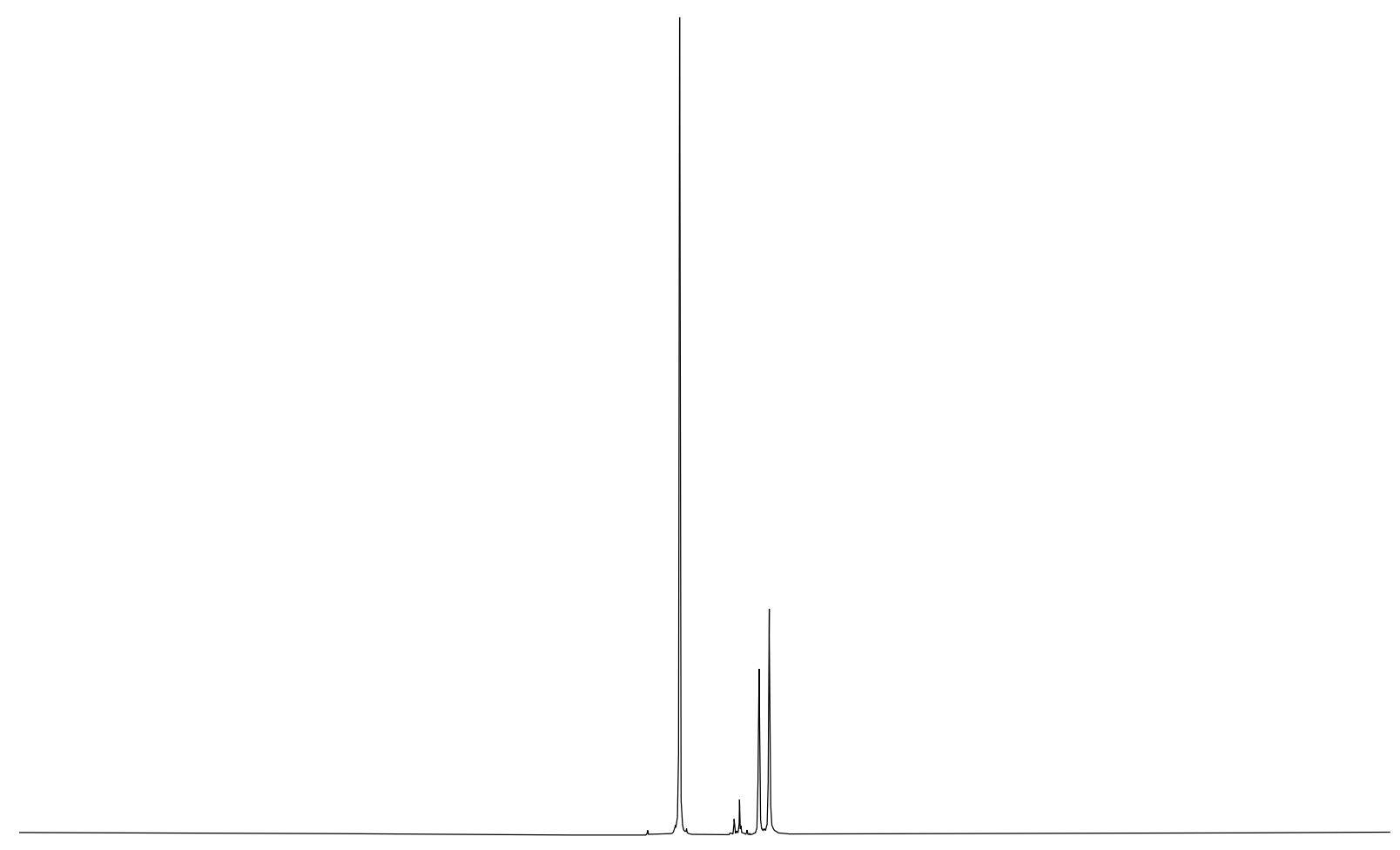

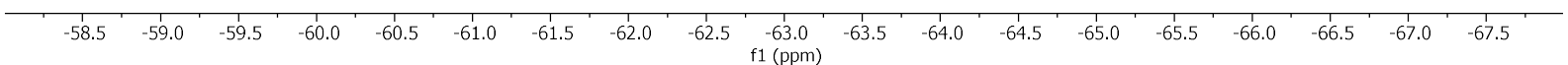

Figure S21: ${ }^{19} \mathrm{~F}\left\{{ }^{1} \mathrm{H}\right\} \mathrm{NMR}$ spectrum of $2^{\mathrm{CF}^{3}}-\mathrm{H}^{+}$in $\mathrm{CD}_{2} \mathrm{Cl}_{2}$ at $300 \mathrm{~K}$.

\subsubsection{Synthesis of $\mathbf{2}^{\mathrm{Me}}-\mathbf{H}^{+}$}
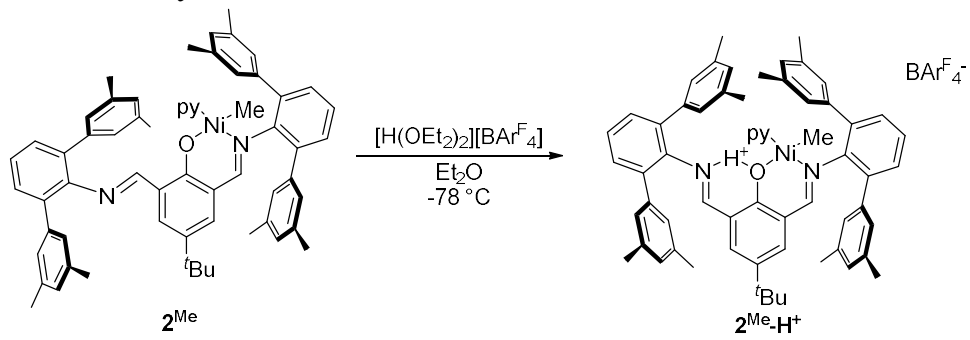

Scheme S9: Synthesis of $\mathbf{2}^{\mathrm{Me}}-\boldsymbol{H}^{+}$.

$\mathbf{2}^{\mathrm{Me}}(100 \mu \mathrm{mol}, 92.3 \mathrm{mg})$ was dissolved in $10 \mathrm{~mL}$ of $\mathrm{Et}_{2} \mathrm{O}$ and cooled to $-78^{\circ} \mathrm{C}$. [H( $\left.\left.\mathrm{OEt}_{2}\right)_{2}\right]\left[\mathrm{BAr}{ }_{4}^{\mathrm{F}}\right](1.0$ equiv. $)$ was dissolved in $2.0 \mathrm{~mL}$ of $\mathrm{Et}_{2} \mathrm{O}$ and added to the complex slowly over one hour. After warming to room temperature, the solvent was removed and the product $\mathbf{2}^{\mathrm{Me}}-\mathbf{H}^{+}$obtained as dark-red solid $(98.1 \mu \mathrm{mol}, 175.4 \mathrm{mg}, 98 \%)$.
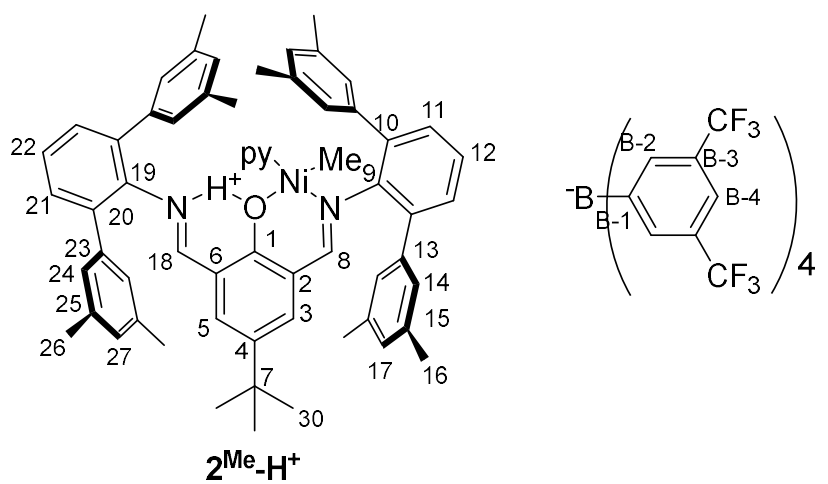
${ }^{1} \mathbf{H}$ NMR $\left(400 \mathrm{MHz}, \mathrm{CD}_{2} \mathrm{Cl}_{2}, 300 \mathrm{~K}\right): \delta(\mathrm{ppm})=12.17\left(\mathrm{~d},{ }^{3} J_{\mathrm{HH}}=14.3 \mathrm{~Hz}, 1 \mathrm{H}, \mathrm{N}^{\cdots} \underline{\mathrm{H}}^{+\cdots} \mathrm{O}\right), 8.04\left(\mathrm{dt},{ }^{3} J_{\mathrm{HH}}=5.0\right.$, $\left.{ }^{4} J_{\mathrm{HH}} 1.6 \mathrm{~Hz}, 2 \mathrm{H}, o-\mathrm{py}\right), 7.72$ (s, 8H, B-2), 7.67 (s, 1H, H-8), $7.63-7.59$ (m, 2H, H-27), 7.55 (s, 4H, B-4), 7.49 (d, $\left.{ }^{3} J_{\mathrm{HH}}=14.3 \mathrm{~Hz}, 1 \mathrm{H}, \mathrm{H}-18\right), 7.45-7.27$ (m, 6H, p-py, H-5, H-21 and H-22), 7.13 (s, 4H, H-24), $7.12-7.04$ (m, 5H, H-17, H-3, H-11), 6.90 (m, 1 H, H-12), 6.88 (t, $\left.{ }^{3} J_{\mathrm{HH}}=5.0,2 \mathrm{H}, m-\mathrm{py}\right), 6.62$ (s, 4H, H-14), 2.34 (s, 12H, H-26), $2.30(\mathrm{~s}, 12 \mathrm{H}, \mathrm{H}-16), 1.16(\mathrm{~s}, 9 \mathrm{H}, \mathrm{H}-30),-0.82\left(\mathrm{~s}, 3 \mathrm{H}, \mathrm{Ni}-\underline{\mathrm{H}}_{3}\right)$.

${ }^{13} \mathbf{C}\left\{{ }^{1} \mathbf{H}\right\}$ NMR $\left(101 \mathrm{MHz}, \mathrm{CD}_{2} \mathrm{Cl}_{2}, 300 \mathrm{~K}\right): \delta(\mathrm{ppm})=169.4(\mathrm{C}-8), 168.9(\mathrm{C}-18), 163.1$ (B-1), $162.6(\mathrm{~B}-1), 162.1$ (B-1), 161.6 (B-1), 150.5 (o-py), 148.6 (C-1), 143.4 (C-3), 139.8 (C-23), 139.6 (C-4), 139.4 (C-20), 138.3 (C-13), 137.9 (p-py), 137.4 (C-5), 136.6 (C-9), 136.2 (C-25), 135.4 (B-2), 134.4 (C-27), 130.9 (C-6), 130.8 (C-10), 130.5 (C-15), 129.11 (C-22) 129.6 (C-17), 128.9 (C-24), 127.8 (C-14), 127.7 (C-11), 127.5 (C-12), 127.4 (C-21), 125.3 $\left(\mathrm{q},{ }^{1} J_{\mathrm{CF}}=270.6 \mathrm{~Hz}, \underline{\mathrm{CF}}_{3}\right) 125.1$ (m-py), $124.4(\mathrm{C}-2), 118.0$ (m, B-4), $115.9(\mathrm{C}-19), 66.23$ (C-7), $30.9(\mathrm{C}-30), 21.8$ (C-16 or C-26), 21.7 (C-16 or C-26), $-5.9\left(\mathrm{Ni}^{-} \underline{\mathrm{H}}_{3}\right)$.

${ }^{11} \mathbf{B}\left\{{ }^{1} \mathbf{H}\right\}$ NMR $\left(128 \mathrm{MHz}, \mathrm{CD}_{2} \mathrm{Cl}_{2}, 300 \mathrm{~K}\right): \delta(\mathrm{ppm})=-6.8\left(\mathrm{~s}, \underline{\mathrm{B} A \mathrm{~F}^{\mathrm{F}}} 4\right)$.

${ }^{19} \mathbf{F}\left\{{ }^{1} \mathbf{H}\right\}$ NMR $\left(376 \mathrm{MHz}, \mathrm{CD}_{2} \mathrm{Cl}_{2}, 300 \mathrm{~K}\right): \delta(\mathrm{ppm})=-62.9\left(\mathrm{~s}, \mathrm{BAr}^{\mathrm{F}}{ }_{4}-\mathrm{CF}_{3}\right)$.

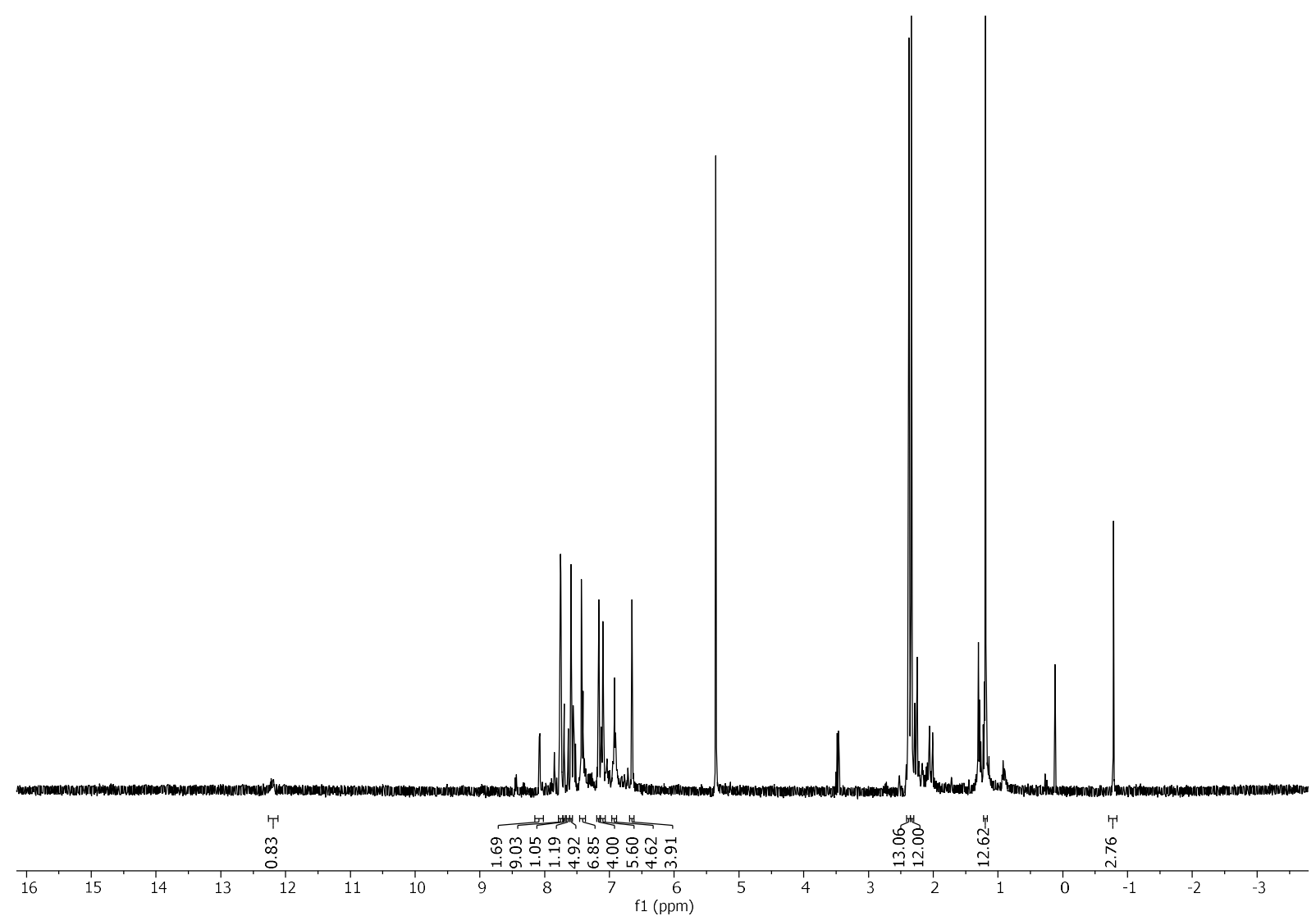

Figure S22: ${ }^{1} \mathrm{H}$ NMR spectrum of $\mathbf{2}^{\mathrm{Me}}-\boldsymbol{H}^{+}$in $\mathrm{CD}_{2} \mathrm{Cl}_{2}$ at $300 \mathrm{~K}$. 


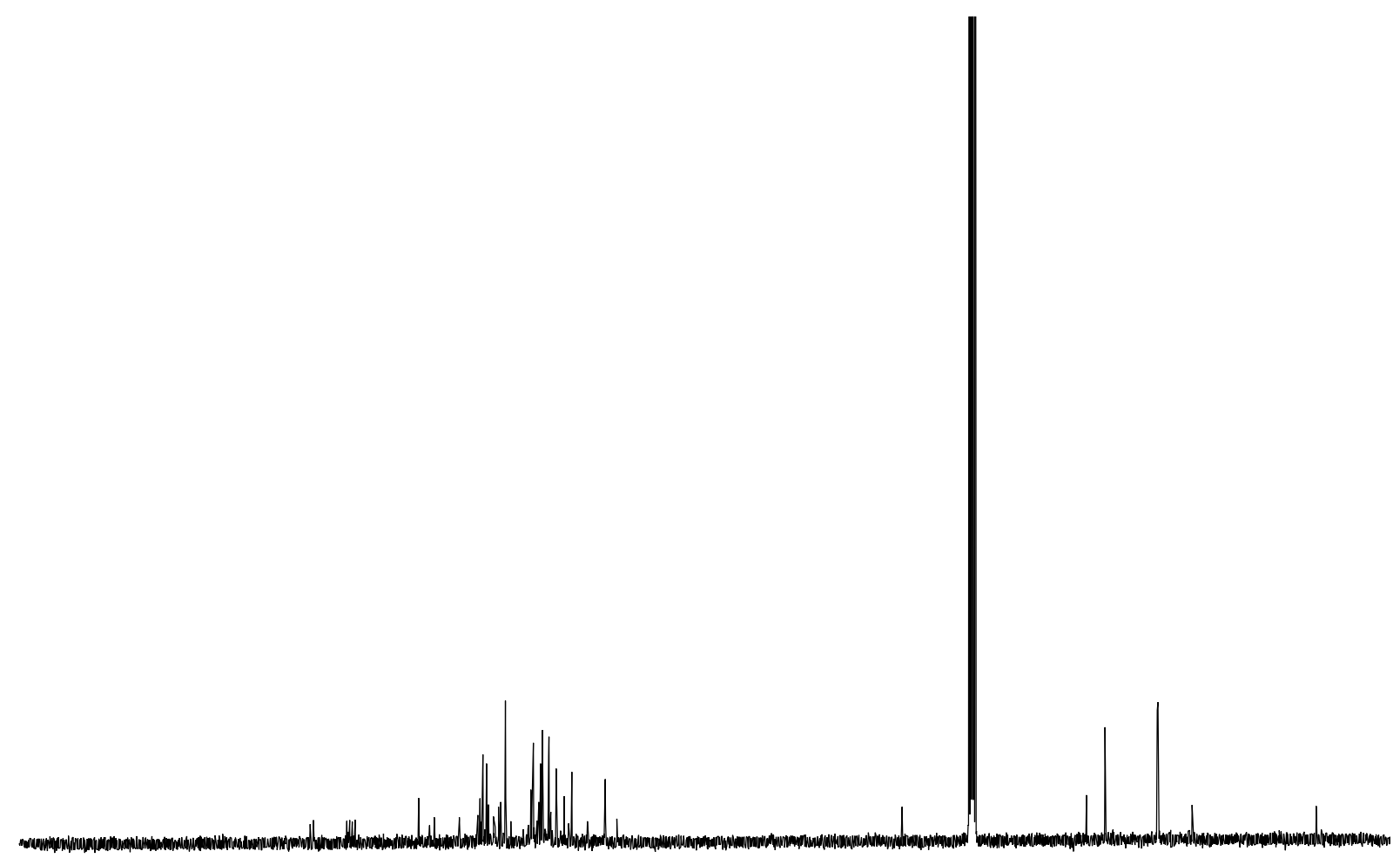

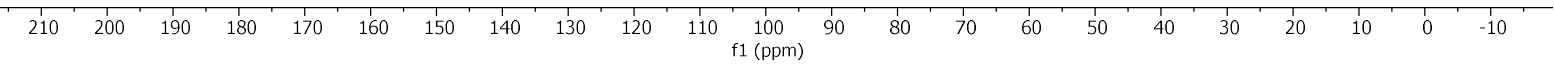

Figure $\left.S 23:{ }^{13} \mathrm{C}_{\{}{ }^{1} \mathrm{H}\right\}$ NMR spectrum of $\mathbf{2}^{\mathrm{Me}}$ - $\mathrm{H}^{+}$in $\mathrm{CD}_{2} \mathrm{Cl}_{2}$ at $300 \mathrm{~K}$.

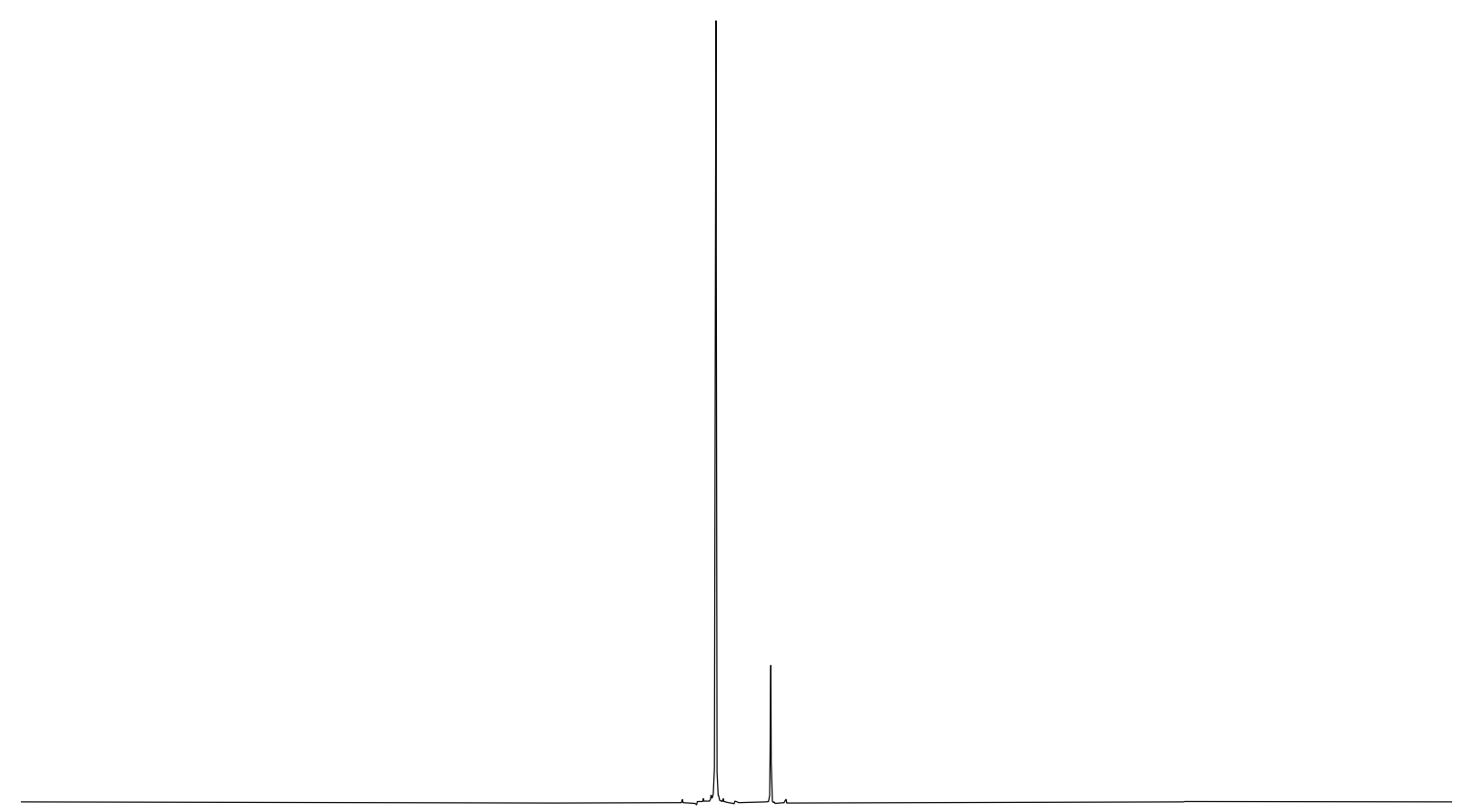

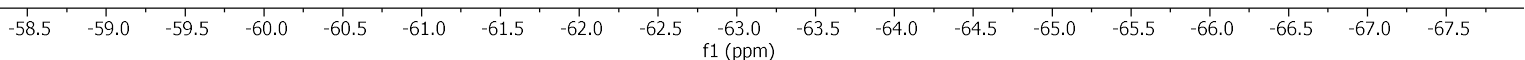

Figure $S 24:{ }^{19} \mathrm{~F}\left\{{ }^{\mathrm{I}} \mathrm{H}\right\}$ NMR spectrum of $\mathbf{2}^{\mathrm{Me}} \boldsymbol{H}^{+}$in $\mathrm{CD}_{2} \mathrm{Cl}_{2}$ at $300 \mathrm{~K}$. 


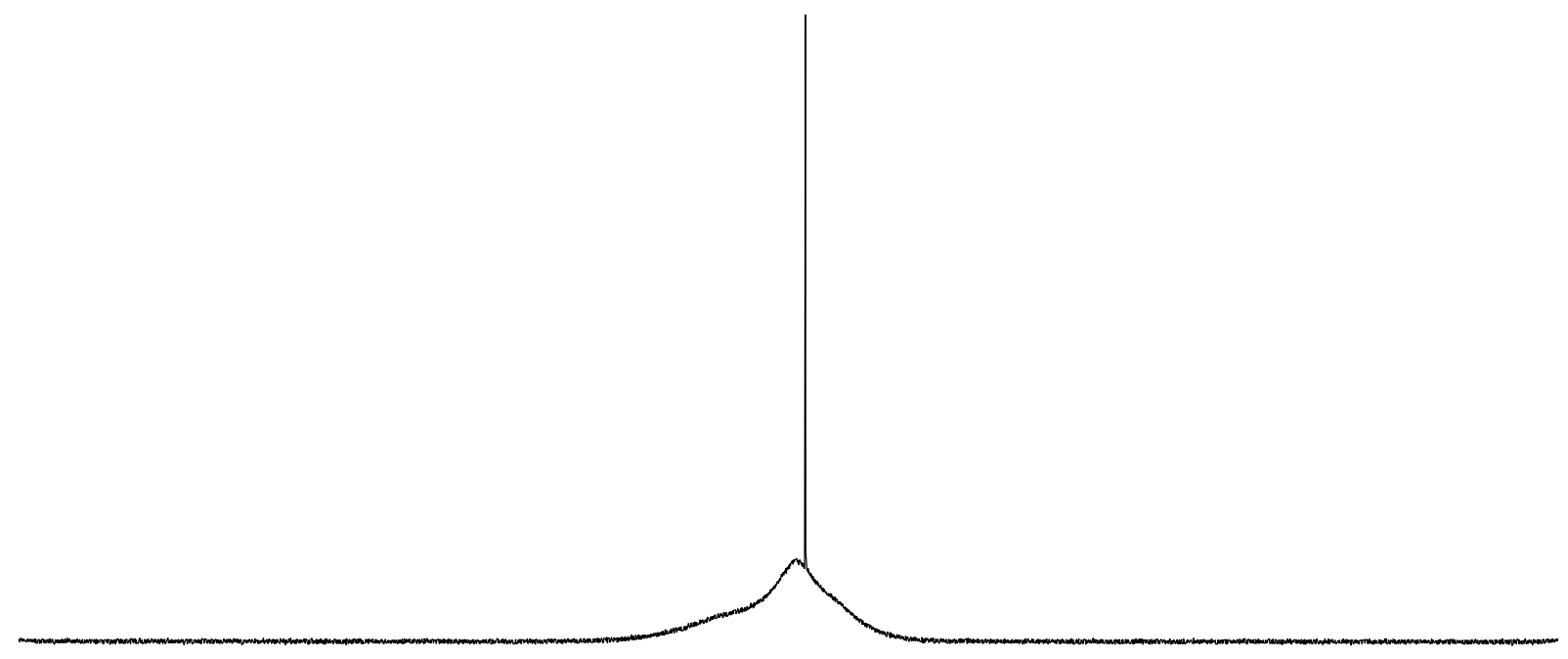

\begin{tabular}{llllllllllllllllllllllllllllllllllll}
\hline 300 & 280 & 260 & 240 & 220 & 200 & 180 & 160 & 140 & 120 & 100 & 80 & 60 & 40 & 20 & 0 & -20 & -40 & -60 & -80 & -100 & -120 & -140 & -160 & -180 & -200 & -220 & -240 & -260 & -280 & -30
\end{tabular} Figure $S 25:{ }^{11} B\left\{{ }^{1} \mathrm{H}\right\}$ NMR spectrum of $\mathbf{2}^{\mathbf{M e}}-\boldsymbol{H}^{+}$in $\mathrm{CD}_{2} \mathrm{Cl}_{2}$ at $300 \mathrm{~K}$.

\subsection{Cyclic Voltammetry}

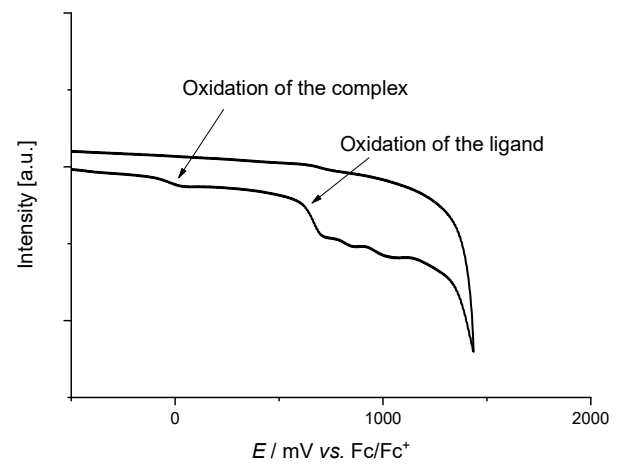

Figure S26: Cyclic voltammogram of $2^{\text {Me }}$. 


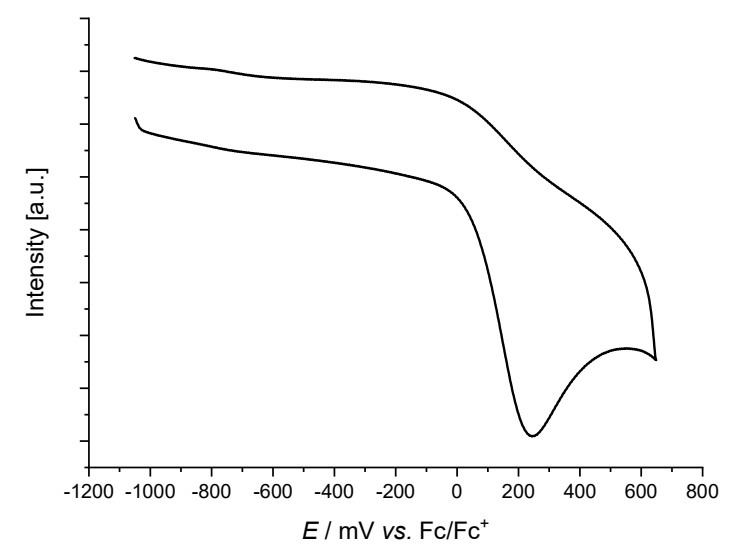

Figure S27: Cyclic voltammogram of $2^{\text {CF } 3}$.

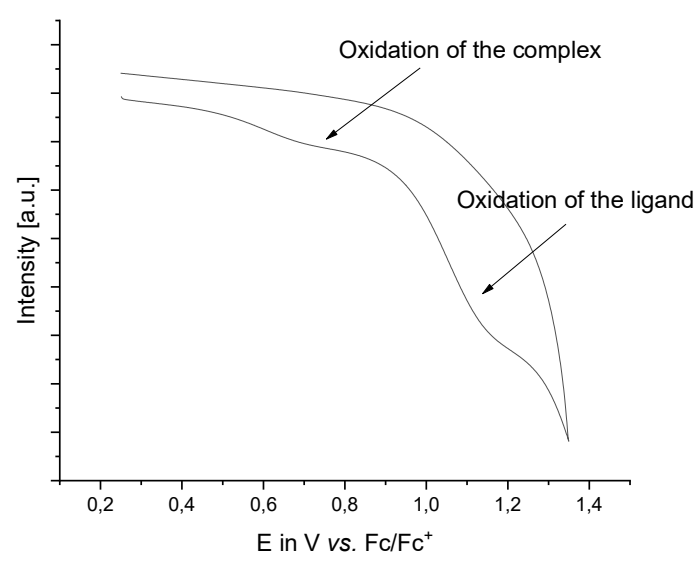

Figure S28: Cyclic voltammogram of $2^{\mathrm{CF}^{3}}-\mathrm{H}^{+}$.

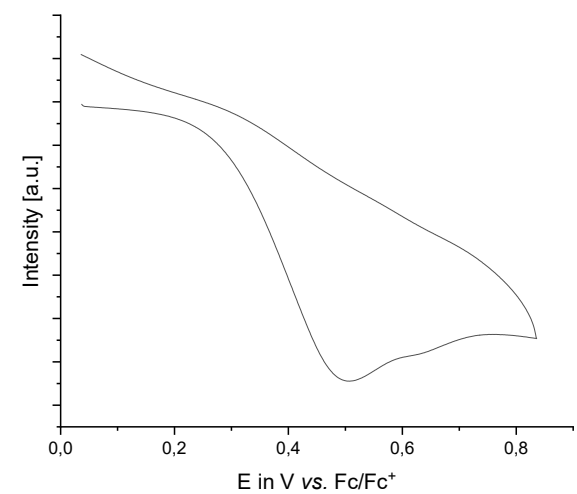

Figure S29: Cyclic voltammogram of $\mathbf{2}^{\mathbf{M e}}-\boldsymbol{H}^{+}$.

$\mathrm{CV}$ was performed in dichloromethane, with an addition of ${ }^{n} \mathrm{Bu}_{4} \mathrm{PF}_{6}$ as electrolyte. The $\mathrm{PF}_{6}{ }^{-}$anions most likely exchange with the $\mathrm{BAr}_{4}^{-}$anions. Yet, there is no significant influence on the forward peak potential of the $\mathrm{Ni}(\mathrm{II}) / \mathrm{Ni}(\mathrm{III})$ pair, proven by reference experiments with complexes with a stronger coordinating counter-ion. 

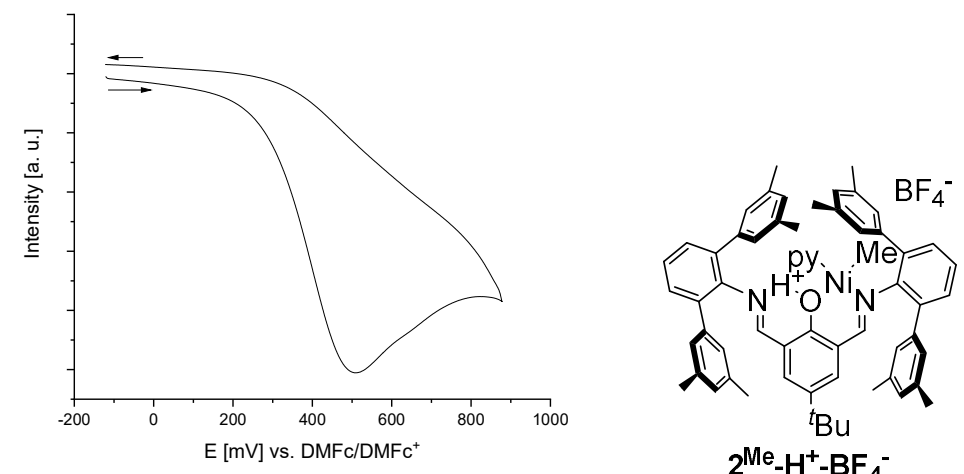

${ }^{t} \mathrm{Bu}$

$2^{\mathrm{Me}}-\mathrm{H}^{+}-\mathrm{BF}_{4}$

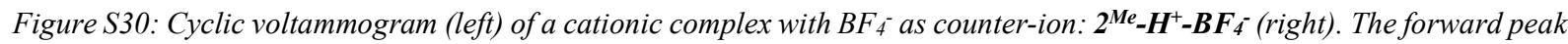
potential for the Ni(II)/Ni(III) pair is $506 \mathrm{mV}$.

\subsection{Crystal Structure Analysis}

\section{$2.3 .1 \mathbf{3}^{\mathrm{Me}}$}
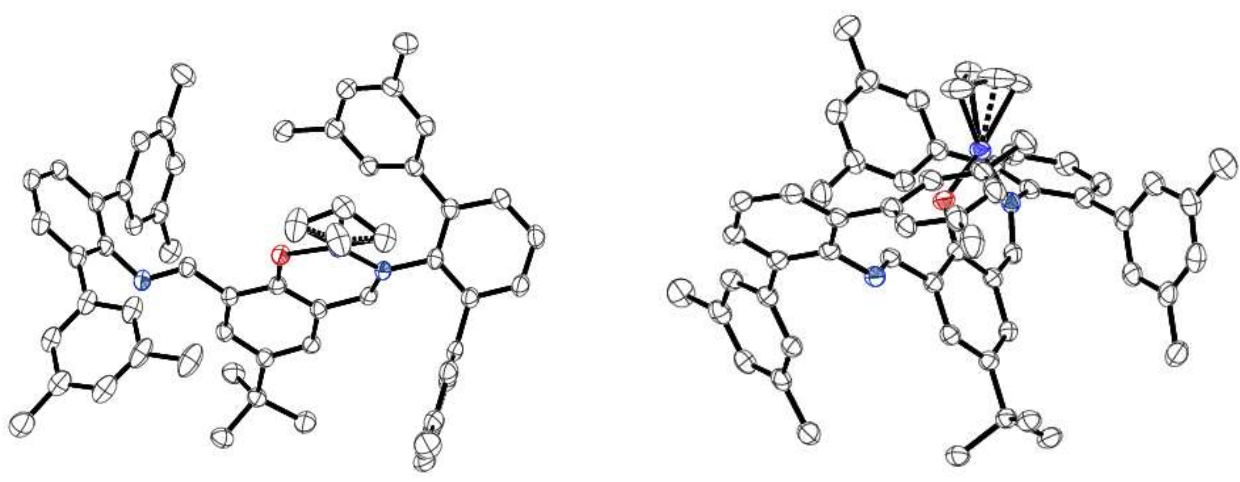

Figure S31: ORTEP-plots of $3^{\text {Me }}$ (30\% probability ellipsoids, hydrogens are omitted for clarity) of structures determined by $X$-ray diffraction from different perspectives.

Table S1: Crystal data and structure refinement for $3^{\text {Me }}$.

\begin{tabular}{|l|l|}
\hline Identification code & $\mathbf{3}^{\mathrm{Me}}$ \\
\hline Deposition number & 2032182 \\
\hline Empirical formula & $\mathrm{C}_{59} \mathrm{H}_{60} \mathrm{~N}_{2} \mathrm{NiO}$ \\
\hline Formula weight & 871.80 \\
\hline Temperature/K & 100 \\
\hline Crystal system & monoclinic \\
\hline Space group & $\mathrm{P} 2{ }_{1} / \mathrm{n}$ \\
\hline $\mathrm{a} / \AA$ & $12.2781(5)$ \\
\hline $\mathrm{b} / \AA$ & $22.6085(10)$ \\
\hline $\mathrm{c} / \AA$ & $18.0159(7)$ \\
\hline$\alpha /{ }^{\circ}$ & 90 \\
\hline$\beta /{ }^{\circ}$ & $106.372(3)$ \\
\hline$\gamma /{ }^{\circ}$ & 90 \\
\hline Volume $/ \AA^{3}$ & $4798.2(4)$ \\
\hline $\mathrm{Z}$ & 4 \\
\hline$\rho_{\text {calcg }} / \mathrm{cm}^{3}$ & 1.207 \\
\hline$\mu / \mathrm{mm}^{-1}$ & 0.447 \\
\hline $\mathrm{F}(000)$ & 1856.0 \\
\hline $\mathrm{Crystal}^{3}$ size $/ \mathrm{mm}^{3}$ & $0.5 \times 0.367 \times 0.1$ \\
\hline Radiation & MoK $\alpha(\lambda=0.71073)$ \\
\hline $2 \Theta$ range for data collection $^{\circ}{ }^{\circ}$ & 2.966 to 54.074 \\
\hline & \\
\hline
\end{tabular}




\begin{tabular}{|l|l|}
\hline Index ranges & $-15 \leq \mathrm{h} \leq 15,-28 \leq \mathrm{k} \leq 28,-23 \leq 1 \leq 22$ \\
\hline Reflections collected & 65484 \\
\hline Independent reflections & $10472\left[\mathrm{R}_{\text {int }}=0.0870, \mathrm{R}_{\text {sigma }}=0.0465\right]$ \\
\hline Data/restraints/parameters & $10472 / 0 / 590$ \\
\hline Goodness-of-fit on $\mathrm{F}^{2}$ & 1.026 \\
\hline Final $\mathrm{R}$ indexes [I $>=2 \sigma(\mathrm{I})]$ & $\mathrm{R}_{1}=0.0522, \mathrm{wR}_{2}=0.1279$ \\
\hline Final $\mathrm{R}$ indexes [all data] & $\mathrm{R}_{1}=0.0822, \mathrm{wR}_{2}=0.1450$ \\
\hline Largest diff. peak/hole $/ \mathrm{e} \AA^{-3}$ & $0.39 /-1.06$ \\
\hline
\end{tabular}

Single crystals of $\mathrm{C}_{59} \mathrm{H}_{60} \mathrm{~N}_{2} \mathrm{NiO}\left(3^{\mathrm{Me}}\right)$ were grown by layering a saturated solution of the complex in benzene with pentanes. A suitable crystal was selected and placed on a STOE IPDS 2 T diffractometer. The crystal was kept at $100 \mathrm{~K}$ during data collection. Using Olex $2^{5}$, the structure was solved with the SHELXT ${ }^{6}$ structure solution program using Intrinsic Phasing and refined with the SHELXL ${ }^{9}$ refinement package using Least Squares minimization.

\subsection{2 $\mathbf{3}^{\mathrm{CF3}}$}
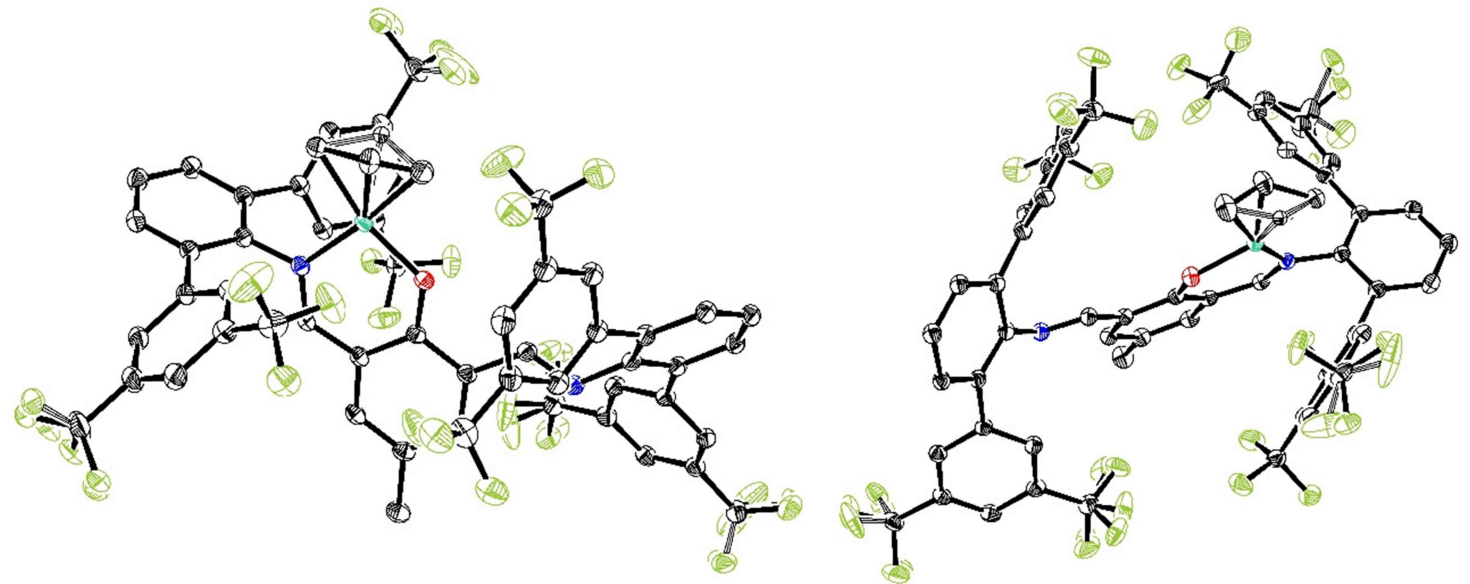

Figure S32: ORTEP-plots of $3^{\text {CF3 }}$ (30\% probability ellipsoids, all hydrogens are omitted for clarity) of structures determined by X-ray diffraction from different perspectives. For the Ni Allyl group is and some of the $\mathrm{CF}_{3}$ groups disorders are modeled.

Table S2: Crystal data and structure refinement for $3^{\text {CF } 3}$.

\begin{tabular}{|l|l|}
\hline Identification code & $\mathbf{3}^{\text {CF3 }}$ \\
\hline Deposition number & 2032183 \\
\hline Empirical formula & $\mathrm{C}_{56} \mathrm{H}_{31} \mathrm{~F}_{24} \mathrm{~N}_{2} \mathrm{NiO}$ \\
\hline Formula weight & 1262.54 \\
\hline Temperature/K & 100 \\
\hline Crystal system & triclinic \\
\hline Space group & $\mathrm{P}-1$ \\
\hline $\mathrm{a} / \AA$ & $13.6061(4)$ \\
\hline $\mathrm{b} / \AA$ & $13.6447(4)$ \\
\hline $\mathrm{c} / \AA$ & $17.5428(6)$ \\
\hline$\alpha /{ }^{\circ}$ & $88.69(3)$ \\
\hline$\beta /{ }^{\circ}$ & $69.978(3)$ \\
\hline$\gamma /{ }^{\circ}$ & $65.608(2)$ \\
\hline Volume/ $\AA^{3}$ & $2760.0(3)$ \\
\hline$Z$ & 2 \\
\hline$\rho_{\text {calcg }} / \mathrm{cm}^{3}$ & 1.519 \\
\hline$\mu / \mathrm{mm}^{-1}$ & 0.474 \\
\hline $\mathrm{F}(000)$ & 1266.0 \\
\hline Crystal size/mm & \\
\hline Radiation & $0.05 \times 0.027 \times 0.01$ \\
\hline $2 \Theta$ range for data collection/ $^{3}$ & 3.31 to 58.786 \\
\hline Index ranges & $-18 \leq \mathrm{h} \leq 18,-18 \leq \mathrm{k} \leq 18,-24 \leq 1 \leq 24$ \\
\hline
\end{tabular}




\begin{tabular}{|l|l|}
\hline Reflections collected & 45030 \\
\hline Independent reflections & $14880\left[\mathrm{R}_{\text {int }}=0.0413, \mathrm{R}_{\text {sigma }}=0.0344\right]$ \\
\hline Data/restraints/parameters & $14880 / 12 / 881$ \\
\hline Goodness-of-fit on $\mathrm{F}^{2}$ & 1.069 \\
\hline Final $\mathrm{R}$ indexes $[\mathrm{I}>=2 \sigma(\mathrm{I})]$ & $\mathrm{R}_{1}=0.0595, \mathrm{wR}_{2}=0.1657$ \\
\hline Final $\mathrm{R}$ indexes [all data] & $\mathrm{R}_{1}=0.0779, \mathrm{wR}_{2}=0.1818$ \\
\hline Largest diff. peak/hole $/ \mathrm{e} \AA^{-3}$ & $0.73 /-0.88$ \\
\hline
\end{tabular}

Single crystals of $\mathrm{C}_{56} \mathrm{H}_{31} \mathrm{~F}_{24} \mathrm{~N}_{2} \mathrm{NiO}\left(3^{\mathrm{Cr} 3}\right)$ were grown by layering a saturated solution of the complex in benzene with pentane. A suitable crystal was selected and placed on a STOE IPDS 2 T diffractometer. The crystal was kept at $100 \mathrm{~K}$ during data collection. Using Olex $2^{5}$, the structure was solved with the SHELXT ${ }^{6}$ structure solution program using Intrinsic Phasing and refined with the SHELXL ${ }^{9}$ refinement package using Least Squares minimization.

\subsection{3 $\mathbf{3}^{\mathrm{Me}}-\mathbf{H}^{+}$}
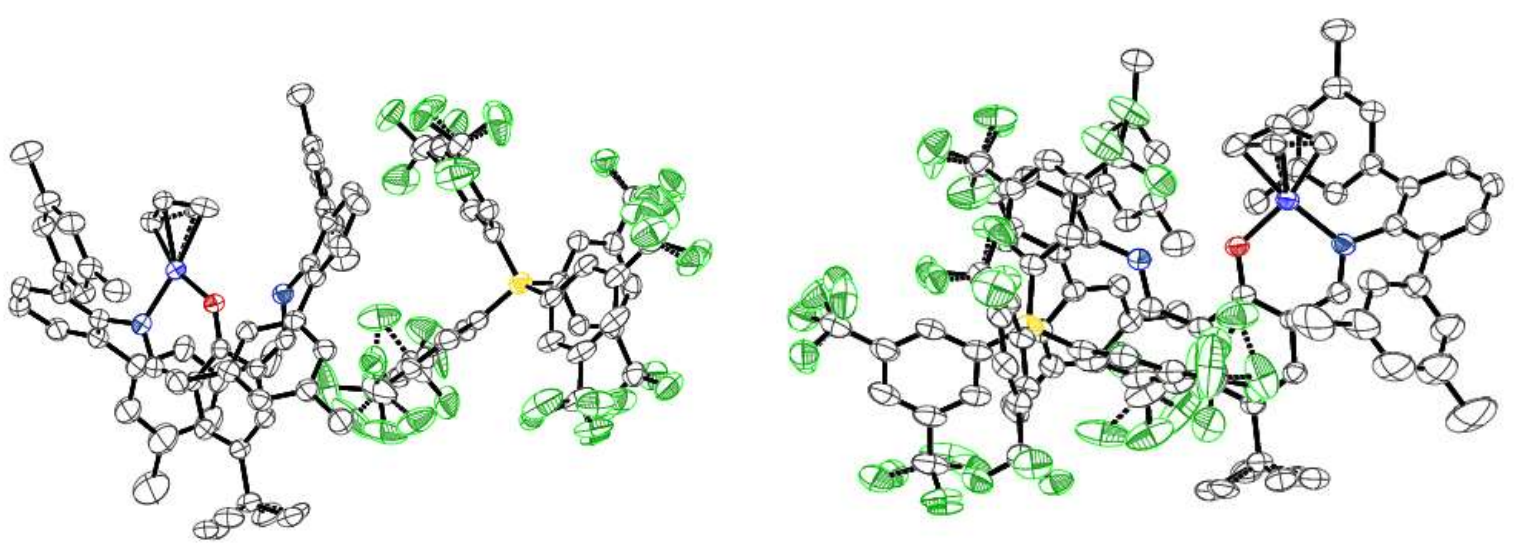

Figure S33: ORTEP-plots of $3^{M_{-}-H^{+}}$(30\% probability ellipsoids, all hydrogens are omitted for clarity) of structures determined by $X$-ray diffraction from different perspectives.

Table S3: Crystal data and structure refinement for $3^{\mathrm{Me}}-\mathrm{H}^{+}$.

\begin{tabular}{|c|c|}
\hline Identification code & $3^{\mathrm{Me}}-\mathrm{H}^{+}$ \\
\hline Deposition number & 2032180 \\
\hline Empirical formula & $\mathrm{C}_{91} \mathrm{H}_{72} \mathrm{BF}_{24} \mathrm{~N}_{2} \mathrm{NiO}$ \\
\hline Formula weight & 1735.02 \\
\hline Temperature/K & 100.0 \\
\hline Crystal system & triclinic \\
\hline Space group & P-1 \\
\hline $\mathrm{a} / \AA \AA$ & $16.7822(5)$ \\
\hline $\mathrm{b} / \AA$ & $18.2187(6)$ \\
\hline $\mathrm{c} / \AA$ & $18.2294(5)$ \\
\hline$\alpha /^{\circ}$ & $109.732(2)$ \\
\hline$\beta /{ }^{\circ}$ & $101.015(2)$ \\
\hline$\gamma /{ }^{\circ}$ & $110.139(2)$ \\
\hline Volume $/ \AA^{3}$ & $4618.2(3)$ \\
\hline $\mathrm{Z}$ & 2 \\
\hline$\rho_{\text {calc }} \mathrm{g} / \mathrm{cm}^{3}$ & 1.248 \\
\hline$\mu / \mathrm{mm}^{-1}$ & 0.302 \\
\hline $\mathrm{F}(000)$ & 1778.0 \\
\hline Crystal size $/ \mathrm{mm}^{3}$ & $0.5 \times 0.367 \times 0.3$ \\
\hline Radiation & Mo $K \alpha(\lambda=0.71073)$ \\
\hline
\end{tabular}




\begin{tabular}{|l|l|}
\hline $2 \Theta$ range for data collection $/{ }^{\circ}$ & 3.68 to 50.648 \\
\hline Index ranges & $-20 \leq \mathrm{h} \leq 20,-21 \leq \mathrm{k} \leq 21,-21 \leq 1 \leq 21$ \\
\hline Reflections collected & 51495 \\
\hline Independent reflections & $51495\left[\mathrm{R}_{\text {int }}=0.0610, \mathrm{R}_{\text {sigma }}=0.0419\right]$ \\
\hline Data/restraints/parameters & $51495 / 0 / 1306$ \\
\hline Goodness-of-fit on $\mathrm{F}^{2}$ & 1.015 \\
\hline Final $\mathrm{R}$ indexes $[\mathrm{I}>=2 \sigma(\mathrm{I})]$ & $\mathrm{R}_{1}=0.0597, \mathrm{wR}_{2}=0.1528$ \\
\hline Final $\mathrm{R}$ indexes [all data] & $\mathrm{R}_{1}=0.0700, \mathrm{wR}_{2}=0.1607$ \\
\hline Largest diff. peak/hole $/ \mathrm{e} \AA^{-3}$ & $0.65 /-0.53$ \\
\hline
\end{tabular}

Single crystals of $\mathrm{C}_{91} \mathrm{H}_{72} \mathrm{BF}_{24} \mathrm{~N}_{2} \mathrm{NiO}\left(3^{\mathrm{Me}}-\mathbf{H}^{+}\right)$were grown from a saturated solution of the complex in benzene. A suitable crystal was selected and placed on a STOE IPDS $2 \mathrm{~T}$ diffractometer. The crystal was kept at $100 \mathrm{~K}$ during data collection. Using Olex $2^{5}$, the structure was solved with the SHELXT ${ }^{6}$ structure solution program using Intrinsic Phasing and refined with the SHELXL ${ }^{9}$ refinement package using Least Squares minimization.

\subsection{4 $\mathbf{2}^{\mathbf{C F} 3}-\mathbf{H}^{+}$}
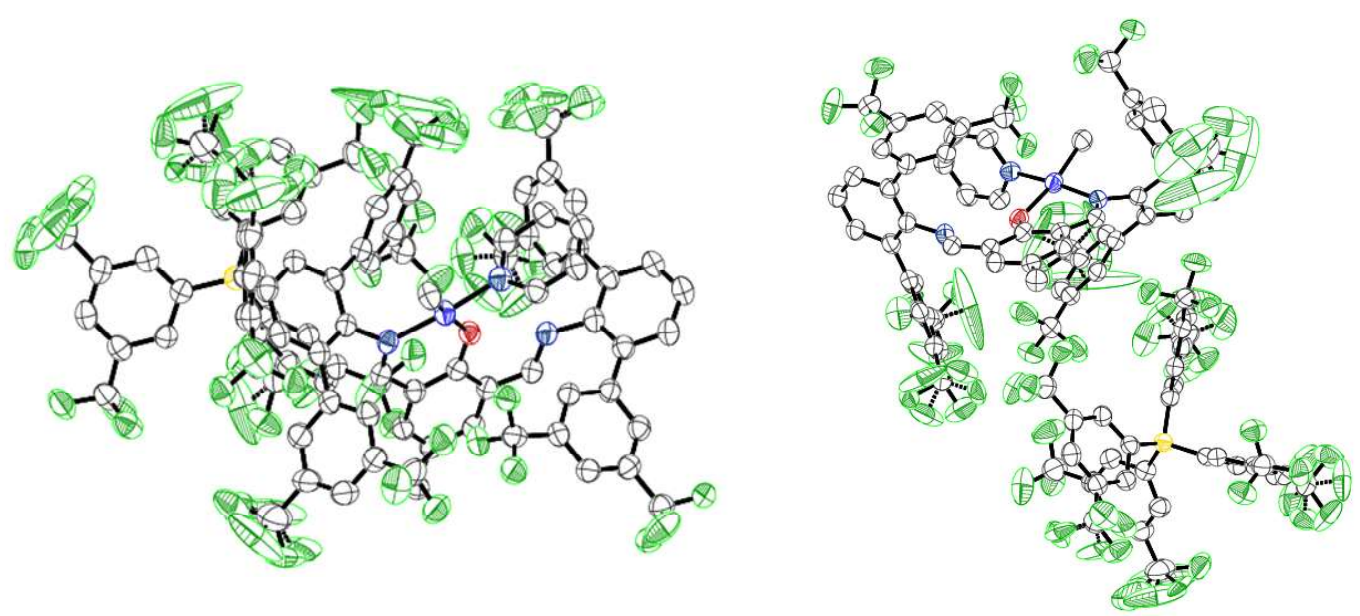

Figure S34: ORTEP-plots of $\mathbf{2}^{\mathbf{C F} 3}-\mathbf{H}^{+}$(30 \% probability ellipsoids, all hydrogens are omitted for clarity) of structures determined by $X$-ray diffraction from different perspectives.

Table S4: Crystal data and structure refinement for $2^{\mathrm{CF}^{3}-\boldsymbol{H}^{+}}$

\begin{tabular}{|l|l|}
\hline Identification code & $\mathbf{2}^{\mathbf{C F} 3}-\mathbf{H}^{+}$ \\
\hline Deposition number & 2032181 \\
\hline Empirical formula & $\mathrm{C}_{91} \mathrm{H}_{45} \mathrm{BF}_{48} \mathrm{~N}_{3} \mathrm{NiO}$ \\
\hline Formula weight & 2177.82 \\
\hline Temperature/K & 100 \\
\hline Crystal system & monoclinic \\
\hline Space group & $\mathrm{C} 2 / \mathrm{c}$ \\
\hline $\mathrm{a} / \AA$ & $44.1023(14)$ \\
\hline $\mathrm{b} / \AA$ & $11.9357(4)$ \\
\hline $\mathrm{c} / \AA$ & $37.7488(10)$ \\
\hline$\alpha /{ }^{\circ}$ & 90 \\
\hline$\beta /{ }^{\circ}$ & $107.621(2)$ \\
\hline$\gamma^{\circ}$ & 90 \\
\hline Volume $/ \AA^{3}$ & $18938.3(10)$ \\
\hline $\mathrm{Z}$ & 8 \\
\hline$\rho_{\text {calc }} \mathrm{g} / \mathrm{cm}^{3}$ & 1.528 \\
\hline$\mu / \mathrm{mm}^{-1}$ & 0.347 \\
\hline $\mathrm{F}(000)$ & 8680.0 \\
\hline Crystal size/mm & \\
\hline Radiation & $0.3 \times 0.2 \times 0.1$ \\
\hline $2 \Theta$ range for data collection $/{ }^{\circ}$ & $\mathrm{MoK} \alpha(\lambda=0.71073)$ \\
\hline Index ranges & 3.396 to 50.778 \\
\hline
\end{tabular}




\begin{tabular}{|l|l|}
\hline Reflections collected & 99181 \\
\hline Independent reflections & $17175\left[\mathrm{R}_{\text {int }}=0.1151, \mathrm{R}_{\text {sigma }}=0.0655\right]$ \\
\hline Data/restraints/parameters & $17175 / 12 / 1453$ \\
\hline Goodness-of-fit on $\mathrm{F}^{2}$ & 1.026 \\
\hline Final $\mathrm{R}$ indexes $[\mathrm{I}>=2 \sigma(\mathrm{I})]$ & $\mathrm{R}_{1}=0.0703, \mathrm{wR}_{2}=0.1823$ \\
\hline Final $\mathrm{R}$ indexes [all data] & $\mathrm{R}_{1}=0.1151, \mathrm{wR}_{2}=0.2087$ \\
\hline Largest diff. peak/hole $/ \mathrm{e} \AA^{-3}$ & $1.33 /-0.44$ \\
\hline
\end{tabular}

Single crystals of $\mathrm{C}_{91} \mathrm{H}_{46} \mathrm{BF}_{48} \mathrm{~N}_{3} \mathrm{NiO}\left(\mathbf{C F}_{3}-\mathbf{H}^{+}\right)$were grown by layering a saturated solution of the complex in dichloromethane with pentanes. A suitable crystal was selected and placed on a STOE IPDS 2T diffractometer using a glass fiber. The crystal was kept at $100 \mathrm{~K}$ during data collection. Using Olex $2^{5}$, the structure was solved with the SHELXT ${ }^{6}$ structure solution program using Intrinsic Phasing and refined with the SHELXL ${ }^{9}$ refinement package using Least Squares minimization.

\subsubsection{Comparison of selected bond lengths}

Bond lengths (and their change) are indications of the bond strength. A change, especially near the catalytic center is a hint for a change in the catalytic property.

Table S5: Comparison of selected bond lengths of the complex $3^{M e}$ and $3^{M e}-H^{+}$.

\begin{tabular}{|c|c|c|}
\hline & $\mathbf{3}^{\mathbf{M e}}$ & $\mathbf{3}^{\mathbf{M e}}-\mathbf{H}^{+}$ \\
\hline $\mathrm{d}(\mathrm{O}-\mathrm{Ni})[\AA]$ & 1.879 & 1.905 \\
\hline $\mathrm{d}(\mathrm{N} 1-\mathrm{Ni})[\AA]$ & 1.915 & 1.914 \\
\hline $\mathrm{d}(\mathrm{Ni}-\mathrm{CH}(\mathrm{allyl}))[\AA]$ & 1.917 & 1.992 \\
\hline $\mathrm{d}(\mathrm{C} 1-\mathrm{O})[\AA]$ & 1.981 & 2.008 \\
\hline
\end{tabular}

\section{Ethylene Polymerizations and Oligomerizations}

\subsection{General Procedure}

Polymerization experiments were performed in a BÜCHI miniclave pressure reactor with a $200 \mathrm{~mL}$ steel vessel, equipped with a heating and cooling jacket and a pitched blade stirrer. The temperature was regulated via a JULABO FB 50 thermostat, controlled via a temperature sensor dipping in the reaction mixture. Prior to polymerization, the reactor was heated to $90{ }^{\circ} \mathrm{C}$ under reduced pressure for at least $30 \mathrm{~min}$. The reactor was filled with nitrogen and evacuated three times at an internal temperature $>60{ }^{\circ} \mathrm{C}$. Afterwards, the reactor was cooled down to the desired reaction temperature. $95 \mathrm{~mL}$ of dry and oxygen-free toluene was transferred to the reactor via cannula in a nitrogen counter stream. The catalyst precursor was dissolved or suspended in $5 \mathrm{~mL}$ of toluene and transferred to the reactor via syringe. The reaction mixture was stirred at $1000 \mathrm{rpm}$ and the reactor was pressurized to 40 bar, while the temperature rose. During polymerization, the temperature of the thermostat was adjusted to achieve the desired internal temperature $\left( \pm 1^{\circ} \mathrm{C}\right)$ and the ethylene mass flow was recorded by a BRONKHORST mass flow meter and Bronkhorst flow plot software. At the end of the predetermined polymerization time, the ethylene flow was stopped and the reactor was carefully vented. A sample was taken for GC analysis. The product mixture was transferred to a round bottom flask and the volatiles removed under reduced pressure. Residual volatiles were removed $60^{\circ} \mathrm{C}$ in a vacuum drying oven.

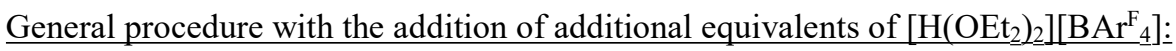

The polymerization was performed analogous to the general procedure for the ethylene polymerization with the following difference: First, the reactor was filled with $90 \mathrm{~mL}$ of toluene (instead of $95 \mathrm{~mL}$ of toluene). Second, 
after the addition of the catalyst precursor, the reactor was pressurized with ethylene at 5 bar for $1 \mathrm{~min}$. The pressure was released and the $\left[\mathrm{H}\left(\mathrm{OEt}_{2}\right)_{2}\right]\left[\mathrm{BAr}_{4}^{\mathrm{F}}\right]$ (suspended in $5 \mathrm{~mL}$ of toluene) was added quickly via syringe. Then, the reactor was pressurized to 40 bar and the polymerization performed as described above.

\subsection{Polymer Analytics}

\subsubsection{NMR Spectroscopy}

3.2.1.1 Determination of the Molecular weight and of the Branching Density

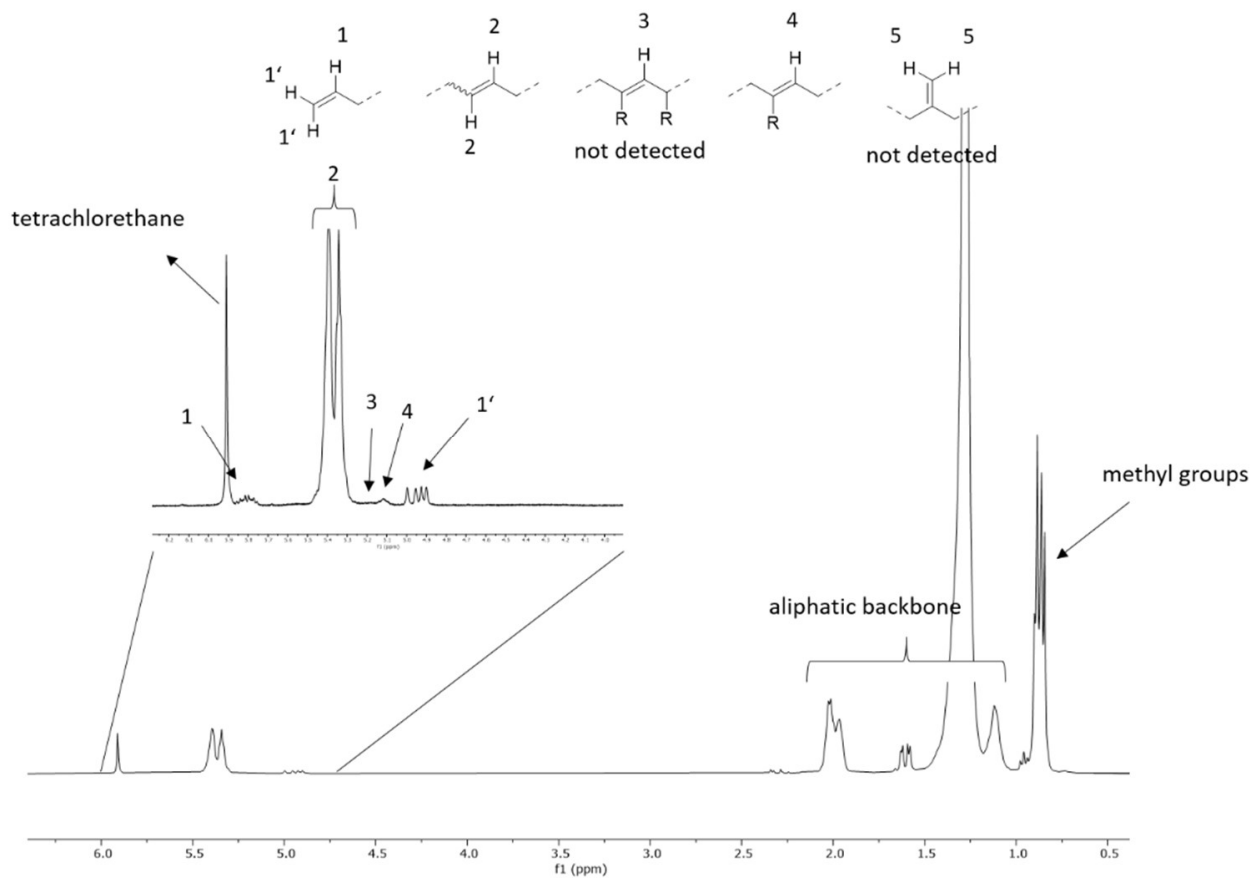

Figure S35: Exemplarily ${ }^{1} H$ NMR spectrum of oligomers obtained with the cationic complexes (Oligomer obtained from an experiment with additional protons in the polymerization mixture, Table 4, entry 2).

The molecular weight from ${ }^{1} \mathrm{H}$ NMR spectra was calculated according to Equation S1:

$$
M_{n}=\frac{\left(\frac{I_{t o t}}{4}\right)}{\left(\frac{I_{2}+2 I_{3}+2 I_{4}+I_{1}+I_{5}}{2}\right)} \cdot 28 \frac{g}{\mathrm{~mol}}
$$

Equation S1: Determination of the molecular weight of the polymerization products from ${ }^{1} H N M R$. Itot is the integral of all signals. 


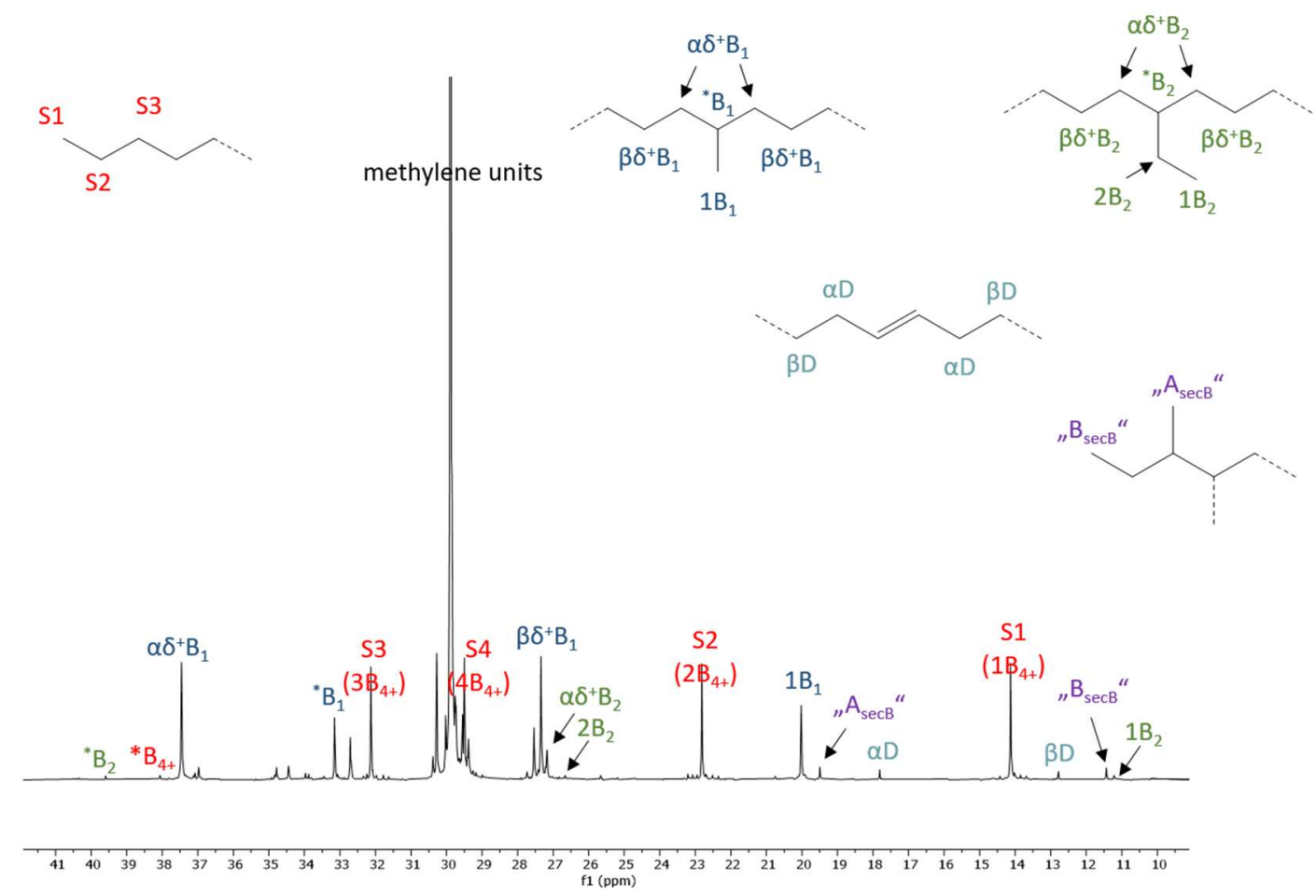

Figure S36: Exemplarily $\left.{ }^{13} C_{\{1}{ }^{1} H\right\} N M R$ spectrum of oligomers obtained with the cationic complexes (oligomer obtained from an experiment with additional protons in the polymerization mixture, Table 4, entry 2). A ${ }^{\text {sec } B u ~ g r o u p ~ c a n ~ b e ~ f o u n d ~ b o t h ~ o n ~ a s ~}$ an end group or as branching motif (giving the same signal), a deviation is only possible using ${ }^{*} B_{4+}$ (which is characteristic for longer branches).

The branching density was calculated according to Equation S2:

$$
\frac{\text { branches }}{1000 C}=\frac{I_{{ }_{B_{1}}}+I_{{ }_{B_{2}}}+I_{{ }_{1} B_{3}}+I_{B_{s e c B}}}{I_{\text {tot }}}
$$

Equation S2: Determination of the branching density of the polymerization products from $\left.{ }^{13} C_{\{}{ }^{1} H\right\} N M R$ (inverse gated). Itot is the integral of all signals.

Percentages of different branch lengths are calculated from the relative intensity ratios of the signals. 


\subsubsection{Complete microstructure analysis}

Table S6: Complete microstructure analysis of the oligomers mentioned in Table 3.

\begin{tabular}{|c|c|c|c|c|c|c|c|c|}
\hline \# & precatalyst & $\begin{array}{l}\mathbf{T} \\
{\left[{ }^{\circ} \mathrm{C}\right]}\end{array}$ & $\begin{array}{l}\mathrm{M}_{\mathrm{n}}(\mathrm{NMR}) \\
{\left[\mathrm{kg} \mathrm{mol}^{-1}\right]^{\mathrm{a}}}\end{array}$ & $\begin{array}{c}\text { Branches/ } \\
1000 \mathrm{C} \\
\text { atoms }^{\mathrm{c}}\end{array}$ & $\% \mathrm{Me}^{\mathrm{c}}$ & $\% \mathbf{E t}^{\mathbf{c}}$ & $\begin{array}{c}\% \\
\text { Prop }^{\mathrm{c}}\end{array}$ & $\begin{array}{c}\% \\
\text { sec } \\
\mathrm{Bu}^{\mathrm{c}}\end{array}$ \\
\hline 1 & $2^{\mathrm{Me}}$ & 30 & 1.5 & 94 & 86 & 7 & 4 & 5 \\
\hline 2 & $2^{\mathrm{Me}}$ & 50 & 1.1 & 96 & 82 & 9 & 3 & 6 \\
\hline 3 & $2^{\mathrm{Me}}-\mathrm{H}^{+}$ & 30 & 0.76 & 23 & 99 & 1 & 0 & 0 \\
\hline 4 & $2^{\mathrm{Me}}-\mathrm{H}^{+}$ & 50 & 0.58 & 33 & 92 & 4 & 4 & 1 \\
\hline 5 & $2^{\mathrm{Me}}-\mathrm{H}^{+}$ & 70 & 0.52 & 35 & 91 & 5 & 4 & 1 \\
\hline 6 & $2^{\mathrm{CF} 3}$ & 30 & i & 5 & 100 & 0 & 0 & 0 \\
\hline 7 & $2^{\mathrm{CF} 3}$ & 50 & i & 15 & 99 & 1 & 0 & 0 \\
\hline 8 & $2^{\mathrm{CF} 3}$ & 70 & $i^{\mathrm{b}}$ & 20 & 97 & 3 & 0 & 0 \\
\hline 9 & $2^{\mathrm{CF} 3}-\mathrm{H}^{+}$ & 30 & 2.4 & 27 & 99 & 1 & 0 & 0 \\
\hline 10 & $2^{\mathrm{CF} 3}-\mathrm{H}^{+}$ & 50 & 4.5 & 23 & 100 & 0 & 0 & 0 \\
\hline 11 & $2^{\mathrm{CF} 3}-\mathrm{H}^{+}$ & 70 & 3.6 & 24 & 99 & 1 & 0 & 0 \\
\hline
\end{tabular}

a) from ${ }^{1} \mathrm{H}$ NMR; determined by comparing the olefinic signals to the backbone; b) no olefinic end groups detectable c) determined by ${ }^{13} \mathrm{C}\left\{{ }^{1} \mathrm{H}\right\} \mathrm{NMR}$.

Table S7: Complete microstructure analysis of the oligomers mentioned in Table 4

\begin{tabular}{|c|c|c|c|c|c|c|c|c|}
\hline \# & precatalyst & $\begin{array}{c}\text { Equiv. } \\
{\left[\mathrm{H}\left(\mathrm{OEt}_{2}\right)_{2}\right]\left[\mathrm{BAr}^{\mathrm{F}}{ }_{4}\right]}\end{array}$ & $\begin{array}{c}\mathrm{M}_{\mathrm{n}}(\mathrm{NMR}) \\
{\left[\mathrm{g} \mathrm{mol}^{-1}\right]^{\mathrm{a}}}\end{array}$ & $\begin{array}{c}\text { Branches/ } \\
1000 \mathrm{C} \text { atoms }\end{array}$ & $\begin{array}{c}\% \\
\mathbf{M e}^{\mathrm{b}}\end{array}$ & $\% \mathbf{E t}^{\mathbf{b}}$ & $\begin{array}{c}\% \\
\text { Prop }^{b}\end{array}$ & $\begin{array}{c}\% \\
\text { sec } \\
\text { Bu }^{b}\end{array}$ \\
\hline 1 & $2^{\mathrm{Me}}-\mathrm{H}^{+}$ & 0.0 & 0.76 & 23 & 99 & 1 & 0 & 0 \\
\hline 2 & $2^{\mathrm{Me}}-\mathrm{H}^{+}$ & 1.0 & 0.62 & 22 & 99 & 1 & 0 & 0 \\
\hline 3 & $2^{\mathrm{CF} 3}-\mathrm{H}^{+}$ & 0.0 & 2.4 & 27 & 99 & 1 & 0 & 0 \\
\hline 4 & $2^{\mathrm{CF} 3}-\mathrm{H}^{+}$ & 1.0 & 0.8 & 39 & 96 & 3 & 0 & 0 \\
\hline 5 & $2^{\mathrm{CF} 3}-\mathrm{H}^{+}$ & 1.5 & 0.8 & 43 & 95 & 4 & 1 & 0 \\
\hline
\end{tabular}

a) determined from ${ }^{1} \mathrm{H}$ NMR; determined by comparing the olefinic signals to the backbone; b) determined from ${ }^{13} \mathrm{C}\left\{{ }^{1} \mathrm{H}\right\} \mathrm{NMR}$.

\subsubsection{DSC Traces}

\subsubsection{DSC Traces for the Polymers from $\mathbf{2}^{\mathrm{CF} 3}$}

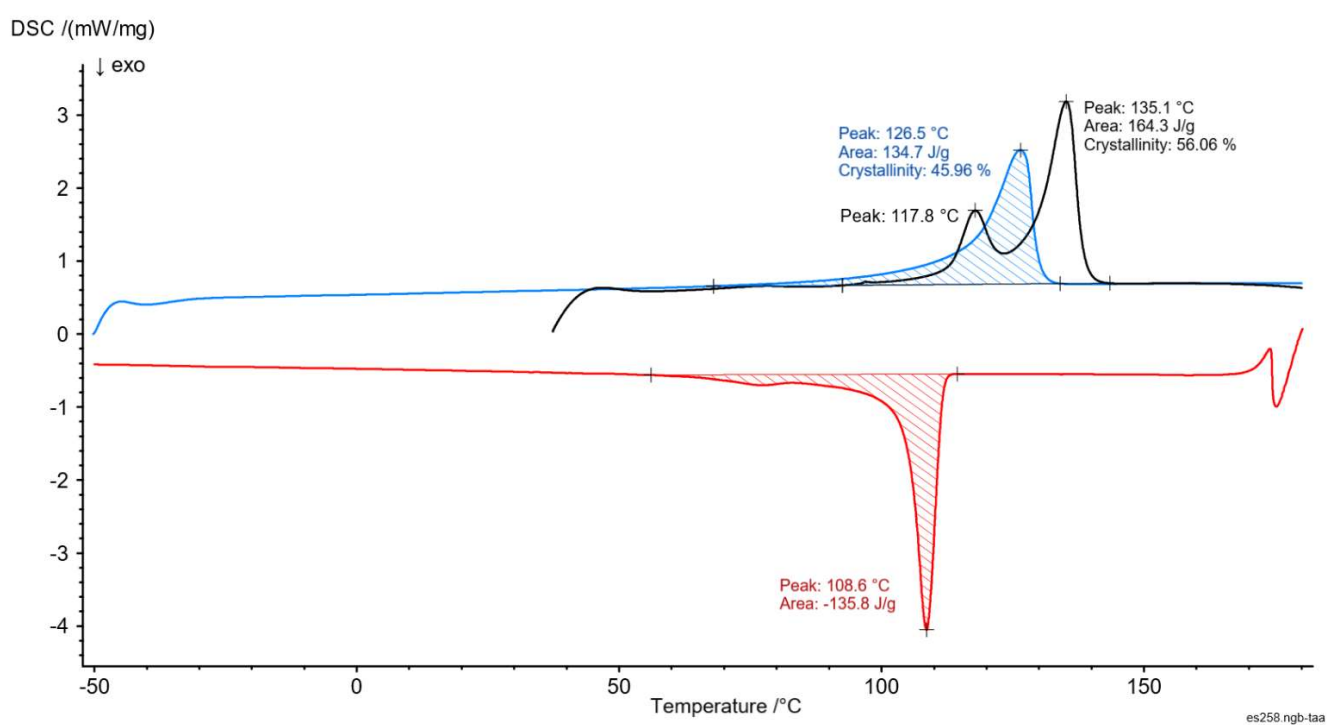

Figure S37: DSC trace for the polymer formed with complex $2^{\mathrm{CF} 3}, \mathrm{~T}=30^{\circ} \mathrm{C}$. 


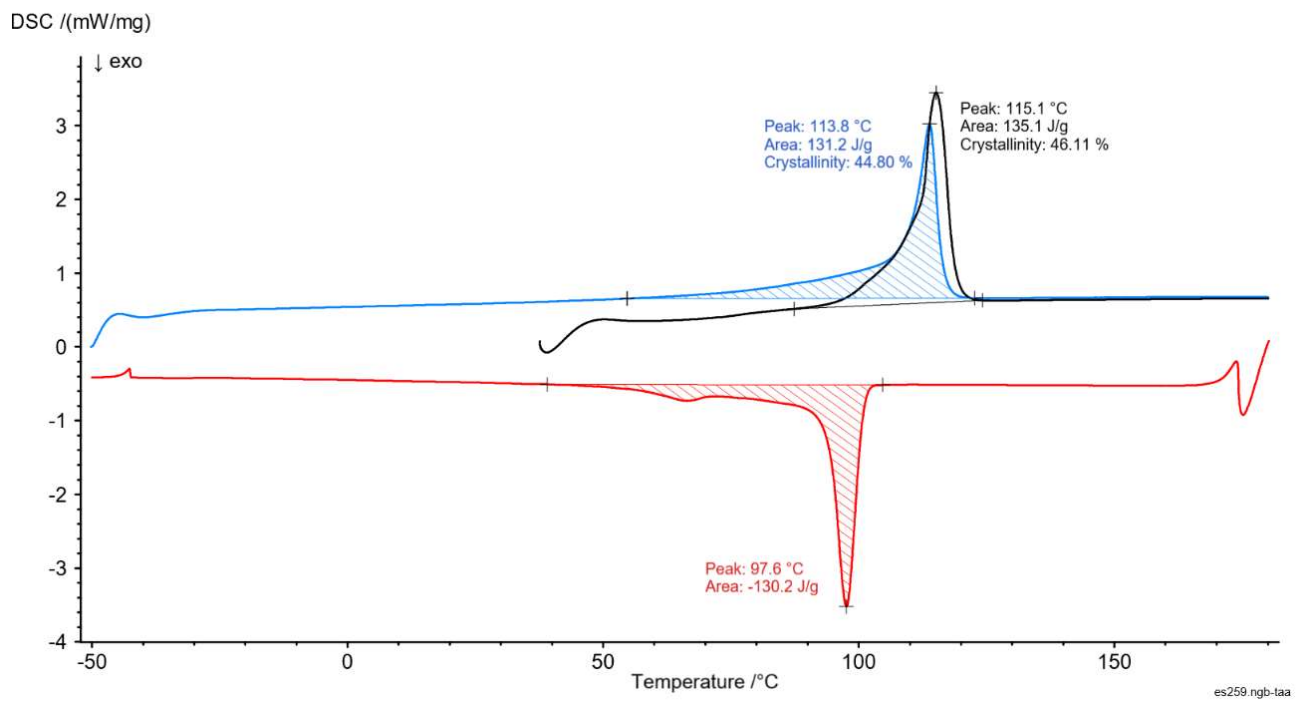

Figure S38: DSC trace for the polymer from the complex $2^{\mathrm{CF} 3}, \mathrm{~T}=50^{\circ} \mathrm{C}$.

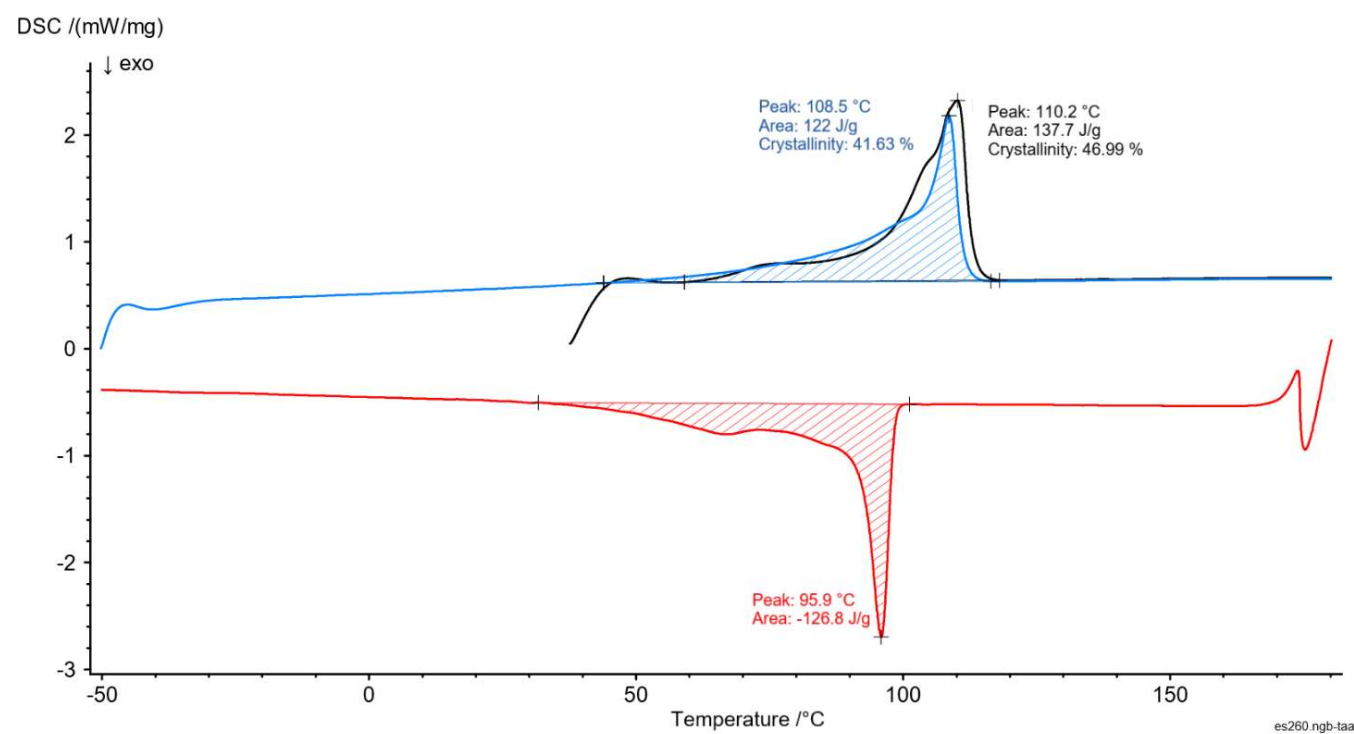

Figure S39: DSC trace for the polymer from the complex $2^{C F 3}, T=70^{\circ} \mathrm{C}$. 
3.2.2.2 DSC traces for the polymers from $\mathbf{2}^{\mathbf{C F 3}}-\mathbf{H}^{+}$

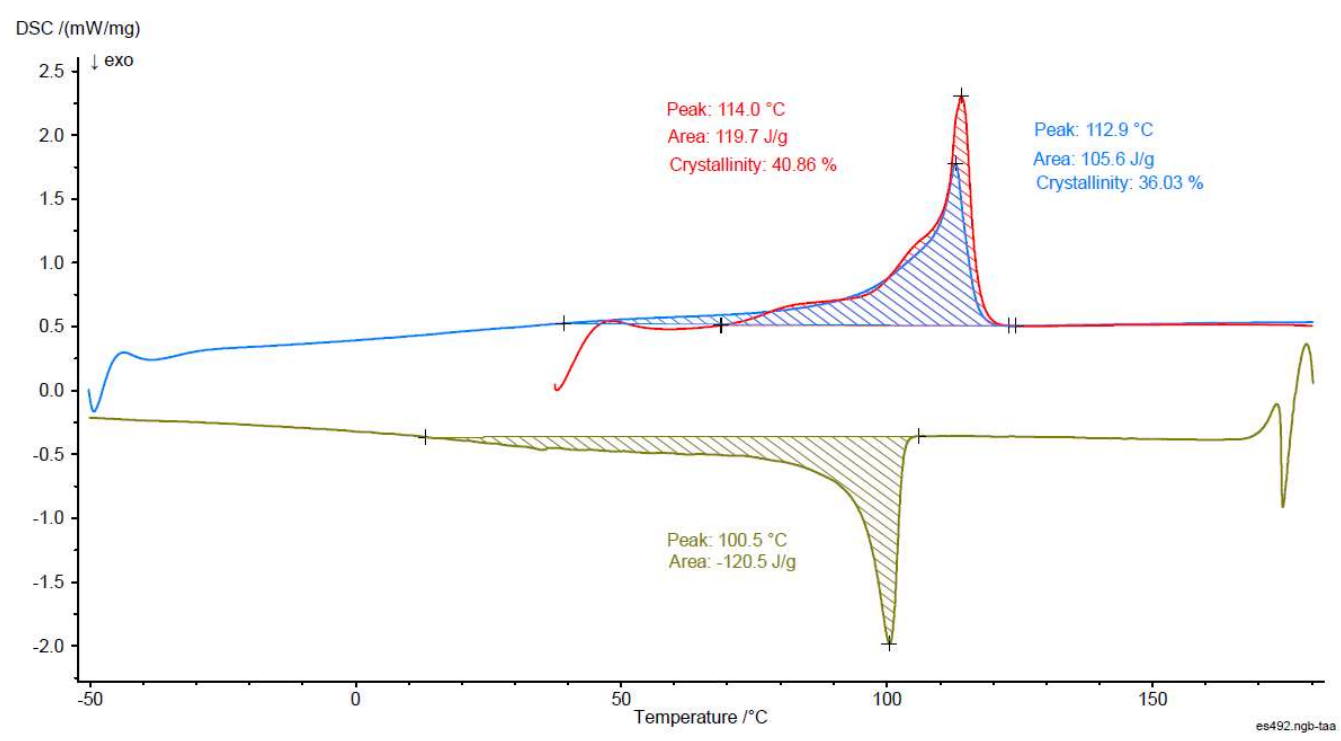

Figure S40: DSC trace for the polymer from the complex $2^{\mathrm{CF} 3}-\mathrm{H}^{+}, \mathrm{T}=30^{\circ} \mathrm{C}$.

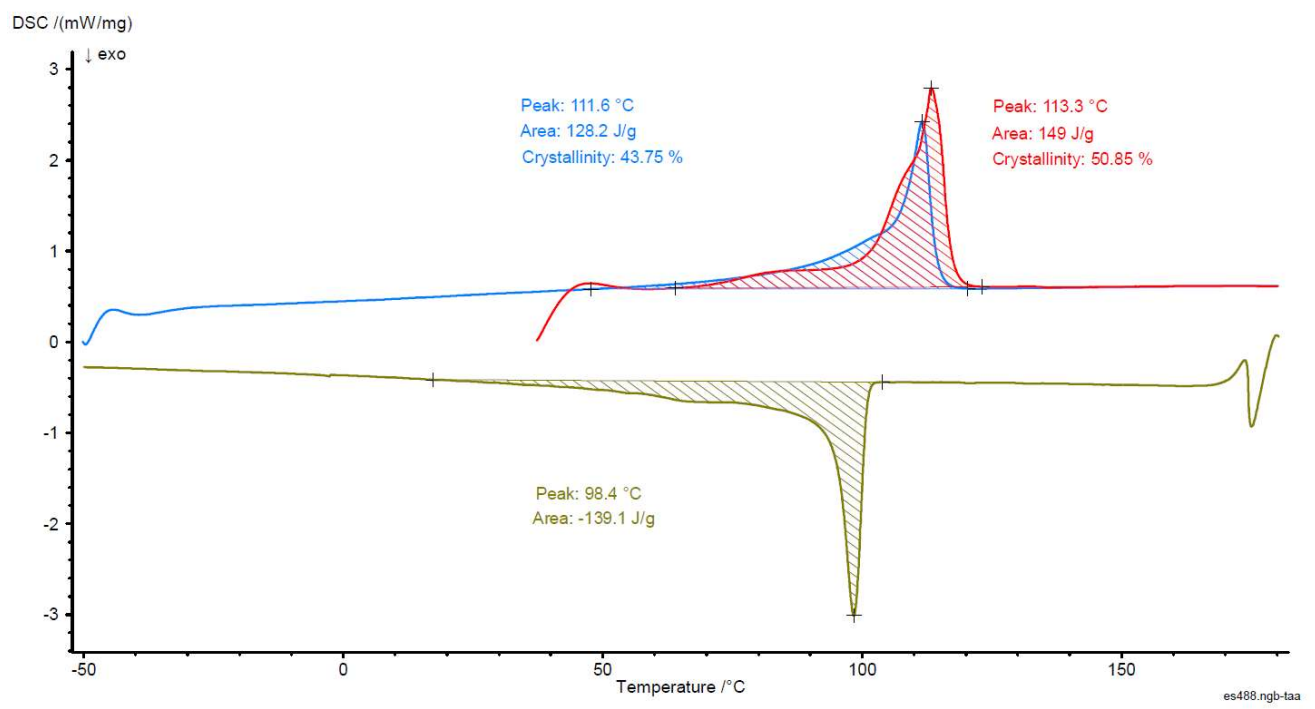

Figure S41: DSC trace for the polymer from the complex $2^{\mathrm{CF}}-\mathrm{H}^{+}, \mathrm{T}=50^{\circ} \mathrm{C}$. 


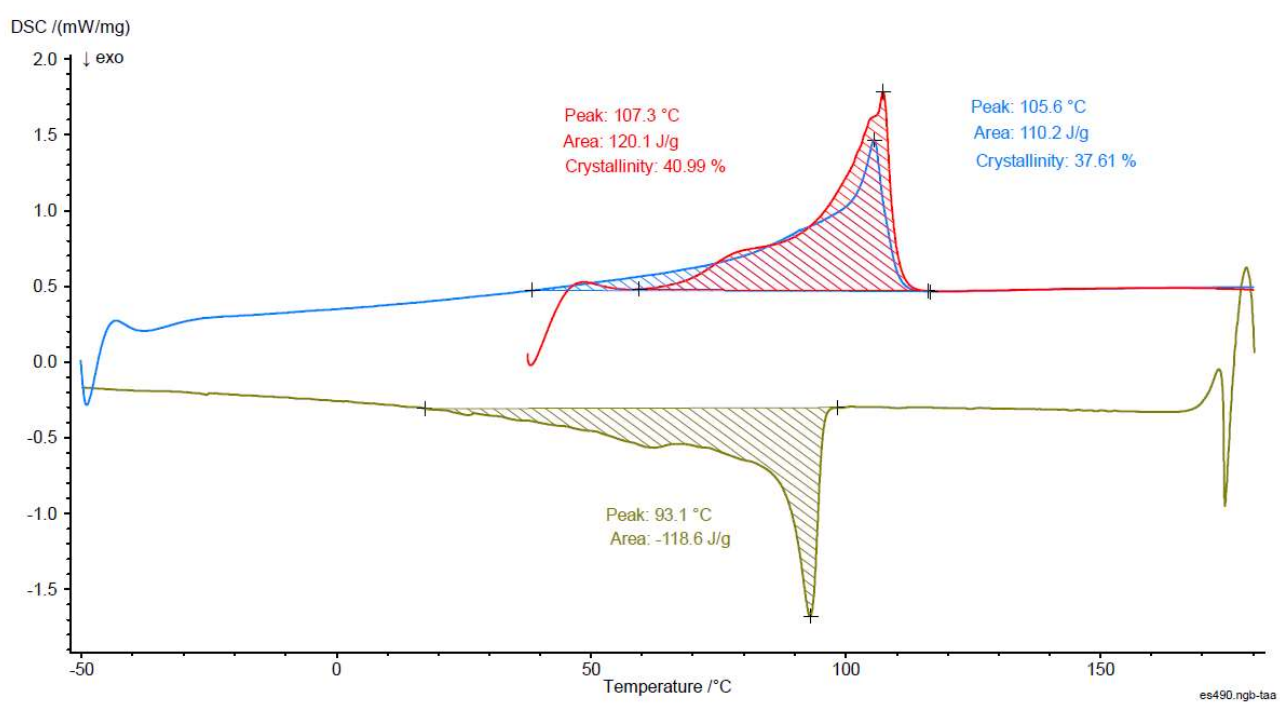

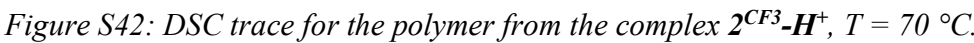

\subsubsection{DSC traces for the polymers from $\mathbf{2}^{\mathrm{Me}}-\mathbf{H}^{+}$}

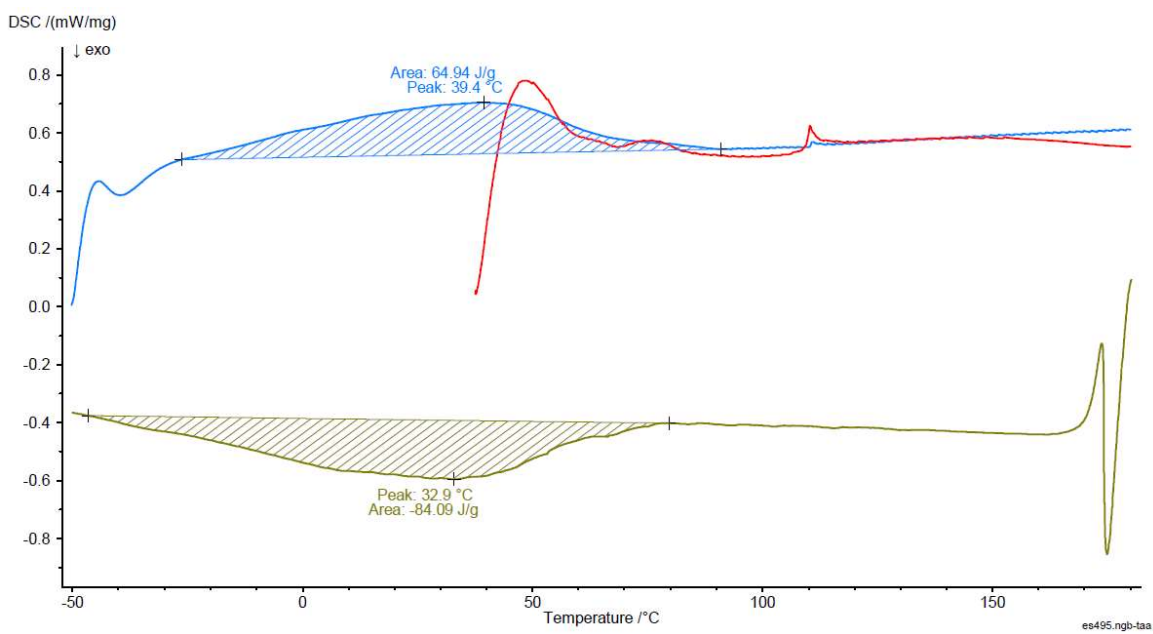

Figure S43: DSC trace for the polymer from the complex $2^{\mathrm{Me}}-\mathrm{H}^{+}, T=30^{\circ} \mathrm{C}$.

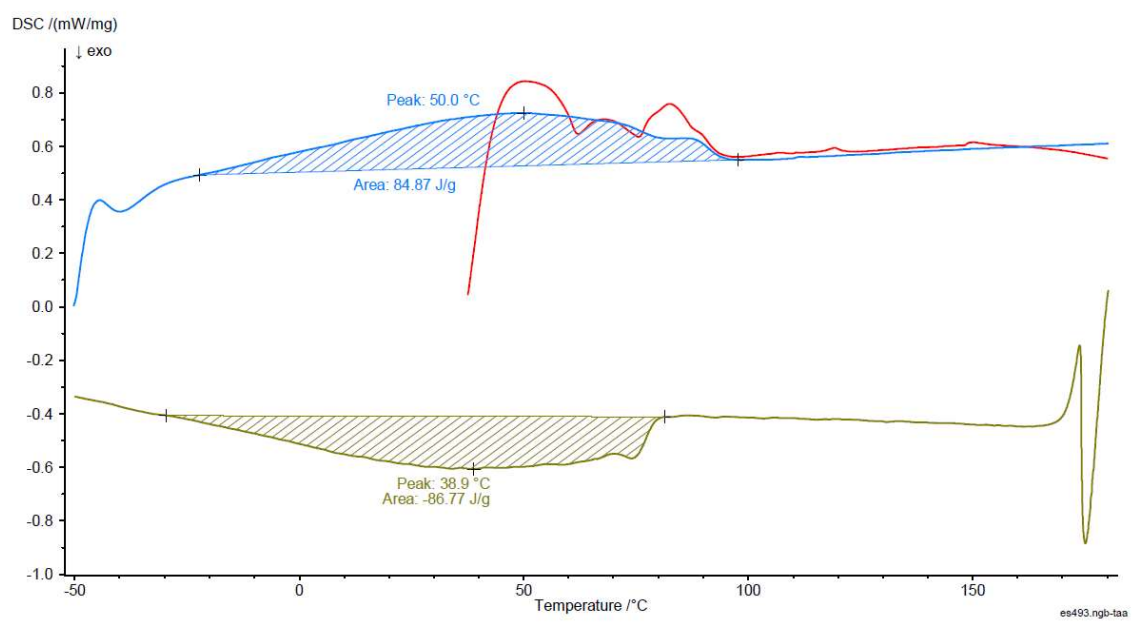

Figure S44: DSC trace for the polymer from the complex $2^{M e_{-}} \boldsymbol{H}^{+}, T=50^{\circ} \mathrm{C}$. 


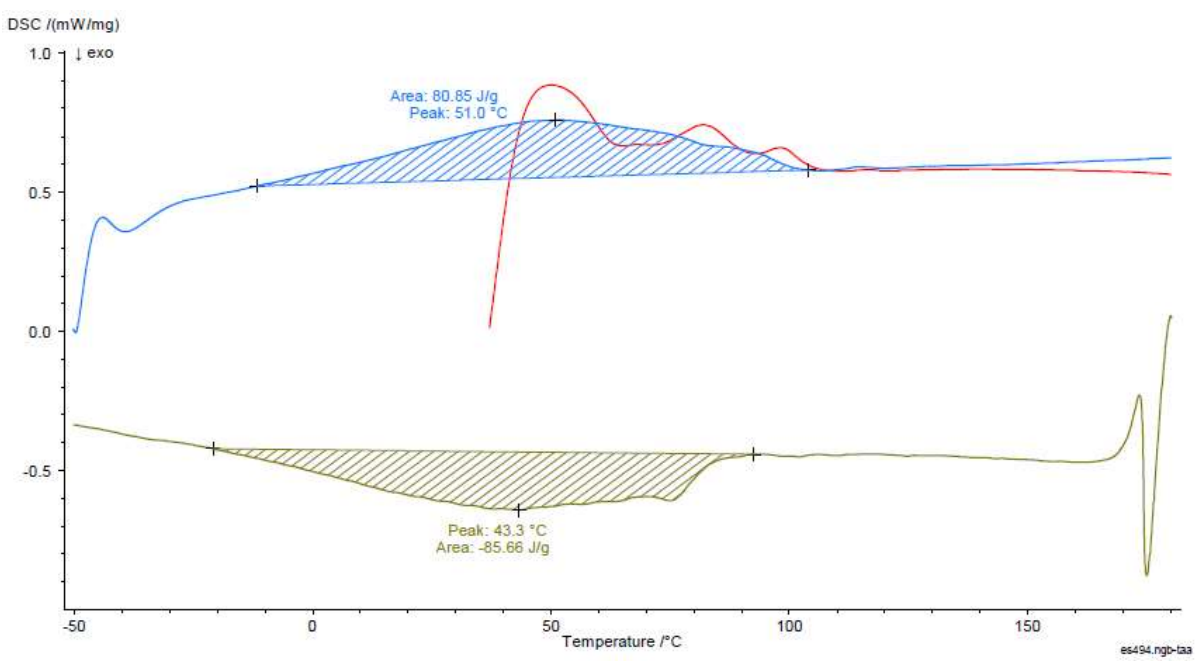

Figure S45: DSC trace for the polymer from the complex $2^{\mathrm{Me}}-\mathrm{H}^{+}, \mathrm{T}=70^{\circ} \mathrm{C}$.

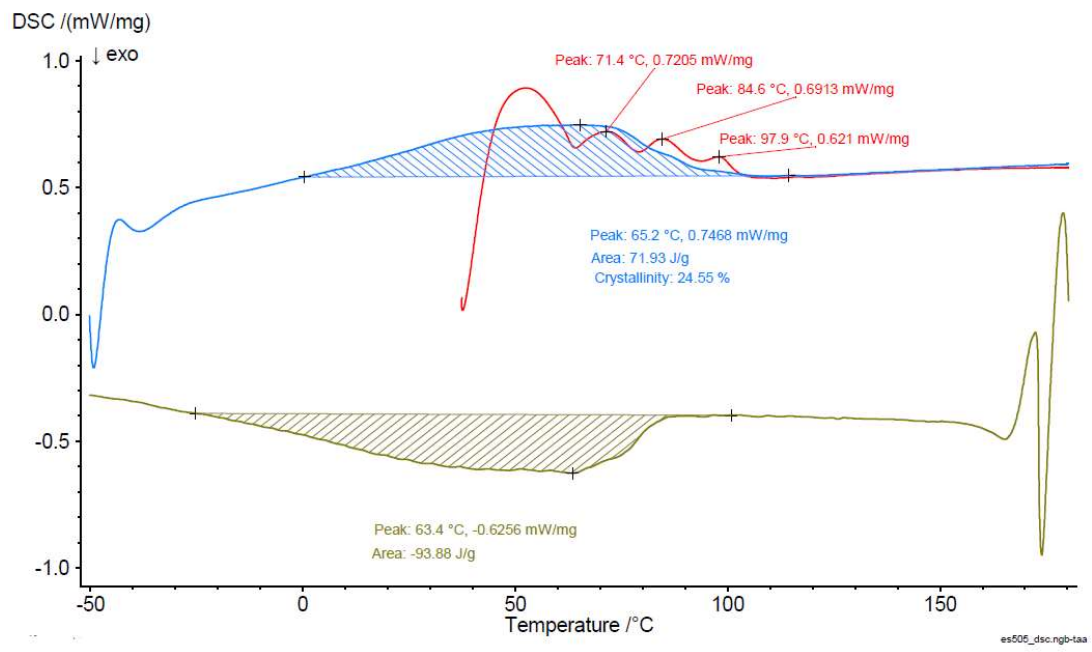

Figure S46: DSC trace for the polymer from the complex $2^{\mathrm{Me}}-\boldsymbol{H}^{+}, T=30^{\circ} \mathrm{C}$, addition of 1.0 equiv. [H(OEt $\left.)_{2}\right]\left[\mathrm{BAr}^{F_{4}}\right]$.

\subsubsection{GPC Traces}

3.2.3.1 GPC traces for the oligomers formed with $2^{\mathrm{Me}}$

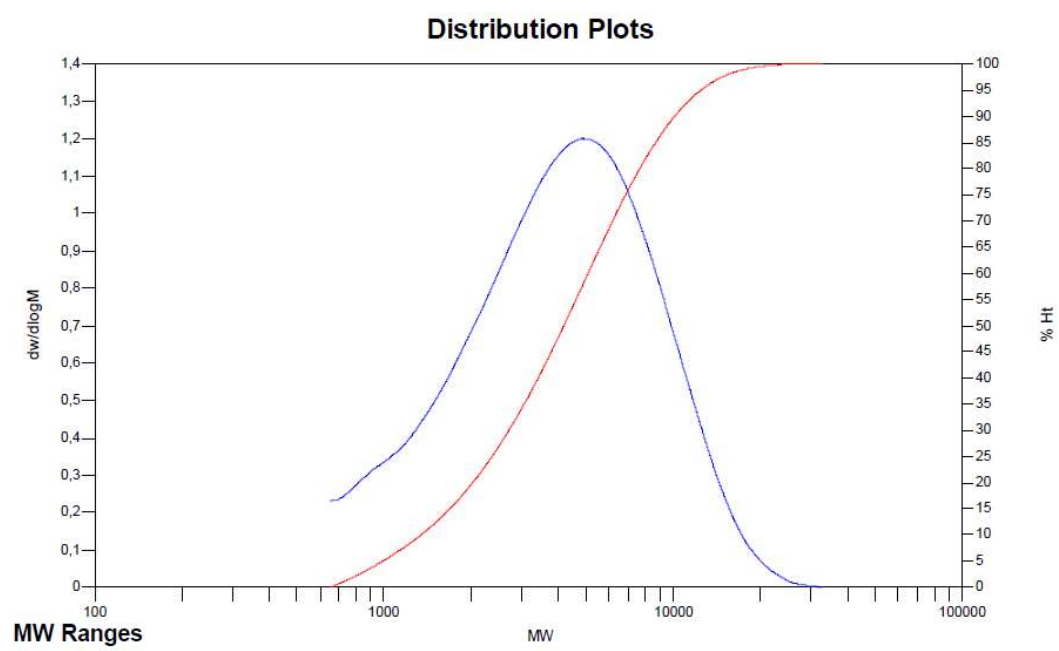

Figure S47: GPC trace for the oligomer formed with $2^{\mathrm{Me}}, \mathrm{T}=30^{\circ} \mathrm{C}$. 


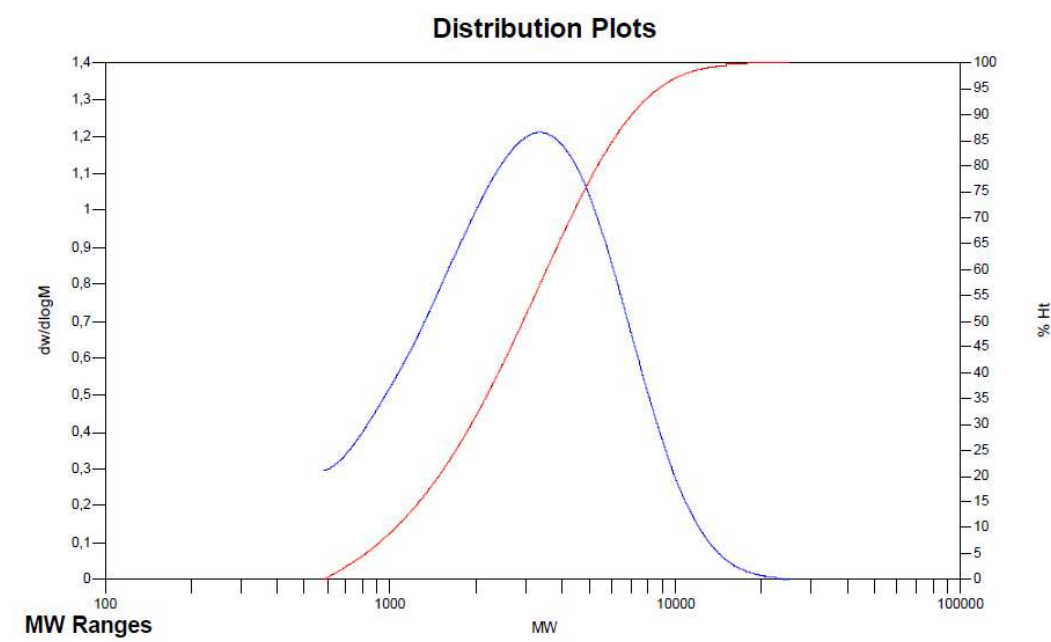

Figure S48: GPC trace for the oligomer formed with $2^{\mathrm{Me}}, T=50^{\circ} \mathrm{C}$.

3.2.3.2 GPC traces for the polymers formed with $2^{\text {CF3 }}$

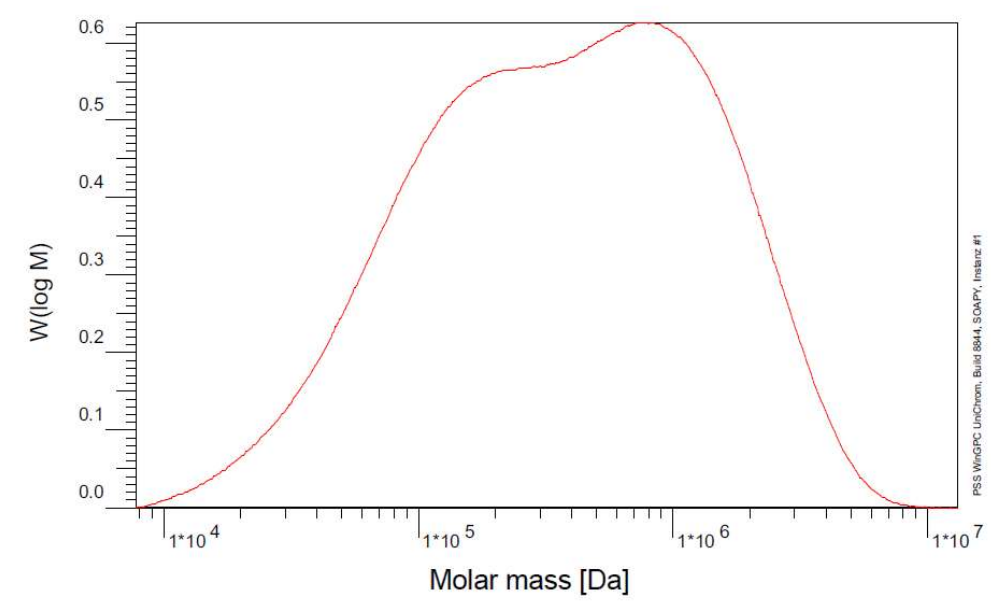

Figure S49: GPC trace for the polymer formed with $2^{C F 3}, T=30^{\circ} \mathrm{C}$.

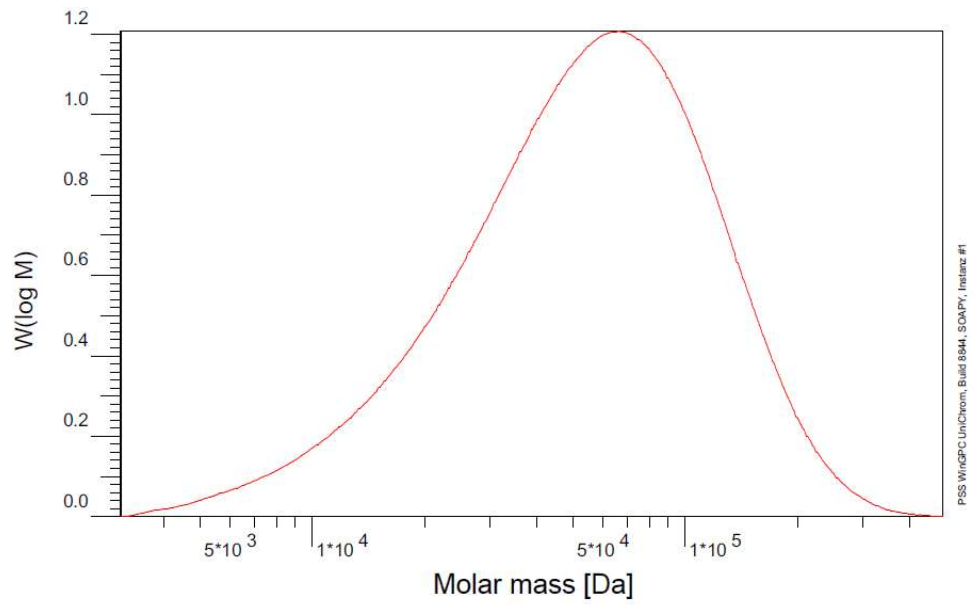

Figure S50: GPC trace for the polymer formed with $2^{C F 3}, T=50^{\circ} \mathrm{C}$. 


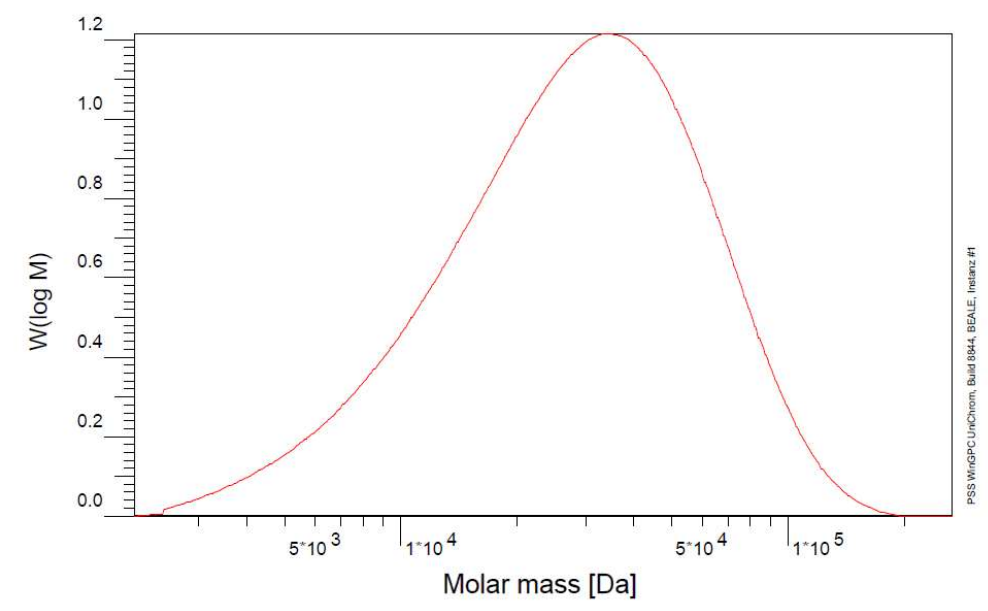

Figure S51: GPC trace for the polymer formed with $2^{\mathrm{CF} 3}, \mathrm{~T}=70^{\circ} \mathrm{C}$.

3.2.3.3 GPC traces for the polymers formed with $\mathbf{2}^{\mathbf{C F 3}}-\mathbf{H}^{+}$

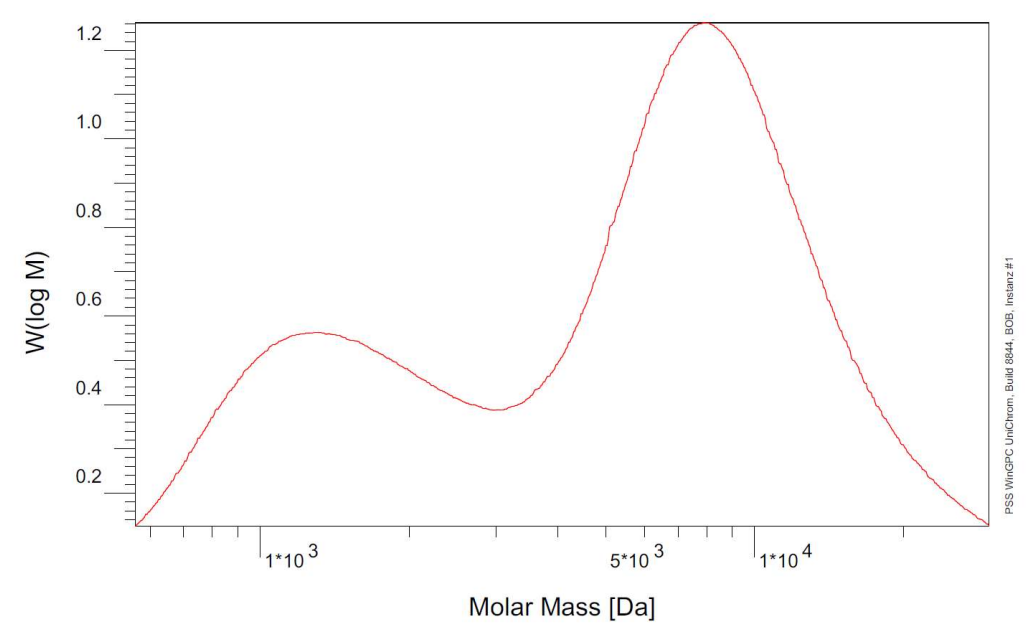

Figure S52: GPC trace for the polymer formed with $2^{C F 3}-H^{+}, T=30^{\circ} \mathrm{C}$.

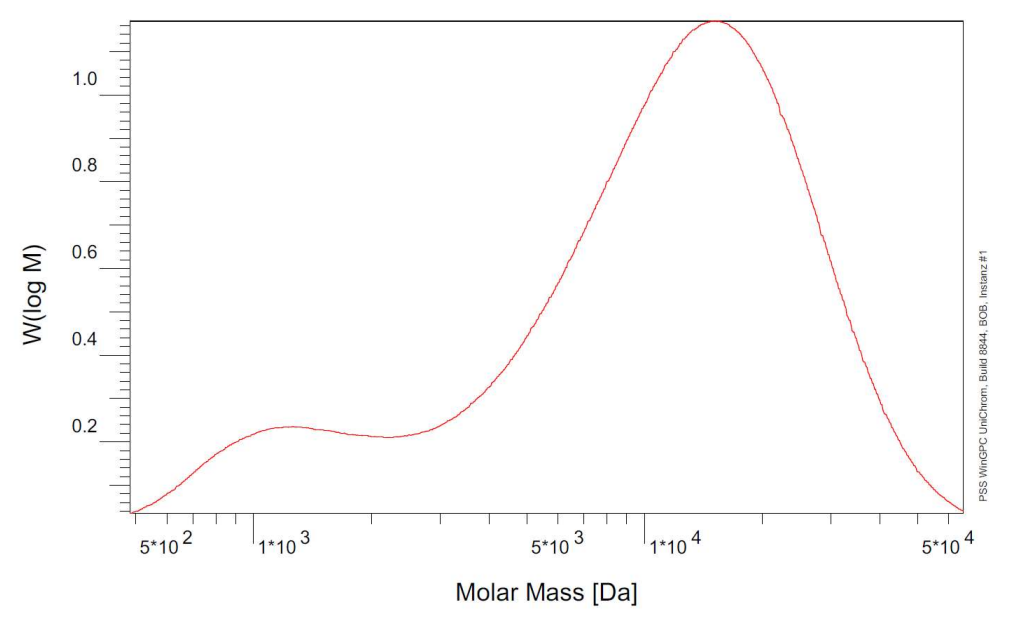

Figure S53: GPC trace for the polymer formed with $2^{C F 3}-H^{+}, T=50^{\circ} \mathrm{C}$. 


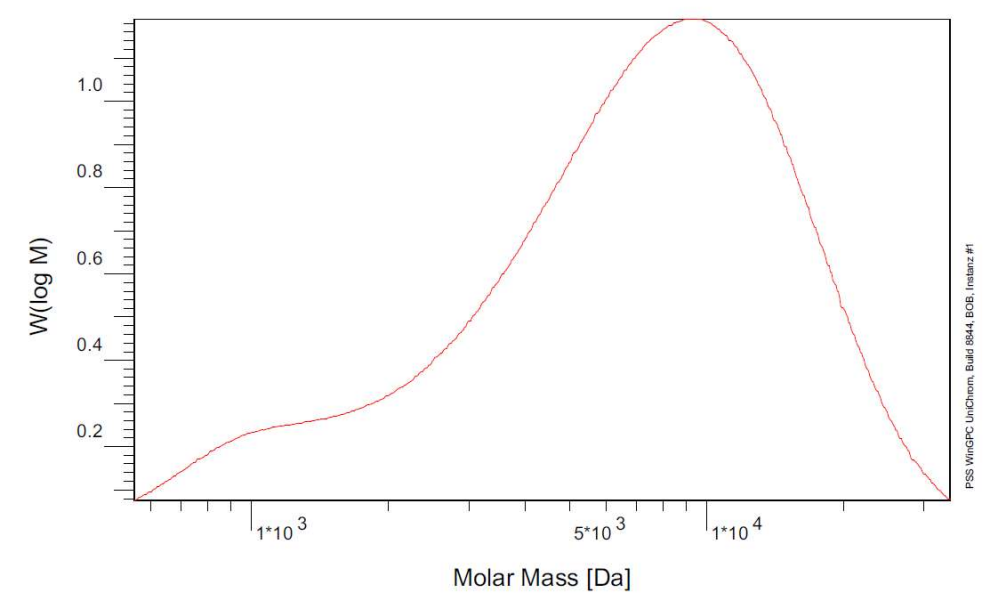

Figure S54: GPC trace for the polymer formed with $2^{\mathrm{CF}}-\mathrm{H}^{+}, T=70^{\circ} \mathrm{C}$.

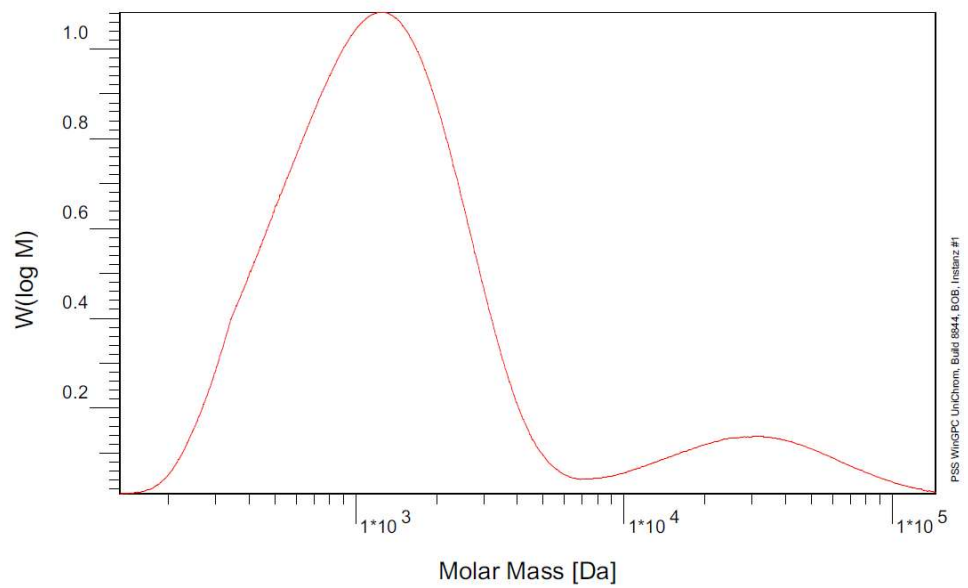

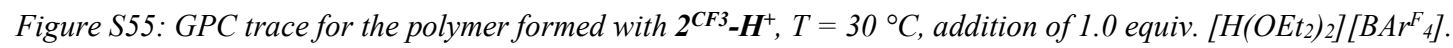

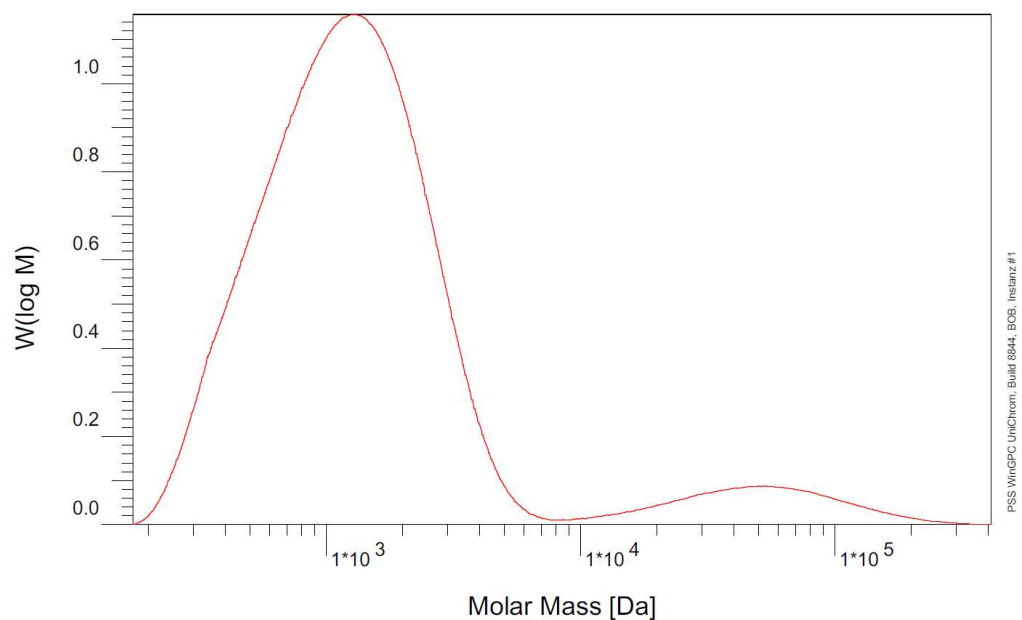

Figure S56: GPC trace for the polymer formed with $2^{C F^{3}}-\mathrm{H}^{+}, T=30^{\circ} \mathrm{C}$, addition of 1.5 equiv. $\mathrm{H}-\mathrm{B}\left(\mathrm{Ar} \mathrm{r}^{\mathrm{F}}\right)_{4}$ 
3.2.3.4 GPC traces for the polymers formed with $\mathbf{2}^{\mathrm{Me}}-\mathbf{H}^{+}$

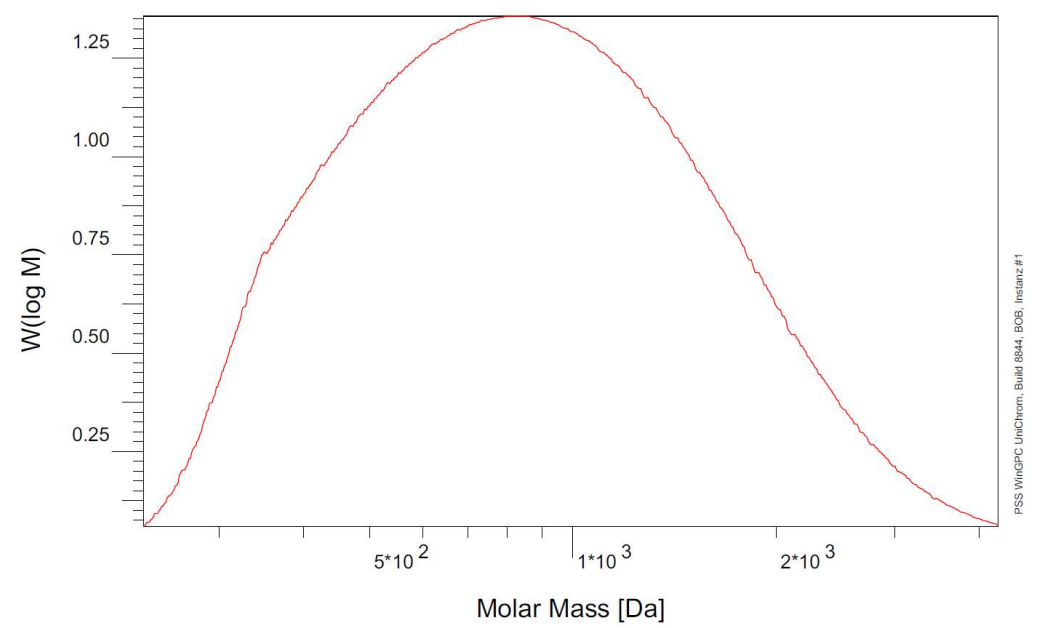

Figure S57: DSC trace for the polymer formed with $2^{M e}-H^{+}, T=30^{\circ} \mathrm{C}$.

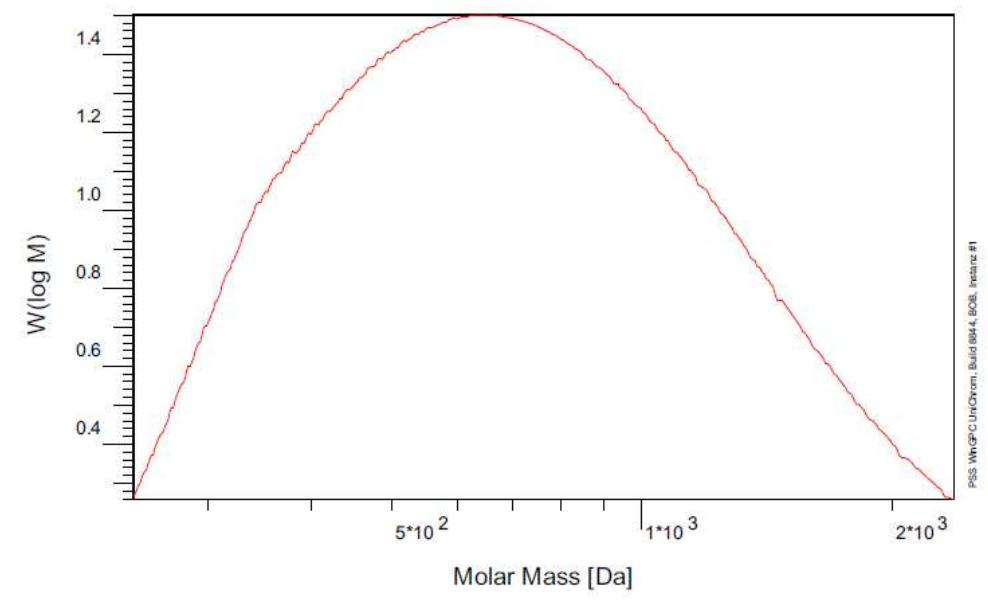

Figure S58: DSC trace for the polymer formed with $2^{\mathrm{Me}}-\mathrm{H}^{+}, T=50^{\circ} \mathrm{C}$.

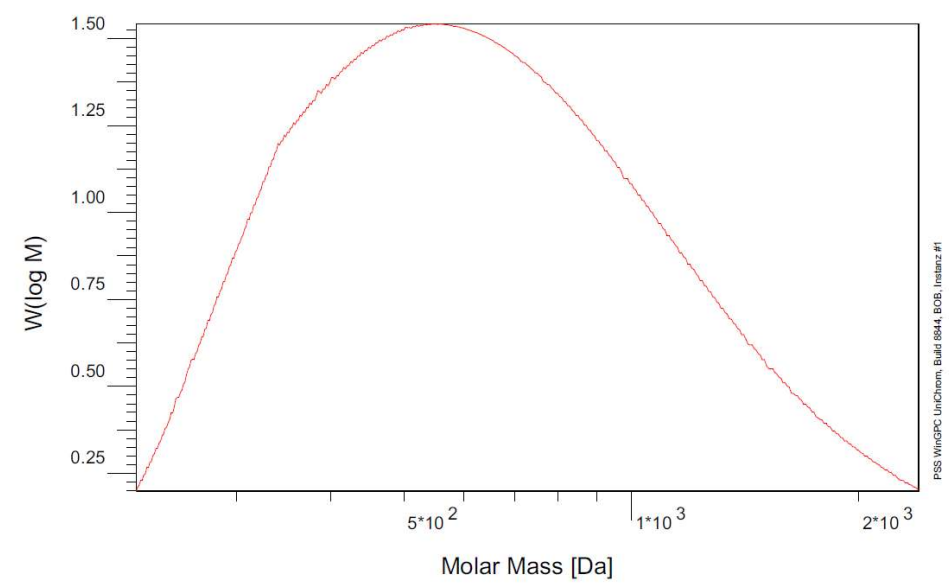

Figure S59: DSC trace for the polymer formed with $2^{\mathrm{Me}}-\boldsymbol{H}^{+}, T=70^{\circ} \mathrm{C}$. 


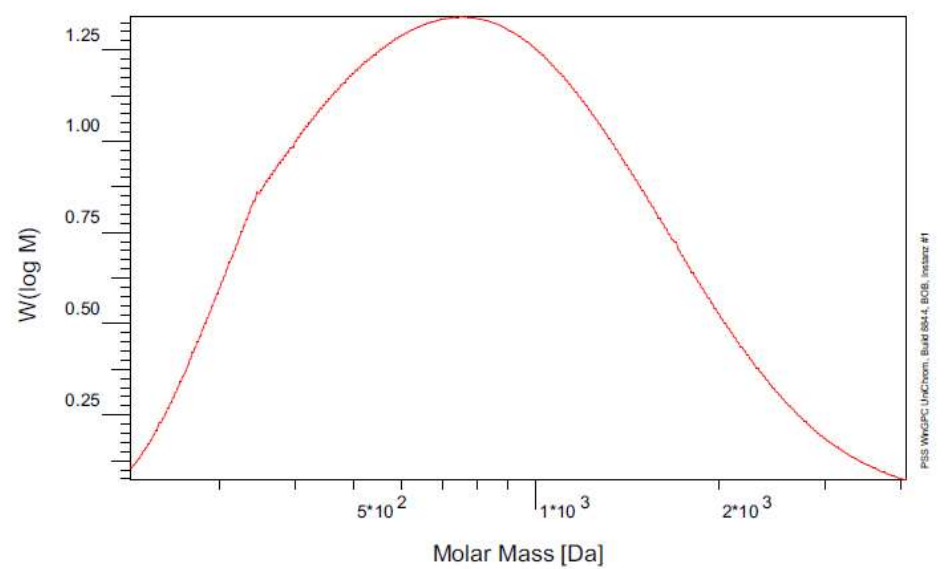

Figure S60: DSC trace for the polymer formed with $2^{\mathrm{Me}}-\mathrm{H}^{+}, \mathrm{T}=30^{\circ} \mathrm{C}$, addition of 1.0 equiv. [H(OEt $\left.)_{2}\right]\left[B A r^{F} 4\right]$.

\subsubsection{Gas Chromatograms}

3.2.4.1 Gas Chromatograms for the polymerizations with $\mathbf{2}^{\mathbf{C F} 3}-\mathbf{H}^{+}$

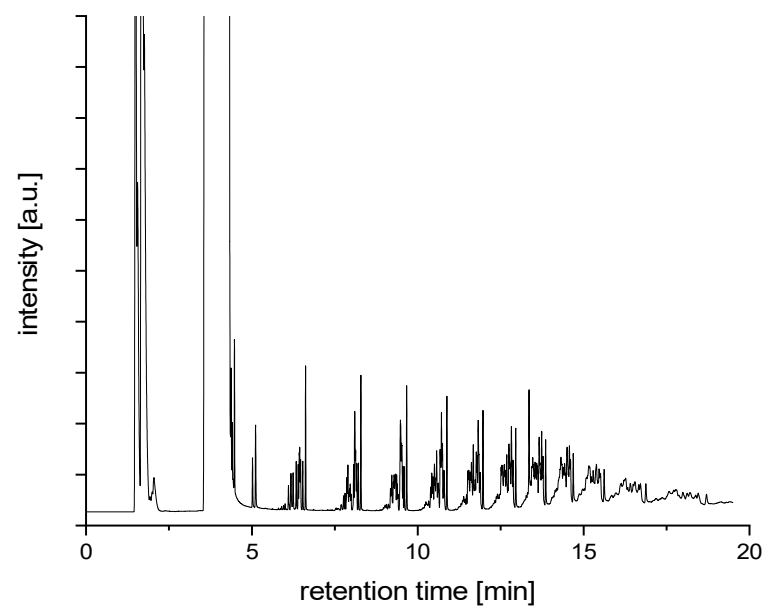

Figure S61: GC trace for the reaction mixture from a polymerization with $2^{\mathrm{CF}^{3}}-\mathrm{H}^{+}, \mathrm{T}=30^{\circ} \mathrm{C}$, addition of 1.0 equiv. [H(OEt $\left.\left.2_{2}\right)_{2}\right]\left[\mathrm{BAr}_{4}{ }_{4}\right]$. 
3.2.4.2 GC-plots for the polymerizations with $\mathbf{2}^{\mathrm{Me}}-\mathbf{H}^{+}$

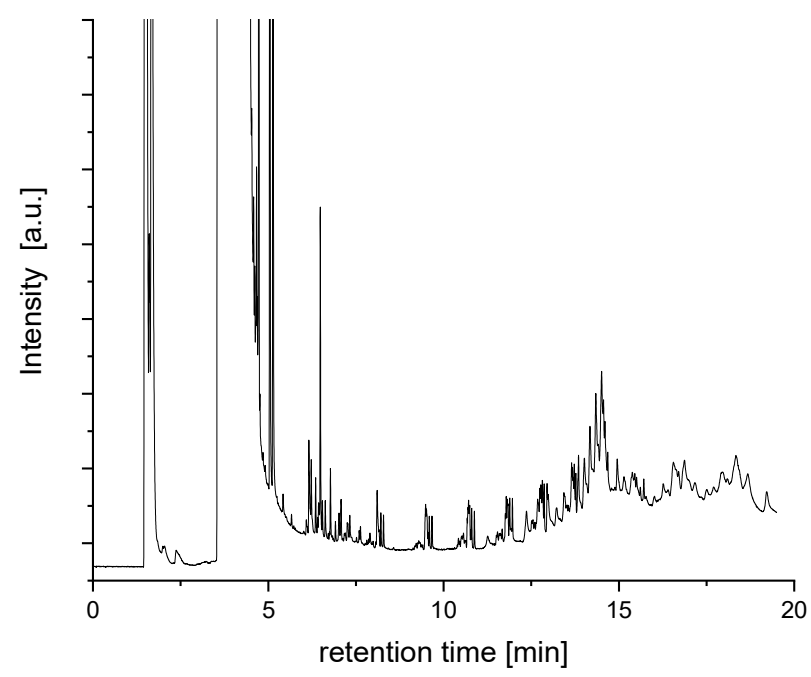

Figure S62: GC trace for the reaction mixture from a polymerization with $\mathbf{2}^{\mathrm{Me}}-\mathrm{H}^{+}, \mathrm{T}=30^{\circ} \mathrm{C}$.

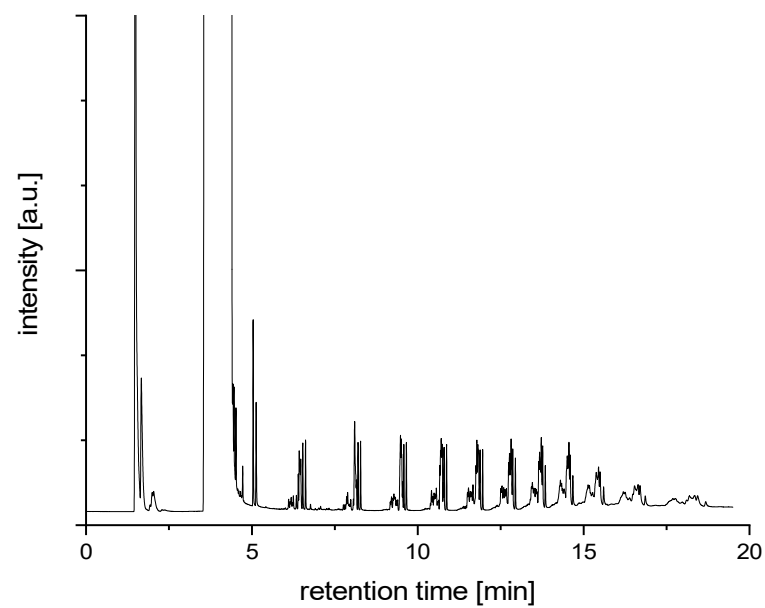

Figure S63: GC trace for the reaction mixture from a polymerization with $2^{\mathrm{Me}}-\mathrm{H}^{+}, T=50^{\circ} \mathrm{C}$. 


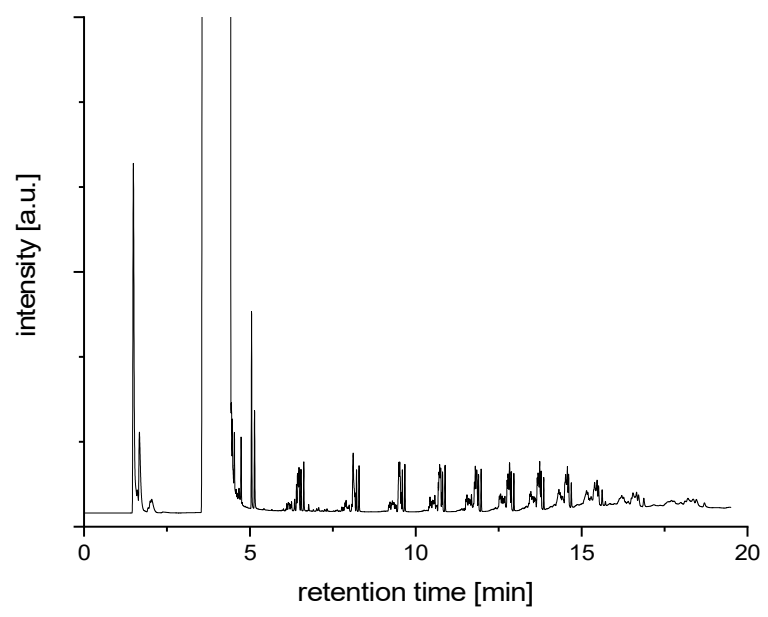

Figure S64: GC trace for the reaction mixture from a polymerization with $\mathbf{2}^{\mathrm{Me}}-\mathrm{H}^{+}, T=70^{\circ} \mathrm{C}$.

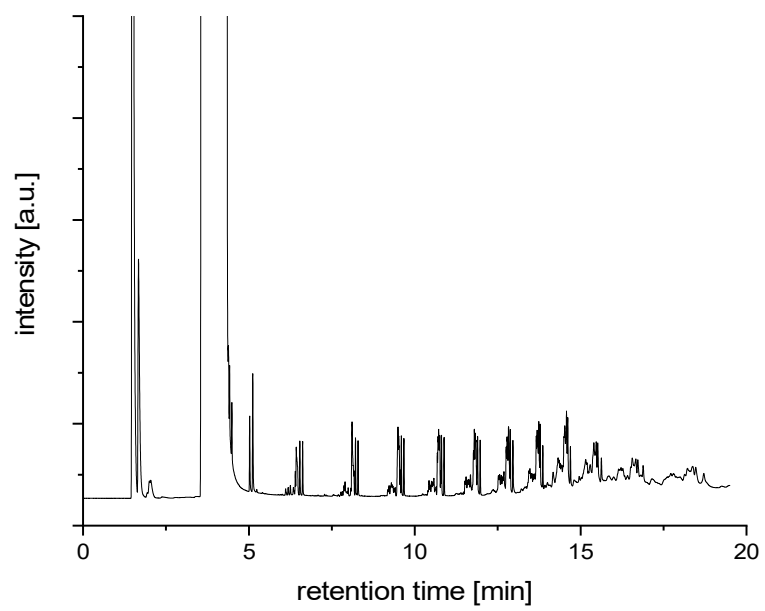

Figure S65: GC trace for the reaction mixture from a polymerization with $2^{\mathrm{Me}}-\mathrm{H}^{+}, \mathrm{T}=30^{\circ} \mathrm{C}$, addition of 1.0 equiv. $\left[\mathrm{H}\left(\mathrm{OEt}_{2}\right)_{2}\right]\left[\mathrm{BAr}_{4}{ }_{4}\right]$.

\subsection{Reference Experiments}

3.3.1 Addition of $\left[\mathrm{H}_{(}\left(\mathrm{OEt}_{2}\right)_{2}\right]\left[\mathrm{BAr}_{4} \mathrm{~F}_{4}\right]$ to Polymerizations with Mono(imino)phenoxy Catalysts

\section{Comparison to another $\mathrm{CF}_{3}$-substituted catalyst}

Reference catalyst precursor: $\mathbf{C F}_{\mathbf{3}}-\mathbf{I 2}^{2,10}$

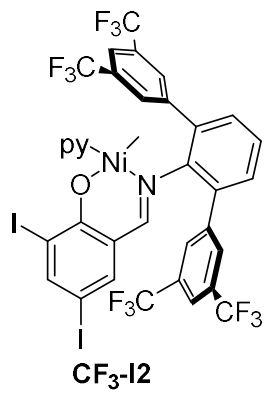

Figure S66: $\mathrm{CF}_{3}$-substituted catalyst precursor: $\boldsymbol{C F}_{3}-\mathbf{I 2}$. 
Table S8: Polymerization results from polymerizations with $\mathbf{C F}_{3}-\mathbf{I 2}$ without or with additional equiv. of $\left.\left[\mathrm{H}_{(\mathrm{OEt}}\right)_{2}\right]\left[\mathrm{BAr}_{4}^{F_{4}}\right]$.

\begin{tabular}{|c|c|c|c|c|}
\hline$\#$ & precatalyst & $\begin{array}{c}\text { equiv. } \\
{\left[\mathbf{H}\left(\mathbf{O E t}_{2}\right)_{\mathbf{2}}\right]\left[\mathbf{B A r}^{\mathbf{F}_{4}} \mathbf{4}\right]}\end{array}$ & yield $[\mathbf{g}]$ & $\mathbf{T}_{\mathbf{m}}\left[{ }^{\mathbf{}} \mathbf{C}^{\mathbf{a}}\right.$ \\
\hline $\mathbf{1}$ & $\mathrm{CF}_{3}$-I2 & 0.0 & 2.0 & 130 \\
\hline $\mathbf{2}$ & $\mathrm{CF}_{3}-\mathrm{I} 2$ & 1.05 & 2.9 & 127 \\
\hline
\end{tabular}

Polymerization conditions: $n$ (precat) $=5 \mu \mathrm{mol}, \mathrm{T}=30^{\circ} \mathrm{C}, t=30 \mathrm{~min}, p=40 \mathrm{bar}, 100 \mathrm{~mL}$ toluene, $1000 \mathrm{rpm}$. Precatalyst solution in toluene saturated with ethylene for $1 \mathrm{~min}$ at 5 bar prior to the addition of $\left[\mathrm{H}\left(\mathrm{OEt}_{2}\right)_{2}\right]\left[\mathrm{BAr}_{4}{ }_{4}\right]$. a) Determined by DSC at a heating rate of $10 \mathrm{~K} / \mathrm{min}$, second heating cycle.

\section{Comparison to another Me-substituted catalyst}

Reference catalyst precursor: Me-I2 $\mathbf{2}^{2,10}$

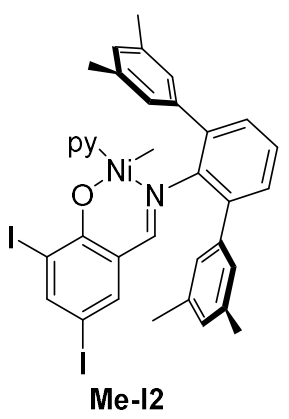

Figure S67: Me-substituted catalyst precursor: Me-I2.

Table S9: Polymerization results from polymerizations with Me-I2 without or with additional equiv. of [H(OEt $\left.)_{2}\right]\left[B A r^{F_{4}}\right]$.

\begin{tabular}{|c|c|c|c|c|c|}
\hline$\#$ & precatalyst & $\begin{array}{c}\text { equiv. } \\
{\left[\mathbf{H}\left(\mathbf{O E t}_{\mathbf{2}}\right)_{2}\right]\left[\mathbf{B A r}_{\mathbf{4}} \mathbf{4}\right]}\end{array}$ & yield $\mathbf{~ [ g ] ~}$ & $\begin{array}{c}\mathbf{M}_{\mathbf{n}} \\
\mathbf{( N M R}^{\mathbf{a}}\end{array}$ & $\begin{array}{c}\text { B /1000 C } \\
\text { atoms }^{\mathbf{b}}\end{array}$ \\
\hline $\mathbf{1}$ & Me-I2 & 0.0 & 2.6 & 2.0 & 73 \\
\hline $\mathbf{2}$ & Me-I2 & 1.05 & 1.5 & 2.2 & 76 \\
\hline
\end{tabular}

Polymerization conditions: $n$ (precat) $=5 \mu \mathrm{mol}, \mathrm{T}=30^{\circ} \mathrm{C}, t=30 \mathrm{~min}, p=40 \mathrm{bar}, 100 \mathrm{~mL}$ toluene, $1000 \mathrm{rpm}$. Precatalyst solution in toluene saturated with ethylene for $1 \mathrm{~min}$ at 5 bar prior to the addition of $\left[\mathrm{H}\left(\mathrm{OEt}_{2}\right)_{2}\right]\left[\mathrm{BAr} \mathrm{F}_{4}\right]_{\text {. a }}$ a) determined by ${ }^{l} H$ NMR comparing the olefinic end groups with the aliphatic signals; b) Branches per $1000 \mathrm{C}$ atoms, by ${ }^{1} H \mathrm{NMR}$.

\subsubsection{Influence of a Substituent para to the Phenol-O}

An analogue of complex $2^{\mathbf{C F 3}}$ with a ${ }^{\text {tert }} \mathrm{Bu}$ substitution in para position to the phenolic oxygen atom $\left(2^{\mathbf{C F} 3}{ }^{t} \mathbf{B u}\right.$; Scheme S10) was also prepared.
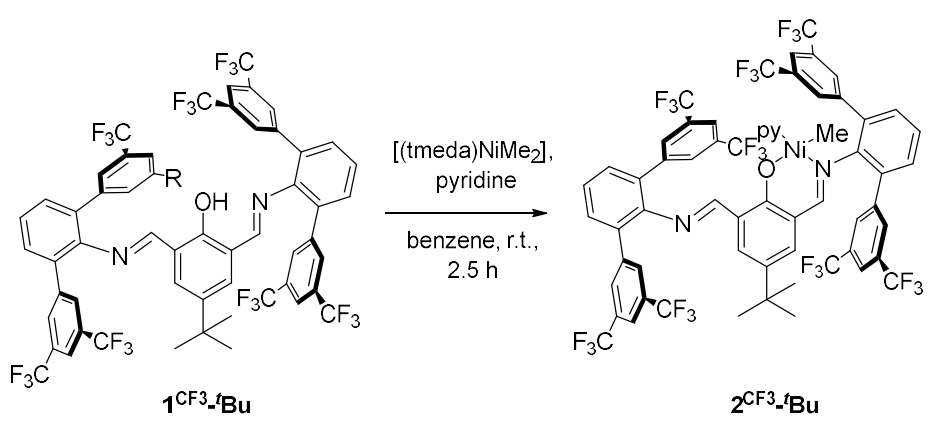

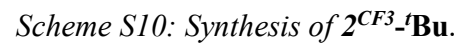


This complex $\left(\mathbf{2}^{\mathbf{C F} 3}{ }^{\mathbf{t}} \mathbf{B u}\right.$ ) was tested in ethylene polymerization experiments using the same conditions as for $\mathbf{2}^{\mathbf{C F 3}}$ (Table S10). The productivity, activity and the microstructure of the obtained polymer does not depend significantly on the substituent para to the phenolic oxygen atom.

Table S10: Ethylene polymerization experiments with $2^{C F 3}$ and $2^{C F 3}{ }_{-}{ }^{B} \boldsymbol{u}$.

\begin{tabular}{|c|c|c|c|c|c|c|c|c|c|c|}
\hline$\#$ & precatalyst & $\begin{array}{c}\mathbf{n} \\
\text { (precat) } \\
{[\mu \mathrm{mol}]}\end{array}$ & $\begin{array}{c}\mathbf{T} \\
{\left[{ }^{\circ} \mathbf{C}\right]}\end{array}$ & $\begin{array}{l}\text { isolated } \\
\text { yield }[\mathrm{g}]\end{array}$ & $\begin{array}{l}\mathrm{M}_{\mathrm{n}}(\mathrm{NMR}) \\
{\left[\mathrm{kg} \mathrm{mol}^{-1}\right]^{\mathrm{a}}}\end{array}$ & $\begin{array}{l}M_{n}(G P C) \\
{\left[\mathrm{kg} \mathrm{mol}^{-1}\right]}\end{array}$ & PDI & $\begin{array}{c}\mathbf{T}_{\mathbf{m}} \\
{\left[{ }^{\circ} \mathbf{C}\right]^{\mathrm{d}}}\end{array}$ & $\mathbf{B}^{\mathrm{e}, \mathrm{f}}$ & $\begin{array}{c}\% \\
\mathbf{M e}^{\mathrm{f}}\end{array}$ \\
\hline 1 & $2^{\mathrm{CF} 3}$ & 5 & 30 & 3.1 & $i^{\mathrm{b}}$ & $126^{\mathrm{c}}$ & $4.6^{c}$ & 127 & 5 & 100 \\
\hline 2 & $2^{\mathrm{CF} 3}$ & 5 & 50 & 9.0 & $j^{\mathrm{b}}$ & $37^{\mathrm{c}}$ & $1.9^{\mathrm{c}}$ & 114 & 15 & 99 \\
\hline 3 & $2^{\mathrm{CF} 3}$ & 5 & 70 & 14.2 & $i^{\mathrm{b}}$ & $20^{\mathrm{c}}$ & $1.8^{\mathrm{c}}$ & 108 & 20 & 97 \\
\hline 4 & $2^{\mathrm{CF} 3{ }_{-}{ }^{t} \mathrm{Bu}}$ & 10 & 30 & 7.5 & $j^{\mathrm{b}}$ & $59.1^{\mathrm{c}}$ & $3.1^{\mathrm{c}}$ & 127 & 8 & 99 \\
\hline 5 & $2^{\mathrm{CF}_{-}{ }^{\prime}} \mathrm{Bu}$ & 10 & 50 & 14.6 & 11.6 & $10.9^{\mathrm{c}}$ & $1.9^{\mathrm{c}}$ & 121 & 22 & 98 \\
\hline
\end{tabular}

Polymerization conditions: 40 bar $\mathrm{C}_{2} \mathrm{H}_{4}, 100 \mathrm{~mL}$ toluene, $1000 \mathrm{rpm}, \mathrm{t}=30 \mathrm{~min}$; a) from ${ }^{1} \mathrm{H} \mathrm{NMR}$; determined by comparing the olefinic signals to the backbone; b) no olefinic end groups detectable c) determined by GPC in dichlorobenzene, universal calibration against polystyrene; d) determined by DSC, second heating cycle, heating rate: $10 \mathrm{~K} \mathrm{~min}^{-1}$; e) Branches/ $1000 \mathrm{C}$ atoms f) determined by ${ }^{13} \mathrm{C}\left\{{ }^{1} \mathrm{H}\right\}$ NMR (inverse gated).

\section{NMR Tube Experiment}

A J. YounG tube was loaded with $21.8 \mathrm{mg}$ of $\mathbf{2}^{\mathbf{C F}}-\mathbf{H}^{+}(10.0 \mu \mathrm{mol})$ and $0.5 \mathrm{~mL}$ of dichloromethane- $d_{2}$. A ${ }^{1} \mathrm{H}$ NMR and ${ }^{19} \mathrm{~F}\left\{{ }^{1} \mathrm{H}\right\}$ NMR spectrum were recorded at $-50{ }^{\circ} \mathrm{C}$. Ethylene (6.6 equiv.) was added at $-196{ }^{\circ} \mathrm{C}$. Another set of spectra at $-50{ }^{\circ} \mathrm{C}$ were measured. No consumption of ethylene was observed.

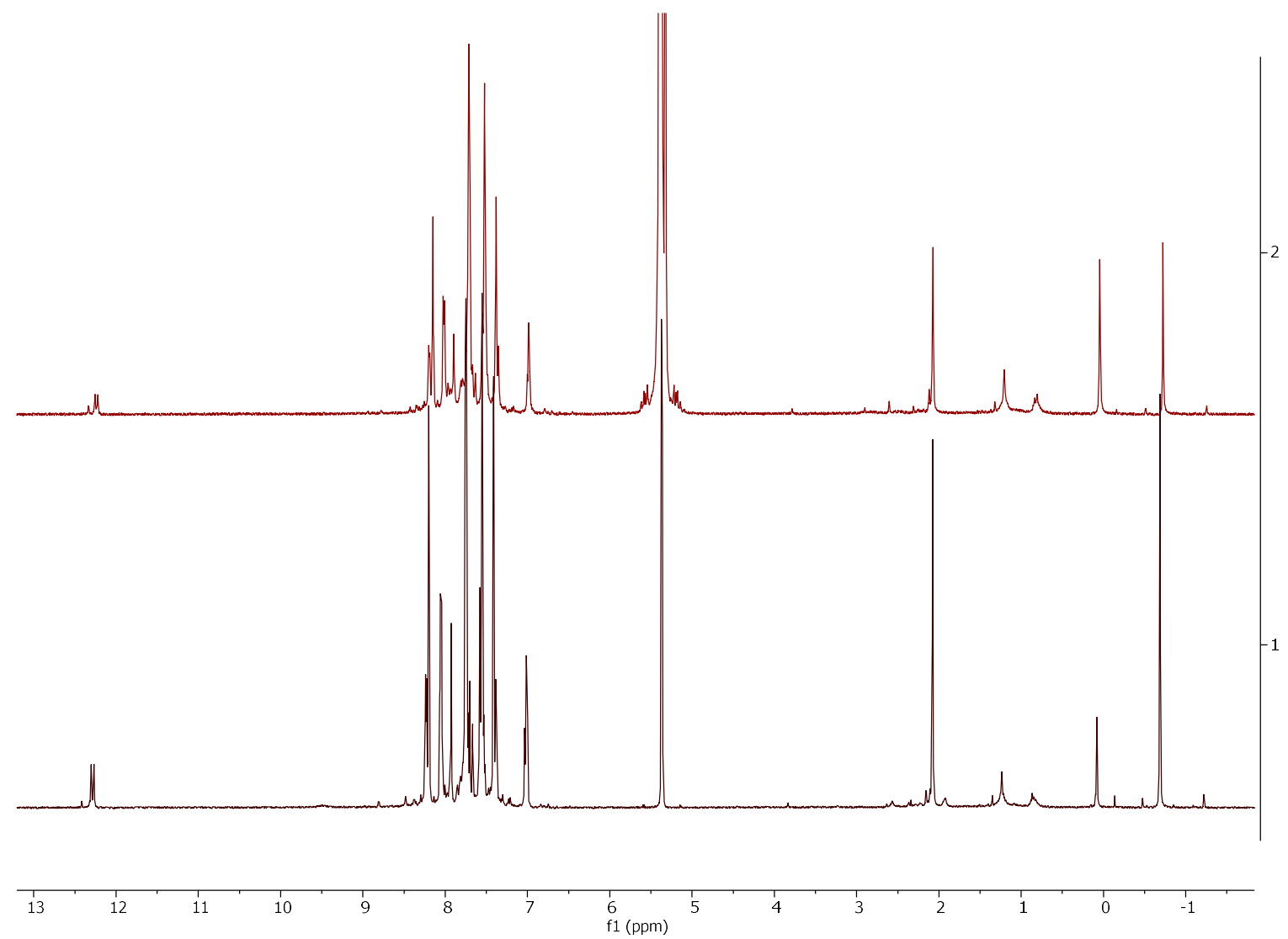

Figure S68: ${ }^{1} \mathrm{H}$ NMR spectra of $\mathbf{2}^{\mathrm{CF}^{3}}-\boldsymbol{H}^{+}$in dichloromethane-d $\mathrm{d}_{2}$ before (bottom) and after (top) the addition of ethylene. 


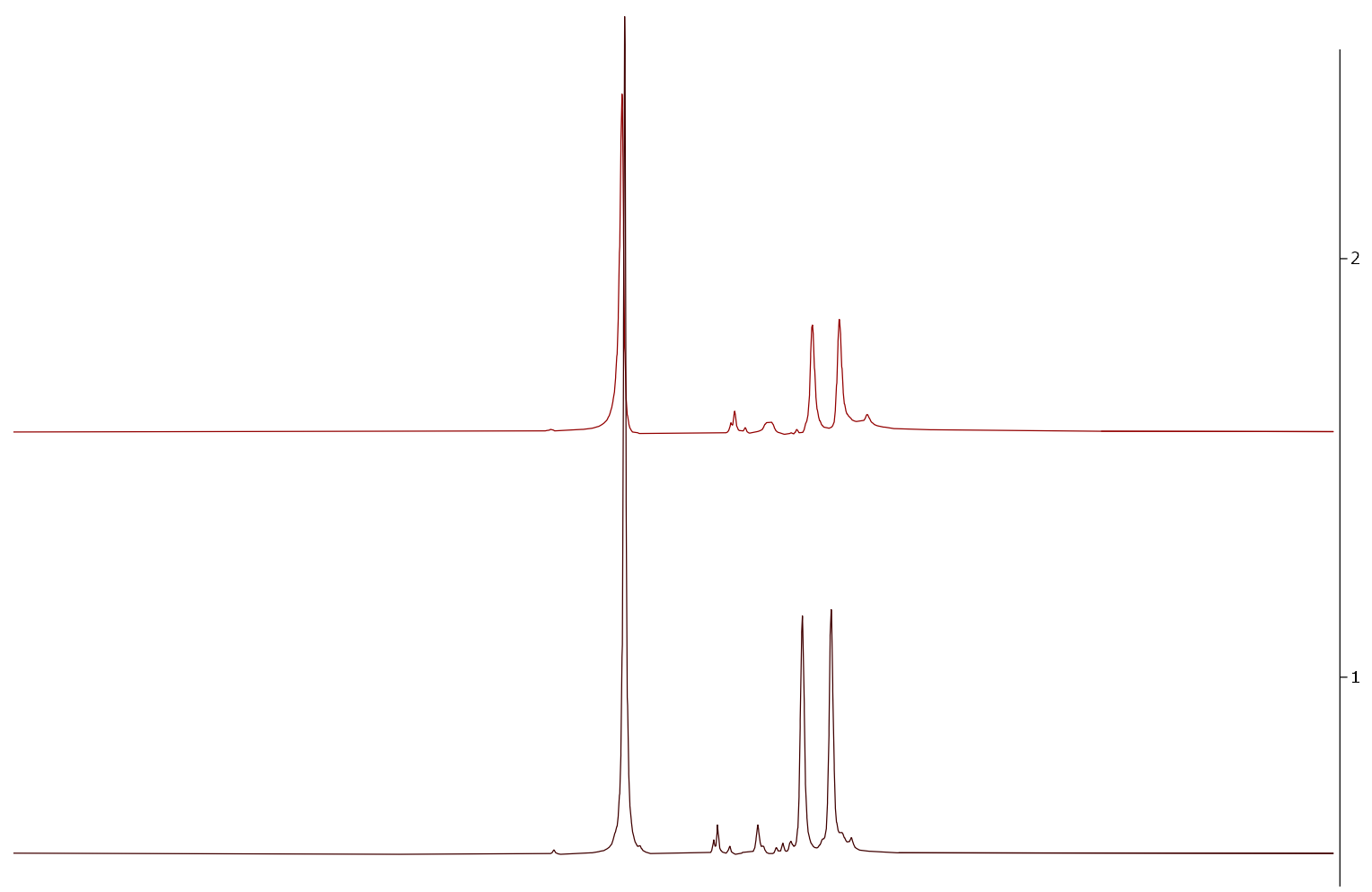

\begin{tabular}{rrrrrrrrrrrrrrrrrrrrrrr}
\hline$j 0.6$ & -60.8 & -61.0 & -61.2 & -61.4 & -61.6 & -61.8 & -62.0 & -62.2 & -62.4 & -62.6 & -62.8 & -63.0 & -63.2 & -63.4 & -63.6 & -63.8 & -64.0 & -64.2 & -64.4 & -64.6 & -64.8
\end{tabular}

Figure S69: ${ }^{19} F\left\{{ }^{1} H\right\}$ NMR spectra of $2^{C F 3}-\boldsymbol{H}^{+}$in dichloromethane- $d_{2}$ before (bottom) and after (top) the addition of ethylene.

The solution was cooled to $-196{ }^{\circ} \mathrm{C}$ and a solution of $\left[\mathrm{H}\left(\mathrm{OEt}_{2}\right)_{2}\right]\left[\mathrm{BAr}{ }_{4}\right]$ in dichloromethane- $d_{2}$ was added (0.72 equiv.). The sample was brought to the NMR machine under constant cooling in a liquid nitrogen bath. It was then put into the NMR machine, where it warmed up to $-50^{\circ} \mathrm{C}$. An immediately measured spectra showed conversion of ethylene. 

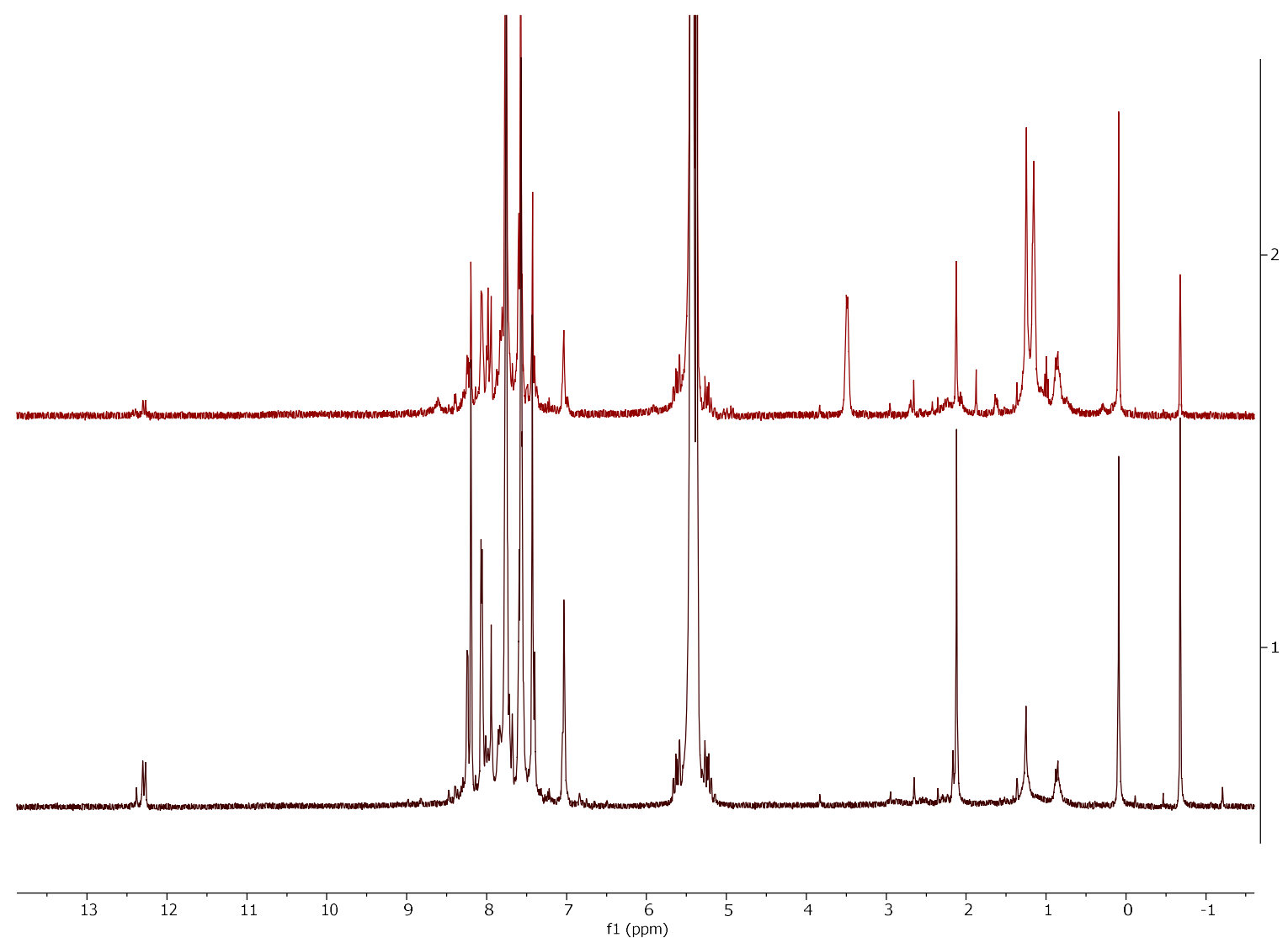

Figure S70: ${ }^{1} \mathrm{H}$ NMR spectra of $2^{\mathrm{CF}^{3}-\mathrm{H}^{+}}$in dichloromethane-d2 before (bottom) and after (top) the addition of $\left[\mathrm{H}\left(\mathrm{OEt_{2 }}\right)_{2}\right]\left[\mathrm{BAr}^{\mathrm{F}} 4\right]$.

Ethylene insertion and elimination (as evidenced by signals for terminal alkenes) took place. 

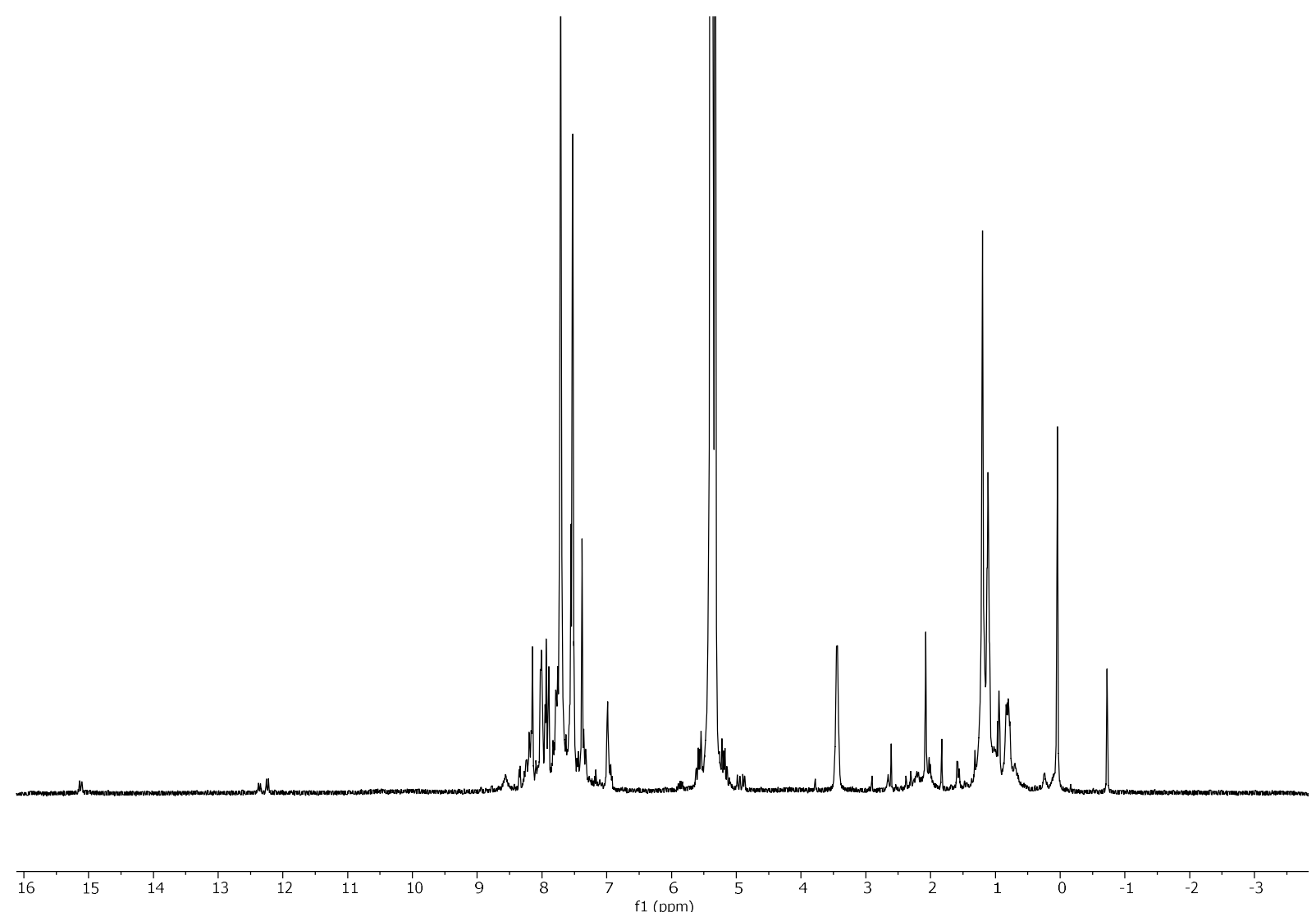

Figure S71: ${ }^{l} H$ NMR spectrum of $2^{C F 3}-\boldsymbol{H}^{+}$in dichloromethane-d $d_{2}$ after the addition of ethylene and $\left[H\left(O E t_{2}\right)_{2}\right]\left[B A r^{F}\right]_{4}$ and a reaction time of $t=30 \mathrm{~min}$.

The original starting complex is still present, along with two other complexes (as evidenced by two additional acidic protons at the characteristic chemical shift). One of these complexes is likely an Ni-ethyl species (which is also detected).

In addition, four new pyridine species are observed. None of those additional pyridine signals are 'free pyridine' signals. However, species are formed, which are also present in a mixture of pyridine and excess $\left[\mathrm{H}\left(\mathrm{OEt}_{2}\right)_{2}\right]\left[\mathrm{BAr}_{4}^{\mathrm{F}}\right]$. 


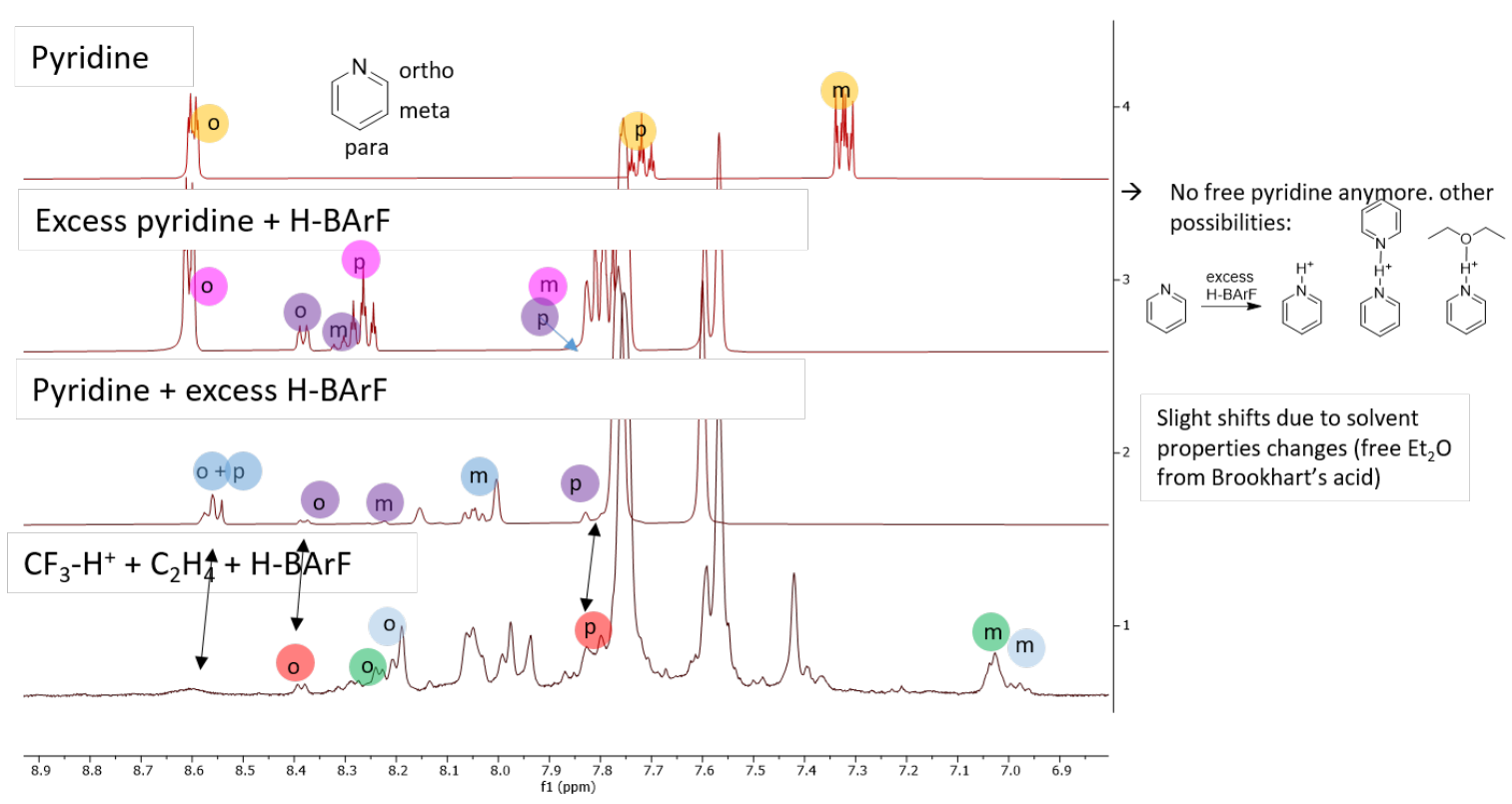

Figure S72: Set of four ${ }^{l} \mathrm{H}$ NMR spectra in dichloromethane-d $\mathrm{d}_{2}$ at $-50{ }^{\circ} \mathrm{C}$ (from top to bottom): pyridine, $\left[\mathrm{H}\left(\mathrm{OEt}_{2}\right)_{2}\right]\left[\mathrm{BAr}^{\mathrm{F}}{ }_{4}\right]$ in the presence of an excess of pyridine; Pyridine in the presence of an excess of $\left.\left[\mathrm{H}_{(\mathrm{OEt}}\right)_{2}\right]\left[\mathrm{BAr}_{4}^{\mathrm{F}}\right] ; \mathbf{2}^{\mathrm{CF}^{3}}-\boldsymbol{H}^{+}$in dichloromethane$d 2$ after the addition of ethylene and $\left[\mathrm{H}_{(\mathrm{OEt}} \mathrm{t}_{2}\right]\left[\mathrm{BAr}_{4}{ }_{4}\right]$ and a reaction time of $t=30 \mathrm{~min}$. Comments to spectrum three and four on the right side.

\section{Natural Bond Population Analysis}

\subsection{Complexes $\mathbf{3}^{\mathrm{CF} 3}, \mathbf{3}^{\mathrm{Me}}, \mathbf{3}^{\mathrm{CF} 3}-\mathbf{H}^{+}$and $\mathbf{3}^{\mathrm{Me}}-\mathbf{H}^{+}$}

Natural bond population analysis (NBO) was performed for $\mathbf{3}^{\mathrm{CF} 3}, \mathbf{3}^{\mathrm{Me}}, \mathbf{3}^{\mathrm{CF3}-} \mathbf{H}^{+}$and $\mathbf{3}^{\mathrm{Me}}-\mathbf{H}^{+}$. Due to the protonation, the $\mathrm{Ni}-\mathrm{O}$ bond is weakened, as indicated by the increased $\mathrm{Ni}-\mathrm{O}$ bond distances for the protonated systems respect to the non-protonated ones, $\left(\mathbf{3}^{\mathrm{Me}}\right.$ : Ni-O $1.885 \AA, \mathbf{3}^{\mathbf{C F 3}}$ : Ni-O $1.882 \AA, \mathbf{3}^{\mathbf{M e}}-\mathbf{H}^{+}: \mathrm{Ni}-\mathrm{O} 1.919 \AA, \mathbf{3}^{\mathrm{Me}}-\mathbf{H}^{+}$: Ni-O 1.913 $\AA$ ). This is in agreement with the structures obtained by X-ray crystallography. Moreover, the protonated systems exhibit a lower positive charge on $\mathrm{Ni}$ and a greater negative charge on the oxygen atom.

The two nitrogen atoms are electronically different in these complexes. In details, for the non-protonated $3^{\mathrm{Me}}$ and $\mathbf{3}^{\mathbf{C F 3}}$ systems the greater electron density is localized on N1 that coordinates to the metal. On the contrary, even though N2 is protonated in case of $\mathbf{3}^{\mathbf{M e}}-\mathbf{H}^{+}$and $\mathbf{3}^{\mathbf{C F}}-\mathbf{H}^{+}$, a greater electron density is present on N2 than on N1. This unusual result can be ascribed to the presence of the $\pi$-conjugated system that is able to delocalize the iminium positive charge. 
Table S11: NBO Charges for $3^{\mathrm{Me}}, 3^{\mathrm{CF} 3}, \mathbf{3}^{\mathrm{Me}-\mathrm{H}^{+}}$and $3^{\mathrm{CF}}-\mathrm{H}^{+}$

\begin{tabular}{|c|c|c|c|c|}
\hline & N1 & N2 & O & Ni \\
\hline $3^{\mathbf{M e}}$ & -0.47939 & -0.44514 & -0.58414 & 0.40857 \\
\hline $3^{\mathbf{M e}}-\mathbf{H}^{+}$ & -0.45075 & -0.48306 & -0.60651 & 0.42562 \\
\hline $\mathbf{3}^{\mathbf{C F 3}}$ & -0.49483 & -0.45978 & -0.58128 & 0.39998 \\
\hline $\mathbf{3}^{\mathbf{C F 3}}-\mathbf{H}^{+}$ & -0.46028 & -0.49156 & -0.60716 & 0.41722 \\
\hline
\end{tabular}

\subsubsection{Complexes $\mathbf{2}^{\mathrm{Me}}, \mathbf{2}^{\mathbf{C F} 3}, \mathbf{2}^{\mathbf{M e}}-\mathbf{H}^{+}$and $\mathbf{2}^{\mathbf{C F} 3}-\mathbf{H}^{+}$}

An electronic analysis with a natural bond population analysis (NBO) was performed on the discussed intermediates and transition states for the two protonated complexes $\mathbf{2}^{\mathrm{Me}}-\mathbf{H}^{+}$and $2^{\mathbf{C F} 3}-\mathbf{H}^{+}$.

\subsubsection{1 $\mathbf{2}^{\mathrm{Me}}-\mathbf{H}^{+}$}

Table S12: NBO Charges and $(\mathrm{O}-\mathrm{H}$ and $\mathrm{Ni-O})$ distances for $\mathbf{2}^{\mathrm{Me}} \mathbf{-} \mathrm{H}^{+}$.

\begin{tabular}{|c|c|c|c|c|c|}
\hline & $\begin{array}{c}\text { Distance } \\
\mathrm{O}-\mathrm{H}(\AA)\end{array}$ & $\mathrm{Ni}-\mathrm{O}(\AA)$ & $\mathrm{Ni}(\mathrm{NBO})$ & $\begin{array}{c}\text { Charge on } \\
(\mathrm{NBO})\end{array}$ & $\begin{array}{c}\text { Charge on O } \\
(\mathrm{NBO})\end{array}$ \\
\hline 2-Coor-T & 1.94339 & 2.04372 & 0.43332 & -0.61770 & 0.46153 \\
\hline 2-Coor-C & 1.87536 & 1.97887 & 0.44627 & -0.61148 & 0.46635 \\
\hline TS-2ins & 1.93332 & 2.01371 & 0.42573 & -0.61699 & 0.45828 \\
\hline 1-B-T & 1.85939 & 1.92229 & 0.43529 & -0.63567 & 0.46884 \\
\hline TS-1 BHE-C & 1.90752 & 1.95767 & 0.28444 & -0.61941 & 0.46490 \\
\hline 1-BHE-C & 1.93220 & 1.96992 & 0.28031 & -0.61778 & 0.46330 \\
\hline
\end{tabular}




\subsubsection{2 $\mathbf{2}^{\mathbf{C F} 3}-\mathbf{H}^{+}$}

Table S13: NBO Charges and (Ni-O and $\mathrm{O}-\mathrm{H}$ ) distances for $\mathbf{2}^{\mathrm{CF} 3}-\mathrm{H}^{+}$.

\begin{tabular}{|c|c|c|c|c|c|}
\hline & $\begin{array}{c}\text { Distance } \\
\mathrm{O}-\mathrm{H}(\AA)\end{array}$ & $\begin{array}{c}\text { Distance } \\
\mathrm{Ni}-\mathrm{O}(\AA)\end{array}$ & $\begin{array}{c}\text { Charge on Ni } \\
(\mathrm{NBO})\end{array}$ & $\begin{array}{c}\text { Charge on O } \\
(\mathrm{NBO})\end{array}$ & $\begin{array}{c}\text { Charge on H } \\
(\mathrm{NBO})\end{array}$ \\
\hline $\mathbf{2 - C o o r - T}$ & 1.85645 & 2.02809 & 0.43666 & -0.62309 & 0.46279 \\
\hline $\mathbf{2 - C o o r - C}$ & 1.84915 & 1.97380 & 0.43470 & -0.61181 & 0.46585 \\
\hline $\mathbf{T S - 2}$ ins & 1.98200 & 2.01420 & 0.42109 & -0.61700 & 0.46313 \\
\hline $\mathbf{1 - \beta}-\mathbf{T}$ & 1.80895 & 1.92411 & 0.42475 & -0.63416 & 0.46984 \\
\hline TS-1 $\mathbf{B H E - C}$ & 1.83693 & 1.95556 & 0.27526 & -0.61926 & 0.46483 \\
\hline $\mathbf{1 - B H E - C}$ & 1.85730 & 1.96900 & 0.27282 & -0.61870 & 0.46458 \\
\hline
\end{tabular}

\section{Computational Details}

\subsection{General Methods}

All the DFT geometry optimizations were performed at the GGA BP86 $6^{11-13}$ level with the Gaussian09 package. ${ }^{14}$ The electronic configuration of the systems was described with the 6-31G basis set for $\mathrm{H}, \mathrm{C}, \mathrm{N}, \mathrm{F}$, and $\mathrm{O}$ while for $\mathrm{Ni}$ the quasi-relativistic LANL2DZ ECP effective core potential was adopted. ${ }^{15}$ All geometries were characterized as minimum or transition state through frequency calculations. The reported free energies were built through single point energy calculations on the BP86/6-31G geometries using the M06 functional and the triple- $\zeta$ TZVP ${ }^{16-18}$ basis set on main group atoms. Solvent effects (toluene) were included with the PCM. ${ }^{19,20}$ To this M06/TZVP electronic energy in solvent, thermal corrections were included from the gas-phase frequency calculations at the BP86/6$31 \mathrm{G}$.

We performed calculations of all important intermediates and transition states involved in the linear chain growth, branch formation and chain transfer reaction during the polymerization (1- $\beta-\mathbf{T}$, as a reference point, Gibbs free energies in kcal $\mathrm{mol}^{-1}$; Scheme S11). 


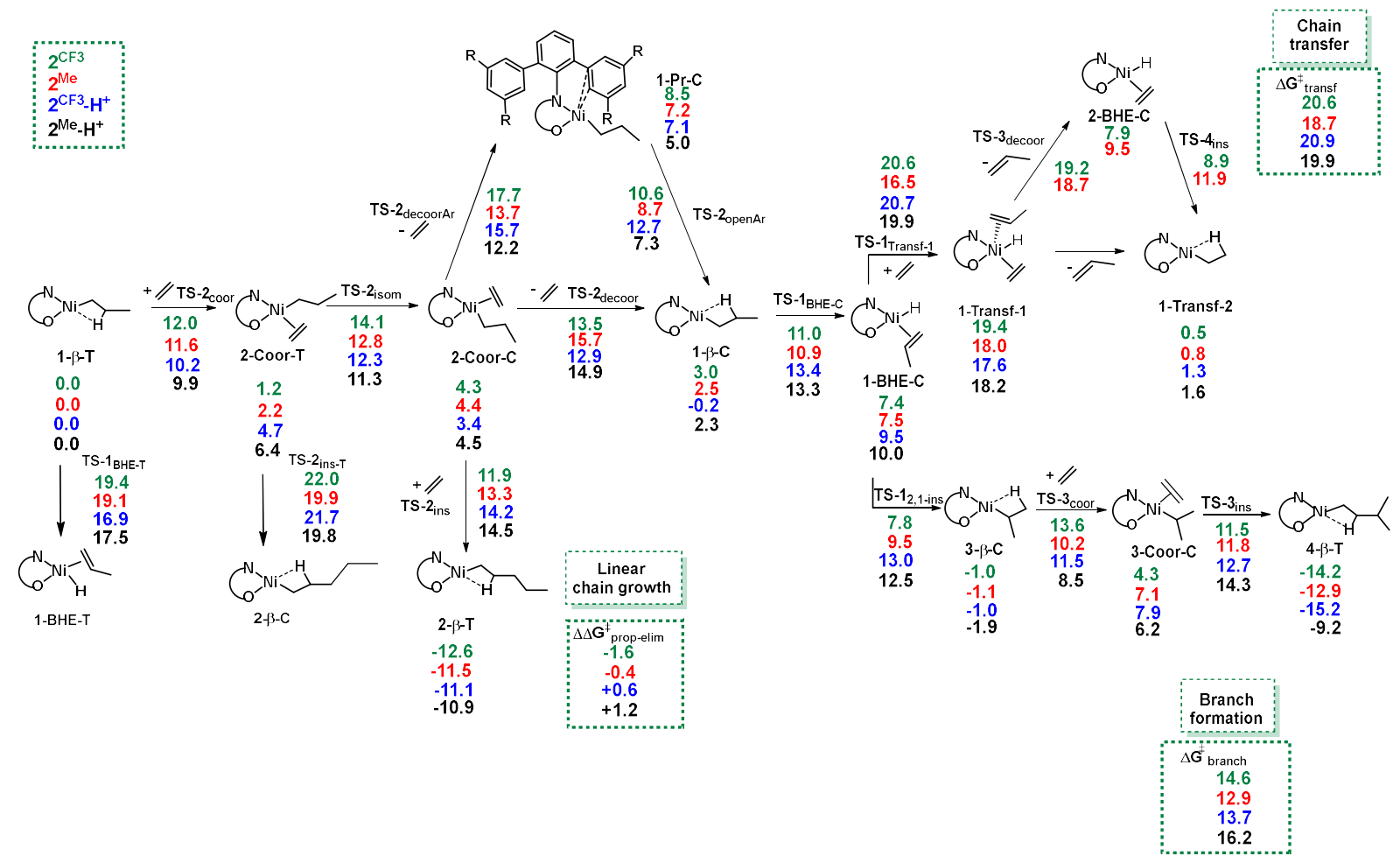

Scheme S11: Gibbs free energies $\left(\Delta G_{T o l}\right.$ in $\left.\mathrm{kcal} \mathrm{mol}^{-1}\right)$ for all the intermediates.

\section{Chain transfer mechanisms.}

The chain transfer reaction occurs prevalently by a two steps mechanism consisting in monomer coordination and then chain decoordination leading to the $\beta$-agostic complex 1-Transf-2 that starts a new polymer chain. A flat reactions profile was obtained for all considered system. In detail, from 1-BHE-C the ethylene coordination leads to a five-coordinated intermediate, 1-Transf-1, with an energy barrier of 13.2, 9.0, 11.2 and $9.9 \mathrm{kcal} \mathrm{mol}^{-1}$ for $\mathbf{2}^{\mathbf{C F 3}}$, $\mathbf{2}^{\mathrm{Me}}, \mathbf{2}^{\mathrm{CF} 3}-\mathbf{H}^{+}$and $\mathbf{2}^{\mathrm{Me}}-\mathbf{H}^{+}$, respectively. From 1-Transf-1, for $\mathbf{2}^{\mathrm{CF} 3}$ and $\mathbf{2}^{\mathrm{Me}}$ the following release of propene occur via TS-3 decoor leads to the 2-BHE-C intermediate with the monomer coordinated in cis position with respect to the oxygen atom. After displacement of propene, ethylene insertion in the $\mathrm{Ni-H}$ bond via TS-4ins yields to the stable $\beta$-agostic complex, 1-Transf-2. Instead for $\mathbf{2}^{\mathbf{C F 3}}-\mathbf{H}^{+}$and $\mathbf{2}^{\mathbf{M e}}-\mathbf{H}^{+}$the decoordination of the unsaturated chain is concerted with monomer insertion and 1-Transf-2 product is obtained through a barrierless mechanism for which it was not possible to locate the corresponding TS. This difference can be ascribed to the effect of the bridge and to the nature of the species lying trans to the oxygen atom for $\mathbf{2}^{\mathbf{M e}}-\mathbf{H}^{+}$and $\mathbf{2}^{\mathbf{C F} 3}-\mathbf{H}^{+}$respect to $\mathbf{2}^{\mathbf{M e}}$ and $\mathbf{2}^{\mathbf{C F} 3}$ systems. Overall, the determining energy barriers for the chain transfer are 18.7 and $20.6 \mathrm{kcal} \mathrm{mol}^{-1}$ for $\mathbf{2}^{\mathbf{M e}}$ and $\mathbf{2}^{\mathbf{C F} 3}$, respectively, and 19.9 and $20.9 \mathrm{kcal} \mathrm{mol}^{-1}$ for $\mathbf{2}^{\mathbf{M e}}-\mathbf{H}^{+}$and $\mathbf{2}^{\mathbf{C F} 3} \mathbf{-} \mathbf{H}^{+}$, respectively, almost $9 \mathrm{kcal} \mathrm{mol}{ }^{-1}$ higher than the monomer insertion barrier for the $\mathrm{CF}_{3}$ substituted non-protonated system and about $6 \mathrm{kcal} \mathrm{mol}^{-1}$ higher than the monomer insertion for the other three systems. This result is in line with the lower molecular weight products observed experimentally with $\mathbf{2}^{\mathrm{Me}}, \mathbf{2}^{\mathrm{Me}}-\mathbf{H}^{+}$and $\mathbf{2}^{\mathrm{CF}}-\mathbf{H}^{+}$compared to $\mathbf{2}^{\mathbf{C F} 3}$. 


\subsection{Compared Ni-O distances for $\mathbf{2}^{\mathbf{C F 3}}, \mathbf{2}^{\mathbf{M e}}, \mathbf{2}^{\mathbf{C F 3}}-\mathbf{H}^{+}$and $\mathbf{2}^{\mathrm{Me}}-\mathbf{H}^{+}$}

Table S14: Ni-O distances for $2^{C F 3}, 2^{M e}, 2^{C F 3}-H^{+}$and $2^{M e}-H^{+}$.

\begin{tabular}{|c|c|}
\hline & $\begin{array}{l}\text { Distance } \\
\mathrm{Ni}-\mathrm{O}(\AA)\end{array}$ \\
\hline $2^{\mathrm{CF} 3}:$ TS-2 decoor & 1.86620 \\
\hline $2^{\mathrm{Me}}:$ TS-2 decoorAr & 1.93094 \\
\hline $2^{\mathrm{CF}}-\mathrm{H}^{+}: \mathrm{TS}-\mathbf{2}_{\text {decoor }}$ & 1.89211 \\
\hline $2^{\mathrm{Me}}-\mathrm{H}^{+}: \mathrm{TS}-\mathbf{2}_{\operatorname{decoorAr}}$ & 1.97636 \\
\hline $2^{\text {CF3 }}:$ TS-1 BHE-C & 1.91054 \\
\hline $2^{\mathrm{Me}}:$ TS-1 ${ }_{\text {BHE-C }}$ & 1.90662 \\
\hline $2^{\mathrm{CF} 3}-\mathrm{H}^{+}:$TS-1 BHE-C & 1.95556 \\
\hline $2^{\mathrm{Me}}-\mathrm{H}^{+}:$TS-1 $\mathbf{B H E}_{\mathrm{C}}$ & 1.95767 \\
\hline
\end{tabular}

\subsection{Cartesian Coordinates}

Internal energies (a.u.), enthalpies (a.u.) and free energies (a.u.) in Toluene are also reported.

\section{$6.3 .13^{\mathrm{Me}}$}

\section{Me1-Allyl}

Zero-point correction $=0.914903$ (Hartree/Particle)

Thermal correction to Energy $=0.976360$

Thermal correction to Enthalpy $=0.977304$

Thermal correction to Gibbs Free Energy= 0.807146

Sum of electronic and zero-point Energies $=-2519.753980$

Sum of electronic and thermal Energies $=\quad-2519.692523$

Sum of electronic and thermal Enthalpies $=-2519.691579$

Sum of electronic and thermal Free Energies $=-2519.861737$

E solvent $=-2519.52873510$

$\begin{array}{lrrr}\mathrm{Ni} & -1.822434000 & 0.459085000 & -1.394416000 \\ \mathrm{C} & -0.931265000 & 0.376892000 & -3.241051000 \\ \mathrm{~N} & -2.880411000 & 0.457178000 & 0.200623000 \\ \mathrm{C} & -2.388475000 & 0.096059000 & 1.375146000 \\ \mathrm{C} & -1.035804000 & -0.252298000 & 1.682624000 \\ \mathrm{C} & 0.020268000 & -0.204441000 & 0.696402000 \\ \mathrm{O} & -0.170947000 & 0.119535000 & -0.552078000 \\ \mathrm{C} & -2.103817000 & 1.167988000 & -3.245920000 \\ \mathrm{H} & -2.038882000 & 2.262878000 & -3.300467000 \\ \mathrm{H} & 0.057099000 & 0.840021000 & -3.324473000 \\ \mathrm{H} & -0.979792000 & -0.700615000 & -3.448228000 \\ \mathrm{H} & -3.091018000 & 0.085054000 & 2.225284000 \\ \mathrm{C} & -0.735536000 & -0.614696000 & 3.026542000 \\ \mathrm{H} & -1.562535000 & -0.640023000 & 3.750021000\end{array}$

$\begin{array}{lrrr}\mathrm{C} & 0.554667000 & -0.936753000 & 3.448874000 \\ \mathrm{C} & 0.850512000 & -1.326624000 & 4.883379000 \\ \mathrm{H} & 1.257705000 & -2.353340000 & 4.951758000 \\ \mathrm{H} & -0.060248000 & -1.287493000 & 5.505995000 \\ \mathrm{H} & 1.599153000 & -0.653519000 & 5.342103000 \\ \mathrm{C} & 1.586341000 & -0.880614000 & 2.481907000 \\ \mathrm{H} & 2.620447000 & -1.114231000 & 2.765935000 \\ \mathrm{C} & 1.359789000 & -0.526986000 & 1.145366000 \\ \mathrm{C} & 2.462058000 & -0.464952000 & 0.180489000 \\ \mathrm{~N} & 3.693027000 & -0.692749000 & 0.515119000 \\ \mathrm{H} & 2.179340000 & -0.198374000 & -0.853634000 \\ \mathrm{C} & 4.701077000 & -0.627503000 & -0.471538000 \\ \mathrm{C} & 4.824356000 & -1.632868000 & -1.479847000 \\ \mathrm{C} & 5.859714000 & -1.510749000 & -2.434376000 \\ \mathrm{C} & 6.754100000 & -0.435479000 & -2.412232000 \\ \mathrm{C} & 6.636365000 & 0.535590000 & -1.409446000 \\ \mathrm{C} & 5.631934000 & 0.454808000 & -0.423299000 \\ \mathrm{C} & 5.558169000 & 1.527045000 & 0.614883000 \\ \mathrm{C} & 5.466879000 & 1.220834000 & 1.992717000 \\ \mathrm{H} & 5.530562000 & 6.057339000 & 1.584428000 \\ \mathrm{C} & 5.554305000 & 3.578299000 & 2.546966000 \\ \mathrm{C} & 5.740827000 & 5.367421000 & 0.748577000 \\ \mathrm{C} & 5.642345000 & 3.918623000 & 1.186963000 \\ \mathrm{C} & 5.644145000 & 2.880649000 & 0.232132000 \\ \mathrm{C} & 3.928907000 & -2.826743000 & -1.553089000 \\ \mathrm{C} & 3.617230000 & -3.591698000 & -0.409061000 \\ \mathrm{C} & 2.848437000 & -4.767146000 & -0.499979000\end{array}$




\begin{tabular}{|c|c|c|c|}
\hline $\mathrm{C}$ & 2.387091000 & -5.178894000 & -1.764529000 \\
\hline $\mathrm{C}$ & 2.668876000 & -4.434179000 & -2.927314000 \\
\hline $\mathrm{C}$ & 3.440086000 & -3.265090000 & -2.805429000 \\
\hline $\mathrm{C}$ & 2.138662000 & -4.880302000 & -4.276814000 \\
\hline $\mathrm{C}$ & 2.514517000 & -5.565540000 & 0.745866000 \\
\hline $\mathrm{C}$ & 5.469192000 & 2.232589000 & 2.965568000 \\
\hline $\mathrm{C}$ & 5.385882000 & 1.893130000 & 4.441652000 \\
\hline $\mathrm{C}$ & -4.531429000 & -4.322388000 & 1.353856000 \\
\hline $\mathrm{C}$ & -3.894905000 & -3.734933000 & 0.245690000 \\
\hline $\mathrm{C}$ & -4.116560000 & -2.368437000 & -0.016661000 \\
\hline $\mathrm{C}$ & -4.964334000 & -1.595026000 & 0.801225000 \\
\hline $\mathrm{C}$ & -5.590215000 & -2.218945000 & 1.906015000 \\
\hline $\mathrm{C}$ & -5.384214000 & -3.578527000 & 2.195126000 \\
\hline $\mathrm{C}$ & -2.988361000 & -4.549750000 & -0.656547000 \\
\hline $\mathrm{C}$ & -5.270181000 & -0.161307000 & 0.500630000 \\
\hline $\mathrm{C}$ & -6.626062000 & 0.239225000 & 0.505970000 \\
\hline $\mathrm{C}$ & -6.998943000 & 1.555471000 & 0.215498000 \\
\hline $\mathrm{C}$ & -6.013078000 & 2.499763000 & -0.093787000 \\
\hline $\mathrm{C}$ & -4.643180000 & 2.156098000 & -0.112206000 \\
\hline $\mathrm{C}$ & -4.275338000 & 0.813237000 & 0.195320000 \\
\hline $\mathrm{C}$ & -3.655381000 & 3.217887000 & -0.478752000 \\
\hline $\mathrm{C}$ & -3.929774000 & 4.041895000 & -1.597706000 \\
\hline $\mathrm{C}$ & -3.061365000 & 5.078624000 & -1.980775000 \\
\hline $\mathrm{C}$ & -1.891781000 & 5.289272000 & -1.221117000 \\
\hline $\mathrm{C}$ & -1.591576000 & 4.498525000 & -0.097430000 \\
\hline $\mathrm{C}$ & -2.480419000 & 3.464077000 & 0.261412000 \\
\hline $\mathrm{C}$ & -0.333227000 & 4.739006000 & 0.713338000 \\
\hline $\mathrm{C}$ & -3.379765000 & 5.962275000 & -3.171683000 \\
\hline $\mathrm{H}$ & -6.236569000 & -1.621542000 & 2.561644000 \\
\hline $\mathrm{H}$ & -4.354957000 & -5.384496000 & 1.571530000 \\
\hline $\mathrm{H}$ & -2.005694000 & -4.060893000 & -0.788394000 \\
\hline $\mathrm{H}$ & -2.811960000 & -5.559032000 & -0.246245000 \\
\hline $\mathrm{H}$ & -3.427231000 & -4.672486000 & -1.665241000 \\
\hline $\mathrm{H}$ & -3.617782000 & -1.896721000 & -0.871498000 \\
\hline $\mathrm{H}$ & -7.391381000 & -0.515182000 & 0.719343000 \\
\hline $\mathrm{H}$ & -8.055587000 & 1.844933000 & 0.226247000 \\
\hline $\mathrm{H}$ & -6.293553000 & 3.537975000 & -0.302800000 \\
\hline $\mathrm{H}$ & -4.834054000 & 3.854789000 & -2.190814000 \\
\hline $\mathrm{H}$ & -4.129343000 & 5.495412000 & -3.834106000 \\
\hline $\mathrm{H}$ & -3.787829000 & 6.939951000 & -2.849401000 \\
\hline $\mathrm{H}$ & -2.477373000 & 6.173201000 & -3.773546000 \\
\hline $\mathrm{H}$ & -1.199909000 & 6.091416000 & -1.511589000 \\
\hline $\mathrm{H}$ & 0.228356000 & 5.611213000 & 0.337133000 \\
\hline $\mathrm{H}$ & 0.340412000 & 3.862832000 & 0.678051000 \\
\hline $\mathrm{H}$ & -0.567975000 & 4.922123000 & 1.778514000 \\
\hline $\mathrm{H}$ & 1.793599000 & -6.099973000 & -1.846512000 \\
\hline $\mathrm{H}$ & 2.633973000 & -4.342519000 & -5.103950000 \\
\hline $\mathrm{H}$ & 2.294007000 & -5.963419000 & -4.435238000 \\
\hline $\mathrm{H}$ & 1.050588000 & -4.695210000 & -4.366801000 \\
\hline $\mathrm{H}$ & 3.660891000 & -2.671477000 & -3.701585000 \\
\hline $\mathrm{H}$ & 2.275449000 & -6.615966000 & 0.502725000 \\
\hline $\mathrm{H}$ & 1.637248000 & -5.140525000 & 1.271241000 \\
\hline $\mathrm{H}$ & 3.353315000 & -5.564232000 & 1.464796000 \\
\hline $\mathrm{H}$ & 5.969456000 & -2.299291000 & -3.187805000 \\
\hline $\mathrm{H}$ & 7.555707000 & -0.368116000 & -3.156179000 \\
\hline $\mathrm{H}$ & 7.348754000 & 1.367436000 & -1.362820000 \\
\hline $\mathrm{H}$ & 5.028216000 & 5.594442000 & -0.065869000 \\
\hline $\mathrm{H}$ & 6.751895000 & 5.608037000 & 0.366586000 \\
\hline $\mathrm{H}$ & 5.693236000 & 3.130277000 & -0.835595000 \\
\hline $\mathrm{H}$ & 5.392021000 & 0.172510000 & 2.299860000 \\
\hline $\mathrm{H}$ & 5.285012000 & 0.805429000 & 4.598728000 \\
\hline $\mathrm{H}$ & 4.519187000 & 2.385187000 & 4.922353000 \\
\hline $\mathrm{H}$ & 3.991357000 & -3.264949000 & 0.567883000 \\
\hline $\mathrm{H}$ & -2.256094000 & 2.855348000 & 1.142918000 \\
\hline $\mathrm{H}$ & 5.547995000 & 4.377611000 & 3.300984000 \\
\hline $\mathrm{H}$ & 6.288274000 & 2.228672000 & 4.987586000 \\
\hline $\mathrm{C}$ & -6.060557000 & -4.235071000 & 3.383003000 \\
\hline$H$ & -6.812157000 & -4.980150000 & 3.058707000 \\
\hline
\end{tabular}

$\begin{array}{lrrr}\mathrm{H} & -5.331870000 & -4.770974000 & 4.019039000 \\ \mathrm{H} & -6.579232000 & -3.493214000 & 4.014549000 \\ \mathrm{C} & -3.303246000 & 0.551981000 & -2.798062000 \\ \mathrm{H} & -4.192063000 & 1.156431000 & -2.595244000 \\ \mathrm{H} & -3.488377000 & -0.510166000 & -3.014742000\end{array}$

\subsection{2 $\mathbf{2}^{\mathrm{Me}}$ \\ Me $1-\beta-T$}

Zero-point correction=

Thermal correction to Energy= 0.998927

Thermal correction to Enthalpy= 0.999871

Thermal correction to Gibbs Free Energy $=\quad 0.826967$

Sum of electronic and zero-point Energies $=\quad-2520.928538$

Sum of electronic and thermal Energies $=\quad-2520.866247$

Sum of electronic and thermal Enthalpies $=\quad-2520.865303$

Sum of electronic and thermal Free Energies $=\quad-2521.038206$

E solvent $=-2520.72365032$

\begin{tabular}{|c|c|c|c|}
\hline & 000 & 0 & \\
\hline & -3.113746000 & 0.627298000 & -2.705664000 \\
\hline & -2.874494000 & 9000 & 00 \\
\hline & -2.362919000 & 000 & \\
\hline & -1.000152000 & 3000 & 000 \\
\hline & 772000 & 8000 & 000 \\
\hline & -0.154664000 & 000 & -0 . \\
\hline & -1.865370000 & & -3 \\
\hline & -0.930945000 & 0.415831000 & -2 \\
\hline & -1.765918000 & -0.720566000 & -4.465352000 \\
\hline & -0.756839000 & -0.822651000 & 58000 \\
\hline & -1.999180000 & & -3.9 \\
\hline & -2.491192000 & & \\
\hline & & & \\
\hline & & & \\
\hline & & & \\
\hline & & & \\
\hline & -0.6 & & \\
\hline & -1.5 & & \\
\hline & 0.6 & & \\
\hline & & & \\
\hline & & & \\
\hline 1 & & & \\
\hline$H_{0}$ & & & \\
\hline 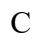 & & & \\
\hline$H_{0}$ & 2.6 & & \\
\hline & & & \\
\hline 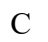 & 2.5 & & \\
\hline $\mathrm{T}$ & 3.7 & & \\
\hline & & & \\
\hline & & & \\
\hline & & & \\
\hline & 5.9 & & -2 \\
\hline $\mathrm{C}$ & 6.80 & -0.3 & -2 \\
\hline $\mathrm{C}$ & & & \\
\hline $\mathrm{C}$ & 5.59 & & 000 \\
\hline $\mathrm{C}$ & 5.423 & & \\
\hline & 5.29 & & \\
\hline & 5.1 & & \\
\hline & & & \\
\hline $\mathrm{C}$ & & & \\
\hline $\mathrm{C}$ & & & \\
\hline $\mathrm{C}$ & & & \\
\hline$e^{2}+{ }_{2}$ & & & \\
\hline $\mathrm{C}$ & & & \\
\hline $\mathrm{C}$ & 3.20 & & \\
\hline & 2.773483000 & & -1.173120000 \\
\hline & 3.002702000 & -4.693193000 & -2.410619 \\
\hline & 2 couk12000 & -3.467903000 & -2.421428 \\
\hline
\end{tabular}

$\begin{array}{llll}\text { C } & 2.497742000 & -5.310313000 & -3.701033000\end{array}$ 


\begin{tabular}{|c|c|c|c|}
\hline & & & 1. \\
\hline & 11235000 & 32848000 & 2.624192000 \\
\hline & 82505000 & 82727000 & 400 \\
\hline & 560000 & 54000 & 700 \\
\hline & 60285000 & 836501000 & 36000 \\
\hline & 951000 & .486074000 & -009279700 \\
\hline & 462000 & 26000 & 0.7 \\
\hline & 6437000 & 2.488073000 & 1.77527800 \\
\hline & 07000 & -3.833436000 & 2.0 \\
\hline C & 80000 & -4.56 & -0.7 \\
\hline $\mathrm{C}$ & 2000 & $038>$ & \\
\hline $\mathrm{C}$ & 56000 & -0.0 & \\
\hline $\mathrm{C}$ & -7.060802000 & & \\
\hline $\mathrm{C}$ & 29000 & 3000 & 5000 \\
\hline $\mathrm{C}$ & 22000 & 3000 & 5000 \\
\hline $\mathrm{C}$ & -4.29 & 0.675 & \\
\hline $\mathrm{C}$ & 03000 & 3.153333000 & -0.3 \\
\hline $\mathrm{C}$ & 01000 & 4.023014000 & -1.428548000 \\
\hline $\mathrm{C}$ & 370000 & 5.150038000 & -1.720724000 \\
\hline$C$ & -2.2 & 5.407385000 & 347000 \\
\hline $\mathrm{C}$ & $-1.8 \mathrm{~s}$ & 4.56 & 28000 \\
\hline C & $-2.7 x$ & & \\
\hline$C$ & -0.6 & & \\
\hline$C$ & -3.7 & 6.0 & \\
\hline & 90000 & -1.954682000 & 2. \\
\hline & -3.9 & -5.542215 & 000 \\
\hline & -1.6 & -3.9 & -0 \\
\hline $\mathrm{H}$ & -2.3 & -5.5 & 000 \\
\hline & -2.9 & -4 . & 000 \\
\hline & -3.3 & & -0 \\
\hline & -7.3 & -0.5 & 34000 \\
\hline & -8.1 & & \\
\hline 1 & -6.4 & & \\
\hline I & -5.0 & & \\
\hline I & -4.4 & & 71000 \\
\hline 1 & -4.2 & & 000 \\
\hline 1 & -2.8 & 00 & 25000 \\
\hline $\mathrm{H}$ & -1.6 & 6.2 & 753000 \\
\hline & -0.2 & & 5300 \\
\hline $\mathrm{H}$ & 0.1 & & 79000 \\
\hline & -0.9 & 4.776 & 500000 \\
\hline & 2.2 & -6.287928000 & 874000 \\
\hline & 54000 & -5.008570000 & 646000 \\
\hline & 2.49 & -6.413908000 & 918000 \\
\hline & & 56000 & 64000 \\
\hline & 3.8 & -2.962 & 69000 \\
\hline & 2.8 & -6.5 & 32000 \\
\hline & 1.9 & 00 & 97000 \\
\hline & 3.70 & 0 & 387000 \\
\hline & 6.1 & -2.3 & 92000 \\
\hline & 7.6 & -03 & 00 \\
\hline & & & \\
\hline & & & 0 \\
\hline & 6.4 & 0 & -0. \\
\hline & 5.53 & 3.2 & 42000 \\
\hline & 5.26 & 0 & 57000 \\
\hline & 5.13 & & 91000 \\
\hline & & 3.090 & 76000 \\
\hline $\mathrm{H}$ & 93000 & -3.061078000 & 0.928731000 \\
\hline $\mathrm{H}$ & -2.45 & 97000 & 315000 \\
\hline 11 & 5.17 & 2000 & 2.679890000 \\
\hline $\mathrm{H}$ & 5.88 & 70000 & 390000 \\
\hline$C$ & 38000 & -4.563569000 & 062000 \\
\hline $\mathrm{H}$ & 16000 & -5.313925000 & 2.830099000 \\
\hline 11 & -5.0 & -5.106985000 & 3.81643800 \\
\hline $\mathrm{H}$ & -6.344375000 & -3.867046000 & 3.85536400 \\
\hline
\end{tabular}

${ }^{\text {MeTS-2 }}$ coor
Zero-point correction $=$

Thermal correction to Energy=

Thermal correction to Enthalpy=

0.987801 (Hartree/Particle)

Thermal correction to Gibbs Free Energy $=0.875553$

Sum of electronic and zero-point Energies $=\quad-2599.450715$ Sum of electronic and thermal Energies $=\quad-2599.384725$

Sum of electronic and thermal Enthalpies $=\quad-2599.383781$

Sum of electronic and thermal Free Energies $=-2599.562964$

E solvent $=-2599.27048225$

$\mathrm{Ni}-1.799901000$

C -3.106451000

$-2.881538000$

0.259279000

$-2.352224000$

$-0.970731000$

$0.598801000-2.661170000$

$-0.118670000 \quad 0.163970000$

$-0.541213000 \quad 1.306812000$

$-0.696717000 \quad 1.649105000$

$\begin{array}{lll}0.103412000 & -0.288909000 & 0.765057000\end{array}$

$\begin{array}{lll}-0.117146000 & 0.167404000 & -0.431678000\end{array}$

$\begin{array}{lll}-2.101890000 & 1.559658000 & -3.263501000\end{array}$

$\begin{array}{lll}-1.071289000 & 1.354466000 & -2.809305000\end{array}$

$\begin{array}{lll}-1.904308000 & 1.458596000 & -4.791469000\end{array}$

$\begin{array}{lll}-1.090579000 & 2.118741000 & -5.142395000\end{array}$

$\begin{array}{lll}-1.661551000 & 0.423125000 & -5.088678000\end{array}$

$-2.834149000 \quad 1.749552000 \quad-5.311477000$

$\begin{array}{lll}-2.326747000 & 2.596106000 & -2.954862000\end{array}$

$-3.279136000 \quad-0.320162000 \quad-3.251524000$

$\begin{array}{lll}-4.052687000 & 1.044283000 & -2.326684000\end{array}$

$\begin{array}{lll}-3.068521000 & -0.766063000 & 2.113450000\end{array}$

$\begin{array}{lll}-0.663527000 & -1.206559000 & 2.942683000\end{array}$

$\begin{array}{lll}-1.499099000 & -1.503932000 & 3.591722000\end{array}$

$\begin{array}{lll}0.645771000 & -1.343789000 & 3.407984000\end{array}$

$\begin{array}{lll}0.948426000 & -1.905810000 & 4.782855000\end{array}$

$\begin{array}{lll}1.521386000 & -2.850564000 & 4.720002000\end{array}$

$\begin{array}{lll}0.021358000 & -2.116304000 & 5.344226000\end{array}$

$\begin{array}{lll}1.553620000 & -1.203642000 & 5.386733000\end{array}$

$\begin{array}{lll}1.689282000 & -0.935816000 & 2.544038000\end{array}$

$\begin{array}{lll}2.735205000 & -1.023889000 & 2.864835000\end{array}$

$\begin{array}{lll}1.457476000 & -0.411323000 & 1.265264000\end{array}$

$\begin{array}{lll}2.565835000 & 0.000895000 & 0.400410000\end{array}$

$\begin{array}{lll}3.808681000 & -0.175720000 & 0.722258000\end{array}$

$\begin{array}{lll}2.275875000 & 0.469880000 & -0.557904000\end{array}$

$\begin{array}{lll}4.821739000 & 0.271155000 & -0.154828000\end{array}$

$\begin{array}{lll}5.566509000 & -0.694947000 & -0.898766000\end{array}$

$\begin{array}{lll}6.578538000 & -0.246922000 & -1.773391000\end{array}$

$\begin{array}{lll}6.878908000 & 1.114266000 & -1.909381000\end{array}$

$\begin{array}{lll}6.164267000 & 2.052426000 & -1.157283000\end{array}$

$\begin{array}{lll}5.133675000 & 1.660388000 & -0.273504000\end{array}$

$\begin{array}{lll}4.435670000 & 2.719645000 & 0.515897000\end{array}$

$\begin{array}{lll}4.223409000 & 2.589148000 & 1.906425000\end{array}$

$\begin{array}{lll}2.299343000 & 6.818980000 & 0.490873000\end{array}$

$\begin{array}{lll}3.257524000 & 4.809052000 & 1.997340000\end{array}$

$\begin{array}{lll}3.068406000 & 6.268109000 & -0.078767000\end{array}$

$\begin{array}{lll}3.453850000 & 4.974375000 & 0.613247000\end{array}$

$\begin{array}{lll}4.039303000 & 3.920155000 & -0.113238000\end{array}$

$\begin{array}{lll}5.291624000 & -2.161190000 & -0.813308000\end{array}$

$\begin{array}{lll}5.162707000 & -2.823455000 & 0.429547000\end{array}$

$\begin{array}{lll}4.971685000 & -4.212736000 & 0.498827000\end{array}$

$\begin{array}{lll}4.904882000 & -4.951093000 & -0.702164000\end{array}$

$\begin{array}{lll}5.026221000 & -4.325898000 & -1.954602000\end{array}$

$\begin{array}{lll}5.218498000 & -2.929780000 & -1.993433000\end{array}$

$\begin{array}{lll}4.965119000 & -5.131012000 & -3.238720000\end{array}$

$\begin{array}{lll}4.828689000 & -4.912191000 & 1.837203000\end{array}$

$\begin{array}{lll}3.635655000 & 3.622056000 & 2.657121000\end{array}$

$\begin{array}{lll}3.395615000 & 3.452978000 & 4.145263000\end{array}$

$\begin{array}{lll}-4.015326000 & -5.190139000 & 0.616776000\end{array}$

$\begin{array}{lll}-4.864796000 & -4.627607000 & 1.589988000\end{array}$

$\begin{array}{lll}-5.208842000 & -3.269811000 & 1.470911000\end{array}$

$\begin{array}{lll}-4.708925000 & -2.471272000 & 0.416793000\end{array}$

$\begin{array}{lll}-3.849498000 & -3.061667000 & -0.531238000\end{array}$

$\begin{array}{lll}-3.500065000 & -4.424525000 & -0.445711000\end{array}$ 


\begin{tabular}{|c|c|c|c|}
\hline C & -5.383386000 & -5.465818000 & 2.742122000 \\
\hline$C$ & -5.181515000 & -1.056191000 & 0.295998000 \\
\hline $\mathrm{C}$ & -6.576798000 & -0.830969000 & 0.321865000 \\
\hline $\mathrm{C}$ & -7.108401000 & 0.458886000 & 0.225912000 \\
\hline $\mathrm{C}$ & -6.243723000 & 1.553747000 & 0.117500000 \\
\hline $\mathrm{C}$ & -4.841109000 & 1.387591000 & 0.097338000 \\
\hline $\mathrm{C}$ & -4.312587000 & 0.065428000 & 0.175537000 \\
\hline $\mathrm{C}$ & -3.994946000 & 2.616977000 & -0.002012000 \\
\hline $\mathrm{C}$ & -4.338303000 & 3.608025000 & -0.951061000 \\
\hline $\mathrm{C}$ & -3.626126000 & 4.818314000 & -1.044173000 \\
\hline $\mathrm{C}$ & -2.547478000 & 5.031406000 & -0.164157000 \\
\hline $\mathrm{C}$ & -2.179975000 & 4.070481000 & 0.797591000 \\
\hline $\mathrm{C}$ & -2.910786000 & 2.868903000 & 0.866563000 \\
\hline $\mathrm{C}$ & -1.010742000 & 4.316116000 & 1.730466000 \\
\hline $\mathrm{C}$ & -4.028901000 & 5.881467000 & -2.048285000 \\
\hline $\mathrm{H}$ & -3.466656000 & -2.450336000 & -1.356543000 \\
\hline $\mathrm{H}$ & -3.749735000 & -6.253698000 & 0.686757000 \\
\hline $\mathrm{H}$ & -5.697572000 & -6.469741000 & 2.403362000 \\
\hline $\mathrm{H}$ & -4.604204000 & -5.615855000 & 3.514487000 \\
\hline $\mathrm{H}$ & -6.247580000 & -4.987428000 & 3.235123000 \\
\hline $\mathrm{H}$ & -5.875304000 & -2.814425000 & 2.214644000 \\
\hline $\mathrm{H}$ & -7.241794000 & -1.698258000 & 0.399262000 \\
\hline $\mathrm{H}$ & -8.193161000 & 0.612020000 & 0.243444000 \\
\hline $\mathrm{H}$ & -6.647864000 & 2.571286000 & 0.078149000 \\
\hline $\mathrm{H}$ & -5.173400000 & 3.422536000 & -1.638465000 \\
\hline $\mathrm{H}$ & -4.580920000 & 5.447484000 & -2.900426000 \\
\hline $\mathrm{H}$ & -4.686680000 & 6.643087000 & -1.585706000 \\
\hline $\mathrm{H}$ & -3.148539000 & 6.415571000 & -2.448331000 \\
\hline $\mathrm{H}$ & -1.982568000 & 5.971543000 & -0.225613000 \\
\hline $\mathrm{H}$ & -0.938467000 & 5.380964000 & 2.015950000 \\
\hline $\mathrm{H}$ & -0.049835000 & 4.038567000 & 1.257004000 \\
\hline $\mathrm{H}$ & -1.100841000 & 3.719915000 & 2.655085000 \\
\hline $\mathrm{H}$ & 4.750650000 & -6.038135000 & -0.657449000 \\
\hline $\mathrm{H}$ & 5.971453000 & -5.266587000 & -3.680823000 \\
\hline $\mathrm{H}$ & 4.543716000 & -6.136647000 & -3.065378000 \\
\hline $\mathrm{H}$ & 4.343208000 & -4.629527000 & -4.002701000 \\
\hline $\mathrm{H}$ & 5.295111000 & -2.424231000 & -2.964692000 \\
\hline $\mathrm{H}$ & 5.507897000 & -5.781484000 & 1.916838000 \\
\hline $\mathrm{H}$ & 3.799872000 & -5.293297000 & 1.985708000 \\
\hline $\mathrm{H}$ & 5.053994000 & -4.229294000 & 2.674424000 \\
\hline $\mathrm{H}$ & 7.151796000 & -0.994316000 & -2.333995000 \\
\hline $\mathrm{H}$ & 7.683656000 & 1.438764000 & -2.578440000 \\
\hline $\mathrm{H}$ & 6.416703000 & 3.117003000 & -1.223985000 \\
\hline $\mathrm{H}$ & 2.673504000 & 6.082915000 & -1.093940000 \\
\hline $\mathrm{H}$ & 3.940114000 & 6.942540000 & -0.188564000 \\
\hline $\mathrm{H}$ & 4.181429000 & 4.026215000 & -1.196296000 \\
\hline $\mathrm{H}$ & 4.525311000 & 1.661668000 & 2.406155000 \\
\hline $\mathrm{H}$ & 4.172078000 & 2.819777000 & 4.610036000 \\
\hline $\mathrm{H}$ & 2.419964000 & 2.967827000 & 4.342676000 \\
\hline $\mathrm{H}$ & 5.210673000 & -2.235862000 & 1.352101000 \\
\hline $\mathrm{H}$ & -2.640569000 & 2.130053000 & 1.627647000 \\
\hline $\mathrm{H}$ & 2.801190000 & 5.623572000 & 2.576426000 \\
\hline $\mathrm{H}$ & 3.389977000 & 4.425554000 & 4.668808000 \\
\hline $\mathrm{C}$ & -2.586263000 & -5.058077000 & -1.476997000 \\
\hline $\mathrm{H}$ & -2.646681000 & -6.159876000 & -1.445429000 \\
\hline $\mathrm{H}$ & -2.843523000 & -4.734343000 & -2.501698000 \\
\hline $\mathrm{H}$ & -1.528440000 & -4.780822000 & -1.306259000 \\
\hline $\mathrm{C}$ & 0.156483000 & -1.213423000 & -3.272601000 \\
\hline $\mathrm{C}$ & -0.830365000 & -1.932965000 & -2.700818000 \\
\hline $\mathrm{H}$ & 1.003567000 & -0.847155000 & -2.683786000 \\
\hline $\mathrm{H}$ & 0.145965000 & -0.964038000 & -4.340365000 \\
\hline $\mathrm{H}$ & -1.674046000 & -2.319519000 & -3.285588000 \\
\hline $\mathrm{H}$ & -0.789212000 & -2.226179000 & -1.646450000 \\
\hline
\end{tabular}

\section{Me2-Coor-T}

Zero-point correction= Thermal correction to Energy= Thermal correction to Enthalpy= 0.990701 (Hartree/Particle) 1.056366 1.057310
2.742122000

0.097338000

$-0.951061000$

$-0.164157000$

0.797591000

866563000

$-2.048285000$

2.403362000

2.214644000

62000

0.078149000

$-1.638465000$

1.585706000

2.015950000

1.257004000

$-0.657449000$

$-3.680823000$

1.985708000

$-2.578440000$

23985000

$-0.188564000$

$-1.196296000$

4.342676000

01000

2.576426000

4.668808000

$-1.445429000$

$-3.272601000$

2.683786000

$-1.646450000$
Thermal correction to Gibbs Free Energy $=0.877256$

Sum of electronic and zero-point Energies $=\quad-2599.473572$ Sum of electronic and thermal Energies $=\quad-2599.407907$

Sum of electronic and thermal Enthalpies $=\quad-2599.406962$

Sum of electronic and thermal Free Energies $=-2599.587017$ E solvent $=-2599.28707369$

$\mathrm{Ni} \quad 1.757493000$

$-0.226118000-1.381319000$

$\begin{array}{llll}\text { C } & 3.271170000 & 0.115855000 & -2.596657000\end{array}$

$\begin{array}{llll}\mathrm{N} & 2.763600000 & 0.266820000 & 0.234073000\end{array}$

$\begin{array}{llll}\text { C } & 2.177787000 & 0.709812000 & 1.338430000\end{array}$

$\begin{array}{llll}\text { C } & 0.785573000 & 0.821094000 & 1.637227000\end{array}$

$\begin{array}{llll}\text { C } & -0.237198000 & 0.346502000 & 0.732400000\end{array}$

O $\quad 0.036672000 \quad-0.149931000 \quad-0.433532000$

$\begin{array}{llll}\text { C } & 3.776054000 & -0.911268000 & -3.610800000\end{array}$

$\mathrm{H} \quad 2.976385000 \quad-1.213783000 \quad-4.314852000$

C $\quad 4.966279000 \quad-0.365912000 \quad-4.432638000$

$\mathrm{H} \quad 5.328677000 \quad-1.118263000 \quad-5.158116000$

$\mathrm{H} \quad 4.678174000 \quad 0.538963000 \quad-4.999257000$

$\begin{array}{llll}\mathrm{H} & 5.811683000 & -0.092729000 & -3.775103000\end{array}$

$\begin{array}{llll}\mathrm{H} & 4.096393000 & -1.829973000 & -3.083025000\end{array}$

H $\quad 2.871604000 \quad 1.016985000 \quad-3.112183000$

$\mathrm{H} \quad 4.106405000 \quad 0.430489000 \quad-1.950097000$

$\begin{array}{llll}\mathrm{H} & 2.851099000 & 1.005469000 & 2.160590000\end{array}$

$\begin{array}{llll}\text { C } & 0.421507000 & 1.367276000 & 2.900985000\end{array}$

$\mathrm{H} \quad 1.225863000 \quad 1.716568000 \quad 3.563484000$

$\begin{array}{llll}\text { C } & -0.906000000 & 1.480042000 & 3.314955000\end{array}$

$\begin{array}{llll}\text { C } & -1.276543000 & 2.083326000 & 4.655013000\end{array}$

$\begin{array}{llll}\mathrm{H} & -1.863881000 & 3.013509000 & 4.534176000\end{array}$

$\mathrm{H} \quad-0.377865000 \quad 2.331874000 \quad 5.245940000$

$\begin{array}{ll}1.390610000 & 5.257616000\end{array}$

$\begin{array}{llll}\text { C } & -1.904681000 & 1.006893000 & 2.429462000\end{array}$

$\begin{array}{llll}\mathrm{H} & -2.963461000 & 1.071254000 & 2.711932000\end{array}$

C $\quad-1.613798000 \quad 0.444359000 \quad 1.180303000$

$\begin{array}{llll}\text { C } & -2.679208000 & -0.040727000 & 0.299154000\end{array}$

$\begin{array}{llll}\mathrm{N} & -3.935931000 & 0.086914000 & 0.587377000\end{array}$

$\mathrm{H} \quad-2.343670000 \quad-0.523007000 \quad-0.636831000$

$\begin{array}{llll}\text { C } & -4.906938000 & -0.439498000 & -0.293031000\end{array}$

$\begin{array}{llll}\text { C } & -5.694526000 & 0.463670000 & -1.070652000\end{array}$

$\begin{array}{llll}\text { C } & -6.669243000 & -0.061803000 & -1.944185000\end{array}$

$\begin{array}{llll}\text { C } & -6.892319000 & -1.440688000 & -2.047018000\end{array}$

$\begin{array}{llll}\text { C } & -6.134219000 & -2.317845000 & -1.264672000\end{array}$

$\begin{array}{llll}\text { C } & -5.136649000 & -1.847066000 & -0.381189000\end{array}$

$\begin{array}{llll}\text { C } & -4.387329000 & -2.846915000 & 0.438259000\end{array}$

$\begin{array}{llll}\text { C } & -4.190756000 & -2.672531000 & 1.826715000\end{array}$

$\begin{array}{llll}\mathrm{H} & -2.036146000 & -6.827150000 & 0.523856000\end{array}$

$\begin{array}{llll}\text { C } & -3.106466000 & -4.834069000 & 1.975043000\end{array}$

C $\quad-2.828123000 \quad-6.330368000 \quad-0.063817000$

$\begin{array}{llll}\text { C } & -3.285500000 & -5.042564000 & 0.594755000\end{array}$

$\begin{array}{llll}\text { C } & -3.922465000 & -4.038712000 & -0.159806000\end{array}$

$\begin{array}{llll}\text { C } & -5.498629000 & 1.944417000 & -1.023820000\end{array}$

$\begin{array}{llll}\text { C } & -5.431713000 & 2.648650000 & 0.201168000\end{array}$

$-5.318712000$

$-5.266869000$

$-5.324615000$

4.047344000

4.753070000

4.085964000

$-5.442506000$

2.680594000

4.852486000

$-5.261575000$

$-5.249718000$

$-3.552245000$

4.793211000

$-3.653631000$

$-3.330274000$

$-3.438713000$

3.367608000

5.405084000

4.321626000

4.987675000

4.810696000

3.672025000

2.775290000

3.414133000

3.226882000

$2.904598000 \quad 4.539122000$

$4.813746000 \quad 5.942084000$

$4.969849000 \quad 1.405218000$

1.405218000
1.298920000

0.232284000

$-0.989436000$

$-2.224146000$

$-2.224679000$

$-3.531594000$

1.551253000

2.604480000

4.089485000

0.639368000

1.589243000

1.513627000

0.512542000

$-0.420682000$

$-0.368288000$

2.660030000

0.449507000

0.525609000 


\begin{tabular}{|c|c|c|c|}
\hline & & & \\
\hline & 6.252703000 & 10910000 & 0.372388000 \\
\hline & 2890000 & 64359000 & 5000 \\
\hline & 04000 & 08361000 & \\
\hline & 3762000 & 61342000 & 1503000 \\
\hline & 3604000 & 1969000 & 0601222000 \\
\hline & 72000 & & \\
\hline & 00 & 000 & \\
\hline 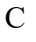 & 2.400141000 & 000 & 210 \\
\hline $\mathrm{C}$ & 29000 & & $18+2$ \\
\hline $\mathrm{C}$ & 1.238130000 & & 7000 \\
\hline C & 4.457182000 & 6720000 & 957000 \\
\hline I & 3.065630000 & 2000 & 238000 \\
\hline & 2.973079000 & & 08000 \\
\hline & 5.460911000 & & 396000 \\
\hline & 3.973604000 & 6.455069000 & 3.163191000 \\
\hline & 5.400969000 & 5.415884000 & 2410000 \\
\hline & 5.541788000 & & 932000 \\
\hline 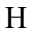 & 6.96 & & \\
\hline & & & \\
\hline 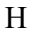 & 6.74 & & \\
\hline & & & \\
\hline 11 & & & \\
\hline$n$ & & & \\
\hline H & 3.62 & & 67000 \\
\hline & 38 & & \\
\hline & 皮 & & \\
\hline & & & \\
\hline & & & 000 \\
\hline & & & 000 \\
\hline & & & \\
\hline & & & \\
\hline & & & \\
\hline 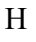 & & & -3 \\
\hline $\mathrm{H}$ & 0 & & 1. \\
\hline 2 & -4 . & & \\
\hline 2 & 00 & & \\
\hline$T$ & -7.2 & & 000 \\
\hline $\mathrm{H}$ & -7.6 & & 28000 \\
\hline $\mathrm{H}$ & & & 59000 \\
\hline 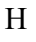 & -2.43 & & 704000 \\
\hline 11 & -3.662972000 & & 033000 \\
\hline 11 & -4.052355000 & 4000 & 625000 \\
\hline $\mathrm{H}$ & & & 99000 \\
\hline $\mathrm{H}$ & 44000 & & 685000 \\
\hline $\mathrm{H}$ & 152000 & & 15000 \\
\hline 11 & & & \\
\hline $\mathrm{H}$ & & & \\
\hline $\mathrm{H}$ & & & \\
\hline $\mathrm{H}$ & & & \\
\hline C & 1.8 & & \\
\hline & & & \\
\hline $\mathrm{H}$ & & & \\
\hline $\mathrm{H}$ & & & 19000 \\
\hline $\mathrm{C}$ & & & 15000 \\
\hline $\mathrm{C}$ & 40000 & & 8200 \\
\hline $\mathrm{H}$ & 306000 & & -1.71888500 \\
\hline $\mathrm{H}$ & 1.746292000 & & \\
\hline$\Gamma$ & 597000 & -0.193631000 & -3.97235300 \\
\hline & $0.378248 c$ & -0.072642000 & -2.7831570 \\
\hline
\end{tabular}

\section{MeTS- $\mathbf{2}_{\text {isom }}$}

Zero-point correction $=\quad 0.989653($ Hartree/Particle $)$ Thermal correction to Energy= $\quad 1.054463$ Thermal correction to Enthalpy= $\quad 1.055407$

Thermal correction to Gibbs Free Energy $=\quad 0.879784$ Sum of electronic and zero-point Energies $=\quad-2599.463791$ Sum of electronic and thermal Energies $=\quad-2599.398981$
Sum of electronic and thermal Enthalpies $=\quad-2599.398037$ Sum of electronic and thermal Free Energies $=-2599.573660$ E solvent $=-2599.27280605$

$-2.829767000$

0.666721000

0.172835000

$\begin{array}{lll}-5.189691000 & -0.890179000 & 1.206991000\end{array}$

$\begin{array}{lll}-5.802882000 & -1.134441000 & 2.458176000\end{array}$

$\begin{array}{lll}-6.273976000 & -0.299220000 & 2.991958000\end{array}$

$-4.564085000 \quad-3.260948000 \quad 1.093685000$

$\begin{array}{lll}-3.197612000 & 3.393382000 & -0.873550000\end{array}$

$\begin{array}{lll}-5.182323000 & -3.467781000 & 2.340534000\end{array}$

$\begin{array}{lll}-4.151009000 & 1.219509000 & 0.138147000\end{array}$

$\begin{array}{lll}-5.809138000 & -2.415313000 & 3.036942000\end{array}$

$\begin{array}{lll}-5.276819000 & 0.483284000 & 0.617768000\end{array}$

$\begin{array}{lll}-1.957794000 & 3.495414000 & -0.206630000\end{array}$

$\begin{array}{lll}-1.787335000 & 2.932423000 & 0.715050000\end{array}$

$\begin{array}{lll}-1.157957000 & 5.082322000 & -1.855704000\end{array}$

$-4.573456000 \quad-1.966099000 \quad 0.537301000$

$\begin{array}{lll}-4.103248000 & -1.790623000 & -0.437370000\end{array}$

$\begin{array}{lll}-0.930072000 & 4.334188000 & -0.687701000\end{array}$

$\begin{array}{lll}-5.626526000 & 3.087672000 & -0.410813000\end{array}$

$\begin{array}{lll}-5.753429000 & 4.113819000 & -0.772506000\end{array}$

$\begin{array}{lll}-3.885321000 & -4.407337000 & 0.370049000\end{array}$

$\begin{array}{lll}-2.783512000 & -4.339135000 & 0.455438000\end{array}$

$\begin{array}{lll}-4.127550000 & -4.406489000 & -0.708012000\end{array}$

$\begin{array}{lll}-4.188552000 & -5.384180000 & 0.785227000\end{array}$

$\begin{array}{lll}-2.387489000 & 5.016575000 & -2.544331000\end{array}$

$\begin{array}{lll}-6.736562000 & 2.367398000 & 0.042053000\end{array}$

$\begin{array}{lll}-7.736775000 & 2.812949000 & 0.004614000\end{array}$

$\begin{array}{lll}-6.489057000 & -2.665538000 & 4.369065000\end{array}$

$\begin{array}{lll}-5.889945000 & -3.336657000 & 5.010832000\end{array}$

$\begin{array}{lll}-7.476838000 & -3.146931000 & 4.232581000\end{array}$

$\begin{array}{lll}-6.655593000 & -1.724792000 & 4.922205000\end{array}$

$\begin{array}{lll}-3.390207000 & 4.169535000 & -2.044360000\end{array}$

$-4.339786000 \quad 4.089329000 \quad-2.588417000$

$\begin{array}{lll}-6.556438000 & 1.077894000 & 0.552686000\end{array}$

$\begin{array}{lll}-7.418020000 & 0.497380000 & 0.900842000\end{array}$

$\begin{array}{lll}-2.623292000 & 5.851863000 & -3.787706000\end{array}$

$\begin{array}{lll}-2.870652000 & 6.899438000 & -3.527160000\end{array}$

$\begin{array}{lll}-3.459808000 & 5.455733000 & -4.389244000\end{array}$

$-1.725952000 \quad 5.883249000 \quad-4.431641000$

$\begin{array}{lll}0.397499000 & 4.401958000 & 0.040020000\end{array}$

$\begin{array}{lll}0.260851000 & 4.639230000 & 1.111346000\end{array}$

$\begin{array}{lll}1.059570000 & 5.169979000 & -0.395226000\end{array}$

$\begin{array}{lll}0.925239000 & 3.431578000 & -0.009975000\end{array}$

$\begin{array}{rrr}3.726526000 & -0.676385000 & 0.724410000\end{array}$

$\begin{array}{lll}5.729786000 & 0.424661000 & -0.136247000\end{array}$

$\begin{array}{lll}4.056830000 & -2.875300000 & -1.264818000\end{array}$

$\begin{array}{lll}3.646170000 & -3.343510000 & -2.534009000\end{array}$

$\begin{array}{lll}3.929382000 & -2.774826000 & -3.428756000\end{array}$

$\begin{array}{lll}2.881902000 & -4.771374000 & -0.232379000\end{array}$

$\begin{array}{lll}5.608831000 & 1.541692000 & 0.848957000\end{array}$

$\begin{array}{lll}2.500316000 & -5.214365000 & -1.513489000\end{array}$

$\begin{array}{lll}4.788791000 & -0.649048000 & -0.205090000\end{array}$

$\begin{array}{lll}2.876344000 & -4.511946000 & -2.675437000\end{array}$

$\begin{array}{lll}4.960746000 & -1.689440000 & -1.169766000\end{array}$

$\begin{array}{lll}5.375589000 & 1.305617000 & 2.223513000\end{array}$

$\begin{array}{lll}5.221956000 & 0.277662000 & 2.567125000\end{array}$

$\begin{array}{lll}5.523490000 & 3.680680000 & 2.683906000\end{array}$

$\begin{array}{lll}3.660139000 & -3.604059000 & -0.122919000\end{array}$

$\begin{array}{lll}3.970981000 & -3.252542000 & 0.867489000\end{array}$

$\begin{array}{lll}5.334290000 & 2.361494000 & 3.148235000\end{array}$

$\begin{array}{lll}6.797747000 & 0.453781000 & -1.057024000\end{array}$

$\begin{array}{lll}7.520407000 & 1.275270000 & -0.992032000\end{array}$

$\begin{array}{lll}2.447441000 & -5.524820000 & 1.010412000\end{array}$

$\begin{array}{lll}3.238894000 & -5.524086000 & 1.781169000\end{array}$

$\begin{array}{lll}1.550726000 & -5.062827000 & 1.467460000\end{array}$

H $\quad 2.196036000 \quad-6.575397000 \quad 0.780997000$ 


\begin{tabular}{|c|c|c|c|}
\hline $\mathrm{C}$ & 5.756229000 & 3.952465000 & 1.324957000 \\
\hline $\mathrm{C}$ & 6.965198000 & -0.552959000 & -2.015887000 \\
\hline $\mathrm{H}$ & 7.815027000 & -0.523244000 & -2.706826000 \\
\hline $\mathrm{C}$ & 2.484297000 & -5.020189000 & -4.050039000 \\
\hline $\mathrm{H}$ & 3.183867000 & -5.801888000 & -4.405663000 \\
\hline $\mathrm{H}$ & 1.474893000 & -5.469995000 & -4.046304000 \\
\hline $\mathrm{H}$ & 2.493610000 & -4.209147000 & -4.799446000 \\
\hline $\mathrm{C}$ & 5.793905000 & 2.872688000 & 0.419931000 \\
\hline $\mathrm{H}$ & 5.951293000 & 3.071698000 & -0.647901000 \\
\hline $\mathrm{C}$ & 6.056159000 & -1.614707000 & -2.060085000 \\
\hline $\mathrm{H}$ & 6.201207000 & -2.430566000 & -2.777517000 \\
\hline $\mathrm{C}$ & 5.978329000 & 5.372930000 & 0.841448000 \\
\hline $\mathrm{H}$ & 7.056722000 & 5.595464000 & 0.721182000 \\
\hline $\mathrm{H}$ & 5.502509000 & 5.547311000 & -0.140603000 \\
\hline $\mathrm{H}$ & 5.569637000 & 6.111238000 & 1.553688000 \\
\hline $\mathrm{C}$ & 5.091500000 & 2.095409000 & 4.621850000 \\
\hline $\mathrm{H}$ & 5.935847000 & 2.449379000 & 5.243497000 \\
\hline $\mathrm{H}$ & 4.185677000 & 2.618048000 & 4.983657000 \\
\hline $\mathrm{H}$ & 4.958971000 & 1.017720000 & 4.819230000 \\
\hline $\mathrm{Ni}$ & -1.731365000 & 0.295350000 & -1.532667000 \\
\hline $\mathrm{C}$ & 2.516477000 & -0.435467000 & 0.328724000 \\
\hline $\mathrm{H}$ & 2.283639000 & -0.183355000 & -0.721453000 \\
\hline $\mathrm{C}$ & -2.361760000 & 0.344679000 & 1.363538000 \\
\hline $\mathrm{H}$ & -3.036803000 & 0.466758000 & 2.230111000 \\
\hline $\mathrm{C}$ & -1.035190000 & -0.102975000 & 1.691294000 \\
\hline $\mathrm{C}$ & -0.792145000 & -0.457001000 & 3.047748000 \\
\hline $\mathrm{C}$ & 0.061029000 & -0.103425000 & 0.740095000 \\
\hline $\mathrm{H}$ & -1.642771000 & -0.437462000 & 3.743650000 \\
\hline $\mathrm{C}$ & 0.470113000 & -0.821538000 & 3.521096000 \\
\hline $\mathrm{C}$ & 1.372977000 & -0.460298000 & 1.247527000 \\
\hline $\mathrm{C}$ & 0.697048000 & -1.210465000 & 4.968127000 \\
\hline $\mathrm{C}$ & 1.538974000 & -0.805103000 & 2.595286000 \\
\hline $\mathrm{H}$ & 2.554057000 & -1.065209000 & 2.921853000 \\
\hline $\mathrm{O}$ & -0.076434000 & 0.224786000 & -0.511431000 \\
\hline $\mathrm{C}$ & -1.377977000 & -1.588790000 & -1.957625000 \\
\hline $\mathrm{C}$ & -2.220773000 & -2.330102000 & -2.982580000 \\
\hline $\mathrm{H}$ & -0.300476000 & -1.605641000 & -2.188930000 \\
\hline $\mathrm{H}$ & -1.546935000 & -1.959968000 & -0.928109000 \\
\hline $\mathrm{C}$ & -2.856280000 & 0.752135000 & -3.121495000 \\
\hline $\mathrm{C}$ & -1.466566000 & 0.793623000 & -3.421173000 \\
\hline $\mathrm{C}$ & -1.783172000 & -3.812840000 & -3.068457000 \\
\hline $\mathrm{H}$ & -3.294606000 & -2.283050000 & -2.718054000 \\
\hline $\mathrm{H}$ & -2.118446000 & -1.876745000 & -3.986046000 \\
\hline $\mathrm{H}$ & -2.392212000 & -4.354821000 & -3.815295000 \\
\hline $\mathrm{H}$ & -0.721909000 & -3.896351000 & -3.363167000 \\
\hline $\mathrm{H}$ & -1.900758000 & -4.324019000 & -2.096387000 \\
\hline $\mathrm{H}$ & -0.984291000 & 0.027156000 & -4.039878000 \\
\hline $\mathrm{H}$ & -0.925501000 & 1.749924000 & -3.364635000 \\
\hline $\mathrm{H}$ & -3.391437000 & 1.679938000 & -2.891210000 \\
\hline $\mathrm{H}$ & -3.482501000 & -0.073685000 & -3.476216000 \\
\hline $\mathrm{H}$ & -0.224604000 & -1.094795000 & 5.564576000 \\
\hline $\mathrm{H}$ & 1.024021000 & -2.263781000 & 5.061073000 \\
\hline $\mathrm{H}$ & 1.481870000 & -0.588569000 & 5.437958000 \\
\hline $\mathrm{H}$ & -5.175367000 & -4.474071000 & 2.780801000 \\
\hline $\mathrm{H}$ & 5.484055000 & 4.513867000 & 3.399317000 \\
\hline $\mathrm{H}$ & 1.895971000 & -6.126993000 & -1.608431000 \\
\hline 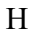 & -0.360743000 & 5.732543000 & -2.240394000 \\
\hline
\end{tabular}

\section{Me2-Coor-C}

Zero-point correction $=\quad 0.990960($ Hartree/Particle $)$ Thermal correction to Energy $=\quad 1.056445$

Thermal correction to Enthalpy= $\quad 1.057390$

Thermal correction to Gibbs Free Energy $=0.878384$

Sum of electronic and zero-point Energies $=\quad-2599.473977$

Sum of electronic and thermal Energies $=\quad-2599.408492$

Sum of electronic and thermal Enthalpies $=\quad-2599.407548$

Sum of electronic and thermal Free Energies $=-2599.586554$

E solvent $=-2599.28477536$

\begin{tabular}{|c|c|c|c|}
\hline & 000 & & \\
\hline & 157000 & .705833000 & 17000 \\
\hline & 699517000 & & \\
\hline & -3.604249000 & 5000 & -0 \\
\hline & -3.025776000 & 2000 & -0 \\
\hline & -5.036849000 & -4.194678000 & 9000 \\
\hline & -4.102677000 & 7000 & -0.1 \\
\hline & -3.944927000 & -4.775787000 & 3000 \\
\hline & -4.345531000 & 0.404106000 & 000 \\
\hline & -3.223782000 & -4.063609000 & 7000 \\
\hline & -5.190065000 & -0.744679000 & 0.17 \\
\hline & -3.085088000 & 3.245058000 & 0.809272000 \\
\hline & -2.839996000 & 2.510255000 & 1.581879000 \\
\hline & -2.726694000 & 5.43 & 000 \\
\hline & & -2.87 & \\
\hline & & -2.4 & \\
\hline & & & \\
\hline & & 1.82 & \\
\hline & & & \\
\hline & & -4.9 & \\
\hline & & $-5.8<-x+1$ & \\
\hline & & -4 & \\
\hline & & & \\
\hline & & & \\
\hline & & & \\
\hline & & & \\
\hline & & -4.7 & -0 \\
\hline & & -4.8 & -1 \\
\hline & & -5.6 & -0 . \\
\hline & -1.1 & -4.0 & 9000 \\
\hline & & & \\
\hline & & & \\
\hline & & -0.5 & 000 \\
\hline & -7. & -1.4 & 000 \\
\hline & -4.0 & 6.24 & 000 \\
\hline & & & \\
\hline & & & \\
\hline & -3.21 & & \\
\hline & & & \\
\hline & & & \\
\hline & & & \\
\hline & & & \\
\hline & & & \\
\hline & & & \\
\hline & & 0 & \\
\hline & & & \\
\hline & & & \\
\hline & & & \\
\hline & & & \\
\hline & & -5 . & \\
\hline & & -0. & \\
\hline & & & \\
\hline & & & 000 \\
\hline & 5.076 & & \\
\hline & & & 00 \\
\hline & 4.882 & & \\
\hline & 4.003882000 & 50000 & 0000 \\
\hline & 88000 & -2.927 & 1000 \\
\hline & 4.887372000 & 3.05 & 2000 \\
\hline & & 00 & 00 \\
\hline & & & \\
\hline & & & \\
\hline & & & \\
\hline & & & \\
\hline & & & \\
\hline & & & \\
\hline & & -0 & -2.03880700 \\
\hline & 7.742169000 & -0.229346000 & -2.7416130 \\
\hline
\end{tabular}




\begin{tabular}{|c|c|c|c|}
\hline & 5.26 & $20084 / 0$ & -0 \\
\hline $\mathrm{H}$ & 5.394907000 & 3.337761000 & 3000 \\
\hline $\mathrm{C}$ & 6.143036000 & -1.446332000 & 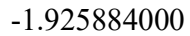 \\
\hline $\mathrm{H}$ & 6.394680000 & -2.324143000 & 25321160 \\
\hline$C$ & 5.083426000 & 5.794338000 & 003060300 \\
\hline 11 & 116162000 & .164992000 & -0.1211080 \\
\hline $\mathrm{H}$ & 4.603368000 & 5.760883000 & -0.9 \\
\hline $\mathrm{H}$ & 4.552481000 & 6.547187000 & \\
\hline $\mathrm{C}$ & 4.710671000 & 2.947966000 & 4. \\
\hline $\mathrm{H}$ & 5.658804000 & 3.154273000 & 394000 \\
\hline $\mathrm{H}$ & 3.966116000 & 3.674862000 & 4.597884000 \\
\hline $\mathrm{H}$ & 4.379633000 & 1.937845000 & 4.522956000 \\
\hline $\mathrm{Ni}$ & -1.734902000 & 0.236671000 & -1.585114000 \\
\hline $\mathrm{C}$ & 2.477979000 & -0.351370000 & 0.3385090 \\
\hline $\mathrm{H}$ & 2.228657000 & -0.189157000 & -0.725261000 \\
\hline $\mathrm{C}$ & -2.448481000 & -0.062181000 & 1.303112000 \\
\hline $\mathrm{H}$ & -3.180600000 & -0.133730000 & 7222000 \\
\hline $\mathrm{C}$ & -1.086826000 & -0.287939000 & \\
\hline $\mathrm{C}$ & -0.824638000 & -0.551283000 & \\
\hline $\mathrm{C}$ & -0.000742000 & -0.225 & \\
\hline $\mathrm{H}$ & -1.6 & 050000 & \\
\hline $\mathrm{C}$ & & -0.746 & \\
\hline $\mathrm{C}$ & & -0.4 & \\
\hline $\mathrm{C}$ & & -1.027703000 & \\
\hline $\mathrm{C}$ & 1.53 & -0.67 & \\
\hline $\mathrm{H}$ & 2.56 & -0.8 & \\
\hline $\mathrm{O}$ & -0.1 & -0 . & -0 . \\
\hline $\mathrm{C}$ & -0.4 & & -3. \\
\hline $\mathrm{C}$ & 0.3 & 1.4 & -3.1 \\
\hline $\mathrm{H}$ & 0.16 & -0.6 & -2. \\
\hline $\mathrm{H}$ & -0.9 & -0.0 & -4 \\
\hline $\mathrm{C}$ & -3.0 & 1. & -2 \\
\hline $\mathrm{C}$ & -3.2 & -0.2 & -2.8 \\
\hline $\mathrm{C}$ & 00 & 1.3 & -4.1 \\
\hline $\mathrm{H}$ & -0.27 & 2.33 & 09600 \\
\hline $\mathrm{H}$ & 0.79 & 1.66 & -2.0 \\
\hline $\mathrm{H}$ & 30000 & 3000 & 18700 \\
\hline $\mathrm{H}$ & 837000 & 0.583 & 5727000 \\
\hline $\mathrm{H}$ & 67000 & 8000 & -5.1 \\
\hline $\mathrm{H}$ & -4.054259000 & -0.721029000 & -2.26707000 \\
\hline $\mathrm{H}$ & -2.823315000 & -0.956664000 & -3.63657600 \\
\hline $\mathrm{H}$ & -2.471709000 & 1.563600000 & -3.70640600 \\
\hline $\mathrm{H}$ & -3.681125000 & 1.755362000 & -2.31645200 \\
\hline $\mathrm{H}$ & 1.226682000 & -2.003502000 & 17000 \\
\hline $\mathrm{H}$ & 893000 & -0.259788000 & 5.4 \\
\hline $\mathrm{H}$ & 367000 & 44000 & 5. \\
\hline $\mathrm{H}$ & & 3000 & 12800 \\
\hline $\mathrm{H}$ & & & 00 \\
\hline $\mathrm{H}$ & & & \\
\hline $\mathrm{H}$ & & & \\
\hline $\mathrm{C}$ & 2.971915000 & -5.485623000 & 2302716 \\
\hline $\mathrm{H}$ & & -4.950683000 & $-4.2780740 \mathrm{C}$ \\
\hline $\mathrm{H}$ & 3.37 & -6.514 & -3.41696 \\
\hline$H_{1}$ & 1.874370000 & -5.570590000 & -3.51009300 \\
\hline
\end{tabular}

\footnotetext{
MeTS-2 ins

Zero-point correction $=\quad 0.991223($ Hartree/Particle $)$

Thermal correction to Energy $=\quad 1.056206$

Thermal correction to Enthalpy= $\quad 1.057150$

Thermal correction to Gibbs Free Energy $=0.876306$

Sum of electronic and zero-point Energies $=\quad-2599.452090$

Sum of electronic and thermal Energies $=\quad-2599.387106$

Sum of electronic and thermal Enthalpies $=\quad-2599.386162$

Sum of electronic and thermal Free Energies $=-2599.567006$

E solvent $=-2599.26857199$

$\begin{array}{lrrr}\mathrm{C} & 1.303698000 & -0.637779000 & 1.255333000 \\ \mathrm{C} & -0.028033000 & -0.323600000 & 0.774928000 \\ \mathrm{C} & -1.094065000 & -0.325771000 & 1.746064000\end{array}$
}

0.449891000

O $\quad-0.222035000$

$\mathrm{Ni} \quad-1.864411000$

N $\quad-2.888064000$

C $\quad-4.271519000$

C $\quad-4.592952000$

$-5.950278000$

$-6.968052000$

$-6.639221000$

$-5.296744000$

$-2.418852000$

$-3.568456000$

$-2.412336000$

$-1.495889000$

$-1.744336000$

$-2.891679000$

$-3.792689000$

$-5.044100000$

$-4.165135000$

$-4.002415000$

$-4.721337000$

$-5.601260000$

$-5.750845000$

$-3.074540000$

$-6.365487000$

$-0.274059000$

$-3.148752000$

0.714740000

2.429819000

3.651184000

$-2.556589000$

$-3.531280000$

$-0.418494000$

0.103127000

$-2.302447000$

$-2.476729000$

$-4.206752000$

$-3.907474000$

0.160794000

$-0.430608000$

0.186928000

1.485157000

$-0.607802000$

2.524950000

1.502232000

1.055505000

$-0.194714000$

$-1.667600000$

$-3.129807000$

$-4.585902000$

$-6.414093000$

$-3.582520000$

$-7.431225000$

$-8.015364000$

$-6.193709000$

$-4.683466000$

$-1.027683000$

$-2.224056000$

2.178038000

4.686626000

5.640698000

6.677263000

6.802596000

5.882344000

4.816203000

5.560083000
$-0.660661000$ $-0.996472000$ $-0.968215000$ $-0.044637000$ 0.308693000 0.427806000 0.841996000 2.211596000 2.601384000 1.674079000 0.334775000 $-0.111632000$ 0.074419000 3.263632000 3.437312000 4.475621000 5.340326000 5.200880000 4.162470000 $-1.559772000$ $-2.355583000$ $-3.729489000$ $-4.305562000$ $-3.540760000$ $-2.174440000$ $-4.568789000$ $-4.179937000$ 4.657972000 6.157206000 $-1.367890000$ $-0.594171000$ $-0.825148000$ 0.226453000 0.499376000 0.368781000 1.799002000 1.025851000 $-0.787366000$ $-0.294329000$ 1.522826000 $-0.356484000$ 0.025244000 2.096862000 1.941462000 2.504654000 $-1.205430000$ $-0.732144000$ $-2.416505000$ $-1.256565000$ $-0.651771000$ 0.129337000 $-5.372590000$ $-1.563048000$ $-1.899073000$ $-0.400731000$ 1.995542000 3.658806000 4.032460000 6.143338000 2.767862000 $-0.334985000$ $-0.778683000$ 0.282532000 0.337046000 $-0.636139000$ $-1.688246000$ $-1.785259000$ 1.362212000
3.102472000 3.552969000 2.604025000 $-0.478936000$ $-1.463098000$ 0.208736000 0.248352000 0.023613000 0.057544000 0.306608000 0.537658000 0.521853000 1.396281000 $-0.261886000$ 0.526563000 0.263938000 $-0.817231000$ $-1.624638000$ $-1.332710000$ 0.811377000 0.047069000 0.311040000

1.374166000 2.165940000

1.872458000 $-0.545985000$ 3.309251000 1.143610000 $-2.773220000$ 4.998424000 0.319921000 0.687528000 $-3.505736000$ $-2.520613000$ $-3.036008000$ $-2.935116000$ $-4.210701000$ $-3.916651000$ $-2.179044000$ $-2.410441000$ $-2.445456000$ $-4.082005000$ $-1.874997000$ $-3.615510000$ $-3.409389000$ 2.912601000 5.444802000 5.095423000 5.614313000 3.812033000 2.237916000 1.597372000 2.497159000 $-0.761041000$ 0.715908000 0.324308000 $-0.093772000$ $-1.960846000$ $-1.036535000$ 1.371675000 $-0.724563000$ $-0.270032000$ $-0.193709000$ $-1.148283000$ $-2.147886000$ $-2.199743000$ $-1.276882000$ 0.836256000 


$\begin{array}{lrrr}\mathrm{H} & 7.408872000 & 1.150354000 & -1.078943000 \\ \mathrm{H} & 7.628902000 & -0.588415000 & -2.865873000 \\ \mathrm{H} & 5.994768000 & -2.478691000 & -2.950807000 \\ \mathrm{C} & 3.894196000 & -2.956455000 & -1.382097000 \\ \mathrm{H} & -2.734180000 & -5.471239000 & -0.008652000 \\ \mathrm{H} & -3.579587000 & -4.909004000 & -1.471095000 \\ \mathrm{H} & -2.180862000 & -3.997174000 & -0.853307000 \\ \mathrm{H} & -6.870903000 & -3.421843000 & 3.932260000 \\ \mathrm{H} & -7.141984000 & -4.875479000 & 2.936817000 \\ \mathrm{H} & -5.696878000 & -4.767053000 & 3.965544000 \\ \mathrm{H} & -3.943413000 & 5.783487000 & -3.442130000 \\ \mathrm{H} & -3.465438000 & 7.152618000 & -2.406091000 \\ \mathrm{H} & -2.239076000 & 6.313911000 & -3.381212000 \\ \mathrm{H} & 0.470539000 & 5.320226000 & 0.669076000 \\ \mathrm{H} & -0.543959000 & 5.108768000 & 2.118302000 \\ \mathrm{H} & 0.217496000 & 3.692469000 & 1.358459000 \\ \mathrm{C} & 3.534426000 & -3.723444000 & -0.252443000 \\ \mathrm{C} & 2.729145000 & -4.870212000 & -0.372127000 \\ \mathrm{C} & 2.281545000 & -5.253895000 & -1.651672000 \\ \mathrm{C} & 2.618849000 & -4.512583000 & -2.800321000 \\ \mathrm{C} & 3.420495000 & -3.366231000 & -2.648625000 \\ \mathrm{H} & 3.892409000 & -3.414764000 & 0.736363000 \\ \mathrm{C} & 2.331856000 & -5.663748000 & 0.858021000 \\ \mathrm{H} & 1.652706000 & -6.148959000 & -1.754850000 \\ \mathrm{C} & 2.148278000 & -4.952020000 & -4.173844000 \\ \mathrm{H} & 3.675625000 & -2.767668000 & -3.532414000 \\ \mathrm{C} & 5.389724000 & 1.071658000 & 2.209689000 \\ \mathrm{C} & 5.383771000 & 2.090973000 & 3.175174000 \\ \mathrm{C} & 5.543349000 & 3.428484000 & 2.753419000 \\ \mathrm{C} & 5.712948000 & 3.753817000 & 1.397071000 \\ \mathrm{C} & 5.718317000 & 2.709279000 & 0.450354000 \\ \mathrm{H} & 5.257522000 & 0.030003000 & 2.519368000 \\ \mathrm{H} & 5.210341000 & 1.767608000 & 4.647066000 \\ \mathrm{H} & 1.233855000 & 1.284462000 & -3.138008000 \\ \mathrm{H} & 5.530822000 & 4.233545000 & 3.501309000 \\ \mathrm{C} & 5.900080000 & 5.193650000 & 0.957994000 \\ \mathrm{H} & 5.827169000 & 2.949236000 & -0.615104000 \\ \mathrm{H} & 2.064427000 & -4.095125000 & -4.865659000 \\ \mathrm{H} & 2.853821000 & -5.672900000 & -4.631701000 \\ \mathrm{H} & 1.162956000 & -5.449284000 & -4.125900000 \\ \mathrm{H} & 2.121686000 & -6.719380000 & 0.610082000 \\ \mathrm{H} & 3.126311000 & -5.644839000 & 1.625090000 \\ \mathrm{H} & 1.418691000 & -5.247448000 & 1.326167000 \\ \mathrm{H} & 5.346183000 & 5.409460000 & 0.026203000 \\ \mathrm{H} & 5.552523000 & 5.900584000 & 1.731760000 \\ \mathrm{H} & 6.08373734000 & 5.421547000 & 0.759325000 \\ \mathrm{H} & 2.097153000 & 5.241476000 \\ \mathrm{H} & 1.852858000 & 2.275796000 & 5.071565000 \\ \mathrm{H} & 0.683041000 & 4.808558000 \\ \mathrm{H} & 1.672992000 & -4.687318000\end{array}$

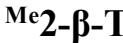

Zero-point correction $=\quad 0.992351($ Hartree/Particle)

Thermal correction to Energy= $\quad 1.057398$

Thermal correction to Enthalpy $=\quad 1.058343$

Thermal correction to Gibbs Free Energy $=0.879813$

Sum of electronic and zero-point Energies $=\quad-2599.495226$

Sum of electronic and thermal Energies $=\quad-2599.430178$

Sum of electronic and thermal Enthalpies $=\quad-2599.429234$

Sum of electronic and thermal Free Energies $=-2599.607764$ E solvent $=-2599.31158214$

$\begin{array}{lrrr}\mathrm{Ni} & -1.827863000 & -0.344240000 & 0.974948000 \\ \mathrm{O} & -0.100211000 & -0.014244000 & 0.305497000 \\ \mathrm{C} & 0.195108000 & 0.368346000 & -0.903048000 \\ \mathrm{C} & -0.811198000 & 0.569894000 & -1.925124000 \\ \mathrm{C} & -2.203034000 & 0.332512000 & -1.696282000 \\ \mathrm{~N} & -2.791296000 & -0.095334000 & -0.586211000\end{array}$

\begin{tabular}{|c|c|c|c|}
\hline & & & \\
\hline & 1800903000 & & 31000 \\
\hline & -0.584227000 & -5.572630000 & 162000 \\
\hline & -0.418863000 & 70000 & 000 \\
\hline & 0.912226000 & 1900000 & 6000 \\
\hline & 1.306964000 & 0000 & 5000 \\
\hline & 2.615816000 & 2000 & 51000 \\
\hline & 3.875081000 & 7000 & 18000 \\
\hline & 4.819763000 & 8000 & 7000 \\
\hline & 4.965208000 & 802000 & 7000 \\
\hline & 5.939855000 & 1.168836000 & 6000 \\
\hline & 6.753855000 & 0.031353000 & 2000 \\
\hline & 6.614219000 & -0.910483000 & 69000 \\
\hline & 5.667476000 & 7000 & 0. \\
\hline & 4.155107000 & & \\
\hline & & & \\
\hline & 3.2364 & & \\
\hline & 2.7460 & & \\
\hline & 2.94 & & \\
\hline & 3.6 & & \\
\hline & 2.41 & & \\
\hline & 3.0 & 0 & \\
\hline & & 00 & \\
\hline & & 0 & \\
\hline & & -2.4 & \\
\hline & & -3.8 & \\
\hline & & -4.1 & \\
\hline & & & \\
\hline & & & 000 \\
\hline & & & -4 \\
\hline & -4.2 & & -0 \\
\hline & 0 & & -0 \\
\hline & & -1.6 & 1000 \\
\hline & -7.0 & -0.5 & 59000 \\
\hline & -6.45 & & 77000 \\
\hline & & & \\
\hline & -3.9 & -2.8 & 9000 \\
\hline & -4.389187000 & -3.82 & \\
\hline E & & & \\
\hline & & & \\
\hline$x$ & & & \\
\hline & & & \\
\hline & & & 00 \\
\hline & & & \\
\hline & & & \\
\hline & & & \\
\hline & & & \\
\hline & -3 . & & \\
\hline & -4 & & \\
\hline & & & -1 \\
\hline & & & -2 \\
\hline & & & \\
\hline & & -0.7 & 00 \\
\hline & -1.9 & -0.7 & 1000 \\
\hline & & -2.1 & 5000 \\
\hline & -2.27 & -3.7 & 0000 \\
\hline & -2.591 & 000 & 3000 \\
\hline & 000 & 2000 & 49000 \\
\hline & -1.2 & & 33000 \\
\hline & & & \\
\hline & & & \\
\hline & & & \\
\hline & & & \\
\hline & & & \\
\hline & & & \\
\hline & & & \\
\hline & & & \\
\hline & 3.785412000 & 2.466701000 & 3.7528780 \\
\hline
\end{tabular}




\begin{tabular}{|c|c|c|c|}
\hline & & & \\
\hline & .497166000 & 23144000 & 600 \\
\hline & 82134000 & 33751000 & 000 \\
\hline & .419869000 & 6.397428000 & 900 \\
\hline & 194681000 & 6.022960000 & 2200 \\
\hline & 347589000 & 5.068482000 & 247862000 \\
\hline & 16000 & & \\
\hline & 497563000 & 3000 & \\
\hline & 000 & -2.3 & \\
\hline & 00 & -0.9 & \\
\hline & 0 & 00 & \\
\hline & 00 & 00 & \\
\hline & 0 & -6.2 & \\
\hline & 000 & 0 & \\
\hline & 000 & & \\
\hline & 6000 & & \\
\hline & 71000 & & \\
\hline & 000 & & \\
\hline & 000 & & \\
\hline & 2000 & -2.3 & \\
\hline & 1000 & -6.1 & \\
\hline & & & \\
\hline & 4000 & -6.9 & 00 \\
\hline & 39000 & -5.7 & 000 \\
\hline & -6.622122000 & -2.6 & 7000 \\
\hline & 95000 & -0.6 & \\
\hline & 79000 & 1.5 & -0 . \\
\hline & 67000 & 2.1 & \\
\hline & -5.7 & 2.8 & -2 \\
\hline & 5000 & 5.0 & 5000 \\
\hline & 1000 & 5.7 & -3 \\
\hline & 7000 & 6.4 & \\
\hline & & 6.0 & \\
\hline & -1 & 4.2 & \\
\hline & -2 . & 4.2 & \\
\hline & & & \\
\hline & & & \\
\hline & & -1.6 & $2 .($ \\
\hline 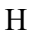 & & & \\
\hline t & & & \\
\hline 11 & & & 3000 \\
\hline 11 & 40000 & -1.5 & 3000 \\
\hline 11 & 42000 & -2.1 & 3000 \\
\hline 11 & 01000 & -3.9 & 7000 \\
\hline $\mathrm{H}$ & 56000 & -4.5 & 534000 \\
\hline & -1.243245000 & -3.757455000 & 6.09756900 \\
\hline & -5.263741000 & -3.635774000 & 1.03846100 \\
\hline & -1.723792000 & -2.922860000 & 3.06434000 \\
\hline
\end{tabular}

MeTS-2

Zero-point correction $=\quad 0.987524($ Hartree/Particle $)$ Thermal correction to Energy $=\quad 1.053252$ Thermal correction to Enthalpy $=\quad 1.054197$

Thermal correction to Gibbs Free Energy= 0.876593 Sum of electronic and zero-point Energies $=\quad-2599.444987$ Sum of electronic and thermal Energies $=\quad-2599.379258$ Sum of electronic and thermal Enthalpies $=\quad-2599.378314$ Sum of electronic and thermal Free Energies $=-2599.555918$ E solvent $=-2599.26490094$

$\begin{array}{lrrr}\mathrm{N} & 2.761363000 & 0.610690000 & 0.195351000 \\ \mathrm{C} & 5.176022000 & 0.099923000 & -0.052862000 \\ \mathrm{C} & 3.401069000 & 3.547102000 & 0.176026000 \\ \mathrm{C} & 3.721950000 & 4.620654000 & 1.041465000 \\ \mathrm{H} & 4.669207000 & 4.594743000 & 1.594667000 \\ \mathrm{C} & 1.267867000 & 4.656263000 & -0.335985000 \\ \mathrm{C} & 4.987562000 & -1.373373000 & 0.110920000 \\ \mathrm{C} & 1.622821000 & 5.707028000 & 0.528502000 \\ \mathrm{C} & 4.119433000 & 1.055182000 & 0.049297000\end{array}$

\begin{tabular}{|c|c|c|c|}
\hline & 0 & & \\
\hline & 2470000 & 0000 & 000 \\
\hline & 000 & 000 & \\
\hline & 3.611971000 & 263833000 & 000 \\
\hline & 4.809564000 & -4.169456000 & 2000 \\
\hline & 165793000 & 582711000 & 3000 \\
\hline & 892974000 & 2.757769000 & 1000 \\
\hline & 4.067987000 & -3.320606000 & 8000 \\
\hline & 493639000 & 0.564591000 & -0.267 \\
\hline & 305925000 & -0.169033000 & -0.310106000 \\
\hline & -0.062283000 & 4.658560000 & -1.063432000 \\
\hline & .734955000 & 000 & 000 \\
\hline & 0.066116000 & & 000 \\
\hline & & & \\
\hline & & & \\
\hline & & 1.9 & -0.3 \\
\hline & 7.80 & 2.2 & \\
\hline & 00 & 6.8 & \\
\hline & 00 & & \\
\hline & 0 & & \\
\hline & 4.03 & 6.5 & \\
\hline & 00 & -2.2 & -0. \\
\hline & & & \\
\hline & & & \\
\hline & & & \\
\hline & & -4.5 & 000 \\
\hline & & -4.9 & -0 . \\
\hline & 6.8 & -4.0 & 3000 \\
\hline & 00 & & 000 \\
\hline & 00 & $-3.8-x-1$ & \\
\hline & 00 & & 000 \\
\hline & 00 & -3.6 & 6000 \\
\hline & 00 & -3.4 & 7000 \\
\hline & 00 & -0.4 & \\
\hline & -4.77 & -2.4 & \\
\hline & & & \\
\hline & & & \\
\hline & & & \\
\hline & & & \\
\hline & & & \\
\hline & & & \\
\hline & & & \\
\hline & & & \\
\hline & & -0.2 & \\
\hline & & & \\
\hline & & & \\
\hline & & -5 . & \\
\hline & & & -2 . \\
\hline & & & -3 . \\
\hline & & -4 & 000 \\
\hline & & & 000 \\
\hline & & -4 & 000 \\
\hline & & & 000 \\
\hline & 000 & & 5000 \\
\hline & 000 & & 7000 \\
\hline & 94000 & 4000 & 5000 \\
\hline & 5000 & 18000 & 5000 \\
\hline & 000 & -2.3 & 1000 \\
\hline & & -2.8 & 000 \\
\hline & & & \\
\hline & & & \\
\hline & & & \\
\hline & & & \\
\hline & & & \\
\hline & & & \\
\hline & & & -1.92216400 \\
\hline & -7.272470000 & -0.318457000 & -2.493304 \\
\hline & -1.805834000 & -6.583538000 & -0.1484580 \\
\hline
\end{tabular}




$\begin{array}{rrrr}\mathrm{H} & -2.441833000 & -7.488555000 & -0.088469000 \\ \mathrm{H} & -1.618941000 & -6.386790000 & -1.218968000 \\ \mathrm{H} & -0.840129000 & -6.835248000 & 0.325632000 \\ \mathrm{C} & -2.770168000 & -3.880778000 & 4.051256000 \\ \mathrm{H} & -3.740862000 & -3.711192000 & 4.552632000 \\ \mathrm{H} & -2.285968000 & -4.746682000 & 4.535695000 \\ \mathrm{H} & -2.143781000 & -2.990855000 & 4.252953000 \\ \mathrm{Ni} & 1.792016000 & -0.506462000 & -1.136207000 \\ \mathrm{C} & -2.592482000 & -0.408793000 & 0.296490000 \\ \mathrm{H} & -2.205906000 & -0.853098000 & -0.638023000 \\ \mathrm{C} & 2.138820000 & 1.023092000 & 1.286230000 \\ \mathrm{H} & 2.736844000 & 1.571539000 & 2.034385000 \\ \mathrm{C} & 0.754496000 & 0.848337000 & 1.622277000 \\ \mathrm{C} & 0.327814000 & 1.340417000 & 2.887679000 \\ \mathrm{C} & -0.210988000 & 0.254905000 & 0.724613000 \\ \mathrm{H} & 1.085031000 & 1.795678000 & 3.541677000 \\ \mathrm{C} & -0.999225000 & 1.280771000 & 3.318428000 \\ \mathrm{C} & -1.587817000 & 0.196483000 & 1.175653000 \\ \mathrm{C} & -1.424019000 & 1.814265000 & 4.671899000 \\ \mathrm{C} & -1.939114000 & 0.703812000 & 2.434145000 \\ \mathrm{H} & -2.996906000 & 0.636435000 & 2.719112000 \\ \mathrm{O} & 0.071766000 & -0.200712000 & -0.466953000 \\ \mathrm{C} & 1.152320000 & -2.052971000 & -2.080154000 \\ \mathrm{C} & 2.601749000 & -2.334629000 & -2.302990000 \\ \mathrm{H} & 0.618383000 & -2.672198000 & -1.345611000 \\ \mathrm{H} & 0.538989000 & -1.818427000 & -2.964183000 \\ \mathrm{C} & 2.901650000 & 1.113634000 & -3.309515000 \\ \mathrm{C} & 1.566352000 & 0.900185000 & -3.225343000 \\ \mathrm{C} & 3.059309000 & -2.517651000 & -3.760698000 \\ \mathrm{H} & 3.214065000 & -1.419600000 & -1.906526000 \\ \mathrm{H} & 2.978110000 & -3.143025000 & -1.652210000 \\ \mathrm{H} & 4.160363000 & -2.553913000 & -3.838930000 \\ \mathrm{H} & 2.654322000 & -3.463031000 & -4.163435000 \\ \mathrm{H} & 2.692900000 & -1.695879000 & -4.400438000 \\ \mathrm{H} & 0.908449000 & 1.531845000 & -2.617632000 \\ \mathrm{H} & 1.058620000 & 0.181390000 & -3.878434000 \\ \mathrm{H} & 3.536726000 & 0.522127000 & -3.979193000 \\ \mathrm{H} & 3.407507000 & 1.879715000 & -2.712431000 \\ \mathrm{H} & -2.198591000 & 2.598411000 & 4.575840000 \\ \mathrm{H} & -1.851970000 & 1.017510000 & 5.309818000 \\ \mathrm{H} & -0.570071000 & 2.254085000 & 5.216067000 \\ \mathrm{H} & 4.738001000 & -5.256858000 & 0.595669000 \\ \mathrm{H} & -5.944369000 & 5.114967000 & -0.835626000 \\ & -1.925597000 & 6.543955000 & 0.669060000 \\ & & & \\ \mathrm{H} & & \end{array}$

Me TS-2 $2_{\text {decoorAr }}$

Zero-point correction= Thermal correction to Energy=

Thermal correction to Enthalpy= $\quad 1.055053$

Thermal correction to Gibbs Free Energy $=0.876241$

Sum of electronic and zero-point Energies $=\quad-2599.453611$

Sum of electronic and thermal Energies $=\quad-2599.387661$

Sum of electronic and thermal Enthalpies $=\quad-2599.386717$

Sum of electronic and thermal Free Energies $=-2599.565530$ E solvent $=-2599.26770583$

$\begin{array}{lrrr}\mathrm{N} & 2.924286000 & -0.135443000 & 0.221112000 \\ \mathrm{C} & 4.650384000 & -1.558893000 & -0.606627000 \\ \mathrm{C} & 5.057367000 & 2.147226000 & 0.460223000 \\ \mathrm{C} & 4.251963000 & 3.001798000 & -0.322349000 \\ \mathrm{H} & 3.774946000 & 2.609301000 & -1.228432000 \\ \mathrm{C} & 5.538887000 & 4.023843000 & 1.972173000 \\ \mathrm{C} & 3.607955000 & -2.624403000 & -0.752755000 \\ \mathrm{C} & 4.713371000 & 4.843944000 & 1.180241000 \\ \mathrm{C} & 4.304108000 & -0.280366000 & -0.080207000 \\ \mathrm{C} & 4.064647000 & 4.351020000 & 0.029988000 \\ \mathrm{C} & 5.308399000 & 0.726712000 & 0.051778000 \\ \mathrm{C} & 2.766646000 & -2.986838000 & 0.365482000\end{array}$

$2.856338000 \quad-2.426331000$ $2.371256000 \quad-5.176783000$ $\begin{array}{ll}5.700651000 & 2.674366000\end{array}$ $6.331965000 \quad 2.014742000$ $2.172361000-4.291601000$ $5.988046000-1.841968000$ $6.244892000-2.842017000$ $6.259479000 \quad 4.583443000$ $5.739511000 \quad 5.468012000$ $\begin{array}{ll}7.289972000 & 4.898333000\end{array}$ $6.341108000 \quad 3.833256000$ $3.137812000-4.825560000$ $6.985819000-0.870683000$ $8.025411000-1.096292000$ $3.166738000 \quad 5.248583000$ $3.474937000 \quad 6.306635000$ $2.116262000 \quad 5.188794000$ 3.175904000 3.734158000 4.348301000 6.639495000 7.402480000 3.292849000 3.689010000 3.978378000 2.320846000 1.357576000 1.095535000 0.415914000 1.905074000 $-3.671705000$

$-5.291204000$ $-4.850347000$ $-4.657859000$ $-4.823764000$ $-4.200939000$ $-4.745454000$ $-4.017930000$ $-4.760492000$ $-4.236609000$ $-5.361455000$ $-4.452436000$ $-4.573566000$ $-3.867410000$ $-4.612057000$ $-4.756212000$ $-4.014168000$ $-6.395725000$ $-6.816559000$ $-3.956514000$ $-4.526346000$ $-4.250723000$ $-2.887863000$ $-4.147836000$ $-6.971956000$ $-7.835421000$ $-4.010191000$ $-3.869891000$ $-3.110352000$ $-4.862400000$ $-4.581805000$ $-4.785440000$ $-6.453373000$ $-6.910782000$ $-4.003253000$ $-3.502779000$

$-3.416144000$ $-4.989664000$
1.304028000 $-0.620788000$ 1.600095000 2.209136000 0.433103000 $-0.937962000$ $-1.303623000$ 3.183300000 3.590875000 2.927754000 3.989961000 $-1.775241000$ $-0.775083000$ $-1.036735000$ $-0.798400000$ $-0.728971000$ $-0.453803000$ $-1.863775000$ $-1.825496000$ $-2.692427000$ $-0.290729000$ $-0.200227000$ $-2.906753000$ $-2.540921000$ $-3.685669000$ $-3.389957000$ 1.649165000 1.644062000 1.687664000 2.586182000 0.649464000 0.277622000 $-1.501697000$ $-2.865316000$ $-3.613006000$ $-0.937894000$

1.385432000 $-2.311991000$ $-0.060856000$ $-3.286322000$ $-1.104680000$ 2.657924000 2.817893000 3.493094000 $-0.547397000$ 0.514673000 3.716214000 $-0.454578000$ $-0.171465000$ 0.093075000 $-0.140500000$ 1.103817000 0.133320000 2.241128000 $-1.495139000$ $-2.040890000$ $-4.757904000$ $-4.939938000$ $-5.138763000$ $-5.375116000$ 1.196893000 0.208299000 $-1.811558000$ $-2.606546000$ 2.017629000 1.056866000 2.823385000 1.988870000 


$\begin{array}{lrrr}\mathrm{C} & -3.687888000 & -1.536769000 & 5.071042000 \\ \mathrm{H} & -2.593865000 & -1.462774000 & 5.225663000 \\ \mathrm{H} & -4.103887000 & -0.519467000 & 5.172988000 \\ \mathrm{H} & -4.089802000 & -2.153422000 & 5.895945000 \\ \mathrm{Ni} & 1.712917000 & -1.449281000 & -0.683685000 \\ \mathrm{C} & -2.473343000 & 0.366827000 & 0.430367000 \\ \mathrm{H} & -2.271961000 & -0.422440000 & -0.315621000 \\ \mathrm{C} & 2.508599000 & 0.694928000 & 1.158622000 \\ \mathrm{H} & 3.260133000 & 1.248768000 & 1.741358000 \\ \mathrm{C} & 1.143186000 & 0.978317000 & 1.488924000 \\ \mathrm{C} & 0.917254000 & 1.940446000 & 2.514486000 \\ \mathrm{C} & 0.016170000 & 0.394094000 & 0.786419000 \\ \mathrm{H} & 1.796359000 & 2.358148000 & 3.025137000 \\ \mathrm{C} & -0.357025000 & 2.378280000 & 2.877322000 \\ \mathrm{C} & -1.302218000 & 0.887667000 & 1.143002000 \\ \mathrm{C} & -0.570122000 & 3.395788000 & 3.980060000 \\ \mathrm{C} & -1.450477000 & 1.837282000 & 2.162736000 \\ \mathrm{H} & -2.474292000 & 2.157026000 & 2.396003000 \\ \mathrm{O} & 0.107620000 & -0.534670000 & -0.122394000 \\ \mathrm{C} & 0.471147000 & -2.660962000 & -1.595695000 \\ \mathrm{C} & -0.359643000 & -2.008902000 & -2.693519000 \\ \mathrm{H} & 1.084637000 & -3.491399000 & -1.981548000 \\ \mathrm{H} & -0.169156000 & -3.011499000 & -0.765453000 \\ \mathrm{C} & -1.461649000 & -2.963289000 & -3.215147000 \\ \mathrm{H} & -0.837055000 & -1.088542000 & -2.309642000 \\ \mathrm{H} & 0.286211000 & -1.715126000 & -3.543372000 \\ \mathrm{H} & -2.052181000 & -2.485969000 & -4.019558000 \\ \mathrm{H} & -1.026354000 & -3.895707000 & -3.620517000 \\ \mathrm{H} & -2.157793000 & -3.242453000 & -2.403466000 \\ \mathrm{H} & -1.146687000 & 4.269537000 & 3.622441000 \\ \mathrm{H} & -1.134479000 & 2.965666000 & 4.829598000 \\ \mathrm{H} & 0.391606000 & 3.767758000 & 4.374508000 \\ \mathrm{H} & 1.927650000 & -6.180152000 & -0.567906000 \\ \mathrm{H} & -3.695310000 & 5.942787000 & -2.626857000 \\ \mathrm{H} & 4.575043000 & 5.896331000 & 1.462374000 \\ \mathrm{H} & -3.525368000 & -4.164801000 & 4.315220000 \\ \mathrm{C} & 2.754961000 & -0.113876000 & -3.343991000 \\ \mathrm{C} & 1.809084000 & 0.662294000 & -2.783845000 \\ \mathrm{H} & 3.808049000 & -0.048199000 & -3.047968000 \\ \mathrm{H} & 2.509653000 & -0.846535000 & -4.122052000 \\ \mathrm{H} & 2.047844000 & 1.397516000 & -2.008383000 \\ \mathrm{H} & 0.757488000 & 0.601770000 & -3.082324000\end{array}$

\section{Me1-Pr-C}

Zero-point correction $=\quad 0.937336($ Hartree/Particle $)$ Thermal correction to Energy $=0.999381$

Thermal correction to Enthalpy= $\quad 1.000325$

Thermal correction to Gibbs Free Energy= 0.830692

Sum of electronic and zero-point Energies $=\quad-2520.929363$

Sum of electronic and thermal Energies $=\quad-2520.867319$

Sum of electronic and thermal Enthalpies $\quad-2520.866374$

Sum of electronic and thermal Free Energies $=-2521.036007$ E solvent $=-2520.71585006$

$\begin{array}{lrrr}\mathrm{N} & 2.996442000 & 0.052342000 & 0.182325000 \\ \mathrm{C} & 4.799468000 & -1.242512000 & -0.690131000 \\ \mathrm{C} & 4.887474000 & 2.537654000 & 0.134129000 \\ \mathrm{C} & 3.887884000 & 3.191176000 & -0.618212000 \\ \mathrm{H} & 3.354002000 & 2.636181000 & -1.399353000 \\ \mathrm{C} & 5.289488000 & 4.623225000 & 1.370213000 \\ \mathrm{C} & 3.834815000 & -2.383331000 & -0.809886000 \\ \mathrm{C} & 4.277177000 & 5.241388000 & 0.611470000 \\ \mathrm{C} & 4.365297000 & 0.019340000 & -0.193489000 \\ \mathrm{C} & 3.568013000 & 4.541520000 & -0.385157000 \\ \mathrm{C} & 5.272656000 & 1.121342000 & -0.162232000 \\ \mathrm{C} & 3.065757000 & -2.838328000 & 0.325982000 \\ \mathrm{H} & 3.159201000 & -2.306066000 & 1.281237000 \\ \mathrm{C} & 2.767908000 & -5.013751000 & -0.725614000 \\ \mathrm{C} & 5.581962000 & 3.268122000 & 1.122562000\end{array}$

\begin{tabular}{|c|c|c|c|}
\hline $\mathrm{H}$ & 6.357906000 & 2.764605000 & 1.713413000 \\
\hline $\mathrm{C}$ & 2.549878000 & -4.178125000 & 0.365229000 \\
\hline $\mathrm{C}$ & 6.145527000 & -1.427263000 & -1.052766000 \\
\hline $\mathrm{H}$ & 6.473101000 & -2.414374000 & -1.396920000 \\
\hline $\mathrm{C}$ & 6.063625000 & 5.405696000 & 2.413134000 \\
\hline $\mathrm{H}$ & 6.923928000 & 5.936454000 & 1.960687000 \\
\hline $\mathrm{H}$ & 6.466214000 & 4.744836000 & 3.200852000 \\
\hline $\mathrm{H}$ & 5.430793000 & 6.169935000 & 2.898283000 \\
\hline $\mathrm{C}$ & 3.464532000 & -4.575132000 & -1.893044000 \\
\hline $\mathrm{C}$ & 7.060820000 & -0.369220000 & -0.957690000 \\
\hline $\mathrm{H}$ & 8.108194000 & -0.517059000 & -1.242619000 \\
\hline $\mathrm{C}$ & 2.466685000 & 5.219444000 & -1.176236000 \\
\hline $\mathrm{H}$ & 2.603319000 & 6.314626000 & -1.205898000 \\
\hline $\mathrm{H}$ & 1.474425000 & 5.021842000 & -0.726852000 \\
\hline $\mathrm{H}$ & 2.429167000 & 4.850217000 & -2.216366000 \\
\hline $\mathrm{C}$ & 3.975262000 & -3.279777000 & -1.921477000 \\
\hline $\mathrm{H}$ & 4.538791000 & -2.937773000 & -2.798341000 \\
\hline $\mathrm{C}$ & 6.617355000 & 0.887646000 & -0.531550000 \\
\hline $\mathrm{H}$ & 7.308909000 & 1.737723000 & -0.518971000 \\
\hline $\mathrm{C}$ & 3.640262000 & -5.519353000 & -3.064050000 \\
\hline $\mathrm{H}$ & 4.090745000 & -6.477594000 & -2.743338000 \\
\hline $\mathrm{H}$ & 4.287469000 & -5.082760000 & -3.843760000 \\
\hline $\mathrm{H}$ & 2.668012000 & -5.763789000 & -3.532779000 \\
\hline $\mathrm{C}$ & 1.810509000 & -4.652091000 & 1.596364000 \\
\hline $\mathrm{H}$ & 1.640826000 & -5.742119000 & 1.572390000 \\
\hline $\mathrm{H}$ & 0.824846000 & -4.156138000 & 1.678131000 \\
\hline $\mathrm{H}$ & 2.369223000 & -4.411403000 & 2.520410000 \\
\hline $\mathrm{N}$ & -3.622666000 & 0.535786000 & 0.634847000 \\
\hline $\mathrm{C}$ & -4.987262000 & -1.489742000 & 0.221673000 \\
\hline $\mathrm{C}$ & -5.152645000 & 2.047828000 & -1.321281000 \\
\hline $\mathrm{C}$ & -5.010049000 & 3.021056000 & -0.304805000 \\
\hline $\mathrm{H}$ & -5.047243000 & 2.704400000 & 0.742617000 \\
\hline $\mathrm{C}$ & -4.913072000 & 3.824575000 & -3.007183000 \\
\hline $\mathrm{C}$ & -4.271580000 & -2.310966000 & 1.244723000 \\
\hline $\mathrm{C}$ & -4.786274000 & 4.765639000 & -1.972067000 \\
\hline $\mathrm{C}$ & -4.665551000 & -0.123598000 & -0.050886000 \\
\hline $\mathrm{C}$ & -4.831307000 & 4.378238000 & -0.615248000 \\
\hline $\mathrm{C}$ & -5.433053000 & 0.614918000 & -1.003250000 \\
\hline $\mathrm{C}$ & -3.977218000 & -1.810387000 & 2.530627000 \\
\hline $\mathrm{H}$ & -4.229025000 & -0.770293000 & 2.767285000 \\
\hline $\mathrm{C}$ & -3.046536000 & -3.949394000 & 3.185483000 \\
\hline $\mathrm{C}$ & -5.100649000 & 2.469379000 & -2.665306000 \\
\hline $\mathrm{H}$ & -5.192676000 & 1.720728000 & -3.462703000 \\
\hline $\mathrm{C}$ & -3.365348000 & -2.616943000 & 3.508725000 \\
\hline $\mathrm{C}$ & -6.051542000 & -2.089680000 & -0.489142000 \\
\hline $\mathrm{H}$ & -6.314716000 & -3.127692000 & -0.255319000 \\
\hline $\mathrm{C}$ & -4.836585000 & 4.247222000 & -4.461978000 \\
\hline $\mathrm{H}$ & -4.860185000 & 5.346179000 & -4.564952000 \\
\hline $\mathrm{H}$ & -3.904188000 & 3.888416000 & -4.939083000 \\
\hline $\mathrm{H}$ & -5.676788000 & 3.834121000 & -5.050435000 \\
\hline $\mathrm{C}$ & -3.329052000 & -4.485016000 & 1.913095000 \\
\hline $\mathrm{C}$ & -6.785113000 & -1.381589000 & -1.446417000 \\
\hline $\mathrm{H}$ & -7.612905000 & -1.864703000 & -1.977401000 \\
\hline $\mathrm{C}$ & -4.690861000 & 5.415292000 & 0.482899000 \\
\hline $\mathrm{H}$ & -5.414865000 & 6.242128000 & 0.356685000 \\
\hline $\mathrm{H}$ & -4.857182000 & 4.970905000 & 1.479404000 \\
\hline $\mathrm{H}$ & -3.681432000 & 5.869939000 & 0.483766000 \\
\hline $\mathrm{C}$ & -3.934049000 & -3.653577000 & 0.954494000 \\
\hline $\mathrm{H}$ & -4.134743000 & -4.047966000 & -0.049654000 \\
\hline $\mathrm{C}$ & -6.473702000 & -0.038763000 & -1.694700000 \\
\hline $\mathrm{H}$ & -7.060912000 & 0.536706000 & -2.419625000 \\
\hline $\mathrm{C}$ & -3.004493000 & -5.931534000 & 1.591784000 \\
\hline $\mathrm{H}$ & -3.830752000 & -6.606531000 & 1.889856000 \\
\hline $\mathrm{H}$ & -2.837899000 & -6.078934000 & 0.510009000 \\
\hline $\mathrm{H}$ & -2.099886000 & -6.273034000 & 2.126239000 \\
\hline $\mathrm{C}$ & -3.051467000 & -2.051570000 & 4.880965000 \\
\hline $\mathrm{H}$ & -3.961699000 & -1.668728000 & 5.379646000 \\
\hline $\mathrm{H}$ & -2.603783000 & -2.815679000 & 5.540090000 \\
\hline
\end{tabular}




$\begin{array}{lrrr}\mathrm{H} & -2.341457000 & -1.205851000 & 4.814145000 \\ \mathrm{Ni} & 1.878287000 & -1.376740000 & -0.617413000 \\ \mathrm{C} & -2.392132000 & 0.214698000 & 0.385119000 \\ \mathrm{H} & -2.131090000 & -0.542436000 & -0.375262000 \\ \mathrm{C} & 2.546841000 & 0.896713000 & 1.091287000 \\ \mathrm{H} & 3.266955000 & 1.542086000 & 1.616931000 \\ \mathrm{C} & 1.170159000 & 1.083542000 & 1.441648000 \\ \mathrm{C} & 0.880292000 & 2.042596000 & 2.453014000 \\ \mathrm{C} & 0.086130000 & 0.422840000 & 0.742322000 \\ \mathrm{H} & 1.728843000 & 2.523880000 & 2.959122000 \\ \mathrm{C} & -0.421629000 & 2.398843000 & 2.808206000 \\ \mathrm{C} & -1.261970000 & 0.831430000 & 1.087427000 \\ \mathrm{C} & -0.704356000 & 3.413831000 & 3.897603000 \\ \mathrm{C} & -1.475523000 & 1.778238000 & 2.098869000 \\ \mathrm{H} & -2.518877000 & 2.029523000 & 2.329152000 \\ \mathrm{O} & 0.240715000 & -0.507562000 & -0.160374000 \\ \mathrm{C} & 0.748104000 & -2.502291000 & -1.728822000 \\ \mathrm{C} & 0.493700000 & -1.687155000 & -2.993222000 \\ \mathrm{H} & 1.258983000 & -3.456068000 & -1.933434000 \\ \mathrm{H} & -0.170792000 & -2.664768000 & -1.139706000 \\ \mathrm{C} & -0.470572000 & -2.410279000 & -3.968248000 \\ \mathrm{H} & 0.058688000 & -0.705253000 & -2.726496000 \\ \mathrm{H} & 1.447721000 & -1.489072000 & -3.523168000 \\ \mathrm{H} & -0.638497000 & -1.807431000 & -4.880250000 \\ \mathrm{H} & -0.063908000 & -3.390701000 & -4.277837000 \\ \mathrm{H} & -1.451084000 & -2.587837000 & -3.490784000 \\ \mathrm{H} & -1.327053000 & 4.248550000 & 3.524602000 \\ \mathrm{H} & -1.251399000 & 2.960555000 & 4.746330000 \\ \mathrm{H} & 0.230066000 & 3.845361000 & 4.296882000 \\ \mathrm{H} & 2.392287000 & -6.045294000 & -0.692244000 \\ \mathrm{H} & -4.646338000 & 5.825831000 & -2.224596000 \\ \mathrm{H} & 4.035567000 & 6.296330000 & 0.799136000 \\ \mathrm{H} & -2.564916000 & -4.584758000 & 3.941453000\end{array}$

\section{${ }^{M e}$ TS-2}

Zero-point correction $=0.935570($ Hartree/Particle $)$

Thermal correction to Energy= $\quad 0.997228$

Thermal correction to Enthalpy= $\quad 0.998173$

Thermal correction to Gibbs Free Energy $=0.828954$

Sum of electronic and zero-point Energies $=\quad-2520.918938$

Sum of electronic and thermal Energies $=-2520.857280$

Sum of electronic and thermal Enthalpies $=\quad-2520.856336$

Sum of electronic and thermal Free Energies $=-2521.025555$

E solvent $=-2520.71174182$

$\begin{array}{lrrr}\mathrm{N} & 2.864386000 & 0.409255000 & -0.534046000 \\ \mathrm{C} & 4.492811000 & 2.200592000 & -0.314340000 \\ \mathrm{C} & 5.136452000 & -1.562797000 & -0.976397000 \\ \mathrm{C} & 4.438650000 & -2.310975000 & -0.004856000 \\ \mathrm{H} & 3.964029000 & -1.789747000 & 0.834988000 \\ \mathrm{C} & 5.656818000 & -3.647293000 & -2.172036000 \\ \mathrm{C} & 3.412839000 & 3.162483000 & 0.070705000 \\ \mathrm{C} & 4.948121000 & -4.362827000 & -1.187039000 \\ \mathrm{C} & 4.228791000 & 0.820831000 & -0.581829000 \\ \mathrm{C} & 4.340885000 & -3.712103000 & -0.095348000 \\ \mathrm{C} & 5.303101000 & -0.081166000 & -0.847157000 \\ \mathrm{C} & 2.132658000 & 3.175187000 & -0.551739000 \\ \mathrm{H} & 1.933296000 & 2.516290000 & -1.401800000 \\ \mathrm{C} & 1.496931000 & 5.146535000 & 0.717195000 \\ \mathrm{C} & 5.741040000 & -2.247560000 & -2.054066000 \\ \mathrm{H} & 6.269235000 & -1.671274000 & -2.824376000 \\ \mathrm{C} & 1.173805000 & 4.174941000 & -0.239443000 \\ \mathrm{C} & 5.821451000 & 2.666446000 & -0.409473000 \\ \mathrm{H} & 6.018119000 & 3.732809000 & -0.256040000 \\ \mathrm{C} & 6.321424000 & -4.370954000 & -3.327061000 \\ \mathrm{H} & 7.304933000 & -4.784501000 & -3.030261000 \\ \mathrm{H} & 6.495019000 & -3.695501000 & -4.182944000 \\ \mathrm{H} & 5.708339000 & -5.219284000 & -3.680737000 \\ \mathrm{C} & 2.756316000 & 5.161461000 & 1.362614000\end{array}$

(1)

\section{(1)}

(1)

$\mathrm{N}-\quad-3.684600000$

C $\quad-5.724453000$

C -3.707948000

C $\quad-3.346322000$

$\mathrm{H} \quad-3.771164000$

C $\quad-2.255876000$

C $\quad-5.755700000$

C -1.918212000

C $\quad-4.692105000$

C $\quad-2.454095000$

C $\quad-4.715632000$

C $\quad-5.601498000$

H $\quad-5.401238000$

C $\quad-5.951939000$

C $\quad-3.147860000$

H $\quad-3.406177000$

C $\quad-5.697170000$

C -6.730936000

$\mathrm{H} \quad-7.522149000$

C $\quad-1.689787000$

$\mathrm{H} \quad-0.705018000$

H $\quad-1.568340000$

H $\quad-2.355941000$

C -6.110062000

C $\quad-6.751666000$

$\mathrm{H} \quad-7.555338000$

C -2.059027000

$\mathrm{H} \quad-2.906805000$

$\mathrm{H} \quad-1.243326000$

$\mathrm{H} \quad-1.703522000$

C -6.007703000

H $\quad-6.108270000$

C $\quad-5.754862000$

$\mathrm{H} \quad-5.782219000$

C $\quad-6.398652000$

$\mathrm{H} \quad-7.461049000$

$\mathrm{H} \quad-5.793417000$

$\mathrm{H}-6.184113000$

C $\quad-5.516770000$

$\mathrm{H} \quad-5.566769000$

H $\quad-6.291967000$

$\mathrm{H} \quad-4.536205000$

$\mathrm{Ni} \quad 1.728514000$

C $\quad-2.468885000$

$\mathrm{H}-2.205768000$

C 2.385409000

$\begin{array}{ll}\mathrm{C} & 2.385409000 \\ \mathrm{H} & 3.074505000\end{array}$

C $\quad 1.026995000$

C $\quad 0.745534000$

C $\quad-0.046803000$
1.799912000 2.181616000 $-4.502526000$ $-5.492141000$ $-3.969002000$ $-4.675168000$ 4.166984000 4.159290000 0.439359000 $-0.256972000$ 6.232720000 6.197501000 7.245001000 6.122860000 4.161788000 5.015204000 3.228165000 4.205355000 $-0.951119000$ 0.311296000 $-2.057811000$ $-3.208340000$ $-3.357485000$ $-2.816623000$ 0.878342000 $-3.952032000$ $-0.571319000$ $-4.160706000$ $-1.082393000$ 0.062601000 $-1.005400000$ 1.977408000 $-1.878652000$ $-0.978743000$ 0.597357000 0.695301000 1.367881000 $-2.618834000$

$-3.106649000$

$-1.547264000$ $-3.050575000$ 2.817949000 0.212622000

0.506169000

$-5.376174000$

$-5.753464000$

$-5.134934000$ $-6.200169000$ 2.253498000 2.900619000 $-0.673996000$ $-1.091135000$ 4.297119000 4.530042000 4.911441000 4.634242000 $-0.285645000$ $-1.355671000$ $-0.088881000$ $-0.110125000$ 1.181980000 $-0.542107000$ 0.133651000 $-0.372313000$ $-0.713437000$ $-0.819134000$ $-1.739932000$ $-0.371547000$
$-0.726319000$ $-0.799463000$ 0.971890000 0.596817000 1.323639000 1.856481000 1.036292000 1.545529000 $-0.925255000$ $-1.112556000$ 2.385434000 3.245675000 1.947117000 2.777657000 $-0.935041000$ $-0.625003000$ $-0.703463000$ $-2.034712000$ $-0.283969000$ 0.184063000 2.479511000 1.745271000 0.745972000 4.316401000 $-1.198154000$ 3.555329000 0.628427000 2.269185000 1.963361000 $-2.342722000$ $-2.209746000$ $-3.786268000$ 3.764048000 4.336773000 $-3.637544000$ 1.094271000 0.743413000 5.709863000 5.820711000 5.949230000 6.482483000 $-2.671290000$ 2.409025000 3.093522000 1.452015000 0.852586000 0.743117000 2.095747000 $-1.383716000$ $-0.502895000$ 2.829422000 3.842767000 $-2.843027000$ $-2.634322000$ $-2.151439000$ $-3.872334000$ $-4.857648000$ $-4.591277000$ $-5.621130000$ $-5.341431000$ 0.832416000 $-0.095476000$ 0.737589000 $-1.483237000$ $-2.272468000$ $-1.601678000$ $-2.651252000$ $-0.735451000$ 


$\begin{array}{rrrr}\mathrm{H} & 1.580271000 & -2.048679000 & -3.296097000 \\ \mathrm{C} & -0.525752000 & -2.267413000 & -2.880978000 \\ \mathrm{C} & -1.360634000 & -0.940345000 & -0.969227000 \\ \mathrm{C} & -0.791503000 & -3.252429000 & -4.001693000 \\ \mathrm{C} & -1.562966000 & -1.849438000 & -2.016232000 \\ \mathrm{H} & -2.582282000 & -2.233852000 & -2.149441000 \\ \mathrm{O} & 0.075356000 & 0.526350000 & 0.208220000 \\ \mathrm{C} & 0.953614000 & 1.794351000 & 2.469989000 \\ \mathrm{C} & 2.243498000 & 1.336924000 & 3.097282000 \\ \mathrm{H} & 0.800534000 & 2.885853000 & 2.425113000 \\ \mathrm{H} & 0.043486000 & 1.231530000 & 2.717170000 \\ \mathrm{C} & 2.924726000 & 2.307776000 & 4.085909000 \\ \mathrm{H} & 2.142434000 & 0.329246000 & 3.538023000 \\ \mathrm{H} & 3.021342000 & 1.190818000 & 2.248063000 \\ \mathrm{H} & 3.915605000 & 1.935443000 & 4.402759000 \\ \mathrm{H} & 3.056342000 & 3.301642000 & 3.626621000 \\ \mathrm{H} & 2.294903000 & 2.423662000 & 4.985617000 \\ \mathrm{H} & -1.181890000 & -4.213444000 & -3.616207000 \\ \mathrm{H} & -1.542615000 & -2.864912000 & -4.715868000 \\ \mathrm{H} & 0.128842000 & -3.470718000 & -4.570940000 \\ \mathrm{H} & 0.759022000 & 5.921554000 & 0.964114000 \\ \mathrm{H} & -1.220251000 & -4.690900000 & 3.972563000 \\ \mathrm{H} & 4.864215000 & -5.454580000 & -1.273539000 \\ \mathrm{H} & -6.025502000 & 2.405152000 & -4.795744000\end{array}$

\section{Me $\mathbf{1 - \beta}-\mathbf{C}$}

Zero-point correction=

Thermal correction to Energy= 0.936116 (Hartree/Particle) Thermal correction to Enthalpy= 0.998475 0.999419

Thermal correction to Gibbs Free Energy $=\quad 0.826957$ Sum of electronic and zero-point Energies $=\quad-2520.925300$ Sum of electronic and thermal Energies $=\quad-2520.862941$ Sum of electronic and thermal Enthalpies $=\quad-2520.861997$ Sum of electronic and thermal Free Energies $=-2521.034459$ E solvent $=-2520.71970544$

$\begin{array}{lr}\mathrm{Ni} & -1.770072000 \\ \mathrm{C} & -1.064275000 \\ \mathrm{~N} & -2.853635000 \\ \mathrm{C} & -2.354276000 \\ \mathrm{C} & -0.982926000 \\ \mathrm{C} & 0.054201000 \\ \mathrm{O} & -0.142071000 \\ \mathrm{C} & -2.509275000 \\ \mathrm{H} & -2.732235000 \\ \mathrm{C} & -3.262771000 \\ \mathrm{H} & -4.357246000 \\ \mathrm{H} & -2.962306000 \\ \mathrm{H} & -3.019379000 \\ \mathrm{H} & -3.099317000 \\ \mathrm{H} & -0.377186000 \\ \mathrm{H} & -0.635015000 \\ \mathrm{H} & -3.046665000 \\ \mathrm{C} & -0.649666000 \\ \mathrm{H} & -1.463078000 \\ \mathrm{C} & 0.651597000 \\ \mathrm{C} & 0.979796000 \\ \mathrm{H} & 1.459131000 \\ \mathrm{H} & 0.071398000 \\ \mathrm{H} & 1.680786000 \\ \mathrm{C} & 1.662816000 \\ \mathrm{H} & 2.705161000 \\ \mathrm{C} & 1.404605000 \\ \mathrm{C} & 2.488561000 \\ \mathrm{~N} & 3.727274000 \\ \mathrm{H} & 2.185659000 \\ \mathrm{C} & 4.717703000 \\ \mathrm{C} & 4.854403000 \\ & 5.872565000 \\ \end{array}$

$0.595543000 \quad-1.240722000$ $0.800730000-3.008107000$ $0.447890000 \quad 0.343438000$ $0.030293000 \quad 1.492750000$ $-0.292832000 \quad 1.754957000$ $-0.202278000 \quad 0.751616000$ $0.156187000 \quad-0.492507000$ $1.047605000-3.248391000$ $2.097432000-3.512414000$ $0.041991000-4.135451000$ $0.171804000-4.069127000$ $0.181766000 \quad-5.188824000$ $-0.994684000 \quad-3.845641000$ $1.006805000-2.201137000$ $1.657766000-3.048639000$ $-0.137360000 \quad-3.390245000$ $-0.065382000 \quad 2.346398000$ $-0.701884000 \quad 3.077461000$ $-0.764863000 \quad 3.813898000$ $-1.022562000 \quad 3.465946000$ $-1.454147000 \quad 4.881231000$ $-2.451111000 \quad 4.902731000$ $-1.507160000 \quad 5.506368000$ $-0.750787000 \quad 5.369576000$ $-0.922049000 \quad 2.482537000$ $-1.152899000 \quad 2.737045000$ $-0.527426000 \quad 1.163174000$ $-0.422519000 \quad 0.180403000$ $-0.648386000 \quad 0.486487000$ $-0.126492000 \quad-0.839650000$ $-0.540794000 \quad-0.514409000$ $-1.532668000-1.534118000$ $-1.373023000$

\section{c \\ (a)} $\mathrm{C}$

c

C

c

$\mathrm{C}$

$-4.090598000$

C $\quad-3.290810000$

C -2.160920000

C -1.830414000

C $\quad-2.651629000$

$\mathrm{H} \quad-6.136703000$

H $\quad-4.003589000$

$\mathrm{H} \quad-3.407434000$

$\mathrm{H} \quad-7.302528000$

$\mathrm{H} \quad-8.106953000$

H $\quad-6.446275000$

$\mathrm{H} \quad-4.963915000$

$\mathrm{H} \quad-1.524679000$

$\mathrm{H} \quad 1.897145000$

$\mathrm{H} \quad 3.690715000$

$\mathrm{H} \quad 5.993009000$

$\mathrm{H} \quad 7.526969000$

H $\quad 7.296348000$

$\mathrm{H} \quad 5.610820000$

$\mathrm{H} \quad 5.410592000$

$\mathrm{H} \quad 4.074446000$

$\mathrm{H} \quad-2.409296000$

H $\quad 5.474874000$

C $\quad 2.239853000$

$\mathrm{H} \quad 2.331034000$

H 2.808742000

$\mathrm{H} \quad 1.177764000$

C 2.659837000

$\mathrm{H} \quad 2.137008000$

$\mathrm{H} \quad 3.587961000$

H $\quad 2.023156000$

C $\quad 5.617151000$

$\mathrm{H} \quad 5.422206000$

$\mathrm{H} \quad 6.610063000$

$\mathrm{H} \quad 4.873352000$

C $\quad 5.384083000$

$\mathrm{H} \quad 5.304643000$

H $\quad 6.286382000$

$\mathrm{H} \quad 4.514031000$

C $\quad-3.633636000$

$-0.274070000$

$-1.467646000$

$0.567223000 \quad-0.469203000$

$1.627833000 \quad 0.579525000$

$1.307313000 \quad 1.955365000$

$3.661090000 \quad 2.530706000$

$4.015537000 \quad 1.173134000$

$2.986594000 \quad 0.208729000$

$-2.749030000-1.608630000$

$-3.527922000 \quad-0.468609000$

$-4.717084000-0.561925000$

$-5.124727000 \quad-1.823685000$

$-4.368968000 \quad-2.984845000$

$-3.186406000-2.860463000$

$\begin{array}{ll}2.309948000 & 2.937496000\end{array}$

$\begin{array}{ll}-4.436725000 & 1.455648000\end{array}$

$\begin{array}{ll}-3.808065000 & 0.367180000\end{array}$

$\begin{array}{ll}-2.456634000 & 0.101293000\end{array}$

$\begin{array}{ll}-1.738316000 & 0.896347000\end{array}$

$\begin{array}{ll}-2.402752000 & 1.981098000\end{array}$

$\begin{array}{ll}-3.748853000 & 2.272910000\end{array}$

$\begin{array}{ll}-0.326363000 & 0.584337000\end{array}$

$\begin{array}{ll}-0.019882000 & 0.530899000\end{array}$

$\begin{array}{ll}1.263437000 & 0.203239000\end{array}$

$2.267223000 \quad-0.088389000$

$2.018770000 \quad-0.050083000$

$\begin{array}{ll}0.710519000 & 0.302864000\end{array}$

$3.145017000 \quad-0.386224000$

$3.967496000 \quad-1.499916000$

$5.070892000-1.841526000$

$5.353523000 \quad-1.045394000$

$4.562811000 \quad 0.069345000$

$3.461132000 \quad 0.387890000$

$-1.847488000 \quad 2.619091000$

$\begin{array}{ll}-5.486794000 & 1.676952000\end{array}$

$-1.952586000-0.737585000$

$\begin{array}{ll}-0.823002000 & 0.724364000\end{array}$

$1.479777000 \quad 0.170125000$

$3.280618000 \quad-0.323778000$

$3.727309000 \quad-2.119624000$

$6.211804000-1.300503000$

$-6.049150000-1.904994000$

$-2.577050000 \quad-3.752166000$

$-2.150547000-3.264625000$

$-0.177812000 \quad-3.234766000$

$\begin{array}{ll}1.535183000 & -1.422663000\end{array}$

$3.247110000 \quad-0.857040000$

$0.254795000 \quad 2.253659000$

$\begin{array}{ll}-3.197947000 & 0.508593000\end{array}$

$2.855366000 \quad 1.266506000$

$4.453238000 \quad 3.292038000$

$-4.830551000 \quad-4.337547000$

$-4.033918000 \quad-5.096369000$

$-5.706034000 \quad-4.706551000$

$-5.134473000 \quad-4.291042000$

$\begin{array}{ll}-5.533831000 & 0.682019000\end{array}$

$\begin{array}{ll}-6.474179000 & 0.434483000\end{array}$

$\begin{array}{ll}-5.796744000 & 1.223097000\end{array}$

$-4.971231000 \quad 1.390785000$

$\begin{array}{ll}5.470144000 & 0.746748000\end{array}$

$6.147890000 \quad 1.596161000$

$5.733202000 \quad 0.333414000$

$5.691767000 \quad-0.040990000$

$1.955005000 \quad 4.411028000$

$0.864205000 \quad 4.558467000$

$2.301646000 \quad 4.950219000$

$2.426541000 \quad 4.905968000$

$5.944987000 \quad-3.032769000$ 


$\begin{array}{lrrr}\mathrm{H} & -2.771274000 & 6.057026000 & -3.715828000 \\ \mathrm{H} & -4.473308000 & 5.525671000 & -3.613600000 \\ \mathrm{H} & -3.924575000 & 6.964235000 & -2.714265000 \\ \mathrm{C} & -0.606320000 & 4.869784000 & 0.909558000 \\ \mathrm{H} & -0.843533000 & 4.860952000 & 1.989101000 \\ \mathrm{H} & 0.187411000 & 4.116109000 & 0.748031000 \\ \mathrm{H} & -0.183914000 & 5.858861000 & 0.662159000 \\ \mathrm{C} & -2.627194000 & -4.563408000 & -0.510367000 \\ \mathrm{H} & -1.678654000 & -4.008269000 & -0.626982000 \\ \mathrm{H} & -2.390117000 & -5.555528000 & -0.088880000 \\ \mathrm{H} & -3.036151000 & -4.722658000 & -1.526570000 \\ \mathrm{C} & -5.830199000 & -4.450190000 & 3.437463000 \\ \mathrm{H} & -6.568150000 & -5.197693000 & 3.087693000 \\ \mathrm{H} & -5.096456000 & -4.992069000 & 4.062139000 \\ \mathrm{H} & -6.365364000 & -3.735504000 & 4.086511000\end{array}$

\section{${ }^{\text {MeTS-1 }}$ BHE-C}

Zero-point correction $=\quad 0.933448($ Hartree/Particle $)$ Thermal correction to Energy $=0.995366$ Thermal correction to Enthalpy $=\quad 0.996310$

Thermal correction to Gibbs Free Energy $=0.825486$ Sum of electronic and zero-point Energies $=-2520.913023$ Sum of electronic and thermal Energies $=\quad-2520.851105$ Sum of electronic and thermal Enthalpies $=\quad-2520.850161$ Sum of electronic and thermal Free Energies $=-2521.020985$ E solvent $=-2520.70487163$

\begin{tabular}{|c|c|c|c|}
\hline $\mathrm{N}$ & -2.930567000 & -0.212183000 & 00 \\
\hline $\mathrm{C}$ & -5.100648000 & 0.948661000 & -0.102329000 \\
\hline $\mathrm{C}$ & .349280000 & -2.836872000 & 0.225001000 \\
\hline $\mathrm{C}$ & -3.290573000 & -3.196316000 & -0.633172000 \\
\hline $\mathrm{H}$ & -2.929432000 & -2.469744000 & -1.370845000 \\
\hline G & -4.221795000 & -5.059463000 & 1.271205000 \\
\hline $\mathrm{C}$ & 1610000 & 2.291751000 & -0.19 \\
\hline $\mathrm{C}$ & 990000 & -5.3 & \\
\hline $\mathrm{C}$ & -4.369492000 & -0.265153000 & 0.046 \\
\hline $\mathrm{C}$ & -2.689261000 & -4.468245000 & -0.5 \\
\hline $\mathrm{C}$ & -5.046969000 & -1.5 & \\
\hline $\mathrm{C}$ & -3.4 & 2.7 & \\
\hline $\mathrm{H}$ & -3.1 & 2.06 & \\
\hline $\mathrm{C}$ & -3.3 & 4.8 & -0. \\
\hline $\mathrm{C}$ & -4.8017 & -3.78 & 00 \\
\hline $\mathrm{H}$ & -5.61 & -3.50 & 1.8 \\
\hline $\mathrm{C}$ & -2.9 & 4.0 & \\
\hline $\mathrm{C}$ & -6.5 & & \\
\hline $\mathrm{H}$ & $-7.0^{\prime}$ & 1.8 & \\
\hline $\mathrm{C}$ & 7000 & -6.06 & 2.2 \\
\hline $\mathrm{H}$ & $-5.328 c$ & -6.8 & 1.8 \\
\hline $\mathrm{H}$ & -5.361 & -5.5 & \\
\hline $\mathrm{H}$ & -3.88 & -6.5 & \\
\hline $\mathrm{C}$ & -4.3 & & \\
\hline $\mathrm{C}$ & 2000 & -0.3 & 9000 \\
\hline $\mathrm{H}$ & 2000 & -0.36 & 0000 \\
\hline $\mathrm{C}$ & -1.5 & -4.82 & -1.4 \\
\hline $\mathrm{H}$ & -1.7 & -4.60 & -2. \\
\hline $\mathrm{H}$ & -1.287615000 & -5.90 & -1.4 \\
\hline $\mathrm{H}$ & 0000 & -4.25 & -1.2 \\
\hline $\mathrm{C}$ & -4.875981000 & 3.1889 & -1.20 \\
\hline $\mathrm{H}$ & -5.627283000 & 2.85678 & -1.9 \\
\hline $\mathrm{C}$ & -6.458748000 & -1.523967000 & \\
\hline $\mathrm{H}$ & -6.976497000 & -2.489149000 & 2000 \\
\hline $\mathrm{C}$ & 1000 & 5.44 & 4000 \\
\hline $\mathrm{H}$ & 2000 & 6.214111000 & 95000 \\
\hline $\mathrm{H}$ & -5.378881000 & 4.912187000 & -3.176542000 \\
\hline $\mathrm{H}$ & -3.977420000 & 5.973541000 & 75000 \\
\hline $\mathrm{C}$ & -1.852544000 & 4.462932000 & 1.626006000 \\
\hline $\mathrm{H}$ & -1.809141000 & 5.563142000 & 1.708663000 \\
\hline $\mathrm{H}$ & & 4.114276000 & 1.314294000 \\
\hline $\mathrm{H}$ & -2.037984000 & 4.051870000 & 2.63439000 \\
\hline
\end{tabular}

3.729594000 4.958968000

5.332351000

5.224494000

5.234518000

5.189860000

4.189781000

5.086262000

4.723702000

5.102431000

5.531581000

3.940386000

4.269193000

2.852469000

5.310922000

5.372702000

3.277282000

5.982588000

6.177876000

5.186904000

4.554578000

6.205425000

4.811016000

3.084696000

6.761078000

7.558723000

4.977323000

5.670149000

5.195547000

3.955022000

3.749224000

3.913912000

6.532135000

7.153347000

2.642088000

3.418139000

2.445453000

1.722915000

3.035647000

3.981480000

2.362260000

2.582873000

$-1.895280000$

2.480154000

2.166281000

$-2.397016000$

$-3.100758000$

$-1.011842000$

$-0.672342000$

0.028882000

$-1.489767000$

0.645615000

1.394830000

0.986773000

1.661583000

2.714662000

$-0.203275000$

$-0.870386000$

$-1.901814000$

$-0.970589000$

0.133915000

$-1.711345000$

$-2.747057000$

$-2.609254000$

$-1.536086000$

$-0.846498000$

1.629983000

$\mathrm{H} \quad 1.535473000$
$-0.005263000$

1.915514000

$-1.883792000$

$-2.573287000$

$-2.000219000$

$-4.039005000$

2.926968000

$-4.691345000$

0.509137000

$-3.971425000$

$-0.403956000$

2.764702000

1.844336000

4.927821000

$-2.634587000$

$-2.109058000$

3.756819000

2.374234000

3.451725000

$-4.823928000$

$-4.338967000$

$-4.904307000$

$-5.851265000$

5.124883000

1.487071000

1.864431000

$-4.699019000$

$-5.558922000$

$-4.028886000$

$-5.098492000$

4.115955000

4.242882000

0.109852000

$-0.597796000$

6.403058000

7.190186000

6.238754000

6.812317000

3.565138000

3.615042000

4.341264000

2.579006000

0.460131000

0.116841000

0.584072000

$-0.664063000$

$-1.017975000$

$-0.725870000$

$-1.259110000$

$-0.257370000$

$-1.608756000$

$-1.357959000$

$-0.354828000$

$-1.935866000$

$-0.896818000$

$-0.955306000$

0.233474000

1.435036000

0.742172000

2.507045000

1.006948000

$-0.607760000$

1.329864000

$-1.240788000$

$-0.465333000$

$-1.148176000$

$-2.831865000$

$-1.207919000$
0.636140000

$-0.321773000$

$-0.910709000$

0.319108000

1.251947000

$-2.091080000$

0.464655000

$-0.850806000$

$-0.224539000$

0.362944000

$-0.969429000$

1.844049000

2.340090000

1.934153000

$-2.104327000$

$-3.065976000$

2.590170000

$-1.181493000$

$-1.230263000$

$-3.389124000$

$-4.155268000$

$-3.816501000$

$-3.239683000$

0.558406000

$-1.931995000$

$-2.581534000$

1.688044000

1.747746000

2.537451000

1.834117000

$-0.161100000$

$-1.238631000$

$-1.820442000$

$-2.381426000$

$-0.127901000$

$-0.055245000$

$-1.202252000$

0.328247000

4.075539000

4.648615000

4.479276000

4.286263000

$-1.328367000$

0.315515000

$-0.635250000$

1.245996000

2.017721000

1.591809000

2.867396000

0.703907000

3.513488000

3.314829000

1.180102000

4.673878000

2.444280000

2.748449000

$-0.479281000$

$-2.779603000$

$-3.432794000$

$-2.569813000$

$-2.699135000$

$-4.093028000$

$-3.816197000$

$-3.986048000$

$-5.178435000$

$-3.673143000$

4.585642000

5.301094000 


$\begin{array}{rrrr}\mathrm{H} & 0.076795000 & -2.231432000 & 5.224566000 \\ \mathrm{H} & -2.934784000 & 5.894803000 & -0.447869000 \\ \mathrm{H} & 4.987632000 & -5.785483000 & -0.826039000 \\ \mathrm{H} & -2.697502000 & -6.376742000 & 0.466364000 \\ \mathrm{H} & 2.326025000 & 5.703218000 & 2.507378000 \\ \mathrm{H} & -3.176464000 & 0.610079000 & -2.044606000\end{array}$

\section{Me1-BHE-C}

Zero-point correction $=\quad 0.934071$ (Hartree/Particle)

Thermal correction to Energy $=0.996651$

Thermal correction to Enthalpy $=0.997595$

Thermal correction to Gibbs Free Energy= 0.824148

Sum of electronic and zero-point Energies $=\quad-2520.918215$

Sum of electronic and thermal Energies $=\quad-2520.855635$

Sum of electronic and thermal Enthalpies $=\quad-2520.854691$

Sum of electronic and thermal Free Energies $=-2521.028138$

E solvent $=-2520.70890341$

\begin{tabular}{|c|c|c|c|}
\hline & -1.947607000 & 0.343744000 & \\
\hline & -1.084807000 & 1.564453000 & 8000 \\
\hline & -2.976369000 & -0.304951000 & 0.069391000 \\
\hline & -2.432098000 & -0.764504000 & 1 \\
\hline & -1.048227000 & -0.800642000 & 567000 \\
\hline & -0.008147000 & 57000 & \\
\hline & -0.247306000 & 2000 & 13000 \\
\hline & -1.157824000 & 2000 & 9000 \\
\hline & & & \\
\hline & & & \\
\hline & 0 & & \\
\hline & -0.3 & & \\
\hline & -2.0 & & 000 \\
\hline & -1.8 & 2.3 & -2 \\
\hline & -0.1 & 1.9 & -2 \\
\hline & -3.1 & -1.1 & \\
\hline & -0.7 & & \\
\hline & -1.5 & -1.7 & \\
\hline & & -1.3 & \\
\hline & & & \\
\hline & & -2 & \\
\hline & & -2.2 & \\
\hline & 00 & -1. & \\
\hline & 1.6 & -0.8 & \\
\hline & 2.6 & -0.9 & 000 \\
\hline & 1.35 & -0.3 & 5000 \\
\hline & 2.43 & & 3000 \\
\hline & 3.68 & & \\
\hline & 2.12 & & -0. \\
\hline & & & \\
\hline & & & \\
\hline & & & \\
\hline & & & \\
\hline & & & \\
\hline & & & \\
\hline & & & \\
\hline & & & \\
\hline & & & -0 . \\
\hline & & & \\
\hline & & & -0.3 \\
\hline & & & \\
\hline & & 4.1 & -0.2 \\
\hline & & & -0.7 \\
\hline & & & 000 \\
\hline & & & 000 \\
\hline $\mathrm{C}$ & & & \\
\hline $\mathrm{C}$ & 5.5 & & \\
\hline $\mathrm{C}$ & 5.53 & 2000 & \\
\hline 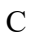 & & 16000 & \\
\hline 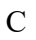 & 000 & 700000 & \\
\hline & 2.916696000 & 3.915940000 & 2.4616340 \\
\hline
\end{tabular}

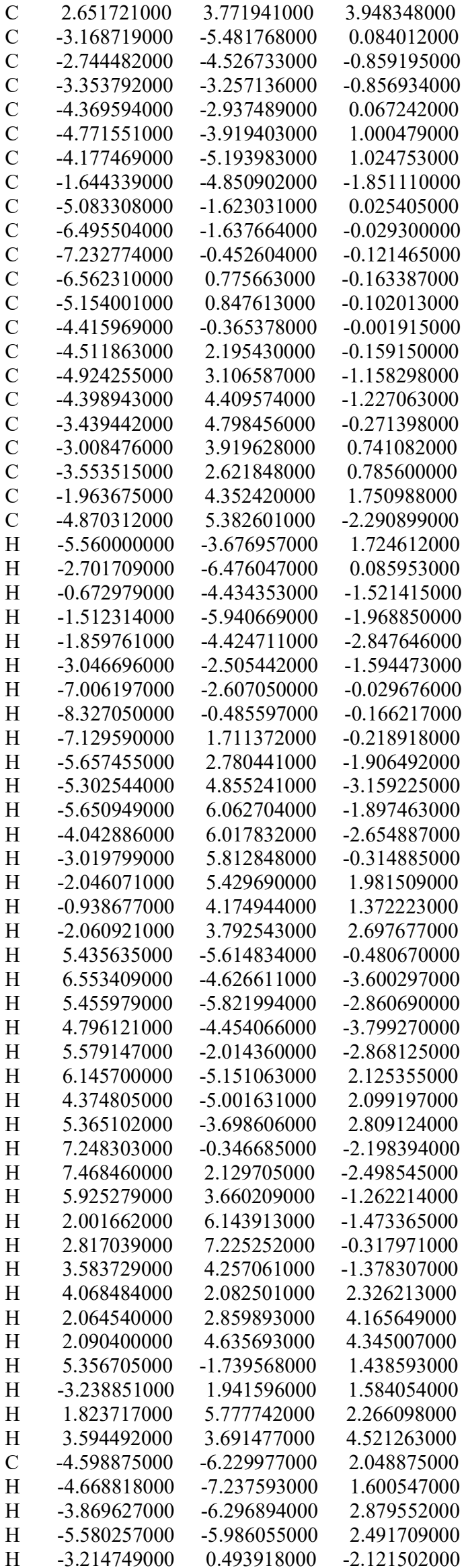

Me TS-1 2,1-ins

Zero-point correction $=0.933105($ Hartree $/$ Particle $)$ 
Thermal correction to Energy $=\quad 0.995203$

Thermal correction to Enthalpy= $\quad 0.996148$

Thermal correction to Gibbs Free Energy $=0.824647$

Sum of electronic and zero-point Energies $\quad-2520.914655$

Sum of electronic and thermal Energies $=\quad-2520.852557$

Sum of electronic and thermal Enthalpies $=\quad-2520.851613$

Sum of electronic and thermal Free Energies $=-2521.023113$

E solvent $=-2520.70620998$

$\begin{array}{lll}-0.323471000 & -1.389545000\end{array}$

$\begin{array}{llll}\mathrm{C} & 1.839577000 & -0.441066000 & -3.459114000\end{array}$

$\begin{array}{llll}\mathrm{N} & 2.947372000 & 0.271909000 & 0.091844000\end{array}$

$\begin{array}{llll}\text { C } & 2.416035000 & 0.692714000 & 1.228129000\end{array}$

$\begin{array}{llll}\text { C } & 1.032286000 & 0.734907000 & 1.583429000\end{array}$

$\begin{array}{llll}\text { C } & -0.012059000 & 0.300925000 & 0.682017000\end{array}$

$\begin{array}{llll}\text { O } & 0.217034000 & -0.130627000 & -0.524668000\end{array}$

C $\quad 0.821146000 \quad-1.226426000 \quad-2.898699000$

$\begin{array}{llll}\text { C } & 0.881513000 & -2.729720000 & -2.799575000\end{array}$

H $\quad 0.334373000 \quad-3.193646000 \quad-3.646390000$

$\mathrm{H} \quad 1.920492000 \quad-3.101131000 \quad-2.813801000$

$\mathrm{H} \quad 0.398102000-3.080240000-1.870993000$

$\mathrm{H} \quad-0.155058000 \quad-0.760302000 \quad-2.722470000$

$\mathrm{H} \quad 1.647163000 \quad 0.603352000$

$\mathrm{H} \quad 2.683439000$

H $\quad 3.121451000$

$-0.910733000$

1.036090000

1.216049000

0.698139000

H $\quad 1.518054000$

C $\quad-0.617498000$

C $\quad-0.953266000$

$\mathrm{H}-1.602316000$

$\mathrm{H} \quad-0.041526000$

H $\quad-1.493608000$

C $\quad-1.636414000$

H $\quad-2.687869000$

C $\quad-1.375103000$

C -2.464802000

N $\quad-3.711691000$

$\mathrm{H} \quad-2.156503000$

C $\quad-4.709114000$

C $\quad-5.472921000$

C -6.476688000

C $\quad-6.748851000$

C $\quad-6.012643000$

C $\quad-4.989772000$

C $\quad-4.262139000$

C $\quad-4.020841000$

$\mathrm{H} \quad-1.898267000$

C $\quad-3.013581000$

C $\quad-2.840887000$

C $\quad-3.238458000$

C $\quad-3.857711000$

C $\quad-5.229801000$

C $\quad-5.118393000$

C $\quad-4.968077000$

C $\quad-4.924353000$

C $\quad-5.021099000$

C $\quad-5.178118000$

C $\quad-4.852026000$

C $\quad-3.397773000$

C $\quad-3.134860000$

C $\quad 3.022005000$

C 2.574002000

C $\quad 3.212313000$

C $\quad 4.280760000$

C $\quad 4.705684000$

C $\quad 4.085815000$

C $\quad 1.421940000$

C $\quad 5.022015000$

C $\quad 6.431629000$
1.542795000

1.290902000

1.813921000

2.708629000

2.093943000

1.059085000

0.858767000

0.899704000

0.370186000

$-0.069391000$

0.017918000

$-0.481095000$

$-0.453895000$

0.489092000

0.012014000

$-1.356162000$

$-2.272019000$

$-1.850861000$

$-2.888651000$

$-2.737371000$

$-6.825212000$

$-4.938983000$

$-6.421143000$

$-5.125508000$

$-4.092045000$

1.961549000

2.635821000

4.030553000

4.762234000

4.124235000

2.723566000

4.743387000

$-3.752446000$

$-3.558733000$

5.446844000

4.492964000

3.237277000

2.931676000

3.914152000

5.173386000

4.798804000

1.634435000

1.683900000
$-3.979497000$

2.003518000

2.880776000

3.535717000

3.339584000

4.721928000

4.672041000

5.277813000

5.324093000

2.457741000

2.770150000

1.170979000

0.294591000

0.634745000

$-0.683364000$

$-0.247916000$

$-1.001336000$

$-1.870389000$

$-1.992644000$

$-1.233807000$

$-0.354388000$

0.436898000

1.818785000

0.268505000

1.916747000

$-0.142987000$

0.538139000

$-0.186372000$

$-0.927549000$

0.310530000

0.370416000

$-0.835634000$

$-2.083850000$

$-2.113027000$

1.704291000

2.569067000

4.050514000

0.221805000

$-0.709843000$

$-0.752730000$

0.113559000

1.037959000

1.106130000

$-1.647260000$

0.028837000

$-0.063306000$
7.195309000 6.554558000

5.149983000

4.384109000

4.545645000

4.975599000

4.499205000

3.567266000

3.118127000

3.616104000

2.103532000

4.995094000

5.530509000

2.528879000

0.536755000

1.116189000

1.689991000

2.877182000

6.918863000

8.286917000

7.143840000

5.688637000

5.402052000

5.802172000

4.187850000

3.184576000

2.054502000

1.088434000

2.347462000

$-4.810134000$

$-5.247575000$

$-5.538421000$

$-3.828312000$

$-5.085663000$

$-7.066204000$

$-7.547642000$

$-6.242069000$

$-2.706024000$

$-3.613838000$

$-4.019547000$

$-4.325079000$

$-2.328820000$

$-2.829885000$

$-5.156246000$

3.288064000

$-2.525661000$

$-4.032493000$

3.189826000

4.540410000

4.770298000

3.756960000

5.445793000

$-4.941390000$

$-5.073847000$

$-3.961191000$

$-5.714435000$
0.518672000 $-0.725349000$ $-0.832904000$ 0.360566000 $-2.199053000$ $-3.107606000$ $-4.429749000$ $-4.841258000$ $-3.965772000$ $-2.647790000$

$-4.419846000$

$-5.400528000$

3.681318000

6.427587000

4.178768000

5.857191000

4.590112000

2.483833000

2.665216000

0.579277000

$-1.646039000$

$-2.765818000$

$-4.871058000$

$-6.047071000$

$-6.069834000$

$-5.870625000$

$-5.520988000$

$-4.055482000$

$-4.032677000$

5.854487000

2.210029000

5.608430000

5.132988000

4.066701000

0.742093000

$-1.703957000$

$-3.342271000$

$-6.282286000$

$-7.202722000$

$-4.213654000$

$-1.808206000$

$-2.819861000$

$-4.502910000$

2.054172000

$-1.969238000$

$-5.735682000$

$-3.183336000$

$-0.470816000$

6.213718000

7.177632000

6.417263000

5.885898000

4.917622000

5.998520000

4.783874000

4.597962000

$-0.187183000$

$-0.221804000$

$-0.126409000$

0.001628000

$-0.168677000$

$-1.164547000$

$-1.209285000$

$-0.234697000$

0.771429000

0.793057000

1.802765000

$-2.264046000$

1.723582000

0.262791000 


\begin{tabular}{|c|c|c|c|}
\hline C & -1.058976000 & 0.864814000 & -3.019688000 \\
\hline iv & -2.924626000 & 0.418148000 & 0.293791000 \\
\hline $\mathrm{C}$ & -2.437267000 & -0.026414000 & 1.438306000 \\
\hline $\mathrm{C}$ & -1.068117000 & -0.354001000 & 1.711311000 \\
\hline $\mathrm{C}$ & -0.013135000 & -0.235237000 & 0.728499000 \\
\hline $\mathrm{O}$ & -0.189902000 & 0.164112000 & -0.506253000 \\
\hline $\mathrm{C}$ & -2.510359000 & 1.114422000 & -3.254447000 \\
\hline $\mathrm{H}$ & -3.145791000 & 1.029267000 & -2.251612000 \\
\hline $\mathrm{H}$ & -2.760547000 & 2.145891000 & -3.557736000 \\
\hline $\mathrm{H}$ & -3.017820000 & 0.371424000 & -3.893031000 \\
\hline $\mathrm{C}$ & -0.060110000 & 1.998376000 & -3.109493000 \\
\hline $\mathrm{H}$ & -0.682028000 & -0.106599000 & -3.377714000 \\
\hline $\mathrm{H}$ & -3.139304000 & -0.145020000 & 2.281418000 \\
\hline $\mathrm{C}$ & -0.756084000 & -0.795810000 & 3.028395000 \\
\hline $\mathrm{H}$ & -1.581695000 & -0.877187000 & 3.749443000 \\
\hline $\mathrm{C}$ & 0.538380000 & -1.127035000 & 3.431243000 \\
\hline $\mathrm{C}$ & 0.841525000 & -1.593908000 & 4.840934000 \\
\hline $\mathrm{H}$ & 1.317770000 & -2.592592000 & 4.846222000 \\
\hline $\mathrm{H}$ & -0.077489000 & -1.659398000 & 5.449139000 \\
\hline $\mathrm{H}$ & 1.536010000 & -0.904779000 & 5.358009000 \\
\hline $\mathrm{C}$ & 1.565203000 & -1.005196000 & 2.467335000 \\
\hline $\mathrm{H}$ & 2.602648000 & -1.246603000 & 2.731882000 \\
\hline $\mathrm{C}$ & 1.328905000 & -0.577857000 & 1.153722000 \\
\hline $\mathrm{C}$ & 2.429758000 & -0.463967000 & 0.191697000 \\
\hline $\mathrm{N}$ & 3.662489000 & -0.699633000 & 0.514738000 \\
\hline $\mathrm{H}$ & 2.145846000 & -0.155768000 & -0.830301000 \\
\hline $\mathrm{C}$ & 4.669117000 & -0.594242000 & -0.469333000 \\
\hline $\mathrm{C}$ & 4.792163000 & -1.560025000 & -1.515880000 \\
\hline $\mathrm{C}$ & 5.829878000 & -1.403408000 & -2.462737000 \\
\hline $\mathrm{C}$ & 6.725702000 & -0.331083000 & -2.398237000 \\
\hline $\mathrm{C}$ & 6.606403000 & 0.602319000 & -1.360442000 \\
\hline $\mathrm{C}$ & 5.600318000 & 0.485242000 & -0.379601000 \\
\hline $\mathrm{C}$ & 5.524572000 & 1.518366000 & 0.697347000 \\
\hline $\mathrm{C}$ & 5.430784000 & 1.162041000 & 2.062921000 \\
\hline $\mathrm{C}$ & 5.517979000 & 3.497712000 & 2.703099000 \\
\hline $\mathrm{C}$ & 5.608086000 & 3.887433000 & 1.356641000 \\
\hline $\mathrm{C}$ & 5.611167000 & 2.885062000 & 0.364535000 \\
\hline $\mathrm{C}$ & 3.892854000 & -2.746804000 & -1.639716000 \\
\hline $\mathrm{C}$ & 3.567667000 & -3.552194000 & -0.528502000 \\
\hline $\mathrm{C}$ & 2.791477000 & -4.718483000 & -0.670418000 \\
\hline $\mathrm{C}$ & 2.333620000 & -5.075930000 & -1.951775000 \\
\hline $\mathrm{C}$ & 2.629521000 & -4.289247000 & -3.083876000 \\
\hline $\mathrm{C}$ & 3.409680000 & -3.133368000 & -2.911985000 \\
\hline $\mathrm{C}$ & 5.431099000 & 2.137600000 & 3.072074000 \\
\hline $\mathrm{C}$ & -4.284126000 & -4.509514000 & 1.219184000 \\
\hline $\mathrm{C}$ & -3.657436000 & -3.842803000 & 0.150895000 \\
\hline $\mathrm{C}$ & -3.961528000 & -2.485066000 & -0.070960000 \\
\hline $\mathrm{C}$ & -4.875876000 & -1.797053000 & 0.751530000 \\
\hline $\mathrm{C}$ & -5.488401000 & -2.498388000 & 1.816268000 \\
\hline $\mathrm{C}$ & -5.203339000 & -3.852187000 & 2.062627000 \\
\hline $\mathrm{C}$ & -5.271520000 & -0.379017000 & 0.481646000 \\
\hline $\mathrm{C}$ & -6.651362000 & -0.081814000 & 0.423143000 \\
\hline $\mathrm{C}$ & -7.108269000 & 1.207179000 & 0.127885000 \\
\hline $\mathrm{C}$ & -6.182865000 & 2.224991000 & -0.127554000 \\
\hline $\mathrm{C}$ & -4.791164000 & 1.986102000 & -0.082027000 \\
\hline $\mathrm{C}$ & -4.335796000 & 0.672800000 & 0.243033000 \\
\hline $\mathrm{C}$ & -3.871939000 & 3.126972000 & -0.383250000 \\
\hline $\mathrm{C}$ & -4.172032000 & 3.970882000 & -1.481189000 \\
\hline $\mathrm{C}$ & -3.376616000 & 5.086132000 & -1.793762000 \\
\hline $\mathrm{C}$ & -2.251541000 & 5.355767000 & -0.986484000 \\
\hline $\mathrm{C}$ & -1.925153000 & 4.544617000 & 0.115314000 \\
\hline $\mathrm{C}$ & -2.741061000 & 3.430821000 & 0.404078000 \\
\hline $\mathrm{H}$ & -6.190378000 & -1.967451000 & 2.471977000 \\
\hline $\mathrm{H}$ & -4.048038000 & -5.566476000 & 1.402756000 \\
\hline $\mathrm{H}$ & -3.475423000 & -1.953253000 & -0.898135000 \\
\hline $\mathrm{H}$ & -7.366524000 & -0.895700000 & 0.586249000 \\
\hline $\mathrm{H}$ & -8.183003000 & 1.416836000 & 0.090166000 \\
\hline $\mathrm{H}$ & -6.531459000 & 3.241519000 & -0.340426000 \\
\hline
\end{tabular}

\begin{tabular}{|c|c|}
\hline $\mathrm{H}$ & -5.038053000 \\
\hline $\mathrm{H}$ & -1.616966000 \\
\hline $\mathrm{H}$ & 1.731462000 \\
\hline $\mathrm{H}$ & 3.639613000 \\
\hline $\mathrm{H}$ & 5.940331000 \\
\hline $\mathrm{H}$ & 7.529078000 \\
\hline $\mathrm{H}$ & 7.318946000 \\
\hline $\mathrm{H}$ & 5.662720000 \\
\hline $\mathrm{H}$ & 5.354963000 \\
\hline $\mathrm{H}$ & 3.934773000 \\
\hline $\mathrm{H}$ & -2.499163000 \\
\hline $\mathrm{H}$ & 5.511555000 \\
\hline $\mathrm{H}$ & 0.291074000 \\
\hline $\mathrm{H}$ & -0.488854000 \\
\hline $\mathrm{H}$ & 0.828340000 \\
\hline $\mathrm{C}$ & 2.100638000 \\
\hline $\mathrm{H}$ & 2.611503000 \\
\hline $\mathrm{H}$ & 2.237161000 \\
\hline $\mathrm{H}$ & 1.016820000 \\
\hline $\mathrm{C}$ & 2.459062000 \\
\hline $\mathrm{H}$ & 1.979565000 \\
\hline $\mathrm{H}$ & 3.365378000 \\
\hline $\mathrm{H}$ & 1.767800000 \\
\hline $\mathrm{C}$ & 5.707763000 \\
\hline $\mathrm{H}$ & 5.504743000 \\
\hline $\mathrm{H}$ & 6.716893000 \\
\hline $\mathrm{H}$ & 4.990298000 \\
\hline $\mathrm{C}$ & 5.340492000 \\
\hline $\mathrm{H}$ & 5.290584000 \\
\hline $\mathrm{H}$ & 6.215292000 \\
\hline $\mathrm{H}$ & 4.441882000 \\
\hline $\mathrm{C}$ & -0.718474000 \\
\hline $\mathrm{H}$ & -1.009148000 \\
\hline $\mathrm{H}$ & 0.007008000 \\
\hline $\mathrm{H}$ & -0.195022000 \\
\hline $\mathrm{C}$ & -3.724041000 \\
\hline $\mathrm{H}$ & -2.825198000 \\
\hline $\mathrm{H}$ & -4.436349000 \\
\hline $\mathrm{H}$ & -4.190213000 \\
\hline $\mathrm{C}$ & -2.667382000 \\
\hline $\mathrm{H}$ & -1.660472000 \\
\hline $\mathrm{H}$ & -2.577318000 \\
\hline $\mathrm{H}$ & -2.971271000 \\
\hline $\mathrm{C}$ & -5.870658000 \\
\hline $\mathrm{H}$ & -6.590753000 \\
\hline $\mathrm{H}$ & -5.131470000 \\
\hline $\mathrm{H}$ & -6.424597000 \\
\hline
\end{tabular}

3.736458000 $6.221250000-1.220514000$ $-5.987057000 \quad-2.072076000$ $-2.506064000 \quad-3.782531000$ $-2.162591000 \quad-3.245669000$ $-0.236900000 \quad-3.137364000$ $1.431622000 \quad-1.281683000$ $3.173633000 \quad-0.693243000$ $0.103217000 \quad 2.331101000$ $-3.264414000 \quad 0.463343000$ $2.805199000 \quad 1.268745000$ $4.268991000 \quad 3.485744000$ $2.154377000 \quad-4.152019000$ $2.951755000 \quad-2.751121000$ $1.768368000 \quad-2.494329000$ $-4.676105000-4.451955000$ $-4.117650000 \quad-5.255649000$ $-5.755400000 \quad-4.649008000$ $-4.467328000 \quad-4.541834000$ $\begin{array}{ll}-5.568284000 & 0.541557000\end{array}$ $\begin{array}{ll}-6.519089000 & 0.250056000\end{array}$ $-5.812059000 \quad 1.126204000$ $\begin{array}{ll}-5.039974000 & 1.225528000\end{array}$ $\begin{array}{ll}5.351226000 & 0.971412000\end{array}$ $6.010561000 \quad 1.833368000$ $\begin{array}{lll}5.603189000 & 0.591660000\end{array}$ $5.609928000 \quad 0.170735000$ $1.744797000 \quad 4.534422000$ $0.648807000 \quad 4.653940000$ $2.106255000 \quad 5.107676000$ $2.176629000 \quad 5.014860000$ $\begin{array}{ll}4.851384000 & 0.980704000\end{array}$ $5.011005000 \quad 2.035974000$ $4.017107000 \quad 0.969051000$ $5.759161000 \quad 0.635010000$ $\begin{array}{ll}5.989851000 & -2.961095000\end{array}$ $6.269985000-3.539830000$ $\begin{array}{ll}5.505348000 & -3.651425000\end{array}$ $6.932632000-2.614735000$ $-4.560591000-0.745737000$ $-4.108492000-0.675857000$ $\begin{array}{ll}-5.626892000 & -0.475503000\end{array}$ $-4.506558000-1.807922000$ $\begin{array}{ll}-4.594793000 & 3.204024000\end{array}$ $\begin{array}{ll}-5.347840000 & 2.830020000\end{array}$ $\begin{array}{ll}-5.137298000 & 3.821858000\end{array}$ $-3.906502000 \quad 3.865718000$

\section{Me TS-3}

Zero-point correction= Thermal correction to Energy=

Thermal correction to Enthalpy= 0.987017 (Hartree/Particle) Thermal correction to Gibbs Free Energy $=\quad 0.872846$ Sum of electronic and zero-point Energies $=\quad-2599.449235$ Sum of electronic and thermal Energies $=\quad-2599.383254$ Sum of electronic and thermal Enthalpies $=\quad-2599.382309$ Sum of electronic and thermal Free Energies $=-2599.563406$ E solvent $=-2599.26990349$

$\begin{array}{lrrr}\mathrm{Ni} & -1.695247000 & 0.627164000 & -1.393203000 \\ \mathrm{C} & -0.777370000 & 1.264724000 & -2.983814000 \\ \mathrm{~N} & -2.865022000 & 0.420568000 & 0.205250000 \\ \mathrm{C} & -2.362927000 & -0.054207000 & 1.331529000 \\ \mathrm{C} & -1.012650000 & -0.474696000 & 1.584086000 \\ \mathrm{C} & 0.078874000 & -0.278550000 & 0.652054000 \\ \mathrm{O} & -0.048398000 & 0.257551000 & -0.530722000 \\ \mathrm{C} & -2.120506000 & 1.875508000 & -3.261061000 \\ \mathrm{H} & -2.899533000 & 1.548998000 & -2.466193000 \\ \mathrm{H} & -2.126357000 & 2.975609000 & -3.191514000\end{array}$




\begin{tabular}{|c|c|c|c|}
\hline H & -2.596070000 & 1.533517000 & -4.197605000 \\
\hline $\mathrm{C}$ & 0.392053000 & 2.183048000 & -2.707084000 \\
\hline $\mathrm{H}$ & -0.518485000 & 0.399531000 & -3.615363000 \\
\hline $\mathrm{H}$ & -3.037175000 & -0.115350000 & 2.202135000 \\
\hline $\mathrm{C}$ & -0.751581000 & -1.041162000 & 2.864046000 \\
\hline $\mathrm{H}$ & -1.602403000 & -1.169793000 & 3.547915000 \\
\hline $\mathrm{C}$ & 0.520421000 & -1.444436000 & 3.274101000 \\
\hline $\mathrm{C}$ & 0.762582000 & -2.061651000 & 4.636933000 \\
\hline $\mathrm{H}$ & 1.184513000 & -3.081386000 & 4.554290000 \\
\hline $\mathrm{H}$ & -0.174213000 & -2.135378000 & 5.216509000 \\
\hline $\mathrm{H}$ & 1.479254000 & -1.465987000 & 5.233540000 \\
\hline $\mathrm{C}$ & 1.581901000 & -1.250954000 & 2.361568000 \\
\hline $\mathrm{H}$ & 2.604207000 & -1.543993000 & 2.633094000 \\
\hline $\mathrm{C}$ & 1.401269000 & -0.686360000 & 1.091462000 \\
\hline $\mathrm{C}$ & 2.537369000 & -0.511449000 & 0.182977000 \\
\hline $\mathrm{N}$ & 3.755388000 & -0.794969000 & 0.526057000 \\
\hline $\mathrm{H}$ & 2.295814000 & -0.118552000 & -0.820901000 \\
\hline $\mathrm{C}$ & 4.792993000 & -0.653521000 & -0.418648000 \\
\hline $\mathrm{C}$ & 4.872729000 & -1.495607000 & -1.571678000 \\
\hline $\mathrm{C}$ & 5.943767000 & -1.310388000 & -2.474778000 \\
\hline $\mathrm{C}$ & 6.915403000 & -0.326010000 & -2.266400000 \\
\hline $\mathrm{C}$ & 6.838609000 & 0.484657000 & -1.126459000 \\
\hline $\mathrm{C}$ & 5.800694000 & 0.332786000 & -0.184235000 \\
\hline $\mathrm{C}$ & 5.771596000 & 1.239517000 & 1.003439000 \\
\hline $\mathrm{C}$ & 5.593933000 & 0.741791000 & 2.315350000 \\
\hline $\mathrm{C}$ & 5.861925000 & 2.972187000 & 3.224477000 \\
\hline $\mathrm{C}$ & 6.038847000 & 3.501613000 & 1.935350000 \\
\hline $\mathrm{C}$ & 5.992025000 & 2.621685000 & 0.834439000 \\
\hline $\mathrm{C}$ & 3.894629000 & -2.590042000 & -1.853550000 \\
\hline $\mathrm{C}$ & 3.500586000 & -3.509474000 & -0.858778000 \\
\hline $\mathrm{C}$ & 2.634130000 & -4.579549000 & -1.151821000 \\
\hline $\mathrm{C}$ & 2.158079000 & -4.724525000 & -2.468443000 \\
\hline $\mathrm{C}$ & 2.533014000 & -3.827769000 & -3.488970000 \\
\hline $\mathrm{C}$ & 3.395562000 & -2.765560000 & -3.165043000 \\
\hline $\mathrm{C}$ & 5.637514000 & 1.593821000 & 3.430141000 \\
\hline $\mathrm{C}$ & -4.710911000 & -4.183613000 & 1.974919000 \\
\hline $\mathrm{C}$ & -4.336301000 & -3.846061000 & 0.658660000 \\
\hline $\mathrm{C}$ & -4.485818000 & -2.512441000 & 0.236043000 \\
\hline $\mathrm{C}$ & -5.003926000 & -1.523264000 & 1.096651000 \\
\hline $\mathrm{C}$ & -5.384422000 & -1.899162000 & 2.402826000 \\
\hline $\mathrm{C}$ & -5.236129000 & -3.224234000 & 2.859442000 \\
\hline $\mathrm{C}$ & -5.261915000 & -0.113961000 & 0.646884000 \\
\hline $\mathrm{C}$ & -6.610210000 & 0.304714000 & 0.654691000 \\
\hline $\mathrm{C}$ & -6.971776000 & 1.607035000 & 0.295657000 \\
\hline $\mathrm{C}$ & -5.972666000 & 2.520281000 & -0.052019000 \\
\hline $\mathrm{C}$ & -4.606013000 & 2.158872000 & -0.077267000 \\
\hline $\mathrm{C}$ & -4.246846000 & 0.813763000 & 0.253697000 \\
\hline $\mathrm{C}$ & -3.621579000 & 3.243332000 & -0.382541000 \\
\hline $\mathrm{C}$ & -3.937816000 & 4.204443000 & -1.375749000 \\
\hline $\mathrm{C}$ & -3.096410000 & 5.296779000 & -1.644082000 \\
\hline $\mathrm{C}$ & -1.900762000 & 5.422508000 & -0.905208000 \\
\hline $\mathrm{C}$ & -1.551948000 & 4.490491000 & 0.088441000 \\
\hline $\mathrm{C}$ & -2.418414000 & 3.405129000 & 0.337636000 \\
\hline $\mathrm{H}$ & -5.798345000 & -1.137987000 & 3.076955000 \\
\hline $\mathrm{H}$ & -4.594675000 & -5.221273000 & 2.316325000 \\
\hline $\mathrm{H}$ & -4.219892000 & -2.245822000 & -0.790770000 \\
\hline $\mathrm{H}$ & -7.376754000 & -0.422957000 & 0.943542000 \\
\hline $\mathrm{H}$ & -8.023031000 & 1.915179000 & 0.309814000 \\
\hline $\mathrm{H}$ & -6.239955000 & 3.560321000 & -0.266966000 \\
\hline $\mathrm{H}$ & -4.854084000 & 4.081425000 & -1.966501000 \\
\hline $\mathrm{H}$ & -1.229995000 & 6.268487000 & -1.106920000 \\
\hline $\mathrm{H}$ & 1.476917000 & -5.553312000 & -2.704944000 \\
\hline $\mathrm{H}$ & 3.678226000 & -2.044939000 & -3.942998000 \\
\hline $\mathrm{H}$ & 6.017747000 & -1.978778000 & -3.340424000 \\
\hline $\mathrm{H}$ & 7.743639000 & -0.207980000 & -2.974006000 \\
\hline $\mathrm{H}$ & 7.609909000 & 1.240024000 & -0.936474000 \\
\hline $\mathrm{H}$ & 6.113488000 & 3.021756000 & -0.180505000 \\
\hline $\mathrm{H}$ & 5.419267000 & -0.329520000 & 2.457581000 \\
\hline
\end{tabular}

$\begin{array}{lr}\mathrm{H} & 3.876542000 \\ \mathrm{H} & -2.158599000 \\ \mathrm{H} & 5.896653000 \\ \mathrm{H} & 0.833312000 \\ \mathrm{H} & 0.084658000 \\ \mathrm{H} & 1.186119000 \\ \mathrm{C} & -2.908984000 \\ \mathrm{C} & -1.868292000 \\ \mathrm{H} & -2.750830000 \\ \mathrm{H} & -3.947784000 \\ \mathrm{H} & -0.845983000 \\ \mathrm{H} & -2.028988000 \\ \mathrm{C} & 6.279950000 \\ \mathrm{H} & 6.195877000 \\ \mathrm{H} & 7.288374000 \\ \mathrm{H} & 5.553318000 \\ \mathrm{C} & 5.435656000 \\ \mathrm{H} & 5.517031000 \\ \mathrm{H} & 6.180937000 \\ \mathrm{H} & 4.436078000 \\ \mathrm{C} & 2.031535000 \\ \mathrm{H} & 2.673282000 \\ \mathrm{H} & 1.006587000 \\ \mathrm{H} & 2.025589000 \\ \mathrm{C} & 2.215858000 \\ \mathrm{H} & 1.611196000 \\ \mathrm{H} & 3.093258000 \\ \mathrm{H} & 1.613095000 \\ \mathrm{C} & -3.808567000 \\ \mathrm{H} & -3.026203000 \\ \mathrm{H} & -4.612486000 \\ \mathrm{H} & -3.375969000 \\ \mathrm{C} & -5.614817000 \\ \mathrm{H} & -5.893230000 \\ \mathrm{H} & -4.773460000 \\ \mathrm{H} & -6.465747000 \\ \mathrm{C} & -3.465856000 \\ \mathrm{H} & -2.596787000 \\ \mathrm{H} & -4.263259000 \\ \mathrm{H} & -3.832405000 \\ \mathrm{C} & -0.270590000 \\ \mathrm{H} & -0.477961000 \\ \mathrm{H} & 0.383012000 \\ \mathrm{H} & 0.299315000\end{array}$

$-3.382288000$ 2.696322000 3.646762000 2.564521000 3.055507000 1.646673000 $-1.283886000$ $-1.610435000$ $-1.013429000$ $-1.273739000$ $-1.702178000$ $-1.974346000$ 4.984434000 5.544825000 5.177955000 5.416745000 1.050146000 $-0.050473000$ 1.459310000 1.316114000 $-4.015878000$ $-4.719966000$ $-4.428147000$ $-3.062305000$ $-5.547182000$ $-6.377028000$ $-5.986931000$ $-5.039918000$ $-4.905915000$ $-5.521597000$ $-5.597095000$ $-4.455648000$ $-3.599356000$ $-4.665144000$ $-3.431266000$ $-2.997258000$ 6.325738000 6.597956000 5.955568000 7.261365000 4.637543000 4.730314000 3.755261000 0.162965000 1.128937000 4.091141000 $-3.652932000$ $-2.102705000$ $-2.160332000$ $-2.964844000$ $-2.150949000$ $-4.015491000$ $-2.615701000$ $-2.532608000$ $-1.129992000$ 1.725374000 2.672808000 1.311937000 1.012125000 4.831772000 4.852418000 5.538667000 5.227306000 $-4.908317000$ $-5.473586000$ $-4.925623000$ $-5.465454000$ $-0.060983000$ $-0.466586000$ 0.448946000 0.715944000 $-0.288970000$ 0.191322000 $-0.607765000$ $-1.198681000$ 4.279245000 4.355267000 4.979476000 4.644409000 $-2.695305000$ $-3.321537000$ $-3.362944000$ $-2.230233000$ 0.885515000 1.968183000 0.757543000 0.575008000

\section{Me3-Coor-C}

Zero-point correction $=0.990712($ Hartree/Particle $)$ Thermal correction to Energy $=\quad 1.056055$ Thermal correction to Enthalpy= 1.056999 Thermal correction to Gibbs Free Energy= 0.880404 Sum of electronic and zero-point Energies $=\quad-2599.470567$ Sum of electronic and thermal Energies $=\quad-2599.405223$ Sum of electronic and thermal Enthalpies $=-2599.404279$ Sum of electronic and thermal Free Energies= -2599.580875 E solvent $=-2599.28243232$

$\begin{array}{lrrr}\mathrm{Ni} & -1.729731000 & 0.127530000 & -1.584395000 \\ \mathrm{C} & -0.402831000 & -0.164543000 & -3.078370000 \\ \mathrm{~N} & -2.871388000 & 0.303663000 & 0.161870000 \\ \mathrm{C} & -2.383125000 & 0.038963000 & 1.358104000 \\ \mathrm{C} & -1.018264000 & -0.194953000 & 1.723736000 \\ \mathrm{C} & 0.049000000 & -0.185343000 & 0.756400000 \\ \mathrm{O} & -0.142024000 & -0.038437000 & -0.525486000 \\ \mathrm{C} & -0.815699000 & -0.784958000 & -4.409082000 \\ \mathrm{H} & -1.250091000 & -1.793672000 & -4.290255000 \\ \mathrm{H} & -1.526157000 & -0.164187000 & -4.988431000 \\ \mathrm{H} & 0.086514000 & -0.898042000 & -5.047491000 \\ \mathrm{C} & 0.343985000 & 1.159378000 & -3.236447000 \\ \mathrm{H} & 0.207215000 & -0.882239000 & -2.504864000\end{array}$




\begin{tabular}{|c|c|c|c|}
\hline H & -3.097346000 & 0.020191000 & 2.200935000 \\
\hline $\mathrm{C}$ & -0.732007000 & -0.400906000 & 3.101133000 \\
\hline $\mathrm{H}$ & -1.574488000 & -0.410067000 & 3.807132000 \\
\hline $\mathrm{C}$ & 0.566996000 & -0.584964000 & 3.579323000 \\
\hline $\mathrm{C}$ & 0.851616000 & -0.812427000 & 5.050443000 \\
\hline $\mathrm{H}$ & 1.303457000 & -1.806646000 & 5.229597000 \\
\hline $\mathrm{H}$ & -0.072507000 & -0.754422000 & 5.651514000 \\
\hline $\mathrm{H}$ & 1.559275000 & -0.061729000 & 5.449576000 \\
\hline $\mathrm{C}$ & 1.614371000 & -0.547899000 & 2.630609000 \\
\hline $\mathrm{H}$ & 2.655369000 & -0.675453000 & 2.954273000 \\
\hline $\mathrm{C}$ & 1.397206000 & -0.353242000 & 1.259012000 \\
\hline $\mathrm{C}$ & 2.520855000 & -0.318750000 & 0.317087000 \\
\hline $\mathrm{N}$ & 3.753111000 & -0.417216000 & 0.704137000 \\
\hline $\mathrm{H}$ & 2.253212000 & -0.194558000 & -0.747500000 \\
\hline $\mathrm{C}$ & 4.788632000 & -0.393961000 & -0.256998000 \\
\hline $\mathrm{C}$ & 5.072122000 & -1.541175000 & -1.060115000 \\
\hline $\mathrm{C}$ & 6.131419000 & -1.470390000 & -1.993008000 \\
\hline $\mathrm{C}$ & 6.898380000 & -0.310575000 & -2.144058000 \\
\hline $\mathrm{C}$ & 6.622864000 & 0.803561000 & -1.341663000 \\
\hline $\mathrm{C}$ & 5.585842000 & 0.784302000 & -0.385583000 \\
\hline $\mathrm{C}$ & 5.344879000 & 2.011339000 & 0.432355000 \\
\hline $\mathrm{C}$ & 5.187906000 & 1.950905000 & 1.837007000 \\
\hline $\mathrm{H}$ & 4.961006000 & 6.631026000 & 0.569541000 \\
\hline $\mathrm{C}$ & 5.031152000 & 4.366617000 & 1.949295000 \\
\hline $\mathrm{C}$ & 5.170186000 & 5.811622000 & -0.140021000 \\
\hline $\mathrm{C}$ & 5.179061000 & 4.463774000 & 0.555877000 \\
\hline $\mathrm{C}$ & 5.336370000 & 3.276612000 & -0.189083000 \\
\hline $\mathrm{C}$ & 4.312802000 & -2.822955000 & -0.945486000 \\
\hline $\mathrm{C}$ & 4.026475000 & -3.407758000 & 0.308701000 \\
\hline $\mathrm{C}$ & 3.394379000 & -4.659347000 & 0.405058000 \\
\hline $\mathrm{C}$ & 3.043008000 & -5.335130000 & -0.781283000 \\
\hline $\mathrm{C}$ & 3.299857000 & -4.777639000 & -2.047158000 \\
\hline $\mathrm{C}$ & 3.937698000 & -3.523859000 & -2.112386000 \\
\hline $\mathrm{C}$ & 2.885075000 & -5.500493000 & -3.314646000 \\
\hline $\mathrm{C}$ & 3.086737000 & -5.267275000 & 1.760233000 \\
\hline $\mathrm{C}$ & 5.035430000 & 3.115972000 & 2.604682000 \\
\hline $\mathrm{C}$ & 4.882248000 & 3.040078000 & 4.111979000 \\
\hline $\mathrm{C}$ & -3.976118000 & -4.640814000 & 1.368669000 \\
\hline $\mathrm{C}$ & -3.296605000 & -3.983951000 & 0.327612000 \\
\hline $\mathrm{C}$ & -3.662151000 & -2.661667000 & 0.004558000 \\
\hline $\mathrm{C}$ & -4.699273000 & -1.998558000 & 0.692535000 \\
\hline $\mathrm{C}$ & -5.359300000 & -2.691839000 & 1.736451000 \\
\hline $\mathrm{C}$ & -5.012141000 & -4.007710000 & 2.084798000 \\
\hline $\mathrm{C}$ & -2.186433000 & -4.676316000 & -0.438967000 \\
\hline $\mathrm{C}$ & -5.168422000 & -0.615073000 & 0.353791000 \\
\hline $\mathrm{C}$ & -6.563962000 & -0.403961000 & 0.270856000 \\
\hline $\mathrm{C}$ & -7.097893000 & 0.865570000 & 0.026878000 \\
\hline $\mathrm{C}$ & -6.238022000 & 1.958444000 & -0.116007000 \\
\hline $\mathrm{C}$ & -4.833044000 & 1.808972000 & -0.052443000 \\
\hline $\mathrm{C}$ & -4.297937000 & 0.502674000 & 0.155848000 \\
\hline $\mathrm{C}$ & -4.000846000 & 3.047515000 & -0.178807000 \\
\hline $\mathrm{C}$ & -4.342445000 & 4.004697000 & -1.166903000 \\
\hline $\mathrm{C}$ & -3.641261000 & 5.214498000 & -1.297671000 \\
\hline $\mathrm{C}$ & -2.572970000 & 5.469937000 & -0.413154000 \\
\hline $\mathrm{C}$ & -2.208371000 & 4.548303000 & 0.583798000 \\
\hline $\mathrm{C}$ & -2.931245000 & 3.342268000 & 0.690971000 \\
\hline $\mathrm{C}$ & -1.050755000 & 4.831175000 & 1.521338000 \\
\hline $\mathrm{C}$ & -4.025909000 & 6.229435000 & -2.356496000 \\
\hline $\mathrm{H}$ & -6.146539000 & -2.178764000 & 2.303073000 \\
\hline $\mathrm{H}$ & -3.686503000 & -5.666354000 & 1.634869000 \\
\hline $\mathrm{H}$ & -1.270604000 & -4.058344000 & -0.468172000 \\
\hline $\mathrm{H}$ & -1.926135000 & -5.647465000 & 0.016258000 \\
\hline $\mathrm{H}$ & -2.480522000 & -4.868536000 & -1.488560000 \\
\hline $\mathrm{H}$ & -3.119930000 & -2.142870000 & -0.794526000 \\
\hline $\mathrm{H}$ & -7.229728000 & -1.265829000 & 0.388983000 \\
\hline $\mathrm{H}$ & -8.182884000 & 1.005353000 & -0.033867000 \\
\hline $\mathrm{H}$ & -6.649784000 & 2.964529000 & -0.251387000 \\
\hline $\mathrm{H}$ & -5.165020000 & 3.785910000 & -1.859727000 \\
\hline
\end{tabular}

$\begin{array}{cr}\mathrm{H} & -4.762561000 \\ \mathrm{H} & -4.472715000 \\ \mathrm{H} & -3.145905000 \\ \mathrm{H} & -2.012654000 \\ \mathrm{H} & -0.746579000 \\ \mathrm{H} & -0.165923000 \\ \mathrm{H} & -1.310114000 \\ \mathrm{H} & 2.557167000 \\ \mathrm{H} & 3.644487000 \\ \mathrm{H} & 2.731941000 \\ \mathrm{H} & 1.935912000 \\ \mathrm{H} & 4.141460000 \\ \mathrm{H} & 3.128264000 \\ \mathrm{H} & 2.072059000 \\ \mathrm{H} & 3.798701000 \\ \mathrm{H} & 6.363464000 \\ \mathrm{H} & 7.723219000 \\ \mathrm{H} & 7.235370000 \\ \mathrm{H} & 4.403973000 \\ \mathrm{H} & 6.143698000 \\ \mathrm{H} & 5.435461000 \\ \mathrm{H} & 5.187864000 \\ \mathrm{H} & 4.921058000 \\ \mathrm{H} & 3.919433000 \\ \mathrm{H} & 4.314892000 \\ \mathrm{H} & -2.663422000 \\ \mathrm{H} & 4.906745000 \\ \mathrm{H} & 5.682174000 \\ \mathrm{C} & -5.736469000 \\ \mathrm{H} & -5.031821000 \\ \mathrm{H} & -6.305749000 \\ \mathrm{H} & -6.454684000 \\ \mathrm{H} & 1.273401000 \\ \mathrm{H} & -0.250445000 \\ \mathrm{H} & 0.640845000 \\ \mathrm{C} & -3.364599000 \\ \mathrm{C} & -2.968294000 \\ \mathrm{H} & -4.190727000 \\ \mathrm{H} & -3.111824000 \\ \mathrm{H} & -3.451478000 \\ \mathrm{H} & -2.397167000\end{array}$

5.814974000

$7.135223000-1.903370000$

$6.561888000 \quad-2.937283000$

$6.410173000-0.504833000$

$5.891350000 \quad 1.479960000$

$4.219908000 \quad 1.260852000$

$\begin{array}{rr}4.591626000 & 2.568659000\end{array}$

$-6.318389000 \quad-0.715767000$

$-5.394205000-4.110377000$

$-6.578864000 \quad-3.132925000$

$-5.097374000 \quad-3.718703000$

$-3.074562000 \quad-3.092782000$

$\begin{array}{ll}-6.370864000 & 1.729089000\end{array}$

$\begin{array}{ll}-4.987962000 & 2.105243000\end{array}$

$\begin{array}{ll}-4.920537000 & 2.529813000\end{array}$

$-2.362154000-2.586545000$

$-0.282986000 \quad-2.864757000$

$1.708379000-1.428370000$

$\begin{array}{ll}5.851442000 & -0.936899000\end{array}$

$6.026715000-0.620771000$

$3.335434000-1.280701000$

$0.973129000 \quad 2.329768000$

$1.996089000 \quad 4.467588000$

$3.473192000 \quad 4.443940000$

$-2.875678000 \quad 1.222192000$

$2.637443000 \quad 1.483549000$

$5.284119000 \quad 2.540965000$

$3.601923000 \quad 4.630636000$

$-4.734378000 \quad 3.201381000$

$-5.286741000 \quad 3.849332000$

$-4.034524000 \quad 3.837569000$

$-5.475910000 \quad 2.800912000$

$1.001731000-3.826213000$

$1.920361000-3.777023000$

$1.577070000-2.260027000$

$-0.275306000-2.755457000$

$\begin{array}{ll}1.060169000 & -2.893670000\end{array}$

$-0.536372000-2.085174000$

$-1.023793000-3.511769000$

$1.853575000-2.314389000$

$1.396885000-3.764552000$

\section{MeTS-3}

Zero-point correction $=\quad 0.989859($ Hartree/Particle $)$

Thermal correction to Energy= 1.054837

Thermal correction to Enthalpy= $\quad 1.055782$

Thermal correction to Gibbs Free Energy= 0.877480

Sum of electronic and zero-point Energies $=\quad-2599.454745$

Sum of electronic and thermal Energies $=\quad-2599.389767$

Sum of electronic and thermal Enthalpies $=\quad-2599.388823$

Sum of electronic and thermal Free Energies $=-2599.567125$

E solvent $=-2599.2720955$

$\begin{array}{lrrr}\mathrm{Ni} & -1.854088000 & 0.487947000 & -1.298230000 \\ \mathrm{C} & -0.583385000 & 1.316548000 & -2.840883000 \\ \mathrm{~N} & -2.873685000 & 0.330996000 & 0.320978000 \\ \mathrm{C} & -2.363226000 & -0.078946000 & 1.472921000 \\ \mathrm{C} & -1.006133000 & -0.417783000 & 1.770901000 \\ \mathrm{C} & 0.053913000 & -0.306067000 & 0.791430000 \\ \mathrm{O} & -0.157794000 & 0.058772000 & -0.437168000 \\ \mathrm{C} & 0.523501000 & 0.480504000 & -3.467203000 \\ \mathrm{H} & 0.785701000 & -0.375436000 & -2.823644000 \\ \mathrm{H} & 0.246708000 & 0.105748000 & -4.471562000 \\ \mathrm{H} & 1.435587000 & 1.098906000 & -3.588879000 \\ \mathrm{C} & -1.034745000 & 2.527410000 & -3.650981000 \\ \mathrm{H} & -0.245458000 & 1.675526000 & -1.850304000 \\ \mathrm{H} & -3.063858000 & -0.146740000 & 2.322151000 \\ \mathrm{C} & -0.706312000 & -0.833297000 & 3.098686000 \\ \mathrm{H} & -1.536733000 & -0.911004000 & 3.814641000\end{array}$




\begin{tabular}{|c|c|c|c|}
\hline 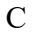 & 0.588757000 & -1.146081000 & 3.515784000 \\
\hline$C$ & 0.884099000 & -1.597824000 & 4.932152000 \\
\hline $\mathrm{H}$ & 1.311786000 & -2.618321000 & 4.954492000 \\
\hline $\mathrm{H}$ & -0.030932000 & -1.607275000 & 5.549737000 \\
\hline $\mathrm{H}$ & 1.615983000 & -0.932659000 & 5.428108000 \\
\hline $\mathrm{C}$ & 1.625003000 & -1.018136000 & 2.561522000 \\
\hline $\mathrm{H}$ & 2.662833000 & -1.236978000 & 2.844146000 \\
\hline $\mathrm{C}$ & 1.399780000 & -0.608906000 & 1.240046000 \\
\hline $\mathrm{C}$ & 2.508623000 & -0.468448000 & 0.293605000 \\
\hline $\mathrm{N}$ & 3.743276000 & -0.685739000 & 0.623499000 \\
\hline $\mathrm{H}$ & 2.229232000 & -0.150729000 & -0.727132000 \\
\hline $\mathrm{C}$ & 4.754206000 & -0.545488000 & -0.352168000 \\
\hline $\mathrm{C}$ & 4.919795000 & -1.511809000 & -1.392309000 \\
\hline $\mathrm{C}$ & 5.958587000 & -1.323557000 & -2.332167000 \\
\hline $\mathrm{C}$ & 6.815732000 & -0.220171000 & -2.266614000 \\
\hline $\mathrm{C}$ & 6.655341000 & 0.713844000 & -1.235007000 \\
\hline $\mathrm{C}$ & 5.645542000 & 0.567196000 & -0.261666000 \\
\hline $\mathrm{C}$ & 5.524152000 & 1.602790000 & 0.808621000 \\
\hline $\mathrm{C}$ & 5.422306000 & 1.250618000 & 2.174522000 \\
\hline $\mathrm{H}$ & 5.319378000 & 6.097349000 & 1.909455000 \\
\hline $\mathrm{C}$ & 5.430765000 & 3.590785000 & 2.804148000 \\
\hline $\mathrm{C}$ & 5.597310000 & 5.440828000 & 1.066442000 \\
\hline $\mathrm{C}$ & 5.528739000 & 3.976598000 & 1.456844000 \\
\hline $\mathrm{C}$ & 5.574236000 & 2.970242000 & 0.470170000 \\
\hline $\mathrm{C}$ & 4.063072000 & -2.729614000 & -1.517051000 \\
\hline $\mathrm{C}$ & 3.754462000 & -3.538414000 & -0.402933000 \\
\hline $\mathrm{C}$ & 3.022211000 & -4.732371000 & -0.543787000 \\
\hline $\mathrm{C}$ & 2.594045000 & -5.117343000 & -1.828084000 \\
\hline $\mathrm{C}$ & 2.874004000 & -4.329228000 & -2.962531000 \\
\hline $\mathrm{C}$ & 3.608459000 & -3.142910000 & -2.791065000 \\
\hline $\mathrm{C}$ & 2.378360000 & -4.746875000 & -4.334089000 \\
\hline $\mathrm{C}$ & 2.695415000 & -5.579674000 & 0.671318000 \\
\hline $\mathrm{C}$ & 5.377731000 & 2.230704000 & 3.178633000 \\
\hline $\mathrm{C}$ & 5.266414000 & 1.841696000 & 4.640600000 \\
\hline $\mathrm{C}$ & -4.261487000 & -4.574989000 & 1.373238000 \\
\hline $\mathrm{C}$ & -3.626040000 & -3.921533000 & 0.302330000 \\
\hline $\mathrm{C}$ & -3.917395000 & -2.563025000 & 0.068165000 \\
\hline $\mathrm{C}$ & -4.834400000 & -1.860348000 & 0.875501000 \\
\hline $\mathrm{C}$ & -5.454996000 & -2.550189000 & 1.944183000 \\
\hline $\mathrm{C}$ & -5.180445000 & -3.903207000 & 2.205198000 \\
\hline $\mathrm{C}$ & -2.645839000 & -4.657351000 & -0.590584000 \\
\hline $\mathrm{C}$ & -5.220765000 & -0.438181000 & 0.607965000 \\
\hline $\mathrm{C}$ & -6.599701000 & -0.124331000 & 0.612976000 \\
\hline $\mathrm{C}$ & -7.053453000 & 1.175551000 & 0.369210000 \\
\hline $\mathrm{C}$ & -6.126863000 & 2.192230000 & 0.113280000 \\
\hline $\mathrm{C}$ & -4.737921000 & 1.935985000 & 0.091163000 \\
\hline $\mathrm{C}$ & -4.286678000 & 0.608005000 & 0.344037000 \\
\hline $\mathrm{C}$ & -3.821281000 & 3.082129000 & -0.192124000 \\
\hline $\mathrm{C}$ & -4.153332000 & 3.978324000 & -1.237350000 \\
\hline $\mathrm{C}$ & -3.371799000 & 5.113650000 & -1.512737000 \\
\hline $\mathrm{C}$ & -2.227401000 & 5.348497000 & -0.722521000 \\
\hline $\mathrm{C}$ & -1.868765000 & 4.483590000 & 0.327071000 \\
\hline $\mathrm{C}$ & -2.671475000 & 3.352059000 & 0.579335000 \\
\hline $\mathrm{C}$ & -0.646921000 & 4.758060000 & 1.182540000 \\
\hline $\mathrm{C}$ & -3.757154000 & 6.074546000 & -2.621022000 \\
\hline $\mathrm{H}$ & -6.153008000 & -2.008850000 & 2.595350000 \\
\hline $\mathrm{H}$ & -4.030677000 & -5.630783000 & 1.569632000 \\
\hline $\mathrm{H}$ & -1.691410000 & -4.107188000 & -0.680845000 \\
\hline $\mathrm{H}$ & -2.420492000 & -5.664294000 & -0.198840000 \\
\hline $\mathrm{H}$ & -3.047291000 & -4.780803000 & -1.614708000 \\
\hline $\mathrm{H}$ & -3.413305000 & -2.043344000 & -0.754608000 \\
\hline $\mathrm{H}$ & -7.317507000 & -0.933127000 & 0.788522000 \\
\hline $\mathrm{H}$ & -8.126560000 & 1.396508000 & 0.378937000 \\
\hline $\mathrm{H}$ & -6.471092000 & 3.219452000 & -0.049506000 \\
\hline $\mathrm{H}$ & -5.035396000 & 3.771808000 & -1.856709000 \\
\hline $\mathrm{H}$ & -4.449725000 & 5.605632000 & -3.341483000 \\
\hline $\mathrm{H}$ & -4.261787000 & 6.973350000 & -2.216208000 \\
\hline $\mathrm{H}$ & -2.871493000 & 6.427002000 & -3.179753000 \\
\hline
\end{tabular}

\begin{tabular}{cr}
$\mathrm{H}$ & -1.602603000 \\
$\mathrm{H}$ & -0.059852000 \\
$\mathrm{H}$ & 0.018360000 \\
$\mathrm{H}$ & -0.931021000 \\
$\mathrm{H}$ & 2.029013000 \\
$\mathrm{H}$ & 2.890341000 \\
$\mathrm{H}$ & 2.542715000 \\
$\mathrm{H}$ & 1.291829000 \\
$\mathrm{H}$ & 3.827246000 \\
$\mathrm{H}$ & 2.420797000 \\
$\mathrm{H}$ & 1.845031000 \\
$\mathrm{H}$ & 3.551548000 \\
$\mathrm{H}$ & 6.102316000 \\
$\mathrm{H}$ & 7.621507000 \\
$\mathrm{H}$ & 7.337821000 \\
$\mathrm{H}$ & 4.920705000 \\
$\mathrm{H}$ & 6.617844000 \\
$\mathrm{H}$ & 5.632677000 \\
$\mathrm{H}$ & 5.375648000 \\
$\mathrm{H}$ & 5.367129000 \\
$\mathrm{H}$ & 4.289091000 \\
$\mathrm{H}$ & 4.101244000 \\
$\mathrm{H}$ & -2.402696000 \\
$\mathrm{H}$ & 5.391767000 \\
$\mathrm{H}$ & 6.046545000 \\
$\mathrm{C}$ & -5.855657000 \\
$\mathrm{H}$ & -5.122738000 \\
$\mathrm{H}$ & -6.399071000 \\
$\mathrm{H}$ & -6.586755000 \\
$\mathrm{H}$ & -0.205426000 \\
$\mathrm{H}$ & -1.289123000 \\
$\mathrm{H}$ & -1.898631000 \\
$\mathrm{C}$ & -2.204988000 \\
$\mathrm{C}$ & -3.368353000 \\
$\mathrm{H}$ & -1.912697000 \\
$\mathrm{H}$ & -2.011220000 \\
$\mathrm{H}$ & -4.071716000 \\
$\mathrm{H}$ & -3.824312000 \\
& \\
\hline
\end{tabular}

6.228035000 5.604883000 3.876783000 5.005209000 $-6.051939000$ $-4.188038000$ $-5.825317000$ $-4.564263000$ $-2.516081000$ $-6.610234000$ $-5.155190000$ $-5.636248000$ $-2.082424000$ $-0.101842000$ 1.567925000 5.667811000 5.727728000 3.255476000 0.191273000 0.750933000 2.141884000 $-3.231066000$ 2.685344000 4.365131000 2.331637000 $-4.628820000$ $-5.177340000$ $-3.928733000$ $-5.374322000$ 3.262462000 2.276303000 3.035120000 0.014678000 0.439796000 $-1.045790000$ 0.429075000 $-0.306797000$ 1.407710000

$-0.928969000$ 0.787577000 1.231274000 2.223237000 $-1.948627000$ $-5.136823000$ $-4.513202000$ $-4.446020000$ $-3.664745000$ 0.385128000 1.239173000 1.367623000 $-3.109980000$ $-2.999637000$ $-1.154902000$ 0.222008000 0.746315000 $-0.588189000$ 2.447401000 4.775295000 5.065249000 0.590187000 1.404574000 3.582794000 5.252852000 3.352824000 3.972741000 4.010830000 2.985022000 $-3.686349000$ $-4.698982000$ $-3.187517000$ $-3.269990000$ $-2.535829000$ $-3.211752000$ $-4.265930000$ $-2.146381000$ $-2.785809000$

\section{Me4- $\boldsymbol{\beta}$-T}

Zero-point correction $=\quad 0.991917$ (Hartree/Particle) Thermal correction to Energy= $\quad 1.056860$

Thermal correction to Enthalpy $=\quad 1.057804$ Thermal correction to Gibbs Free Energy $=0.880124$ Sum of electronic and zero-point Energies $=\quad-2599.496754$ Sum of electronic and thermal Energies $=\quad-2599.431811$ Sum of electronic and thermal Enthalpies $=\quad-2599.430867$ Sum of electronic and thermal Free Energies $=-2599.608546$ E solvent $=-2599.31400347$

$\begin{array}{lrrr}\mathrm{Ni} & 1.813404000 & 0.391008000 & 1.105003000 \\ \mathrm{C} & 3.128480000 & 0.572131000 & 2.472532000 \\ \mathrm{~N} & 2.827453000 & 0.385140000 & -0.444902000 \\ \mathrm{C} & 2.286243000 & 0.099228000 & -1.622244000 \\ \mathrm{C} & 0.910212000 & -0.144834000 & -1.925824000 \\ \mathrm{C} & -0.132711000 & -0.100818000 & -0.921264000 \\ \mathrm{O} & 0.113737000 & 0.127522000 & 0.336664000 \\ \mathrm{C} & 1.892192000 & 0.471096000 & 3.317579000 \\ \mathrm{H} & 1.675495000 & 1.412055000 & 3.861987000 \\ \mathrm{C} & 1.753175000 & -0.759298000 & 4.250346000 \\ \mathrm{C} & 2.733073000 & -0.629274000 & 5.432535000 \\ \mathrm{H} & 2.034325000 & -1.652683000 & 3.656518000 \\ \mathrm{C} & 0.303381000 & -0.935280000 & 4.738803000 \\ \mathrm{H} & 0.943370000 & 0.417870000 & 2.624095000 \\ \mathrm{H} & 3.830266000 & -0.276978000 & 2.521602000 \\ \mathrm{H} & 3.636115000 & 1.546743000 & 2.455850000 \\ \mathrm{H} & 2.980570000 & 0.065659000 & -2.477533000 \\ \mathrm{C} & 0.571053000 & -0.416978000 & -3.282358000 \\ \mathrm{H} & 1.384276000 & -0.449161000 & -4.020964000\end{array}$




\begin{tabular}{|c|c|c|c|}
\hline & 0 & 0 & \\
\hline & 000 & 6669000 & \\
\hline & 157680 & & \\
\hline & -0.169853000 & -0.950761000 & \\
\hline & -1.763840000 & -0.177610000 & 000 \\
\hline & -1.756117000 & 21000 & -2 \\
\hline $\mathrm{H}$ & -2.804002000 & -0.754758000 & -2 \\
\hline & -1.492937000 & -0.323070000 & -1 \\
\hline & -2.573190000 & -0.267305000 & -0.37 \\
\hline & -3.817593000 & -0.436826000 & -0.690978000 \\
\hline & -2.258793000 & -0.064701000 & 0.665025000 \\
\hline & -4.807062000 & -0.3 & \\
\hline & -5.066937000 & & \\
\hline & & & \\
\hline$C$ & & & \\
\hline 乙 & & & \\
\hline & & & \\
\hline & 0 & & -0. \\
\hline & & & \\
\hline & & & -0 \\
\hline & 0 & & \\
\hline $\mathrm{C}$ & -5. & & \\
\hline C & -5. & & \\
\hline & & & \\
\hline & 4. & -2.8 & \\
\hline $\mathrm{C}$ & & -3.4 & \\
\hline $\mathrm{C}$ & -3.4 & -4.6 & \\
\hline t & -3.0 & & \\
\hline & & & \\
\hline & 00 & & \\
\hline & $-2.8+x-y$ & -5.4 & \\
\hline & -3.1 & -5.2 & -1 \\
\hline & -5.1 & & -2.5 \\
\hline $\mathrm{C}$ & & & \\
\hline & & -4.5 & \\
\hline & & & \\
\hline C & & & \\
\hline C & & & \\
\hline $\mathrm{C}$ & & & \\
\hline & & & \\
\hline c & & & \\
\hline & & & \\
\hline & & & \\
\hline & & & \\
\hline $\mathrm{C}$ & & & \\
\hline & & & -0 . \\
\hline & & & -0 . \\
\hline & & & \\
\hline & & & \\
\hline $\mathrm{C}$ & & & 1.5 \\
\hline$C$ & & & 000 \\
\hline $\mathrm{C}$ & & & -0 \\
\hline $\mathrm{C}$ & & & -0 \\
\hline $\mathrm{C}$ & & & -1.2 \\
\hline C & 3.92 & & 000 \\
\hline-1 & & -2.0 & 2000 \\
\hline & 3.65 & & -1.8 \\
\hline & 1.366 & & \\
\hline $\mathrm{H}$ & 2.04 & & \\
\hline 3. & & & \\
\hline & & & \\
\hline 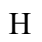 & & & \\
\hline II & & & \\
\hline & & & \\
\hline & & & \\
\hline & & & \\
\hline & & 6.875094 & 2.27177700 \\
\hline & 3.042442000 & 6.411846000 & 3.17213900 \\
\hline
\end{tabular}

\begin{tabular}{cr}
$\mathrm{H}$ & 1.799013000 \\
$\mathrm{H}$ & 0.526979000 \\
$\mathrm{H}$ & -0.091062000 \\
$\mathrm{H}$ & 0.949919000 \\
$\mathrm{H}$ & -2.584464000 \\
$\mathrm{H}$ & -3.350447000 \\
$\mathrm{H}$ & -3.046978000 \\
$\mathrm{H}$ & -1.750398000 \\
$\mathrm{H}$ & -4.087941000 \\
$\mathrm{H}$ & -3.109111000 \\
$\mathrm{H}$ & -2.218055000 \\
$\mathrm{H}$ & -3.967424000 \\
$\mathrm{H}$ & -6.298789000 \\
$\mathrm{H}$ & -7.626810000 \\
$\mathrm{H}$ & -7.180000000 \\
$\mathrm{H}$ & -4.412558000 \\
$\mathrm{H}$ & -6.138696000 \\
$\mathrm{H}$ & -5.384161000 \\
$\mathrm{H}$ & -5.292010000 \\
$\mathrm{H}$ & -5.103319000 \\
$\mathrm{H}$ & -4.094190000 \\
$\mathrm{H}$ & -4.377821000 \\
$\mathrm{H}$ & 2.493686000 \\
$\mathrm{H}$ & -5.000244000 \\
$\mathrm{H}$ & -5.862068000 \\
$\mathrm{C}$ & 5.556757000 \\
$\mathrm{H}$ & 6.277170000 \\
$\mathrm{H}$ & 4.805210000 \\
$\mathrm{H}$ & 6.107167000 \\
$\mathrm{H}$ & 0.211089000 \\
$\mathrm{H}$ & -0.030129000 \\
$\mathrm{H}$ & -0.394421000 \\
$\mathrm{H}$ & 2.683890000 \\
$\mathrm{H}$ & 3.775096000 \\
$\mathrm{H}$ & 2.484784000 \\
& \\
\hline
\end{tabular}

6.295168000 $5.975505000-1.140651000$ $4.325222000 \quad-0.875562000$ $4.661441000 \quad-2.275998000$ $-6.311789000 \quad 0.776101000$ $\begin{array}{ll}-5.073558000 & 4.268576000\end{array}$ $-6.555488000 \quad 3.318503000$ $\begin{array}{ll}-5.359504000 & 3.540058000\end{array}$ $-3.038492000 \quad 3.164633000$ $-6.379140000-1.633262000$ $-4.914747000-2.117564000$ $\begin{array}{ll}-5.023051000 & -2.419484000\end{array}$ $\begin{array}{ll}-2.307384000 & 2.735836000\end{array}$ $\begin{array}{ll}-0.212495000 & 3.045885000\end{array}$ $1.757958000 \quad 1.568179000$ $\begin{array}{ll}5.856296000 & 0.950232000\end{array}$ $6.081031000 \quad 0.592708000$ $3.367831000 \quad 1.321749000$ $0.956280000 \quad-2.263270000$ $1.949754000 \quad-4.423980000$ $3.421260000-4.457562000$ $-2.878441000 \quad-1.145709000$ $2.797894000-1.503691000$ $\begin{array}{ll}5.262720000 & -2.543806000\end{array}$ $3.557602000-4.579439000$ $-4.645193000-3.537317000$ $-5.406515000 \quad-3.180504000$ $-5.176513000-4.149496000$ $-3.954796000 \quad-4.199846000$ $\begin{array}{ll}-1.817164000 & 5.397841000\end{array}$ $\begin{array}{ll}-0.051388000 & 5.315602000\end{array}$ $\begin{array}{ll}-1.071803000 & 3.892478000\end{array}$ $-1.519680000 \quad 6.085107000$ $\begin{array}{ll}-0.516261000 & 5.084365000\end{array}$ $0.254128000 \quad 6.051652000$

${ }^{M e}$ TS-1 $1_{\text {Transf-1 }}$

Zero-point correction $=\quad 0.984659($ Hartree/Particle $)$ Thermal correction to Energy= $\quad 1.051190$ Thermal correction to Enthalpy $=\quad 1.052134$ Thermal correction to Gibbs Free Energy $=0.869924$

Sum of electronic and zero-point Energies $=\quad-2599.438589$ Sum of electronic and thermal Energies $=\quad-2599.372058$ Sum of electronic and thermal Enthalpies $=\quad-2599.371114$ Sum of electronic and thermal Free Energies $=-2599.553324$ E solvent $=-2599.25709616$

$\begin{array}{lrrr}\mathrm{Ni} & -1.900964000 & 0.292064000 & -1.290258000 \\ \mathrm{~N} & -2.921387000 & -0.097775000 & 0.296005000 \\ \mathrm{O} & -0.172361000 & 0.179892000 & -0.440652000 \\ \mathrm{~N} & 3.788073000 & -0.018102000 & 0.625109000 \\ \mathrm{C} & -0.953023000 & -0.439016000 & 1.763590000 \\ \mathrm{C} & 5.333459000 & -2.102419000 & -0.696567000 \\ \mathrm{C} & 1.454906000 & -0.224912000 & 1.252254000 \\ \mathrm{C} & 0.080652000 & -0.147556000 & 0.790280000 \\ \mathrm{C} & -5.139307000 & -1.229260000 & 0.363662000 \\ \mathrm{C} & -3.436715000 & -4.485932000 & 1.516320000 \\ \mathrm{C} & -2.350150000 & -0.352637000 & 1.459523000 \\ \mathrm{H} & -3.027134000 & -0.482723000 & 2.321026000 \\ \mathrm{C} & 2.535065000 & 0.100704000 & 0.317184000 \\ \mathrm{H} & 2.215399000 & 0.460444000 & -0.677455000 \\ \mathrm{C} & -4.364102000 & -0.036836000 & 0.300094000 \\ \mathrm{C} & 5.265455000 & -2.623053000 & 0.616806000 \\ \mathrm{H} & 5.319545000 & -1.933017000 & 1.465121000 \\ \mathrm{C} & -2.621632000 & 4.178713000 & 0.826528000 \\ \mathrm{C} & -3.664382000 & -5.285456000 & 0.382150000 \\ \mathrm{C} & -4.564615000 & -2.615509000 & 0.388205000 \\ \mathrm{C} & 5.051180000 & -4.872172000 & -0.259475000 \\ \mathrm{C} & 1.736508000 & -0.596823000 & 2.573094000 \\ \mathrm{H} & 2.794958000 & -0.650801000 & 2.858915000 \\ \mathrm{C} & 5.255453000 & -3.001373000 & -1.779638000 \\ \mathrm{H} & 5.284752000 & -2.606549000 & -2.803382000\end{array}$




\begin{tabular}{|c|c|c|c|}
\hline C & -5.002046000 & 1.231681000 & 0.174828000 \\
\hline $\mathrm{C}$ & -3.895969000 & -3.152470000 & 1.505964000 \\
\hline 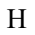 & -3.759214000 & -2.539438000 & 2.403525000 \\
\hline C & -3.250171000 & 2.923804000 & 0.941544000 \\
\hline $\mathrm{H}$ & -2.954751000 & 2.258221000 & 1.759245000 \\
\hline $\mathrm{C}$ & 5.546083000 & -0.646327000 & -0.955690000 \\
\hline $\mathrm{C}$ & -4.261623000 & 2.523281000 & 0.043276000 \\
\hline $\mathrm{C}$ & 5.017545000 & 1.745590000 & -0.599609000 \\
\hline$C$ & 5.128978000 & -4.000714000 & 0.848455000 \\
\hline $\mathrm{C}$ & -6.412431000 & 1.277383000 & 0.135471000 \\
\hline $\mathrm{H}$ & -6.899180000 & 2.256882000 & 0.070169000 \\
\hline $\mathrm{C}$ & 4.769839000 & 0.367142000 & -0.314615000 \\
\hline 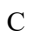 & -0.595812000 & -0.791454000 & 3.095854000 \\
\hline $\mathrm{H}$ & -1.406743000 & -0.996497000 & 3.809552000 \\
\hline $\mathrm{C}$ & -6.547962000 & -1.127471000 & 0.305729000 \\
\hline $\mathrm{H}$ & -7.136740000 & -2.050520000 & 0.353093000 \\
\hline $\mathrm{C}$ & 6.762585000 & 1.085792000 & -2.194039000 \\
\hline $\mathrm{H}$ & 7.542207000 & 1.364020000 & -2.911881000 \\
\hline $\mathrm{C}$ & 0.729443000 & -0.890170000 & 3.522472000 \\
\hline$c$ & 5.113849000 & -4.389794000 & -1.577343000 \\
\hline $\mathrm{C}$ & 6.018490000 & 2.074295000 & -1.541552000 \\
\hline $\mathrm{H}$ & 6.223807000 & 39000 & -1.735869000 \\
\hline $\mathrm{C}$ & -7.185295000 & 0.112597000 & 0.201095000 \\
\hline $\mathrm{H}$ & -8.278997000 & 0.172463000 & 0.175188000 \\
\hline $\mathrm{C}$ & 5.067878000 & 2000 & 000 \\
\hline $\mathrm{H}$ & 5.119306000 & -3.740434000 & 3000 \\
\hline $\mathrm{H}$ & 5.903134000 & -5.247311000 & 22000 \\
\hline $\mathrm{H}$ & 4.131115000 & -5.112134000 & 6000 \\
\hline $\mathrm{C}$ & -4.797155000 & -3.456495000 & -0.723986000 \\
\hline $\mathrm{H}$ & -5.340221000 & -3.052105000 & -1.587426000 \\
\hline $\mathrm{C}$ & 6.523736000 & -0.262014000 & 82000 \\
\hline $\mathrm{H}$ & 7.118819000 & -1.045551000 & -2.379992000 \\
\hline $\mathrm{C}$ & -4.344823000 & -4.788243000 & -0.747574000 \\
\hline $\mathrm{C}$ & 4.285128000 & 2.860780000 & 91000 \\
\hline $\mathrm{C}$ & -1.520302000 & 4.581500000 & 1.787827000 \\
\hline $\mathrm{H}$ & -1.603958000 & 4.041723000 & 2.747272000 \\
\hline $\mathrm{H}$ & -1.546450000 & 5.665048000 & 2.001953000 \\
\hline $\mathrm{H}$ & -0.519667000 & 000 & 1.372670000 \\
\hline C & -2.722257000 & -5.039619000 & 2.733895000 \\
\hline $\mathrm{H}$ & -3.360885000 & -4.989629000 & 3.636037000 \\
\hline $\mathrm{H}$ & -1.803558000 & 8000 & 20000 \\
\hline $\mathrm{H}$ & -2.431988000 & -6.093979000 & 2.586581000 \\
\hline $\mathrm{C}$ & 3.208319000 & 5.069290000 & -0.080684000 \\
\hline $\mathrm{C}$ & 5.036976000 & -5.336350000 & -2.760128000 \\
\hline $\mathrm{H}$ & 6.016187000 & -5.431003000 & -3.268345000 \\
\hline $\mathrm{H}$ & 4.315104000 & -4.980856000 & -3.518471000 \\
\hline $\mathrm{H}$ & 4.727730000 & -6.348966000 & -2.447383000 \\
\hline C & 4.085099000 & 2.882036000 & 0000 \\
\hline $\mathrm{H}$ & 4.424798000 & 2.029356000 & 2.068803000 \\
\hline $\mathrm{C}$ & 3.025222000 & 5.055524000 & 1.316914000 \\
\hline $\mathrm{C}$ & 3.833938000 & 3.965335000 & 5000 \\
\hline $\mathrm{H}$ & 3.962555000 & 3.950442000 & -1.775610000 \\
\hline $\mathrm{C}$ & -3.023709000 & 5.043914000 & -0.209189000 \\
\hline C & -4.640545000 & 3.419477000 & -0.983408000 \\
\hline $\mathrm{H}$ & -5.416089000 & 3.113757000 & -1.696755000 \\
\hline $\mathrm{C}$ & -4.034248000 & 4.681319000 & -1.121670000 \\
\hline $\mathrm{C}$ & 1.085854000 & -1.287867000 & 4.940722000 \\
\hline $\mathrm{H}$ & 1.658916000 & -2.234265000 & 4.966786000 \\
\hline $\mathrm{H}$ & 0.181602000 & -1.429550000 & 5.558178000 \\
\hline $\mathrm{H}$ & 1.711735000 & -0.521043000 & 5.434932000 \\
\hline $\mathrm{C}$ & 2.759145000 & 6.257530000 & -0.909529000 \\
\hline $\mathrm{H}$ & 2.573170000 & 5.971397000 & -1.959750000 \\
\hline $\mathrm{H}$ & 3.526779000 & 7.056013000 & -0.918863000 \\
\hline $\mathrm{H}$ & 1.832127000 & 6.705861000 & -0.509140000 \\
\hline $\mathrm{C}$ & 3.458308000 & 3.971481000 & 2.103690000 \\
\hline $\mathrm{C}$ & -4.554736000 & -5.657946000 & -1.970716000 \\
\hline $\mathrm{H}$ & -3.697873000 & -5.573314000 & -2.666924000 \\
\hline $\mathrm{H}$ & -5.460599000 & -5.362537000 & -2.529097000 \\
\hline $\mathrm{H}$ & -4.652087000 & -6.724051000 & -1.699355000 \\
\hline $\mathrm{C}$ & 3.252420000 & 3.967653000 & 3.606650000 \\
\hline $\mathrm{H}$ & 4.209943000 & 3.842738000 & 4.146183000 \\
\hline . & 2.595498000 & 3.134524000 & 3.919995000 \\
\hline $\mathrm{H}$ & 2.791561000 & 4.908586000 & 3.954312000 \\
\hline $\mathrm{C}$ & -4.471176000 & 5.638944000 & -2.213850000 \\
\hline & -5.133989000 & 6.430669000 & -1.813644000 \\
\hline $\mathrm{H}$ & -5.027192000 & 5.116168000 & -3.011368000 \\
\hline I & -3.606750000 & 6.146885000 & -2.678343000 \\
\hline
\end{tabular}

$\begin{array}{lrrr}\mathrm{C} & -1.368895000 & 1.933632000 & -2.356431000 \\ \mathrm{H} & -2.298628000 & 2.502730000 & -2.462060000 \\ \mathrm{H} & -0.571967000 & 2.402398000 & -1.765711000 \\ \mathrm{C} & -1.076903000 & 0.839736000 & -3.181252000 \\ \mathrm{H} & -3.180328000 & 0.350304000 & -2.015447000 \\ \mathrm{H} & -1.841651000 & 0.531546000 & -3.907026000 \\ \mathrm{C} & 0.319615000 & 0.327574000 & -3.438522000 \\ \mathrm{H} & 0.997315000 & 0.566001000 & -2.604654000 \\ \mathrm{H} & 0.336377000 & -0.764831000 & -3.598794000 \\ \mathrm{H} & 0.719819000 & 0.796511000 & -4.361107000 \\ \mathrm{C} & -1.986192000 & -2.513989000 & -2.826959000 \\ \mathrm{C} & -1.263444000 & -2.698999000 & -1.708047000 \\ \mathrm{H} & -1.517702000 & -2.263945000 & -3.786534000 \\ \mathrm{H} & -3.077406000 & -2.608527000 & -2.822733000 \\ \mathrm{H} & -0.172149000 & -2.598918000 & -1.700467000 \\ \mathrm{H} & -1.740605000 & -2.963080000 & -0.757758000 \\ \mathrm{H} & 2.533948000 & 5.909154000 & 1.803673000 \\ \mathrm{H} & 4.935401000 & -5.951351000 & -0.088217000 \\ \mathrm{H} & -3.308887000 & -6.324516000 & 0.379589000 \\ \mathrm{H} & -2.540173000 & 6.025692000 & -0.305711000\end{array}$

\section{Me 1-Transf-1}

Zero-point correction $=0.986473($ Hartree/Particle $)$ Thermal correction to Energy= 1.052407 Thermal correction to Enthalpy= $\quad 1.053351$ Thermal correction to Gibbs Free Energy $=\quad 0.874185$ Sum of electronic and zero-point Energies $=\quad-2599.44443$ Sum of electronic and thermal Energies $=\quad-2599.378498$ Sum of electronic and thermal Enthalpies $=\quad-2599.377553$ Sum of electronic and thermal Free Energies $=-2599.556719$ E solvent $=-2599.25881587$

$\begin{array}{lrrr}\mathrm{Ni} & -1.960155000 & -0.147894000 & -1.353829000 \\ \mathrm{~N} & -2.857150000 & 0.690896000 & 0.267712000 \\ \mathrm{O} & -0.166775000 & -0.101181000 & -0.526933000 \\ \mathrm{~N} & 3.718129000 & -0.791005000 & 0.576560000 \\ \mathrm{C} & -0.900176000 & 0.322069000 & 1.748330000 \\ \mathrm{C} & 3.574917000 & -3.495513000 & -0.658947000 \\ \mathrm{C} & 1.437674000 & -0.274119000 & 1.220736000 \\ \mathrm{C} & 0.087994000 & -0.025337000 & 0.744242000 \\ \mathrm{C} & -5.322768000 & 0.441572000 & 0.267432000 \\ \mathrm{C} & -4.733818000 & -2.994409000 & 1.872402000 \\ \mathrm{C} & -2.249295000 & 0.715790000 & 1.429674000 \\ \mathrm{H} & -2.827575000 & 1.106425000 & 2.287933000 \\ \mathrm{C} & 2.475466000 & -0.619390000 & 0.246869000 \\ \mathrm{H} & 2.135565000 & -0.722966000 & -0.799274000 \\ \mathrm{C} & -4.171443000 & 1.279664000 & 0.209851000 \\ \mathrm{C} & 3.335295000 & -3.835760000 & 0.689979000 \\ \mathrm{H} & 3.858175000 & -3.283396000 & 1.479269000 \\ \mathrm{C} & -1.179149000 & 4.801472000 & 0.737488000 \\ \mathrm{C} & -5.379531000 & -3.816521000 & 0.930315000 \\ \mathrm{C} & -5.269427000 & -1.036377000 & 0.487788000 \\ \mathrm{C} & 1.798000000 & -5.579704000 & 0.008375000 \\ \mathrm{C} & 1.735408000 & -0.195964000 & 2.589043000 \\ \mathrm{H} & 2.773184000 & -0.400213000 & 2.882894000 \\ \mathrm{C} & 2.899542000 & -4.222633000 & -1.664681000 \\ \mathrm{H} & 3.072330000 & -3.962146000 & -2.716817000 \\ \mathrm{C} & -4.320653000 & 2.687244000 & 0.014590000 \\ \mathrm{C} & -4.688595000 & -1.605444000 & 1.638743000 \\ \mathrm{H} & -4.217541000 & -0.955698000 & 2.384616000 \\ \mathrm{C} & -2.189430000 & 3.826973000 & 0.870675000 \\ \mathrm{H} & -2.200335000 & 3.206756000 & 1.771926000 \\ \mathrm{C} & 4.583419000 & -2.459076000 & -1.036874000 \\ \mathrm{C} & -3.192737000 & 3.667207000 & -0.105637000 \\ \mathrm{C} & 5.680493000 & -0.255746000 & -0.777867000 \\ \mathrm{C} & 2.451645000 & -4.873659000 & 1.037124000 \\ \mathrm{C} & -5.626597000 & 3.212179000 & -0.132068000 \\ \mathrm{H} & -5.735840000 & 4.294680000 & -0.262120000 \\ \mathrm{C} & 4.645053000 & -1.172361000 & -0.416945000 \\ \mathrm{H} & -0.525303000 & 0.391966000 & 3.118086000 \\ \mathrm{H} & -6.601010000 & 1.018936000 & 0.108305000 \\ \mathrm{H} & -7.532471000 & -1.877598000 & -2.408748000 \\ \mathrm{H} & -269638000 & -2.154137000 & -3.170728000 \\ \mathrm{H} & & & \\ \mathrm{H} & & \end{array}$




\begin{tabular}{|c|c|c|c|}
\hline$C$ & 2.005697000 & -5.262207000 & -1.347836000 \\
\hline $\mathrm{C}$ & 6.599207000 & -0.626845000 & -1.781420000 \\
\hline . & 7.393598000 & 0.079812000 & -2.047767000 \\
\hline C & -6.758832000 & 2.394042000 & -0.093286000 \\
\hline $\mathrm{H}$ & -7.759220000 & 2.827751000 & -0.200612000 \\
\hline $\mathrm{C}$ & 2.200227000 & -5.216032000 & 2.493276000 \\
\hline $\mathrm{H}$ & 3.110689000 & -5.080852000 & 3.104019000 \\
\hline $\mathrm{H}$ & 1.861596000 & -6.260546000 & 2.611984000 \\
\hline $\mathrm{H}$ & 1.418080000 & -4.564463000 & 2.929255000 \\
\hline $\mathrm{C}$ & -5.915591000 & -1.891695000 & -0.434295000 \\
\hline $\mathrm{H}$ & -6.372107000 & -1.456440000 & -1.331855000 \\
\hline 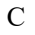 & 5.536939000 & -2.783556000 & -2.027677000 \\
\hline $\mathrm{H}$ & 5.499744000 & -3.781667000 & -2.479382000 \\
\hline $\mathrm{C}$ & -5.974555000 & -3.282305000 & -0.231152000 \\
\hline C & 5.804037000 & 1.099316000 & -0.160074000 \\
\hline $\mathrm{C}$ & -0.126703000 & 4.962047000 & 1.817426000 \\
\hline $\mathrm{H}$ & -0.520271000 & 5.531423000 & 2.681989000 \\
\hline $\mathrm{H}$ & 0.757603000 & 5.504664000 & 1.440933000 \\
\hline $\mathrm{H}$ & 0.211776000 & 3.982237000 & 2.198496000 \\
\hline $\mathrm{C}$ & -4.088656000 & -3.589934000 & 3.108697000 \\
\hline $\mathrm{H}$ & -4.167678000 & -2.909923000 & 3.975509000 \\
\hline $\mathrm{H}$ & -3.010814000 & -3.782383000 & 2.944329000 \\
\hline $\mathrm{H}$ & -4.555013000 & -4.551656000 & 3.385323000 \\
\hline 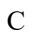 & 6.225330000 & 3.509052000 & -0.431055000 \\
\hline $\mathrm{C}$ & 72000 & -6.009 & 0728000 \\
\hline $\mathrm{H}$ & 1.822056000 & -5.989290000 & -3.394321000 \\
\hline $\mathrm{H}$ & 0.270837000 & -5.560991000 & -2.632409000 \\
\hline $\mathrm{H}$ & 1.096771000 & -7.0661 & -2.166513000 \\
\hline $\mathrm{C}$ & 5.767552000 & 1.284128000 & 1.241772000 \\
\hline $\mathrm{H}$ & 5.585248000 & 0.418461000 & 6515000 \\
\hline $\mathrm{C}$ & 17000 & 3.65 & 593000 \\
\hline $\mathrm{C}$ & 6.031746000 & 2.224226000 & -0.978874000 \\
\hline $\mathrm{H}$ & 6.041196000 & 2.096910000 & -2.069097000 \\
\hline $\mathrm{C}$ & -1.184605000 & 5.629988000 & -0.398382000 \\
\hline $\mathrm{C}$ & -3.177600000 & 4.536900000 & -1.223134000 \\
\hline $\mathrm{H}$ & -3.952308000 & 4.425596000 & -1.992311000 \\
\hline$C$ & -2.182139000 & 5.514510000 & -1.388095000 \\
\hline $\mathrm{C}$ & 000 & 0.19 & 8000 \\
\hline $\mathrm{H}$ & 1.410490000 & -0.801441000 & 6767000 \\
\hline $\mathrm{H}$ & 0.294249000 & 0.572184000 & 5.645089000 \\
\hline $\mathrm{H}$ & 2.001726000 & 0.8595 & 336000 \\
\hline C & 6.476720000 & 4.701000000 & -1.334941000 \\
\hline $\mathrm{H}$ & 5.721393000 & 4.766685000 & -2.140054000 \\
\hline $\mathrm{H}$ & 7.465880000 & 4.6351 & -1.827889000 \\
\hline $\mathrm{H}$ & 6.450807000 & 5.648867000 & -0.769572000 \\
\hline $\mathrm{C}$ & 5.959876000 & 2.550770000 & 1.815182000 \\
\hline $\mathrm{C}$ & -6.653284000 & -4.190297000 & -1.238571000 \\
\hline $\mathrm{H}$ & -5.913526000 & -4.783714000 & -1.809347000 \\
\hline $\mathrm{H}$ & -7.247653000 & -3.612645000 & -1.967325000 \\
\hline $\mathrm{H}$ & -7.329411000 & -4.910461000 & -0.742367000 \\
\hline $\mathrm{C}$ & 5.915991000 & 2.737641000 & 3.319939000 \\
\hline $\mathrm{H}$ & 6.785273000 & 3.316366000 & 3.683954000 \\
\hline $\mathrm{H}$ & 5.911700000 & 1.767319000 & 3.846065000 \\
\hline $\mathrm{H}$ & 5.008546000 & 3.2896 & 3.632864000 \\
\hline $\mathrm{C}$ & -2.161272000 & 6.406344000 & -2.614333000 \\
\hline $\mathrm{H}$ & -3.129455000 & 6.388171000 & -3.144050000 \\
\hline $\mathrm{H}$ & -1.384537000 & 6.083213000 & -3.333932000 \\
\hline $\mathrm{H}$ & -1.937878000 & 7.455522000 & -2.348279000 \\
\hline $\mathrm{C}$ & -1.695349000 & 1.661968000 & -2.435904000 \\
\hline $\mathrm{H}$ & -2.715447000 & 2.043768000 & -2.539087000 \\
\hline $\mathrm{H}$ & -1.014062000 & 2.245244000 & -1.806122000 \\
\hline $\mathrm{C}$ & -1.214630000 & 0.679632000 & -3.292073000 \\
\hline $\mathrm{H}$ & -3.258988000 & -0.2482 & 25615000 \\
\hline $\mathrm{H}$ & -1.911813000 & 0.259979000 & -4.030301000 \\
\hline $\mathrm{C}$ & 0.249339000 & 0.397120000 & -3.519842000 \\
\hline $\mathrm{H}$ & 0.850265000 & 0.664652000 & -2.636583000 \\
\hline $\mathrm{H}$ & 0.433677000 & -0.664392000 & -3.763060000 \\
\hline $\mathrm{H}$ & 0.608223000 & 0.991841000 & -4.384521000 \\
\hline $\mathrm{C}$ & -1.552477000 & -2.088709000 & -2.088120000 \\
\hline $\mathrm{C}$ & -2.197112000 & -2.191385000 & -0.857348000 \\
\hline $\mathrm{H}$ & -0.459195000 & -2.099901000 & -2.143752000 \\
\hline 11 & -2.100850000 & -2.252233000 & -3.022695000 \\
\hline $\mathrm{H}$ & -1.620116000 & -2.280275000 & 0.069131000 \\
\hline $\mathrm{H}$ & -3.264786000 & -2.419001000 & -0.794713000 \\
\hline & 6.336976000 & 4.650705000 & 1.405489000 \\
\hline$\Pi$ & 1.111699000 & -6.396755000 & 0.269922000 \\
\hline . & -5.426351000 & -4.899642000 & 1.107567000 \\
\hline
\end{tabular}

${ }^{\text {Me }}$ TS-3 Decoor $_{\text {D }}$

Zero-point correction= 0.985627 (Hartree/Particle)

Thermal correction to Energy= $\quad 1.051396$

Thermal correction to Enthalpy= $\quad 1.052340$

Thermal correction to Gibbs Free Energy $=\quad 0.874248$

Sum of electronic and zero-point Energies $=\quad-2599.440856$

Sum of electronic and thermal Energies= $\quad-2599.375087$

Sum of electronic and thermal Enthalpies $=\quad-2599.374143$

Sum of electronic and thermal Free Energies $=-2599.552236$

E solvent $=-2599.257649$

c

t.

\section{(c)}

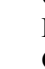 \\ clons}

$c$
$c$
$c$

c

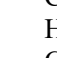

\section{tong}

辛

\section{政}

\section{$c$
$c$
$c$
$c$}

1.932968000

2.916196000

0.187301000

$-3.776964000$

0.908883000

$-3.830175000$

$-1.473052000$

$-0.094661000$

5.076795000

2.892013000

2.313179000

2.968085000

$-2.515883000$

$-2.160536000$

4.361148000

$-3.655112000$

$-4.147727000$

3.025643000

3.388279000

4.427319000

$-2.266901000$

$-1.790265000$

$-2.850874000$

$-3.197729000$

$-3.319029000$

5.063689000

3.421283000

3.054900000

3.556199000

3.310569000

$-4.740967000$

4.420139000

$-5.667974000$

$-2.879883000$

6.477962000

7.010770000

$-4.713132000$

0.517369000

1.305656000

6.488575000

7.032124000

$-6.614415000$

$-7.357995000$

$-0.814277000$

$-2.409776000$

$-6.595369000$

$-7.328853000$

7.189927000

8.285459000

$-2.703472000$

$-3.533286000$

$-2.656669000$

$-1.765828000$

4.902028000

5.677815000

$-5.699174000$

$-5.732828000$

4.393202000

$-5.694190000$

2.111894000

2.667674000

1.638768000
0.165306000

$\begin{array}{ll}0.316515000 & 0.295447000\end{array}$

$0.153028000-0.470337000$

$\begin{array}{ll}0.281832000 & 0.569589000\end{array}$

$\begin{array}{ll}-0.507229000 & 1.742657000\end{array}$

$3.147199000 \quad-0.248298000$

$\begin{array}{ll}-0.112736000 & 1.215099000\end{array}$

$\begin{array}{ll}-0.145927000 & 0.761638000\end{array}$

$0.887418000 \quad 0.307501000$

$3.955790000 \quad 1.088141000$

$-0.527077000 \quad 1.450470000$

$\begin{array}{ll}-0.710742000 & 2.320497000 \\ 0.287278000 & 0.26770600\end{array}$

$\begin{array}{ll}0.287278000 & 0.267706000\end{array}$

$\begin{array}{rr}0.598920000 & -0.731085000\end{array}$

$\begin{array}{rr}-0.345730000 & 0.328978000\end{array}$

$3.305084000 \quad 1.145274000$

$2.601296000 \quad 1.826018000$

$-4.762726000 \quad 1.112740000$

$\begin{array}{ll}4.853243000 & 0.123246000\end{array}$

$\begin{array}{ll}2.232752000 & 0.239760000\end{array}$

$\begin{array}{ll}5.255805000 & 0.778375000\end{array}$

$\begin{array}{ll}-0.447902000 & 2.538220000\end{array}$

$\begin{array}{ll}-0.413861000 & 2.818822000\end{array}$

$4.066488000-1.112575000$

$3.949151000 \quad-2.197069000$

$\begin{array}{ll}-1.585363000 & 0.328435000\end{array}$

$2.651417000 \quad 1.135580000$

$1.963080000 \quad 1.903847000$

$\begin{array}{ll}-3.460026000 & 1.218551000\end{array}$

$\begin{array}{ll}-2.863544000 & 2.102887000\end{array}$

$2.101534000 \quad-0.806823000$

$\begin{array}{ll}-2.936481000 & 0.236603000\end{array}$

$-0.192085000 \quad-0.903139000$

$\begin{array}{rr}4.351996000 & 1.671532000\end{array}$

$\begin{array}{ll}-1.561854000 & 0.323635000\end{array}$

$\begin{array}{ll}-2.519326000 & 0.332284000\end{array}$

$\begin{array}{ll}0.736247000 & -0.383360000\end{array}$

$\begin{array}{ll}-0.819639000 & 3.075107000\end{array}$

$\begin{array}{ll}-1.079653000 & 3.796344000\end{array}$

$0.850992000 \quad 0.312770000$

$\begin{array}{ll}1.802161000 & 0.329213000\end{array}$

$\begin{array}{ll}1.585846000 & -2.303640000\end{array}$

$1.916059000 \quad-3.037544000$

$\begin{array}{rr}1.916059000 & -3.495293000\end{array}$

$5.122715000 \quad-0.615115000$

$0.254953000-1.866984000$

$\begin{array}{ll}-0.460437000 & -2.256237000\end{array}$

$\begin{array}{ll}-0.359268000 & 0.324330000\end{array}$

$\begin{array}{ll}-0.364151000 & 0.339702000\end{array}$

$\begin{array}{ll}4.507283000 & 3.170005000\end{array}$

$4.035980000 \quad 3.725126000$

$5.571144000 \quad 3.464997000$

$\begin{array}{ll}4.031372000 & 3.516993000 \\ 3.162913000 & -0.714741000\end{array}$

$3.162913000 \quad-0.714741000$

$2.848108000-1.423748000$

$2.497248000-1.766755000$

$3.551199000-2.065991000$

$\begin{array}{ll}4.472169000 & -0.787913000\end{array}$

$\begin{array}{ll}-1.630277000 & -0.498076000\end{array}$

$-5.305272000 \quad 2.194831000$

$\begin{array}{ll}-5.501790000 & 3.131916000\end{array}$

1.885118000 


\begin{tabular}{|c|c|c|c|}
\hline $\mathrm{H}$ & 1.307709000 & -4.587676000 & 000 \\
\hline & 1.801390000 & 4.383402000 & 2.051046000 \\
\hline & 1.749423000 & 3.712834000 & 926277000 \\
\hline & 0.806481000 & 4.366911000 & 565273000 \\
\hline & 1.966662000 & 9000 & 7000 \\
\hline & -5.911681000 & -3.996516000 & 402000 \\
\hline & -1.721848000 & 6.086087000 & -1.563731000 \\
\hline 11 & -2.365307000 & 6.335075000 & 4000 \\
\hline & -0.787492000 & 5.654078000 & -1.971901000 \\
\hline & -1.453513000 & 95000 & 2000 \\
\hline $\mathrm{C}$ & 445000 & 50000 & 5000 \\
\hline $\mathrm{H}$ & 07000 & 359000 & 2000 \\
\hline 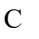 & -5.86 & -4.3 & 000 \\
\hline 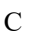 & -5.82 & -2.6 & 7000 \\
\hline $\mathrm{H}$ & 0000 & 000 & 000 \\
\hline 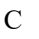 & & & \\
\hline $\mathrm{C}$ & 4.7 & -3.7 & 000 \\
\hline $\mathrm{H}$ & 5.4 & -3.3 & 000 \\
\hline $\mathrm{C}$ & 000 & -5.0 & 000 \\
\hline $\mathrm{C}$ & -1.2 & -1.1 & 000 \\
\hline $\mathrm{H}$ & -1.8 & -0.3 & 000 \\
\hline $\mathrm{H}$ & -0.3 & -1.3 & \\
\hline $\mathrm{H}$ & 00 & -2.0 & \\
\hline $\mathrm{C}$ & -6.0 & -5.0 & -2.2 \\
\hline $\mathrm{H}$ & & -4.8 & \\
\hline $\mathrm{H}$ & -7.1 & -5. & 000 \\
\hline $\mathrm{H}$ & -5.8 & & \\
\hline C & -5.7 & & \\
\hline$c$ & 0 & & 00 \\
\hline $\mathrm{H}$ & & & \\
\hline $\mathrm{H}$ & & & 00 \\
\hline $\mathrm{H}$ & 5.1 & 6.4 & 00 \\
\hline $\mathrm{C}$ & & & \\
\hline $\mathrm{H}$ & -6.6 & -4.2 & 000 \\
\hline $\mathrm{H}$ & -5.5 & -2.9 & 00 \\
\hline $\mathrm{H}$ & -4.8 & -4.5 & 000 \\
\hline $\mathrm{C}$ & 4.6 & -5.9 & -2.1 \\
\hline $\mathrm{H}$ & & & \\
\hline $\mathrm{H}$ & 3.7 & -5.8 & 000 \\
\hline $\mathrm{H}$ & & & \\
\hline $\mathrm{C}$ & & -2.3 & \\
\hline $\mathrm{H}$ & 2.8 & -2.1 & 00 \\
\hline $\mathrm{H}$ & 1.8 & -2.7 & 000 \\
\hline $\mathrm{C}$ & & & \\
\hline $\mathrm{H}$ & & & 00 \\
\hline $\mathrm{H}$ & 0.78 & -1.7 & \\
\hline $\mathrm{C}$ & & & \\
\hline $\mathrm{H}$ & -0.61 & -2.8 & 5000 \\
\hline $\mathrm{H}$ & -1.22 & -1.4 & \\
\hline $\mathrm{H}$ & -1.2 & -3.1 & -3.0 \\
\hline $\mathrm{C}$ & 1.118195000 & 1.061 & 8000 \\
\hline $\mathrm{C}$ & 1.6 & 2.0 & -2.0 \\
\hline $\mathrm{H}$ & 0.05 & 0.78 & 000 \\
\hline $\mathrm{H}$ & 000 & 0.77 & 000 \\
\hline $\mathrm{H}$ & & & 00 \\
\hline $\mathrm{H}$ & 0000 & 2.49 & 77000 \\
\hline $\mathrm{H}$ & -5.925422000 & -5.412458000 & 0.497399000 \\
\hline $\mathrm{H}$ & & & 1.179646000 \\
\hline $\mathrm{H}$ & 8000 & 9000 & 0.083781000 \\
\hline $\mathrm{H}$ & 2.967176000 & -6.561325000 & -0.091547000 \\
\hline
\end{tabular}

\section{Me2-BHE-C}

Zero-point correction $=\quad 0.906639$ (Hartree/Particle $)$ Thermal correction to Energy= $\quad 0.967636$ Thermal correction to Enthalpy $=\quad 0.968580$

Thermal correction to Gibbs Free Energy $=\quad 0.799327$ Sum of electronic and zero-point Energies $=\quad-2481.630555$ Sum of electronic and thermal Energies $=\quad-2481.569558$ Sum of electronic and thermal Enthalpies $=\quad-2481.568614$ Sum of electronic and thermal Free Energies $=-2481.737867$ E solvent $=-2481.40917615$

$\begin{array}{lrrc}\mathrm{Ni} & -1.952360000 & -0.295237000 & -1.430367000 \\ \mathrm{~N} & -2.966950000 & 0.393217000 & 0.016650000 \\ \mathrm{O} & -0.252514000 & -0.176211000 & -0.563238000 \\ \mathrm{~N} & 3.679218000 & -0.175659000 & 0.606150000\end{array}$

$\begin{array}{lll}1.365285000 & 0.342780000 & 1.105024000\end{array}$

$0.002262000 \quad 0.316803000 \quad 0.613111000$

$\begin{array}{lll}-5.185908000 & -0.679604000 & -0.132395000\end{array}$

$\begin{array}{lll}-3.169123000 & -3.807944000 & 0.813260000\end{array}$

$\begin{array}{lll}-2.408093000 & 0.856315000 & 1.124498000\end{array}$

$\begin{array}{lll}-3.094526000 & 1.273394000 & 1.880284000\end{array}$

$2.430638000 \quad-0.207554000 \quad 0.261605000$

$\begin{array}{lll}2.102472000 & -0.650929000 & -0.695672000\end{array}$

$\begin{array}{lll}-4.402793000 & 0.506347000 & -0.062810000\end{array}$

$\begin{array}{lll}3.548899000 & -3.051146000 & 1.659864000\end{array}$

$\begin{array}{lll}3.994412000 & -2.228523000 & 2.230960000\end{array}$

$\begin{array}{lll}-3.957063000 & 5.349868000 & 0.802284000\end{array}$

$-3.629838000 \quad-4.698442000 \quad-0.175858000$

$\begin{array}{lll}-4.595910000 & -2.052061000 & -0.148323000\end{array}$

$\begin{array}{lll}2.205508000 & -5.065276000 & 1.570706000\end{array}$

$\begin{array}{lll}1.645613000 & 0.889826000 & 2.363161000\end{array}$

$2.695773000 \quad 0.890578000 \quad 2.682318000$

$3.222408000 \quad-4.205155000 \quad-0.449249000$

$3.399133000 \quad-4.274282000 \quad-1.530293000$

$\begin{array}{lll}-5.019929000 & 1.789405000 & -0.075678000\end{array}$

$\begin{array}{lll}-3.660993000 & -2.488427000 & 0.815312000\end{array}$

$-3.324904000 \quad-1.798018000 \quad 1.596213000$

$\begin{array}{lll}-4.606136000 & 4.102267000 & 0.823594000\end{array}$

$\begin{array}{lll}-5.398485000 & 3.917240000 & 1.560215000\end{array}$

$\begin{array}{lll}4.712078000 & -2.179094000 & -0.422356000\end{array}$

$\begin{array}{lll}-4.252978000 & 3.074076000 & -0.078853000\end{array}$

$\begin{array}{lll}5.588280000 & 0.086701000 & -0.894645000\end{array}$

$\begin{array}{lll}2.757107000 & -4.005948000 & 2.320667000\end{array}$

$\begin{array}{lll}-6.430239000 & 1.857415000 & -0.134766000\end{array}$

$-6.902943000 \quad 2.845393000 \quad-0.165309000$

$\begin{array}{lll}4.644389000 & -0.763105000 & -0.240797000\end{array}$

$\begin{array}{lll}-0.668901000 & 1.397775000 & 2.748376000\end{array}$

$\begin{array}{lll}-1.471989000 & 1.805373000 & 3.378034000\end{array}$

$\begin{array}{lll}-6.590214000 & -0.554876000 & -0.201944000\end{array}$

$\begin{array}{lll}-7.193251000 & -1.469084000 & -0.234657000\end{array}$

$\begin{array}{lll}6.618263000 & -1.878539000 & -1.938271000\end{array}$

$\begin{array}{lll}7.390196000 & -2.310785000 & -2.584646000\end{array}$

$\begin{array}{lll}0.646738000 & 1.428684000 & 3.210035000\end{array}$

$2.423690000 \quad-5.176393000 \quad 0.184964000$

$\begin{array}{lll}6.552888000 & -0.492740000 & -1.744404000\end{array}$

$\begin{array}{lll}7.275679000 & 0.166702000 & -2.238473000\end{array}$

$\begin{array}{lll}-7.212468000 & 0.699278000 & -0.195889000\end{array}$

$\begin{array}{lll}-8.304536000 & 0.773188000 & -0.245368000\end{array}$

$\begin{array}{lll}2.493442000 & -3.893172000 & 3.810089000\end{array}$

$3.349841000 \quad-3.439314000 \quad 4.339564000$

$\begin{array}{lll}2.296671000 & -4.881005000 & 4.263367000\end{array}$

$\begin{array}{lll}1.610787000 & -3.256426000 & 4.014331000\end{array}$

$\begin{array}{lll}-5.038267000 & -2.974460000 & -1.123686000\end{array}$

$\begin{array}{lll}-5.753402000 & -2.641277000 & -1.886096000\end{array}$

$\begin{array}{lll}5.708470000 & -2.707532000 & -1.273927000\end{array}$

$\begin{array}{lll}5.774564000 & -3.795964000 & -1.385352000\end{array}$

$\begin{array}{lll}-4.566400000 & -4.299393000 & -1.149709000\end{array}$

$\begin{array}{lll}5.563346000 & 1.573810000 & -0.749178000\end{array}$

$\begin{array}{lll}-4.357659000 & 6.452914000 & 1.762751000\end{array}$

$-4.971104000 \quad 7.224237000 \quad 1.257882000$

$\begin{array}{lll}-3.473452000 & 6.967896000 & 2.180419000\end{array}$

$\begin{array}{lll}-4.952053000 & 6.059942000 & 2.605939000\end{array}$

$\begin{array}{lll}-2.150216000 & -4.253719000 & 1.843920000\end{array}$

$\begin{array}{lll}-2.219594000 & -3.651122000 & 2.766484000\end{array}$

$\begin{array}{lll}-1.116458000 & -4.147424000 & 1.462072000\end{array}$

$\begin{array}{lll}-2.289774000 & -5.314806000 & 2.117915000\end{array}$

$\begin{array}{lll}5.702669000 & 3.794324000 & -1.800543000\end{array}$

$\begin{array}{lll}1.805067000 & -6.308250000 & -0.613398000\end{array}$

$2.531446000 \quad-6.753297000 \quad-1.317838000$

$\begin{array}{lll}0.946162000 & -5.956112000 & -1.216912000\end{array}$

$\begin{array}{lll}1.436502000 & -7.113127000 & 0.046421000\end{array}$

$5.509405000 \quad 2.198509000 \quad 0.518662000$

$\begin{array}{lll}5.430692000 & 1.575131000 & 1.415217000\end{array}$

$\begin{array}{lll}5.652005000 & 4.381016000 & -0.525275000\end{array}$

$\begin{array}{lll}5.658444000 & 2.388113000 & -1.895760000\end{array}$

$\begin{array}{lll}5.679435000 & 1.913819000 & -2.885422000\end{array}$

$\begin{array}{lll}-2.934319000 & 5.558135000 & -0.144754000\end{array}$

$\begin{array}{lll}-3.227197000 & 3.316894000 & -1.015218000\end{array}$

$\begin{array}{lll}-2.953161000 & 2.527284000 & -1.725771000\end{array}$

$\begin{array}{lll}-2.558319000 & 4.555433000 & -1.058362000\end{array}$ 


$\begin{array}{lrrr}\mathrm{C} & 1.005966000 & 2.012671000 & 4.561528000 \\ \mathrm{H} & 1.506642000 & 1.266817000 & 5.207434000 \\ \mathrm{H} & 0.108865000 & 2.369802000 & 5.096423000 \\ \mathrm{H} & 1.699639000 & 2.868933000 & 4.462762000 \\ \mathrm{C} & 5.808008000 & 4.647358000 & -3.050360000 \\ \mathrm{H} & 5.037963000 & 4.372672000 & -3.795097000 \\ \mathrm{H} & 6.790754000 & 4.523516000 & -3.544861000 \\ \mathrm{H} & 5.686156000 & 5.719737000 & -2.818337000 \\ \mathrm{C} & 5.556895000 & 3.595656000 & 0.644169000 \\ \mathrm{C} & -5.071932000 & -5.284075000 & -2.186855000 \\ \mathrm{H} & -4.271894000 & -5.969535000 & -2.519482000 \\ \mathrm{H} & -5.469668000 & -4.766373000 & -3.077213000 \\ \mathrm{H} & -5.888231000 & -5.912734000 & -1.780503000 \\ \mathrm{C} & 5.504523000 & 4.257126000 & 2.008260000 \\ \mathrm{H} & 6.357526000 & 4.945073000 & 2.159577000 \\ \mathrm{H} & 5.527487000 & 3.509224000 & 2.819596000 \\ \mathrm{H} & 4.582520000 & 4.857143000 & 2.131990000 \\ \mathrm{C} & -1.441130000 & 4.791549000 & -2.056185000 \\ \mathrm{H} & -1.676859000 & 4.346500000 & -3.039626000 \\ \mathrm{H} & -0.493737000 & 4.333772000 & -1.711965000 \\ \mathrm{H} & -1.251829000 & 5.868832000 & -2.206012000 \\ \mathrm{H} & -3.208854000 & -0.391856000 & -2.191761000 \\ \mathrm{C} & -1.017045000 & -0.340541000 & -3.261899000 \\ \mathrm{C} & -1.169452000 & -1.638268000 & -2.772350000 \\ \mathrm{H} & -0.083227000 & 0.207284000 & -3.092587000 \\ \mathrm{H} & -1.703300000 & 0.068553000 & -4.011401000 \\ \mathrm{H} & -0.358793000 & -2.115099000 & -2.210609000 \\ \mathrm{H} & -1.988720000 & -2.282859000 & -3.107687000 \\ \mathrm{H} & 5.684359000 & 5.475777000 & -0.436789000 \\ \mathrm{H} & 1.597209000 & -5.824471000 & 2.081143000 \\ \mathrm{H} & -3.252656000 & -5.730248000 & -0.185623000 \\ \mathrm{H} & -2.415658000 & 6.526133000 & -0.170006000\end{array}$

\section{${ }^{M e}$ TS-4}

Zero-point correction $=0.905901($ Hartree $/$ Particle $)$

Thermal correction to Energy=

Thermal correction to Enthalpy=

0.966322

Thermal correction to Gibbs Free Energy $=\quad 0.799148$

Sum of electronic and zero-point Energies $=\quad-2481.625651$

Sum of electronic and thermal Energies $=\quad-2481.565231$

Sum of electronic and thermal Enthalpies $=\quad-2481.564287$

Sum of electronic and thermal Free Energies $=-2481.732405$

E solvent $=-2481.405251$

$\begin{array}{lrrr}\mathrm{Ni} & -1.946791000 & -0.322347000 & -1.399620000 \\ \mathrm{~N} & -2.948878000 & 0.408625000 & 0.044715000 \\ \mathrm{O} & -0.256654000 & -0.228012000 & -0.520867000 \\ \mathrm{~N} & 3.686453000 & -0.167325000 & 0.592520000 \\ \mathrm{C} & -1.003622000 & 0.895601000 & 1.488654000 \\ \mathrm{C} & 3.808955000 & -3.130392000 & 0.281494000 \\ \mathrm{C} & 1.376836000 & 0.360481000 & 1.104829000 \\ \mathrm{C} & 0.007405000 & 0.318081000 & 0.631551000 \\ \mathrm{C} & -5.170384000 & -0.663198000 & -0.084781000 \\ \mathrm{C} & -3.085930000 & -3.771199000 & 0.788627000 \\ \mathrm{C} & -2.389577000 & 0.895726000 & 1.140904000 \\ \mathrm{H} & -3.072399000 & 1.337271000 & 1.885828000 \\ \mathrm{C} & 2.433977000 & -0.204229000 & 0.261317000 \\ \mathrm{H} & 2.097107000 & -0.660351000 & -0.686819000 \\ \mathrm{C} & -4.384683000 & 0.523630000 & -0.031020000 \\ \mathrm{C} & 3.577513000 & -3.032903000 & 1.673459000 \\ \mathrm{H} & 4.023671000 & -2.200827000 & 2.229947000 \\ \mathrm{C} & -3.948796000 & 5.387646000 & 0.751536000 \\ \mathrm{C} & -3.581183000 & -4.671148000 & -0.176344000 \\ \mathrm{C} & -4.581252000 & -2.036294000 & -0.106349000 \\ \mathrm{C} & 2.238065000 & -5.050978000 & 1.619958000 \\ \mathrm{C} & 1.673046000 & 0.939217000 & 2.345810000 \\ \mathrm{H} & 2.726996000 & 0.946105000 & 2.652147000 \\ \mathrm{C} & 3.230647000 & -4.208615000 & -0.420024000 \\ \mathrm{H} & 3.391468000 & -4.287074000 & -1.502976000 \\ \mathrm{C} & -5.001736000 & 1.807394000 & -0.055196000 \\ \mathrm{C} & -3.595059000 & -2.458891000 & 0.812164000 \\ \mathrm{H} & -3.227608000 & -1.762157000 & 1.572217000 \\ \mathrm{C} & -4.597735000 & 4.141282000 & 0.794120000 \\ \mathrm{H} & -5.397579000 & 3.973304000 & 1.526730000 \\ \mathrm{C} & 4.712581000 & -2.177137000 & -0.431461000 \\ \mathrm{C} & -4.234316000 & 3.091586000 & -0.080161000\end{array}$

\begin{tabular}{|c|c|c|}
\hline 578691000 & 0.086599000 & -0.930036000 \\
\hline 2.797036000 & -3.981682000 & 2.352973000 \\
\hline 6.412573000 & 1.875285000 & -0.103951000 \\
\hline-6.885241000 & 2.862924000 & -0.144418000 \\
\hline 4.643398000 & -0.759935000 & -0.259422000 \\
\hline-0.634513000 & 1.467784000 & 2.739238000 \\
\hline-1.429688000 & 1.898156000 & 3.364102000 \\
\hline-6.575449000 & -0.537274000 & -0.139936000 \\
\hline-7.180561000 & -1.450594000 & -0.155152000 \\
\hline 6.599942000 & -1.883833000 & -1.972389000 \\
\hline 7.365020000 & -2.319222000 & -2.624801000 \\
\hline 0.685780000 & 1.502854000 & 3.188783000 \\
\hline 2.443415000 & -5.177438000 & 0.234900000 \\
\hline 6.534325000 & -0.496833000 & -1.787065000 \\
\hline 7.250192000 & 0.160594000 & -2.293772000 \\
\hline-7.196428000 & 0.717380000 & -0.141781000 \\
\hline-8.288878000 & 0.791676000 & 444000 \\
\hline 2.549025000 & -3.857284000 & 19000 \\
\hline 3.282547000 & -3.183468000 & 4.319721000 \\
\hline 2.608676000 & -4.838326000 & 341000 \\
\hline 1.541989000 & -3.446318000 & 4.051578000 \\
\hline-5.060090000 & -2.968764000 & -1.054557000 \\
\hline-5.815327000 & -2.648426000 & 5000 \\
\hline 5.699953000 & -2.709685000 & -1.291 \\
\hline 5.767354000 & -3.798823000 & 86000 \\
\hline-4.573210000 & -4.288632000 & 58000 \\
\hline 5.554253000 & 1.574534000 & 975000 \\
\hline-4.347107000 & 40000 & 5000 \\
\hline-4.746885000 & 24000 & 3000 \\
\hline-3.482406000 & 6.871587000 & 1000 \\
\hline-5.123577000 & 6.181355000 & 92000 \\
\hline-2.012538000 & -4.200708000 & 324000 \\
\hline-2.022063000 & -3.572 & 18000 \\
\hline-1.001869000 & -4.116377000 & 58000 \\
\hline & -5.2 & 2.079876000 \\
\hline 5.678480000 & 3.789354000 & 00000 \\
\hline 1.837294000 & -6.329 & 42000 \\
\hline 2.616456000 & -6.936104000 & -1.043265000 \\
\hline 1.155596000 & -5.9 & 19000 \\
\hline 1.260166000 & -7.002498000 & 3000 \\
\hline 5.517382000 & 2.206150000 & 0.472101000 \\
\hline 5.450692000 & 12000 & 69000 \\
\hline 5.645329000 & 1000 & -0.5 \\
\hline 5.633502000 & 2.382587000 & 73000 \\
\hline & & \\
\hline-2.917907000 & 06000 & 96000 \\
\hline-3.198933000 & 3.313857000 & -1.010438000 \\
\hline-2.912808000 & 2.508088000 & 861000 \\
\hline-2.531345000 & 4.552516000 & 391000 \\
\hline 1.059625000 & 2.118864000 & 067000 \\
\hline 1.551553000 & 1.383927000 & 95000 \\
\hline 0.170129000 & 2.504223000 & 5.050192000 \\
\hline 1.765569000 & 2.961624000 & 4.397356000 \\
\hline 5.764894000 & 4.635670000 & -3.113926000 \\
\hline 4.976331000 & 9000 & 5000 \\
\hline 6.735476000 & 4.500394000 & -3.628987000 \\
\hline 5.657779000 & 5.710149000 & -2.884338000 \\
\hline 5.566454000 & 3.603903000 & 0.589367000 \\
\hline-5.121873000 & -5.283647000 & -2.105322000 \\
\hline-4.352089000 & -6.011539000 & -2.417560000 \\
\hline-5.502056000 & -4.778715000 & -3.010805000 \\
\hline-5.962718000 & -5.863745000 & -1.677490000 \\
\hline 5.533144000 & 4.272844000 & 1.950417000 \\
\hline 6.392863000 & 4.955104000 & 2.089391000 \\
\hline 5.558905000 & 3.529017000 & 2.765400000 \\
\hline 4.617029000 & 4.880460000 & 2.080382000 \\
\hline-1.406798000 & 4.765617000 & -2.071100000 \\
\hline-1.653156000 & 4.334460000 & -3.058323000 \\
\hline-0.473321000 & 4.278551000 & -1.729486000 \\
\hline-1.188201000 & 5.838399000 & -2.212614000 \\
\hline-3.217858000 & -0.303656000 & -2.137794000 \\
\hline-0.886511000 & -0.974749000 & -3.028494000 \\
\hline-2.103005000 & -1.666690000 & -2.965084000 \\
\hline 0.014516000 & -1.364503000 & -2.547238000 \\
\hline-0.747650000 & -0.161196000 & -3.751288000 \\
\hline-2.194645000 & -2.607547000 & -2.406860000 \\
\hline 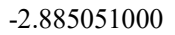 & 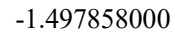 & 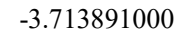 \\
\hline
\end{tabular}




$\begin{array}{rrrr}\mathrm{H} & 5.678481000 & 5.478209000 & -0.503402000 \\ \mathrm{H} & 1.633976000 & -5.804056000 & 2.144361000 \\ \mathrm{H} & -3.190001000 & -5.697344000 & -0.203615000 \\ \mathrm{H} & -2.401572000 & 6.544687000 & -0.236490000\end{array}$

\section{Me1-Transf-2}

Zero-point correction= 0.908498 (Hartree/Particle)

$\begin{array}{lc}\text { Thermal correction to Energy }= & 0.969354 \\ \text { Thermal correction to Enthalpy }= & 0.970298\end{array}$

Thermal correction to Enthalpy $=\quad 0.970298$
Thermal correction to Gibbs Free Energy $=0.801222$

Sum of electronic and zero-point Energies $=-2481.640859$

Sum of electronic and thermal Energies $=\quad-2481.580003$

Sum of electronic and thermal Enthalpies $=\quad-2481.579059$

Sum of electronic and thermal Free Energies $=-2481.748135$

E solvent $=-2481.42507582$

C $\quad 4.931002000$

C 4.635895000

C $\quad 5.133861000$

$\mathrm{H} \quad 5.860238000$

C $\quad 3.244573000$

C $\quad 4.130807000$

C $\quad 3.761597000$

C 4.353862000

C 4.710052000

C $\quad 5.174611000$

C $\quad 4.545756000$

H $\quad 5.407313000$

C 2.752404000

C $\quad 3.691253000$

$\mathrm{H} \quad 3.309899000$

C $\quad 3.868317000$

C 6.331961000

$\mathrm{H} \quad 6.770487000$

C $\quad 2.311848000$

C 7.148084000

H 8.232672000

C 3.010471000

H 2.673039000

C $\quad 6.569348000$

$\mathrm{H} \quad 7.203029000$

N $\quad-3.697309000$

C $\quad-4.737127000$

C $\quad-5.521091000$

C $\quad-5.486456000$

H $\quad-5.434704000$

C $\quad-5.607299000$

C $\quad-3.856101000$

C $\quad-5.577604000$

C $\quad-4.649915000$

C $\quad-5.518471000$

C $\quad-5.563723000$

C $\quad-3.644692000$

H -4.089410000

C $\quad-2.327573000$

C $\quad-5.580091000$

$\mathrm{H} \quad-5.585585000$

C $\quad-2.885524000$

C $\quad-5.719265000$

$\mathrm{H} \quad-5.799673000$

C $\quad-2.512587000$

C $\quad-6.598345000$

$\mathrm{H} \quad-7.360286000$

C $\quad-3.279741000$

$\mathrm{H} \quad-3.424609000$

C $\quad-6.516042000$

$\mathrm{H} \quad-7.216150000$

$\mathrm{Ni} \quad 1.880626000$
0.394936000

1.861846000

$-1.998713000$

$-2.930484000$

$-2.594595000$

$-3.784957000$

3.125346000

$-4.681136000$

0.556974000

$-4.270909000$

$-0.603743000$

4.168662000

4.005489000

5.579480000

$-2.448343000$

$-1.755497000$

5.399190000

1.975047000

2.977476000

4.563371000

0.843580000

0.953986000

3.339754000

2.537674000

$-0.430067000$

$-0.195782000$

$-2.199259000$

1.559240000

2.202641000

3.765372000

$-3.155026000$

4.370719000

$-0.781387000$

3.601888000

0.070480000

$-3.043313000$

$-2.199409000$

$-5.076513000$

2.357326000

1.868437000

$-3.992670000$

$-2.727934000$

$-3.817177000$

$-5.216854000$

$-1.897503000$

$-2.329651000$

$-4.248144000$

$-4.337335000$

$-0.509599000$

0.151769000

$-0.360966000$
$-1.321662000$

1.592042000
0.091913000

$-0.091281000$

$-0.160022000$

$-1.103120000$

$-1.854143000$

0.801670000

$-0.031534000$

$-0.152168000$

$-0.046636000$

$-0.169979000$

0.828751000

1.488685000

0.025627000

0.788026000

1.544341000

0.868765000

$-0.235145000$

$-0.291476000$

$-0.841482000$

$-0.341993000$

$-0.451385000$

$-0.859242000$

$-1.527624000$

$-0.314184000$

$-0.453708000$

$-0.867905000$

0.391411000

1.298669000

$-1.954760000$

0.283673000

$-0.687685000$

$-0.297337000$

0.495109000

$-0.990269000$

1.677566000

2.217141000

1.668680000

$-2.028128000$

$-3.010868000$

2.380236000

$-1.321338000$

$-1.414408000$

0.282160000

$-2.024314000$

$-2.682547000$

$-0.396036000$

$-1.480392000$

$-1.853421000$

$-2.376761000$

$-1.328092000$
$-1.111015000$

$-0.374665000$

0.564174000

$-2.442591000$

$-2.098623000$

2.379697000

3.045481000

0.985450000

0.600549000

$-0.020681000$

1.390595000

$-0.722871000$

$-1.392682000$

$-1.105823000$

$-1.701828000$

$-2.758624000$

0.220767000

$-1.817192000$

$-1.594826000$

$-0.220825000$

2.210256000

$-5.597739000$

3.420228000

$-1.738703000$

2.966376000

1.209100000

2.668360000

0.764495000

0.550561000

3.268723000

3.174055000

$-1.903675000$

$-1.356742000$

$-2.677658000$

$-1.192865000$

$-2.657575000$

$-3.411067000$

$-2.701481000$

$-1.662474000$

5.268151000

5.935150000

4.463523000

5.854344000

2.209652000

1.182234000

2.310381000

2.298299000

1.101662000

1.335058000

0.270742000

0.731137000

$-5.668838000$

$-4.853365000$

$-6.620212000$

$-5.586300000$

$-5.488792000$

$-6.345895000$

$-5.522391000$

$-4.570376000$

4.324581000

3.487626000

5.112477000

4.733353000

$-0.232363000$ $-0.679381000$ 0.874887000 1.332933000 0.843476000 1.383833000 0.292913000 1.798924000 1.408612000 0.319693000 1.991021000 0.866336000 0.862240000 $-0.220704000$ 2.831557000 1.237640000 2.370155000 6.534456000 5.467051000 $-5.725245000$ $-5.829212000$ $-0.325823000$ $-1.098707000$ $-1.030076000$ $-2.089405000$ $-0.395365000$ $-0.591056000$ $-2.003493000$ $-6.382337000$

$-7.061475000$ $-6.978183000$ $-6.036065000$ $-3.851861000$ $-3.190710000$ $-4.830160000$ $-3.416609000$ $-5.259619000$ $-5.996218000$

$-5.834225000$

$-4.751917000$

$-4.238284000$

$-4.075687000$

$-5.314289000$

$-3.678344000$

4.766268000

4.542052000

4.098182000

5.804602000

4.600677000

4.337965000

4.444980000 
Sum of electronic and thermal Free Energies $=-2599.554626$ E solvent $=-2599.25682447$

\begin{tabular}{|c|c|c|c|}
\hline & -1.121501000 & & \\
\hline & 1000 & 4000 & 3000 \\
\hline & 3000 & 000 & 0 \\
\hline & 2000 & 0000 & 6000 \\
\hline & -0.849768000 & -0.3099 & 5000 \\
\hline & 0.178441000 & 0.286209000 & 689000 \\
\hline & -0.046299000 & -0.009966000 & -0.470037000 \\
\hline & -3.422847000 & 0.947796000 & 6271000 \\
\hline & -3.846345000 & 0.140184000 & 351000 \\
\hline & -4.282593000 & 1000 & \\
\hline & -4.358097000 & 5000 & \\
\hline & -5.30 & & \\
\hline & & & \\
\hline & -2.4 & & \\
\hline & -4.2 & & \\
\hline & -3.0 & & \\
\hline & -2.8 & & \\
\hline & -0.5 & -0.6 & 00 \\
\hline & -1.3 & & \\
\hline & 0.7 & -0 . & \\
\hline & 1.12 & 00 & \\
\hline & & & \\
\hline & 0.2 & -1. & \\
\hline & 1.9 & -0. & \\
\hline & 1.79 & & \\
\hline & 2.8 & & \\
\hline & 1.5 & & \\
\hline & 2.60 & & \\
\hline & 3.85 & & \\
\hline & 2.25 & -0.2 & 000 \\
\hline & 4.82 & -0.7 & \\
\hline & 4.87 & & \\
\hline & $5.8^{\prime}$ & & \\
\hline C & 6.80 & -0. & \\
\hline & & & \\
\hline & & & \\
\hline & & & \\
\hline & & & \\
\hline & & & \\
\hline & & & \\
\hline & 6.1 & & \\
\hline & 6.0 & & \\
\hline & 5.9 & & \\
\hline & 3.9 & 0 & \\
\hline & 3.6 & 0 & \\
\hline & 2.8 & & -0 . \\
\hline & 2.2 & 00 & -1 \\
\hline $\mathrm{C}$ & & & \\
\hline $\mathrm{C}$ & & & \\
\hline & & & 000 \\
\hline & & & 000 \\
\hline $\mathrm{C}$ & & & 000 \\
\hline $\mathrm{C}$ & 5.79 & & 000 \\
\hline$C$ & -4.91 & 2000 & 9000 \\
\hline $\mathrm{C}$ & -5.62 & 5000 & 1000 \\
\hline $\mathrm{C}$ & -5.66 & -1.7 & 26000 \\
\hline $\mathrm{C}$ & -5.0 & -1.3 & \\
\hline $\mathrm{C}$ & & & \\
\hline $\mathrm{C}$ & & & \\
\hline $\mathrm{C}$ & & & \\
\hline $\mathrm{C}$ & & & \\
\hline 0 & & & \\
\hline $\mathrm{C}$ & & & \\
\hline $\mathrm{C}$ & & 2.864816000 & 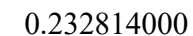 \\
\hline & & 2354233000 & 0.16857100 \\
\hline
\end{tabular}

C $\quad-4.057647000$

C -3.161589000

C $\quad-3.382705000$

C -2.418356000

C -1.199197000

C $\quad-0.944796000$

C -1.934370000

C $\quad 0.373461000$

C -2.680951000

$\mathrm{H} \quad-3.830280000$

$\mathrm{H} \quad-4.879061000$

$\mathrm{H} \quad-6.982805000$

$\mathrm{H} \quad-5.563447000$

$\mathrm{H} \quad-6.892036000$

H $\quad-6.196625000$

$\mathrm{H} \quad-7.297791000$

$\mathrm{H} \quad-7.679201000$

$\mathrm{H} \quad-5.733494000$

H $\quad-4.329019000$

$\mathrm{H} \quad-3.564409000$

$\mathrm{H} \quad-2.864635000$

$\mathrm{H} \quad-1.817435000$

$\mathrm{H} \quad-0.431083000$

$\mathrm{H} \quad 0.963344000$

$\mathrm{H} \quad 0.988772000$

$\mathrm{H} \quad 0.218906000$

$\mathrm{H} \quad 1.623700000$

$\mathrm{H} \quad 2.578909000$

$\mathrm{H} \quad 0.943814000$

$\mathrm{H} \quad 1.936541000$

$\mathrm{H} \quad 3.569296000$

$\mathrm{H} \quad 3.446438000$

$\mathrm{H} \quad 1.865177000$

$\mathrm{H} \quad 2.016293000$

$\mathrm{H} \quad 5.920877000$

$\mathrm{H} \quad 7.578158000$

$\mathrm{H} \quad 7.507079000$

H $\quad 5.417267000$

$\mathrm{H} \quad 7.155140000$

$\mathrm{H} \quad 5.948186000$

$\mathrm{H} \quad 5.649705000$

$\mathrm{H} \quad 6.717101000$

$\mathrm{H} \quad 5.677293000$

$\mathrm{H} \quad 4.066000000$

$\mathrm{H} \quad-1.740660000$

$\mathrm{H} \quad 6.014935000$

H 4.949773000

C $\quad-3.495757000$

$\mathrm{H} \quad-3.771082000$

$\mathrm{H} \quad-3.683317000$

$\mathrm{H} \quad-2.404561000$

C $\quad-0.880977000$

C -2.264524000

$\mathrm{H} \quad-0.119316000$

$\mathrm{H} \quad-0.515927000$

$\mathrm{H} \quad-2.816954000$

$\mathrm{H} \quad-2.544548000$

0.967628000 3.313755000 4.280585000 5.249055000 5.244563000 4.307652000 3.351031000 4.305096000 6.284144000 $-1.896063000$ $-5.090418000$ $-4.448825000$ $-3.953872000$ $-2.796938000$ $-1.051715000$ 0.047536000 2.468950000 3.934804000 4.263605000 6.022686000 7.284066000 6.386306000 5.989966000 5.213062000 3.430560000 4.249368000 $-6.075919000$ $-5.741438000$ $-5.326224000$ $-4.105120000$ $-2.759004000$ $-5.953169000$ $-6.439694000$ $-4.937762000$ $-2.476452000$ $-0.605039000$ 1.153702000 5.480332000 5.425883000 2.982847000 0.070525000 2.136326000 0.726700000 $-3.331715000$ 2.640109000 4.275206000 2.322970000 $-4.586309000$ $-5.630160000$ $-4.417444000$ $-4.488727000$ $-0.813553000$ $-1.218666000$ $-1.442879000$ $-0.150687000$ $-1.268852000$ $-2.058173000$

0.443578000 $-0.164446000$ $-1.177692000$ $-1.501481000$ $-0.792937000$ 0.223420000 0.530021000 0.971586000 $-2.578290000$ $-0.524091000$ 2.192126000 3.800931000 4.748522000 4.472144000 2.990299000 1.098881000 0.596264000 0.056165000 $-1.731615000$ $-3.186511000$ $-2.139795000$ $-3.260646000$ $-1.039352000$ 0.757664000 0.689085000 2.064717000 $-1.689233000$ $-4.605695000$ $-4.043671000$ $-4.890073000$ $-3.664386000$ 1.325761000 0.657633000 1.611681000 $-3.249189000$ $-3.302154000$ $-1.520474000$ $-0.183398000$ 0.183938000 $-0.949821000$ 2.228027000 4.862387000 4.521421000 0.591395000 1.337920000 3.174992000 4.850758000 $-0.120769000$ 0.109835000 $-1.197079000$ 0.037942000 $-2.882460000$ $-2.896545000$ $-2.410592000$ $-3.679584000$ $-3.842618000$ $-2.240433000$

\section{Me TS-1}

Zero-point correction= Thermal correction to Energy= Thermal correction to Enthalpy= 0.932892 (Hartree/Particle)

Thermal correction to Gibbs Free Energy $=\quad 0.828608$ Sum of electronic and zero-point Energies $=\quad-2520.903663$ Sum of electronic and thermal Energies $=\quad-2520.842689$ Sum of electronic and thermal Enthalpies $=\quad-2520.841744$ Sum of electronic and thermal Free Energies $=-2521.007947$ E solvent $=-2520.69489719$ 


\begin{tabular}{|c|c|c|c|}
\hline & t & 0 & \\
\hline & -3.252770000 & 0.928770000 & -2.630372000 \\
\hline & -2.865932000 & 0.522078000 & 0.290437000 \\
\hline & -2.372207000 & -0.093609000 & 08000 \\
\hline- & -1.046679000 & -0.615147000 & 56000 \\
\hline $\mathrm{C}$ & 0.047460000 & 821000 & 7000 \\
\hline U & -0.071966000 & 0.438384000 & 53000 \\
\hline$C$ & -2.202057000 & 0.126786000 & 17000 \\
\hline $\mathrm{H}$ & -0.839539000 & 1.409152000 & 17000 \\
\hline $\mathrm{C}$ & -2.138796000 & -1.380385000 & 49000 \\
\hline $\mathrm{H}$ & -1.095893000 & -1.734093000 & -2.945086000 \\
\hline & -2.695159000 & -1.750243000 & -2.143850000 \\
\hline 1 & -2.578846000 & -1.842776000 & -3.930103000 \\
\hline $\mathrm{H}$ & -1.609454000 & 0000 & 07000 \\
\hline $\mathrm{H}$ & -4.122784000 & 2000 & 3000 \\
\hline H & -3.398911000 & 1.9 & 9000 \\
\hline 1 & -3.028109 & -0.1 & 1000 \\
\hline C & -0.7 & -1.3 & 6000 \\
\hline$H$ & -1.651088 & -1.5 & 8000 \\
\hline $\mathrm{C}$ & 0.46 & -1.8 & 000 \\
\hline $\mathrm{C}$ & 0.69 & -2.7 & 000 \\
\hline & 1.0 & -3.7 & 000 \\
\hline & -0.2 & -2.8 & 000 \\
\hline & 1.48 & -2.2 & 0000 \\
\hline & 1.52 & -1.5 & 000 \\
\hline & 2.5 & -1.5 & 3000 \\
\hline & 1.3 & -0 . & \\
\hline & 2.49 & -0. & 000 \\
\hline $\mathrm{N}$ & 3.70 & -0.8 & 3000 \\
\hline $\mathrm{H}$ & 2.26 & & 6000 \\
\hline $\mathrm{C}$ & 4.743928000 & -0.5 & 15000 \\
\hline $\mathrm{C}$ & 4.77 & & 2000 \\
\hline $\mathrm{C}$ & 5.85 & -0.8 & 3000 \\
\hline $\mathrm{C}$ & 6.876423000 & & 3000 \\
\hline $\mathrm{C}$ & 6.845826000 & & 5000 \\
\hline C & 5.804308 & & 1000 \\
\hline $\mathrm{C}$ & 5.826864000 & & 39000 \\
\hline C & 5.641356000 & 7000 & 95000 \\
\hline $\mathrm{H}$ & 6.330770000 & & 7000 \\
\hline $\mathrm{C}$ & 6.005390000 & 2.0 & 9000 \\
\hline $\mathrm{C}$ & 6.503837000 & & 8000 \\
\hline $\mathrm{C}$ & 6.195070 & 2.8 & 0000 \\
\hline $\mathrm{C}$ & 6.102480 & & \\
\hline $\mathrm{C}$ & 3.7 & & 000 \\
\hline $\mathrm{C}$ & 3.30 & & \\
\hline $\mathrm{C}$ & 2.38 & & \\
\hline $\mathrm{C}$ & 1.8 & & -3 \\
\hline $\mathrm{C}$ & 2.31 & $-2.8+x-y$ & -4.2 \\
\hline I & 3.2 & -1.9 & -3.6 \\
\hline $\mathrm{C}$ & 1.7 & -2.6 & -5.6 \\
\hline $\mathrm{C}$ & 1.957029000 & 4000 & 37000 \\
\hline $\mathrm{C}$ & & & 06000 \\
\hline $\mathrm{C}$ & 578000 & -0.1 & 4.967565000 \\
\hline $\mathrm{C}$ & -5.268106000 & 28000 & 1.767094000 \\
\hline $\mathrm{C}$ & -4.817288000 & 4000 & 0.506014000 \\
\hline $\mathrm{C}$ & -4.804909000 & -2.07 & 63000 \\
\hline $\mathrm{C}$ & -5.226 & -1.1 & \\
\hline$C_{0}$ & -5.676149000 & -1.608354000 & 2.451309000 \\
\hline
\end{tabular}

\begin{tabular}{|c|c|c|c|}
\hline & 00 & 00 & \\
\hline & -4.332298000 & -4.451295000 & 29667000 \\
\hline & -5.283545000 & 2385000 & 0 \\
\hline 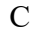 & -6.527390000 & 0.976296000 & \\
\hline C & -6.689798000 & 2.339069000 & 0.8208780 \\
\hline $\mathrm{C}$ & -5.603117000 & 3.078684000 & 28660 \\
\hline $\mathrm{C}$ & -4.337619000 & 2.487130000 & 50340 \\
\hline $\mathrm{C}$ & -4.172125000 & 1.100269000 & $+41 / 320$ \\
\hline $\mathrm{C}$ & -3.242407000 & 3.343695000 & -0.4127170 \\
\hline $\mathrm{C}$ & -3.547796000 & 4.262545000 & -1.4471000 \\
\hline $\mathrm{C}$ & -2.566513000 & 5.096648000 & -2.0075890 \\
\hline $\mathrm{C}$ & -1.243692000 & 5.001486000 & -1.5248810 \\
\hline $\mathrm{C}$ & -0.901243000 & 95000 & \\
\hline $\mathrm{C}$ & -1.910295000 & & \\
\hline $\mathrm{C}$ & & & \\
\hline $\mathrm{C}$ & & & \\
\hline $\mathrm{H}$ & -6. & -0.8 & \\
\hline$H$ & -5.2 & 00 & \\
\hline 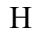 & -3.2 & 00 & -0 \\
\hline 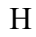 & & 00 & \\
\hline $\mathrm{H}$ & -4.5 & $-4 .($ & \\
\hline 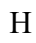 & -4 & -1.7 & \\
\hline 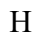 & & & \\
\hline $\mathrm{H}$ & -7.6 & & \\
\hline $\mathrm{H}$ & -5 . & & \\
\hline $\mathrm{H}$ & -4 & & -1 . \\
\hline $\mathrm{H}$ & -3 & 5.9 & \\
\hline $\mathrm{H}$ & -2.8 & 7.1 & -2 \\
\hline$U_{-}$ & -2.207 & & \\
\hline $\mathrm{H}$ & -0.4 & 00 & -1 . \\
\hline $\mathrm{H}$ & 1.20 & 4.6 & -0.5 \\
\hline $\mathrm{H}$ & $0.881^{7}$ & $2.9^{\prime}$ & -0.0 \\
\hline $\mathrm{H}$ & 0.5790 & 4.29 & 1.0 \\
\hline $\mathrm{H}$ & 1.172765000 & -4.65 & -3.8 \\
\hline $\mathrm{H}$ & 1.723318000 & -1.6 & -5 . \\
\hline $\mathrm{H}$ & & & \\
\hline $\mathrm{H}$ & & & -5.7 \\
\hline $\mathrm{H}$ & 3.544809000 & & -4 \\
\hline $\mathrm{H}$ & & & \\
\hline $\mathrm{H}$ & & & \\
\hline 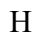 & & & \\
\hline $\mathrm{H}_{2}$ & & & \\
\hline & & & \\
\hline & & & \\
\hline F & & & \\
\hline $\mathrm{H}$ & & & 2.5 \\
\hline $\mathrm{H}$ & & & 0.5 \\
\hline $\mathrm{H}$ & & 00 & 2. \\
\hline & & & 4.7 \\
\hline & & & 5.4 \\
\hline $\mathrm{H}$ & 3.68 & -3.3 & -0.56673600 \\
\hline 11 & -1.65 & & 52600 \\
\hline 11 & 6.072478000 & 2.52 & 500 \\
\hline $\mathrm{H}$ & 1000 & 0.0 & 5. \\
\hline C & -6.215302000 & -3.475987000 & 4.09022700 \\
\hline 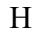 & -7.295053000 & -3.718061000 & 4.0 \\
\hline & -5.690684000 & -4.393194000 & 4.4116090 \\
\hline & -6.085064000 & -2.713802000 & 4.87848800 \\
\hline
\end{tabular}

\section{$6.3 .3 \mathbf{3}^{\mathrm{CF} 3}$}

\section{CF31-Allyl}

Zero-point correction $=\quad 0.735766($ Hartree/Particle $)$ Thermal correction to Energy $=\quad 0.812018$ Thermal correction to Enthalpy $=0.812962$ Thermal correction to Gibbs Free Energy $=0.606236$

Sum of electronic and zero-point Energies $=\quad-4901.706717$ Sum of electronic and thermal Energies $=\quad-4901.630465$ Sum of electronic and thermal Enthalpies $=\quad-4901.629521$ Sum of electronic and thermal Free Energies $=-4901.836247$ E solvent $=-4901.66506329$

$\mathrm{Ni} \quad 1.859553000 \quad 1.193398000 \quad 1.066690000$ 


\begin{tabular}{|c|c|c|c|}
\hline & & & \\
\hline & 5000 & 540000 & 3000 \\
\hline & 2413000 & 8611000 & 1000 \\
\hline & 010000 & 19000 & 28100 \\
\hline & 12000 & 88000 & 000 \\
\hline & 74000 & 000 & 1000 \\
\hline & 5000 & 6000 & \\
\hline & 6000 & 21000 & \\
\hline & 924346000 & 0000 & \\
\hline & 5000 & 8000 & \\
\hline & 3000 & 1000 & -2 \\
\hline & 0.318099000 & 6000 & -3 \\
\hline & 0.975106000 & -0.093004000 & -3.977238000 \\
\hline & -0.911878000 & -0.897660000 & -3.303798000 \\
\hline & -1.370869000 & -1.370432000 & -4.668516000 \\
\hline & -1.156621000 & -2.446697000 & -4.816134000 \\
\hline & -0.86 & -0.8 & \\
\hline & & & \\
\hline & & & \\
\hline & & & \\
\hline & -1 & -0 & \\
\hline & 00 & & \\
\hline & -3.3 & & \\
\hline & -1.8 & & \\
\hline & -4.0 & & \\
\hline & & & \\
\hline & & & \\
\hline & & -2 & \\
\hline & & & \\
\hline & & & \\
\hline & & & \\
\hline & & & \\
\hline & -83 & & \\
\hline & -72 & & \\
\hline & 7000 & & \\
\hline & -7.4 & & \\
\hline & 1000 & & \\
\hline & -2.156 & 74000 & \\
\hline & -1.64 & & \\
\hline & -0.35 & & \\
\hline & & & \\
\hline & & & \\
\hline & & & \\
\hline & & & \\
\hline & & & \\
\hline & -6.4 & & \\
\hline & & & \\
\hline & & & \\
\hline & & & \\
\hline & & & \\
\hline & & & -1 \\
\hline & & & \\
\hline & & & -2 \\
\hline & & & \\
\hline & & & \\
\hline $\mathrm{C}$ & & & -1.6 \\
\hline$C$ & & & -1.4 \\
\hline $\mathrm{C}$ & & 00 & $-1 .($ \\
\hline $\mathrm{C}$ & 3.97 & & -0 . \\
\hline $\mathrm{C}$ & 00 & 1 & -0 \\
\hline $\mathrm{C}$ & 2.8 & 0000 & -0.3 \\
\hline $\mathrm{C}$ & 3.09 & 5.2 & \\
\hline $\mathrm{C}$ & 2.07 & 6.062 & \\
\hline $\mathrm{C}$ & 0.77 & 5.9 & \\
\hline $\mathrm{C}$ & & & \\
\hline & & & \\
\hline & & & -0.847592000 \\
\hline & 2.397762000 & 7.128435000 & 2.17497600 \\
\hline
\end{tabular}

\begin{tabular}{cc} 
H & 5.876375000 \\
H & 5.155156000 \\
F & 3.095356000 \\
F & 3.862056000 \\
F & 5.174977000 \\
H & 4.134070000 \\
H & 7.100306000 \\
H & 7.265601000 \\
H & 5.276031000 \\
H & 4.101195000 \\
F & 3.321767000 \\
F & 2.918989000 \\
F & 1.294696000 \\
H & -0.028066000 \\
F & -1.585337000 \\
F & -1.600952000 \\
F & -0.931700000 \\
H & 1.457765000 \\
F & 0.150893000 \\
F & 1.553650000 \\
F & 1.774069000 \\
H & -1.705484000 \\
F & 1.065274000 \\
F & 0.863328000 \\
F & -0.797553000 \\
H & -3.974762000 \\
H & -6.337297000 \\
H & -7.279161000 \\
F & -7.925688000 \\
F & -9.614759000 \\
H & -6.979159000 \\
H & -5.243512000 \\
F & -5.026324000 \\
F & -6.334202000 \\
H & -2.264326000 \\
H & 1.302146000 \\
H & -7.660897000 \\
F & -7.185251000 \\
C & 6.190466000 \\
F & 7.534120000 \\
F & 5.643922000 \\
F & 6.016185000 \\
C & 1.281598000 \\
H & 1.626740000 \\
H & 0.228848000 \\
& \\
\hline
\end{tabular}

$-0.317087000$ $-4.188496000$ $-2.714263000$ $-4.548701000$ $-3.324710000$ $-0.578189000$ 1.313741000 3.801622000 5.117547000 5.312314000 6.688166000 8.241596000 7.518626000 6.594516000 5.975325000 3.944751000 4.276519000 $-4.609498000$ $-2.682293000$ $-4.223188000$ $-2.152726000$ $-2.249739000$ $-6.144464000$ $-4.362927000$ $-5.786335000$ $-3.267604000$ $-2.457503000$ $-1.325567000$ 2.981941000 1.884885000 0.822669000 $-2.110619000$ $-1.734667000$ $-0.164641000$ $-4.028092000$ 3.416688000 1.103802000 $-2.074456000$ $-2.985133000$ $-3.166171000$ $-4.211264000$ $-2.240240000$ 1.066162000 0.057446000 1.284418000

$-3.419960000$ $-1.622461000$ 1.392269000 0.450606000 1.712207000 0.532062000 $-1.917798000$ $-1.725119000$ $-0.988473000$ 1.113192000 3.077283000 1.583658000 2.869693000 1.006788000 $-0.869264000$ $-0.041633000$ $-2.105410000$ 2.705647000 5.983629000 5.279799000 4.602910000 4.485806000 0.758224000 $-0.504693000$ $-0.354534000$ 4.475985000 4.503830000 2.482873000 0.566302000 $-0.299327000$ 1.352101000 $-1.306718000$ $-3.768828000$ $-4.587340000$ 0.579991000 $-1.599996000$ $-2.910721000$ $-3.927393000$ $-3.848845000$ $-3.693693000$ $-4.073042000$ $-4.977494000$ 3.034902000 3.294646000 3.241816000

\section{$6.3 .4 \quad \mathbf{2}^{\text {CF3 }}$}

\section{CF31- $\beta-T$}

Zero-point correction= Thermal correction to Energy= 0.834487 Thermal correction to Enthalpy $=0.835431$

Thermal correction to Gibbs Free Energy $=0.625616$ Sum of electronic and zero-point Energies $=\quad-4902.882534$ Sum of electronic and thermal Energies $=\quad-4902.805498$ Sum of electronic and thermal Enthalpies $=\quad-4902.804553$ Sum of electronic and thermal Free Energies= -4903.014369 E solvent $=-4902.86138094$

$\begin{array}{lrrr}\mathrm{Ni} & 1.962278000 & 0.984154000 & 1.020989000 \\ \mathrm{C} & 3.349225000 & 1.616828000 & 2.167525000 \\ \mathrm{~N} & 2.593511000 & 1.404212000 & -0.668283000 \\ \mathrm{C} & 1.955858000 & 0.996910000 & -1.764102000 \\ \mathrm{C} & 0.703501000 & 0.319913000 & -1.856826000 \\ \mathrm{C} & -0.076025000 & -0.043177000 & -0.689558000 \\ \mathrm{O} & 0.334950000 & 0.176386000 & 0.524242000 \\ \mathrm{C} & 2.460132000 & 0.957967000 & 3.183924000 \\ \mathrm{H} & 1.480826000 & 0.573902000 & 2.659111000\end{array}$




\begin{tabular}{|c|c|c|c|}
\hline & & & \\
\hline & 000 & 09268000 & (1000 \\
\hline & 779574000 & -0.984629000 & 197172000 \\
\hline & 818663000 & 0.048813000 & \\
\hline & & 1.688836000 & \\
\hline & 340165000 & 0000 & 2.02 \\
\hline & 395956000 & & \\
\hline & 2.442641000 & & \\
\hline & 204251000 & & \\
\hline I & 6000 & & \\
\hline & .023643000 & 2000 & 200 \\
\hline & 545087000 & -0.902876000 & 900 \\
\hline & 465260000 & 1618000 & 2210 \\
\hline & .980425000 & 2575000 & 352570 \\
\hline & -2.611056000 & -0.629614000 & $-4.8569270 c$ \\
\hline & -1.785180000 & 3450000 & -2.22977800 \\
\hline & -2.767308000 & 282000 & \\
\hline & -1.3 & & \\
\hline & -2.204969000 & & \\
\hline & -3.3233 & & \\
\hline & -1.847884 & -0 . & \\
\hline & $-4.12-x-2 \cdot$ & & \\
\hline & -3.6 & & \\
\hline & -4.5 & -2. & \\
\hline & -5.8 & & \\
\hline & -6.3 & & \\
\hline & -5.4 & & \\
\hline & -6.0 & & \\
\hline & & & \\
\hline & & & \\
\hline & & & \\
\hline & -8.1 & & \\
\hline & -7.29 & & \\
\hline & -6.78 & & \\
\hline & -2.29 & & 2000 \\
\hline & -1.776291000 & & 2000 \\
\hline 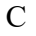 & -0.50 & & 1000 \\
\hline $\mathrm{C}$ & 0.273 & -4.4 & 38000 \\
\hline $\mathrm{C}$ & -0.241583000 & & 743000 \\
\hline$C$ & -1.5 & -3. & 7000 \\
\hline C & & & \\
\hline 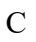 & & & \\
\hline $\mathrm{C}$ & & & \\
\hline & & & \\
\hline & & & \\
\hline & & & \\
\hline & & & \\
\hline C & & & \\
\hline $\mathrm{C}$ & & & \\
\hline $\mathrm{C}$ & & & \\
\hline $\mathrm{C}$ & 0 & & \\
\hline$C$ & & & \\
\hline C & & & \\
\hline $\mathrm{C}$ & & & 100 \\
\hline C & & & 00 \\
\hline 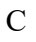 & & & 000 \\
\hline $\mathrm{C}$ & & & 13000 \\
\hline 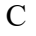 & & & 0000 \\
\hline 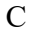 & & & 7000 \\
\hline 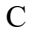 & 00 & & 000 \\
\hline 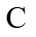 & 00 & & 000 \\
\hline $\mathrm{C}$ & 00 & 5.0 & 9000 \\
\hline$c$ & 000 & 4.2 & 262000 \\
\hline & -0.95 & & -0. \\
\hline & & & \\
\hline & & & Ecrop \\
\hline & & & \\
\hline & 2.804004000 & -3.049569000 & 0.75939600 \\
\hline
\end{tabular}

\begin{tabular}{cc} 
F & 4.356203000 \\
F & 4.785814000 \\
H & 4.181794000 \\
H & 7.024508000 \\
H & 7.185658000 \\
H & 5.221562000 \\
H & 4.133571000 \\
F & 3.452069000 \\
F & 2.940479000 \\
F & 1.403325000 \\
H & -0.004745000 \\
F & -1.683272000 \\
F & -1.565899000 \\
F & -1.031178000 \\
H & 1.268919000 \\
F & 0.112723000 \\
F & 1.877605000 \\
F & 0.437084000 \\
H & -1.889931000 \\
F & 0.987652000 \\
F & 0.650313000 \\
F & -0.926625000 \\
H & -4.188893000 \\
H & -6.518951000 \\
H & -7.345035000 \\
F & -7.753349000 \\
F & -9.467172000 \\
H & -6.953614000 \\
H & -5.188994000 \\
F & -5.271184000 \\
F & -5.218321000 \\
H & -2.369851000 \\
H & 1.222618000 \\
H & -7.429508000 \\
F & -7.104775000 \\
C & 6.007117000 \\
F & 7.359780000 \\
F & 5.465072000 \\
F & 5.761199000 \\
& \\
\hline
\end{tabular}

$-4.539090000$ $-2.862977000$ $-0.530708000$ 1.493703000 3.968242000 5.224330000 5.307388000 6.587453000 8.206804000 7.389460000 6.553865000 5.971423000 3.848875000 4.487629000 $-4.921705000$ $-4.500028000$ $-3.867359000$ $-2.372468000$ $-2.572437000$ $-6.258335000$ $-4.470909000$ $-5.981750000$ $-3.531307000$ $-2.629336000$ $-1.329836000$ 3.133536000 2.096103000 0.855006000 $-1.930804000$ $-1.833287000$ 0.294262000

$-4.131456000$ 3.526663000 1.475669000 $-0.812773000$ $-2.716985000$ $-2.883736000$ $-3.940655000$ $-1.926926000$

0.310327000 1.669416000 0.430136000 $-2.029707000$ $-1.694260000$ $-0.807838000$ 1.319282000 3.372313000 1.986086000 3.321494000 1.473518000 $-0.255862000$ 0.285403000 $-1.744055000$ 2.588224000 5.852541000 4.712443000 5.442071000 4.391242000 0.526900000 $-0.699069000$ $-0.521197000$ 4.252737000 4.259087000 2.289696000 0.614794000 $-0.273730000$ 1.314044000 $-1.481671000$ $-3.854517000$ $-4.395755000$ 0.384287000 $-1.352841000$ $-2.939730000$ $-4.514098000$ $-4.162179000$ $-4.089109000$ $-4.409911000$ $-5.245858000$

\section{${ }^{\text {CF3 TS-2 }}$ coor}

Zero-point correction $=\quad 0.808225($ Hartree/Particle $)$ Thermal correction to Energy= 0.889095 Thermal correction to Enthalpy $=0.890039$

Thermal correction to Gibbs Free Energy $=0.674048$ Sum of electronic and zero-point Energies $=\quad-4981.404547$ Sum of electronic and thermal Energies $=\quad-4981.323677$ Sum of electronic and thermal Enthalpies $=\quad-4981.322733$ Sum of electronic and thermal Free Energies $=-4981.538724$ E solvent $=-4981.40740381$

$\mathrm{Ni} \quad 1.952759000$

C $\quad 3.353103000$

$\mathrm{N} \quad 2.654158000$

C $\quad 1.914959000$

C $\quad 0.589416000$

C -0.114491000

O 0.374184000

C $\quad 2.920871000$

$\mathrm{H} \quad 1.889093000$

C $\quad 2.784248000$

$\mathrm{H} \quad 2.360439000$

$\mathrm{H} \quad 2.136414000$

$\mathrm{H} \quad 3.777271000$

$\mathrm{H} \quad 3.569178000$

$\mathrm{H} \quad 3.144957000$

$\mathrm{H} \quad 4.378720000$

$\mathrm{H} \quad 2.401179000$

C $\quad-0.017742000$
0.559652000 0.798509000 1.461482000 1.678571000 1.222186000 0.300071000 $-0.090182000$ $-0.510575000$ $-0.793298000$ $-0.492153000$ $-1.438424000$ 0.338188000 $-0.349367000$ $-1.343457000$ 1.694843000 0.822767000 2.251424000 1.644436000
$-1.441589000$ $-2.720597000$ 0.037898000 1.124275000 1.402327000 0.529663000 $-0.608184000$ $-3.352772000$ $-2.951235000$ $-4.890941000$ $-5.271685000$ $-5.222148000$ $-5.352182000$ $-3.029672000$ $-3.333793000$ $-2.325016000$ 1.930882000 2.620249000 


\begin{tabular}{|c|c|c|c|}
\hline $\mathrm{H}$ & 0.537164000 & 2.346586000 & 2000 \\
\hline $\mathrm{C}$ & -1.284533000 & 1.213785000 & 3.020470000 \\
\hline $\mathrm{C}$ & -1.925244000 & 1.686075000 & 4.310176000 \\
\hline $\mathrm{H}$ & -2.995633000 & 1.914466000 & 4.161404000 \\
\hline $\mathrm{H}$ & -1.432065000 & 2.593626000 & 4.699397000 \\
\hline $\mathrm{H}$ & -1.866762000 & 0.911686000 & 5.099241000 \\
\hline $\mathrm{C}$ & -1.958082000 & 0.303036000 & 2.174467000 \\
\hline $\mathrm{H}$ & -2.964097000 & -0.042233000 & 2.441522000 \\
\hline $\mathrm{C}$ & -1.410905000 & -0.171590000 & 0.973243000 \\
\hline $\mathrm{C}$ & -2.149830000 & -1.094189000 & 0.115270000 \\
\hline $\mathrm{N}$ & -3.331605000 & -1.542929000 & 0.415304000 \\
\hline $\mathrm{H}$ & -1.648049000 & -1.376692000 & -0.828615000 \\
\hline $\mathrm{C}$ & -3.992630000 & -2.411302000 & -0.472607000 \\
\hline $\mathrm{C}$ & -5.228915000 & -1.978478000 & -1.046155000 \\
\hline $\mathrm{C}$ & -5.889986000 & -2.816775000 & -1.965714000 \\
\hline $\mathrm{C}$ & -5.374275000 & -4.074307000 & -2.307075000 \\
\hline $\mathrm{C}$ & -4.186547000 & -4.514789000 & -1.712160000 \\
\hline $\mathrm{C}$ & -3.484716000 & -3.707157000 & -0.791087000 \\
\hline $\mathrm{C}$ & -2.265007000 & -4.265285000 & -0.135775000 \\
\hline $\mathrm{C}$ & -2.136878000 & -4.273815000 & 1.271171000 \\
\hline $\mathrm{C}$ & -0.002242000 & -5.440855000 & 1.113026000 \\
\hline $\mathrm{C}$ & -0.115804000 & -5.425237000 & -0.283104000 \\
\hline $\mathrm{C}$ & -1.240672000 & -4.853120000 & -0.904187000 \\
\hline $\mathrm{C}$ & -5.811287000 & -0.642531000 & -0.725518000 \\
\hline $\mathrm{C}$ & -5.888492000 & -0.172123000 & 0.604215000 \\
\hline $\mathrm{C}$ & -6.446338000 & 1.081893000 & 0.888992000 \\
\hline $\mathrm{C}$ & -6.953390000 & 1.892863000 & -0.140077000 \\
\hline $\mathrm{C}$ & -6.895369000 & 1.427005000 & -1.461180000 \\
\hline $\mathrm{C}$ & -6.327191000 & 0.174935000 & -1.752550000 \\
\hline $\mathrm{C}$ & -1.023472000 & -4.862531000 & 1.885163000 \\
\hline $\mathrm{C}$ & 1.551139000 & 6.591842000 & 0.107848000 \\
\hline $\mathrm{C}$ & 2.573435000 & 6.516042000 & 1.064603000 \\
\hline $\mathrm{C}$ & 3.459228000 & 5.427476000 & 1.071473000 \\
\hline $\mathrm{C}$ & 3.337315000 & 4.386787000 & 0.125011000 \\
\hline $\mathrm{C}$ & 2.301605000 & 4.464134000 & -0.828913000 \\
\hline $\mathrm{C}$ & 1.425112000 & 5.562168000 & -0.837222000 \\
\hline $\mathrm{C}$ & 4.353267000 & 3.290358000 & 0.124744000 \\
\hline $\mathrm{C}$ & 5.716811000 & 3.663497000 & 0.165954000 \\
\hline $\mathrm{C}$ & 6.735763000 & 2.706286000 & 0.185103000 \\
\hline $\mathrm{C}$ & 6.402158000 & 1.346293000 & 0.191905000 \\
\hline $\mathrm{C}$ & 5.056112000 & 0.928359000 & 0.168527000 \\
\hline $\mathrm{C}$ & 4.023060000 & 1.906620000 & 0.108146000 \\
\hline $\mathrm{C}$ & 4.764259000 & -0.536095000 & 0.214358000 \\
\hline $\mathrm{C}$ & 5.378478000 & -1.403283000 & -0.712580000 \\
\hline $\mathrm{C}$ & 5.136547000 & -2.786675000 & -0.671762000 \\
\hline $\mathrm{C}$ & 4.302630000 & -3.337094000 & 0.312660000 \\
\hline $\mathrm{C}$ & 3.723276000 & -2.483057000 & 1.263800000 \\
\hline $\mathrm{C}$ & 3.941402000 & -1.098077000 & 1.212032000 \\
\hline $\mathrm{H}$ & 2.195085000 & 3.669673000 & -1.574175000 \\
\hline $\mathrm{H}$ & 0.869544000 & 7.445516000 & 0.090137000 \\
\hline $\mathrm{H}$ & 4.254203000 & 5.383811000 & 1.823285000 \\
\hline $\mathrm{H}$ & 5.968052000 & 4.729930000 & 0.155130000 \\
\hline $\mathrm{H}$ & 7.785269000 & 3.018107000 & 0.203745000 \\
\hline $\mathrm{H}$ & 7.189221000 & 0.585536000 & 0.243916000 \\
\hline $\mathrm{H}$ & 6.036421000 & -0.990941000 & -1.484925000 \\
\hline $\mathrm{H}$ & 4.101380000 & -4.410647000 & 0.334039000 \\
\hline $\mathrm{H}$ & -7.368806000 & 2.879135000 & 0.082437000 \\
\hline $\mathrm{H}$ & -6.254529000 & -0.153185000 & -2.794393000 \\
\hline $\mathrm{H}$ & -6.839119000 & -2.480099000 & -2.398606000 \\
\hline $\mathrm{H}$ & -5.912117000 & -4.719272000 & -3.009814000 \\
\hline $\mathrm{H}$ & -3.800006000 & -5.516530000 & -1.933639000 \\
\hline $\mathrm{H}$ & -1.306926000 & -4.834135000 & -1.997437000 \\
\hline $\mathrm{H}$ & -2.913539000 & -3.807942000 & 1.885000000 \\
\hline $\mathrm{H}$ & -5.488501000 & -0.786521000 & 1.413255000 \\
\hline $\mathrm{H}$ & 3.481583000 & -0.456192000 & 1.968318000 \\
\hline $\mathrm{H}$ & 0.882906000 & -5.861971000 & 1.593407000 \\
\hline $\mathrm{C}$ & -0.314781000 & 0.767817000 & -3.540397000 \\
\hline $\mathrm{C}$ & 0.097088000 & 1.934853000 & -3.005318000 \\
\hline
\end{tabular}

$\begin{array}{crrr}\text { H } & -0.873227000 & 0.037760000 & -2.945389000 \\ \text { H } & -0.114467000 & 0.507587000 & -4.586343000 \\ \text { H } & 0.627589000 & 2.688077000 & -3.598472000 \\ \text { H } & -0.166073000 & 2.217827000 & -1.981381000 \\ \text { C } & 5.732892000 & -3.664598000 & -1.748426000 \\ \text { F } & 5.751102000 & -4.975569000 & -1.393944000 \\ \text { F } & 5.017241000 & -3.569926000 & -2.912373000 \\ \text { F } & 7.011485000 & -3.294269000 & -2.044921000 \\ \text { C } & 2.698755000 & 7.580760000 & 2.132732000 \\ \text { F } & 2.091683000 & 7.193260000 & 3.291706000 \\ \text { F } & 4.004555000 & 7.833189000 & 2.434189000 \\ \text { F } & 2.127179000 & 8.754792000 & 1.750072000 \\ \text { C } & 0.316290000 & 5.613508000 & -1.864984000 \\ \text { F } & -0.696428000 & 4.754948000 & -1.545232000 \\ \text { F } & -0.227185000 & 6.854475000 & -1.974966000 \\ \text { F } & 0.767597000 & 5.250542000 & -3.102819000 \\ \text { C } & -7.484221000 & 2.259924000 & -2.577468000 \\ \text { F } & -8.791830000 & 1.936060000 & -2.803186000 \\ \text { F } & -7.447578000 & 3.591642000 & -2.290863000 \\ \text { F } & -6.821668000 & 2.072513000 & -3.754448000 \\ \text { C } & -6.545606000 & 1.540728000 & 2.326235000 \\ \text { F } & -5.498924000 & 1.081455000 & 3.080452000 \\ \text { F } & -6.555478000 & 2.899012000 & 2.427882000 \\ \text { F } & -7.684529000 & 1.085299000 & 2.922683000 \\ \text { C } & 0.970309000 & -6.018232000 & -1.150391000 \\ \text { F } & 0.565740000 & -7.176834000 & -1.745131000 \\ \text { F } & 1.325205000 & -5.164851000 & -2.156181000 \\ \text { F } & 2.101877000 & -6.303447000 & -0.440914000 \\ \text { C } & -0.952789000 & -4.915155000 & 3.395594000 \\ \text { F } & 0.326601000 & -5.040929000 & 3.845401000 \\ \text { F } & -1.484330000 & -3.797623000 & 3.965078000 \\ \text { F } & -1.653924000 & -5.981192000 & 3.884539000 \\ \text { C } & 2.811535000 & -3.033622000 & 2.338416000 \\ \text { F } & 2.969281000 & -4.377698000 & 2.498491000 \\ \text { F } & 3.048801000 & -2.443452000 & 3.543293000 \\ \text { F } & 1.501516000 & -2.806180000 & 2.036294000\end{array}$

\section{CF32-Coor-T}

Zero-point correction $=\quad 0.811170($ Hartree/Particle $)$ Thermal correction to Energy $=0.891692$

Thermal correction to Enthalpy $=0.892636$ Thermal correction to Gibbs Free Energy $=0.675441$ Sum of electronic and zero-point Energies $=-4981.426834$ Sum of electronic and thermal Energies $=\quad-4981.346313$ Sum of electronic and thermal Enthalpies $=\quad-4981.345368$ Sum of electronic and thermal Free Energies $=-4981.562564$ E solvent $=-4981.42596512$

$\begin{array}{lrrr}\mathrm{Ni} & 1.939120000 & 0.761577000 & -1.299076000 \\ \mathrm{C} & 3.182568000 & 1.770712000 & -2.454240000 \\ \mathrm{~N} & 2.255385000 & 1.907778000 & 0.280571000 \\ \mathrm{C} & 1.399103000 & 1.976575000 & 1.298186000 \\ \mathrm{C} & 0.195129000 & 1.248734000 & 1.521563000 \\ \mathrm{C} & -0.265171000 & 0.220184000 & 0.614340000 \\ \mathrm{O} & 0.371954000 & -0.086520000 & -0.469368000 \\ \mathrm{C} & 4.225691000 & 1.116809000 & -3.364931000 \\ \mathrm{H} & 3.758587000 & 0.410023000 & -4.077378000 \\ \mathrm{C} & 5.005620000 & 2.176788000 & -4.176203000 \\ \mathrm{H} & 5.765598000 & 1.700976000 & -4.822921000 \\ \mathrm{H} & 4.326339000 & 2.761896000 & -4.823082000 \\ \mathrm{H} & 5.524866000 & 2.888408000 & -3.508052000 \\ \mathrm{H} & 4.945720000 & 0.527186000 & -2.765478000 \\ \mathrm{H} & 2.430089000 & 2.320794000 & -3.061583000 \\ \mathrm{H} & 3.685819000 & 2.493358000 & -1.791456000 \\ \mathrm{H} & 1.664095000 & 2.678201000 & 2.107333000 \\ \mathrm{C} & -0.546883000 & 1.546047000 & 2.702114000 \\ \mathrm{H} & -0.168754000 & 2.336659000 & 3.365187000 \\ \mathrm{C} & -1.729859000 & 0.884164000 & 3.030439000 \\ \mathrm{C} & -2.520617000 & 1.212373000 & 4.280374000\end{array}$




\begin{tabular}{|c|c|c|c|}
\hline $\mathrm{H}$ & -3.599652000 & 1.287366000 & 4.057470000 \\
\hline $\mathrm{H}$ & -2.194731000 & 2.168195000 & 4.725751000 \\
\hline 11 & -2.402082000 & 0.427696000 & 5.052207000 \\
\hline $\mathrm{C}$ & -2.178625000 & -0.118551000 & 2.138143000 \\
\hline $\mathrm{H}$ & -3.115926000 & -0.647979000 & 2.348480000 \\
\hline $\mathrm{C}$ & -1.493626000 & -0.468039000 & 0.966304000 \\
\hline $\mathrm{C}$ & -2.023687000 & -1.484076000 & 0.060038000 \\
\hline $\mathrm{N}$ & -3.110345000 & -2.148074000 & 0.316995000 \\
\hline $\mathrm{H}$ & -1.454824000 & -1.642354000 & -0.874355000 \\
\hline $\mathrm{C}$ & -3.582877000 & -3.112373000 & -0.589908000 \\
\hline $\mathrm{C}$ & -4.894498000 & -2.938290000 & -1.133247000 \\
\hline $\mathrm{C}$ & -5.384134000 & -3.875930000 & -2.063843000 \\
\hline $\mathrm{C}$ & -4.624275000 & -4.990157000 & -2.443968000 \\
\hline $\mathrm{C}$ & -3.357968000 & -5.182027000 & -1.881021000 \\
\hline $\mathrm{C}$ & -2.818487000 & -4.264092000 & -0.953300000 \\
\hline $\mathrm{C}$ & -1.485156000 & -4.564156000 & -0.353406000 \\
\hline $\mathrm{C}$ & -1.280521000 & -4.528863000 & 1.042977000 \\
\hline $\mathrm{C}$ & 1.030616000 & -5.258305000 & 0.769032000 \\
\hline $\mathrm{C}$ & 0.836449000 & -5.292209000 & -0.619994000 \\
\hline $\mathrm{C}$ & -0.407694000 & -4.954605000 & -1.176844000 \\
\hline $\mathrm{C}$ & -5.742575000 & -1.764722000 & -0.771140000 \\
\hline $\mathrm{C}$ & -5.907184000 & -1.356331000 & 0.571143000 \\
\hline $\mathrm{C}$ & -6.733804000 & -0.270720000 & 0.892532000 \\
\hline $\mathrm{C}$ & -7.426421000 & 0.428106000 & -0.110459000 \\
\hline $\mathrm{C}$ & -7.277459000 & 0.022555000 & -1.443995000 \\
\hline $\mathrm{C}$ & -6.440931000 & -1.058205000 & -1.772262000 \\
\hline $\mathrm{C}$ & -0.038344000 & -4.877362000 & 1.594096000 \\
\hline $\mathrm{C}$ & -0.557894000 & 6.177729000 & -0.301299000 \\
\hline $\mathrm{C}$ & 0.248741000 & 6.555965000 & 0.783751000 \\
\hline $\mathrm{C}$ & 1.465895000 & 5.900679000 & 1.031465000 \\
\hline $\mathrm{C}$ & 1.903346000 & 4.850960000 & 0.195800000 \\
\hline $\mathrm{C}$ & 1.091436000 & 4.483463000 & -0.895703000 \\
\hline $\mathrm{C}$ & -0.125017000 & 5.138947000 & -1.138333000 \\
\hline $\mathrm{C}$ & 3.224623000 & 4.196158000 & 0.440607000 \\
\hline $\mathrm{C}$ & 4.351341000 & 5.021016000 & 0.652684000 \\
\hline $\mathrm{C}$ & 5.622127000 & 4.472796000 & 0.856074000 \\
\hline $\mathrm{C}$ & 5.783122000 & 3.082604000 & 0.844921000 \\
\hline $\mathrm{C}$ & 4.685178000 & 2.217720000 & 0.644680000 \\
\hline $\mathrm{C}$ & 3.392534000 & 2.780334000 & 0.448883000 \\
\hline $\mathrm{C}$ & 4.938940000 & 0.746962000 & 0.620069000 \\
\hline $\mathrm{C}$ & 6.014468000 & 0.252442000 & -0.149434000 \\
\hline $\mathrm{C}$ & 6.298997000 & -1.121321000 & -0.194930000 \\
\hline $\mathrm{C}$ & 5.523429000 & -2.033871000 & 0.535592000 \\
\hline $\mathrm{C}$ & 4.463832000 & -1.548576000 & 1.315585000 \\
\hline $\mathrm{C}$ & 4.171367000 & -0.175641000 & 1.360705000 \\
\hline $\mathrm{H}$ & 1.418123000 & 3.686531000 & -1.569196000 \\
\hline $\mathrm{H}$ & -1.504031000 & 6.688811000 & -0.495323000 \\
\hline $\mathrm{H}$ & 2.077806000 & 6.205310000 & 1.886966000 \\
\hline $\mathrm{H}$ & 4.220413000 & 6.108605000 & 0.626949000 \\
\hline $\mathrm{H}$ & 6.485772000 & 5.126478000 & 1.017104000 \\
\hline $\mathrm{H}$ & 6.772158000 & 2.644742000 & 1.019998000 \\
\hline $\mathrm{H}$ & 6.614662000 & 0.946765000 & -0.746443000 \\
\hline $\mathrm{H}$ & 5.736071000 & -3.104351000 & 0.491697000 \\
\hline $\mathrm{H}$ & -8.067308000 & 1.276412000 & 0.143830000 \\
\hline $\mathrm{H}$ & -6.310535000 & -1.336968000 & -2.822806000 \\
\hline $\mathrm{H}$ & -6.391989000 & -3.737476000 & -2.471826000 \\
\hline $\mathrm{H}$ & -5.030131000 & -5.720023000 & -3.152218000 \\
\hline $\mathrm{H}$ & -2.776288000 & -6.076081000 & -2.134382000 \\
\hline $\mathrm{H}$ & -0.538666000 & -4.977404000 & -2.263473000 \\
\hline $\mathrm{H}$ & -2.098553000 & -4.219463000 & 1.700363000 \\
\hline $\mathrm{H}$ & -5.380889000 & -1.892493000 & 1.363266000 \\
\hline $\mathrm{H}$ & 3.348544000 & 0.173267000 & 1.989322000 \\
\hline $\mathrm{H}$ & 2.004066000 & -5.499395000 & 1.201534000 \\
\hline $\mathrm{C}$ & 2.222420000 & -1.036255000 & -2.250531000 \\
\hline $\mathrm{C}$ & 1.314842000 & -0.274416000 & -2.988302000 \\
\hline $\mathrm{H}$ & 1.865144000 & -1.749876000 & -1.501564000 \\
\hline $\mathrm{H}$ & 3.270204000 & -1.126368000 & -2.551496000 \\
\hline $\mathrm{H}$ & 1.616272000 & 0.243952000 & -3.904188000 \\
\hline
\end{tabular}

$\begin{array}{lrrr}\text { H } & 0.238799000 & -0.380798000 & -2.818831000 \\ \text { C } & 2.003944000 & -5.597983000 & -1.528559000 \\ \text { F } & 2.695099000 & -4.458285000 & -1.847688000 \\ \text { F } & 2.895245000 & -6.447192000 & -0.946679000 \\ \text { F } & 1.604329000 & -6.157633000 & -2.704653000 \\ \text { C } & 0.122645000 & -4.878756000 & 3.098589000 \\ \text { F } & -0.494089000 & -3.806451000 & 3.669814000 \\ \text { F } & -0.431674000 & -5.996356000 & 3.654558000 \\ \text { F } & 1.431683000 & -4.851691000 & 3.475485000 \\ \text { C } & -8.061285000 & 0.717875000 & -2.534269000 \\ \text { F } & -8.337220000 & 2.013411000 & -2.214812000 \\ \text { F } & -7.387202000 & 0.719152000 & -3.719456000 \\ \text { F } & -9.257822000 & 0.098901000 & -2.759419000 \\ \text { C } & -6.820242000 & 0.197049000 & 2.327177000 \\ \text { F } & -8.012852000 & 0.794897000 & 2.600012000 \\ \text { F } & -6.657425000 & -0.826803000 & 3.210491000 \\ \text { F } & -5.843631000 & 1.118681000 & 2.607285000 \\ \text { C } & -0.961776000 & 4.679975000 & -2.312952000 \\ \text { F } & -2.038217000 & 5.482303000 & -2.525787000 \\ \text { F } & -0.227606000 & 4.656429000 & -3.463348000 \\ \text { F } & -1.425923000 & 3.410692000 & -2.118914000 \\ \text { C } & -0.224842000 & 7.630224000 & 1.738666000 \\ \text { F } & -1.051822000 & 8.522087000 & 1.127897000 \\ \text { F } & -0.914459000 & 7.092811000 & 2.786464000 \\ \text { F } & 0.820881000 & 8.328255000 & 2.266997000 \\ \text { C } & 7.467530000 & -1.597114000 & -1.029101000 \\ \text { F } & 7.499263000 & -0.963447000 & -2.239179000 \\ \text { F } & 8.657303000 & -1.333450000 & -0.415542000 \\ \text { F } & 7.418700000 & -2.935133000 & -1.265060000 \\ \text { C } & 3.576794000 & -2.495017000 & 2.093479000 \\ \text { F } & 2.307690000 & -2.495246000 & 1.587330000 \\ \text { F } & 4.032828000 & -3.778292000 & 2.053224000 \\ \text { F } & 3.480666000 & -2.128575000 & 3.400722000\end{array}$

\section{${ }^{\text {CF3 TS-2 }}$ isom}

Zero-point correction $=0.809554$ (Hartree/Particle)

Thermal correction to Energy $=\quad 0.889596$

Thermal correction to Enthalpy $=\quad 0.890540$

Thermal correction to Gibbs Free Energy $=\quad 0.674671$

Sum of electronic and zero-point Energies $=\quad-4981.410562$

Sum of electronic and thermal Energies $=\quad-4981.330521$

Sum of electronic and thermal Enthalpies $=-4981.329576$

Sum of electronic and thermal Free Energies $=-4981.545445$

E solvent $=-4981.40358656$

$\begin{array}{lrrr}\mathrm{N} & -1.211223000 & -2.333158000 & 0.523953000 \\ \mathrm{C} & -3.322560000 & -3.630690000 & 0.511610000 \\ \mathrm{C} & 0.196961000 & -4.609397000 & -0.727450000 \\ \mathrm{C} & 1.224013000 & -4.502855000 & 0.231822000 \\ \mathrm{H} & 0.975347000 & -4.414782000 & 1.292635000 \\ \mathrm{C} & 1.912466000 & -4.762334000 & -2.468175000 \\ \mathrm{C} & -4.116296000 & -2.506293000 & 1.096101000 \\ \mathrm{C} & 2.927834000 & -4.646811000 & -1.507056000 \\ \mathrm{C} & -1.920593000 & -3.551433000 & 0.263449000 \\ \mathrm{C} & 2.573880000 & -4.524903000 & -0.155256000 \\ \mathrm{C} & -1.253132000 & -4.643308000 & -0.361659000 \\ \mathrm{C} & -3.802217000 & -1.905429000 & 2.332858000 \\ \mathrm{H} & -2.936953000 & -2.247611000 & 2.905580000 \\ \mathrm{C} & -5.761169000 & -0.459479000 & 2.183828000 \\ \mathrm{C} & 0.562159000 & -4.755523000 & -2.082653000 \\ \mathrm{H} & -0.219940000 & -4.836272000 & -2.844262000 \\ \mathrm{C} & -4.617898000 & -0.896396000 & 2.869136000 \\ \mathrm{C} & -4.018362000 & -4.803975000 & 0.144674000 \\ \mathrm{H} & -5.091209000 & -4.864777000 & 0.360109000 \\ \mathrm{C} & -6.088952000 & -1.057469000 & 0.958535000 \\ \mathrm{C} & -3.363899000 & -5.882000000 & -0.460736000 \\ \mathrm{H} & -3.919401000 & -6.786890000 & -0.728227000 \\ \mathrm{C} & -5.279710000 & -2.072024000 & 0.423155000 \\ \mathrm{H} & -5.553606000 & -2.533240000 & -0.531541000\end{array}$




\begin{tabular}{|c|c|c|c|}
\hline & & & \\
\hline & 62065000 & -6.631283000 & \\
\hline & 231191000 & 2.771242000 & 0.253957000 \\
\hline & 2727000 & 2096000 & .26349900 \\
\hline & 9000 & & \\
\hline & 2066000 & & 8060 \\
\hline & 907269000 & & \\
\hline & .518680000 & & \\
\hline & 5587000 & 00 & \\
\hline & 3.313220000 & 172000 & \\
\hline & 1.795022000 & 20000 & -0 . \\
\hline & 2.711236000 & 3000 & 3000 \\
\hline & 0.931708000 & 4.925705000 & \\
\hline & 4.088758000 & 1.798992000 & 206000 \\
\hline & 3.237169000 & 1.526203000 & -0.981089000 \\
\hline & 6.159448000 & 5236000 & \\
\hline & -1.144444000 & 5.072022000 & \\
\hline & -0.5 & & \\
\hline & 5.05 & 92000 & \\
\hline & & & \\
\hline & 4.8 & 5000 & \\
\hline & 6.2 & & \\
\hline & & & \\
\hline & & & \\
\hline & & & \\
\hline & & & \\
\hline & & & \\
\hline & & & \\
\hline & & & \\
\hline & & & \\
\hline & & & \\
\hline & & & \\
\hline & & & \\
\hline & & & \\
\hline & 00 & & \\
\hline & 00 & & \\
\hline & & 9377000 & \\
\hline & 00 & -0.289116000 & \\
\hline & & & \\
\hline & & & \\
\hline & & & \\
\hline & & & \\
\hline & & & \\
\hline & & & \\
\hline & & & \\
\hline & & & \\
\hline & & & \\
\hline & & & \\
\hline & & & \\
\hline & & & \\
\hline & & 0 & \\
\hline & & & \\
\hline & & & \\
\hline & & & \\
\hline & & & \\
\hline & & & -3. \\
\hline & & & 00000 \\
\hline & & -1.823561000 & 7000 \\
\hline $\mathrm{H}$ & 5000 & -2.007787000 & -3 \\
\hline & 785000 & 0.029609000 & \\
\hline & 227000 & 0836000 & 2000 \\
\hline & 143000 & -1.533679000 & \\
\hline & 4000 & -4.638452000 & \\
\hline & & & \\
\hline & & & \\
\hline & & & \\
\hline & & & \\
\hline & -8.205801000 & 0.038173000 & 0.96634900 \\
\hline
\end{tabular}

$\begin{array}{cccc}\text { F } & -6.919120000 & 0.326456000 & -0.787826000 \\ \text { F } & -7.911340000 & -1.601658000 & -0.471172000 \\ \text { C } & -4.226347000 & -0.252792000 & 4.181810000 \\ \text { F } & -5.291786000 & 0.325619000 & 4.801831000 \\ \text { F } & -3.692731000 & -1.166813000 & 5.044642000 \\ \text { F } & -3.287328000 & 0.717300000 & 4.001595000 \\ \text { C } & -3.133824000 & 5.328729000 & -3.745836000 \\ \text { F } & -3.310932000 & 6.682505000 & -3.769349000 \\ \text { F } & -2.336887000 & 5.018294000 & -4.809442000 \\ \text { F } & -4.351399000 & 4.759468000 & -3.964233000 \\ \text { C } & -3.555846000 & 3.208466000 & 0.804279000 \\ \text { F } & -4.770605000 & 3.811604000 & 0.952192000 \\ \text { F } & -3.813494000 & 1.907140000 & 0.449974000 \\ \text { F } & -2.958108000 & 3.179869000 & 2.023200000 \\ \text { C } & 2.269103000 & -4.938522000 & -3.927722000 \\ \text { F } & 1.326810000 & -4.378971000 & -4.744404000 \\ \text { F } & 3.466494000 & -4.367788000 & -4.229442000 \\ \text { F } & 2.352751000 & -6.257086000 & -4.266848000 \\ \text { C } & 3.647876000 & -4.484607000 & 0.910015000 \\ \text { F } & 3.295051000 & -3.677515000 & 1.950052000 \\ \text { F } & 3.865953000 & -5.728699000 & 1.432054000 \\ \text { F } & 4.835866000 & -4.041506000 & 0.420893000 \\ \text { C } & 4.890738000 & -0.565072000 & -0.574270000 \\ \text { F } & 3.722640000 & -1.130690000 & -0.141964000 \\ \text { F } & 4.839295000 & -0.569174000 & -1.940747000 \\ \text { F } & 5.905218000 & -1.389183000 & -0.203138000 \\ \text { C } & 7.463973000 & 2.883003000 & 2.101043000 \\ \text { F } & 7.073777000 & 3.602482000 & 3.193722000 \\ \text { F } & 8.171458000 & 1.809277000 & 2.547245000 \\ \text { F } & 8.326151000 & 3.674100000 & 1.398162000\end{array}$

\section{CF32-Coor-C}

Zero-point correction $=\quad 0.811820($ Hartree/Particle $)$ Thermal correction to Energy= $\quad 0.892032$ Thermal correction to Enthalpy $=\quad 0.892976$ Thermal correction to Gibbs Free Energy $=0.678589$

Sum of electronic and zero-point Energies $=\quad-4981.425160$ Sum of electronic and thermal Energies $=\quad-4981.344948$ Sum of electronic and thermal Enthalpies $=\quad-4981.344004$ Sum of electronic and thermal Free Energies $=-4981.558392$ E solvent $=-4981.42424077$

$\begin{array}{lrrr}\mathrm{N} & 2.239361000 & 2.040185000 & 0.005497000 \\ \mathrm{C} & 4.669798000 & 2.524823000 & 0.157590000 \\ \mathrm{C} & 1.667183000 & 4.951845000 & -0.177036000 \\ \mathrm{C} & 0.653428000 & 4.425506000 & -1.008124000 \\ \mathrm{H} & 0.841999000 & 3.529816000 & -1.605621000 \\ \mathrm{C} & 0.102831000 & 6.713968000 & 0.504322000 \\ \mathrm{C} & 5.049035000 & 1.086050000 & 0.318147000 \\ \mathrm{C} & -0.891183000 & 6.189977000 & -0.333823000 \\ \mathrm{C} & 3.324593000 & 2.980889000 & 0.017321000 \\ \mathrm{C} & -0.603484000 & 5.041759000 & -1.088129000 \\ \mathrm{C} & 3.050916000 & 4.379698000 & -0.105768000 \\ \mathrm{C} & 4.344000000 & 0.180391000 & 1.139339000 \\ \mathrm{H} & 3.465916000 & 0.510266000 & 1.697776000 \\ \mathrm{C} & 5.894543000 & -1.621083000 & 0.575240000 \\ \mathrm{C} & 1.365515000 & 6.103570000 & 0.582711000 \\ \mathrm{H} & 2.123475000 & 6.525115000 & 1.251122000 \\ \mathrm{C} & 4.764320000 & -1.155605000 & 1.260264000 \\ \mathrm{C} & 5.713065000 & 3.479374000 & 0.151071000 \\ \mathrm{H} & 6.742671000 & 3.135609000 & 0.297758000 \\ \mathrm{C} & 6.608928000 & -0.726938000 & -0.236881000 \\ \mathrm{C} & 5.455334000 & 4.844710000 & -0.000813000 \\ \mathrm{H} & 6.280978000 & 5.563893000 & -0.011081000 \\ \mathrm{C} & 6.192825000 & 0.605807000 & -0.363257000 \\ \mathrm{H} & 6.754324000 & 1.278307000 & -1.019862000 \\ \mathrm{C} & 4.134402000 & 5.286632000 & -0.125113000 \\ \mathrm{H} & 3.926759000 & 6.354421000 & -0.254671000 \\ \mathrm{~N} & -2.904691000 & -2.219297000 & 0.468451000\end{array}$




\begin{tabular}{|c|c|c|c|}
\hline $\mathrm{C}$ & -2.575589000 & -4.389519000 & -0.679693000 \\
\hline $\mathrm{C}$ & -5.602328000 & -2.016947000 & -0.514623000 \\
\hline $\mathrm{C}$ & -6.376938000 & -1.397560000 & -1.517461000 \\
\hline $\mathrm{H}$ & -6.286084000 & -1.732048000 & -2.555917000 \\
\hline $\mathrm{C}$ & -6.572680000 & -0.467789000 & 1.111091000 \\
\hline $\mathrm{C}$ & -1.201454000 & -4.573821000 & -0.126185000 \\
\hline $\mathrm{C}$ & -7.339313000 & 0.145597000 & 0.106779000 \\
\hline $\mathrm{C}$ & -3.378126000 & -3.251672000 & -0.358962000 \\
\hline $\mathrm{C}$ & -7.238987000 & -0.331629000 & -1.207953000 \\
\hline $\mathrm{C}$ & -4.718711000 & -3.172256000 & -0.848380000 \\
\hline $\mathrm{C}$ & -0.931419000 & -4.415887000 & 1.249463000 \\
\hline $\mathrm{H}$ & -1.735902000 & -4.110660000 & 1.925399000 \\
\hline $\mathrm{C}$ & 1.410294000 & -5.004560000 & 0.907539000 \\
\hline $\mathrm{C}$ & -5.719399000 & -1.538476000 & 0.808895000 \\
\hline $\mathrm{H}$ & -5.134260000 & -2.008090000 & 1.602385000 \\
\hline $\mathrm{C}$ & 0.358848000 & -4.634939000 & 1.758098000 \\
\hline $\mathrm{C}$ & -3.110217000 & -5.391165000 & -1.519119000 \\
\hline $\mathrm{H}$ & -2.497873000 & -6.272764000 & -1.742134000 \\
\hline $\mathrm{C}$ & 1.149140000 & -5.165213000 & -0.463150000 \\
\hline $\mathrm{C}$ & -4.407729000 & -5.293303000 & -2.033864000 \\
\hline $\mathrm{H}$ & -4.808744000 & -6.085963000 & -2.674089000 \\
\hline $\mathrm{C}$ & -0.141332000 & -4.961423000 & -0.974462000 \\
\hline $\mathrm{H}$ & -0.323655000 & -5.081050000 & -2.046447000 \\
\hline $\mathrm{C}$ & -5.202934000 & -4.190934000 & -1.692546000 \\
\hline $\mathrm{H}$ & -6.232810000 & -4.124795000 & -2.061973000 \\
\hline $\mathrm{Ni}$ & 1.723257000 & 0.902611000 & -1.654116000 \\
\hline $\mathrm{C}$ & -1.896691000 & -1.490074000 & 0.096820000 \\
\hline $\mathrm{H}$ & -1.406893000 & -1.629893000 & -0.883644000 \\
\hline $\mathrm{C}$ & 1.474602000 & 2.103906000 & 1.084291000 \\
\hline $\mathrm{H}$ & 1.768763000 & 2.824840000 & 1.868021000 \\
\hline $\mathrm{C}$ & 0.312711000 & 1.330596000 & 1.387716000 \\
\hline $\mathrm{C}$ & -0.335066000 & 1.582495000 & 2.630625000 \\
\hline $\mathrm{C}$ & -0.197200000 & 0.319929000 & 0.492833000 \\
\hline $\mathrm{H}$ & 0.076187000 & 2.371576000 & 3.275660000 \\
\hline $\mathrm{C}$ & -1.464442000 & 0.875891000 & 3.045849000 \\
\hline $\mathrm{C}$ & -1.348382000 & -0.433778000 & 0.945881000 \\
\hline $\mathrm{C}$ & -2.160553000 & 1.160169000 & 4.360985000 \\
\hline $\mathrm{C}$ & -1.941954000 & -0.133866000 & 2.181101000 \\
\hline $\mathrm{H}$ & -2.828804000 & -0.714178000 & 2.462918000 \\
\hline $\mathrm{O}$ & 0.312185000 & 0.067388000 & -0.680043000 \\
\hline $\mathrm{C}$ & 1.030098000 & -0.212234000 & -3.126124000 \\
\hline $\mathrm{C}$ & 1.409194000 & -1.673010000 & -2.910837000 \\
\hline $\mathrm{H}$ & -0.053825000 & -0.032183000 & -3.018263000 \\
\hline $\mathrm{H}$ & 1.375476000 & 0.164718000 & -4.106043000 \\
\hline $\mathrm{C}$ & 3.434700000 & 1.077695000 & -2.755968000 \\
\hline $\mathrm{C}$ & 2.594257000 & 2.167177000 & -3.012242000 \\
\hline $\mathrm{C}$ & 0.806801000 & -2.585188000 & -4.007051000 \\
\hline $\mathrm{H}$ & 2.509057000 & -1.799219000 & -2.913716000 \\
\hline $\mathrm{H}$ & 1.056729000 & -2.007581000 & -1.918956000 \\
\hline $\mathrm{H}$ & 1.117246000 & -3.633774000 & -3.850543000 \\
\hline $\mathrm{H}$ & -0.297794000 & -2.544691000 & -3.999061000 \\
\hline $\mathrm{H}$ & 1.145875000 & -2.279656000 & -5.014165000 \\
\hline $\mathrm{H}$ & 2.786880000 & 3.127650000 & -2.520306000 \\
\hline $\mathrm{H}$ & 1.959752000 & 2.208574000 & -3.904682000 \\
\hline $\mathrm{H}$ & 3.500352000 & 0.228277000 & -3.443357000 \\
\hline $\mathrm{H}$ & 4.261789000 & 1.174001000 & -2.047186000 \\
\hline $\mathrm{H}$ & -3.255561000 & 1.210577000 & 4.225068000 \\
\hline $\mathrm{H}$ & -1.961173000 & 0.365370000 & 5.105112000 \\
\hline $\mathrm{H}$ & -1.824909000 & 2.116511000 & 4.797994000 \\
\hline $\mathrm{H}$ & 6.212186000 & -2.661931000 & 0.670773000 \\
\hline $\mathrm{H}$ & -7.999693000 & 0.983499000 & 0.344215000 \\
\hline $\mathrm{H}$ & -1.870014000 & 6.670813000 & -0.402966000 \\
\hline $\mathrm{H}$ & 2.419583000 & -5.141020000 & 1.302692000 \\
\hline $\mathrm{C}$ & 0.583481000 & -4.503399000 & 3.248714000 \\
\hline $\mathrm{F}$ & 0.005609000 & -3.372822000 & 3.743465000 \\
\hline $\mathrm{F}$ & 0.031514000 & -5.554946000 & 3.923814000 \\
\hline $\mathrm{F}$ & 1.905687000 & -4.468239000 & 3.572725000 \\
\hline $\mathrm{C}$ & 2.297425000 & -5.483171000 & -1.392351000 \\
\hline
\end{tabular}

$\begin{array}{cccc}\text { F } & 3.070904000 & -4.378260000 & -1.623253000 \\ \text { F } & 3.120982000 & -6.435261000 & -0.871753000 \\ \text { F } & 1.869312000 & -5.928781000 & -2.609574000 \\ \text { C } & 7.786427000 & -1.215493000 & -1.052115000 \\ \text { F } & 8.739387000 & -0.248425000 & -1.183197000 \\ \text { F } & 8.375923000 & -2.305269000 & -0.490162000 \\ \text { F } & 7.401816000 & -1.567836000 & -2.313104000 \\ \text { C } & 3.978759000 & -2.080946000 & 2.165660000 \\ \text { F } & 2.647369000 & -2.034468000 & 1.877008000 \\ \text { F } & 4.384020000 & -3.377012000 & 2.045177000 \\ \text { F } & 4.114745000 & -1.729184000 & 3.474489000 \\ \text { C } & -1.674938000 & 4.418385000 & -1.958306000 \\ \text { F } & -2.599628000 & 5.334865000 & -2.356400000 \\ \text { F } & -1.143237000 & 3.852339000 & -3.080361000 \\ \text { F } & -2.341073000 & 3.433509000 & -1.292403000 \\ \text { C } & -0.187418000 & 7.913229000 & 1.380669000 \\ \text { F } & -1.271842000 & 8.611150000 & 0.948048000 \\ \text { F } & -0.433291000 & 7.535631000 & 2.668714000 \\ \text { F } & 0.871948000 & 8.772224000 & 1.416486000 \\ \text { C } & -8.099089000 & 0.271422000 & -2.295708000 \\ \text { F } & -8.427742000 & 1.565795000 & -2.025281000 \\ \text { F } & -7.471876000 & 0.248573000 & -3.506134000 \\ \text { F } & -9.270533000 & -0.415406000 & -2.443668000 \\ \text { C } & -6.604932000 & 0.067913000 & 2.524198000 \\ \text { F } & -7.757737000 & 0.740113000 & 2.793554000 \\ \text { F } & -6.479372000 & -0.926278000 & 3.447628000 \\ \text { F } & -5.572797000 & 0.942666000 & 2.746708000\end{array}$

\section{${ }^{\text {CF3 TS-2 }}$ ins}

Zero-point correction $=0.811249($ Hartree/Particle $)$ Thermal correction to Energy= $\quad 0.891280$ Thermal correction to Enthalpy $=\quad 0.892224$

Thermal correction to Gibbs Free Energy $=0.675845$ Sum of electronic and zero-point Energies $=\quad-4981.406379$ Sum of electronic and thermal Energies $=\quad-4981.326349$ Sum of electronic and thermal Enthalpies $=-4981.325405$ Sum of electronic and thermal Free Energies $=-4981.541784$ E solvent $=-4981.40941267$

$\begin{array}{lrrr}\mathrm{Ni} & 1.954494000 & 1.098801000 & 1.159476000 \\ \mathrm{C} & 0.700021000 & 1.349510000 & 2.860919000 \\ \mathrm{C} & 0.092412000 & 2.720427000 & 2.585110000 \\ \mathrm{C} & -1.218746000 & 2.916159000 & 3.384679000 \\ \mathrm{H} & 0.099513000 & 0.510367000 & 2.481947000 \\ \mathrm{H} & 0.868949000 & 1.189785000 & 3.935464000 \\ \mathrm{H} & -0.124480000 & 2.833415000 & 1.506904000 \\ \mathrm{H} & 0.800397000 & 3.527803000 & 2.855572000 \\ \mathrm{H} & -1.653208000 & 3.912619000 & 3.185695000 \\ \mathrm{H} & -1.039404000 & 2.834934000 & 4.472547000 \\ \mathrm{H} & -1.971691000 & 2.156889000 & 3.107136000 \\ \mathrm{C} & 2.911320000 & 1.357040000 & 3.073354000 \\ \mathrm{C} & 3.726905000 & 1.554065000 & 1.938507000 \\ \mathrm{H} & 2.931981000 & 0.401366000 & 3.608896000 \\ \mathrm{H} & 2.682760000 & 2.231030000 & 3.692567000 \\ \mathrm{H} & 4.028322000 & 2.572637000 & 1.669006000 \\ \mathrm{H} & 4.406744000 & 0.760021000 & 1.604183000 \\ \mathrm{O} & 0.318135000 & 0.281564000 & 0.507622000 \\ \mathrm{~N} & 2.675095000 & 1.174155000 & -0.671456000 \\ \mathrm{C} & 2.088716000 & 0.653644000 & -1.744653000 \\ \mathrm{H} & 2.625042000 & 0.772421000 & -2.701934000 \\ \mathrm{C} & 0.837149000 & -0.023275000 & -1.824380000 \\ \mathrm{C} & 0.409098000 & -0.519670000 & -3.088364000 \\ \mathrm{H} & 1.072662000 & -0.377868000 & -3.953063000 \\ \mathrm{C} & -0.813883000 & -1.172105000 & -3.261190000 \\ \mathrm{C} & -1.263419000 & -1.702131000 & -4.607990000 \\ \mathrm{H} & -1.118659000 & -2.797336000 & -4.681814000 \\ \mathrm{H} & -2.337538000 & -1.506083000 & -4.774424000 \\ \mathrm{H} & -0.698056000 & -1.237907000 & -5.434745000 \\ \mathrm{C} & -1.636152000 & -1.321090000 & -2.120182000\end{array}$




\begin{tabular}{|c|c|c|c|}
\hline & & & \\
\hline & 9000 & 37162000 & 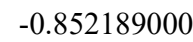 \\
\hline & .010590000 & -0.164957000 & 4 \\
\hline & 180141000 & -0.965435000 & 0 . \\
\hline & .877261000 & -0.417656000 & \\
\hline & 66000 & 2911000 & 0 . \\
\hline & 000 & 9000 & \\
\hline & 69000 & -1.328642000 & \\
\hline & 0000 & & \\
\hline & -5.835 & 796000 & 1000 \\
\hline & -4.50 & 251000 & 7000 \\
\hline & 000 & 75000 & 000 \\
\hline & -2.23 & -2.734005000 & 4000 \\
\hline & -6.013907000 & -0.815710000 & -0.040051000 \\
\hline & -6.853988000 & 0.315323000 & -0.063985000 \\
\hline & -7.077437000 & 0.845903000 & 0.867221000 \\
\hline & -7.377 & & -1.2 \\
\hline & -8.32 & & \\
\hline & -7.0 & & \\
\hline & & & \\
\hline & -6.2 & -0 & \\
\hline & & & 7000 \\
\hline & & & -1.2 \\
\hline & -5.0 & & -1.2 \\
\hline & 0 & & \\
\hline & -2.3 & & \\
\hline & -0.4 & & \\
\hline & 0 & & \\
\hline & & & \\
\hline & & & \\
\hline & 00 & & \\
\hline & & & \\
\hline & & & \\
\hline & & & \\
\hline & 00 & & \\
\hline & 4.0 & & \\
\hline & 5.27 & & 3000 \\
\hline & 6.41 & & 27000 \\
\hline & 00 & & 37000 \\
\hline & 5.0 & & \\
\hline & 7.20 & & \\
\hline & & & \\
\hline & & & \\
\hline & 2.8 & & \\
\hline & & & \\
\hline & & & \\
\hline & -8 & & \\
\hline 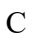 & 5.1 & & \\
\hline & & & \\
\hline E & & & -0 \\
\hline & 2.98 & & 00 \\
\hline & 1.9 & & \\
\hline & & & 00 \\
\hline & 0.5 & 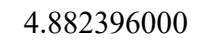 & -0.9 \\
\hline & & & -1.2 \\
\hline & & 02000 & -3.251462000 \\
\hline 11 & 3.8 & & 0.273230000 \\
\hline $\mathrm{C}$ & 3.6 & 15000 & 5600000 \\
\hline $\mathrm{H}$ & 5.17 & 28000 & 19000 \\
\hline $\mathrm{C}$ & 6.6 & 99000 & -3. \\
\hline 11 & $3.92-r a$ & 59000 & 531000 \\
\hline $\mathrm{C}$ & 2.04 & 49000 & 662000 \\
\hline & -0.1 & 45000 & \\
\hline $\mathrm{C}$ & -0.6 & & -1.7 \\
\hline & & & \\
\hline & & 90000 & -3.4 \\
\hline $\mathrm{F}$ & & & \\
\hline & 6.626407000 & -2.238887000 & -4.91164300 \\
\hline
\end{tabular}

\begin{tabular}{|c|c|}
\hline & $2.298 / 57000$ \\
\hline & 3.945678000 \\
\hline & 3.844268000 \\
\hline & 1.900061000 \\
\hline & 1.111101000 \\
\hline & 3.274021000 \\
\hline & -1.7886661000 \\
\hline & -0.885682000 \\
\hline & -0.685437000 \\
\hline & -7.359190000 \\
\hline & -6.506806000 \\
\hline & -4.134790000 \\
\hline & 0.326911000 \\
\hline & 1.004839000 \\
\hline & 2.053712000 \\
\hline & 1.304943000 \\
\hline & -0.729966000 \\
\hline & -0.184479000 \\
\hline & -8.341178000 \\
\hline & -9.606013000 \\
\hline & -7.988176000 \\
\hline & -5.094047000 \\
\hline & -6.93012100 \\
\hline & -5.08925600 \\
\hline
\end{tabular}

$-3.437072000$ $-4.888798000$ $-3.218006000$ 6.092367000 7.780078000 7.380758000 5.097811000 3.646764000 5.798869000 $-1.075516000$ $-1.861409000$ $-2.627555000$ $-1.010763000$ $-3.044703000$ $-1.876027000$ $-5.760481000$ $-6.580536000$ $-5.190527000$ 2.641870000 1.585301000 2.882221000 $-2.720859000$ $-1.921954000$ $-0.735274000$

$-0.009309000$ 0.101475000 1.528208000 3.211431000 2.047955000 2.090111000 $-0.940272000$ $-2.326162000$ $-2.700913000$ 2.279246000 4.490874000 4.701291000 5.612417000 6.083401000 4.557579000 1.527269000 1.611198000 0.010919000 $-2.447478000$ $-0.999510000$ $-0.289331000$ $-3.617335000$ $-4.529436000$ $-4.554291000$

\section{CF32- $\beta$ - T}

Zero-point correction $=0.812956($ Hartree $/$ Particle $)$

$\begin{array}{lc}\text { Thermal correction to Energy= } & 0.892747 \\ \text { Thermal correction to Enthalpy= } & 0.893691 \\ \text { Thermal correction to Gibbs Free Energy= } & 0.678343 \\ \text { Sum of electronic and zero-point Energies }= & -4981.449235 \\ \text { Sum of electronic and thermal Energies }= & -4981.369444 \\ \text { Sum of electronic and thermal Enthalpies }= & -4981.368500 \\ \text { Sum of electronic and thermal Free Energies }= & -4981.583848 \\ \text { E solvent }=-4981.45077006 & \end{array}$

$\begin{array}{lrrr}\mathrm{Ni} & 1.835904000 & 0.948624000 & -0.971126000 \\ \mathrm{O} & 0.328050000 & 0.013680000 & -0.337972000 \\ \mathrm{C} & -0.352906000 & 0.288428000 & 0.734406000 \\ \mathrm{C} & 0.023861000 & 1.348485000 & 1.648825000 \\ \mathrm{C} & 1.156831000 & 2.187653000 & 1.430604000 \\ \mathrm{~N} & 2.031074000 & 2.151109000 & 0.426129000 \\ \mathrm{C} & -1.543794000 & -0.471358000 & 1.053736000 \\ \mathrm{C} & -2.268179000 & -0.183514000 & 2.219161000 \\ \mathrm{C} & -0.757312000 & 1.582710000 & 2.818481000 \\ \mathrm{C} & -1.893259000 & 0.833775000 & 3.128281000 \\ \mathrm{C} & -2.704240000 & 1.086021000 & 4.383266000 \\ \mathrm{C} & -1.984778000 & -1.502734000 & 0.117398000 \\ \mathrm{~N} & -3.006150000 & -2.271480000 & 0.350477000 \\ \mathrm{C} & -3.377703000 & -3.249032000 & -0.588620000 \\ \mathrm{C} & -4.711531000 & -3.215371000 & -1.103609000 \\ \mathrm{C} & -5.103206000 & -4.164932000 & -2.067679000 \\ \mathrm{C} & -4.221832000 & -5.160713000 & -2.509422000 \\ \mathrm{C} & -2.929485000 & -5.218808000 & -1.976324000 \\ \mathrm{C} & -2.486525000 & -4.281514000 & -1.017868000 \\ \mathrm{C} & -5.686976000 & -2.168923000 & -0.679062000 \\ \mathrm{C} & -5.887136000 & -1.853389000 & 0.682530000 \\ \mathrm{C} & -6.830624000 & -0.887478000 & 1.061657000 \\ \mathrm{C} & -7.604386000 & -0.220312000 & 0.098112000 \\ \mathrm{C} & -7.420182000 & -0.536478000 & -1.255617000 \\ \mathrm{C} & -6.469401000 & -1.496591000 & -1.641200000 \\ \mathrm{C} & -1.110427000 & -4.435982000 & -0.459773000 \\ \mathrm{C} & -0.876338000 & -4.461925000 & 0.931063000 \\ \mathrm{C} & 0.419069000 & -4.652260000 & 1.437186000 \\ \mathrm{C} & 1.511034000 & -4.811565000 & 0.571544000 \\ \mathrm{C} & 1.285398000 & -4.791748000 & -0.814193000 \\ \mathrm{C} & -0.009831000 & -4.613254000 & -1.325444000 \\ \mathrm{C} & 3.063746000 & 3.153649000 & 0.466051000 \\ \mathrm{C} & 4.415488000 & 2.781689000 & 0.719071000\end{array}$




\begin{tabular}{|c|c|c|c|}
\hline & & & \\
\hline & 64000 & & \\
\hline & & & \\
\hline & 2.719641000 & 6000 & 100 \\
\hline & 4.864726000 & & \\
\hline & 6000 & 2000 & 8000 \\
\hline & 583000 & 5000 & 8000 \\
\hline & 591000 & 7000 & 9000 \\
\hline & 4.632119000 & 70000 & 6000 \\
\hline & 186000 & 3000 & 000 \\
\hline & 1.333513000 & 4.968907000 & -0.065755000 \\
\hline & 0.594356000 & 33000 & -1.096508000 \\
\hline & -0.685758000 & 29000 & 358000 \\
\hline & -1.257089000 & 74000 & -0.758807000 \\
\hline & -0.5 & & \\
\hline & & & \\
\hline$C$ & & & \\
\hline$C$ & & & \\
\hline & & & \\
\hline & & & \\
\hline & 4.6 & & \\
\hline & & & \\
\hline & -0 . & & \\
\hline & -2.5 & & \\
\hline & -2.4 & & \\
\hline & & & \\
\hline & -3.1 & & \\
\hline & -1.4 & & \\
\hline & & & \\
\hline & & & \\
\hline & & & \\
\hline & & & \\
\hline & -5.2 & & 000 \\
\hline & -8.3 & & 000 \\
\hline & 2.5 & -4 & 000 \\
\hline & -0.1 & & \\
\hline & -1.7 & -4 & 000 \\
\hline & 3.26 & & \\
\hline & & & \\
\hline & & & \\
\hline & & & \\
\hline & & & \\
\hline & & & \\
\hline & & & \\
\hline & & & \\
\hline & & & \\
\hline & & & \\
\hline & & & \\
\hline & & & \\
\hline & & & -3 \\
\hline & & & \\
\hline & & & \\
\hline & & & \\
\hline & & & \\
\hline & & & -4 \\
\hline & & & \\
\hline & 000 & -1. & 2000 \\
\hline & 5000 & & 0000 \\
\hline & 3000 & 00 & 70000 \\
\hline & 0 & & \\
\hline & & & \\
\hline & & & \\
\hline & & & \\
\hline & & & \\
\hline $\mathrm{F}$ & & & \\
\hline & & & \\
\hline & & & -2.54759700 \\
\hline & -8.686382000 & 1.368618000 & $-1.9235850 \mathrm{C}$ \\
\hline
\end{tabular}

$\begin{array}{lrrr}\text { F } & -7.632041000 & 0.247045000 & -3.495733000 \\ \text { C } & -6.955042000 & -0.517944000 & 2.521488000 \\ \text { F } & -6.796576000 & -1.598117000 & 3.335900000 \\ \text { F } & -5.994027000 & 0.391843000 & 2.883996000 \\ \text { F } & -8.159855000 & 0.046026000 & 2.810000000 \\ \text { C } & -1.429705000 & 4.152459000 & -2.577973000 \\ \text { F } & -2.777004000 & 4.286834000 & -2.448012000 \\ \text { F } & -1.081467000 & 4.704449000 & -3.776846000 \\ \text { F } & -1.151391000 & 2.819034000 & -2.660618000 \\ \text { C } & -1.092861000 & 7.730436000 & 0.979818000 \\ \text { F } & -2.442718000 & 7.822704000 & 0.840771000 \\ \text { F } & -0.814615000 & 7.692444000 & 2.315004000 \\ \text { F } & -0.554663000 & 8.888135000 & 0.497038000 \\ \text { C } & 7.786793000 & -0.767641000 & -0.429586000 \\ \text { F } & 8.155750000 & 0.124394000 & -1.389663000 \\ \text { F } & 8.823721000 & -0.871719000 & 0.448777000 \\ \text { F } & 7.654937000 & -1.987936000 & -1.030860000 \\ \text { C } & 3.812632000 & -1.933730000 & 2.458948000 \\ \text { F } & 2.562692000 & -2.055834000 & 1.923179000 \\ \text { F } & 4.381624000 & -3.170831000 & 2.459922000 \\ \text { F } & 3.652080000 & -1.549169000 & 3.755070000\end{array}$

\section{${ }^{\text {CF3 TS-2 }}$ decoor}

Zero-point correction $=\quad 0.808172$ (Hartree/Particle)

$\begin{array}{lc}\text { Thermal correction to Energy }= & 0.888943 \\ \text { Thermal correction to Enthalpy= } & 0.889887 \\ \text { Thermal correction to Gibbs Free Energy }= & 0.673464 \\ \text { Sum of electronic and zero-point Energies }= & -4981.397553 \\ \text { Sum of electronic and thermal Energies }= & -4981.316782 \\ \text { Sum of electronic and thermal Enthalpies }= & -4981.315838 \\ \text { Sum of electronic and thermal Free Energies }= & -4981.532261 \\ \text { E solvent }=-4981.40446449 & \end{array}$

E solvent $=-4981.40446449$

$\begin{array}{lrrr}\mathrm{N} & 2.255570000 & 1.902333000 & 0.077133000 \\ \mathrm{C} & 4.673679000 & 2.457243000 & 0.051333000 \\ \mathrm{C} & 1.602990000 & 4.808623000 & -0.177156000 \\ \mathrm{C} & 1.309537000 & 5.980328000 & 0.555185000 \\ \mathrm{H} & 2.084882000 & 6.437941000 & 1.178320000 \\ \mathrm{C} & -0.701756000 & 4.823516000 & -1.000663000 \\ \mathrm{C} & 5.101089000 & 1.041711000 & 0.256820000 \\ \mathrm{C} & -0.982476000 & 5.990125000 & -0.273405000 \\ \mathrm{C} & 3.308133000 & 2.871272000 & -0.002947000 \\ \mathrm{C} & 0.032977000 & 6.562860000 & 0.506202000 \\ \mathrm{C} & 2.996957000 & 4.261661000 & -0.149803000 \\ \mathrm{C} & 4.461420000 & 0.166572000 & 1.163211000 \\ \mathrm{H} & 3.598009000 & 0.507511000 & 1.737995000 \\ \mathrm{C} & 6.057675000 & -1.612481000 & 0.652006000 \\ \mathrm{C} & 0.571487000 & 4.235924000 & -0.951323000 \\ \mathrm{H} & 0.766661000 & 3.323792000 & -1.519430000 \\ \mathrm{C} & 4.935420000 & -1.143251000 & 1.351237000 \\ \mathrm{C} & 5.690150000 & 3.432314000 & -0.063467000 \\ \mathrm{H} & 6.735441000 & 3.116183000 & 0.021483000 \\ \mathrm{C} & 6.700756000 & -0.750363000 & -0.249292000 \\ \mathrm{C} & 5.390775000 & 4.786425000 & -0.236504000 \\ \mathrm{H} & 6.195343000 & 5.523232000 & -0.329440000 \\ \mathrm{C} & 6.225834000 & 0.555046000 & -0.448606000 \\ \mathrm{H} & 6.729031000 & 1.199779000 & -1.176215000 \\ \mathrm{C} & 4.053333000 & 5.191481000 & -0.275117000 \\ \mathrm{H} & 3.808516000 & 6.248666000 & -0.425274000 \\ \mathrm{~N} & -3.097521000 & -2.121928000 & 0.370693000 \\ \mathrm{C} & -2.648324000 & -4.299619000 & -0.732122000 \\ \mathrm{C} & -5.787294000 & -2.074651000 & -0.635967000 \\ \mathrm{C} & -5.951998000 & -1.590068000 & 0.679283000 \\ \mathrm{H} & -5.361192000 & -2.024916000 & 1.488718000 \\ \mathrm{C} & -7.478602000 & -0.466549000 & -1.380194000 \\ \mathrm{C} & -1.275005000 & -4.418144000 & -0.157494000 \\ \mathrm{C} & -7.624935000 & 0.019701000 & -0.072406000 \\ \mathrm{C} & -3.508055000 & -3.195160000 & -0.436481000 \\ \mathrm{C} & -6.855497000 & -0.552328000 & 0.952534000\end{array}$




\begin{tabular}{|c|c|c|c|}
\hline & & & \\
\hline C & 000 & 40719000 & \\
\hline & 5937000 & 978781000 & \\
\hline & 339085000 & -4.711583000 & 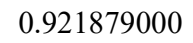 \\
\hline & 6.567722000 & 8719000 & \\
\hline $\mathrm{H}$ & 1546000 & 08000 & \\
\hline 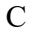 & 0.257089000 & 6000 & \\
\hline $\mathrm{C}$ & -3.125110000 & & \\
\hline $\mathrm{H}$ & -2.46 & 000 & \\
\hline $\mathrm{C}$ & 000 & 000 & \\
\hline$C$ & -4.42012 & 000 & -2 \\
\hline $\mathrm{H}$ & -4.775 & 2000 & -2. \\
\hline $\mathrm{C}$ & -0.1836 & 000 & -0.9 \\
\hline$\pi$ & -0.3400 & 8000 & -2.05976 \\
\hline C & -5.271564000 & -4.244722000 & -1.77371700 \\
\hline 11 & -6.29 & 8000 & \\
\hline INI & 1.96 & & -1.2 \\
\hline C & -2.0 & & \\
\hline $\mathrm{H}$ & & & \\
\hline$C$ & & & \\
\hline 11 & & & \\
\hline C & 0.21 & & \\
\hline$C$ & -0.5 & & \\
\hline C & -0.3 & & \\
\hline $\mathrm{H}$ & -0.0 & & \\
\hline $\mathrm{C}$ & -1.7 & & \\
\hline$C$ & -1.5 & & \\
\hline 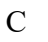 & -2.4 & & \\
\hline 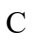 & -2.1 & & \\
\hline $\mathrm{H}$ & -3.1 & & \\
\hline $\mathrm{O}$ & & & -0.6 \\
\hline$c$ & & & \\
\hline $\mathrm{C}$ & & & \\
\hline $\mathrm{H}$ & & & \\
\hline $\mathrm{H}$ & & & \\
\hline $\mathrm{C}$ & 00 & & \\
\hline $\mathrm{C}$ & & & \\
\hline $\mathrm{C}$ & 4.16 & & \\
\hline $\mathrm{H}$ & & & \\
\hline $\mathrm{H}$ & 4.11 & & \\
\hline $\mathrm{H}$ & & & \\
\hline $\mathrm{H}$ & & & \\
\hline $\mathrm{H}$ & & & \\
\hline $\mathrm{H}$ & & & \\
\hline $\mathrm{H}$ & & & \\
\hline $\mathrm{H}$ & & & \\
\hline $\mathrm{H}$ & & & \\
\hline $\mathrm{H}$ & -3 & & \\
\hline $\mathrm{H}$ & & & \\
\hline $\mathrm{H}$ & -2.1 & & \\
\hline 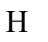 & & & 0 \\
\hline$H$ & & & \\
\hline 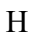 & & & 03 \\
\hline 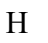 & & & \\
\hline a & & & -1.3 \\
\hline 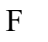 & & & \\
\hline $\mathrm{F}$ & & & -1.0 \\
\hline $\mathrm{F}$ & & & -2.6 \\
\hline 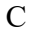 & & & \\
\hline$\Gamma$ & 00 & 000 & 3.5 \\
\hline$\Gamma$ & -0.42 & 000 & 3.9 \\
\hline $\mathrm{F}$ & 1.71 & 000 & 3.6 \\
\hline $\mathrm{C}$ & 00 & & \\
\hline $\mathrm{F}$ & 0 & -0.2 & -1.2 \\
\hline $\mathrm{F}$ & & & -0.5 \\
\hline 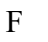 & & & -2.3 \\
\hline 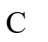 & & & \\
\hline & 2.868881000 & -1.971992000 & 2.17026500 \\
\hline
\end{tabular}

$\begin{array}{cc}\text { F } & 4.589932000 \\ \text { F } & 4.475630000 \\ \text { C } & -0.253800000 \\ \text { F } & -1.339277000 \\ \text { F } & -0.498784000 \\ \text { F } & 0.805405000 \\ \text { C } & -1.786791000 \\ \text { F } & -2.830278000 \\ \text { F } & -1.309156000 \\ \text { F } & -2.282718000 \\ \text { C } & -6.944545000 \\ \text { F } & -8.000738000 \\ \text { F } & -7.061239000 \\ \text { F } & -5.814328000 \\ \text { C } & -8.339882000 \\ \text { F } & -8.716333000 \\ \text { F } & -7.692623000 \\ \text { F } & -9.485382000\end{array}$

$-3.342770000$ $-1.666962000$ 7.782021000 8.470113000 7.434237000 8.641518000 4.153512000 4.990237000 3.726953000 3.057309000 $-0.030583000$ 0.808008000 $-1.043912000$ 0.667482000 0.091740000 1.378084000 0.073636000 $-0.635889000$

2.212010000 3.628163000 1.355698000 0.909187000 2.652396000 1.370251000 $-1.816861000$ $-2.063668000$ $-3.025843000$ $-1.179874000$ 2.367873000 2.548113000 3.271957000 2.709202000 $-2.490431000$ $-2.244011000$ $-3.690432000$ $-2.647565000$

\section{${ }^{\text {CF3 }}$ TS-2 $2_{\text {decoorar }}$}

Zero-point correction $=\quad 0.809101($ Hartree/Particle $)$

Thermal correction to Energy $=\quad 0.889862$

Thermal correction to Enthalpy $=\quad 0.890807$

Thermal correction to Gibbs Free Energy= $\quad 0.675601$

Sum of electronic and zero-point Energies $=\quad-4981.403713$

Sum of electronic and thermal Energies $=\quad-4981.322951$

Sum of electronic and thermal Enthalpies $=\quad-4981.322007$

Sum of electronic and thermal Free Energies $=-4981.537213$

E solvent $=-4981.39981639$

$\begin{array}{lrrr}\mathrm{N} & 2.336255000 & 1.882835000 & 0.061844000 \\ \mathrm{C} & 4.603691000 & 2.006112000 & -0.661018000 \\ \mathrm{C} & 2.288588000 & 4.999357000 & 0.103879000 \\ \mathrm{C} & 1.140506000 & 4.873276000 & -0.706770000 \\ \mathrm{H} & 1.112373000 & 4.098771000 & -1.478221000 \\ \mathrm{C} & 1.207952000 & 6.868756000 & 1.252850000 \\ \mathrm{C} & 4.556748000 & 0.517010000 & -0.793395000 \\ \mathrm{C} & 0.066103000 & 6.729533000 & 0.450296000 \\ \mathrm{C} & 3.450907000 & 2.711681000 & -0.217077000 \\ \mathrm{C} & 0.042093000 & 5.726548000 & -0.532717000 \\ \mathrm{C} & 3.486255000 & 4.136947000 & -0.134934000 \\ \mathrm{C} & 4.135695000 & -0.314797000 & 0.313603000 \\ \mathrm{H} & 3.812759000 & 0.154790000 & 1.248388000 \\ \mathrm{C} & 5.344831000 & -2.226251000 & -0.642035000 \\ \mathrm{C} & 2.310991000 & 6.013767000 & 1.083186000 \\ \mathrm{H} & 3.185565000 & 6.121603000 & 1.733243000 \\ \mathrm{C} & 4.598994000 & -1.669186000 & 0.384523000 \\ \mathrm{C} & 5.795067000 & 2.700831000 & -0.935162000 \\ \mathrm{H} & 6.686099000 & 2.139561000 & -1.236988000 \\ \mathrm{C} & 5.677093000 & -1.437461000 & -1.779545000 \\ \mathrm{C} & 5.853634000 & 4.095030000 & -0.795865000 \\ \mathrm{H} & 6.782806000 & 4.633305000 & -1.009642000 \\ \mathrm{C} & 5.299023000 & -0.100904000 & -1.848778000 \\ \mathrm{H} & 5.602239000 & 0.503992000 & -2.709019000 \\ \mathrm{C} & 4.705765000 & 4.796925000 & -0.411744000 \\ \mathrm{H} & 4.725321000 & 5.891749000 & -0.366145000 \\ \mathrm{~N} & -3.096158000 & -1.966122000 & 0.609184000 \\ \mathrm{C} & -2.887303000 & -4.374293000 & 0.033109000 \\ \mathrm{C} & -5.547904000 & -1.738485000 & -0.857224000 \\ \mathrm{C} & -6.049338000 & -1.290800000 & -2.096618000 \\ \mathrm{H} & -5.839761000 & -1.865232000 & -3.004602000 \\ \mathrm{C} & -6.579512000 & 0.210584000 & 0.200900000 \\ \mathrm{C} & -1.639771000 & -4.566758000 & 0.828573000 \\ \mathrm{C} & -7.075534000 & 0.650630000 & -1.037832000 \\ \mathrm{C} & -3.563346000 & -3.117347000 & -0.044962000 \\ \mathrm{C} & -6.807618000 & -0.110535000 & -2.184271000 \\ \mathrm{C} & -4.792703000 & -3.022973000 & -0.769648000 \\ \mathrm{C} & -1.515554000 & -4.080528000 & 2.150112000 \\ \mathrm{H} & -2.338704000 & -3.517048000 & 2.598114000 \\ & & & \end{array}$




\begin{tabular}{|c|c|c|c|}
\hline & & & \\
\hline & 000 & 9488000 & 000 \\
\hline & 000 & 304901000 & $\cos x$ \\
\hline & & 20894000 & \\
\hline & & 7794000 & (1) \\
\hline & 4000 & 5000 & 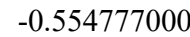 \\
\hline & 000 & 00 & \\
\hline & .613204000 & -5. & \\
\hline & -5.023437000 & -6.26 & \\
\hline & -0.5657 & & \\
\hline & 0000 & -5. & -0 . \\
\hline & -5.285306000 & 9000 & 000 \\
\hline & -6.228706000 & 53000 & 00 \\
\hline & 2.370385000 & 0.070985000 & -0.80201800 \\
\hline & -1.945979000 & -1.442053000 & 0.31096300 \\
\hline & -1.299657000 & -1.866267000 & -0.478221000 \\
\hline & 1.457302000 & & \\
\hline & & & \\
\hline & 0.262 & & 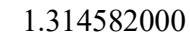 \\
\hline & -0.58 & & \\
\hline & -0.1 & & 0. \\
\hline & -0.2 & & \\
\hline & -1.82 & & \\
\hline & -1.4 & -0.2 & \\
\hline & -2.7 & & \\
\hline & -2.22 & & \\
\hline & -3.2 & & \\
\hline & 0.5 & & \\
\hline & 2.3 & & \\
\hline & 1.2 & & \\
\hline & 3.30 & & \\
\hline & 2.17 & & \\
\hline & 1.19 & & \\
\hline & & & \\
\hline & 0000 & & \\
\hline & 3000 & & \\
\hline & 7000 & & -3 \\
\hline & 1.089205000 & -4.0 & 765000 \\
\hline & -3.759894000 & & \\
\hline & -2.73 & & \\
\hline & -2.375159000 & & \\
\hline & & & \\
\hline & & & \\
\hline & & & \\
\hline & & & 000 \\
\hline & & & \\
\hline & & & \\
\hline & & & \\
\hline & & & \\
\hline & 0 & & \\
\hline & 0 & & -3 \\
\hline & & & \\
\hline & & & \\
\hline & & & \\
\hline & & 0 & \\
\hline & & & -3.534508000 \\
\hline & & & 7000 \\
\hline & & & 2000 \\
\hline & 2000 & -0.6 & 57000 \\
\hline$C$ & 000 & & 97000 \\
\hline & -7.98 & & 784000 \\
\hline & 8000 & 00 & 13000 \\
\hline & -5.82 & 1.9 & 22000 \\
\hline & & & \\
\hline & & & \\
\hline & & & \\
\hline & & & -1.766012000 \\
\hline & 1.281892000 & 7.975486000 & 2.28348400 \\
\hline
\end{tabular}

$\begin{array}{cccc}\text { F } & 0.045868000 & 8.395410000 & 2.667668000 \\ \text { F } & 1.949810000 & 7.572277000 & 3.401815000 \\ \text { F } & 1.945417000 & 9.061879000 & 1.793690000 \\ \text { C } & 6.387780000 & -2.090222000 & -2.940896000 \\ \text { F } & 7.024362000 & -1.179638000 & -3.729545000 \\ \text { F } & 7.315285000 & -2.993763000 & -2.517412000 \\ \text { F } & 5.508481000 & -2.766971000 & -3.739658000 \\ \text { C } & 4.247408000 & -2.474015000 & 1.613219000 \\ \text { F } & 2.908792000 & -2.749653000 & 1.658771000 \\ \text { F } & 4.908491000 & -3.660063000 & 1.663764000 \\ \text { F } & 4.542692000 & -1.784478000 & 2.754153000 \\ \text { C } & 1.749604000 & -6.266654000 & 0.383668000 \\ \text { F } & 2.400311000 & -5.462738000 & -0.515138000 \\ \text { F } & 2.675416000 & -6.689315000 & 1.285447000 \\ \text { F } & 1.320964000 & -7.358346000 & -0.312996000\end{array}$

\section{CF31-Pr-C}

Zero-point correction $=\quad 0.757373($ Hartree/Particle $)$

Thermal correction to Energy= $\quad 0.834620$

Thermal correction to Enthalpy= $\quad 0.835564$

Thermal correction to Gibbs Free Energy= $\quad 0.626387$

Sum of electronic and zero-point Energies $=\quad-4902.878630$

Sum of electronic and thermal Energies $=\quad-4902.801383$

Sum of electronic and thermal Enthalpies $=\quad-4902.800439$

Sum of electronic and thermal Free Energies $=\quad-4903.009615$

E solvent $=-4902.84852681$

N

c

th

c

(a)

(a)

$\mathrm{C}$

c

C

c

H

2.040369000

\subsection{0}

5.131461000

4.865345000

4.043512000

7.009409000

0.777721000

6.737456000

2.923520000

5.659566000

4.348184000

$-0.052200000$

0.420227000

$-1.938299000$

6.214903000

6.434533000

$-1.395599000$

3.030617000

2.516161000

$-1.150682000$

4.431731000

5.019859000

0.169057000

0.769464000

5.073848000

6.167774000

$-2.062933000$

$-4.389548000$

$-2.022867000$

$-1.360366000$

$-1.703449000$

$-0.476644000$

$-4.512168000$

0.180470000

$-3.215904000$

$-0.270966000$

$-3.210317000$

$-4.170006000$

$-3.747806000$

$-4.889108000$

$-1.562777000$

$-2.042208000$
0.096180000

$-0.692466000$

$-0.084810000$

$-0.869216000$

$-1.591561000$

0.932906000

$-0.776006000$

0.157240000

$-0.262153000$

$-0.745865000$

$-0.279640000$

0.359722000

1.301910000

$-0.620140000$

0.814402000

1.434713000

0.423443000

$-1.028908000$

$-1.320464000$

$-1.775835000$

$-0.973546000$

$-1.236980000$

$-1.851789000$

$-2.734119000$

$-0.619220000$

$-0.647889000$

0.510593000

$-0.231107000$

$-0.899029000$

0.306069000

1.243086000

$-2.082239000$

0.570658000

$-0.884331000$

$-0.210826000$

0.308655000

$-0.916444000$

1.940338000

2.413114000

2.110055000

$-2.091769000$

$-3.042912000$ 


\begin{tabular}{|c|c|c|c|}
\hline & & & \\
\hline $\mathrm{C}$ & 000 & 5000 & \\
\hline & -2.254410000 & 407426000 & \\
\hline & 1.133409000 & -5.228295000 & 4046 \\
\hline & -4.064980000 & & 7200 \\
\hline $\mathrm{H}$ & 921000 & & \\
\hline 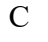 & 00 & 11000 & -0.01 \\
\hline $\mathrm{H}$ & 000 & -5.31 & \\
\hline C & 523000 & & \\
\hline $\mathrm{H}$ & -5.83554 & -4.327516000 & \\
\hline $\mathrm{Ni}$ & 206000 & 000 & \\
\hline $\mathrm{C}$ & -1.806312000 & 8000 & \\
\hline 1 & -1.131609000 & -1.784958000 & -0 \\
\hline & 1.38 & 2.366936000 & \\
\hline 11 & 1.48 & 3.327297000 & 1.52 \\
\hline & 0.23 & 561000 & \\
\hline 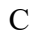 & -0.6 & & \\
\hline & -0.1( & & \\
\hline & -0.3 & & \\
\hline & & & \\
\hline C & -1.3 & -0.2 & \\
\hline C & -2.7 & & \\
\hline$C$ & -2.2 & & \\
\hline $\mathrm{H}$ & -3.1 & 0 & \\
\hline $\mathrm{O}$ & & 0 & \\
\hline $\mathrm{C}$ & & & \\
\hline 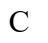 & & & \\
\hline $\mathrm{H}$ & & & \\
\hline$\Pi$ & & & \\
\hline $\mathrm{C}$ & & & \\
\hline $\mathrm{H}$ & & & \\
\hline $\mathrm{H}$ & & -0.0 & \\
\hline $\mathrm{H}$ & & & \\
\hline $\mathrm{H}$ & 0 & & \\
\hline $\mathrm{H}$ & & -2.8 & \\
\hline $\mathrm{H}$ & 0 & & \\
\hline $\mathrm{H}$ & -2.7 & & \\
\hline $\mathrm{H}$ & -2.5 & & \\
\hline 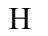 & & & \\
\hline $\mathrm{H}$ & -7.9 & & \\
\hline $\mathrm{H}$ & & & \\
\hline $\mathrm{H}$ & & & \\
\hline $\mathrm{C}$ & & -1 & \\
\hline $\mathrm{F}$ & & & \\
\hline $\mathrm{F}$ & & 0 & \\
\hline 1 & & U & \\
\hline C & & & \\
\hline $\mathrm{F}$ & & & \\
\hline $\mathrm{F}$ & & & \\
\hline $\mathrm{F}$ & & & \\
\hline 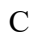 & & & \\
\hline $\mathrm{F}$ & & & \\
\hline 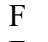 & & & 27 \\
\hline 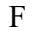 & & & \\
\hline $\mathrm{C}$ & & & 000 \\
\hline te & & & 4000 \\
\hline 1 & & & 0000 \\
\hline $\mathrm{F}$ & & $9.0^{\prime}$ & 000 \\
\hline 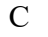 & & & 00( \\
\hline $\mathrm{F}$ & -8.08 & 7000 & 6000 \\
\hline 1 & & 2000 & 000 \\
\hline$\Gamma$ & & 36000 & 300( \\
\hline $\mathrm{C}$ & & 62000 & -3 \\
\hline $\mathrm{F}$ & & & \\
\hline $\mathrm{F}$ & & & \\
\hline $\mathrm{F}$ & & & \\
\hline & & & \\
\hline & 3.092263000 & -4.679239000 & -0.4881990 \\
\hline
\end{tabular}

$\begin{array}{cccc}\text { F } & 3.237772000 & -6.329592000 & 0.949822000 \\ \text { F } & 2.125771000 & -6.596121000 & -0.929491000 \\ \text { C } & -0.027569000 & -4.057433000 & 4.180523000 \\ \text { F } & -0.773401000 & -2.949653000 & 4.453392000 \\ \text { F } & -0.574205000 & -5.087815000 & 4.890764000 \\ \text { F } & 1.222587000 & -3.846781000 & 4.678511000\end{array}$

\section{${ }^{\text {CF3 TS-2 }}$ openAr}

Zero-point correction $=0.756036$ (Hartree/Particle)

Thermal correction to Energy $=\quad 0.832563$

Thermal correction to Enthalpy $=\quad 0.833508$

Thermal correction to Gibbs Free Energy= $\quad 0.626917$

Sum of electronic and zero-point Energies $=\quad-4902.873138$

Sum of electronic and thermal Energies $=\quad-4902.796611$

Sum of electronic and thermal Enthalpies $=\quad-4902.795667$

Sum of electronic and thermal Free Energies $=-4903.002257$

E solvent $=-4902.84584654$

\begin{tabular}{|c|c|c|c|}
\hline & 8097000 & -0.043798000 & \\
\hline & 4.226457000 & 1.444387000 & \\
\hline & 5.142119000 & -1.968899000 & -0 \\
\hline & 5.617136000 & -3.183596000 & \\
\hline & 5.926045000 & -3.229866000 & -2 \\
\hline & 4.835264000 & -3.092834000 & 0 \\
\hline & 3.139212000 & 2.472253000 & 750 \\
\hline & 5.309108000 & -4.300321000 & 5500 \\
\hline & 4.058266000 & & \\
\hline & & & \\
\hline & & & \\
\hline & 1.77 & & \\
\hline & 1.50 & & \\
\hline & 1.21 & & \\
\hline & 4.7 & -1.9 & \\
\hline & 4.3 & & \\
\hline & 0.8 & & \\
\hline & 5.4 & & \\
\hline & 5.5 & & \\
\hline & 0 & & \\
\hline & 0 & & \\
\hline & & & \\
\hline & & & \\
\hline & 4.5 & & \\
\hline & 000 & -0.3 & -2 \\
\hline & 7.200 & -1.0 & -2 \\
\hline & -3.48 & -0.6 & 7500 \\
\hline & -5.73 & & \\
\hline & -2.88 & & \\
\hline & -2.28 & & 00 \\
\hline & -2.73 & & \\
\hline & & & \\
\hline & & & \\
\hline & & & \\
\hline & & & \\
\hline & & & \\
\hline & & & \\
\hline & & & \\
\hline & -5 & & 0.8 \\
\hline & -6. & -2 & -2.2 \\
\hline & -2.2 & & 3.7 \\
\hline & -2.7 & & 3.6 \\
\hline & -6.07 & 000 & -1.0 \\
\hline & & & 1.3 \\
\hline & & & \\
\hline & & & \\
\hline & & & \\
\hline & -7.2 & 000 & 2. \\
\hline & -6.707 & 01000 & \\
\hline & 44278000 & 0.820861000 & \\
\hline & & & a \\
\hline
\end{tabular}




\begin{tabular}{|c|c|c|c|}
\hline $\mathrm{H}$ & -5.014858000 & 2.842776000 & 3.773378000 \\
\hline $\mathrm{Ni}$ & 1.829403000 & 1.501651000 & -0.537402000 \\
\hline $\mathrm{C}$ & -2.337623000 & -0.205638000 & 0.590500000 \\
\hline $\mathrm{H}$ & -2.080017000 & 0.865822000 & 0.515134000 \\
\hline $\mathrm{C}$ & 2.210695000 & -1.234493000 & -1.336885000 \\
\hline $\mathrm{H}$ & 2.772129000 & -2.041221000 & -1.835169000 \\
\hline $\mathrm{C}$ & 0.904824000 & -1.585095000 & -0.866060000 \\
\hline $\mathrm{C}$ & 0.528445000 & -2.953893000 & -0.989439000 \\
\hline $\mathrm{C}$ & -0.035736000 & -0.628406000 & -0.309562000 \\
\hline $\mathrm{H}$ & 1.259739000 & -3.647226000 & -1.428014000 \\
\hline $\mathrm{C}$ & -0.712213000 & -3.443190000 & -0.577575000 \\
\hline $\mathrm{C}$ & -1.329971000 & -1.143139000 & 0.095245000 \\
\hline $\mathrm{C}$ & -1.073369000 & -4.910581000 & -0.691092000 \\
\hline $\mathrm{C}$ & -1.625767000 & -2.507918000 & -0.043309000 \\
\hline $\mathrm{H}$ & -2.628534000 & -2.838892000 & 0.251632000 \\
\hline $\mathrm{O}$ & 0.183584000 & 0.651905000 & -0.177271000 \\
\hline $\mathrm{C}$ & 1.283831000 & 3.056923000 & 0.439045000 \\
\hline $\mathrm{C}$ & 2.702702000 & 3.121738000 & 0.939860000 \\
\hline $\mathrm{H}$ & 0.968473000 & 3.849153000 & -0.256932000 \\
\hline $\mathrm{H}$ & 0.512348000 & 2.799208000 & 1.177886000 \\
\hline $\mathrm{C}$ & 2.900438000 & 2.843744000 & 2.439259000 \\
\hline $\mathrm{H}$ & 3.326193000 & 2.327560000 & 0.370715000 \\
\hline $\mathrm{H}$ & 3.198995000 & 4.055186000 & 0.609632000 \\
\hline $\mathrm{H}$ & 3.970580000 & 2.814459000 & 2.710163000 \\
\hline $\mathrm{H}$ & 2.410830000 & 3.630746000 & 3.036763000 \\
\hline $\mathrm{H}$ & 2.446878000 & 1.876601000 & 2.718360000 \\
\hline $\mathrm{H}$ & 5.387490000 & -5.194377000 & 1.318221000 \\
\hline $\mathrm{H}$ & 0.426619000 & 1.546185000 & 5.408370000 \\
\hline $\mathrm{H}$ & -6.969794000 & -3.203148000 & -3.067948000 \\
\hline $\mathrm{H}$ & 0.482365000 & 5.346216000 & -2.974377000 \\
\hline $\mathrm{C}$ & -0.488590000 & 4.001738000 & 4.560745000 \\
\hline $\mathrm{F}$ & -1.367960000 & 4.852513000 & 5.160899000 \\
\hline $\mathrm{F}$ & 0.626065000 & 3.942701000 & 5.341320000 \\
\hline $\mathrm{F}$ & -0.121916000 & 4.590993000 & 3.379280000 \\
\hline $\mathrm{C}$ & -0.385816000 & -1.024094000 & 5.014436000 \\
\hline $\mathrm{F}$ & 0.202909000 & -0.849486000 & 6.232479000 \\
\hline $\mathrm{F}$ & -1.243676000 & -2.075799000 & 5.116828000 \\
\hline $\mathrm{F}$ & 0.602320000 & -1.391975000 & 4.146782000 \\
\hline $\mathrm{C}$ & -5.781288000 & -4.423746000 & -0.930384000 \\
\hline $\mathrm{F}$ & -6.820955000 & -5.085644000 & -0.347734000 \\
\hline $\mathrm{F}$ & -5.536952000 & -5.034093000 & -2.123502000 \\
\hline $\mathrm{F}$ & -4.686364000 & -4.635096000 & -0.132827000 \\
\hline $\mathrm{C}$ & -7.681435000 & -0.619040000 & -3.647654000 \\
\hline $\mathrm{F}$ & -8.605685000 & 0.347453000 & -3.376021000 \\
\hline $\mathrm{F}$ & -6.756553000 & -0.057645000 & -4.479365000 \\
\hline $\mathrm{F}$ & -8.309147000 & -1.603331000 & -4.350044000 \\
\hline $\mathrm{C}$ & 2.937561000 & 6.274358000 & -2.137112000 \\
\hline $\mathrm{F}$ & 2.022103000 & 7.179401000 & -2.572218000 \\
\hline $\mathrm{F}$ & 3.087675000 & 6.464047000 & -0.788243000 \\
\hline $\mathrm{F}$ & 4.136431000 & 6.586479000 & -2.707926000 \\
\hline $\mathrm{C}$ & -0.580316000 & 2.881164000 & -3.389735000 \\
\hline $\mathrm{F}$ & -1.403614000 & 2.934556000 & -2.302721000 \\
\hline $\mathrm{F}$ & -1.069684000 & 3.773311000 & -4.298462000 \\
\hline $\mathrm{F}$ & -0.697287000 & 1.636114000 & -3.927014000 \\
\hline $\mathrm{C}$ & 4.367112000 & -3.056558000 & 2.674680000 \\
\hline $\mathrm{F}$ & 3.074168000 & -3.472297000 & 2.779746000 \\
\hline $\mathrm{F}$ & 5.117619000 & -3.874435000 & 3.464941000 \\
\hline $\mathrm{F}$ & 4.433569000 & -1.800421000 & 3.197129000 \\
\hline $\mathrm{C}$ & 6.153528000 & -5.640759000 & -1.272903000 \\
\hline $\mathrm{F}$ & 5.111748000 & -6.297415000 & -1.861127000 \\
\hline $\mathrm{F}$ & 7.091485000 & -5.435218000 & -2.240712000 \\
\hline $\mathrm{F}$ & 6.689498000 & -6.482428000 & -0.347659000 \\
\hline $\mathrm{H}$ & -2.156755000 & -5.043983000 & -0.851469000 \\
\hline $\mathrm{H}$ & -0.538439000 & -5.396941000 & -1.525788000 \\
\hline $\mathrm{H}$ & -0.811622000 & -5.462752000 & 0.232436000 \\
\hline
\end{tabular}

\section{${ }^{\mathrm{CF}} \mathbf{1}-\boldsymbol{\beta}-\mathrm{C}$}

Zero-point correction=

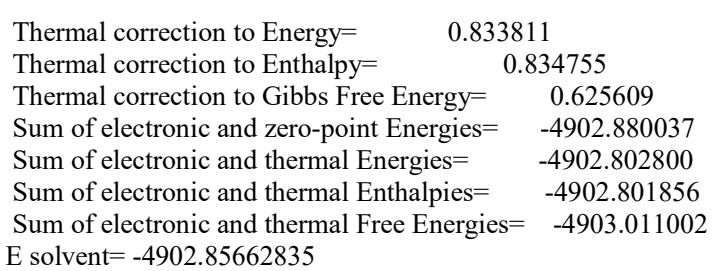

E solvent $=-4902.85662835$

1.484036000

$\mathrm{N} \quad 2.586798000 \quad 1.307955000 \quad-0.893576000$

$\begin{array}{llll}\text { C } & 2.006786000 & 0.697086000 & -1.916582000\end{array}$

$\begin{array}{llll}\text { C } & 0.743266000 & 0.027001000 & -1.901932000\end{array}$

C $\quad-0.070132000 \quad-0.091844000 \quad-0.711027000$

$\begin{array}{llll}\text { O } & 0.263541000 & 0.366173000 & 0.466824000\end{array}$

$\begin{array}{llll}\text { C } & 2.671090000 & 2.157247000 & 2.691681000\end{array}$

$\begin{array}{llll}\mathrm{H} & 2.606815000 & 3.249668000 & 2.848074000\end{array}$

$\begin{array}{llll}\text { C } & 3.815257000 & 1.508225000 & 3.489554000\end{array}$

$\begin{array}{llll}\mathrm{H} & 4.798936000 & 1.930973000 & 3.219757000\end{array}$

$\mathrm{H} \quad 3.651364000 \quad 1.679653000 \quad 4.567513000$

$\begin{array}{llll}\mathrm{H} & 3.843864000 & 0.418513000 & 3.319963000\end{array}$

$\begin{array}{llll}\mathrm{H} & 3.083196000 & 2.161106000 & 1.556835000\end{array}$

$\begin{array}{llll}\mathrm{H} & 0.451590000 & 2.109583000 & 2.831316000\end{array}$

$\begin{array}{llll}\mathrm{H} & 1.285035000 & 0.508981000 & 3.226351000\end{array}$

H $\quad 2.528754000 \quad 0.724077000 \quad-2.888792000$

C $\quad 0.274891000 \quad-0.514734000 \quad-3.133529000$

$\mathrm{H} \quad 0.915346000 \quad-0.400552000 \quad-4.019564000$

C $\quad-0.953936000 \quad-1.166108000 \quad-3.256833000$

$\begin{array}{llll}\text { C } & -1.429590000 & -1.742927000 & -4.575116000\end{array}$

$\mathrm{H} \quad-1.175597000 \quad-2.817287000 \quad-4.661627000$

$\mathrm{H} \quad-0.968130000 \quad-1.226073000 \quad-5.434787000$

$\mathrm{H} \quad-2.526070000 \quad-1.661256000 \quad-4.672717000$

C $\quad-1.745475000 \quad-1.274549000 \quad-2.091633000$

$\begin{array}{llll}\mathrm{H} & -2.731329000 & -1.751455000 & -2.148092000\end{array}$

$\begin{array}{llll}\text { C } & -1.343580000 & -0.768614000 & -0.846535000\end{array}$

$\begin{array}{llll}\text { C } & -2.202703000 & -0.889841000 & 0.329311000\end{array}$

$\mathrm{N} \quad-3.314568000 \quad-1.561997000 \quad 0.311522000$

$\mathrm{H} \quad-1.853496000 \quad-0.366123000 \quad 1.237064000$

$\begin{array}{llll}\text { C } & -4.090187000 & -1.674308000 & 1.476825000\end{array}$

$\begin{array}{llll}\text { C } & -3.575930000 & -2.230616000 & 2.689875000\end{array}$

$\begin{array}{llll}\text { C } & -4.416235000 & -2.303098000 & 3.822239000\end{array}$

$\begin{array}{llll}\text { C } & -5.738729000 & -1.848032000 & 3.779143000\end{array}$

$\begin{array}{llll}\text { C } & -6.249456000 & -1.329506000 & 2.581506000\end{array}$

$\begin{array}{llll}\mathrm{C} & -5.454421000 & -1.248749000 & 1.421702000\end{array}$

$\begin{array}{llll}\text { C } & -6.040882000 & -0.679182000 & 0.173160000\end{array}$

$\begin{array}{llll}\text { C } & -5.864433000 & -1.310212000 & -1.078205000\end{array}$

$\begin{array}{llll}\text { C } & -7.214709000 & 0.397585000 & -2.180336000\end{array}$

$\begin{array}{llll}\text { C } & -7.406579000 & 1.022038000 & -0.940154000\end{array}$

$\begin{array}{llll}\text { C } & -6.823572000 & 0.492389000 & 0.224140000\end{array}$

$\begin{array}{llll}\text { C } & -2.191950000 & -2.781102000 & 2.792885000\end{array}$

$\begin{array}{llll}\text { C } & -1.686430000 & -3.688380000 & 1.836985000\end{array}$

$\begin{array}{llll}\text { C } & -0.403092000 & -4.238229000 & 1.977120000\end{array}$

$\begin{array}{llll}\text { C } & 0.407728000 & -3.895223000 & 3.069605000\end{array}$

$\begin{array}{llll}\text { C } & -0.089526000 & -2.997764000 & 4.027391000\end{array}$

$\begin{array}{llll}\text { C } & -1.374382000 & -2.447650000 & 3.894021000\end{array}$

$\begin{array}{llll}\text { C } & -6.439195000 & -0.772660000 & -2.238245000\end{array}$

$\begin{array}{llll}\text { C } & 5.045007000 & -3.185550000 & -1.716100000\end{array}$

C $\quad 4.510812000 \quad-2.528094000 \quad-0.597124000$

C $\quad 4.459591000 \quad-1.127161000 \quad-0.547985000$

$\begin{array}{llll}\text { C } & 4.950513000 & -0.351499000 & -1.618981000\end{array}$

$\begin{array}{llll}\text { C } & 5.491087000 & -1.018112000 & -2.738821000\end{array}$

$\begin{array}{llll}\text { C } & 5.533915000 & -2.421022000 & -2.786386000\end{array}$

C $\quad 4.981927000 \quad 1.141099000 \quad-1.544538000$

$\begin{array}{llll}\text { C } & 6.205501000 & 1.793893000 & -1.813313000\end{array}$

$\begin{array}{llll}\text { C } & 6.205501000 & 1.793893000 & -1.813313000 \\ \text { C } & 6.334334000 & 3.180971000 & -1.680635000\end{array}$

$\begin{array}{llll}\text { C } & 5.234424000 & 3.938559000 & -1.260713000\end{array}$

$\begin{array}{llll}\text { C } & 3.988774000 & 3.332189000 & -0.994106000\end{array}$

$\begin{array}{llll}\text { C } & 3.855473000 & 1.922749000 & -1.154119000\end{array}$ 


\begin{tabular}{|c|c|c|c|}
\hline & & & \\
\hline & 89721000 & 39802000 & $0 \Omega 0$ \\
\hline & 6063000 & 807000 & 5000 \\
\hline & 000 & & 000 \\
\hline & 000 & & 1000 \\
\hline & 29000 & & 1000 \\
\hline & & & \\
\hline & 0000 & 469000 & 7960 \\
\hline & 01000 & 31210000 & 0.3 \\
\hline & 563000 & & \\
\hline & 909000 & 564000 & \\
\hline & 887000 & 165000 & 4000 \\
\hline & 701000 & 7000 & 400 \\
\hline & -0.0 & & 3000 \\
\hline & 1.408754000 & -4.320628000 & 34000 \\
\hline & -1.740958000 & -1.741134000 & 406000 \\
\hline & -4.023128000 & -2.755410000 & 257000 \\
\hline & & 00 & \\
\hline & -7.2 & & \\
\hline & & & \\
\hline & & & -1 \\
\hline & & & \\
\hline & & & \\
\hline & & & \\
\hline & & & \\
\hline & & & \\
\hline & & & \\
\hline & & & \\
\hline & & & \\
\hline & & & \\
\hline & & & \\
\hline & & & \\
\hline & & & \\
\hline & & & -0 . \\
\hline & & & \\
\hline & & & \\
\hline & & & 18000 \\
\hline & 97000 & & 8000 \\
\hline & & & 6000 \\
\hline & 00 & & 9000 \\
\hline & 47000 & & 17000 \\
\hline & 86000 & & 53000 \\
\hline & & & 16000 \\
\hline & 29000 & 20000 & 5000 \\
\hline & & & 46000 \\
\hline & & & \\
\hline & & & \\
\hline & & & \\
\hline & & & \\
\hline $\mathrm{F}$ & & & \\
\hline 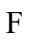 & & & \\
\hline $\mathrm{F}$ & & & \\
\hline 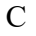 & & & -3 \\
\hline $\mathrm{F}$ & & & \\
\hline & & -2.116234 & \\
\hline & 8496 & -2.466015000 & -3.7272990 \\
\hline
\end{tabular}

\section{${ }^{\text {CF3 }}$ TS-1 $1_{\text {BHE-C }}$}

Zero-point correction=

Thermal correction to Energy=

0.754209 (Hartree/Particle)

Thermal correction to Enthalpy=

0.830859 0.831803

Thermal correction to Gibbs Free Energy $=\quad 0.625784$

Sum of electronic and zero-point Energies $=\quad-4902.868184$ Sum of electronic and thermal Energies $=\quad-4902.791533$

Sum of electronic and thermal Enthalpies $=\quad-4902.790589$

Sum of electronic and thermal Free Energies $=-4902.996608$ E solvent $=-4902.84395765$
$4.739408000 \quad 2.436037000$ $1.759625000 \quad 4.853159000$ $0.926284000 \quad 4.347156000$ 1.264861000 0.039836000 5.100516000 $-0.785985000$ 3.397822000 $-0.331452000$ 3.129821000 4.474859000 3.676948000 5.908342000 1.298715000 1.918840000 4.881469000 5.784683000 6.813960000 $-0.408456000$ $-0.033114000$ 0.136568000

$-1.762366000$ 6.535196000 5.527133000 6.352689000 $-1.174169000$ $-0.557410000$ $-2.395337000$ $-1.372452000$ 6.139840000 6.630224000 4.208837000 4.000086000 7.586452000 8.528792000 7.031993000 8.228756000 4.187575000 4.582631000 2.834204000

4.436876000 $-2.925689000$ $-2.476730000$ $-5.616937000$ $-5.766940000$ $-5.160854000$ $-7.318194000$ $-1.100404000$ $-7.450088000$ $-3.337110000$ $-6.667152000$ $-4.677761000$ $-0.007601000$ $-0.165193000$ 1.511140000 $-6.409390000$ $-6.303837000$ 1.281845000 $-2.955151000$ $-2.296839000$ $-8.097670000$ $-7.344748000$ $-8.498153000$ $-9.211632000$ 0.428804000 $-4.254041000$ $-4.611161000$ $-6.834687000$
0.022695000 $3.494297000-1.591969000$ $\begin{array}{ll}6.518622000 & 0.529928000\end{array}$ $0.987767000 \quad 0.417530000$ $6.005691000-0.483413000$ $2.900980000 \quad 0.320022000$ $4.918006000-1.242980000$ $\begin{array}{ll}4.299351000 & 0.246925000\end{array}$ $\begin{array}{ll}0.038105000 & 1.251236000\end{array}$ $\begin{array}{ll}0.345428000 & 1.932091000\end{array}$ $\begin{array}{ll}-1.736146000 & 0.379056000\end{array}$ $\begin{array}{ll}5.950506000 & 0.781778000\end{array}$ $6.351732000 \quad 1.589939000$ $\begin{array}{ll}-1.307264000 & 1.231387000\end{array}$ $3.382775000 \quad 0.478147000$ $3.021481000 \quad 0.581090000$ $\begin{array}{ll}7.729486000 & 1.319524000\end{array}$ $8.887418000 \quad 0.702657000$ $\begin{array}{ll}7.748188000 & 2.569082000\end{array}$ $7.769735000 \quad 1.457429000$ $-0.796706000 \quad-0.454430000$ $\begin{array}{ll}4.758111000 & 0.444135000\end{array}$ $\begin{array}{ll}5.475312000 & 0.500288000\end{array}$ $4.335976000-2.356635000$ $4.479454000 \quad-3.567229000$ $4.923600000 \quad-2.445656000$ $2.995069000 \quad-2.178799000$ $0.548547000-0.433070000$ $1.264522000-1.100310000$ $5.208112000 \quad 0.320162000$ $\begin{array}{ll}6.280879000 & 0.241869000\end{array}$ $-1.246272000-1.444776000$ $-0.281960000-1.649966000$ $-1.524846000 \quad-2.661266000$ $-2.371496000-1.028529000$ $-2.283102000 \quad 2.155988000$ $-3.570106000 \quad 1.934921000$ $-2.233917000 \quad 1.990909000$ $\begin{array}{ll}-1.998555000 & 3.464330000\end{array}$ $\begin{array}{ll}-2.195072000 & 0.323855000\end{array}$ $-4.249353000 \quad-0.998435000$ $\begin{array}{ll}-2.049043000 & -0.663270000\end{array}$ $\begin{array}{ll}-1.684569000 & 0.693561000\end{array}$ $-2.184680000 \quad 1.451622000$ $-0.383842000-1.239650000$ $-4.422472000 \quad-0.445386000$ $\begin{array}{ll}-0.018120000 & 0.106714000\end{array}$ $-3.184845000-0.584841000$ $\begin{array}{ll}-0.678289000 & 1.069219000\end{array}$ $-3.131435000-1.079373000$ $-4.651872000-1.310362000$ $-4.651580000 \quad-2.393268000$ $\begin{array}{ll}-4.847783000 & 0.589287000\end{array}$ $-1.386110000-1.622328000$ $-1.641400000-2.681881000$ $-4.855942000 \quad-0.796798000$ $-5.197945000-1.928011000$ $-6.024002000-2.221648000$ $0.351472000-2.306380000$ $\begin{array}{ll}1.327520000 & -2.892709000\end{array}$ $-0.489714000-3.303480000$ $0.952393000-1.802205000$ $\begin{array}{ll}-4.633467000 & 1.453186000\end{array}$ $-5.121298000 \quad-2.443359000$ $-5.873850000-3.154147000$ $\begin{array}{ll}-5.342390000 & 2.533422000\end{array}$ 


$\begin{array}{lrrr}\text { F } & -7.257587000 & 0.939186000 & 2.717524000 \\ \mathrm{~F} & -7.746024000 & -1.156601000 & 3.138372000 \\ \mathrm{~F} & -5.660599000 & -0.488742000 & 3.224535000 \\ \mathrm{C} & -0.862480000 & -4.418768000 & 0.944593000 \\ \mathrm{H} & -1.694990000 & -4.245061000 & 1.633548000 \\ \mathrm{C} & -5.106309000 & -4.095347000 & -2.012963000 \\ \mathrm{H} & -6.136655000 & -4.049258000 & -2.384211000 \\ \mathrm{C} & 0.628618000 & -4.600060000 & 2.952863000 \\ \mathrm{~F} & 0.391191000 & -3.356605000 & 3.457834000 \\ \mathrm{~F} & -0.234543000 & -5.450180000 & 3.583455000 \\ \mathrm{~F} & 1.890912000 & -4.956349000 & 3.315794000 \\ \mathrm{C} & 2.460532000 & -5.019137000 & -1.727392000 \\ \mathrm{~F} & 3.226628000 & -3.879593000 & -1.765583000 \\ \mathrm{~F} & 3.288215000 & -6.022645000 & -1.324132000 \\ \mathrm{~F} & 2.080122000 & -5.283570000 & -3.007129000 \\ \mathrm{Ni} & 2.046819000 & 0.768468000 & -1.170996000 \\ \mathrm{C} & -1.893976000 & -1.454342000 & 0.049997000 \\ \mathrm{H} & -1.339288000 & -1.562061000 & -0.899559000 \\ \mathrm{C} & 1.465392000 & 2.047855000 & 1.318301000 \\ \mathrm{H} & 1.718456000 & 2.785698000 & 2.098351000 \\ \mathrm{C} & 0.278703000 & 1.290880000 & 1.544730000 \\ \mathrm{C} & -0.461612000 & 1.555414000 & 2.732985000 \\ \mathrm{C} & -0.184635000 & 0.286839000 & 0.611083000 \\ \mathrm{H} & -0.086070000 & 2.333803000 & 3.412062000 \\ \mathrm{C} & -1.637304000 & 0.874629000 & 3.053438000 \\ \mathrm{C} & -1.396300000 & -0.427749000 & 0.961427000 \\ \mathrm{C} & -2.413508000 & 1.162137000 & 4.323060000 \\ \mathrm{C} & -2.080224000 & -0.114805000 & 2.145214000 \\ \mathrm{H} & -3.010475000 & -0.658425000 & 2.350137000 \\ \mathrm{O} & 0.424480000 & 0.011853000 & -0.503380000 \\ \mathrm{C} & 1.881208000 & -0.642411000 & -2.641910000 \\ \mathrm{C} & 2.351307000 & 0.513618000 & -3.274778000 \\ \mathrm{H} & 2.562600000 & -1.462599000 & -2.387953000 \\ \mathrm{H} & 0.810089000 & -0.866626000 & -2.618801000 \\ \mathrm{C} & 1.464546000 & 1.510770000 & -3.986839000 \\ \mathrm{H} & 3.409535000 & 0.540993000 & -3.567012000 \\ \mathrm{H} & 1.858944000 & 2.538247000 & -3.902992000 \\ \mathrm{H} & 1.438727000 & 1.263655000 & -5.067648000 \\ \mathrm{H} & 0.430061000 & 1.500993000 & -3.607111000 \\ \mathrm{H} & -3.500458000 & 1.065312000 & 4.155740000 \\ \mathrm{H} & -2.145903000 & 0.452546000 & 5.129914000 \\ \mathrm{H} & -2.212849000 & 2.180217000 & 4.699931000 \\ \mathrm{H} & 6.214344000 & -2.784726000 & 0.362889000 \\ \mathrm{H} & -8.146678000 & 0.770874000 & 0.401223000 \\ \mathrm{H} & -1.771367000 & 6.439541000 & -0.670483000 \\ & 2.517728000 & -4.988625000 & 0.988915000 \\ & 3.300518000 & 1.315812000 & -1.714893000\end{array}$

\section{CF3 1-BHE-C}

Zero-point correction $=0.754834($ Hartree/Particle $)$

Thermal correction to Energy

Thermal correction to Enthalpy= $\quad 0.833092$

Thermal correction to Gibbs Free Energy $=\quad 0.625077$

Sum of electronic and zero-point Energies $=\quad-4902.873800$

Sum of electronic and thermal Energies $=\quad-4902.796486$

Sum of electronic and thermal Enthalpies $=\quad-4902.795542$

Sum of electronic and thermal Free Energies $=-4903.003557$

E solvent $=-4902.84908147$

$\begin{array}{lrrr}\mathrm{Ni} & 2.062204000 & 0.842104000 & -1.276652000 \\ \mathrm{C} & 2.167154000 & -0.653775000 & -2.673788000 \\ \mathrm{~N} & 2.411795000 & 1.964570000 & 0.220718000 \\ \mathrm{C} & 1.588504000 & 2.064588000 & 1.257379000 \\ \mathrm{C} & 0.382146000 & 1.340258000 & 1.489994000 \\ \mathrm{C} & -0.099138000 & 0.318660000 & 0.580580000 \\ \mathrm{O} & 0.499943000 & 0.018720000 & -0.532383000 \\ \mathrm{C} & 1.553167000 & 0.436945000 & -3.295552000 \\ \mathrm{C} & 0.070553000 & 0.587247000 & -3.524819000 \\ \mathrm{H} & -0.158860000 & 0.311202000 & -4.574714000\end{array}$

H $\quad-0.259124000$

H 2.190439000

H $\quad 3.236969000$

H $\quad 1.566002000$

H $\quad 1.879087000$

C $\quad-0.345532000$

$\mathrm{H} \quad 0.044393000$

C $\quad-1.525802000$

C $\quad-2.291673000$

$\mathrm{H} \quad-3.381441000$

H $\quad-2.047201000$

H $\quad-2.058147000$

C $\quad-1.984440000$

$\mathrm{H} \quad-2.918821000$

C $\quad-1.312301000$

C -1.832631000

$\mathrm{N} \quad-2.894451000$

$\mathrm{H} \quad-1.270947000$

C $\quad-3.333296000$

C $\quad-4.660055000$

C $\quad-5.108434000$

C $\quad-4.291161000$

C $\quad-3.008734000$

$-2.512645000$

$-1.162417000$

$-0.979809000$

1.394962000

1.220976000

$-0.045076000$

$-5.562143000$

$-5.717891000$

$-6.584292000$

$-7.326996000$

$-7.188927000$

$-6.313836000$

0.282212000

$-0.280762000$

0.061405000

1.262641000

2.148148000

1.802009000

0.600452000

3.457509000

4.630787000

5.896814000

6.008798000

4.863809000

3.579194000

5.031050000

5.757010000

5.914637000

5.373479000

4.679231000

4.499322000

2.467641000

$-1.220214000$

1.521219000

4.536682000

6.794732000

6.994937000

6.175034000

5.482291000

$-7.997783000$

$-6.202958000$

$-6.127765000$

$-4.664369000$

$\mathrm{H} \quad-2.379710000$
$-0.057224000$

1.631788000

1.109611000

$-0.848000000$

$-1.468855000$

2.778137000

1.634699000

2.421462000

0.974469000

1.298711000

1.249565000

2.307747000

0.579331000

$-0.031224000$

$-0.560473000$

$-0.379171000$

$-1.419984000$

$-2.113210000$

$-1.579339000$

$-3.109841000$

$-3.010824000$

$-3.976860000$

$-5.046686000$

$-5.168423000$

$-4.220351000$

$-4.438444000$

$-4.443833000$

$-4.931028000$

$-4.930016000$

$-4.695300000$

$-1.883350000$

$-1.484729000$

$-0.437336000$

0.231658000

$-0.166721000$

$-1.210515000$

$-4.692847000$

6.319194000

5.218351000

4.525046000

4.918170000

6.028649000

6.722212000

4.224780000

5.009587000

4.419378000

3.022676000

2.203042000

2.807337000

0.718998000

0.131442000

$-1.261225000$

$-2.093584000$

$-1.510696000$

$-0.118538000$

6.343738000

6.853425000

3.674993000

6.100141000

5.044790000

2.547714000

0.768595000

$-3.178316000$

1.051962000

$-1.491657000$

$-3.896432000$

$-5.798078000$

$-6.027938000$
$-2.852851000$

$-3.390870000$

$-3.885772000$

$-2.808320000$

$-2.255072000$

2.047240000

2.679621000

3.340501000

3.023055000

4.289830000

4.119228000

4.664843000

5.098472000

2.140719000

2.363871000

0.960382000

0.077589000

0.360262000

$-0.860770000$

$-0.528854000$

$-1.052346000$

$-1.974569000$

$-2.364424000$

$-1.816810000$

$-0.896572000$

$-0.297132000$

1.103015000

0.838761000

$-0.552185000$

$-1.117105000$

$-0.675659000$

0.670733000

1.012014000

0.024348000

$-1.312333000$

$-1.660496000$

1.660929000

$-0.408949000$

$-1.209144000$

$-0.998038000$

0.026978000

0.825260000

0.606265000

0.215818000

0.282725000

0.359083000

0.359993000

0.316814000

0.256536000

0.287170000

$-0.769626000$

$-0.841968000$

0.150252000

1.220298000

1.286579000

1.635655000

$-0.571642000$

$-1.638222000$

0.231201000

0.400374000

0.413691000

$-1.555579000$

0.084679000

0.292040000

$-2.712899000$

$-2.369601000$

$-3.068125000$

$-2.076822000$ 


$\begin{array}{lrrr}\text { H } & -0.160528000 & -4.680733000 & -2.206472000 \\ \text { H } & -1.834795000 & -4.248947000 & 1.758019000 \\ \text { H } & -5.142981000 & -1.991333000 & 1.448466000 \\ \text { H } & 3.951120000 & 0.315269000 & 2.128424000 \\ \text { H } & 2.381557000 & -5.091598000 & 1.276457000 \\ \text { H } & 3.253263000 & 1.433010000 & -1.910579000 \\ \text { C } & 2.391424000 & -5.174563000 & -1.475707000 \\ \text { F } & 2.280280000 & -6.366667000 & -2.127766000 \\ \text { F } & 2.470543000 & -4.208037000 & -2.440876000 \\ \text { F } & 3.582990000 & -5.185061000 & -0.812739000 \\ \text { C } & 0.427989000 & -4.658944000 & 3.166965000 \\ \text { F } & 1.643900000 & -5.110181000 & 3.578654000 \\ \text { F } & 0.272727000 & -3.395391000 & 3.654016000 \\ \text { F } & -0.523099000 & -5.431009000 & 3.770852000 \\ \text { C } & 6.593155000 & -1.858773000 & -2.053207000 \\ \text { F } & 7.029379000 & -3.125946000 & -1.827194000 \\ \text { F } & 5.734257000 & -1.911095000 & -3.119031000 \\ \text { F } & 7.663143000 & -1.113113000 & -2.449279000 \\ \text { C } & 4.070973000 & -2.368789000 & 2.306998000 \\ \text { F } & 4.455763000 & -3.673388000 & 2.209873000 \\ \text { F } & 4.422391000 & -1.927260000 & 3.546966000 \\ \text { F } & 2.707437000 & -2.340160000 & 2.249095000 \\ \text { C } & -0.888172000 & 4.721479000 & -2.276473000 \\ \text { F } & -1.574613000 & 3.619063000 & -1.853666000 \\ \text { F } & -1.805960000 & 5.659965000 & -2.629154000 \\ \text { F } & -0.216617000 & 4.351731000 & -3.408496000 \\ \text { C } & 0.281172000 & 7.947012000 & 1.435883000 \\ \text { F } & 0.804511000 & 7.855163000 & 2.691624000 \\ \text { F } & 0.797799000 & 9.076043000 & 0.869975000 \\ \text { F } & -1.061180000 & 8.138403000 & 1.559475000 \\ \text { C } & -6.758803000 & -0.066336000 & 2.467009000 \\ \text { F } & -5.595297000 & -0.225765000 & 3.173073000 \\ \text { F } & -7.153007000 & 1.228196000 & 2.620174000 \\ \text { F } & -7.694778000 & -0.846681000 & 3.078874000 \\ \text { C } & -7.923267000 & 0.578236000 & -2.404255000 \\ \text { F } & -8.314573000 & -0.259789000 & -3.407469000 \\ \text { F } & -9.037260000 & 1.205374000 & -1.933096000 \\ \text { F } & -7.134449000 & 1.535332000 & -2.974307000\end{array}$

${ }^{\text {CF3 }}$ TS-1 2,1-ins

Zero-point correction $=0.753503($ Hartree $/$ Particle $)$

Thermal correction to Energy $=\quad 0.830472$

Thermal correction to Enthalpy $=\quad 0.831416$

Thermal correction to Gibbs Free Energy= $\quad 0.622697$

Sum of electronic and zero-point Energies $=-4902.869993$

Sum of electronic and thermal Energies $=\quad-4902.793024$

Sum of electronic and thermal Enthalpies $=\quad-4902.792079$

Sum of electronic and thermal Free Energies $=-4903.000798$

E solvent $=-4902.8461084$

$\begin{array}{lrrr}\mathrm{Ni} & 1.828361000 & 1.149638000 & -1.329965000 \\ \mathrm{C} & 1.894280000 & 1.255525000 & -3.402908000 \\ \mathrm{~N} & 2.197798000 & 2.180936000 & 0.232509000 \\ \mathrm{C} & 1.419420000 & 2.174368000 & 1.306740000 \\ \mathrm{C} & 0.281005000 & 1.349661000 & 1.550857000 \\ \mathrm{C} & -0.165658000 & 0.350081000 & 0.603200000 \\ \mathrm{O} & 0.375167000 & 0.177654000 & -0.566883000 \\ \mathrm{C} & 1.504811000 & -0.032725000 & -3.000386000 \\ \mathrm{C} & 2.406731000 & -1.238491000 & -3.061808000 \\ \mathrm{H} & 2.168468000 & -1.839972000 & -3.963257000 \\ \mathrm{H} & 3.474146000 & -0.965068000 & -3.113406000 \\ \mathrm{H} & 2.256464000 & -1.900030000 & -2.191343000 \\ \mathrm{H} & 0.434134000 & -0.224723000 & -2.862039000 \\ \mathrm{H} & 1.140137000 & 2.029688000 & -3.597260000 \\ \mathrm{H} & 2.858537000 & 1.410086000 & -3.903611000 \\ \mathrm{H} & 1.696464000 & 2.866670000 & 2.120290000 \\ \mathrm{C} & -0.405318000 & 1.515439000 & 2.788392000 \\ \mathrm{H} & -0.047209000 & 2.294363000 & 3.476159000 \\ \mathrm{C} & -1.506326000 & 0.737479000 & 3.148639000\end{array}$

$\mathrm{H}-3.332237000$

$-3.332237000$

H $\quad-2.014697000$

C $\quad-1.916682000$

$\mathrm{H} \quad-2.778560000$

C $\quad-1.281964000$

C $\quad-1.740606000$

$\mathrm{N} \quad-2.725142000$

$\mathrm{H}-1.198258000$

C $\quad-3.098188000$

C $\quad-4.404539000$

C $\quad-4.788711000$

C $\quad-3.926957000$

C $\quad-2.663594000$

C $\quad-2.228435000$

C $\quad-0.890412000$

C $\quad-0.725429000$

C $\quad 1.643487000$

C $\quad 1.489594000$

C $\quad 0.237824000$

C $\quad-5.354267000$

C $\quad-5.584802000$

C $\quad-6.496465000$

C $\quad-7.208122000$

C $\quad-6.995930000$

C $\quad-6.076967000$

C $\quad 0.526210000$

C $\quad-0.848612000$

C $\quad-0.405873000$

C $\quad 0.848148000$

1.691666000

1.245544000

$-0.013479000$

3.058839000

4.161976000

5.473562000

5.701753000

4.629095000

3.298194000

4.921505000

5.768287000

6.048180000

5.504454000

4.686612000

4.391241000

1.882771000

$-1.823722000$

1.183846000

3.977027000

6.317029000

6.725160000

6.190305000

5.709110000

$-7.910937000$

$-5.899515000$

$-5.793477000$

$-4.250710000$

$-2.001287000$

0.138521000

$-1.582730000$

$-5.040303000$

3.753125000

2.621655000

2.953085000

6.874269000

7.945170000

7.341477000
0.931740000 0.937297000

1.882777000

0.115572000

$-0.261769000$

$-0.895491000$

$-0.482763000$

$-1.533037000$

$-2.328895000$

$-1.610882000$

$-3.340399000$

$-3.289202000$

$-4.283147000$

$-5.335548000$

$-5.407156000$

$-4.428351000$

$-4.595416000$

$-4.485813000$

$-5.031246000$

$-5.138340000$

$-4.928496000$

$-2.184166000$

$-1.775587000$

$-0.748559000$

$-0.111683000$

$-0.521799000$

$-1.542931000$

$-4.706314000$

6.271028000

5.207926000

4.622263000

5.086994000

6.158426000

6.742087000

4.511692000

5.391457000

4.906637000

3.524662000

2.610585000

3.108279000

1.147908000

0.658558000

$-0.713602000$

$-1.623357000$

$-1.137901000$

0.231504000

6.539653000

6.732012000

3.798618000

6.471089000

5.603577000

3.134359000

1.355246000

$-2.692453000$

0.693890000

$-1.824173000$

$-4.238158000$

$-6.110964000$

$-6.252592000$

$-5.007308000$

$-4.218257000$

$-2.262199000$

0.586092000

$-5.172010000$

1.857320000

$-1.203627000$

$-0.392292000$

$-2.465101000$
4.460999000

4.307794000

4.944459000

5.174583000

2.236233000

2.477684000

1.005843000

0.099757000

0.390461000

$-0.859869000$

$-0.512570000$

$-1.090382000$

$-2.011924000$

$-2.349223000$

$-1.752241000$

$-0.831775000$

$-0.191020000$

1.206642000

1.019040000

$-0.371870000$

$-0.971919000$

$-0.768495000$

0.562573000

0.849283000

$-0.179121000$

$-1.504121000$

$-1.796994000$

1.801496000

$-0.376316000$

$-1.179469000$

$-0.959511000$

0.070799000

0.871128000

0.648375000

0.246390000

0.312825000

0.365511000

0.338200000

0.293331000

0.264802000

0.230878000

$-0.785436000$

$-0.887439000$

0.032253000

1.063031000

1.161201000

1.676024000

$-0.553220000$

$-1.597460000$

0.280523000

0.410073000

0.374717000

$-1.517388000$

$-0.056484000$

0.047655000

$-2.839923000$

$-2.447786000$

$-3.051332000$

$-1.972168000$

$-2.059179000$

1.831477000

1.375054000

1.975579000

1.483593000

$-1.969405000$

$-2.054732000$

$-2.286331000$

$-1.863688000$ 


\begin{tabular}{|c|c|c|c|}
\hline \multicolumn{4}{|c|}{ CF33- $\boldsymbol{\beta}-\mathrm{C}$} \\
\hline \multicolumn{2}{|c|}{ Zero-point correction $=$} & \multicolumn{2}{|c|}{0.756250 (Hartree/Particle) } \\
\hline \multicolumn{3}{|c|}{ Thermal correction to Energy $=$} & 0.833704 \\
\hline \multicolumn{3}{|c|}{ Thermal correction to Enthalpy $=$} & 0.834648 \\
\hline \multicolumn{3}{|c|}{ Thermal correction to Gibbs Free Energy= } & 0.624584 \\
\hline \multicolumn{3}{|c|}{ Sum of electronic and zero-point Energies= } & \\
\hline \multicolumn{3}{|c|}{ Sum of electronic and thermal Energies $=$} & 2.8064 \\
\hline \multicolumn{3}{|c|}{ Sum of electronic and thermal Enthalpies $=$} & \\
\hline \multirow{2}{*}{\multicolumn{4}{|c|}{$\begin{array}{l}\text { Sum of electronic and thermal Free Energies }=-4903.01561 \\
\text { E solvent }=-4902.86188152\end{array}$}} \\
\hline & & & \\
\hline $\mathrm{i}$ & 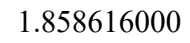 & 1.30 & 0 \\
\hline$C$ & 1.38 & 0 & \\
\hline $1 \mathrm{v}$ & 2.6 & & \\
\hline$C$ & 2 & & \\
\hline $\mathrm{C}$ & 0 & & \\
\hline $\mathrm{C}$ & -0.00 & & \\
\hline $\mathrm{O}$ & 03 & & \\
\hline $\mathrm{C}$ & 2.71 & & \\
\hline $\mathrm{H}$ & & & \\
\hline $\mathrm{H}$ & 2.6 & & \\
\hline $\mathrm{H}$ & 3.5 & & \\
\hline $\mathrm{C}$ & 0.129 & 2. & \\
\hline $\mathrm{H}$ & 1.37 & 0.46 & 3000 \\
\hline $\mathrm{H}$ & 2.623 & $0.70^{\circ}$ & 0000 \\
\hline $\mathrm{C}$ & 0.372 & -0.522 & 8000 \\
\hline $\mathrm{H}$ & 1.02 & -0.41 & 9000 \\
\hline $\mathrm{C}$ & -0.855 & -1.17 & 60000 \\
\hline $\mathrm{C}$ & -1.310711 & -1.755939 & \\
\hline $\mathrm{H}$ & & -2 & \\
\hline $\mathrm{H}$ & -0.84 & -1 & \\
\hline $\mathrm{H}$ & -2.40 & -1 & \\
\hline $\mathrm{C}$ & & -1. & \\
\hline $\mathrm{H}$ & -2.6 & -1.75 & -2 . \\
\hline $\mathrm{C}$ & -1.27 & -0.76 & 20000 \\
\hline $\mathrm{C}$ & -2.15 & -0.88 & 19000 \\
\hline $\mathrm{N}$ & & & \\
\hline $\mathrm{H}$ & -1.816 & -0.37 & 1.239266000 \\
\hline $\mathrm{C}$ & -4.060067000 & -1.670317000 & 1.442216000 \\
\hline
\end{tabular}

C

$-3.560778000 \quad-2.223759000$ $-4.415288000 \quad-2.293963000$ $-5.737453000 \quad-1.839815000$ $-6.233898000 \quad-1.325871000$ $-5.424449000-1.247654000$ $-5.996676000 \quad-0.685281000$ $-5.803522000$ $-7.124897000$ $-7.336068000$ $-6.774914000$ $-2.178634000$ $-1.664103000$ $-0.383288000$ 0.415934000 $-0.090078000$ $-1.372638000$ $-6.354570000$ 5.095186000 4.553186000 4.507237000 5.016673000 5.564375000 5.597250000 5.068590000 6.306191000 6.453365000 5.357036000 4.098107000 3.946282000 2.973740000

3.186736000 2.155072000 0.898783000 0.686951000 1.705574000 5.963813000 5.125109000 4.072382000

7.170087000

7.421995000 5.459213000 4.161277000 0.099287000

1.415220000

$-1.746444000$

$-4.033719000$

$-6.387530000$

$-7.278884000$

$-6.917213000$

$-5.199451000$

$-2.274222000$

1.516234000

$-7.536845000$

$-0.063146000$

0.194091000

$-0.748694000$

2.397817000

1.461473000

2.371341000

3.620996000

$-0.678272000$

$-1.345132000$

$-0.588994000$

$-1.456405000$

4.082988000

2.968404000

$-1.322561000$

0.388829000

1.018032000

0.490015000

$-2.775521000$

$-3.686459000$

$-4.238099000$

$-3.892915000$

$-2.991119000$

$-2.439876000$

$-0.785459000$

$-3.231976000$

$-2.566126000$

$-1.164769000$

$-0.396932000$

$-1.071699000$

$-2.475156000$

1.095326000

1.729443000

3.114810000

3.889586000

3.301729000

1.893960000

4.177314000

5.069044000

5.905623000

5.891591000

5.025204000

4.168778000

$-0.496334000$

$-4.324037000$

$-0.662652000$

1.109510000

3.587453000

4.976074000

5.089380000

6.550552000

$-4.319401000$

$-1.731044000$

$-2.744063000$

$-1.913777000$

$-1.001786000$

1.018083000

$-2.230819000$

$-3.969274000$

3.508592000

0.815764000

2.387182000

3.295828000

1.775216000

6.744762000

7.715227000

5.963713000

7.344007000

4.958916000

6.141656000

4.652068000

4.002799000

$-3.353927000$

$-2.798434000$

3.792266000

$-4.643314000$

5.039583000
2.663016000

3.784860000

3.723957000

2.518289000

1.368380000

0.110022000

$-1.135583000$

$-2.266374000$

$-1.031621000$

0.144153000

2.783931000

1.836504000

1.992444000

3.092604000

4.041922000

3.893124000

$-2.307265000$

$-1.614819000$

$-0.504388000$

$-0.462660000$

$-1.530880000$

$-2.641953000$

$-2.684140000$

$-1.458256000$

$-1.706443000$

$-1.574648000$

$-1.177432000$

$-0.933093000$

$-1.090546000$

$-0.487336000$

0.584100000

1.042017000

0.420302000

$-0.665068000$

$-1.109609000$

$-3.483697000$

$-1.645528000$

0.407466000

$-1.971306000$

$-1.768034000$

$-1.077282000$

1.083738000

0.768219000

3.206852000

4.638604000

4.708825000

4.602025000

2.451142000

1.092549000

$-1.183583000$

0.973620000

$-1.959429000$

$-3.184353000$

4.118834000

2.600926000

2.589109000

2.274994000

2.435779000

3.402368000

2.244736000

$-1.316556000$

$-1.207817000$

$-2.640543000$

$-0.733572000$

0.699544000

1.258124000

0.385963000

1.674093000 


\section{CF3 TS-3}

Zero-point correction 0.807869 (Hartree/Particle) Thermal correction to Energy= Thermal correction to Enthalpy= 0.888736 0.889680 Thermal correction to Gibbs Free Energy $=0.673416$ Sum of electronic and zero-point Energies $=\quad-4981.402888$ Sum of electronic and thermal Energies $=\quad-4981.322020$ Sum of electronic and thermal Enthalpies $=\quad-4981.321076$ Sum of electronic and thermal Free Energies $=-4981.537341$ E solvent $=-4981.40419568$

$\begin{array}{lrrr}\mathrm{Ni} & 1.944893000 & 1.097948000 & 1.058919000 \\ \mathrm{C} & 1.202886000 & 1.890486000 & 2.670463000 \\ \mathrm{~N} & 2.809479000 & 0.781388000 & -0.714156000 \\ \mathrm{C} & 2.119271000 & 0.225964000 & -1.700388000 \\ \mathrm{C} & 0.779521000 & -0.277904000 & -1.662653000 \\ \mathrm{C} & -0.077396000 & -0.187578000 & -0.498054000 \\ \mathrm{O} & 0.279738000 & 0.331691000 & 0.642892000 \\ \mathrm{C} & 2.495821000 & 2.647888000 & 2.654852000 \\ \mathrm{H} & 3.155816000 & 2.317884000 & 1.759313000 \\ \mathrm{H} & 2.377813000 & 3.734025000 & 2.504789000 \\ \mathrm{H} & 3.153920000 & 2.440606000 & 3.516364000 \\ \mathrm{C} & -0.084906000 & 2.653756000 & 2.452049000 \\ \mathrm{H} & 1.123527000 & 1.105983000 & 3.438968000 \\ \mathrm{H} & 2.611542000 & 0.153286000 & -2.684528000 \\ \mathrm{C} & 0.286353000 & -0.875959000 & -2.857584000 \\ \mathrm{H} & 0.969407000 & -0.945271000 & -3.716055000 \\ \mathrm{C} & -1.006126000 & -1.393777000 & -2.972229000 \\ \mathrm{C} & -1.498600000 & -2.048433000 & -4.247341000 \\ \mathrm{H} & -1.349088000 & -3.145449000 & -4.222982000 \\ \mathrm{H} & -0.963579000 & -1.664898000 & -5.133680000 \\ \mathrm{H} & -2.578258000 & -1.872506000 & -4.394086000 \\ \mathrm{C} & -1.842940000 & -1.290205000 & -1.840013000 \\ \mathrm{H} & -2.876725000 & -1.651858000 & -1.894556000 \\ \mathrm{C} & -1.424145000 & -0.712317000 & -0.631184000 \\ \mathrm{C} & -2.336942000 & -0.605209000 & 0.502379000 \\ \mathrm{~N} & -3.523756000 & -1.137143000 & 0.502021000 \\ \mathrm{H} & -1.963591000 & -0.028629000 & 1.367617000 \\ \mathrm{C} & -4.353199000 & -1.012282000 & 1.627658000 \\ \mathrm{C} & -3.959877000 & -1.454156000 & 2.930255000 \\ \mathrm{C} & -4.851734000 & -1.286577000 & 4.012294000 \\ \mathrm{C} & -6.109741000 & -0.700086000 & 3.836815000 \\ \mathrm{C} & -6.503285000 & -0.288179000 & 2.556359000 \\ \mathrm{C} & -5.654988000 & -0.447942000 & 1.443584000 \\ \mathrm{C} & -6.117995000 & 0.019291000 & 0.104070000 \\ \mathrm{C} & -5.974788000 & -0.784172000 & -1.048337000 \\ \mathrm{C} & -7.045169000 & 0.922765000 & -2.425414000 \\ & -7.203581000 & 1.720680000 & -1.283678000\end{array}$

$\mathrm{C}$
$\mathrm{C}$
$\mathrm{C}$
$\mathrm{C}$
$\mathrm{C}$
$\mathrm{C}$

$-6.742890000$ $-2.652702000$ $-2.201481000$ $-1.000868000$ $-0.216202000$

$-0.656461000$

$-1.862471000$

$-6.427672000$

4.064240000

4.202296000

4.522150000

4.712149000

4.601604000

4.267246000

5.096347000

6.443575000

6.886847000

5.966887000

4.606652000

4.165143000

3.686069000

4.111896000

3.298003000

2.039823000

1.609411000

2.415989000

4.768599000

3.802574000

4.628898000

7.144975000

7.935519000

6.291679000

5.082500000

1.410206000

0.716399000

$-2.186953000$

$-4.553789000$

$-6.791326000$

$-7.499045000$

$-6.840659000$

$-5.486911000$

$-2.800182000$

2.063497000

$-7.379716000$

$-0.380082000$

0.000265000

$-0.906659000$

4.076406000

2.880514000

4.343736000

4.828900000

2.173629000

2.638511000

3.730023000

3.228577000

3.286929000

5.085847000

0.233160000

$-0.198577000$

0.209084000

$-0.688123000$

$-7.909705000$

$-7.811305000$

$-9.241443000$

$-7.401352000$

$-6.287416000$

$-6.284179000$

$-5.125084000$
1.276125000

$-2.126515000$

$-3.199014000$

$-3.859850000$

$-3.465990000$

$-2.401236000$

$-1.740303000$

$-0.332537000$

$-3.989558000$

$-3.575806000$

$-2.241953000$

$-1.295500000$

$-1.725211000$

$-3.054840000$

0.132003000

0.485924000

1.801018000

2.785472000

2.480730000

1.128734000

3.614038000

4.646785000

5.762485000

5.879368000

4.860834000

3.738855000

$-1.009146000$

$-5.023805000$

$-1.944253000$

$-0.293679000$

2.061971000

3.829283000

4.570828000

6.753320000

$-3.987062000$

$-0.906465000$

$-1.655416000$

$-0.590103000$

0.143461000

1.929914000

$-1.757491000$

$-3.522315000$

2.975996000

1.280304000

3.198932000

3.394790000

1.963859000

$-0.236884000$

$-0.856097000$

0.254322000

$-0.191215000$

$-0.946190000$

$-1.433810000$

6.791527000

8.025095000

6.450960000

6.899392000

4.934337000

6.219159000

4.362895000

4.274213000

3.053742000

3.586210000

2.935085000

3.964869000

$-1.233770000$

$-0.532795000$

$-1.959195000$
$-0.032455000$

3.188282000

2.386640000

2.682457000

3.778951000

4.578990000

4.290973000

$-2.296083000$

$-2.345059000$

$-1.012385000$

$-0.703969000$

$-1.728522000$

$-3.067372000$

$-3.370995000$

$-1.450663000$

$-1.671966000$

$-1.489444000$

$-1.115915000$

$-0.887670000$

$-1.024282000$

$-0.579173000$

0.286284000

0.535966000

$-0.074460000$

$-0.938032000$

$-1.185560000$

$-3.879660000$

$-2.580358000$

0.342826000

$-1.989927000$

$-1.665770000$

$-1.041721000$

0.786385000

0.111750000

4.009847000

4.921898000

5.000772000

4.686730000

2.402446000

0.840218000

$-0.964153000$

1.530279000

$-1.882276000$

$-3.402485000$

3.374354000

1.637702000

2.197211000

2.423219000

2.262559000

3.366017000

1.627165000

3.093597000

1.364867000

1.555415000

1.277774000

2.805122000

1.630165000

$-1.563395000$

$-1.690498000$

$-2.798576000$

$-0.799641000$

$-1.387319000$

$-2.637653000$

$-1.107202000$

$-0.509254000$

$-3.501311000$

$-4.668305000$

$-3.457025000$ 


$\begin{array}{lrrr}\text { F } & -7.305001000 & -2.138466000 & -3.577398000 \\ \text { C } & 0.206034000 & -1.905290000 & 5.714278000 \\ \text { F } & -0.535004000 & -1.423703000 & 6.749255000 \\ \text { F } & 1.025334000 & -2.873860000 & 6.202619000 \\ \text { F } & 1.019676000 & -0.870813000 & 5.306245000 \\ \text { C } & -0.515864000 & -4.967427000 & 1.772804000 \\ \text { F } & 0.229200000 & -4.470439000 & 0.742710000 \\ \text { F } & 0.267067000 & -5.862657000 & 2.437534000 \\ \text { F } & -1.552251000 & -5.656706000 & 1.218107000 \\ \text { C } & 4.053657000 & -4.569843000 & 0.120352000 \\ \text { F } & 3.264622000 & -4.073130000 & 1.115688000 \\ \text { F } & 3.508623000 & -5.743387000 & -0.294567000 \\ \text { F } & 5.263762000 & -4.855823000 & 0.682884000 \\ \text { C } & 4.060691000 & -3.439936000 & -4.818841000 \\ \text { F } & 5.044593000 & -2.933362000 & -5.615227000 \\ \text { F } & 4.035067000 & -4.787398000 & -4.995639000 \\ \text { F } & 2.877335000 & -2.943680000 & -5.292008000\end{array}$

\section{CF33-Coor-C}

Zero-point correction 0.810830 (Hartree/Particle)

Thermal correction to Energy=

Thermal correction to Enthalpy= 0.891301

Sum of electronic and zero-point Energies $=\quad-4981.423511$

Sum of electronic and thermal Energies $=\quad-4981.343040$

Sum of electronic and thermal Enthalpies $=\quad-4981.342095$

Sum of electronic and thermal Free Energies $=-4981.558467$

E solvent $=-4981.42141544$

\begin{tabular}{|c|c|c|c|}
\hline $\mathrm{Ni}$ & -1.920535000 & 0.828392000 & -1.383135000 \\
\hline $\mathrm{C}$ & -1.303882000 & -0.346208000 & -2.906947000 \\
\hline $\mathrm{N}$ & -2.563970000 & 1.555847000 & 0.482413000 \\
\hline $\mathrm{C}$ & -1.970807000 & 1.206237000 & 1.612419000 \\
\hline $\mathrm{C}$ & -0.727047000 & 0.523500000 & 1.787389000 \\
\hline $\mathrm{C}$ & 0.055057000 & 0.038767000 & 0.674444000 \\
\hline $\mathrm{O}$ & -0.320554000 & 0.142233000 & -0.566935000 \\
\hline $\mathrm{C}$ & -2.318403000 & -0.819992000 & -3.938 \\
\hline $\mathrm{H}$ & -3.200873000 & -1.293769000 & 96000 \\
\hline $\mathrm{H}$ & -2.653504000 & -0.019979000 & -4.626093000 \\
\hline $\mathrm{H}$ & -1.836573000 & -1.596342000 & -4.568 \\
\hline $\mathrm{C}$ & -0.034335000 & 0.233625000 & -3.522613000 \\
\hline $\mathrm{H}$ & -1.058442000 & -1.169025000 & 82000 \\
\hline $\mathrm{H}$ & -2.472697000 & 026000 & 3000 \\
\hline $\mathrm{C}$ & -0.2581 & 0.3538 & 5000 \\
\hline $\mathrm{H}$ & -0.88226 & 7000 & 000 \\
\hline $\mathrm{C}$ & 0.960109000 & -0.261160000 & 3.417 \\
\hline $\mathrm{C}$ & 1.447033000 & -0.4366 & 4.8415 \\
\hline $\mathrm{H}$ & 1.190976000 & -1.43 & 000 \\
\hline $\mathrm{H}$ & 0.99513 & & \\
\hline $\mathrm{H}$ & 2.5444 & -0.3353 & 4.9022 \\
\hline $\mathrm{C}$ & 1.728517000 & -0.723168000 & 53000 \\
\hline $\mathrm{H}$ & 2.704700000 & -1.187400000 & 1000 \\
\hline $\mathrm{C}$ & 1.316938000 & -0.600572000 & 0.989 \\
\hline $\mathrm{C}$ & 2.16058 & -1.0 & -0.0 \\
\hline $\mathrm{N}$ & 3.270023000 & -1.731753000 & 56000 \\
\hline $\mathrm{H}$ & 1.805255000 & -0.885592000 & -1.122562000 \\
\hline $\mathrm{C}$ & 4.055429000 & -2.208346000 & -0.935850000 \\
\hline $\mathrm{C}$ & 3.575706000 & -3.170135000 & -1.879 \\
\hline $\mathrm{C}$ & 4.435816000 & -3.600154000 & 12000 \\
\hline $\mathrm{C}$ & 5.738823000 & -3.106612000 & -3.03 \\
\hline $\mathrm{C}$ & 6.214845000 & -2.183627000 & -2.094900000 \\
\hline $\mathrm{C}$ & 5.403148000 & -1.738900000 & -1.033600000 \\
\hline $\mathrm{C}$ & 5.959113000 & -0.759768000 & -0.053973000 \\
\hline $\mathrm{C}$ & 5.807654000 & -0.946304000 & 1000 \\
\hline $\mathrm{C}$ & 7.088647000 & 1.079651000 & 1.793780000 \\
\hline $\mathrm{C}$ & 7.253868000 & 1.265572000 & 0.414187000 \\
\hline $\mathrm{C}$ & 6.693172000 & 0.357699000 & -0.501083000 \\
\hline $\mathrm{C}$ & 2.213246000 & -3.772467000 & -1.794302000 \\
\hline , & 1.685286000 & -4.251823000 & -0.574580000 \\
\hline
\end{tabular}

\begin{tabular}{|c|c|c|c|}
\hline & & & \\
\hline & -0.348151000 & -5.003055000 & 21000 \\
\hline & 0.173066000 & 000 & \\
\hline & 7000 & 000 & 00 \\
\hline & 6.361775000 & 2000 & \\
\hline & -5.056891000 & 8000 & 200 \\
\hline & -4.506896000 & -2.227068000 & 1000 \\
\hline & -4.457884000 & -0.8385 & 500( \\
\hline & -4.956007000 & 37000 & 00( \\
\hline & -5.5138 & 1535000 & 00 \\
\hline & -5.563848000 & -1.891738000 & 2.694 \\
\hline & -4.961219000 & 1.527395000 & 1.1 \\
\hline & -6.169047000 & 5433000 & \\
\hline & -6.25 & & \\
\hline & -5.1 & & \\
\hline & -3.8 & & \\
\hline & -3.8 & & \\
\hline & -2.72 & & \\
\hline & -2.9 & & \\
\hline & -1.8 & & \\
\hline & -0.6 & & \\
\hline & -0.3 & & \\
\hline & -1.4 & & \\
\hline & -5.8 & & \\
\hline & -5.0 & & \\
\hline & -4.0 & & \\
\hline & -7.0 & & \\
\hline & -7.1 & & \\
\hline & -5.1 & & \\
\hline & -3.9 & & \\
\hline & & & \\
\hline & -1.3 & & \\
\hline & & & \\
\hline & 4.0 & -4 & \\
\hline & 6.3 & 8000 & \\
\hline & & & \\
\hline & 6.8 & & \\
\hline & 5.242285000 & & \\
\hline & 2.2 & & \\
\hline & & & \\
\hline & & & \\
\hline & & & \\
\hline & & & \\
\hline & & & \\
\hline & -3 & & \\
\hline & -2 & & \\
\hline & -4 & & \\
\hline & -4 & & \\
\hline & & & \\
\hline & $-2 .($ & & \\
\hline & 6.2 & & 000 \\
\hline & & & \\
\hline & & & \\
\hline & & 19000 & \\
\hline & & & 000 \\
\hline & 8.90 & & \\
\hline & 8.60 & & 0000 \\
\hline & 7.102717000 & 3.523352000 & 000 \\
\hline & -2.142 & 269000 & -2.5 \\
\hline & -1.228 & & 8000 \\
\hline & -2.07 & & \\
\hline & -3.3 & & \\
\hline & & & \\
\hline & & & \\
\hline & & & \\
\hline & & & \\
\hline & & & \\
\hline & -6.067585000 & -1.620390000 & 5.0078840 \\
\hline
\end{tabular}




$\begin{array}{llll}\text { F } & -7.558576000 & -2.629226000 & 3.755667000 \\ \text { F } & -5.697409000 & -3.663846000 & 4.280585000 \\ \text { C } & -3.937616000 & -3.116560000 & -0.533098000 \\ \text { F } & -2.601323000 & -2.888599000 & -0.706500000 \\ \text { F } & -4.093495000 & -4.438509000 & -0.262416000 \\ \text { F } & -4.534527000 & -2.868867000 & -1.738525000 \\ \text { C } & -0.149630000 & -5.289396000 & 0.803191000 \\ \text { F } & -0.777664000 & -4.255809000 & 1.436957000 \\ \text { F } & -1.067435000 & -6.287125000 & 0.662833000 \\ \text { F } & 0.822345000 & -5.739107000 & 1.646312000 \\ \text { C } & -0.607935000 & -4.739820000 & -4.190904000 \\ \text { F } & -0.396438000 & -3.716582000 & -5.076614000 \\ \text { F } & -0.242534000 & -5.888423000 & -4.829611000 \\ \text { F } & -1.948400000 & -4.813912000 & -3.965918000\end{array}$

\section{${ }^{{ }^{C F} 3}$ TS-3 3 ins}

Zero-point correction $=\quad 0.810490($ Hartree/Particle $)$

Thermal correction to Energy= $\quad 0.890267$

Thermal correction to Enthalpy= $\quad 0.891211$

Thermal correction to Gibbs Free Energy $=0.677394$

Sum of electronic and zero-point Energies $=\quad-4981.408665$

Sum of electronic and thermal Energies $=\quad-4981.328889$

Sum of electronic and thermal Enthalpies $=\quad-4981.327944$

Sum of electronic and thermal Free Energies $=-4981.541762$

E solvent $=-4981.41157084$

$\begin{array}{lrrr}\mathrm{Ni} & 1.915024000 & 1.257549000 & 0.946922000 \\ \mathrm{C} & 0.753662000 & 1.893804000 & 2.655722000 \\ \mathrm{~N} & 2.651952000 & 1.221334000 & -0.828980000 \\ \mathrm{C} & 2.029075000 & 0.683034000 & -1.873316000 \\ \mathrm{C} & 0.745127000 & 0.062355000 & -1.919107000 \\ \mathrm{C} & -0.090978000 & -0.074680000 & -0.743446000 \\ \mathrm{O} & 0.267133000 & 0.353698000 & 0.426649000 \\ \mathrm{C} & -0.035704000 & 0.855444000 & 3.438780000 \\ \mathrm{H} & -0.171237000 & -0.066764000 & 2.851802000 \\ \mathrm{H} & 0.451434000 & 0.597340000 & 4.397043000 \\ \mathrm{H} & -1.041237000 & 1.258731000 & 3.675364000 \\ \mathrm{C} & 1.028359000 & 3.193710000 & 3.405380000 \\ \mathrm{H} & 0.214400000 & 2.143307000 & 1.720309000 \\ \mathrm{H} & 2.557753000 & 0.740390000 & -2.840104000 \\ \mathrm{C} & 0.282581000 & -0.403189000 & -3.183000000 \\ \mathrm{H} & 0.940469000 & -0.271616000 & -4.053732000 \\ \mathrm{C} & -0.964990000 & -1.007427000 & -3.352457000 \\ \mathrm{C} & -1.443070000 & -1.502501000 & -4.702662000 \\ \mathrm{H} & -1.262482000 & -2.588328000 & -4.823294000 \\ \mathrm{H} & -0.923736000 & -0.988708000 & -5.530444000 \\ \mathrm{H} & -2.528309000 & -1.339978000 & -4.824593000 \\ \mathrm{C} & -1.773334000 & -1.151814000 & -2.202447000 \\ \mathrm{H} & -2.769025000 & -1.602209000 & -2.295235000 \\ \mathrm{C} & -1.378278000 & -0.718319000 & -0.927493000 \\ \mathrm{C} & -2.250450000 & -0.898548000 & 0.228422000 \\ \mathrm{~N} & -3.377766000 & -1.542010000 & 0.159639000 \\ \mathrm{H} & -1.896377000 & -0.453284000 & 1.175656000 \\ \mathrm{C} & -4.153189000 & -1.735045000 & 1.313829000 \\ \mathrm{C} & -3.655068000 & -2.436381000 & 2.456726000 \\ \mathrm{C} & -4.489357000 & -2.588768000 & 3.585676000 \\ \mathrm{C} & -5.790804000 & -2.074910000 & 3.604866000 \\ \mathrm{C} & -6.287723000 & -1.416327000 & 2.471936000 \\ \mathrm{C} & -5.498600000 & -1.250288000 & 1.316937000 \\ \mathrm{C} & -6.072412000 & -0.539716000 & 0.136845000 \\ \mathrm{C} & -5.918503000 & -1.045043000 & -1.173085000 \\ \mathrm{C} & -7.208364000 & 0.812092000 & -2.090007000 \\ \mathrm{C} & -7.379717000 & 1.311958000 & -0.791236000 \\ \mathrm{C} & -6.814985000 & 0.646513000 & 0.310458000 \\ \mathrm{C} & -2.297802000 & -3.057492000 & 2.480205000 \\ \mathrm{C} & -1.840207000 & -3.863620000 & 1.413105000 \\ \mathrm{C} & -0.585623000 & -4.485159000 & 1.471611000 \\ \mathrm{C} & 0.248951000 & -4.315283000 & 2.589030000 \\ \mathrm{C} & -0.198848000 & -3.521494000 & 3.653842000\end{array}$

-1.459869000
-6.473810000
4.795725000

$-2.901658000$ $-0.371840000$ $-3.431028000$ $4.339591000-2.694653000$ $4.377906000-1.293541000$ $\begin{array}{ll}4.886093000 & -0.591344000\end{array}$ $5.348074000-1.336533000$ $5.297624000-2.740786000$ 5.012264000 6.260660000 6.470539000 5.431209000 4.164238000 3.949049000 3.113101000 3.465090000 2.516200000 1.192712000 0.835642000 1.779916000 5.728631000 4.745208000 3.998839000 7.079853000 7.443540000 5.585040000 4.489503000 0.453380000 1.230819000 $-1.789959000$ $-4.110684000$ $-6.426382000$ $-7.318923000$ $-6.926094000$ $-5.341803000$ $-2.474295000$ 1.475196000 $-7.623178000$ 0.068289000 1.449794000 1.700919000 2.684108000 3.563668000

2.661733000 2.549846000 4.380426000 3.783859000 $-6.316366000$ $-6.384700000$ $-5.116027000$ $-7.285928000$ $-8.212599000$ $-9.521836000$ $-7.780822000$ $-8.186816000$ 2.914349000 2.082465000 2.882814000

4.183343000

$-0.603236000$

$-1.269832000$

$-0.696500000$ $-1.278223000$ 5.840660000 5.672802000 7.178283000 0.899119000 1.462711000 2.844651000 3.684410000 3.168393000 1.763312000 4.121436000 5.129434000 6.060826000 6.009433000 5.015562000 4.081283000 $-0.816955000$ $-4.522944000$ $-0.741702000$ 0.792997000 3.265996000 4.768873000 5.177144000 6.735224000 $-4.792122000$ $-2.278934000$ $-3.151202000$ $-2.214284000$ $-1.044776000$ 1.074396000 $-1.958942000$ $-4.005165000$ 3.336485000 1.345329000 3.730731000 3.024594000 3.875561000 1.012572000 1.684847000 $-0.088751000$ 1.413387000 1.132190000 2.747852000 $-0.957102000$ $-0.002149000$ $-1.601442000$ $-1.876442000$ 2.553889000 2.239732000 3.265077000 3.386119000 7.060188000 8.135613000 6.489934000 7.519864000 4.887754000 6.071811000 4.445832000 3.991243000 $-3.513704000$ $-2.829767000$ $-3.748317000$ 5.232774000 $-4.723449000$ $3.870069000 \quad-3.399028000$

3.603708000 $-2.270203000$ $-1.523730000$ $-0.419371000$ $-0.429545000$ $-1.541560000$ $-2.646712000$ $-2.637628000$ $-1.526442000$ $-1.872995000$ $-1.820033000$ $-1.403566000$ $-1.053557000$ $-1.133757000$ $-0.591405000$ 0.332570000 0.784278000 0.323084000 $-0.600978000$ $-1.055495000$ $-3.532232000$ $-1.523007000$ 0.434104000 $-2.157742000$ $-2.093639000$ $-1.372905000$ 0.716205000 0.671050000 2.623224000 4.441654000 4.447156000 4.485597000 2.464014000 1.311918000 $-1.329083000$ 0.533435000 $-1.793382000$ $-2.949322000$ 3.543574000 4.414602000 2.857753000 2.832971000 1.910329000 2.830906000 3.843388000 1.429014000 2.080859000 $-3.655212000$ $-4.623676000$ $-3.799999000$ $-3.928530000$ $-0.566787000$ $-0.336772000$ 0.513724000 $-1.645484000$ 1.846571000 1.876423000 3.089743000 1.656625000 $-1.050310000$ $-0.967402000$ $-2.334464000$ $-0.268747000$ $-3.820345000$ $-4.988139000$ $-3.685141000$ $-3.957298000$ 0.834225000 


$\begin{array}{cc}\text { F } & 2.859152000 \\ \text { F } & 3.419200000 \\ \text { F } & 4.879555000 \\ \text { C } & -0.092252000 \\ \text { F } & 0.619246000 \\ \text { F } & 0.727604000 \\ \text { F } & -1.120361000 \\ \text { C } & 0.708439000 \\ \text { F } & 0.008427000 \\ \text { F } & 1.641398000 \\ \text { F } & 1.390258000\end{array}$

$-2.712005000$ $-4.657213000$ $-3.505949000$ $-5.292224000$ $-4.516041000$ $-6.308609000$ $-5.840574000$ $-3.257889000$ $-3.157366000$ $-4.234841000$ $-2.077559000$
1.446545000 0.583171000 1.746191000 0.290910000 $-0.578730000$ 0.680396000 $-0.415879000$ 4.832991000 5.998745000 4.992745000 4.684370000

\section{CF3 4- $\beta$ - T}

Zero-point correction $=\quad 0.811947($ Hartree/Particle $)$ $\begin{array}{lr}\text { Thermal correction to Energy }= & 0.891956 \\ \text { The } & 0.892901\end{array}$ $\begin{array}{ll}\text { Thermal correction to Enthalpy }= & 0.892901 \\ \text { Thermal correction to Gibbs Free Energy }= & 0.675888\end{array}$ Sum of electronic and zero-point Energies $=-4981.450475$ Sum of electronic and thermal Energies $=\quad-4981.370466$

Sum of electronic and thermal Enthalpies $=\quad-4981.369522$ Sum of electronic and thermal Free Energies $=-4981.586535$ E solvent $=-4981.45093006$

$\begin{array}{lrrr}\mathrm{Ni} & -1.849185000 & 1.063236000 & -1.025940000 \\ \mathrm{C} & -3.212109000 & 1.651574000 & -2.224245000 \\ \mathrm{~N} & -2.489479000 & 1.613963000 & 0.623327000 \\ \mathrm{C} & -1.918987000 & 1.207168000 & 1.755186000 \\ \mathrm{C} & -0.730071000 & 0.433765000 & 1.913868000 \\ \mathrm{C} & 0.065026000 & -0.019372000 & 0.788444000 \\ \mathrm{O} & -0.284701000 & 0.186620000 & -0.446710000 \\ \mathrm{C} & -2.321470000 & 0.914873000 & -3.180273000 \\ \mathrm{H} & -1.876494000 & 1.582850000 & -3.945170000 \\ \mathrm{C} & -2.864961000 & -0.391466000 & -3.813519000 \\ \mathrm{C} & -3.965122000 & -0.056967000 & -4.839053000 \\ \mathrm{H} & -3.311184000 & -0.992408000 & -2.995906000 \\ \mathrm{C} & -1.732518000 & -1.213936000 & -4.453445000 \\ \mathrm{H} & -1.336901000 & 0.583161000 & -2.624362000 \\ \mathrm{H} & -4.203295000 & 1.206852000 & -2.035896000 \\ \mathrm{H} & -3.256879000 & 2.745432000 & -2.332032000 \\ \mathrm{H} & -2.407066000 & 1.533883000 & 2.688006000 \\ \mathrm{C} & -0.303038000 & 0.145236000 & 3.243105000 \\ \mathrm{H} & -0.926121000 & 0.503581000 & 4.074465000 \\ \mathrm{C} & 0.869848000 & -0.557569000 & 3.521546000 \\ \mathrm{C} & 1.305090000 & -0.866183000 & 4.939807000 \\ \mathrm{H} & 1.052957000 & -1.906554000 & 5.222848000 \\ \mathrm{H} & 0.814216000 & -0.198090000 & 5.668723000 \\ \mathrm{H} & 2.397714000 & -0.756208000 & 5.053665000 \\ \mathrm{C} & 1.656228000 & -0.969056000 & 2.420021000 \\ \mathrm{H} & 2.607734000 & -1.484080000 & 2.597837000 \\ \mathrm{C} & 1.296613000 & -0.718829000 & 1.087944000 \\ \mathrm{C} & 2.172843000 & -1.107026000 & -0.016796000 \\ \mathrm{~N} & 3.264522000 & -1.784414000 & 0.169755000 \\ \mathrm{H} & 1.865157000 & -0.763526000 & -1.021446000 \\ \mathrm{C} & 4.111157000 & -2.095958000 & -0.908736000 \\ \mathrm{C} & 3.732982000 & -3.003039000 & -1.945644000 \\ \mathrm{C} & 4.650314000 & -3.268375000 & -2.988104000 \\ \mathrm{C} & 5.910728000 & -2.664287000 & -3.026757000 \\ \mathrm{C} & 6.286384000 & -1.792467000 & -1.996125000 \\ \mathrm{C} & 5.415827000 & -1.509889000 & -0.925872000 \\ \mathrm{C} & 5.864325000 & -0.576525000 & 0.149105000 \\ \mathrm{C} & 5.692340000 & -0.891381000 & 1.515691000 \\ \mathrm{C} & 6.787149000 & 1.186477000 & 2.176695000 \\ \mathrm{C} & 6.973974000 & 1.499753000 & 0.823595000 \\ \mathrm{C} & 6.515075000 & 0.629713000 & -0.180867000 \\ \mathrm{C} & 2.426429000 & -3.723047000 & -1.955276000 \\ \mathrm{C} & 1.897255000 & -4.322894000 & -0.791426000 \\ \mathrm{C} & 0.702592000 & -5.057406000 & -0.842451000 \\ \mathrm{C} & 0.005104000 & -5.210302000 & -2.050633000 \\ \mathrm{C} & 0.523113000 & -4.617689000 & -3.212722000\end{array}$

1.717557000

6.143551000

$-5.378794000$

$-4.796102000$

$-4.604095000$

$-4.998315000$

$-5.588351000$

$-5.772316000$

$-4.866843000$

$-5.995469000$

$-5.955672000$

$-4.780805000$

$-3.624080000$

$-3.668058000$

$-2.415605000$

$-2.574039000$

$-1.471984000$

$-0.186328000$

$-0.020689000$

$-1.119018000$

$-5.895614000$

$-5.515971000$

$-4.136315000$

$-6.921217000$

$-6.839528000$

$-4.737469000$

$-3.568667000$

0.671374000

$-0.935848000$

2.091162000

4.369881000

6.608713000

7.284855000

6.651137000

5.191225000

2.421332000

$-0.960173000$

7.133659000

$-2.111957000$

$-1.260511000$

$-0.944688000$

$-4.390634000$

$-4.789481000$

$-3.554397000$

7.602354000

8.454491000

8.308179000

6.658851000

5.990123000

5.799048000

4.925690000

7.092662000

$-1.670082000$

$-0.709348000$

$-1.625195000$

$-2.881075000$

1.366449000

2.200052000

1.341653000

1.932784000

$-4.426873000$

$-3.300045000$

$-4.203796000$

$-5.418412000$

$-0.192807000$

0.108912000

0.141341000

$-1.549597000$
$-3.882179000$ $-0.016949000$

$-2.333155000$

$-1.948520000$

$-0.594652000$

0.412271000

0.021059000

$-1.339232000$

1.861354000

2.692701000

4.048395000

4.591517000

3.802837000

2.427809000

4.448556000

5.282698000

5.929952000

5.763167000

4.943436000

4.292036000

0.783780000

$-3.389874000$

$-0.313921000$

2.249843000

4.679187000

5.656267000

5.418213000

6.273589000

$-5.765862000$

$-3.405868000$

$-3.991419000$

$-2.894045000$

$-1.340228000$

0.901531000

$-1.821236000$

$-4.207977000$

3.680668000

1.869936000

$-2.167112000$

$-0.659223000$

$-1.457010000$

$-0.981095000$

0.518492000

0.543721000

2.819888000

3.276627000

2.719712000

3.786898000

$-0.395146000$

0.698371000

$-1.234182000$

$-1.042133000$

6.749746000

7.702875000

5.962619000

7.376379000

4.700352000

5.749078000

4.494294000

3.595515000

$-2.992155000$

$-2.638341000$

$-4.208955000$

$-3.147111000$

$-4.818949000$

$-3.841250000$

$-6.009565000$

$-4.824055000$
$-3.167031000$

2.513341000

2.249553000

1.030348000

0.724945000

1.631646000

2.850452000

3.155790000

1.289389000

1.463521000

1.120983000

0.588781000

0.402662000

0.768757000

$-0.191311000$

$-1.319911000$

$-1.900968000$

$-1.365628000$

$-0.238882000$

0.344232000

3.574497000

2.490874000

$-0.223347000$

1.847527000

1.262454000

0.334038000

$-1.757165000$

$-1.811365000$

$-2.081316000$

$-4.078661000$

$-3.762554000$

$-3.838546000$

$-1.997427000$

$-1.232658000$

1.792109000

0.161651000

1.234966000

2.956484000

$-4.857545000$

$-5.286928000$

$-3.717192000$

$-5.269197000$

$-4.381287000$

$-5.673040000$

0.436820000

1.397778000

$-0.726268000$

0.246110000

3.968713000

4.758571000

4.168342000

4.443078000

$-3.156737000$

$-3.298771000$

$-4.272126000$

$-3.162389000$

0.316531000

0.072280000

1.662945000

$-0.248608000$

$-0.001250000$

$-0.689275000$

0.560940000

$-0.926653000$

$-4.528880000$

$-5.431820000$

$-5.104768000$

$-4.370738000$ 


$\begin{array}{cccc}\text { C } & 0.197760000 & -5.743235000 & 0.408446000 \\ \text { F } & 0.474911000 & -5.019487000 & 1.528837000 \\ \text { F } & -1.149215000 & -5.944332000 & 0.368645000 \\ \text { F } & 0.780392000 & -6.967428000 & 0.571490000 \\ \text { C } & -6.368008000 & -1.705567000 & 4.497636000 \\ \text { F } & -6.649520000 & -3.032453000 & 4.592527000 \\ \text { F } & -5.517262000 & -1.392428000 & 5.517754000 \\ \text { F } & -7.524913000 & -1.018783000 & 4.726701000\end{array}$

\section{CF3 TS-1}

Zero-point correction $=0.805641($ Hartree/Particle $)$ $\begin{array}{lc}\text { Thermal correction to Energy= } & 0.886826 \\ \text { Thermal correction to Enthalpy= } & 0.887770 \\ \text { Thermal correction to Gibbs Free Energy= } & 0.668841 \\ \text { Sum of electronic and zero-point Energies }= & -4981.389876 \\ \text { Sum of electronic and thermal Energies }= & -4981.308691 \\ \text { Sum of electronic and thermal Enthalpies }= & -4981.307747 \\ \text { Sum of electronic and thermal Free Energies }= & -4981.526676\end{array}$ E solvent $=-4981.38844262$

\begin{tabular}{|c|c|c|c|}
\hline $\mathrm{N}$ & 2.074012000 & 2.205233000 & -0.063348000 \\
\hline $\mathrm{C}$ & 4.266500000 & 3.335270000 & -0.308920000 \\
\hline $\mathrm{C}$ & 0.711712000 & 4.868116000 & -0.370539000 \\
\hline $\mathrm{C}$ & -0.238302000 & 4.040681000 & -1.003759000 \\
\hline H & 0.095972000 & 3.160661000 & -1.558094000 \\
\hline $\mathrm{C}$ & -1.131905000 & 6.265008000 & 0.443034000 \\
\hline C & 5.031401000 & 2.099502000 & 638000 \\
\hline $\mathrm{C}$ & -2.068338000 & 5.444598000 & -0.204 \\
\hline 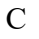 & 2.842720000 & 3.400411000 & 3000 \\
\hline $\mathrm{C}$ & -1.609896000 & 4.333308000 & -0.926433000 \\
\hline $\mathrm{C}$ & 2.190235000 & 4.655763000 & -0.479880000 \\
\hline $\mathrm{C}$ & 4.752387000 & 1.343412000 & 604000 \\
\hline $\mathrm{H}$ & 3.928408000 & 1.6317 & 9000 \\
\hline 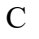 & 6.623 & 6000 & 0000 \\
\hline $\mathrm{C}$ & 0.239713000 & 60000 & 40000 \\
\hline $\mathrm{H}$ & 0.953129000 & 6.630675000 & 1000 \\
\hline $\mathrm{C}$ & 5.538337000 & 0.230562000 & 1.519805000 \\
\hline C & 5.004849000 & 5000 & 9000 \\
\hline $\mathrm{H}$ & 6.099 & 7000 & 000 \\
\hline $\mathrm{C}$ & 6.909685000 & 0000 & 6000 \\
\hline $\mathrm{C}$ & 4.368999000 & 3000 & 65000 \\
\hline $\mathrm{H}$ & 4.956972000 & 6.608792000 & -1.111293000 \\
\hline$C^{\prime}$ & 6.123375000 & 7000 & -0.778815000 \\
\hline $\mathrm{H}$ & 6.353959000 & 2.27 & 208000 \\
\hline $\mathrm{C}$ & 2.976429 & 5.792 & 71000 \\
\hline $\mathrm{H}$ & 2.468699000 & 33000 & -0.976027000 \\
\hline $\mathrm{N}$ & -2.630812000 & 78000 & 0.472744000 \\
\hline $\mathrm{C}$ & -2.352678000 & -4.670 & 93000 \\
\hline $\mathrm{C}$ & -5.302378000 & -2.206339000 & -0.537032000 \\
\hline $\mathrm{C}$ & -5.434917000 & -1.789295000 & 0.806270000 \\
\hline $\mathrm{H}$ & -4.878937000 & -2.311746000 & 1.587459000 \\
\hline $\mathrm{C}$ & -6.881882000 & -0.447806000 & -1.180755000 \\
\hline $\mathrm{C}$ & -0.981284000 & -4.937533000 & -0.268341000 \\
\hline $\mathrm{C}$ & -7.002574000 & 6325000 & 67000 \\
\hline $\mathrm{C}$ & -3.119581000 & -3.524025000 & -0.416270000 \\
\hline $\mathrm{C}$ & -6.271453000 & -0.716113000 & 1.142576000 \\
\hline $\mathrm{C}$ & -4.452598000 & -3.374514000 & -0.912639000 \\
\hline $\mathrm{C}$ & -0.650400000 & -4.763806000 & 1.093805000 \\
\hline $\mathrm{H}$ & -1.396330000 & -4.358822000 & 1.783590000 \\
\hline $\mathrm{C}$ & 1.602177000 & -5.621708000 & 0.706876000 \\
\hline $\mathrm{C}$ & -6.038050000 & -1.518456000 & -1.523909000 \\
\hline $\mathrm{H}$ & -5.932463000 & -1.804410000 & -2.575373000 \\
\hline $\mathrm{C}$ & 0.624536000 & -5.104982000 & 1.571501000 \\
\hline $\mathrm{C}$ & -2.925423000 & -5.615528000 & -1.673258000 \\
\hline $\mathrm{H}$ & -2.348408000 & -6.511208000 & -1.930426000 \\
\hline $\mathrm{C}$ & 1.284894000 & -5.792262000 & -0.649393000 \\
\hline $\mathrm{C}$ & -4.215739000 & -5.452155000 & -2.186051000 \\
\hline $\mathrm{H}$ & -4.642575000 & -6.204338000 & -2.857608000 \\
\hline & & & \\
\hline
\end{tabular}

\section{c}

C $\quad-4.970197000$

H $\quad-5.994937000$

$\mathrm{Ni} \quad 2.146537000$

C $\quad-1.667093000$

H $\quad-1.201931000$

1.298982000

1.402695000

0.320141000

$-0.361316000$

$-0.044518000$

$-0.057298000$

$-1.399381000$

$-1.164448000$

$-2.113671000$

$-1.786317000$

$-2.624676000$

0.568576000

3.341610000

2.675921000

4.373745000

2.982232000

1.555082000

3.201715000

0.882405000

1.986464000

0.955952000

$-3.209571000$

$-1.855521000$

$-1.847560000$

7.238555000

$-7.651616000$

$-3.137071000$

2.593742000

3.323333000

0.553366000

1.624759000

0.557831000

$-0.376702000$

2.532328000

1.625477000

8.018637000

8.644211000

8.963165000

7.530317000

5.159148000

4.192759000

6.219605000

4.661029000

$-1.608989000$

$-2.765359000$

$-1.851047000$

$-0.682457000$

$-2.588627000$

$-2.564844000$

$-2.279402000$

$-3.866274000$

$-7.702546000$

$-8.015622000$

$-7.044142000$

$-8.881153000$

$-6.336942000$

$-7.560427000$

$-6.065037000$

$-5.423789000$

2.343985000

3.080639000

3.224990000
$-5.584227000$

$-4.335622000$

$-4.214460000$

0.663073000

1.754170000

$-1.817712000$

2.203487000

3.062018000

1.235040000

1.451910000

0.112714000

2.312992000

0.631551000

$-0.696828000$

0.872449000

$-0.429810000$

$-1.075838000$

$-0.181189000$

$-0.973579000$

$-1.098600000$

$-0.612303000$

$-1.517574000$

$-2.080011000$

$-0.734084000$

$-1.762771000$

$-3.058309000$

$-2.226803000$

0.843247000

0.099918000

1.853611000

$-1.013262000$

0.802329000

5.664954000

$-5.883885000$

1.265956000

1.331860000

2.145973000

0.331908000

1.630172000

1.842429000

3.163428000

0.162749000

1.232579000

$-0.577805000$

$-0.600901000$

$-0.605402000$

$-1.516165000$

$-1.302921000$

0.160115000

7.421260000

7.956527000

7.024698000

8.419557000

3.463425000

3.742353000

2.137531000

3.630826000

0.227714000

1.511967000

0.256130000

$-0.428803000$

$-0.238975000$

0.271835000

$-1.236682000$

0.757024000

$-6.280837000$

$-5.242425000$

$-7.126440000$
$-2.194923000$

$-1.802914000$

$-2.172528000$

$-1.290638000$

0.120697000

$-0.880021000$

1.012384000

1.698552000

1.396168000

2.627755000

0.552577000

3.239407000

3.072975000

0.995534000

4.387061000

2.224464000

2.512404000

$-0.553234000$

$-1.158100000$

$-2.387773000$

$-1.116709000$

$-0.275283000$

$-2.625292000$

$-3.281588000$

$-3.441628000$

$-2.914797000$

$-1.713931000$

4.252869000

5.136536000

4.816883000

0.983810000

0.419089000

$-0.144774000$

1.084588000

$-1.927484000$

$-3.595880000$

$-3.690229000$

$-4.041121000$

$-3.101628000$

$-4.223243000$

$-3.282079000$

$-1.369771000$

$-1.938492000$

$-0.728817000$

$-2.391307000$

2.722518000

2.398718000

3.213212000

3.733204000

1.295214000

0.815805000

2.578130000

1.353872000

$-1.685439000$

$-3.021202000$

$-1.558391000$

$-1.255698000$

$-2.256358000$

$-1.926708000$

$-3.450086000$

$-2.469783000$

2.575346000

2.887265000

3.461706000

2.808871000

$-1.610947000$

$-2.118939000$

$-1.009655000$ 


$\begin{array}{llll}\text { F } & 1.801181000 & -6.934787000 & -2.676105000 \\ \text { C } & 0.918397000 & -4.971958000 & 3.050260000 \\ \text { F } & 0.329781000 & -3.863740000 & 3.580772000 \\ \text { F } & 0.446953000 & -6.045641000 & 3.749367000 \\ \text { F } & 2.255686000 & -4.888346000 & 3.297344000\end{array}$

\section{CF3 1 -Transf-1}

Zero-point correction $=0.807122($ Hartree/Particle $)$ $\begin{array}{lr}\text { Thermal correction to Energy= } & 0.887902 \\ \text { Thermal correction to Enthalpy= } & 0.8888 \\ \text { Thermal correction to Gibbs Free Energy }= & 0.672\end{array}$ $\begin{array}{lc}\text { Thermal correction to Gibbs Free Energy= } & 0.672293 \\ \text { Sum of electronic and zero-point Energies }= & -4981.395041 \\ \text { Sum of electronic and thermal Energies }= & -4981.314261\end{array}$ $\begin{array}{ll}\text { Sum of electronic and thermal Energies= } & -4981.314261 \\ \text { Sum of electronic and thermal Enthalpies }= & -4981.313317\end{array}$ Sum of electronic and thermal Free Energies $=-4981.529870$ E solvent $=-4981.39383078$

\begin{tabular}{|c|c|c|c|}
\hline $\mathrm{N}$ & -2.176244000 & -2.132343000 & -0.171383000 \\
\hline$C$ & -4.270867000 & -3.359785000 & -0.688588000 \\
\hline C & -0.684534000 & -4.750024000 & -0.302177000 \\
\hline $\mathrm{C}$ & 0.293909000 & -3.988611000 & -0.972631000 \\
\hline $\mathrm{H}$ & -0.019165000 & -3.211835000 & -1.671862000 \\
\hline G & 1.114800000 & -5.977168000 & 0.816960000 \\
\hline $\mathrm{C}$ & -5.146528000 & -2.180436000 & -0.409655000 \\
\hline 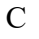 & 2.080897000 & -5.216185000 & 0.144399000 \\
\hline $\mathrm{C}$ & -2.864349000 & -3.356569000 & -0.464411000 \\
\hline $\mathrm{C}$ & 1.658903000 & -4.223938000 & -0.755452000 \\
\hline $\mathrm{C}$ & -2.148986000 & -4.592047000 & -0.573269000 \\
\hline 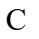 & -5.377829000 & -1.765 & 0.917092000 \\
\hline $\mathrm{H}$ & -4.860191000 & 88000 & 1.740495000 \\
\hline $\mathrm{C}$ & -6.973595000 & -0.086863000 & 0.152181000 \\
\hline $\mathrm{C}$ & -0.254281000 & -5.750858000 & 0.594153000 \\
\hline $\mathrm{H}$ & -0.996382000 & 87000 & 1.127710000 \\
\hline $\mathrm{C}$ & -6.291254000 & 7000 & 1.194516000 \\
\hline $\mathrm{C}$ & -4.918461000 & 61000 & -1.094105000 \\
\hline $\mathrm{H}$ & -6.001830000 & -4.517516000 & -1.257463000 \\
\hline $\mathrm{C}$ & -6.744037000 & -0.494820000 & -1.170745000 \\
\hline$C$ & -4.211313000 & -5.741945000 & -1.244227000 \\
\hline $\mathrm{H}$ & -4.725138000 & -6.65 & -1.56 \\
\hline $\mathrm{C}$ & -5.850102000 & -1.542535000 & -1.450529000 \\
\hline $\mathrm{H}$ & -5.696744000 & -1.866675000 & 4700000 \\
\hline $\mathrm{C}$ & -2.843258000 & -5.761032000 & -0.959619000 \\
\hline $\mathrm{H}$ & -2.275120000 & -6.692043000 & -1.064734000 \\
\hline $\mathrm{N}$ & 2.733021000 & 2.470341000 & 0.470190000 \\
\hline $\mathrm{C}$ & 2.654759 & & 40000 \\
\hline $\mathrm{C}$ & 5.305457000 & 1.860363000 & -0.713770000 \\
\hline $\mathrm{C}$ & 5.431370000 & 16000 & 0.601696000 \\
\hline $\mathrm{H}$ & 4.956266000 & 1.900 & 1.426006000 \\
\hline $\mathrm{C}$ & 6.667371000 & -0.020851000 & -1.492627000 \\
\hline $\mathrm{C}$ & 1.366637000 & 5.047238000 & -0.043213000 \\
\hline $\mathrm{C}$ & 6.777453000 & -0.513674000 & -0.184055000 \\
\hline $\mathrm{C}$ & 3.299132000 & 3.407760000 & -0.414413000 \\
\hline $\mathrm{C}$ & 6.153319000 & 0.188440000 & 0.859113000 \\
\hline $\mathrm{C}$ & 4.572172000 & 3.127843000 & -1.000710000 \\
\hline $\mathrm{C}$ & 1.122054000 & 4.824492000 & 1.329620000 \\
\hline $\mathrm{H}$ & 1.862855000 & 4.287601000 & 1.928467000 \\
\hline $\mathrm{C}$ & -1.033879000 & 5.956645000 & 1.180426000 \\
\hline $\mathrm{C}$ & 5.936407000 & 1.149389000 & -1.756602000 \\
\hline $\mathrm{H}$ & 5.833885000 & 1.497882000 & -2.789376000 \\
\hline $\mathrm{C}$ & -0.062610000 & 5.277642000 & 1.931045000 \\
\hline $\mathrm{C}$ & 3.274664000 & 5.565554000 & -1.568270000 \\
\hline $\mathrm{H}$ & 2.791296000 & 6.531879000 & -1.752354000 \\
\hline $\mathrm{C}$ & -0.804548000 & 6.173682000 & -0.187761000 \\
\hline $\mathrm{C}$ & 4.502135000 & 5.277649000 & -2.173545000 \\
\hline $\mathrm{H}$ & 4.971391000 & 6.004855000 & -2.844272000 \\
\hline $\mathrm{C}$ & 0.378808000 & 5.725421000 & -0.793310000 \\
\hline $\mathrm{H}$ & 0.530964000 & 5.893563000 & -1.863562000 \\
\hline $\mathrm{C}$ & 5.143840000 & 4.067087000 & -1.882943000 \\
\hline $\mathrm{H}$ & 6.124494000 & 3.849407000 & -2.321586000 \\
\hline
\end{tabular}

c

C

政

$-7.405337000$

$-7.781207000$

$-8.507847000$

$-6.546023000$

$-6.478077000$

$-5.512708000$

$-7.681514000$

$-6.399415000$

1.528654000

2.835555000

1.356719000

0.778434000

2.683569000

2.930561000

2.257385000

3.871187000

7.381948000

7.578344000

6.686531000

8.611784000

6.198762000

7.207167000

6.365338000

5.031923000

$-1.877626000$

$-2.986047000$

$-2.301707000$

$-1.456604000$

$-0.262169000$

$-0.550140000$ 1.679888000

1.724639000

$-2.108827000$

$-2.953337000$

$-1.138033000$

$-1.310246000$

$-0.116431000$

$-2.107607000$

$-0.531216000$

0.670504000

$-0.742274000$

0.452511000

1.077327000

0.109942000

0.828045000

1.498297000

0.251688000

1.062330000

2.622440000

1.372675000

2.671108000

3.590381000

2.528890000

$-0.854403000$

0.114951000

$-1.646878000$

0.716747000

$-1.431255000$

$-5.391096000$

6.301833000

$-0.970220000$

$-0.075761000$

$-1.389648000$

0.140866000

0.738283000

$-2.241852000$

$-1.585651000$

0.231569000

$-0.627517000$

0.922149000

1.134119000

$-0.272897000$

0.622969000

0.335254000

$-1.313923000$

$-7.018593000$

$-7.371612000$

$-6.562655000$

$-8.152098000$

$-3.449953000$

$-4.052171000$

$-2.181978000$

$-3.353749000$

$-0.723232000$

$-2.045325000$

$-0.632863000$

$-0.173973000$

$-0.342941000$

$-1.239339000$

0.658323000

$-0.980430000$

6.859565000

6.063254000

8.007858000

7.177822000

5.089502000

3.938966000
$-1.479603000$

0.069426000

$-0.963192000$

0.924340000

1.626078000

1.321797000

2.616018000

0.432176000

3.252358000

3.100045000

0.938064000

4.476499000

2.230935000

2.554757000

$-0.766127000$

$-0.186760000$

$-1.397388000$

0.106478000

0.605613000

$-1.715218000$

$-2.072926000$

$-2.791829000$

$-1.452815000$

$-1.143915000$

4.417223000

5.145630000

4.959137000

0.368718000

0.018154000

0.318685000

1.652165000

$-2.075982000$

$-3.442040000$

$-3.160320000$

$-4.043558000$

$-3.313432000$

$-3.525869000$

$-2.819341000$

$-2.320558000$

$-3.308687000$

$-1.926666000$

$-2.889648000$

2.623774000

2.980857000

2.808104000

3.499817000

1.833741000

1.701903000

3.108733000

1.715979000

$-1.556568000$

$-2.755444000$

$-1.830366000$

$-0.900974000$

$-2.625262000$

$-2.362103000$

$-3.794647000$

$-2.853196000$

2.273774000

2.455789000

3.183870000

2.602929000

$-1.001298000$

$-1.137745000$

$-0.402806000$

$-2.255395000$

3.419328000

3.865421000 
$\begin{array}{lrrr}\text { F } & 0.291636000 & 6.114995000 & 4.129553000 \\ \text { F } & -1.584075000 & 5.042939000 & 3.750011000\end{array}$

${ }^{\text {CF3 TS-3 }}$ decoord

Zero-point correction $=\quad 0.806132($ Hartree/Particle $)$

Thermal correction to Energy= $\quad 0.886667$

Thermal correction to Enthalpy $=\quad 0.887611$

Thermal correction to Gibbs Free Energy= 0.671817

Sum of electronic and zero-point Energies $=\quad-4981.393475$

Sum of electronic and thermal Energies= $\quad-4981.312940$

Sum of electronic and thermal Enthalpies $=\quad-4981.311996$

Sum of electronic and thermal Free Energies $=-4981.527790$

E solvent $=-4981.393674$

\begin{tabular}{|c|c|c|c|}
\hline & 2.077861000 & 5511000 & \\
\hline & 4.197904000 & 56874000 & \\
\hline & 0.567722000 & 4.770544000 & 12000 \\
\hline & -0.311995000 & 3.911737000 & \\
\hline & 0.098613000 & 87810000 & 98000 \\
\hline & -1.374193000 & 6.007999000 & $\mathrm{gen}$ \\
\hline & 5.042920000 & 2.286232000 & > \\
\hline & -2.242615000 & & \\
\hline 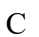 & & & \\
\hline C & -1.7 & & \\
\hline$C$ & & & \\
\hline$C$ & & & \\
\hline & 00 & & \\
\hline & 6.80 & & \\
\hline & 00 & & \\
\hline & & & \\
\hline & & & \\
\hline & & & \\
\hline & & & \\
\hline & & & \\
\hline & & & \\
\hline & & & \\
\hline & & & \\
\hline & & & -2 \\
\hline & 00 & & $-1 .($ \\
\hline$\Pi$ & 2.1 & & \\
\hline $\mathrm{N}$ & -2.6 & 00 & \\
\hline $\mathrm{C}$ & -2.4 & & \\
\hline $\mathrm{C}$ & -5.3 & & \\
\hline C & -5.40 & & \\
\hline $\mathrm{H}$ & & & \\
\hline $\mathrm{C}$ & & & \\
\hline $\mathrm{C}$ & & & \\
\hline $\mathrm{C}$ & & & \\
\hline $\mathrm{C}$ & & & \\
\hline $\mathrm{C}$ & -6.1 & & \\
\hline $\mathrm{C}$ & -4. & & \\
\hline $\mathrm{C}$ & -0.7 & & \\
\hline & -1. & & \\
\hline$C$ & & & \\
\hline$C$ & -6. & & -1 \\
\hline & & & -2 \\
\hline$C$ & & & 1. \\
\hline 0 & & & -1 \\
\hline & & & \\
\hline & & & -0 . \\
\hline$C$ & -4.4 & & \\
\hline $\mathrm{H}$ & -4.8 & 000 & \\
\hline$C$ & 000 & 000 & -1 . \\
\hline $\mathrm{H}$ & -0.3 & 6000 & 700 \\
\hline $\mathrm{C}$ & 000 & 3000 & 700 \\
\hline & & 13000 & -2.07 \\
\hline & & & -1.54239400 \\
\hline & & & - \\
\hline & -1.347844000 & -1.723985000 & -0.9264690 \\
\hline
\end{tabular}

(1)

(1)

H 2.445972000

H 3.084918000

C $\quad 1.513650000$

C $\quad 0.928877000$

$\mathrm{H} \quad 2.443624000$

H 0.924255000

$\mathrm{H} \quad 1.389964000$

H $\quad-0.122029000$

7.624497000

8.526463000

8.318066000

6.871088000

5.980248000

5.004960000

7.165905000

5.747158000

$-1.920242000$

$-3.229834000$

$-1.822264000$

$-1.223688000$

$-2.601502000$

$-2.499236000$

$-2.262087000$

$-3.909224000$

$-7.701076000$

$-7.967324000$

$-7.074918000$

$-8.903786000$

$-6.184130000$

$-7.327667000$

$-6.039976000$

$-5.144722000$

2.127315000

3.411350000

1.941280000

2.029323000

0.811735000

0.029965000

0.630011000

2.112797000
2.182081000 3.024262000 1.199416000 1.338708000 0.166754000 2.146501000 0.506902000 $-0.680733000$ 0.678067000 $-0.497522000$ $-1.165929000$ $-0.016980000$ $-0.930372000$ $-1.504779000$ $-0.113865000$ $-1.359180000$ $-2.750046000$ $-1.084108000$ $-2.650382000$ $-3.603925000$ $-3.017382000$ 0.733964000 $-0.172377000$ 1.598793000 $-0.588601000$ 1.021587000 5.303197000 $-5.960164000$ 1.367258000 $-0.137106000$ 1.137811000 $-0.321927000$ $-1.012543000$ 1.969474000 1.254677000 $-0.013415000$ 0.872560000 $-1.078792000$ $-0.452942000$ 0.358024000 $-0.572197000$ $-0.215641000$ 1.382796000 7.108918000 7.371323000 6.777609000 8.270368000 3.211861000 3.491445000 1.895825000 3.340164000 0.458451000 1.747796000 0.479703000 $-0.161500000$ $-0.086242000$ 0.575493000 $-1.124742000$ 0.774447000 $-6.435170000$ $-6.260319000$ $-7.775122000$ $-5.863188000$ $-5.064290000$ $-4.145083000$ $-6.255126000$ $-4.703828000$
0.952757000 1.653906000 1.358106000 2.662459000 0.466735000 3.300231000 3.153982000 0.982565000 4.540299000 2.285891000 2.619235000 $-0.742737000$ $-0.263137000$ $-1.420052000$ 0.203027000 0.309944000 $-2.020225000$

$-1.929874000$

$-3.109993000$

$-1.886634000$ $-1.545476000$ 4.497120000 5.199615000 5.023867000 0.954326000 0.610892000 $-0.356102000$ 1.070275000 $-2.214438000$ $-3.330452000$ $-3.328245000$ $-3.878255000$ $-3.039429000$ $-3.873204000$ $-3.040076000$ $-1.737403000$ $-2.246581000$ $-1.258851000$ $-2.793967000$ 3.036295000 3.245420000 3.379864000 3.904738000 1.173299000 0.916125000 2.493655000 1.006324000 $-1.988102000$ $-3.320118000$ $-1.839142000$ $-1.642746000$ $-2.063817000$ $-1.713572000$ $-3.274741000$ $-2.251040000$ 2.720862000 3.051307000 3.591093000 2.959576000

$-1.613881000$ $-1.185401000$ $-1.782995000$ $-2.850587000$ 3.056232000 3.686312000 3.698272000 3.250869000 
CF32-BHE-C

Zero-point correction= 0.727153 (Hartree/Particle)

Thermal correction to Energy=

Thermal correction to Enthalpy=

0.803046

0.803991

Thermal correction to Gibbs Free Energy=

0.597356

Sum of electronic and zero-point Energies=

Sum of electronic and thermal Energies $=$

$-4863.585259$

$-4863.509366$

$\begin{array}{lc}\text { Sum of electronic and thermal Enthalpies }= & -4863.508421 \\ \text { Sum of electronic and thermal Free Energies }= & -4863.715056\end{array}$

E solvent $=-4863.548870$

$\mathrm{Ni} \quad-2.056738000$

$\mathrm{N} \quad-2.379528000$

$\mathrm{O}-0.510294000$

N 2.846427000

C $\quad-0.357552000$

1.085680000

0.878170000

2.023228000

0.033359000

$-2.154288000$

1.362299000

$-4.464406000$

1.307339000

$-0.384671000$

0.102579000

$-4.822987000$

0.329695000

2.342518000

$-4.719415000$

$-1.547351000$

$-1.391291000$

2.112577000

$-1.815209000$

2.841996000

1.806227000

1.247785000

$-1.431444000$

$-1.566188000$

2.903638000

0.906165000

1.757753000

$-4.439813000$

$-4.211762000$

$-0.401880000$

$-5.471528000$

6.708479000

$-1.933005000$

0.865976000

$-5.041965000$

$-1.461062000$

1.990018000

2.917487000

$-0.032186000$

0.081299000

$-3.348182000$

$-4.497618000$

$-3.904692000$

$-1.626775000$

$\mathrm{H} \quad-2.277097000$

C $\quad 2.432555000$

C $\quad-2.015454000$

C $\quad 4.589707000$

C $\quad-0.351384000$

C $\quad-4.493589000$

$\mathrm{H} \quad-4.362418000$

3.267698000

0.380983000

0.008481000

$-4.968457000$

$-0.046999000$

$-0.589861000$

$-4.749693000$

$-4.765457000$

4.315789000

$-0.006356000$

0.393946000

6.058432000

6.396744000

$-4.256107000$

4.961961000

$-3.066900000$

$-4.697646000$

5.140517000

6.226969000

$-3.153805000$

1.647267000

2.442714000

3.201884000

2.761763000

$-6.940867000$

4.190003000

4.549258000

1.550882000

$-1.291945000$

5.020516000

6.036262000

$-5.778781000$

$-6.654710000$

$-0.480860000$

0.157460000

$-1.777774000$

0.085004000

$-5.824591000$

$-5.099924000$

$-5.855314000$

0.967139000

$-4.989010000$

$-4.037016000$

$-3.964888000$

4.593744000

5.249566000

$-4.705522000$

$-5.784785000$

$-4.756794000$

$-3.596214000$

0.319459000

0.983043000

$-5.209431000$

$-6.063961000$

$-1.066424000$
$-1.289240000$

$-0.554759000$

0.319992000

1.459210000

$-0.323823000$

0.923427000

0.552280000

0.281181000

1.118784000

1.224987000

2.008124000

$-0.910216000$

0.221623000

1.075694000

1.724273000

0.552896000

0.066459000

0.241572000

0.829183000

2.100088000

2.319058000

$-1.136673000$

$-2.225570000$

0.173896000

1.204398000

2.032218000

0.776399000

1.589898000

$-0.933934000$

$-0.021304000$

$-1.111669000$

1.642581000

0.239504000

0.183267000

$-0.575161000$

2.645048000

3.305428000

0.324602000

$-2.418394000$

$-3.125043000$

2.983840000

$-0.564001000$

$-2.037861000$

$-2.443536000$

0.321072000

0.362923000

3.150399000

3.692408000

3.558921000

3.704561000

$-0.796786000$

$-1.556251000$

$-1.858025000$

$-2.110847000$

$-0.888282000$
0.193713000

0.033824000

0.380561000

$5.505924000 \quad-1.947929000$ $-0.036968000$ $-0.530567000$

1.311491000

$-0.545691000$

$-4.086401000$

$-4.398719000$

$-2.725104000$

$-4.482319000$

7.123220000

$-2.498094000$

$-2.171938000$

$-3.101837000$

$-3.445487000$

5.682813000

5.117378000

7.280448000

6.242778000

6.103157000

0.459527000

$-1.149161000$

$-1.439978000$

0.075225000

2.328808000

2.094756000

2.096094000

3.416770000

7.935138000

7.269774000

9.113761000

8.248078000

6.553156000

$-6.761151000$

$-7.203766000$

$-5.946579000$

$-7.836709000$

6.740762000

7.679387000

5.582751000

7.139381000

1.002914000

0.316052000

1.658929000

1.947406000

$-3.213075000$

$-1.475754000$

$-2.305240000$

$-0.386564000$

$-1.860825000$

$-1.880746000$

$-3.367542000$

7.943150000

$-2.443737000$

$-5.618345000$

1.416767000

7.919947000

9.065450000

8.074033000

7.836136000

$-2.288228000$

$-1.887617000$

$-2.263232000$

$-3.586620000$

$-0.230621000$

$-5.171619000$

$-5.722158000$

$-3.967588000$

$-5.961196000$

$-1.554006000$

$-2.060214000$

0.165283000

$-1.272958000$

$-1.539926000$

6.275420000

4.538608000

3.699267000

5.188351000

1.280360000

0.560082000

2.290190000

1.221794000

0.442032000

0.465685000

$-0.212808000$

1.728011000

$-0.505858000$

$-1.619762000$

$-2.888082000$

$-1.648980000$

$-0.850277000$

$-0.130755000$

$-0.912233000$

$-0.285233000$

1.163007000

4.659774000

4.312289000

3.540657000

5.572098000

1.502310000

0.298167000

$-0.676723000$

0.215096000

1.027890000

$-1.537238000$

$-0.737662000$

0.995206000

$-5.137378000$

$-3.012264000$

6.775738000 


\begin{tabular}{|c|c|c|c|}
\hline & & & \\
\hline & 5411000 & -2.237099000 & \\
\hline & -0.320113000 & 1.311104000 & 08118000 \\
\hline & 8641000 & -4.454590000 & 038765800 \\
\hline & 000 & -0.457158000 & \\
\hline & 111346000 & .273296000 & $507-2$ \\
\hline & -4.723042000 & 54075 & \\
\hline & -4.928068000 & -1.185353000 & \\
\hline & -1.480701000 & & \\
\hline I & -1.717356000 & & \\
\hline & 1.792082000 & -1.49 & \\
\hline & 1.225172000 & -1.599391000 & -0.9 \\
\hline & -3.373163000 & 2.990707000 & \\
\hline & 0.841056000 & -4.53505 & 00 \\
\hline & 1.721031000 & -4.436929000 & 1.657435000 \\
\hline & -0.0044 & 6.588627000 & 0.56 \\
\hline & -5.7945 & -1.655112000 & \\
\hline & -5.077 & & \\
\hline & -1.5656 & -4.84742 & \\
\hline & 2.007 & -0.1 & \\
\hline & 2.92 & -0.7 & \\
\hline & -0.1 & -4.5 & \\
\hline & -0.0 & -4.5 & \\
\hline & -3.0 & & \\
\hline & -4.5 & & \\
\hline & -3.8 & 0.5 & \\
\hline & -1.2 & & \\
\hline & -1.9 & & \\
\hline & 2.3 & -4.2 & \\
\hline & -1.7 & & \\
\hline & 4.53 & & \\
\hline & -0.41 & & \\
\hline C & -4.16 & & \\
\hline & -3.94 & & \\
\hline & 3.21 & -3.22 & \\
\hline & $0.42>$ & & \\
\hline $\mathrm{H}$ & 0.07 & & \\
\hline$C$ & -5.764 & 3.49 & \\
\hline & & & \\
\hline$C$ & 4.05 & & \\
\hline $\mathrm{H}_{2}$ & & & \\
\hline & & & \\
\hline & & & \\
\hline & & & \\
\hline$H_{-2}$ & 5.93 & -4.0 & -2.5 \\
\hline & 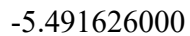 & & \\
\hline & & & \\
\hline & 05 & & \\
\hline & & & \\
\hline $\mathrm{F}$ & 0 & & \\
\hline$F_{1} \rightarrow$ & -1.3 & -3 & \\
\hline $\mathrm{C}$ & & & \\
\hline & & & \\
\hline 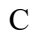 & & 0 & \\
\hline $\mathrm{H}$ & 2.10 & & \\
\hline $\mathrm{C}$ & -6.311 & -0.7 & \\
\hline $\mathrm{C}$ & & -2.0 & -0. \\
\hline - & 00 & & \\
\hline 1 & 00 & & \\
\hline 1 & 00 & $7.8^{\prime}$ & \\
\hline$\Gamma$ & 000 & 7.80 & \\
\hline 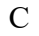 & 000 & -2.1 & 000 \\
\hline $\mathrm{F}$ & -4.98 & -1.9 & \\
\hline $\mathrm{F}$ & & & \\
\hline $\mathrm{F}$ & & & \\
\hline & & & \\
\hline & & & \\
\hline & -2.396215000 & -5.332408000 & -2.7115680 \\
\hline
\end{tabular}

$\begin{array}{cccc}\text { F } & -3.135921000 & -3.528151000 & -1.707017000 \\ \text { F } & -3.672079000 & -5.506018000 & -0.928363000 \\ \text { C } & 5.677118000 & -1.714341000 & 0.564756000 \\ \text { H } & 5.096259000 & -2.211747000 & 1.344043000 \\ \text { C } & 7.324651000 & -0.035267000 & -0.083311000 \\ \text { C } & 6.241557000 & -1.420154000 & -1.772284000 \\ \text { H } & 6.091421000 & -1.668078000 & -2.827841000 \\ \text { C } & 0.867349000 & 6.066461000 & -0.401449000 \\ \text { C } & -0.822274000 & 4.409826000 & -0.988814000 \\ \text { H } & -1.133045000 & 3.559621000 & -1.604449000 \\ \text { C } & 0.447450000 & 4.974410000 & -1.177229000 \\ \text { C } & 2.375023000 & 1.160764000 & 4.284266000 \\ \text { H } & 2.114630000 & 0.454352000 & 5.096252000 \\ \text { H } & 2.178590000 & 2.180444000 & 4.658894000 \\ \text { H } & 3.460556000 & 1.062919000 & 4.107423000 \\ \text { C } & 7.982600000 & 0.253707000 & -2.500735000 \\ \text { F } & 7.319779000 & 0.305356000 & -3.691210000 \\ \text { F } & 9.145541000 & -0.427256000 & -2.723903000 \\ \text { F } & 8.326661000 & 1.528620000 & -2.164487000 \\ \text { C } & 6.579204000 & -0.697137000 & 0.906578000 \\ \text { C } & -7.152970000 & -1.224459000 & -1.988236000 \\ \text { F } & -7.701188000 & -2.445816000 & -1.759540000 \\ \text { F } & -6.394499000 & -1.326604000 & -3.126413000 \\ \text { F } & -8.165439000 & -0.357232000 & -2.270945000 \\ \text { C } & 6.780634000 & -0.345247000 & 2.362896000 \\ \text { F } & 7.706245000 & -1.153721000 & 2.954127000 \\ \text { F } & 5.623715000 & -0.484430000 & 3.083555000 \\ \text { F } & 7.207706000 & 0.937989000 & 2.523778000 \\ \text { C } & 1.385240000 & 4.352043000 & -2.189804000 \\ \text { F } & 0.711120000 & 3.922838000 & -3.296045000 \\ \text { F } & 2.026050000 & 3.267082000 & -1.669637000 \\ \text { F } & 2.341894000 & 5.227943000 & -2.602560000 \\ \text { H } & -3.228971000 & 1.457720000 & -1.905893000 \\ \text { C } & -1.829852000 & -0.365985000 & -2.809079000 \\ \text { C } & -2.969276000 & 0.432156000 & -3.106214000 \\ \text { H } & -1.962924000 & -1.391074000 & -2.446167000 \\ \text { H } & -0.859295000 & -0.126072000 & -3.258881000 \\ \text { H } & -3.970799000 & -0.012187000 & -3.031091000 \\ \text { H } & -2.883152000 & 1.237406000 & -3.848587000 \\ \text { H } & 8.011624000 & 0.771774000 & 0.184312000 \\ \text { H } & -2.551780000 & -4.975200000 & 1.237305000 \\ \text { H } & -6.052739000 & -2.714899000 & 0.045791000 \\ \text { H } & 1.859330000 & 6.501642000 & -0.547056000\end{array}$

\section{${ }^{\mathrm{CF}}$ 1-Transf-2}

Zero-point correction $=$ Thermal correction to Energy= Thermal correction to Enthalpy= 0.728846 (Hartree/Particle)

Thermal correction to Gibbs Free Energy $=0.599958$ Sum of electronic and zero-point Energies $=\quad-4863.595395$ Sum of electronic and thermal Energies $=\quad-4863.519618$ Sum of electronic and thermal Enthalpies $=\quad-4863.518674$ Sum of electronic and thermal Free Energies $=-4863.724283$ E solvent $=-4863.56323948$

$\begin{array}{lr}\mathrm{N} & 2.279727000 \\ \mathrm{C} & 3.025692000 \\ \mathrm{C} & 5.070959000 \\ \mathrm{C} & 6.059864000 \\ \mathrm{H} & 6.516738000 \\ \mathrm{C} & 4.885979000 \\ \mathrm{C} & 1.632577000 \\ \mathrm{C} & 5.852788000 \\ \mathrm{C} & 3.329584000 \\ \mathrm{C} & 6.436968000 \\ \mathrm{C} & 4.688290000 \\ \mathrm{C} & 1.195325000 \\ \mathrm{H} & 1.859008000 \\ \mathrm{C} & -0.961485000\end{array}$

2.061403000 4.424417000 1.165152000 0.692310000 1.382949000 $-1.102416000$ 4.939882000 $-1.568119000$ 3.035709000 $-0.659103000$ 2.607289000 6.057830000 6.510415000 6.023108000
0.227775000 0.044859000 0.271230000 $-0.619487000$ $-1.336024000$ 1.170008000 $-0.125317000$ 0.267428000 0.173797000 $-0.627760000$ 0.219013000 0.613621000 1.358324000 $-0.522619000$ 


\begin{tabular}{|c|c|c|c|}
\hline C & 4.490806000 & 0.246228000 & 1.171419000 \\
\hline 11 & 3.736174000 & 0.582814000 & 1.886813000 \\
\hline $\mathrm{C}$ & -0.090630000 & 6.590885000 & 0.417124000 \\
\hline $\mathrm{C}$ & 4.086356000 & 5.356436000 & 0.017469000 \\
\hline $\mathrm{H}$ & 3.849170000 & 6.419522000 & -0.101433000 \\
\hline $\mathrm{C}$ & -0.528950000 & 4.913390000 & -1.268357000 \\
\hline $\mathrm{C}$ & 5.420641000 & 4.941848000 & 0.092154000 \\
\hline $\mathrm{H}$ & 6.229695000 & 5.679328000 & 0.068884000 \\
\hline $\mathrm{C}$ & 0.750310000 & 4.376174000 & -1.073196000 \\
\hline $\mathrm{H}$ & 1.064783000 & 3.504583000 & -1.655496000 \\
\hline $\mathrm{C}$ & 5.713964000 & 3.576007000 & 0.178144000 \\
\hline $\mathrm{H}$ & 6.755825000 & 3.242633000 & 0.242718000 \\
\hline $\mathrm{N}$ & -2.810604000 & -2.259029000 & 0.327438000 \\
\hline $\mathrm{C}$ & -2.317993000 & -4.341354000 & -0.935135000 \\
\hline $\mathrm{C}$ & -5.481584000 & -2.162141000 & -0.715029000 \\
\hline $\mathrm{C}$ & -5.671444000 & -1.770979000 & 0.628663000 \\
\hline $\mathrm{H}$ & -5.089344000 & -2.256170000 & 1.414815000 \\
\hline $\mathrm{C}$ & -7.153971000 & -0.500315000 & -1.378191000 \\
\hline $\mathrm{C}$ & -0.944316000 & -4.478782000 & -0.366049000 \\
\hline $\mathrm{C}$ & -7.324281000 & -0.106314000 & -0.043496000 \\
\hline $\mathrm{C}$ & -3.195471000 & -3.276414000 & -0.562536000 \\
\hline $\mathrm{C}$ & -6.576144000 & -0.751198000 & 0.955737000 \\
\hline $\mathrm{C}$ & -4.527166000 & -3.248825000 & -1.082343000 \\
\hline $\mathrm{C}$ & -0.715710000 & -4.436194000 & 1.025384000 \\
\hline $\mathrm{H}$ & -1.555055000 & -4.255080000 & 1.704176000 \\
\hline $\mathrm{C}$ & 1.668294000 & -4.829466000 & 0.694458000 \\
\hline $\mathrm{C}$ & -6.240290000 & -1.514824000 & -1.711970000 \\
\hline $\mathrm{H}$ & -6.091460000 & -1.780399000 & -2.763459000 \\
\hline $\mathrm{C}$ & 0.575575000 & -4.615682000 & 1.546498000 \\
\hline $\mathrm{C}$ & -2.772298000 & -5.320557000 & -1.844962000 \\
\hline $\mathrm{H}$ & -2.100271000 & -6.146431000 & -2.106236000 \\
\hline $\mathrm{C}$ & 1.448379000 & -4.873676000 & -0.691677000 \\
\hline $\mathrm{C}$ & -4.063361000 & -5.271778000 & -2.382749000 \\
\hline $\mathrm{H}$ & -4.401516000 & -6.047005000 & -3.078262000 \\
\hline $\mathrm{C}$ & 0.157741000 & -4.708157000 & -1.217698000 \\
\hline $\mathrm{H}$ & 0.006347000 & -4.736440000 & -2.301396000 \\
\hline $\mathrm{C}$ & -4.931347000 & -4.241511000 & -1.996237000 \\
\hline $\mathrm{H}$ & -5.954933000 & -4.212888000 & -2.387495000 \\
\hline $\mathrm{Ni}$ & 2.034358000 & 0.732388000 & -1.155196000 \\
\hline $\mathrm{C}$ & -1.787935000 & -1.508061000 & 0.048015000 \\
\hline $\mathrm{H}$ & -1.214335000 & -1.631687000 & -0.887916000 \\
\hline $\mathrm{C}$ & 1.420057000 & 2.178494000 & 1.230304000 \\
\hline $\mathrm{H}$ & 1.608740000 & 2.973918000 & 1.971670000 \\
\hline $\mathrm{C}$ & 0.265424000 & 1.368431000 & 1.464279000 \\
\hline $\mathrm{C}$ & -0.523432000 & 1.670069000 & 2.612084000 \\
\hline $\mathrm{C}$ & -0.127914000 & 0.284967000 & 0.589925000 \\
\hline $\mathrm{H}$ & -0.198964000 & 2.503216000 & 3.251397000 \\
\hline $\mathrm{C}$ & -1.682712000 & 0.965568000 & 2.941696000 \\
\hline $\mathrm{C}$ & -1.335169000 & -0.437110000 & 0.933841000 \\
\hline $\mathrm{C}$ & -2.504285000 & 1.298460000 & 4.170989000 \\
\hline $\mathrm{C}$ & -2.066873000 & -0.084425000 & 2.077356000 \\
\hline $\mathrm{H}$ & -2.988814000 & -0.642871000 & 2.278879000 \\
\hline $\mathrm{O}$ & 0.526670000 & -0.083510000 & -0.480131000 \\
\hline $\mathrm{H}$ & -3.579610000 & 1.128424000 & 3.988914000 \\
\hline $\mathrm{H}$ & -2.217504000 & 0.664952000 & 5.032767000 \\
\hline $\mathrm{H}$ & -2.369396000 & 2.350667000 & 4.476940000 \\
\hline $\mathrm{H}$ & -1.963329000 & 6.434271000 & -0.669744000 \\
\hline $\mathrm{H}$ & -8.013238000 & 0.702789000 & 0.212609000 \\
\hline $\mathrm{H}$ & 6.141140000 & -2.621642000 & 0.256772000 \\
\hline $\mathrm{H}$ & 2.674798000 & -4.938830000 & 1.103700000 \\
\hline $\mathrm{H}$ & 3.148395000 & 1.204008000 & -3.805921000 \\
\hline $\mathrm{C}$ & 2.077346000 & -0.373305000 & -2.716025000 \\
\hline $\mathrm{C}$ & 3.243932000 & 0.515186000 & -2.949524000 \\
\hline $\mathrm{H}$ & 2.272226000 & -1.420697000 & -2.450678000 \\
\hline $\mathrm{H}$ & 1.182196000 & -0.219579000 & -3.334043000 \\
\hline $\mathrm{H}$ & 3.381931000 & 1.290138000 & -2.051497000 \\
\hline $\mathrm{H}$ & 4.227622000 & 0.016711000 & -2.939314000 \\
\hline $\mathrm{C}$ & 2.627959000 & -5.010640000 & -1.624364000 \\
\hline
\end{tabular}

\begin{tabular}{cc} 
F & 3.209969000 \\
F & 3.603777000 \\
F & 2.273067000 \\
C & 0.758697000 \\
F & 0.221178000 \\
F & 0.131403000 \\
F & 2.069067000 \\
C & -7.986195000 \\
F & -8.338916000 \\
F & -7.322082000 \\
F & -9.144334000 \\
C & -6.780176000 \\
F & -7.196758000 \\
F & -7.714146000 \\
F & -5.626548000 \\
C & 7.395976000 \\
F & 6.741215000 \\
F & 8.382619000 \\
F & 7.985615000 \\
C & 4.214291000 \\
F & 4.669726000 \\
F & 4.420173000 \\
F & 2.865271000 \\
C & -1.425829000 \\
F & -1.204508000 \\
F & -2.742573000 \\
F & -1.206232000 \\
C & -0.541705000 \\
F & 0.445263000 \\
$F$ & -1.635444000 \\
F & -0.863124000 \\
& \\
\hline
\end{tabular}

$-3.792199000$ $-5.802535000$ $-5.532158000$ $-4.590165000$ $-3.464047000$ $-5.651119000$ $-4.642354000$ 0.143570000 1.420376000 0.184491000 $-0.547770000$ $-0.378017000$ 0.910797000 $-1.170501000$ $-0.516961000$ $-1.142800000$ $-1.338345000$ $-0.234032000$ $-2.320216000$ $-2.047983000$ $-3.326443000$ $-1.667477000$ $-2.067656000$ 4.330317000 2.996506000 4.498389000 4.928948000 7.748556000 8.681043000 8.374145000 7.330578000

$-1.876425000$ $-1.100108000$ $-2.830982000$ 3.048339000 3.596072000 3.637746000 3.413053000 $-2.464261000$ $-2.144445000$ $-3.654331000$ $-2.680287000$ 2.406143000 2.548255000 3.005268000 3.133228000 $-1.691103000$ $-2.878861000$ $-1.932252000$ $-1.357710000$ 2.142294000 2.011546000 3.433380000 1.943857000 $-2.339988000$ $-2.511292000$ $-2.041303000$ $-3.546960000$ 1.280914000 1.415033000 0.768968000 2.539764000

\section{${ }^{\text {CF3 }}$ TS-2}

Zero-point correction $=$ Thermal correction to Energy 0.891107 Enthalpy $=\quad 0.892051$

Thermal correction to Gibbs Free Energy $=0.677796$ Sum of electronic and zero-point Energies $=\quad-4981.394449$ Sum of electronic and thermal Energies $=\quad-4981.314538$ Sum of electronic and thermal Enthalpies $=\quad-4981.313593$ Sum of electronic and thermal Free Energies $=\quad-4981.527849$ E solvent $=-4981.39527899$

$\begin{array}{lrrr}\mathrm{Ni} & 1.717009000 & 0.835076000 & -1.370529000 \\ \mathrm{C} & 3.493577000 & 1.318103000 & -2.491925000 \\ \mathrm{~N} & 2.255936000 & 1.826818000 & 0.330099000 \\ \mathrm{C} & 1.446616000 & 1.863341000 & 1.382378000 \\ \mathrm{C} & 0.242926000 & 1.126341000 & 1.605901000 \\ \mathrm{C} & -0.265969000 & 0.173240000 & 0.649341000 \\ \mathrm{O} & 0.327262000 & -0.099947000 & -0.474805000 \\ \mathrm{C} & 4.316001000 & 0.030874000 & -2.620139000 \\ \mathrm{H} & 3.836377000 & -0.681785000 & -3.318296000 \\ \mathrm{C} & 5.749128000 & 0.324299000 & -3.113996000 \\ \mathrm{H} & 6.382451000 & -0.577537000 & -3.058613000 \\ \mathrm{H} & 5.741209000 & 0.679910000 & -4.161074000 \\ \mathrm{H} & 6.230612000 & 1.109414000 & -2.502791000 \\ \mathrm{H} & 4.352071000 & -0.485733000 & -1.644459000 \\ \mathrm{H} & 3.715999000 & 2.027018000 & -3.307128000 \\ \mathrm{H} & 3.742530000 & 1.874418000 & -1.574994000 \\ \mathrm{H} & 1.734924000 & 2.534072000 & 2.211098000 \\ \mathrm{C} & -0.448217000 & 1.345861000 & 2.830460000 \\ \mathrm{H} & -0.028977000 & 2.077768000 & 3.535415000 \\ \mathrm{C} & -1.629113000 & 0.675743000 & 3.160077000 \\ \mathrm{C} & -2.347465000 & 0.911130000 & 4.473548000 \\ \mathrm{H} & -3.442691000 & 0.888468000 & 4.338098000 \\ \mathrm{H} & -2.075947000 & 1.886167000 & 4.914522000 \\ \mathrm{H} & -2.095910000 & 0.130563000 & 5.217622000 \\ \mathrm{C} & -2.135884000 & -0.240878000 & 2.211785000 \\ \mathrm{H} & -3.081488000 & -0.759538000 & 2.410991000\end{array}$




\begin{tabular}{|c|c|c|c|}
\hline C & -1.502338000 & -0.505588000 & 0.987473000 \\
\hline$C$ & -2.090641000 & -1.418255000 & 0.012690000 \\
\hline $\mathrm{N}$ & -3.172178000 & -2.101839000 & 0.247686000 \\
\hline $\mathrm{H}$ & -1.566946000 & -1.477657000 & -0.958123000 \\
\hline $\mathrm{C}$ & -3.673801000 & -2.969411000 & -0.737241000 \\
\hline $\mathrm{C}$ & -5.038654000 & -2.822169000 & -1.139443000 \\
\hline $\mathrm{C}$ & -5.553174000 & -3.661993000 & -2.147311000 \\
\hline $\mathrm{C}$ & -4.764359000 & -4.653961000 & -2.745187000 \\
\hline $\mathrm{C}$ & -3.439814000 & -4.824531000 & -2.325003000 \\
\hline $\mathrm{C}$ & -2.880049000 & -4.003401000 & -1.324236000 \\
\hline $\mathrm{C}$ & -1.480416000 & -4.269706000 & -0.875464000 \\
\hline $\mathrm{C}$ & -1.189734000 & -4.534503000 & 0.479083000 \\
\hline $\mathrm{C}$ & 1.179369000 & -4.803294000 & -0.032976000 \\
\hline $\mathrm{C}$ & 0.895310000 & -4.545922000 & -1.382928000 \\
\hline $\mathrm{C}$ & -0.421515000 & -4.297130000 & -1.804732000 \\
\hline $\mathrm{C}$ & -5.918077000 & -1.770595000 & -0.550155000 \\
\hline $\mathrm{C}$ & -5.979602000 & -1.553514000 & 0.844368000 \\
\hline $\mathrm{C}$ & -6.834352000 & -0.579470000 & 1.379406000 \\
\hline $\mathrm{C}$ & -7.656286000 & 0.195114000 & 0.544013000 \\
\hline $\mathrm{C}$ & -7.609195000 & -0.021001000 & -0.840231000 \\
\hline $\mathrm{C}$ & -6.746764000 & -0.989541000 & -1.382408000 \\
\hline $\mathrm{C}$ & 0.123281000 & -4.807752000 & 0.891344000 \\
\hline $\mathrm{C}$ & -0.666380000 & 5.903878000 & -0.671758000 \\
\hline $\mathrm{C}$ & 0.072589000 & 6.372156000 & 0.425253000 \\
\hline $\mathrm{C}$ & 1.302185000 & 5.782263000 & 0.761824000 \\
\hline $\mathrm{C}$ & 1.818765000 & 4.708255000 & 0.006518000 \\
\hline $\mathrm{C}$ & 1.073181000 & 4.248278000 & -1.097305000 \\
\hline $\mathrm{C}$ & -0.154571000 & 4.839971000 & -1.429868000 \\
\hline $\mathrm{C}$ & 3.155658000 & 4.131235000 & 0.346596000 \\
\hline $\mathrm{C}$ & 4.228286000 & 5.023035000 & 0.555717000 \\
\hline $\mathrm{C}$ & 5.502319000 & 4.549938000 & 0.888092000 \\
\hline $\mathrm{C}$ & 5.716775000 & 3.174038000 & 1.000292000 \\
\hline $\mathrm{C}$ & 4.677940000 & 2.234703000 & 0.790222000 \\
\hline $\mathrm{C}$ & 3.374021000 & 2.725953000 & 0.478893000 \\
\hline $\mathrm{C}$ & 5.030961000 & 0.784704000 & 0.901055000 \\
\hline $\mathrm{C}$ & 6.289325000 & 0.363403000 & 0.405431000 \\
\hline $\mathrm{C}$ & 6.699957000 & -0.972270000 & 0.499189000 \\
\hline $\mathrm{C}$ & 5.863290000 & -1.932902000 & 1.089161000 \\
\hline $\mathrm{C}$ & 4.615820000 & -1.530079000 & 1.582556000 \\
\hline $\mathrm{C}$ & 4.200047000 & -0.189328000 & 1.496213000 \\
\hline $\mathrm{H}$ & 1.457515000 & 3.416470000 & -1.696556000 \\
\hline $\mathrm{H}$ & -1.620560000 & 6.365767000 & -0.936414000 \\
\hline $\mathrm{H}$ & 1.861974000 & 6.156504000 & 1.625319000 \\
\hline $\mathrm{H}$ & 4.052239000 & 6.098571000 & 0.442277000 \\
\hline $\mathrm{H}$ & 6.325179000 & 5.251368000 & 1.061324000 \\
\hline $\mathrm{H}$ & 6.706174000 & 2.806338000 & 1.292171000 \\
\hline $\mathrm{H}$ & 6.949039000 & 1.085924000 & -0.082721000 \\
\hline $\mathrm{H}$ & 6.176899000 & -2.977679000 & 1.159042000 \\
\hline $\mathrm{H}$ & -8.317760000 & 0.956296000 & 0.965325000 \\
\hline $\mathrm{H}$ & -6.698547000 & -1.118897000 & -2.468507000 \\
\hline $\mathrm{H}$ & -6.601498000 & -3.545671000 & -2.445778000 \\
\hline $\mathrm{H}$ & -5.188909000 & -5.307829000 & -3.514156000 \\
\hline $\mathrm{H}$ & -2.824860000 & -5.624675000 & -2.753727000 \\
\hline $\mathrm{H}$ & -0.625517000 & -4.092575000 & -2.861103000 \\
\hline $\mathrm{H}$ & -1.999047000 & -4.519195000 & 1.216124000 \\
\hline $\mathrm{H}$ & -5.348802000 & -2.148374000 & 1.507981000 \\
\hline $\mathrm{H}$ & 3.226294000 & 0.086650000 & 1.903196000 \\
\hline $\mathrm{H}$ & 2.205169000 & -4.978678000 & 0.297117000 \\
\hline $\mathrm{C}$ & 0.784661000 & 0.209869000 & -2.970351000 \\
\hline $\mathrm{C}$ & 1.754521000 & 1.070713000 & -3.558801000 \\
\hline $\mathrm{H}$ & -0.229859000 & 0.579478000 & -2.786165000 \\
\hline $\mathrm{H}$ & 0.898986000 & -0.878435000 & -3.052435000 \\
\hline $\mathrm{H}$ & 2.426333000 & 0.637224000 & -4.308711000 \\
\hline $\mathrm{H}$ & 1.456268000 & 2.101338000 & -3.789267000 \\
\hline $\mathrm{C}$ & 2.012311000 & -4.436950000 & -2.392687000 \\
\hline $\mathrm{F}$ & 2.319007000 & -3.122514000 & -2.650671000 \\
\hline $\mathrm{F}$ & 3.158668000 & -5.033123000 & -1.969471000 \\
\hline $\mathrm{F}$ & 1.670983000 & -4.993763000 & -3.589449000 \\
\hline
\end{tabular}

\begin{tabular}{lr} 
C & 0.362943000 \\
F & -0.095281000 \\
F & -0.299897000 \\
F & 1.679600000 \\
C & -8.525319000 \\
F & -8.882680000 \\
F & -7.941299000 \\
F & -9.680113000 \\
C & -6.810382000 \\
F & -7.976473000 \\
F & -6.582820000 \\
F & -5.811457000 \\
C & -0.929468000 \\
F & -1.957089000 \\
F & -0.122434000 \\
F & -1.454625000 \\
C & -0.482850000 \\
F & -1.372336000 \\
F & -1.130876000 \\
F & 0.507633000 \\
C & 8.017041000 \\
F & 7.838148000 \\
F & 8.881594000 \\
F & 8.630243000 \\
C & 3.715650000 \\
F & 2.407550000 \\
F & 4.032597000 \\
F & 3.820029000 \\
& \\
\hline
\end{tabular}

$-5.114670000$ $-4.111331000$ $-6.249025000$ $-5.302310000$ 0.757850000 1.958058000 1.004196000 0.073060000 $-0.315225000$ 0.229519000 $-1.450339000$ 0.565744000 4.284006000 5.089626000 4.115821000 3.055561000 7.478066000 8.256819000 6.973077000 8.290276000 $-1.411932000$ $-1.976425000$ $-0.371479000$ $-2.348707000$ $-2.537510000$ $-2.329928000$ $-3.816003000$

2.353579000 3.153631000 2.729418000 2.638900000 $-1.756729000$ $-1.219342000$ $-2.964325000$ $-2.008591000$ 2.867039000 3.311512000 3.584211000 3.196498000 $-2.603139000$ $-2.974511000$ $-3.697423000$ $-2.316028000$ 1.296513000 0.622071000 2.385451000 1.765291000 $-0.101130000$ $-1.333932000$ $-0.257363000$ 0.673912000 2.266361000 1.951655000 1.907269000 3.622261000

\section{${ }^{\text {CF3 TS-1 }}$ BHE-T}

Zero-point correction= Thermal correction to Energy=

Thermal correction to Enthalpy= 0.754228 (Hartree/Particle) 0.830827

Thermal correction to Gibbs Free Energy $=0.625081$

Sum of electronic and zero-point Energies $=\quad-4902.855211$ Sum of electronic and thermal Energies $=\quad-4902.778612$ Sum of electronic and thermal Enthalpies $=\quad-4902.777668$ Sum of electronic and thermal Free Energies $=-4902.984358$ E solvent $=-4902.82994547$

$\mathrm{Ni} \quad 1.863417000 \quad 1.204919000$

$\begin{array}{lll}\text { C } & 3.379593000 & 2.025643000\end{array}$

$2.727681000 \quad 1.120576000$

$2.099361000 \quad 0.595490000$

$0.806633000 \quad-0.009505000$

$-0.057185000 \quad-0.076243000$

$\begin{array}{ll}0.268441000 & 0.369584000\end{array}$

$\begin{array}{ll}2.928960000 & 0.883187000\end{array}$

$1.010950000 \quad 1.572793000$

$3.622892000 \quad-0.456879000$

$2.917929000-1.288711000$

$4.409003000 \quad-0.487458000$

$4.102702000 \quad-0.645796000$

$\begin{array}{ll}2.268494000 & 1.017469000\end{array}$

4.307838000

3.058642000

2.621920000

0.361665000

1.040417000

$-0.887404000$

$-1.333420000$

$-1.089682000$

$-0.841164000$

$-2.425418000$

$-1.732040000$

$-2.735054000$

$-1.361510000$

$-2.279862000$

$-3.420637000$
1.167849000 2.229562000

$-0.647814000$

$-1.692676000$

$-1.731103000$

$-0.574542000$

0.604121000

2.924454000

2.294717000

2.877105000

2.705908000

2.104136000

3.858327000

3.789306000

1.647766000

2.551999000

$-2.664348000$

$-2.979397000$

$-3.840722000$

$-3.141334000$

$-4.469470000$

$-4.542620000$

$-5.318451000$

$-4.598308000$

$-2.007581000$

$-2.100966000$

$-0.755860000$

0.381123000

0.338381000 


$\begin{array}{lrrr}\mathrm{H} & -1.951717000 & -0.151314000 & 1.276439000 \\ \mathrm{C} & -4.271244000 & -1.311532000 & 1.457057000 \\ \mathrm{C} & -3.866371000 & -1.782998000 & 2.744297000 \\ \mathrm{C} & -4.778823000 & -1.711568000 & 3.819794000 \\ \mathrm{C} & -6.067734000 & -1.194371000 & 3.649950000 \\ \mathrm{C} & -6.472305000 & -0.758348000 & 2.381041000 \\ \mathrm{C} & -5.602903000 & -0.822874000 & 1.275003000 \\ \mathrm{C} & -6.077059000 & -0.334370000 & -0.053147000 \\ \mathrm{C} & -5.875525000 & -1.087073000 & -1.230814000 \\ \mathrm{~F} & -7.934229000 & 3.227968000 & -2.692246000 \\ \mathrm{C} & -7.022191000 & 0.606760000 & -2.561859000 \\ \mathrm{C} & -8.018751000 & 2.647046000 & -1.462614000 \\ \mathrm{C} & -7.239472000 & 1.352787000 & -1.395060000 \\ \mathrm{C} & -6.769307000 & 0.890080000 & -0.153942000 \\ \mathrm{C} & -2.528091000 & -2.396329000 & 2.995098000 \\ \mathrm{C} & -2.026392000 & -3.432931000 & 2.178628000 \\ \mathrm{C} & -0.802507000 & -4.052160000 & 2.476834000 \\ \mathrm{C} & -0.043964000 & -3.642877000 & 3.583868000 \\ \mathrm{C} & -0.531145000 & -2.606182000 & 4.395100000 \\ \mathrm{C} & -1.761769000 & -1.993477000 & 4.109919000 \\ \mathrm{C} & 0.315488000 & -2.087294000 & 5.533082000 \\ \mathrm{C} & -0.335874000 & -5.205974000 & 1.616136000 \\ \mathrm{C} & -6.337036000 & -0.616099000 & -2.468260000 \\ \mathrm{C} & -6.127293000 & -1.461748000 & -3.703021000 \\ \mathrm{C} & 4.793195000 & -3.468821000 & -2.047685000 \\ \mathrm{C} & 4.165300000 & -2.887725000 & -0.934614000 \\ \mathrm{C} & 4.236187000 & -1.505131000 & -0.713020000 \\ \mathrm{C} & 4.946696000 & -0.663500000 & -1.596215000 \\ \mathrm{C} & 5.568491000 & -1.256779000 & -2.717233000 \\ \mathrm{C} & 5.493344000 & -2.641828000 & -2.937509000 \\ \mathrm{C} & 3.442346000 & -3.765757000 & 0.065491000 \\ \mathrm{C} & 5.091790000 & 0.809064000 & -1.372858000 \\ \mathrm{C} & 6.361910000 & 1.383889000 & -1.610690000 \\ \mathrm{C} & 6.584989000 & 2.755644000 & -1.458597000 \\ \mathrm{C} & 5.528237000 & 3.585504000 & -1.068809000 \\ \mathrm{C} & 4.243766000 & 3.061089000 & -0.808943000 \\ \mathrm{C} & 4.025486000 & 1.658662000 & -0.945564000 \\ \mathrm{C} & 3.167341000 & 4.007541000 & -0.388616000 \\ \mathrm{C} & 3.452733000 & 4.979877000 & 0.593027000 \\ \mathrm{C} & 2.473596000 & 5.896414000 & 1.009250000 \\ \mathrm{C} & 1.193206000 & 5.877476000 & 0.439502000 \\ \mathrm{C} & 0.907250000 & 4.929292000 & -0.555747000 \\ \mathrm{C} & 1.877175000 & 4.001341000 & -0.964093000 \\ \mathrm{C} & -0.486364000 & 4.860230000 & -1.142773000\end{array}$

\begin{tabular}{cc} 
C & 2.799730000 \\
H & 6.092299000 \\
H & 4.718273000 \\
F & 2.410740000 \\
F & 2.935829000 \\
F & 4.280048000 \\
H & 3.719264000 \\
H & 7.190389000 \\
H & 7.577617000 \\
H & 5.683184000 \\
H & 4.443435000 \\
F & 2.792254000 \\
F & 4.042555000 \\
F & 1.908125000 \\
H & 0.432730000 \\
F & -1.085290000 \\
F & -1.287150000 \\
F & -0.473939000 \\
H & 0.911478000 \\
F & -0.441495000 \\
F & 1.123940000 \\
F & 1.136636000 \\
H & -2.124587000 \\
F & 0.978450000 \\
F & -0.500911000 \\
F & -1.047746000 \\
H & -4.469518000 \\
H & -6.763570000 \\
H & -7.490910000 \\
F & -7.574678000 \\
F & -9.345122000 \\
H & -6.913316000 \\
H & -5.335232000 \\
F & -4.919214000 \\
F & -6.158126000 \\
H & -2.599676000 \\
H & 1.629013000 \\
H & -7.364194000 \\
F & -7.080939000 \\
C & 6.212116000 \\
F & 7.533443000 \\
F & 5.684261000 \\
F & 6.153708000 \\
& \\
\hline
\end{tabular}

6.844256000 $-0.628046000$ $-4.543816000$ $-3.100208000$ $-4.881689000$ $-4.174407000$ $-1.075488000$ 0.727468000 3.177229000 4.666758000 5.003156000 6.188066000 7.387565000 7.866228000 6.595268000 6.083789000 4.032112000 4.385001000 $-4.123064000$ $-1.589074000$ $-3.052294000$ $-1.068475000$ $-1.182221000$ $-5.500575000$ $-4.939366000$ $-6.340815000$ $-2.099260000$ $-1.157543000$ $-0.382317000$ 3.553214000 2.443309000 1.505268000 $-2.034460000$ $-2.110699000$ $-0.720273000$ $-3.756997000$ 3.287983000 0.980100000 $-2.428438000$ $-3.241920000$ $-3.445718000$ $-4.443439000$ $-2.420705000$
2.141093000 $-3.444510000$ $-2.230304000$ 0.663233000 $-0.520321000$ 1.062225000 0.146588000 $-1.898199000$ $-1.648337000$ $-0.983211000$ 1.059031000 3.340510000 1.998164000 2.232105000 0.757279000 $-1.161097000$ $-0.413036000$ $-2.419608000$ 3.808293000 6.549179000 6.051862000 5.118306000 4.749188000 1.820672000 0.291006000 1.882471000 4.797469000 4.494637000 2.230378000 $-0.545941000$ $-1.207325000$ 0.740117000 $-1.174603000$ $-3.670860000$ $-4.844315000$

1.304530000 $-1.752791000$ $-3.530350000$ $-3.823836000$ $-4.126825000$ $-3.853961000$ $-4.486372000$ $-5.214233000$

\subsection{5 $\mathbf{3}^{\mathrm{Me}}-\mathbf{H}^{+}$}

\section{Me-H+ 1-Allyl}

Zero-point correction= Thermal correction to Energy= 0.928885 (Hartree/Particle) Thermal correction to Enthalpy= 0.990149 Thermal correction to Gibbs Free Energy $=\quad 0.824288$ Sum of electronic and zero-point Energies $=-2520.172444$ Sum of electronic and thermal Energies $=\quad-2520.111180$ Sum of electronic and thermal Enthalpies $=\quad-2520.110236$ Sum of electronic and thermal Free Energies $=-2520.277041$ E solvent $=-2519.97923744$

$\begin{array}{lrr}\mathrm{Ni} & 1.271739000 & -1.114855000 \\ \mathrm{C} & 2.595501000 & -2.634118000 \\ \mathrm{~N} & 2.505050000 & 0.288267000 \\ \mathrm{C} & 2.177921000 & 1.560050000 \\ \mathrm{C} & 0.895675000 & 2.120416000 \\ \mathrm{C} & -0.269023000 & 1.314933000 \\ \mathrm{O} & -0.265516000 & 0.011329000 \\ \mathrm{C} & 1.372021000 & -3.111495000 \\ \mathrm{H} & 1.325024000 & -3.439782000\end{array}$

0.114586000 $-0.206427000$ 0.578622000 0.512977000 0.152973000 $-0.126632000$ $-0.113862000$ 0.328511000 1.375173000
2.728866000 3.514269000 2.953393000 0.795248000 1.702124000 $-0.396826000$ $-0.430527000$ 0.233712000 $-0.093793000$ $-1.448361000$ $-1.530014000$ $-2.483353000$ $-1.505565000$ $-2.733666000$ $-2.878239000$ $-3.640133000$ $-4.119146000$ $-5.277236000$ $-6.464822000$ $-6.506025000$
$-2.562678000$ $-2.674039000$ 2.295707000 3.527786000 4.110519000 4.219520000 5.732915000 6.127622000 6.172397000 6.106631000 3.444014000 3.939469000 2.021071000 1.341405000 0.021299000 1.935356000 $-0.683701000$ $-0.245299000$ $-0.988644000$ $-2.140907000$
$-1.295806000$ 0.385251000 0.779309000 0.108581000 0.320303000 $-0.198070000$ $-0.241623000$ $-1.032529000$ 0.715031000 $-0.442902000$ $-0.452177000$ $-0.677913000$ $-0.422626000$ $-0.666885000$ $-0.720960000$ $-0.824429000$ $-0.848251000$ $-0.142690000$ $-0.301200000$ $-1.096356000$ 


\begin{tabular}{|c|c|c|c|}
\hline C & -5.353756000 & -2.565385000 & -1.765635000 \\
\hline$C$ & -4.143445000 & -1.844566000 & -1.671957000 \\
\hline $\mathrm{C}$ & -2.980289000 & -2.313275000 & -2.482079000 \\
\hline $\mathrm{C}$ & -2.641186000 & -3.686407000 & -2.476624000 \\
\hline $\mathrm{H}$ & 0.468877000 & -1.349325000 & -5.391828000 \\
\hline $\mathrm{C}$ & -0.931097000 & -3.292035000 & -4.142161000 \\
\hline $\mathrm{C}$ & -0.544585000 & -0.987811000 & -5.145730000 \\
\hline $\mathrm{C}$ & -1.249316000 & -1.920264000 & -4.180865000 \\
\hline $\mathrm{C}$ & -2.269536000 & -1.440715000 & -3.335228000 \\
\hline $\mathrm{C}$ & -5.313823000 & 0.927477000 & 0.788486000 \\
\hline $\mathrm{C}$ & -4.490226000 & 0.989102000 & 1.934249000 \\
\hline $\mathrm{C}$ & -4.583224000 & 2.067505000 & 2.833656000 \\
\hline $\mathrm{C}$ & -5.506155000 & 3.097984000 & 2.559747000 \\
\hline $\mathrm{C}$ & -6.345401000 & 3.063079000 & 1.430592000 \\
\hline $\mathrm{C}$ & -6.235663000 & 1.968189000 & 0.548869000 \\
\hline $\mathrm{C}$ & -7.358942000 & 4.160995000 & 1.178671000 \\
\hline $\mathrm{C}$ & -3.733350000 & 2.111413000 & 4.087420000 \\
\hline $\mathrm{C}$ & -1.620458000 & -4.191785000 & -3.302108000 \\
\hline $\mathrm{C}$ & -1.281950000 & -5.668942000 & -3.305548000 \\
\hline $\mathrm{C}$ & 4.651854000 & 2.832878000 & -3.424971000 \\
\hline $\mathrm{C}$ & 3.936321000 & 1.622487000 & -3.329741000 \\
\hline $\mathrm{C}$ & 4.004924000 & 0.888623000 & -2.131258000 \\
\hline $\mathrm{C}$ & 4.791885000 & 1.333926000 & -1.047415000 \\
\hline $\mathrm{C}$ & 5.504748000 & 2.545770000 & -1.180235000 \\
\hline $\mathrm{C}$ & 5.445565000 & 3.307335000 & -2.363522000 \\
\hline $\mathrm{C}$ & 3.132087000 & 1.110616000 & -4.508320000 \\
\hline $\mathrm{C}$ & 4.953570000 & 0.530447000 & 0.205834000 \\
\hline $\mathrm{C}$ & 6.270918000 & 0.249461000 & 0.633677000 \\
\hline $\mathrm{C}$ & 6.519162000 & -0.507643000 & 1.783062000 \\
\hline $\mathrm{C}$ & 5.444519000 & -1.002822000 & 2.529635000 \\
\hline $\mathrm{C}$ & 4.106120000 & -0.755680000 & 2.150546000 \\
\hline $\mathrm{C}$ & 3.869648000 & 0.019704000 & 0.978417000 \\
\hline $\mathrm{C}$ & 3.022003000 & -1.325921000 & 3.006685000 \\
\hline $\mathrm{C}$ & 3.129168000 & -2.668906000 & 3.436859000 \\
\hline $\mathrm{C}$ & 2.170608000 & -3.250739000 & 4.289205000 \\
\hline $\mathrm{C}$ & 1.084516000 & -2.459612000 & 4.712503000 \\
\hline $\mathrm{C}$ & 0.956293000 & -1.112184000 & 4.320975000 \\
\hline $\mathrm{C}$ & 1.923949000 & -0.560740000 & 3.459617000 \\
\hline $\mathrm{C}$ & -0.184122000 & -0.263602000 & 4.847532000 \\
\hline $\mathrm{C}$ & 2.318010000 & -4.683965000 & 4.761061000 \\
\hline $\mathrm{H}$ & 6.109840000 & 2.905197000 & -0.338162000 \\
\hline $\mathrm{H}$ & 4.591655000 & 3.418091000 & -4.352283000 \\
\hline $\mathrm{H}$ & 2.338185000 & 0.413973000 & -4.186402000 \\
\hline $\mathrm{H}$ & 2.657688000 & 1.937888000 & -5.065563000 \\
\hline $\mathrm{H}$ & 3.776518000 & 0.566600000 & -5.225581000 \\
\hline $\mathrm{H}$ & 3.449487000 & -0.053158000 & -2.043737000 \\
\hline $\mathrm{H}$ & 7.104007000 & 0.620917000 & 0.027632000 \\
\hline $\mathrm{H}$ & 7.548248000 & -0.711362000 & 2.097371000 \\
\hline $\mathrm{H}$ & 5.629893000 & -1.572471000 & 3.446454000 \\
\hline $\mathrm{H}$ & 3.973977000 & -3.276429000 & 3.088334000 \\
\hline $\mathrm{H}$ & 2.887176000 & -5.295777000 & 4.039755000 \\
\hline $\mathrm{H}$ & 2.859142000 & -4.729715000 & 5.725709000 \\
\hline $\mathrm{H}$ & 1.335864000 & -5.163030000 & 4.918372000 \\
\hline $\mathrm{H}$ & 0.328422000 & -2.899528000 & 5.376437000 \\
\hline $\mathrm{H}$ & -1.133198000 & -0.829041000 & 4.869694000 \\
\hline $\mathrm{H}$ & -0.333903000 & 0.639865000 & 4.231010000 \\
\hline $\mathrm{H}$ & 0.013619000 & 0.073267000 & 5.883172000 \\
\hline $\mathrm{H}$ & -5.578109000 & 3.947382000 & 3.251947000 \\
\hline $\mathrm{H}$ & -8.335534000 & 3.910862000 & 1.635831000 \\
\hline $\mathrm{H}$ & -7.033202000 & 5.121653000 & 1.613530000 \\
\hline $\mathrm{H}$ & -7.536026000 & 4.314004000 & 0.099737000 \\
\hline $\mathrm{H}$ & -6.873711000 & 1.924673000 & -0.342973000 \\
\hline $\mathrm{H}$ & -4.321916000 & 1.818047000 & 4.977550000 \\
\hline $\mathrm{H}$ & -3.346543000 & 3.128256000 & 4.278998000 \\
\hline $\mathrm{H}$ & -2.873177000 & 1.423509000 & 4.019888000 \\
\hline $\mathrm{H}$ & -7.355480000 & -0.663543000 & 0.246374000 \\
\hline $\mathrm{H}$ & -7.438455000 & -2.706496000 & -1.195135000 \\
\hline $\mathrm{H}$ & -5.386450000 & -3.447124000 & -2.413724000 \\
\hline
\end{tabular}

$\begin{array}{cr}\mathrm{H} & -0.456738000 \\ \mathrm{H} & -1.101223000 \\ \mathrm{H} & -2.548186000 \\ \mathrm{H} & -3.179380000 \\ \mathrm{H} & -1.601896000 \\ \mathrm{H} & -0.197664000 \\ \mathrm{H} & -3.786498000 \\ \mathrm{H} & 1.840867000 \\ \mathrm{H} & -0.134815000 \\ \mathrm{H} & -1.786949000 \\ \mathrm{C} & 6.232922000 \\ \mathrm{H} & 7.219018000 \\ \mathrm{H} & 5.705445000 \\ \mathrm{H} & 6.421082000 \\ \mathrm{H} & -1.987215000 \\ \mathrm{C} & 0.193466000 \\ \mathrm{H} & 0.193467000 \\ \mathrm{H} & -0.785707000\end{array}$

0.034116000 $-0.909702000$ $-0.381447000$ $-4.368733000$ $-6.162163000$ $-5.837149000$ 0.172899000 0.492675000 $-3.674307000$ $-6.188671000$ 4.595537000 4.411877000 5.328959000 5.063648000 $-0.505814000$ $-2.788754000$ $-2.727724000$ $-2.920831000$

$-4.736808000$ $-6.099405000$ $-3.383473000$ $-1.807188000$ $-2.371755000$ $-3.428816000$ 2.139782000 3.172813000 $-4.794610000$ $-4.142193000$ $-2.498377000$ $-2.966691000$ $-3.133262000$ $-1.516488000$ $-0.625914000$ $-0.383902000$ $-1.480586000$ 0.089177000

\section{$6.3 .6 \quad \mathbf{2}^{\mathrm{Me}}-\mathrm{H}^{+}$ \\ Me-H+1- $\beta-$ T \\ Zero-point correction=} 0.950553 (Hartree/Particle)

Energy $=\quad 1.012604$

Thermal correction to Enthalpy $=\quad 1.013548$

Thermal correction to Gibbs Free Energy $=0.844733$

Sum of electronic and zero-point Energies $=\quad-2521.348248$

Sum of electronic and thermal Energies $=\quad-2521.286197$

Sum of electronic and thermal Enthalpies $=\quad-2521.285253$

Sum of electronic and thermal Free Energies $=-2521.454068$ E solvent $=-2521.17653872$

$\begin{array}{lrrr}\mathrm{Ni} & 1.414222000 & -0.344453000 & -0.762661000 \\ \mathrm{C} & 2.720397000 & -0.933473000 & -2.010549000 \\ \mathrm{~N} & 2.383893000 & 0.900225000 & 0.221824000 \\ \mathrm{C} & 1.820037000 & 1.636272000 & 1.157248000 \\ \mathrm{C} & 0.462515000 & 1.569736000 & 1.643420000 \\ \mathrm{C} & -0.529123000 & 0.667350000 & 1.104779000 \\ \mathrm{O} & -0.277968000 & -0.159876000 & 0.130447000 \\ \mathrm{C} & 1.535562000 & -1.700487000 & -2.504967000 \\ \mathrm{H} & 0.618517000 & -1.507060000 & -1.781916000 \\ \mathrm{C} & 1.007597000 & -1.353934000 & -3.907871000 \\ \mathrm{H} & 0.036011000 & -1.837551000 & -4.108105000 \\ \mathrm{H} & 0.884597000 & -0.263996000 & -4.029662000 \\ \mathrm{H} & 1.728003000 & -1.701917000 & -4.668052000 \\ \mathrm{H} & 1.641852000 & -2.789736000 & -2.351354000 \\ \mathrm{H} & 3.104504000 & -0.129856000 & -2.658411000 \\ \mathrm{H} & 3.509903000 & -1.503464000 & -1.500935000 \\ \mathrm{H} & 2.461168000 & 2.388342000 & 1.643923000 \\ \mathrm{C} & 0.112230000 & 2.431002000 & 2.706493000 \\ \mathrm{H} & 0.886896000 & 3.106752000 & 3.093970000 \\ \mathrm{C} & -1.171272000 & 2.468764000 & 3.292958000 \\ \mathrm{C} & -1.478424000 & 3.414503000 & 4.435393000 \\ \mathrm{H} & -1.273168000 & 4.464158000 & 4.156389000 \\ \mathrm{H} & -0.861622000 & 3.187515000 & 5.324532000 \\ \mathrm{H} & -2.536888000 & 3.348326000 & 4.737625000 \\ \mathrm{C} & -2.134463000 & 1.603815000 & 2.767178000 \\ \mathrm{H} & -3.150972000 & 1.612528000 & 3.181527000 \\ \mathrm{C} & -1.857228000 & 0.713383000 & 1.692473000 \\ \mathrm{C} & -2.933048000 & -0.084373000 & 1.206845000 \\ \mathrm{~N} & -2.838274000 & -0.954941000 & 0.205771000 \\ \mathrm{H} & -3.917208000 & 0.019655000 & 1.674482000 \\ \mathrm{C} & -3.904812000 & -1.700029000 & -0.385500000 \\ \mathrm{C} & -3.599937000 & -3.001400000 & -0.877529000 \\ \mathrm{C} & -4.638081000 & -3.742671000 & -1.481554000 \\ \mathrm{C} & -5.936330000 & -3.230667000 & -1.576158000 \\ \mathrm{C} & -6.214399000 & -1.945326000 & -1.093054000 \\ \mathrm{C} & -5.211527000 & -1.144662000 & -0.510538000 \\ & -5.579190000 & 0.254875000 & -0.122245000\end{array}$




\begin{tabular}{|c|c|c|c|}
\hline & U & & \\
\hline & -6 . & & \\
\hline & -6.992174000 & & \\
\hline & -6.593767000 & & \\
\hline & -2.251356000 & & \\
\hline & 2000 & 5000 & 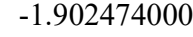 \\
\hline & -0.475640000 & 4000 & 3000 \\
\hline & 000 & 1000 & -0 \\
\hline & -0.341104000 & 6650000 & 000 \\
\hline & -1.560697000 & -3.712886000 & 9000 \\
\hline & -5.344871000 & 2.680674000 & -0.400677000 \\
\hline & 1.232438000 & 5.429113000 & -1.980087000 \\
\hline & 46000 & & \\
\hline & 1.91 & & \\
\hline & & & \\
\hline & & & \\
\hline & & & \\
\hline & & & \\
\hline & 5.42 & & \\
\hline & 6.4 & & \\
\hline & 6.12 & & \\
\hline & & & \\
\hline & 3.7 & & \\
\hline & & & \\
\hline & & & \\
\hline & & & \\
\hline & & & \\
\hline & & & \\
\hline & & & \\
\hline & & & \\
\hline & & & \\
\hline & & & \\
\hline & 5.66 & & \\
\hline & 7.4 & & -0 . \\
\hline & 6.92 & & 000 \\
\hline & & & -0 . \\
\hline & 4.17 & & \\
\hline & & & \\
\hline & & & \\
\hline & & & \\
\hline & & & \\
\hline & & & \\
\hline & & & \\
\hline & & & \\
\hline & & & \\
\hline & & & \\
\hline & & & \\
\hline & & & \\
\hline & & & \\
\hline & & & 000 \\
\hline & & & 00 \\
\hline & & & \\
\hline & & & \\
\hline & & & -2 \\
\hline & -0.30 & & -3.0 \\
\hline 11 & -0.2 & 7000 & 2000 \\
\hline 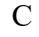 & -4.713 & 000 & 6000 \\
\hline $\mathrm{H}$ & -3.72 & 7000 & 2000 \\
\hline 1 & -5.3 & 4.23 & -1 \\
\hline $\mathrm{H}$ & -4.57 & 5000 & \\
\hline 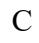 & & & \\
\hline & & & \\
\hline & & & \\
\hline & & & \\
\hline $\mathrm{C}$ & & & \\
\hline & & & \\
\hline & & & -0.6 \\
\hline & 3.166367000 & 7.340100000 & 0.10388100 \\
\hline
\end{tabular}

$\begin{array}{lrrr}\mathrm{C} & 5.679923000 & -4.636791000 & 0.833628000 \\ \mathrm{H} & 6.671458000 & -4.774315000 & 1.306349000 \\ \mathrm{H} & 5.079471000 & -5.530408000 & 1.079272000 \\ \mathrm{H} & 5.839433000 & -4.619547000 & -0.258533000 \\ \mathrm{C} & 2.955251000 & -2.172511000 & 4.346599000 \\ \mathrm{H} & 2.176263000 & -1.390764000 & 4.382879000 \\ \mathrm{H} & 2.474649000 & -3.145535000 & 4.549642000 \\ \mathrm{H} & 3.654565000 & -1.976377000 & 5.182117000 \\ \mathrm{C} & -0.172807000 & 3.725998000 & -3.236247000 \\ \mathrm{H} & -0.414045000 & 2.650435000 & -3.167144000 \\ \mathrm{H} & -1.075489000 & 4.298610000 & -2.956555000 \\ \mathrm{H} & 0.031853000 & 3.950106000 & -4.300798000\end{array}$

\section{Me-H+ TS-2}

Zero-point correction $=\quad 1.001679($ Hartree/Particle $)$

Thermal correction to Energy $=\quad 1.067357$

Thermal correction to Enthalpy $=\quad 1.068301$

Thermal correction to Gibbs Free Energy $=\quad 0.892744$

Sum of electronic and zero-point Energies $=\quad-2599.872757$

Sum of electronic and thermal Energies $=\quad-2599.807078$

Sum of electronic and thermal Enthalpies $=\quad-2599.806134$

Sum of electronic and thermal Free Energies $=-2599.981691$

E solvent $=-2599.72542349$

$\begin{array}{llll}\mathrm{N} & 2.467889000 & -0.573917000 & 0.199536000\end{array}$

$\begin{array}{llll}\text { C } & 4.287772000 & -1.783256000 & -0.986338000\end{array}$

$\begin{array}{llll}\text { C } & 4.498574000 & 1.171476000 & 1.519601000\end{array}$

$\begin{array}{llll}\text { C } & 3.512024000 & 2.156715000 & 1.298359000\end{array}$

$\begin{array}{llll}\mathrm{H} & 2.917898000 & 2.126861000 & 0.376466000\end{array}$

$\begin{array}{llll}\text { C } & 5.030370000 & 2.225921000 & 3.679306000\end{array}$

$\begin{array}{llll}\text { C } & 3.371247000 & -2.809465000 & -1.569066000\end{array}$

$\begin{array}{llll}\text { C } & 4.040993000 & 3.193833000 & 3.423882000\end{array}$

$\begin{array}{llll}\text { C } & 3.877686000 & -0.758232000 & -0.090627000\end{array}$

$\begin{array}{llll}\text { C } & 3.281965000 & 3.179399000 & 2.236474000\end{array}$

$\begin{array}{llll}\text { C } & 4.831916000 & 0.111760000 & 0.515222000\end{array}$

$\begin{array}{llll}\text { C } & 2.533561000 & -3.602681000 & -0.756762000\end{array}$

$\mathrm{H} \quad 2.499463000 \quad-3.422843000 \quad 0.323808000$

$\begin{array}{llll}\text { C } & 1.850905000 & -4.901529000 & -2.685643000\end{array}$

$\begin{array}{llll}\text { C } & 5.245732000 & 1.220412000 & 2.717316000\end{array}$

$\begin{array}{llll}\mathrm{H} & 6.003218000 & 0.449326000 & 2.906387000\end{array}$

C $\quad 1.777826000 \quad-4.660010000 \quad-1.299935000$

C $\quad 5.660277000 \quad-1.886377000 \quad-1.305286000$

H $\quad 5.978626000 \quad-2.688626000 \quad-1.979522000$

$\begin{array}{llll}\text { C } & 5.856432000 & 2.275045000 & 4.949234000\end{array}$

$\begin{array}{llll}\mathrm{H} & 6.742734000 & 2.926096000 & 4.822347000\end{array}$

H $\quad 6.225660000 \quad 1.274600000 \quad 5.234473000$

$\mathrm{H} \quad 5.275661000 \quad 2.680937000 \quad 5.796007000$

C $\quad 2.676872000 \quad-4.129678000 \quad-3.528264000$

C $\quad 6.605881000 \quad-1.019356000 \quad-0.748110000$

$\mathrm{H} \quad 7.664614000 \quad-1.114750000 \quad-1.011509000$

$\begin{array}{llll}\text { C } & 2.252173000 & 4.259573000 & 1.971103000\end{array}$

$\begin{array}{llll}\mathrm{H} & 2.732817000 & 5.192413000 & 1.618400000\end{array}$

$\mathrm{H} \quad 1.689197000 \quad 4.515382000 \quad 2.886731000$

$\begin{array}{llll}\mathrm{H} & 1.527294000 & 3.948143000 & 1.198797000\end{array}$

$\begin{array}{llll}\text { C } & 3.431579000 & -3.089173000 & -2.953999000\end{array}$

$\begin{array}{llll}\mathrm{H} & 4.081916000 & -2.479841000 & -3.594412000\end{array}$

$\begin{array}{llll}\text { C } & 6.192363000 & -0.042155000 & 0.162763000\end{array}$

$\begin{array}{llrr}\mathrm{H} & 6.923806000 & 0.641705000 & 0.606098000\end{array}$

$\begin{array}{llll}\text { C } & 2.766213000 & -4.430435000 & -5.011361000\end{array}$

$\begin{array}{llll}\mathrm{C} & 2.766213000 & -4.430435000 & -5.011361000 \\ \mathrm{H} & 3.424315000 & -5.299681000 & -5.201869000\end{array}$

$\mathrm{H} \quad 3.178593000 \quad-3.576431000 \quad-5.575760000$

$\mathrm{H} \quad 1.776831000 \quad-4.676611000 \quad-5.436137000$

$\begin{array}{llll}\text { C } & 0.930708000 & -5.544117000 & -0.405716000\end{array}$

$\begin{array}{llll}\mathrm{H} & 1.496577000 & -6.436573000 & -0.075760000\end{array}$

$\begin{array}{llll}\mathrm{H} & 1.496577000 & -6.436573000 & -0.075760000 \\ \mathrm{H} & 0.031074000 & -5.909375000 & -0.931586000\end{array}$

$\mathrm{H} \quad 0.605555000 \quad-5.010470000 \quad 0.504793000$

$\begin{array}{llll}\mathrm{N} & -2.949842000 & 0.680854000 & 0.222938000\end{array}$

$\begin{array}{llll}\text { C } & -4.289724000 & 1.949054000 & -1.380615000\end{array}$

$\begin{array}{llll}\text { C } & -5.182892000 & -1.299699000 & 0.552748000\end{array}$ 


\begin{tabular}{|c|c|c|c|}
\hline $\mathrm{C}$ & -6.135010000 & -1.459824000 & 1.581912000 \\
\hline $\mathrm{H}$ & -6.878009000 & -0.670549000 & 1.753217000 \\
\hline $\mathrm{C}$ & -4.215876000 & -3.478209000 & 1.130938000 \\
\hline $\mathrm{C}$ & -3.241287000 & 3.002398000 & -1.522982000 \\
\hline $\mathrm{C}$ & -5.169265000 & -3.600831000 & 2.161886000 \\
\hline $\mathrm{C}$ & -4.183639000 & 0.848489000 & -0.485593000 \\
\hline $\mathrm{C}$ & -6.141372000 & -2.609790000 & 2.396742000 \\
\hline $\mathrm{C}$ & -5.246968000 & -0.086020000 & -0.322725000 \\
\hline $\mathrm{C}$ & -2.831185000 & 3.407610000 & -2.813658000 \\
\hline $\mathrm{H}$ & -3.213686000 & 2.870422000 & -3.690490000 \\
\hline $\mathrm{C}$ & -1.470554000 & 5.160831000 & -1.849258000 \\
\hline $\mathrm{C}$ & -4.227283000 & -2.316194000 & 0.336565000 \\
\hline $\mathrm{H}$ & -3.499817000 & -2.211504000 & -0.478047000 \\
\hline $\mathrm{C}$ & -1.949217000 & 4.489061000 & -2.993631000 \\
\hline $\mathrm{C}$ & -5.479959000 & 2.085916000 & -2.127066000 \\
\hline $\mathrm{H}$ & -5.575765000 & 2.941273000 & -2.803758000 \\
\hline $\mathrm{C}$ & -3.222800000 & -4.591637000 & 0.865479000 \\
\hline $\mathrm{H}$ & -3.684547000 & -5.401137000 & 0.268320000 \\
\hline $\mathrm{H}$ & -2.344769000 & -4.229565000 & 0.303286000 \\
\hline $\mathrm{H}$ & -2.865709000 & -5.048316000 & 1.805521000 \\
\hline $\mathrm{C}$ & -1.859644000 & 4.784102000 & -0.548860000 \\
\hline $\mathrm{C}$ & -6.542105000 & 1.189480000 & -1.968434000 \\
\hline $\mathrm{H}$ & -7.463722000 & 1.322381000 & -2.544712000 \\
\hline $\mathrm{C}$ & -7.183339000 & -2.784881000 & 3.483024000 \\
\hline $\mathrm{H}$ & -8.058399000 & -3.347952000 & 3.105582000 \\
\hline $\mathrm{H}$ & -6.782499000 & -3.349073000 & 4.343105000 \\
\hline $\mathrm{H}$ & -7.555783000 & -1.813578000 & 3.852021000 \\
\hline $\mathrm{C}$ & -2.738209000 & 3.693435000 & -0.398666000 \\
\hline $\mathrm{H}$ & -3.078721000 & 3.414919000 & 0.605729000 \\
\hline $\mathrm{C}$ & -6.425933000 & 0.123363000 & -1.067226000 \\
\hline $\mathrm{H}$ & -7.243930000 & -0.595724000 & -0.954413000 \\
\hline $\mathrm{C}$ & -1.389897000 & 5.569081000 & 0.659650000 \\
\hline $\mathrm{H}$ & -1.278438000 & 4.923698000 & 1.548525000 \\
\hline $\mathrm{H}$ & -0.421946000 & 6.064417000 & 0.471011000 \\
\hline $\mathrm{H}$ & -2.115654000 & 6.362590000 & 0.922433000 \\
\hline $\mathrm{C}$ & -1.553685000 & 4.947106000 & -4.383628000 \\
\hline $\mathrm{H}$ & -2.195108000 & 5.783638000 & -4.721222000 \\
\hline $\mathrm{H}$ & -0.511105000 & 5.309773000 & -4.413612000 \\
\hline $\mathrm{H}$ & -1.657143000 & 4.136745000 & -5.125669000 \\
\hline $\mathrm{Ni}$ & 1.275643000 & 0.198810000 & -1.025501000 \\
\hline $\mathrm{C}$ & -2.821957000 & 0.274318000 & 1.483367000 \\
\hline $\mathrm{H}$ & -3.747759000 & 0.188763000 & 2.062281000 \\
\hline $\mathrm{C}$ & 2.119683000 & -0.785134000 & 1.454283000 \\
\hline $\mathrm{H}$ & 2.912514000 & -1.148344000 & 2.126845000 \\
\hline $\mathrm{C}$ & 0.824690000 & -0.613351000 & 2.071175000 \\
\hline $\mathrm{C}$ & 0.711787000 & -0.956781000 & 3.435640000 \\
\hline $\mathrm{C}$ & -0.335988000 & -0.111607000 & 1.367642000 \\
\hline $\mathrm{H}$ & 1.615182000 & -1.311692000 & 3.950166000 \\
\hline $\mathrm{C}$ & -0.492263000 & -0.871628000 & 4.169910000 \\
\hline $\mathrm{C}$ & -1.588830000 & -0.067437000 & 2.106015000 \\
\hline $\mathrm{C}$ & -0.537638000 & -1.247421000 & 5.636448000 \\
\hline $\mathrm{C}$ & -1.626705000 & -0.442366000 & 3.479363000 \\
\hline $\mathrm{H}$ & -2.591593000 & -0.397216000 & 4.001059000 \\
\hline $\mathrm{O}$ & -0.294888000 & 0.272832000 & 0.125813000 \\
\hline $\mathrm{C}$ & 2.504047000 & 0.607722000 & -2.424412000 \\
\hline $\mathrm{C}$ & 1.485815000 & 1.639396000 & -2.827135000 \\
\hline $\mathrm{H}$ & 3.467614000 & 0.998834000 & -2.064124000 \\
\hline $\mathrm{H}$ & 2.620214000 & -0.273135000 & -3.074604000 \\
\hline $\mathrm{C}$ & -0.887002000 & -0.834995000 & -3.021081000 \\
\hline $\mathrm{C}$ & -0.086869000 & -1.680307000 & -2.336706000 \\
\hline $\mathrm{C}$ & 1.929200000 & 3.105220000 & -2.688634000 \\
\hline $\mathrm{H}$ & 1.049551000 & 1.427010000 & -3.821752000 \\
\hline $\mathrm{H}$ & 0.545909000 & 1.511205000 & -2.159343000 \\
\hline $\mathrm{H}$ & 1.076656000 & 3.797022000 & -2.795471000 \\
\hline $\mathrm{H}$ & 2.395515000 & 3.284055000 & -1.703900000 \\
\hline $\mathrm{H}$ & 2.676097000 & 3.346757000 & -3.464804000 \\
\hline $\mathrm{H}$ & -0.343719000 & -2.020164000 & -1.327794000 \\
\hline $\mathrm{H}$ & 0.792409000 & -2.147218000 & -2.793516000 \\
\hline
\end{tabular}

$\begin{array}{rrrr} & & & \\ \mathrm{H} & -0.667852000 & -0.542919000 & -4.054961000 \\ \mathrm{H} & -1.802129000 & -0.417081000 & -2.584718000 \\ \mathrm{H} & -0.153423000 & -2.270005000 & 5.805103000 \\ \mathrm{H} & 0.080967000 & -0.563134000 & 6.246330000 \\ \mathrm{H} & -1.567078000 & -1.206284000 & 6.029820000 \\ \mathrm{H} & 3.856440000 & 3.980476000 & 4.167726000 \\ \mathrm{H} & -0.789640000 & 6.012998000 & -1.975876000 \\ \mathrm{H} & -5.159881000 & -4.499524000 & 2.792770000 \\ \mathrm{H} & 1.256987000 & -5.717070000 & -3.119229000 \\ \mathrm{H} & -2.046499000 & 0.809213000 & -0.274156000\end{array}$

\section{Me-H+2-Coor-T}

Zero-point correction $=1.004657($ Hartree/Particle $)$ Thermal correction to Energy= $\quad 1.070156$ Thermal correction to Enthalpy $=\quad 1.071100$ Thermal correction to Gibbs Free Energy $=\quad 0.894916$ Sum of electronic and zero-point Energies $=\quad-2599.888682$ Sum of electronic and thermal Energies $=\quad-2599.823184$ Sum of electronic and thermal Enthalpies $=\quad-2599.822240$ Sum of electronic and thermal Free Energies $=-2599.998423$ E solvent $=-2599.7331635$

2.415548000

$-0.726330000$

$\begin{array}{lll}\text { C } & 4.613990000 & -0.048025000\end{array}$

C $\quad 3.866088000 \quad 1.136261000$

$\mathrm{H} \quad 3.386627000 \quad 1.610249000$

C $\quad 5.105114000 \quad-0.061975000$

C $\quad 3.038288000 \quad-2.112737000$

C $\quad 4.352505000 \quad 1.119603000$

C $\quad 3.794396000 \quad-1.026833000$

3.737606000

4.837328000

1.964505000

1.860935000

1.204933000

5.225990000

5.799663000

1.049624000

5.433913000

5.655895000

5.784588000

6.762107000

5.974744000

5.177198000

2.267520000

6.470808000

7.511723000

2.974081000

3.663028000

2.388318000

2.279455000

3.177463000

4.010914000

6.170158000

6.975343000

2.438864000

2.903119000

3.085919000

1.468128000

$-0.056359000$

0.247156000

$-0.970763000$

$-0.317062000$

$-3.057646000$

$-4.327920000$

$-5.454779000$

C -6.391930000

$\mathrm{H} \quad-7.046458000$
0.043510000

$-1.529236000$

1.957130000

2.132853000

1.268177000

4.369395000

$-2.501114000$

4.506767000

$-0.292255000$

3.399026000

0.615405000

$-2.120182000$

$-1.073713000$

$-4.419229000$

3.087951000

2.964811000

$-3.071745000$

$-1.851986000$

$-2.794688000$

5.568235000

5.769667000

5.409322000

6.483992000

$-4.836498000$

$-0.982168000$

$-1.252884000$

3.567818000

3.611552000

4.504388000

2.727186000

$-3.866213000$

$-4.173626000$

0.240781000

0.925442000

$-6.298564000$

$-6.863089000$

$-6.426098000$

$-6.780535000$

$-2.661609000$

$-2.812380000$

$-3.260711000$

$-1.594733000$

0.352484000

$-0.583458000$

0.060136000

1.021031000

1.504409000 


\begin{tabular}{|c|c|c|c|}
\hline $\mathrm{C}$ & -4.703900000 & -3.537553000 & -0.233060000 \\
\hline $\mathrm{C}$ & -3.204198000 & 3.400685000 & -0.372016000 \\
\hline $\mathrm{C}$ & -5.639905000 & -3.939419000 & 0.741704000 \\
\hline $\mathrm{C}$ & -4.289024000 & 1.077698000 & -0.180321000 \\
\hline $\mathrm{C}$ & -6.497075000 & -3.018476000 & 1.372765000 \\
\hline $\mathrm{C}$ & -5.423846000 & 0.227477000 & -0.318734000 \\
\hline $\mathrm{C}$ & -2.811066000 & 4.254719000 & -1.426300000 \\
\hline $\mathrm{H}$ & -3.272748000 & 4.133483000 & -2.413962000 \\
\hline $\mathrm{C}$ & -1.238140000 & 5.369515000 & 0.035916000 \\
\hline $\mathrm{C}$ & -4.613074000 & -2.171095000 & -0.558916000 \\
\hline $\mathrm{H}$ & -3.897212000 & -1.843887000 & -1.323344000 \\
\hline $\mathrm{C}$ & -1.830970000 & 5.247801000 & -1.236613000 \\
\hline $\mathrm{C}$ & -5.520119000 & 2.930074000 & -1.160516000 \\
\hline $\mathrm{H}$ & -5.562281000 & 3.983291000 & -1.456475000 \\
\hline $\mathrm{C}$ & -3.837606000 & -4.564262000 & -0.933438000 \\
\hline $\mathrm{H}$ & -4.413460000 & -5.110651000 & -1.704629000 \\
\hline $\mathrm{H}$ & -2.975558000 & -4.094477000 & -1.436981000 \\
\hline $\mathrm{H}$ & -3.451929000 & -5.319869000 & -0.225779000 \\
\hline $\mathrm{C}$ & -1.611303000 & 4.539627000 & 1.112709000 \\
\hline $\mathrm{C}$ & -6.646389000 & 2.113021000 & -1.300532000 \\
\hline $\mathrm{H}$ & -7.566437000 & 2.515363000 & -1.737244000 \\
\hline $\mathrm{C}$ & -7.524800000 & -3.480506000 & 2.386043000 \\
\hline $\mathrm{H}$ & -8.474675000 & -3.756445000 & 1.889207000 \\
\hline $\mathrm{H}$ & -7.177921000 & -4.371032000 & 2.938412000 \\
\hline $\mathrm{H}$ & -7.759731000 & -2.689872000 & 3.119753000 \\
\hline $\mathrm{C}$ & -2.589799000 & 3.551381000 & 0.892773000 \\
\hline $\mathrm{H}$ & -2.914057000 & 2.925625000 & 1.732441000 \\
\hline $\mathrm{C}$ & -6.597489000 & 0.780941000 & -0.870657000 \\
\hline $\mathrm{H}$ & -7.469963000 & 0.130035000 & -0.988699000 \\
\hline $\mathrm{C}$ & -1.002408000 & 4.735524000 & 2.486818000 \\
\hline $\mathrm{H}$ & -1.485524000 & 5.577091000 & 3.019196000 \\
\hline $\mathrm{H}$ & -1.120597000 & 3.837097000 & 3.116783000 \\
\hline $\mathrm{H}$ & 0.074594000 & 4.971827000 & 2.423526000 \\
\hline $\mathrm{C}$ & -1.440830000 & 6.177259000 & -2.368447000 \\
\hline $\mathrm{H}$ & -2.093052000 & 7.071436000 & -2.389120000 \\
\hline $\mathrm{H}$ & -0.402659000 & 6.536250000 & -2.260778000 \\
\hline $\mathrm{H}$ & -1.534625000 & 5.683945000 & -3.351491000 \\
\hline $\mathrm{Ni}$ & 1.329633000 & 0.673554000 & -0.879074000 \\
\hline $\mathrm{C}$ & -2.944213000 & -0.262726000 & 1.382706000 \\
\hline $\mathrm{H}$ & -3.873319000 & -0.502153000 & 1.910950000 \\
\hline $\mathrm{C}$ & 1.981768000 & -1.386808000 & 1.094690000 \\
\hline $\mathrm{H}$ & 2.695432000 & -2.078746000 & 1.570990000 \\
\hline $\mathrm{C}$ & 0.676173000 & -1.377249000 & 1.709494000 \\
\hline $\mathrm{C}$ & 0.515721000 & -2.173366000 & 2.863320000 \\
\hline $\mathrm{C}$ & -0.452524000 & -0.656488000 & 1.165499000 \\
\hline $\mathrm{H}$ & 1.400226000 & -2.698864000 & 3.248678000 \\
\hline $\mathrm{C}$ & -0.711526000 & -2.327906000 & 3.543758000 \\
\hline $\mathrm{C}$ & -1.731652000 & -0.849020000 & 1.840546000 \\
\hline $\mathrm{C}$ & -0.812882000 & -3.180429000 & 4.791157000 \\
\hline $\mathrm{C}$ & -1.815914000 & -1.667905000 & 3.002765000 \\
\hline $\mathrm{H}$ & -2.797387000 & -1.780724000 & 3.481889000 \\
\hline $\mathrm{O}$ & -0.358227000 & 0.113716000 & 0.128157000 \\
\hline $\mathrm{C}$ & 2.940529000 & 1.397667000 & -1.755469000 \\
\hline $\mathrm{C}$ & 3.042190000 & 2.759203000 & -2.438051000 \\
\hline $\mathrm{H}$ & 3.658297000 & 1.343951000 & -0.917343000 \\
\hline $\mathrm{H}$ & 3.152530000 & 0.575963000 & -2.462935000 \\
\hline $\mathrm{C}$ & 0.167567000 & 2.259241000 & -1.509480000 \\
\hline $\mathrm{C}$ & 0.148900000 & 1.219715000 & -2.442719000 \\
\hline $\mathrm{C}$ & 4.457951000 & 2.959948000 & -3.030415000 \\
\hline $\mathrm{H}$ & 2.305467000 & 2.866154000 & -3.257773000 \\
\hline $\mathrm{H}$ & 2.845385000 & 3.576762000 & -1.717523000 \\
\hline $\mathrm{H}$ & 4.539091000 & 3.948616000 & -3.517266000 \\
\hline $\mathrm{H}$ & 5.232736000 & 2.902848000 & -2.245130000 \\
\hline $\mathrm{H}$ & 4.686989000 & 2.188126000 & -3.786959000 \\
\hline $\mathrm{H}$ & -0.636405000 & 0.454267000 & -2.421950000 \\
\hline $\mathrm{H}$ & 0.745781000 & 1.261169000 & -3.360026000 \\
\hline $\mathrm{H}$ & 0.776111000 & 3.150584000 & -1.670518000 \\
\hline $\mathrm{H}$ & -0.604756000 & 2.351770000 & -0.740089000 \\
\hline
\end{tabular}

$\begin{array}{rrrr}\mathrm{H} & -0.418229000 & -4.198306000 & 4.619069000 \\ \mathrm{H} & -0.232906000 & -2.743592000 & 5.625279000 \\ \mathrm{H} & -1.858706000 & -3.277670000 & 5.127330000 \\ \mathrm{H} & 4.244832000 & 1.573806000 & 5.500834000 \\ \mathrm{H} & -0.473302000 & 6.140793000 & 0.196874000 \\ \mathrm{H} & -5.708601000 & -5.002316000 & 1.008763000 \\ \mathrm{H} & 0.488576000 & -3.426141000 & -5.165556000 \\ \mathrm{H} & -2.155195000 & 0.819761000 & -0.093549000\end{array}$

\section{Me-H+TS-2}

Zero-point correction $=1.003317($ Hartree/Particle) Thermal correction to Energy= $\quad 1.068163$ Thermal correction to Enthalpy= $\quad 1.069107$

Thermal correction to Gibbs Free Energy= 0.894560 Sum of electronic and zero-point Energies $=\quad-2599.879755$ Sum of electronic and thermal Energies $=\quad-2599.814910$ Sum of electronic and thermal Enthalpies $=\quad-2599.813966$ Sum of electronic and thermal Free Energies $=-2599.988513$ E solvent $=-2599.72504594$ $\begin{array}{llll}\text { C } & -3.579336000 & 0.096083000 & 2.778461000\end{array}$ C $\quad-5.125160000 \quad-1.051551000 \quad-0.594391000$

$\begin{array}{llll}\text { C } & -5.871169000 & -2.226099000 & -0.834812000\end{array}$

$\begin{array}{llll}\mathrm{H} & -6.239513000 & -2.808693000 & 0.019172000\end{array}$

$\begin{array}{llll}\text { C } & -4.919329000 & -0.723389000 & -3.018245000\end{array}$

$\begin{array}{llll}\text { C } & -2.290522000 & 0.365814000 & 3.487403000\end{array}$

$\begin{array}{llll}\text { C } & -5.663883000 & -1.902884000 & -3.222066000\end{array}$

$\begin{array}{llll}\text { C } & -3.666060000 & -0.313120000 & 1.413889000\end{array}$

C $\quad-6.150967000 \quad-2.664809000 \quad-2.144025000$

$\begin{array}{llll}\text { C } & -4.933284000 & -0.598745000 & 0.821001000\end{array}$

$\begin{array}{llll}\text { C } & -1.187636000 & -0.515010000 & 3.426293000\end{array}$

$\mathrm{H} \quad-1.256393000 \quad-1.428306000 \quad 2.826227000$

$\begin{array}{llll}\text { C } & 0.057056000 & 0.893463000 & 4.959071000\end{array}$

C $\quad-4.652236000 \quad-0.311077000 \quad-1.699459000$

$\mathrm{H} \quad-4.087955000 \quad 0.613569000 \quad-1.525176000$

$\begin{array}{llll}\text { C } & -0.015661000 & -0.269100000 & 4.167554000\end{array}$

$\begin{array}{llll}\mathrm{C} & -4.775046000 & 0.226278000 & 3.519796000\end{array}$

$\mathrm{H} \quad-4.706621000 \quad 0.509465000 \quad 4.575532000$

C $\quad-4.417871000 \quad 0.076459000 \quad-4.204435000$

$\mathrm{H} \quad-3.542896000 \quad-0.408529000 \quad-4.679312000$

$\begin{array}{llll}\mathrm{H} & -4.114829000 & 1.095099000 & -3.906873000\end{array}$

$\begin{array}{llll}\mathrm{H} & -5.194033000 & 0.171003000 & -4.984780000\end{array}$

$\begin{array}{llll}\text { C } & -1.024184000 & 1.791969000 & 5.049939000\end{array}$

$\begin{array}{llll}\text { C } & -6.022773000 & -0.035805000 & 2.945718000\end{array}$

$\begin{array}{llll}\mathrm{H} & -6.936708000 & 0.072580000 & 3.539263000\end{array}$

C $\quad-6.983610000 \quad-3.908346000 \quad-2.384527000$

$\mathrm{H} \quad-6.691480000 \quad-4.418167000 \quad-3.319209000$

$\mathrm{H} \quad-8.057765000 \quad-3.656328000 \quad-2.474361000$

$\mathrm{H} \quad-6.887952000 \quad-4.630433000 \quad-1.554864000$

$\begin{array}{llll}\mathrm{C} & -2.188803000 & 1.511790000 & 4.311370000\end{array}$

$\begin{array}{llll}\mathrm{H} & -3.034928000 & 2.208471000 & 4.366043000\end{array}$

$\begin{array}{llll}\text { C } & -6.094334000 & -0.447550000 & 1.610458000\end{array}$

$\mathrm{H} \quad-7.066599000 \quad-0.644077000 \quad 1.146119000$

$\begin{array}{llll}\text { C } & -0.945047000 & 3.015430000 & 5.941330000\end{array}$

$\begin{array}{llll}\mathrm{H} & -1.245965000 & 2.771433000 & 6.978359000\end{array}$

$\mathrm{H} \quad-1.612760000 \quad 3.820447000 \quad 5.588666000$

$\begin{array}{lrrr}\mathrm{H} & -1.612760000 & 3.820447000 & 5.588666000 \\ \mathrm{C} & 0.082113000 & 3.417647000 & 5.989189000\end{array}$

C $\quad 1.134363000 \quad-1.256051000 \quad 4.145866000$

$\begin{array}{llll}\mathrm{C} & 1.134363000 & -1.256051000 & 4.145866000 \\ \mathrm{H} & 1.100672000 & -1.927541000 & 5.025526000\end{array}$

$\mathrm{H} \quad 2.111572000 \quad-0.740747000 \quad 4.172852000$

$\mathrm{H} \quad 1.103407000 \quad-1.894350000 \quad 3.245567000$

$\mathrm{N} \quad 2.895592000 \quad 0.016341000 \quad-0.675451000$

$\begin{array}{llll}\text { C } & 4.350660000 & 1.957191000 & -0.443918000\end{array}$

$\begin{array}{lrrr}\text { C } & 4.350660000 & 1.957191000 & -0.443918000 \\ \text { C } & 5.101691000 & -1.766579000 & 0.378288000\end{array}$

$\begin{array}{llll}\text { C } & 5.986283000 & -2.588895000 & -0.354537000\end{array}$

$\begin{array}{llll}\mathrm{C} & 5.986283000 & -2.588895000 & -0.354537000 \\ \mathrm{H} & 6.695599000 & -2.124768000 & -1.051249000\end{array}$

$\begin{array}{llll}\mathrm{C} & 4.161109000 & -3.760968000 & 1.450500000\end{array}$

$\begin{array}{llll}\text { C } & 3.374769000 & 2.845497000 & -1.143157000\end{array}$ 


\begin{tabular}{|c|c|c|c|}
\hline & & & \\
\hline & 3407000 & .552045000 & 3000 \\
\hline & 75676000 & -3.988364000 & .200413000 \\
\hline & 201156000 & -0.279143000 & 0.217298000 \\
\hline & .922298000 & .034131000 & -0.525715000 \\
\hline & 86000 & 4000 & 0.51 \\
\hline & 7000 & 0000 & -2.5 \\
\hline & 4.194719000 & -2.3613 & 1.27 \\
\hline $\mathrm{H}$ & 368000 & -1.725 & 1.8 \\
\hline & 2.141479000 & & -1.230980000 \\
\hline & 7000 & & 6000 \\
\hline I & 4000 & 3.607 & -0 \\
\hline & 60000 & 0000 & 2000 \\
\hline & 3.095985000 & -3.815 & 3.349647000 \\
\hline & 2.169604000 & -4.468559000 & 49000 \\
\hline & 3.494516000 & -5.423879000 & 2.686114000 \\
\hline & 66000 & 7000 & -3.2 \\
\hline & & & \\
\hline & & & \\
\hline & & & \\
\hline & & & \\
\hline & 6.48 & -5.8 & \\
\hline & 7.3 & -4 & \\
\hline & 3.02 & & \\
\hline & & & \\
\hline & & & \\
\hline & & -0 . & \\
\hline & & & \\
\hline & & & \\
\hline & & & \\
\hline & & & \\
\hline & & & \\
\hline & 2.3 & & \\
\hline & & & \\
\hline & & & \\
\hline & 000 & & \\
\hline & 1000 & -1.1 & -1 \\
\hline & 0000 & -1.746 & 000 \\
\hline & 6000 & -1.562 & 0000 \\
\hline & -2.96 & -2.3 & 4000 \\
\hline & -1.02 & & \\
\hline & & & \\
\hline & & & \\
\hline & & & \\
\hline & & & \\
\hline & & & \\
\hline & & & \\
\hline & & & \\
\hline & & & \\
\hline $\mathrm{O}$ & & & \\
\hline & 0 & & \\
\hline & & & \\
\hline & & & 00 \\
\hline & & & -2 \\
\hline & & & \\
\hline 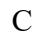 & & & \\
\hline $\mathrm{C}$ & & & -3.6 \\
\hline 11 & 0000 & & -1 . \\
\hline $\mathrm{H}$ & 000 & & 000 \\
\hline 11 & 3000 & 000 & -3 \\
\hline 11 & 2000 & 3.703 & -3 \\
\hline 11 & 06000 & 2.437 & -4 \\
\hline & -0.46 & & \\
\hline & -0.2 & 2.787 & \\
\hline & & & \\
\hline & & & \\
\hline & & & 10710100 \\
\hline & -0.585157000 & -5.143601000 & -3.4775410 \\
\hline
\end{tabular}

$\begin{array}{rrrr}\mathrm{H} & 1.003679000 & -5.567363000 & -2.792417000 \\ \mathrm{H} & -5.876795000 & -2.230140000 & -4.248519000 \\ \mathrm{H} & 1.234491000 & 5.426321000 & -3.147126000 \\ \mathrm{H} & 5.034977000 & -5.643391000 & 0.834592000 \\ \mathrm{H} & 0.972431000 & 1.097195000 & 5.530672000 \\ \mathrm{H} & 2.033920000 & 0.541139000 & -0.417705000\end{array}$

\section{$\mathrm{Me}-\mathrm{H}+\mathbf{2}-\mathrm{CoOr}-\mathrm{C}$}

Zero-point correction $=$ 1.004483 (Hartree/Particle) Thermal correction to Energy $=\quad 1.069922$ Thermal correction to Enthalpy $=\quad 1.070867$ Thermal correction to Gibbs Free Energy $=\quad 0.895988$ Sum of electronic and zero-point Energies $=\quad-2599.891181$ Sum of electronic and thermal Energies $=\quad-2599.825741$ Sum of electronic and thermal Enthalpies $=\quad-2599.824797$ Sum of electronic and thermal Free Energies $=-2599.999676$ E solvent $=-2599.73736125$

c

c

c

c

2.555472000 4.172599000 4.854331000 4.003282000 3.384973000 5.597731000 3.106490000 4.736190000 3.923324000 3.941808000 5.003591000 2.189415000 2.201007000 1.320930000 5.643194000 6.297600000 1.302095000 5.506073000 5.698133000 6.468871000 7.418072000 6.731920000 5.969013000 2.213546000 6.571151000 7.598126000 3.060592000 3.626967000 2.662423000 2.205152000 3.098380000 3.802529000 6.317778000 7.144938000 2.239912000 2.842248000 2.683299000 1.226173000 0.373066000 $-0.532809000$ 0.057620000 0.869313000 $-2.885875000$ $-4.222769000$ $-5.280588000$ $-6.159258000$ $-6.805753000$ $-4.485661000$ $-3.073268000$ $-5.364617000$ $-4.154800000$
0.081894000 1.839618000 $-1.754618000$ $-2.155451000$ $-1.407728000$ $-4.089414000$ 2.746122000 $-4.451333000$ 0.524245000 $-3.496975000$ $-0.329535000$ 2.320852000 1.278549000 4.574353000 $-2.738193000$ $-2.441200000$ 3.228106000 2.301946000 3.305172000 $-5.125728000$ $-5.270998000$ $-4.824684000$ $-6.109189000$ 5.032698000 1.491350000 1.869776000 $-3.904939000$ $-3.899165000$ $-4.926473000$ $-3.217440000$ 4.107035000 4.447625000 0.187221000 $-0.456515000$ 6.494224000 7.090301000 6.634660000 6.932019000 2.769450000 3.398060000 1.720508000 2.827667000 $-0.601511000$ 0.307175000 $-0.531489000$ $-1.543337000$ $-2.048794000$ $-0.240211000$ 0.241832000 $-1.264609000$ $-0.148073000$
$-0.497757000$ $-1.110522000$ 0.196342000 1.247492000 1.756273000 $-0.049862000$ $-1.636399000$ 1.003475000 $-0.623216000$ 1.668498000 $-0.243779000$ $-2.623547000$ $-2.960920000$ $-2.823502000$ $-0.442708000$ $-1.271943000$ $-3.235499000$ $-1.156322000$ $-1.551400000$ $-0.731308000$ $-0.180588000$ $-1.760217000$ $-0.778102000$ $-1.832082000$ $-0.748616000$ $-0.786970000$ 2.832545000 3.783785000 2.700657000 2.955953000 $-1.249693000$ $-0.480332000$ $-0.311524000$ 0.005845000 $-1.430821000$ $-2.143015000$ $-0.429999000$ $-1.421433000$ $-4.342246000$ $-4.397732000$ $-4.199439000$ $-5.330108000$ 0.541895000 2.373889000 $-1.268259000$ $-1.709818000$ $-0.981203000$ $-3.570605000$ 3.324569000 $-3.978183000$ 1.027321000 


\begin{tabular}{|c|c|c|c|}
\hline $\mathrm{C}$ & -6.214312000 & -1.920101000 & -3.067149000 \\
\hline $\mathrm{C}$ & -5.294747000 & -0.126461000 & 0.173243000 \\
\hline $\mathrm{C}$ & -2.748352000 & 1.378096000 & 4.099035000 \\
\hline $\mathrm{H}$ & -3.292740000 & 2.315206000 & 3.929406000 \\
\hline $\mathrm{C}$ & -1.037971000 & 0.120863000 & 5.260227000 \\
\hline $\mathrm{C}$ & -4.447244000 & 0.112518000 & -2.208771000 \\
\hline $\mathrm{H}$ & -3.778106000 & 0.916358000 & -1.877622000 \\
\hline $\mathrm{C}$ & -1.732824000 & 1.332907000 & 5.073198000 \\
\hline $\mathrm{C}$ & -5.457525000 & 0.801895000 & 2.845637000 \\
\hline $\mathrm{H}$ & -5.521372000 & 1.138407000 & 3.885616000 \\
\hline $\mathrm{C}$ & -3.623403000 & 0.482349000 & -4.585897000 \\
\hline $\mathrm{H}$ & -3.198044000 & -0.217490000 & -5.327221000 \\
\hline $\mathrm{H}$ & -4.213981000 & 1.229097000 & -5.150237000 \\
\hline $\mathrm{H}$ & -2.788972000 & 1.019591000 & -4.102963000 \\
\hline $\mathrm{C}$ & -1.344490000 & -1.034939000 & 4.513639000 \\
\hline $\mathrm{C}$ & -6.593191000 & 0.814277000 & 2.028797000 \\
\hline $\mathrm{H}$ & -7.545949000 & 1.187753000 & 2.418518000 \\
\hline $\mathrm{C}$ & -2.357855000 & -0.957918000 & 3.539544000 \\
\hline $\mathrm{H}$ & -2.626954000 & -1.858556000 & 2.975007000 \\
\hline $\mathrm{C}$ & -6.509630000 & 0.345803000 & 0.711335000 \\
\hline $\mathrm{H}$ & -7.387948000 & 0.375543000 & 0.058200000 \\
\hline $\mathrm{C}$ & -0.630375000 & -2.343400000 & 4.787809000 \\
\hline $\mathrm{H}$ & -1.124395000 & -2.901450000 & 5.606300000 \\
\hline $\mathrm{H}$ & -0.627079000 & -3.001000000 & 3.901088000 \\
\hline $\mathrm{H}$ & 0.416156000 & -2.177225000 & 5.098138000 \\
\hline $\mathrm{C}$ & -1.414005000 & 2.550528000 & 5.917860000 \\
\hline $\mathrm{H}$ & -2.054698000 & 2.586097000 & 6.819840000 \\
\hline $\mathrm{H}$ & -0.366228000 & 2.542027000 & 6.264782000 \\
\hline $\mathrm{H}$ & -1.584800000 & 3.488754000 & 5.361627000 \\
\hline $\mathrm{Ni}$ & 1.184574000 & 1.087940000 & 0.666323000 \\
\hline $\mathrm{C}$ & -2.679016000 & -1.640607000 & -0.259540000 \\
\hline $\mathrm{H}$ & -3.555701000 & -2.251314000 & -0.500919000 \\
\hline $\mathrm{C}$ & 2.260895000 & -1.000463000 & -1.177902000 \\
\hline $\mathrm{H}$ & 3.054495000 & -1.443479000 & -1.802815000 \\
\hline $\mathrm{C}$ & 0.994354000 & -1.697410000 & -1.237990000 \\
\hline $\mathrm{C}$ & 0.960270000 & -2.886402000 & -1.997021000 \\
\hline $\mathrm{C}$ & -0.210607000 & -1.220071000 & -0.600897000 \\
\hline $\mathrm{H}$ & 1.897789000 & -3.219541000 & -2.462838000 \\
\hline $\mathrm{C}$ & -0.201222000 & -3.667841000 & -2.182178000 \\
\hline $\mathrm{C}$ & -1.416944000 & -2.011702000 & -0.805919000 \\
\hline $\mathrm{C}$ & -0.162383000 & -4.944813000 & -2.995240000 \\
\hline $\mathrm{C}$ & -1.373212000 & -3.203628000 & -1.583068000 \\
\hline $\mathrm{H}$ & -2.304867000 & -3.770504000 & -1.710771000 \\
\hline $\mathrm{O}$ & -0.269346000 & -0.137104000 & 0.117418000 \\
\hline $\mathrm{C}$ & -0.247270000 & 2.270450000 & 1.262883000 \\
\hline $\mathrm{C}$ & -0.721997000 & 2.930882000 & -0.025915000 \\
\hline $\mathrm{H}$ & -0.997600000 & 1.618742000 & 1.744234000 \\
\hline $\mathrm{H}$ & 0.106050000 & 3.011560000 & 1.999892000 \\
\hline $\mathrm{C}$ & 2.533848000 & 2.159714000 & 1.771347000 \\
\hline $\mathrm{C}$ & 1.918254000 & 1.151427000 & 2.536165000 \\
\hline $\mathrm{C}$ & -1.826759000 & 3.982506000 & 0.257361000 \\
\hline $\mathrm{H}$ & 0.118896000 & 3.436469000 & -0.538897000 \\
\hline $\mathrm{H}$ & -1.113139000 & 2.169119000 & -0.725287000 \\
\hline $\mathrm{H}$ & -2.154782000 & 4.460405000 & -0.683305000 \\
\hline $\mathrm{H}$ & -2.709507000 & 3.519191000 & 0.733264000 \\
\hline $\mathrm{H}$ & -1.456644000 & 4.776290000 & 0.930320000 \\
\hline $\mathrm{H}$ & 2.423970000 & 0.187727000 & 2.679222000 \\
\hline $\mathrm{H}$ & 1.112760000 & 1.365662000 & 3.247689000 \\
\hline $\mathrm{H}$ & 2.216871000 & 3.204822000 & 1.856028000 \\
\hline $\mathrm{H}$ & 3.532083000 & 2.000613000 & 1.351824000 \\
\hline $\mathrm{H}$ & 0.467640000 & -5.712661000 & -2.508991000 \\
\hline $\mathrm{H}$ & -1.171408000 & -5.371758000 & -3.121030000 \\
\hline $\mathrm{H}$ & 0.257481000 & -4.770275000 & -4.002558000 \\
\hline $\mathrm{H}$ & 0.629580000 & 5.287416000 & -3.291873000 \\
\hline $\mathrm{H}$ & -5.393304000 & -1.553055000 & -5.037325000 \\
\hline $\mathrm{H}$ & 4.684941000 & -5.502519000 & 1.316971000 \\
\hline $\mathrm{H}$ & -0.248300000 & 0.070851000 & 6.021727000 \\
\hline $\mathrm{C}$ & -7.180965000 & -2.988455000 & -3.536913000 \\
\hline
\end{tabular}

$\begin{array}{llll}\mathrm{H} & -7.413081000 & -3.711035000 & -2.735329000 \\ \mathrm{H} & -8.140290000 & -2.540246000 & -3.859487000 \\ \mathrm{H} & -6.778997000 & -3.548023000 & -4.399355000 \\ \mathrm{H} & -2.019509000 & -0.081866000 & 0.788871000\end{array}$

\section{${ }^{\mathrm{Me}-\mathrm{H}+} \mathbf{T S}-\mathbf{2}_{\text {ins }}$}

Zero-point correction $=\quad 1.004811($ Hartree/Particle $)$

$\begin{array}{ll}\text { Thermal correction to Energy= } & 1.069673\end{array}$

Thermal correction to Enthalpy $=\quad 1.070617$

Thermal correction to Gibbs Free Energy $=\quad 0.896888$

Sum of electronic and zero-point Energies $=\quad-2599.869306$

Sum of electronic and thermal Energies $=\quad-2599.804444$

Sum of electronic and thermal Enthalpies $=\quad-2599.803499$

Sum of electronic and thermal Free Energies= -2599.977229

E solvent $=-2599.72232284$

C $\quad-4.178282000$

C $\quad-4.837101000$

C -3.994549000

$\mathrm{H} \quad-3.381785000$

C $\quad-5.553145000$

C -3.108554000

C $\quad-4.705842000$

$\begin{array}{ll}\text { C } & -4.705842000 \\ \text { C } & -3.925713000\end{array}$

C $\quad-3.929621000$

C -4.999528000

C -2.151998000

$\mathrm{H}-2.143455000$

C -1.282847000

C $\quad-5.606654000$

$\begin{array}{ll}\mathrm{C} & -5.606654000 \\ \mathrm{H} & -6.251985000\end{array}$

C $\quad-1.243360000$

$\begin{array}{ll}\mathrm{C} & -1.243360000 \\ \mathrm{C} & -5.517619000\end{array}$

H $\quad-5.711992000$

C $\quad-6.401849000$

$\mathrm{H}-7.356487000$

$\mathrm{H}-6.654884000$

$\begin{array}{ll}\mathrm{H} & -6.654884000 \\ \mathrm{H} & -5.888646000\end{array}$

C $\quad-2.219059000$

C $\quad-6.582100000$

H $\quad-7.613973000$

C $\quad-3.059986000$

$\mathrm{H} \quad-3.654016000$

$\mathrm{H} \quad-2.593437000$

$\begin{array}{ll}\mathrm{H} & -2.593437000 \\ \mathrm{H} & -2.254914000\end{array}$

C -3.125330000

$\mathrm{H} \quad-3.865456000$

C -6.320548000

H $\quad-7.146730000$

C -2.274059000

$\mathrm{H}-2.949034000$

$\begin{array}{ll}\mathrm{H} & -2.949034000 \\ \mathrm{H} & -2.652421000\end{array}$

H -1.280148000

C $\quad-0.268306000$

$\mathrm{H} \quad 0.682517000$

$\mathrm{H} \quad-0.041159000$

$\begin{array}{ll}\mathrm{H} & -0.041159000 \\ \mathrm{H} & -0.681712000\end{array}$

N 2.898104000

$\begin{array}{ll}\mathrm{N} & 2.898104000 \\ \mathrm{C} & 4.261768000\end{array}$

C $\quad 5.281692000$

C $\quad 4.467905000$

$\mathrm{H} \quad 3.827502000$

C $\quad 6.159776000$

$\begin{array}{ll}\text { C } & 3.111933000\end{array}$

C $\quad 5.330100000$

C $\quad 4.176143000$

C $\quad 4.489275000$

C $\quad 5.313595000$

0.121618000 $-1.234334000$ 1.591529000 1.534322000 0.642819000 3.846052000 $-1.922979000$ 3.750739000 $-0.218123000$ 2.600204000 0.456022000 $-1.202658000$ $-0.107689000$ $-3.269134000$ 2.758749000 2.822567000 $-1.862358000$ $-1.599052000$ $-2.368329000$ 5.080878000 5.016611000 5.209621000 5.996005000 $-4.021048000$

$-0.973690000$ $-1.273860000$ 2.507117000 2.205898000 3.478688000 1.761686000 $-3.332664000$ $-3.902779000$ 0.048921000 0.543624000 $-5.530969000$ $-5.836582000$ $-6.004621000$ $-5.957017000$ $-1.073190000$ $-1.617652000$ $-0.089633000$ $-0.884448000$ 0.358236000 $-1.156710000$ 1.010914000 0.727252000 $-0.163413000$ 2.991443000 $-1.505195000$ 2.685312000 $-0.225848000$ 1.554053000 0.104430000
$-0.481483000$

$-1.765114000$ 0.816348000 1.947516000 2.123158000 1.501514000 $-2.550840000$ 2.621569000 $-0.801600000$ 2.864084000 $-0.148836000$ $-3.300079000$ $-3.256738000$ $-4.220975000$ 0.608788000 $-0.276376000$ $-4.149113000$ $-2.024770000$ $-2.779761000$ 1.272424000 1.829185000 0.205698000

1.616621000 $-3.483883000$ $-1.366027000$ $-1.577055000$ 4.102093000 4.986369000 4.343960000 3.977766000 $-2.654291000$ $-2.078155000$ $-0.449139000$ 0.072272000 $-3.609673000$ $-4.432156000$ $-2.686957000$ $-3.831695000$ $-5.000708000$ $-5.139782000$ $-4.552957000$ $-6.010156000$ 0.713795000 2.066796000 $-0.991455000$ $-2.109527000$ $-2.099589000$ $-2.149280000$ 2.953189000 $-3.245041000$ 0.992806000 $-3.248558000$ 0.200416000 


\begin{tabular}{|c|c|c|c|}
\hline $\mathrm{C}$ & 2.320142000 & -0.513432000 & 3.572715000 \\
\hline $\mathrm{H}$ & 2.530436000 & 0.545920000 & 3.384407000 \\
\hline $\mathrm{C}$ & 1.065354000 & -2.222701000 & 4.744550000 \\
\hline $\mathrm{C}$ & 6.124029000 & 2.142807000 & -1.024680000 \\
\hline $\mathrm{H}$ & 6.755920000 & 2.367953000 & -0.156142000 \\
\hline $\mathrm{C}$ & 1.298178000 & -0.859248000 & 4.479193000 \\
\hline $\mathrm{C}$ & 5.514215000 & -1.752878000 & 2.329358000 \\
\hline $\mathrm{H}$ & 5.592490000 & -2.453967000 & 3.166628000 \\
\hline $\mathrm{C}$ & 7.085919000 & 4.190329000 & -2.187061000 \\
\hline $\mathrm{H}$ & 6.646368000 & 5.025433000 & -2.759991000 \\
\hline $\mathrm{H}$ & 7.322210000 & 4.555871000 & -1.172743000 \\
\hline $\mathrm{H}$ & 8.046251000 & 3.933727000 & -2.674007000 \\
\hline $\mathrm{C}$ & 1.841227000 & -3.238989000 & 4.148119000 \\
\hline $\mathrm{C}$ & 6.647997000 & -1.421096000 & 1.580446000 \\
\hline $\mathrm{H}$ & 7.613674000 & -1.882502000 & 1.811990000 \\
\hline $\mathrm{C}$ & 3.651592000 & 1.221473000 & -4.466842000 \\
\hline $\mathrm{H}$ & 4.253025000 & 0.691295000 & -5.229941000 \\
\hline $\mathrm{H}$ & 2.798972000 & 0.569457000 & -4.209847000 \\
\hline $\mathrm{H}$ & 3.254469000 & 2.133625000 & -4.946337000 \\
\hline $\mathrm{C}$ & 2.858067000 & -2.864259000 & 3.251706000 \\
\hline $\mathrm{H}$ & 3.460341000 & -3.640521000 & 2.764078000 \\
\hline $\mathrm{C}$ & 6.544654000 & -0.497512000 & 0.532550000 \\
\hline $\mathrm{H}$ & 7.420764000 & -0.254379000 & -0.077593000 \\
\hline $\mathrm{C}$ & 1.598219000 & -4.697020000 & 4.484764000 \\
\hline $\mathrm{H}$ & 1.953960000 & -5.366351000 & 3.682505000 \\
\hline $\mathrm{H}$ & 2.132170000 & -4.984412000 & 5.410819000 \\
\hline $\mathrm{H}$ & 0.526736000 & -4.902726000 & 4.655212000 \\
\hline $\mathrm{C}$ & 0.499381000 & 0.216871000 & 5.186882000 \\
\hline $\mathrm{H}$ & 0.329357000 & 1.092646000 & 4.535907000 \\
\hline $\mathrm{H}$ & -0.482521000 & -0.160540000 & 5.520905000 \\
\hline $\mathrm{H}$ & 1.032021000 & 0.579701000 & 6.086879000 \\
\hline $\mathrm{Ni}$ & -1.331405000 & -1.173120000 & 0.333050000 \\
\hline $\mathrm{C}$ & 2.680236000 & 1.620496000 & 0.356559000 \\
\hline $\mathrm{H}$ & 3.554784000 & 2.279514000 & 0.355815000 \\
\hline $\mathrm{C}$ & -2.239954000 & 1.368836000 & -0.760903000 \\
\hline $\mathrm{H}$ & -3.026106000 & 1.984305000 & -1.226882000 \\
\hline $\mathrm{C}$ & -0.984562000 & 2.047622000 & -0.556856000 \\
\hline $\mathrm{C}$ & -0.937472000 & 3.423426000 & -0.860586000 \\
\hline $\mathrm{C}$ & 0.204717000 & 1.364698000 & -0.104505000 \\
\hline $\mathrm{H}$ & -1.864864000 & 3.904918000 & -1.199669000 \\
\hline $\mathrm{C}$ & 0.230324000 & 4.209887000 & -0.743379000 \\
\hline $\mathrm{C}$ & 1.420285000 & 2.164692000 & -0.015001000 \\
\hline $\mathrm{C}$ & 0.210822000 & 5.689508000 & -1.064982000 \\
\hline $\mathrm{C}$ & 1.389478000 & 3.555343000 & -0.326967000 \\
\hline $\mathrm{H}$ & 2.325716000 & 4.122235000 & -0.239426000 \\
\hline $\mathrm{O}$ & 0.221352000 & 0.101531000 & 0.194419000 \\
\hline $\mathrm{C}$ & 0.234320000 & -2.549279000 & 0.602678000 \\
\hline $\mathrm{C}$ & 0.555880000 & -2.778866000 & -0.872568000 \\
\hline $\mathrm{H}$ & 0.827178000 & -1.777693000 & 1.116946000 \\
\hline $\mathrm{H}$ & 0.383808000 & -3.477880000 & 1.170020000 \\
\hline $\mathrm{C}$ & -1.734704000 & -2.992475000 & 1.439676000 \\
\hline $\mathrm{C}$ & -2.846422000 & -2.192806000 & 1.088322000 \\
\hline $\mathrm{C}$ & 1.936614000 & -3.460613000 & -1.039396000 \\
\hline $\mathrm{H}$ & -0.218827000 & -3.417954000 & -1.336429000 \\
\hline $\mathrm{H}$ & 0.547307000 & -1.826499000 & -1.431177000 \\
\hline $\mathrm{H}$ & 2.157743000 & -3.629164000 & -2.108464000 \\
\hline $\mathrm{H}$ & 2.749189000 & -2.842198000 & -0.616404000 \\
\hline $\mathrm{H}$ & 1.962943000 & -4.441125000 & -0.530344000 \\
\hline $\mathrm{H}$ & -3.521014000 & -2.534649000 & 0.297017000 \\
\hline $\mathrm{H}$ & -3.289316000 & -1.508548000 & 1.824134000 \\
\hline $\mathrm{H}$ & -1.332879000 & -2.951512000 & 2.459042000 \\
\hline $\mathrm{H}$ & -1.636764000 & -3.962526000 & 0.940547000 \\
\hline $\mathrm{H}$ & -0.450641000 & 6.243482000 & -0.373336000 \\
\hline $\mathrm{H}$ & 1.218722000 & 6.130520000 & -0.988390000 \\
\hline $\mathrm{H}$ & -0.160992000 & 5.876007000 & -2.089150000 \\
\hline $\mathrm{H}$ & -0.574620000 & -3.791991000 & -4.877492000 \\
\hline $\mathrm{H}$ & 5.345350000 & 3.341367000 & -4.125448000 \\
\hline $\mathrm{H}$ & -4.649750000 & 4.592576000 & 3.324530000 \\
\hline
\end{tabular}

$\begin{array}{llll}\mathrm{H} & 0.271447000 & -2.500915000 & 5.450533000 \\ \mathrm{H} & 2.041933000 & -0.226658000 & 0.756130000\end{array}$

\section{Me-H+2-ק-T}

Zero-point correction $=1.006304($ Hartree/Particle $)$

Thermal correction to Energy= $\quad 1.070968$

Thermal correction to Enthalpy= $\quad 1.071913$

Thermal correction to Gibbs Free Energy $=\quad 0.898190$

Sum of electronic and zero-point Energies $=\quad-2599.914350$

Sum of electronic and thermal Energies $=\quad-2599.849686$

Sum of electronic and thermal Enthalpies $=\quad-2599.848742$

Sum of electronic and thermal Free Energies $=\quad-2600.022464$

E solvent $=-2599.76413493$

\begin{tabular}{|c|c|c|c|}
\hline & -1.325846000 & 0.730530000 & \\
\hline & 0.319658000 & -0.259824000 & 7239000 \\
\hline & .420306000 & -1.553473000 & 8000 \\
\hline & -0.694792000 & -2.400400000 & م \\
\hline & -2.000838000 & -1.890005000 & ter \\
\hline & & & \\
\hline & 045000 & -2.205106000 & \\
\hline & 1.809408000 & & \\
\hline & 1.198154000 & & \\
\hline & 000 & & \\
\hline & 0.72 & & \\
\hline & 000 & 00 & \\
\hline & 000 & & \\
\hline & 2.9 & & \\
\hline & & & \\
\hline & & & \\
\hline $\mathrm{C}$ & & & \\
\hline & & & 13000 \\
\hline & & & \\
\hline & & & \\
\hline & & & \\
\hline & & & \\
\hline & & & \\
\hline & & & \\
\hline & 6.6 & & r \\
\hline & & & \\
\hline & & & \\
\hline & & & \\
\hline 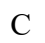 & & & \\
\hline$C_{1}$ & & & \\
\hline 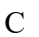 & & & \\
\hline & & & \\
\hline & & & \\
\hline 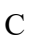 & & & \\
\hline $\mathrm{C}$ & & & \\
\hline & & & \\
\hline & & & \\
\hline & -4 & & \\
\hline & & & \\
\hline & & & \\
\hline & & & 00 \\
\hline 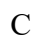 & & & 00 \\
\hline $\mathrm{C}$ & & & \\
\hline $\mathrm{C}$ & -3.0 & & 000 \\
\hline $\mathrm{C}$ & -2.10 & & 000 \\
\hline $\mathrm{C}$ & 00 & 000 & 000 \\
\hline $\mathrm{C}$ & & 000 & 000 \\
\hline $\mathrm{C}$ & & & 3000 \\
\hline & & 00 & \\
\hline & & & \\
\hline 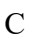 & & & 0 \\
\hline 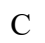 & & & \\
\hline & & & \\
\hline & & & \\
\hline & -5.277466000 & -3.880880000 & -2.18660900 \\
\hline
\end{tabular}




\begin{tabular}{|c|c|c|c|}
\hline C & -5.353961000 & -3.062887000 & -1.043013000 \\
\hline$C$ & -6.026043000 & -5.197258000 & -2.251835000 \\
\hline $\mathrm{C}$ & -3.034864000 & -1.728953000 & -4.449435000 \\
\hline $\mathrm{C}$ & -2.587052000 & 2.137040000 & -0.300907000 \\
\hline $\mathrm{C}$ & -1.339572000 & 2.842856000 & -0.725822000 \\
\hline $\mathrm{C}$ & -0.881862000 & 4.034820000 & 0.143681000 \\
\hline $\mathrm{C}$ & -1.324618000 & 6.481249000 & 0.764451000 \\
\hline $\mathrm{C}$ & -1.766340000 & 5.276845000 & -0.082423000 \\
\hline $\mathrm{H}$ & -2.728245000 & -2.643473000 & 0.924343000 \\
\hline $\mathrm{H}$ & -1.376791000 & -4.416269000 & 0.577472000 \\
\hline $\mathrm{H}$ & 0.435518000 & -6.342306000 & 1.070128000 \\
\hline $\mathrm{H}$ & 1.897669000 & -6.269044000 & 0.051077000 \\
\hline $\mathrm{H}$ & 0.290229000 & -6.436128000 & -0.699957000 \\
\hline $\mathrm{H}$ & 2.792274000 & -4.075820000 & -0.444486000 \\
\hline $\mathrm{H}$ & 3.830129000 & -2.017656000 & -0.743620000 \\
\hline $\mathrm{H}$ & 7.375790000 & 0.906472000 & 0.077631000 \\
\hline $\mathrm{H}$ & 7.264127000 & 2.911844000 & -1.413976000 \\
\hline $\mathrm{H}$ & 5.131301000 & 3.457397000 & -2.599100000 \\
\hline $\mathrm{H}$ & 7.043656000 & -1.772953000 & -0.441546000 \\
\hline $\mathrm{H}$ & 3.960143000 & -0.097293000 & 2.097551000 \\
\hline $\mathrm{H}$ & 3.141203000 & -1.399243000 & 4.026119000 \\
\hline $\mathrm{H}$ & 4.659980000 & -1.509479000 & 4.948325000 \\
\hline $\mathrm{H}$ & 3.847407000 & -2.995981000 & 4.411755000 \\
\hline $\mathrm{H}$ & 5.990252000 & -3.731879000 & 3.266485000 \\
\hline $\mathrm{H}$ & 7.422023000 & -4.898432000 & 1.579696000 \\
\hline $\mathrm{H}$ & 8.669247000 & -3.631382000 & 1.581283000 \\
\hline $\mathrm{H}$ & 7.874503000 & -4.081061000 & 0.055160000 \\
\hline $\mathrm{H}$ & 0.966721000 & 0.624072000 & -6.223452000 \\
\hline $\mathrm{H}$ & 0.639684000 & -0.481255000 & -4.867902000 \\
\hline $\mathrm{H}$ & -0.576505000 & 0.737795000 & -5.350853000 \\
\hline $\mathrm{H}$ & -0.139286000 & 3.197200000 & -4.902606000 \\
\hline $\mathrm{H}$ & -0.251414000 & 5.391332000 & -3.605642000 \\
\hline $\mathrm{H}$ & 1.101173000 & 5.845021000 & -2.526849000 \\
\hline $\mathrm{H}$ & 1.319799000 & 5.852944000 & -4.292051000 \\
\hline $\mathrm{H}$ & 2.840718000 & 4.198964000 & -1.933796000 \\
\hline $\mathrm{H}$ & 2.567112000 & 0.154517000 & -3.463732000 \\
\hline $\mathrm{H}$ & 0.045004000 & -0.422307000 & 5.933168000 \\
\hline $\mathrm{H}$ & 0.392295000 & -1.019032000 & 4.291647000 \\
\hline $\mathrm{H}$ & -1.757060000 & -0.864664000 & 3.194289000 \\
\hline $\mathrm{H}$ & -0.275582000 & 2.531658000 & 5.410245000 \\
\hline $\mathrm{H}$ & -1.279580000 & 4.784814000 & 4.962349000 \\
\hline $\mathrm{H}$ & -2.834223000 & 4.360622000 & 5.712587000 \\
\hline $\mathrm{H}$ & -2.797982000 & 4.929409000 & 4.028424000 \\
\hline $\mathrm{H}$ & -5.549309000 & 1.167766000 & 3.456611000 \\
\hline $\mathrm{H}$ & -7.458028000 & 0.239657000 & 2.137769000 \\
\hline $\mathrm{H}$ & -7.000784000 & -1.133528000 & 0.098514000 \\
\hline $\mathrm{H}$ & -3.376186000 & -0.453174000 & -2.045667000 \\
\hline $\mathrm{H}$ & -5.943181000 & -3.397680000 & -0.179842000 \\
\hline $\mathrm{H}$ & -6.196507000 & -5.619734000 & -1.246309000 \\
\hline $\mathrm{H}$ & -5.478729000 & -5.946150000 & -2.850884000 \\
\hline $\mathrm{H}$ & -7.018959000 & -5.067804000 & -2.723941000 \\
\hline $\mathrm{H}$ & -4.426973000 & -4.066700000 & -4.171471000 \\
\hline $\mathrm{H}$ & -2.223290000 & -1.039030000 & -4.158081000 \\
\hline $\mathrm{H}$ & -3.685796000 & -1.188651000 & -5.163795000 \\
\hline $\mathrm{H}$ & -2.585235000 & -2.574779000 & -4.998992000 \\
\hline $\mathrm{H}$ & -3.331740000 & 1.897153000 & -1.075230000 \\
\hline $\mathrm{H}$ & -3.033769000 & 2.460696000 & 0.649823000 \\
\hline $\mathrm{H}$ & -1.322193000 & 3.082804000 & -1.805631000 \\
\hline $\mathrm{H}$ & -0.429657000 & 2.086424000 & -0.687384000 \\
\hline $\mathrm{H}$ & -2.818563000 & 5.020371000 & 0.148652000 \\
\hline $\mathrm{H}$ & -1.744730000 & 5.548821000 & -1.156707000 \\
\hline $\mathrm{H}$ & 0.171532000 & 4.282363000 & -0.091027000 \\
\hline $\mathrm{H}$ & -1.974877000 & 7.355040000 & 0.584571000 \\
\hline $\mathrm{H}$ & -1.366080000 & 6.248146000 & 1.844223000 \\
\hline $\mathrm{H}$ & -0.287180000 & 6.781607000 & 0.527858000 \\
\hline $\mathrm{H}$ & -3.894547000 & 2.900495000 & 3.078445000 \\
\hline $\mathrm{H}$ & -0.915239000 & 3.736435000 & 1.209360000 \\
\hline 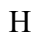 & 2.019371000 & 0.318347000 & -0.729557000 \\
\hline
\end{tabular}

\author{
Me-H+TS-2 ${ }_{\text {decoor }}$ \\ Zero-point correction $=\quad 1.001551($ Hartree/Particle $)$ \\ Thermal correction to Energy $=\quad 1.067154$ \\ Thermal correction to Enthalpy= $\quad 1.068098$ \\ Thermal correction to Gibbs Free Energy $=\quad 0.892114$ \\ Sum of electronic and zero-point Energies $=\quad-2599.864780$ \\ Sum of electronic and thermal Energies $=\quad-2599.799178$ \\ Sum of electronic and thermal Enthalpies $=\quad-2599.798234$ \\ Sum of electronic and thermal Free Energies $=-2599.974218$ \\ E solvent $=-2599.71686159$
}

\begin{tabular}{|c|c|c|c|}
\hline & 000 & 00 & \\
\hline & 4.732190000 & 0.388246000 & 1000 \\
\hline & 2.8907 & 000 & -0.646 \\
\hline & 2.872571000 & 4.701678000 & 000 \\
\hline & 3.557416000 & 7000 & 0000 \\
\hline & 1.180444000 & 1000 & -1.79 \\
\hline & 4.618080000 & -0.972534000 & 7000 \\
\hline & 1.168903000 & 5.996830000 & -0.71 \\
\hline & 3.663694000 & 1.333450000 & 67000 \\
\hline & 2.017084000 & 5.819503000 & 0.400206000 \\
\hline & 3.907410000 & 2.660904000 & -0.632907000 \\
\hline & 3.938012000 & & 1.7 \\
\hline & 3.441307000 & & 8000 \\
\hline & 4.605 & & \\
\hline & & & \\
\hline & & & \\
\hline & 3.9 & & \\
\hline & 6.00 & & \\
\hline & & & \\
\hline & 0.31 & & \\
\hline & 0.0 & & \\
\hline & 0.8 & & \\
\hline & & & \\
\hline & & & \\
\hline & 6.2 & & \\
\hline & & & \\
\hline & 2.0 & & \\
\hline & 1.0 & & \\
\hline & 2.5 & & \\
\hline & 2.5 & & \\
\hline & 5.2 & & 000 \\
\hline & 5.81 & & -1 . \\
\hline & 5.19 & & 2000 \\
\hline & 5.37 & & 7000 \\
\hline & 6.05 & -4.4 & 5000 \\
\hline & 7.02 & & 1000 \\
\hline & 6.27 & & -1.2 \\
\hline & & & 2000 \\
\hline & & & \\
\hline & & & \\
\hline & & & \\
\hline & & & \\
\hline & & & \\
\hline & -5.2 & & \\
\hline & -2. & & \\
\hline & -2. & & -1 . \\
\hline & -2.4 & -4.8 & -2.1 \\
\hline & -1.0 & -4.6 & 1.5 \\
\hline & -5.4 & 0.4 & -0.5 \\
\hline & -0.50 & -5.5 & 2000 \\
\hline & & & 9000 \\
\hline & & 7000 & 107000 \\
\hline & & & \\
\hline & & & \\
\hline & & & \\
\hline & 000 & 3.2 & -0.2 \\
\hline & 000 & -3.828671000 & 04000 \\
\hline
\end{tabular}




\begin{tabular}{|c|c|c|c|}
\hline & & & \\
\hline & 768099000 & 92413000 & 0.38288900 \\
\hline & 6.255568000 & -1.770192000 & -1.2598880 \\
\hline & 7.173766000 & -1.242450000 & -1.537967000 \\
\hline & -0.535794000 & -4.608497000 & 2.950500000 \\
\hline & 548599000 & 4.394983000 & 2.97260200 \\
\hline & 5116000 & 33846000 & \\
\hline & 1000 & -5.577669000 & 00 \\
\hline & 607000 & 07000 & 900 \\
\hline & 0000 & 7000 & 1000 \\
\hline & 41000 & -3.692 & -1.98790200 \\
\hline & 26000 & -6.62( & 17143070 \\
\hline H & -0.8 & 0000 & 49000 \\
\hline I & & & \\
\hline $\mathrm{H}$ & 0.6 & -6.7 & \\
\hline & -4.570631000 & & \\
\hline I & -3.73 & 6000 & 300 \\
\hline & -4.92 & -3.79 & \\
\hline I & -4.82 & -4.87 & -1.37383200 \\
\hline & -3.91 & $3.7^{\prime}$ & -1.730721000 \\
\hline I & 07000 & 4.39 & -1.001053000 \\
\hline & -3.1 & $3.27^{\prime}$ & -2.3 \\
\hline & -4.50 & 4.469394000 & -2.355286000 \\
\hline & -7.96 & 2.77 & 1.172 \\
\hline & -7.77 & 3.76 & \\
\hline & -8.8 & 2.8 & \\
\hline & -8.2 & $2.0^{\circ}-x-1$ & \\
\hline & 1.32 & -0.5 & -0.6 \\
\hline & -3.00 & -0.0 & 1.1 \\
\hline & -4.0 & 0.0 & 1.6 \\
\hline & 1.7 & 1.6 & \\
\hline & 2.3 & 2.3 & \\
\hline & 0.4 & & \\
\hline C & 0.0 & 2.4 & \\
\hline & -0.5 & 0.6 & \\
\hline H & & & \\
\hline $\mathrm{C}$ & -1.2 & 2.4 & \\
\hline $\mathrm{C}$ & -1.5 & 0 & \\
\hline c & & & \\
\hline $\mathrm{C}$ & -2.2 & 00 & 000 \\
\hline $\mathrm{H}$ & -3.2 & 8000 & 3.1 \\
\hline $\mathrm{O}$ & -0.3 & -0.2 & 0.1 \\
\hline C & 0.74 & -2.2 & 03000 \\
\hline $\mathrm{C}$ & 2.20 & -2.3 & 69000 \\
\hline & 18000 & -2.77 & -0.602973000 \\
\hline & 262000 & 70000 & -2.319492000 \\
\hline C & 03000 & 72000 & -3.478610000 \\
\hline $\mathrm{C}$ & 0.6 & 00 & -2 \\
\hline $\mathrm{C}$ & 2000 & -2.8 & -2 \\
\hline I & 2.73 & -1.3 & 0000 \\
\hline & 2.64 & -3.0 & 2000 \\
\hline & 3.83 & -2.7 & 33000 \\
\hline & 2.4 & -3.8 & -3 \\
\hline & 000 & -2.1 & -3 \\
\hline & & & -1.9 \\
\hline & & & -2. \\
\hline & & -0.08 & -4.29132300 \\
\hline & 2.64 & 1.21 & -3.293833000 \\
\hline 11 & -2.62 & 3.396811000 & 4.67624400 \\
\hline $\mathrm{H}$ & -0.9 & 3.20 & 5.302706000 \\
\hline $\mathrm{H}$ & -1.327444000 & 0000 & 4.112919000 \\
\hline $\mathrm{H}$ & 4.603646000 & -4.526 & 2.160777000 \\
\hline $\mathrm{H}$ & 79000 & 08000 & 349000 \\
\hline $\mathrm{H}$ & 04000 & 6.863887000 & -0.738151000 \\
\hline $\mathrm{H}$ & -6.101939000 & 4.278222000 & -0.16523000 \\
\hline 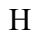 & -1.941843000 & -1.204417000 & -0.07137400 \\
\hline
\end{tabular}

Me-H+ ${ }^{+}$TS-2 $2_{\text {decoorAr }}$

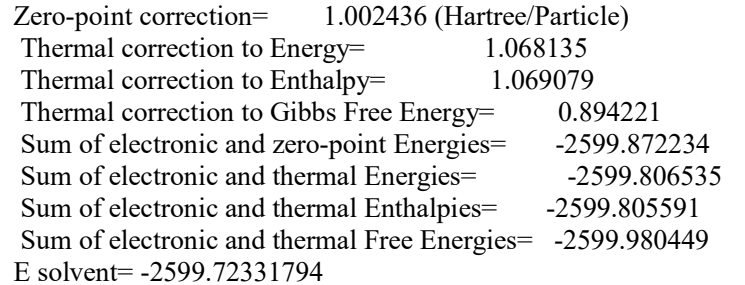

E solvent $=-2599.72331794$

\begin{tabular}{|c|c|c|}
\hline & & \\
\hline ( & 3000 & لخ \\
\hline 000 & 000 & 000 \\
\hline 4.983180000 & .877510000 & 0000 \\
\hline 4.405443000 & 1.663703000 & 4000 \\
\hline 6.542799000 & 2.415662000 & 1.587779000 \\
\hline 2.267016000 & -3.177113000 & -0.730316000 \\
\hline 6.090049000 & 000 & 000 \\
\hline 3.796959000 & -1.209683000 & 32000 \\
\hline 2000 & & \\
\hline & & \\
\hline & & \\
\hline & & \\
\hline & & \\
\hline & & \\
\hline & & \\
\hline & & \\
\hline & & \\
\hline & & \\
\hline & & \\
\hline 7.2 & & \\
\hline & & \\
\hline & & \\
\hline & & \\
\hline & & \\
\hline & & \\
\hline & & \\
\hline & & \\
\hline & & \\
\hline & & \\
\hline 00 & -4.1 & \\
\hline 2.5 & & 000 \\
\hline & & \\
\hline & & \\
\hline & & \\
\hline & & \\
\hline & & \\
\hline & & \\
\hline & & \\
\hline & & \\
\hline & & \\
\hline & & \\
\hline & & \\
\hline & & -0 . \\
\hline & & \\
\hline & & \\
\hline & & \\
\hline & & \\
\hline & 000 & 7000 \\
\hline 000 & 2000 & 74000 \\
\hline 000 & 000 & 000 \\
\hline 000 & -2.2 & 000 \\
\hline 4000 & -0.35 & 1000 \\
\hline 2000 & 2000 & 5000 \\
\hline & 8000 & -0.15 \\
\hline-2 & 5.545113000 & -1.77759400 \\
\hline & & $0<6500700$ \\
\hline & & \\
\hline-3.823436000 & 608419000 & .053810 \\
\hline
\end{tabular}




\begin{tabular}{|c|c|c|c|}
\hline & & & \\
\hline & -6.434586000 & 641679000 & 8000 \\
\hline & 1295000 & -5.322207000 & 500 \\
\hline & 34000 & .642321000 & \\
\hline & 177000 & 5031000 & -0.02708200 \\
\hline & 3710000 & -6.028870000 & 89840 \\
\hline & & & \\
\hline & 39000 & & \\
\hline & 875000 & & \\
\hline & 377000 & & \\
\hline & 345000 & & \\
\hline & 108000 & -2.7 & 4 \\
\hline & 04000 & -1.0 & 4 \\
\hline & 16000 & & 100( \\
\hline & -2.313637000 & & -2.16965000 \\
\hline & -6.252160000 & -0.412653000 & -0.31225800 \\
\hline & 287000 & -1.2 & 28000 \\
\hline & 52000 & & \\
\hline & -0.6 & & \\
\hline & & & \\
\hline & 0.1 & & -2 \\
\hline & -4. & & \\
\hline & & & \\
\hline & 0000 & & \\
\hline & -5. & & \\
\hline & & & \\
\hline & 0 & & \\
\hline & -2 & & \\
\hline & & & \\
\hline & & & \\
\hline & & & \\
\hline & 1.7 & & \\
\hline & & & \\
\hline & & & \\
\hline & 7000 & & \\
\hline & 4000 & & \\
\hline & & & \\
\hline & -0.5 & & 2. \\
\hline & & & 2. \\
\hline U & -0 . & & 0000 \\
\hline & -0.7 & & 100000 \\
\hline & & & 13000 \\
\hline & & & \\
\hline & & & \\
\hline & & & \\
\hline & -1 & & - \\
\hline & & & \\
\hline & & & \\
\hline & & & \\
\hline & & & \\
\hline & & & \\
\hline & & & \\
\hline & & & \\
\hline & -0.4 & & -0.2 \\
\hline & & & \\
\hline $\mathrm{H}$ & & & 1.00 \\
\hline $\mathrm{H}$ & & & -2.043970000 \\
\hline $\mathrm{C}$ & 2.127526000 & -0.50 & -3.257403000 \\
\hline 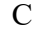 & 8000 & & 8000 \\
\hline & 56000 & -0.8 & 5000 \\
\hline & 691000 & -1.109 & 0000 \\
\hline & 2.120016000 & 76000 & \\
\hline & 0.544805000 & 0.883450000 & -2.897039000 \\
\hline & -2.163420000 & 0.053851000 & 0.20819500 \\
\hline
\end{tabular}

\section{Me-H+ $1-P r-C$}

Zero-point correction= Thermal correction to Energy=
0.951256 (Hartree/Particle) 1.013236

Thermal correction to Enthalpy=

1.014181

Thermal correction to Gibbs Free Energy= 0.846926 Sum of electronic and zero-point Energies $=\quad-2521.348509$ Sum of electronic and thermal Energies $=\quad-2521.286529$ Sum of electronic and thermal Enthalpies $=\quad-2521.285585$ Sum of electronic and thermal Free Energies $=-2521.452840$ E solvent $=-2521.17076158$

N

c

C

c

(a)

th

H

(c)

\section{(a)}

H

(n)

C

H

C

C

\subsection{0}

3.509042000

5.325433000

4.678602000

3.910872000

6.687494000

2.157123000

6.015475000

3.729348000

5.019854000

5.013443000

1.472415000

1.922431000

0.187248000

6.330507000

6.835728000

0.490902000

4.581714000

4.409928000

0.792300000

5.860977000

6.694381000

1.750433000

2.253462000

6.061283000

7.043887000

$-2.683069000$

$-4.439118000$

$-4.466812000$

$-3.985222000$

$-3.851922000$

$-4.430613000$

$-3.579451000$

$-3.951926000$

$-4.008509000$

$-3.732583000$

$-4.851078000$

$-4.032000000$

$-4.983200000$

$-2.058929000$

$-4.680964000$

$-5.043509000$

$-3.280469000$

$-5.748141000$

$-6.085441000$

$-1.586708000$

$-6.586832000$

$-7.594346000$

$-2.353122000$

$-2.005429000$

$-6.138211000$

$-6.801480000$

0.841768000

$-2.099994000$

$-2.730626000$

2.663540000

3.657607000

1.563690000

1.885938000

0.199981000

2.930360000
$-0.737984000$ $-2.665022000$ 0.697270000 1.733444000 1.480860000 2.370135000 $-3.305485000$ 3.380445000 $-1.350414000$ 3.080780000 $-0.739758000$ $-3.439288000$ $-2.996438000$ $-5.307997000$ 1.027201000 0.224041000 $-4.467702000$ $-3.397410000$ $-4.423084000$ $-5.159710000$ $-2.831815000$ $-3.406114000$ $-4.162946000$ $-4.059676000$ $-1.515772000$ $-1.044976000$ 0.964087000 2.176500000 $-1.296729000$

$-1.156419000$ $-0.155024000$ $-3.739416000$ 3.373868000 $-3.563067000$ 1.023002000 $-2.282912000$ $-0.108318000$ 4.649780000 4.737899000 5.667165000 $-2.596278000$ $-2.713061000$ 5.809157000 2.170352000 3.047162000 4.408589000 1.057545000 1.072592000 3.266451000 2.281308000

$-0.071101000$ $-0.930069000$ $-1.580540000$ 1.927385000 2.787105000 0.242960000 0.541334000 1.044890000 2.102046000 0.888178000 2.186682000
0.217157000 $-0.832202000$ $-0.180851000$ $-0.889910000$ $-1.632108000$ 0.993316000 $-0.747982000$ 0.280022000 $-0.337558000$ $-0.673154000$ $-0.472064000$ 0.517243000 1.415242000 $-0.360646000$ 0.751634000 1.302914000 0.708353000 $-1.374215000$ $-1.716773000$ $-1.645564000$ $-1.448428000$ $-1.866365000$ $-1.823184000$ $-2.792016000$ $-1.014378000$ $-1.127268000$ 0.374104000 $-0.885326000$ 0.823844000 2.144019000 2.569475000 1.090328000 $-1.145864000$ 2.405337000 $-0.171400000$ 2.950788000 0.005789000 $-0.747695000$ $-0.207530000$ $-1.709787000$ 0.310550000 $-0.718016000$ $-1.024089000$

$-1.411156000$ $-1.973559000$ $-2.134069000$ $-1.267827000$ $-1.696705000$ $-1.837003000$ $-2.172208000$ $-0.572611000$ $-0.427223000$ $-0.302780000$ 1.077457000 1.331061000 1.084643000 1.446002000 1.571076000 2.449282000 1.114500000 2.778977000 


\begin{tabular}{|c|c|c|c|}
\hline & & & \\
\hline & 748278000 & 14224000 & \\
\hline & 68876000 & 65448000 & 0700 \\
\hline & 2000 & 849000 & \\
\hline & & 708340000 & \\
\hline & 218397000 & -0.094910000 & \\
\hline C & -0.792158000 & 1000 & 1.10 \\
\hline C & 612000 & 3000 & 24375 \\
\hline 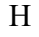 & -0.720776000 & 5000 & \\
\hline $\mathrm{I}$ & -1.619080000 & 9000 & -0 \\
\hline C & -2.171589000 & 8000 & -3.2 \\
\hline & -0.884200000 & 9000 & -2 \\
\hline & -0.008920000 & 5000 & -3.0 \\
\hline & -2.221231000 & -1.030709000 & $-4.1664950 c$ \\
\hline & -2.192166000 & $-2.707 c$ & -3.54996400 \\
\hline & -3.079401000 & -1.4 & \\
\hline & 0.56 & & \\
\hline & 1.60 & & \\
\hline & 2.27 & & \\
\hline & & & \\
\hline & -3.760643000 & -4.4 & \\
\hline & 6.28 & 4.4 & \\
\hline & -1.4 & & \\
\hline & -2.0 & & \\
\hline & 7.7 & & \\
\hline & & & \\
\hline & 7.8 & & \\
\hline & 8.7 & & \\
\hline & & & -1.4 \\
\hline & & & \\
\hline & & & -1.8 \\
\hline & 4.1 & & -0.8 \\
\hline 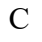 & 0.40 & -6.0 & -2.7 \\
\hline & & & \\
\hline & & & \\
\hline & 000 & & $-3 .($ \\
\hline & -0.1 & & 2.0 \\
\hline $\mathrm{H}$ & -1.00 & & 2.1 \\
\hline & -0.55 & & 2.18757600 \\
\hline 11 & & & 2.88 \\
\hline 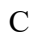 & 351000 & & 0.54267100 \\
\hline$\Pi$ & -3.9 & & \\
\hline $\mathrm{H}$ & 527000 & 000 & \\
\hline H & -4 & & \\
\hline C & & & \\
\hline $\mathrm{H}$ & & & \\
\hline & -2.6 & & \\
\hline & -2.6 & & 4.5 \\
\hline & -0.2 & & 0 \\
\hline & & & -2.52267500 \\
\hline & 0.10 & & -2.90042900 \\
\hline & & & -3.98121000 \\
\hline & & 7.17 & -0.61239800 \\
\hline & & 7.665 & -1.44279900 \\
\hline & & & \\
\hline & -2.9559330 & 7.849270000 & $-0.3316450 c$ \\
\hline
\end{tabular}

\section{Me-H+ TS-2 ${ }_{\text {openAr }}$ \\ Zero-point correction $=$ $\begin{array}{ll}\text { Thermal correction to Energy= } & 1.011032\end{array}$ Thermal correction to Enthalpy= $\quad 1.011976$ Thermal correction to Gibbs Free Energy $=0.847497$ Sum of electronic and zero-point Energies $=\quad-2521.342087$ Sum of electronic and thermal Energies $=\quad-2521.280879$ Sum of electronic and thermal Enthalpies $=\quad-2521.279935$ Sum of electronic and thermal Free Energies $=-2521.444414$ E solvent $=-2521.16759667$}

$\mathrm{N} \quad-2.571499000 \quad 0.180551000 \quad-0.583334000$
-3.724315000
-5.295361000
-6.065127000

$-6.452688000$

$-5.049586000$

$-2.416261000$

$-5.811231000$

$-3.817311000$

$-6.333604000$

$-5.088393000$

$-1.264618000$

$-1.335298000$

$-0.089265000$

$-4.792458000$

$-4.206957000$

$-0.104876000$

$-4.906901000$

$-4.834556000$

$-4.542833000$

$-4.183559000$

$-3.715515000$

$-5.345458000$

$-1.206751000$

$-6.156122000$

$-7.065489000$

$-7.183281000$

$-6.895956000$

$-8.253323000$

$-7.100721000$

$-2.350219000$

$-3.222661000$

$-6.240379000$

$-7.218492000$

$-1.165449000$

$-1.066023000$

$-2.081245000$

$-0.300772000$

1.074806000

0.795901000

1.912361000

1.439713000

2.866344000

4.469393000

4.993190000

5.776441000

6.502519000

3.898584000

3.471462000

4.685166000

4.201691000

5.634946000

5.213546000

3.314733000

3.854721000

1.782823000

4.057969000

3.462192000

2.476221000

5.770233000

5.989018000

2.923020000

3.446758000

2.168285000

2.392226000

1.915139000

6.780495000

7.787135000

6.497876000
$-1.298844000$

1.159479000

2.326798000

2.559700000

1.725313000

$-1.902536000$

2.891425000

$-0.275644000$

3.204057000

0.214843000

$-1.116324000$

$-0.023316000$

$-3.090964000$

0.871276000

$-0.042796000$

$-1.708144000$

$-1.756718000$

$-2.508899000$

1.388985000

2.289419000

0.658880000

0.948490000

$-3.907163000$

$-1.241149000$

$-1.603317000$

4.441884000

5.252436000

4.224100000

4.825652000

$-3.302422000$

$-3.922438000$

$-0.280087000$

0.082456000

$-5.396201000$

$-5.595268000$

$-5.901433000$

$-5.877118000$

$-0.836025000$

$-0.092308000$

$-1.433012000$

$-0.273121000$

0.293740000

$-1.475547000$

1.797796000

2.916996000

2.826195000

3.138204000

$-2.224288000$

4.241444000

$-0.221236000$

4.148592000

0.496435000

$-3.615616000$

$-4.105749000$

$-3.723731000$

1.921370000

1.049794000

$-4.379981000$

$-2.010399000$

$-2.966399000$

3.257423000

3.194293000

2.452426000

4.225905000

$-2.338994000$

$-1.315914000$

$-1.741393000$

5.337932000
$-2.113502000$

0.443233000

0.256363000

$-0.743621000$

2.818543000

$-2.523649000$

2.595978000

$-1.122086000$

1.326944000

$-0.699723000$

$-2.824611000$

$-2.802243000$

$-3.616707000$

1.731098000

1.889376000

$-3.395470000$

$-2.730094000$

$-3.522464000$

4.207155000

4.736683000

4.172398000

4.829146000

$-3.308874000$

$-2.364119000$

$-2.855106000$

1.119374000

1.811588000

1.300957000

0.087500000

$-2.766996000$

$-2.527650000$

$-1.349709000$

$-1.015766000$

$-3.585620000$

$-4.669236000$

$-3.235237000$

$-3.092315000$

$-3.768737000$

$-4.538960000$

$-4.167039000$

$-2.889137000$

0.637069000

1.175211000

$-0.850143000$

$-0.495605000$

0.322177000

$-2.589587000$

1.994293000

$-2.201304000$

0.559912000

$-1.165851000$

$-0.141679000$

1.799850000

0.980376000

3.669052000

$-1.900261000$

$-2.198535000$

2.631650000

1.053409000

1.540181000

$-3.743437000$

$-4.716376000$

$-3.722973000$

$-3.725885000$

3.891950000

0.380012000

0.310620000

$-0.795395000$ 


\begin{tabular}{|c|c|c|c|}
\hline & & & \\
\hline & 5011000 & 8177000 & 0 \\
\hline & 68843000 & 3000 & 00 \\
\hline & .756455000 & 1.597965000 & 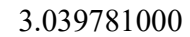 \\
\hline & .895508000 & -0.526642000 & 516200 \\
\hline & 503138000 & 35000 & -0.199823000 \\
\hline & 91000 & 000 & 000 \\
\hline & 202321000 & 5000 & .000 \\
\hline & & & \\
\hline & 1.005 & & \\
\hline & 0.236 & -2 & 5.2 \\
\hline & 00 & 00 & \\
\hline & 1.31 & -6 & 2. \\
\hline & 2.58 & & \\
\hline & 3.02 & & \\
\hline & -1.1 & & \\
\hline & 00 & & \\
\hline & 00 & & \\
\hline & 00 & & \\
\hline & & & \\
\hline & -1.17 & & \\
\hline & & & \\
\hline & 00 & & \\
\hline & 2.18 & & 0 . \\
\hline & 0115 & 3000 & 0.9 \\
\hline & 000 & & \\
\hline & -0.254 & & 1.2 \\
\hline & 00 & & \\
\hline & 99 & & \\
\hline $\mathrm{O}$ & 00 & 000 & 4000 \\
\hline & -0.15 & 0000 & 600 \\
\hline & -1.4 & & \\
\hline & & -3 . & -0 \\
\hline & 0 & -2 & \\
\hline & & & \\
\hline & & & 0.2 \\
\hline & -1 . & & $-0 .(1+y-x$ \\
\hline & -2.6 & -3.6 & 2.3 \\
\hline 1 & & & 2.318822000 \\
\hline 1 & & & \\
\hline $\mathrm{H}$ & 2.05 & & 6000 \\
\hline $\mathrm{H}$ & $-6.008<>0$ & 5000 & 05000 \\
\hline $\mathrm{H}$ & 4.559 & 1000 & 70000 \\
\hline $\mathrm{H}$ & 1.130 & 80000 & 471000 \\
\hline 1 & 0.803 & 095000 & -4.0 \\
\hline & 0.723 & 1000 & 1.4 \\
\hline & 1000 & 6.210339000 & 0.41307500 \\
\hline & -0.910175000 & 5.813489000 & 2.13359900 \\
\hline
\end{tabular}

\section{Me-H+ $1-\boldsymbol{\beta}-\mathbf{C}$}

Zero-point correction $=$ Thermal correction to Energy= Thermal correction to Enthalpy= 0.950183 (Hartree/Particle) $\quad 1.013264$ Thermal correction to Gibbs Free Energy $=\quad 0.843957$ Sum of electronic and zero-point Energies $=\quad-2521.345364$ Sum of electronic and thermal Energies $=\quad-2521.283227$ Sum of electronic and thermal Enthalpies $=\quad-2521.282283$ Sum of electronic and thermal Free Energies $=-2521.451590$ E solvent $=-2521.17212432$

$\begin{array}{ll}\mathrm{N} & 2.402997000 \\ \mathrm{C} & 4.754267000 \\ \mathrm{C} & 3.154875000 \\ \mathrm{C} & 3.412053000 \\ \mathrm{H} & 4.292195000 \\ \mathrm{C} & 1.149700000 \\ \mathrm{C} & 4.453254000 \\ \mathrm{C} & 1.429458000 \\ \mathrm{C} & 3.778780000\end{array}$

0.913580000 0.171251000 3.515125000 4.828600000 5.017645000 4.327870000 $-1.132890000$ 5.626869000 1.183010000
0.289933000 0.169421000 $-0.962884000$ $-0.516758000$ 0.111064000 $-2.121356000$ 0.835621000 $-1.649814000$ $-0.057327000$
2.556729000 4.135084000 3.791067000 3.440305000 4.107404000 2.014624000 1.810015000 3.623680000 6.088515000 6.845892000 $-0.043402000$ $-0.314404000$ 0.167450000 $-0.928995000$ 4.771075000 6.456448000 7.498103000 2.857486000 1.934200000 3.490972000 3.399751000 4.937119000 5.456761000 5.487334000 5.760512000 5.317510000 6.234741000 5.577475000 4.591539000 2.980086000 2.545980000 2.178846000 3.722987000 $-2.827152000$ $-5.179281000$ $-2.450783000$ $-1.915584000$ $-2.397779000$ $-0.667814000$ $-5.445665000$ $-0.159404000$ $-3.930314000$ $-0.772408000$ $-3.725809000$ $-6.481881000$ $-7.046057000$ $-6.050944000$ $-1.811971000$ $-2.239547000$ $-6.797986000$ $-6.226457000$ $-7.185302000$ $-0.001556000$ 1.051990000 $-0.519015000$ 0.002454000 $-5.020855000$ $-6.044728000$ $-6.871551000$ $-0.231850000$ $-0.690716000$ $-0.447244000$ 0.859833000 $-4.721275000$ $-3.929171000$ $-4.802849000$ $-4.659090000$ $-4.275751000$
5.897267000 2.427945000 $-1.198436000$ $-0.276512000$ $-3.599637000$ 3.279290000 2.262767000 $-2.423633000$ 0.429180000 $-0.336829000$ 4.076667000 3.006431000 4.388556000 4.648218000 $-3.573332000$ 1.649442000 1.831241000 7.306686000 7.898713000 7.843802000 7.309137000 $-2.333524000$ $-2.293823000$ 2.636945000 3.585949000 $-4.848762000$ $-5.179315000$ $-4.713019000$ $-5.678587000$ $-2.462717000$ $-3.453796000$ $-1.708686000$ $-2.251294000$ $-0.927755000$ $-0.968669000$ $-3.720738000$ $-4.482350000$ $-4.441596000$ $-4.559785000$ 0.472941000 $-5.305112000$ $-1.624537000$ $-5.284975000$ $-2.975266000$ 0.809186000 0.007473000 3.162236000 $-3.767355000$ $-3.218095000$ 2.154812000 $-1.711655000$ $-1.210137000$ $-4.629688000$ $-4.298009000$ $-3.994120000$ $-5.664201000$ 2.858414000 $-3.040979000$ $-3.594283000$ $-6.127088000$ $-7.134419000$ $-5.671763000$ $-6.268502000$ 1.506161000

1.252095000

$-3.657455000$

$-4.703226000$ 3.966355000
$-0.851823000$ $-0.651454000$ 2.079820000 2.556171000 2.151011000 $-1.761422000$ $-2.121315000$ 2.755550000 $-0.214698000$ $-0.016321000$ $-3.022242000$ $-3.048323000$ $-4.063154000$ $-2.690358000$ 0.906371000 $-0.793330000$ $-1.078465000$ $-0.382085000$ $-0.256466000$ $-1.114040000$ 0.579624000 0.263440000 $-0.702470000$ $-1.003263000$ $-1.476947000$ 0.295394000 0.818953000 $-0.768535000$ 0.367443000 4.128029000 4.346149000 4.224202000 4.921033000 0.180842000 $-0.615774000$ $-0.598239000$ $-1.660962000$ $-2.645392000$ 0.868680000 $-0.306396000$ $-0.215293000$ $-0.410004000$ $-1.483464000$ $-0.811129000$ 0.590553000 1.083681000 0.226457000 0.662312000 1.509197000 0.866769000 $-1.197362000$ $-1.364662000$ 2.227707000 2.172695000 2.966261000 2.618514000 $-0.686588000$ $-1.599557000$ $-2.056999000$ $-2.621852000$ $-2.621634000$ $-3.604042000$ $-2.539785000$ $-0.939278000$ $-1.654722000$ $-1.417781000$ $-1.707974000$ $-1.403334000$ 


$\begin{array}{lrrr}\mathrm{H} & -4.116897000 & 4.838445000 & -0.744725000 \\ \mathrm{H} & -3.290922000 & 3.625503000 & -1.766985000 \\ \mathrm{H} & -4.845797000 & 4.323850000 & -2.282144000 \\ \mathrm{C} & -7.930008000 & 2.508116000 & 1.810100000 \\ \mathrm{H} & -7.722199000 & 3.441144000 & 2.362528000 \\ \mathrm{H} & -8.874219000 & 2.664566000 & 1.254074000 \\ \mathrm{H} & -8.114533000 & 1.706395000 & 2.546010000 \\ \mathrm{Ni} & 1.401985000 & -0.408385000 & -0.686652000 \\ \mathrm{C} & -2.909715000 & -0.028546000 & 1.157227000 \\ \mathrm{H} & -3.901335000 & 0.111588000 & 1.599299000 \\ \mathrm{C} & 1.852781000 & 1.692875000 & 1.189574000 \\ \mathrm{H} & 2.478346000 & 2.470340000 & 1.658289000 \\ \mathrm{C} & 0.483198000 & 1.619888000 & 1.649280000 \\ \mathrm{C} & 0.105177000 & 2.495091000 & 2.690016000 \\ \mathrm{C} & -0.491088000 & 0.704688000 & 1.103104000 \\ \mathrm{H} & 0.870867000 & 3.178361000 & 3.082249000 \\ \mathrm{C} & -1.190218000 & 2.539412000 & 3.249302000 \\ \mathrm{C} & -1.834321000 & 0.762224000 & 1.655243000 \\ \mathrm{C} & -1.523520000 & 3.499340000 & 4.372093000 \\ \mathrm{C} & -2.138444000 & 1.667789000 & 2.711334000 \\ \mathrm{H} & -3.165481000 & 1.681419000 & 3.098519000 \\ \mathrm{O} & -0.248545000 & -0.155812000 & 0.147417000 \\ \mathrm{C} & 0.776669000 & -1.731916000 & -1.924619000 \\ \mathrm{C} & 2.200752000 & -1.611444000 & -2.322085000 \\ \mathrm{H} & 0.459085000 & -2.643103000 & -1.398064000 \\ \mathrm{H} & 0.025361000 & -1.321968000 & -2.617393000 \\ \mathrm{C} & 2.512380000 & -1.055853000 & -3.721762000 \\ \mathrm{H} & 2.757109000 & -0.842785000 & -1.576786000 \\ \mathrm{H} & 2.793266000 & -2.510502000 & -2.073711000 \\ \mathrm{H} & 3.577701000 & -0.791150000 & -3.831172000 \\ \mathrm{H} & 2.268351000 & -1.819980000 & -4.479949000 \\ \mathrm{H} & 1.908766000 & -0.157093000 & -3.935022000 \\ \mathrm{H} & -2.589321000 & 3.438982000 & 4.648762000 \\ \mathrm{H} & -0.929474000 & 3.281661000 & 5.278917000 \\ \mathrm{H} & -1.308700000 & 4.545111000 & 4.085803000 \\ \mathrm{H} & 3.982915000 & -4.559069000 & 2.670967000 \\ \mathrm{H} & 0.731523000 & -5.928679000 & -0.063110000 \\ \mathrm{H} & 0.753398000 & 6.450089000 & -1.916912000 \\ \mathrm{H} & -6.283977000 & 4.214502000 & 0.436722000 \\ \mathrm{H} & -1.864845000 & -1.094921000 & -0.170911000\end{array}$

\begin{tabular}{|c|c|c|c|}
\hline \multicolumn{4}{|c|}{${ }^{\mathrm{Me}-\mathrm{H}+}$ TS-1 $\mathbf{B H E}_{\text {BHC }}$} \\
\hline \multicolumn{4}{|c|}{0.947413 (Hartree/Particle) } \\
\hline \multicolumn{4}{|c|}{ Thermal correction to Energy= } \\
\hline \multicolumn{4}{|c|}{ Thermal correction to Enthalpy= } \\
\hline \multicolumn{2}{|c|}{ Thermal correction to Gibbs Free Energy= } & ibbs Free Energy= & 0.842999 \\
\hline \multicolumn{4}{|c|}{ Sum of electronic and zero-point Energies= } \\
\hline \multicolumn{4}{|c|}{ Sum of electronic and thermal Energies $=$} \\
\hline \multicolumn{4}{|c|}{ Sum of electronic and thermal Enthalpies $=$} \\
\hline & f electronic and & nermal Free Energi & $e s=-2521.434435$ \\
\hline \multicolumn{4}{|c|}{ E solvent $=-2521.15361382$} \\
\hline $\mathrm{N}$ & 2.510608000 & 0.699151000 & 0.164945000 \\
\hline $\mathrm{C}$ & 4.815416000 & -0.185403000 & 0.057322000 \\
\hline $\mathrm{C}$ & 3.482536000 & 3.329289000 & -0.896623000 \\
\hline $\mathrm{C}$ & 2.307076000 & 3.251557000 & -1.673206000 \\
\hline $\mathrm{H}$ & 1.998139000 & 2.282802000 & -2.084226000 \\
\hline $\mathrm{C}$ & 3.117294000 & 5.747264000 & -0.629729000 \\
\hline $\mathrm{C}$ & 4.416057000 & -1.481924000 & 0.684373000 \\
\hline $\mathrm{C}$ & 1.949651000 & 5.633095000 & -1.408616000 \\
\hline $\mathrm{C}$ & 3.913658000 & 0.894401000 & -0.144725000 \\
\hline $\mathrm{C}$ & 1.537609000 & 4.397735000 & -1.946192000 \\
\hline $\mathrm{C}$ & 4.364403000 & 2.142507000 & -0.660858000 \\
\hline $\mathrm{C}$ & 3.818372000 & -1.525153000 & 1.962426000 \\
\hline $\mathrm{H}$ & 3.589511000 & -0.589181000 & 2.485163000 \\
\hline $\mathrm{C}$ & 3.894690000 & -3.945748000 & 1.938573000 \\
\hline $\mathrm{C}$ & 3.872901000 & 4.585325000 & -0.381943000 \\
\hline $\mathrm{H}$ & 4.779606000 & 4.653384000 & 0.232487000 \\
\hline $\mathrm{C}$ & 3.567992000 & -2.750081000 & 2.609570000 \\
\hline
\end{tabular}

C

H.

5.206616000

5.732988000

6.079103000

4.871382000

5.909291000

4.818184000

4.217975000

3.007031000

2.450569000

2.328072000

3.817852000

$-2.868121000$

$-5.234362000$

$-2.694969000$

$-2.034123000$

$-2.408018000$

$-1.138424000$

$-5.398212000$

$-0.501696000$

$-4.027248000$

$-0.939068000$

$-3.921847000$

$-6.388152000$

$-6.990711000$

$-5.815616000$

$-2.231977000$

$-2.731942000$

$-6.610050000$

$-6.341338000$

$-7.268552000$

$-0.674390000$

$-1.202562000$

$-0.870842000$

0.405716000

$-4.829938000$

$-6.257394000$

$-7.129417000$

$-0.250552000$

0.828649000

$-0.685089000$

$-0.338628000$

$-4.623753000$

$-3.867089000$

$-5.055408000$

$-4.987987000$

$-4.034860000$

$-3.826041000$

$-3.071748000$

$-4.593412000$

$-7.693450000$

$-7.879501000$

$-7.432113000$

$\mathrm{H} \quad-8.651386000$

Ni 1.460678000
$-0.009702000$ $-0.836575000$ 7.092018000

7.671341000 6.980274000

7.706519000

$-3.939714000$

1.204042000

1.322965000

4.315059000

4.577227000

5.015428000

3.296692000

$-2.699081000$

$-2.672191000$

2.267566000

3.216737000

$-5.237080000$

$-5.527523000$

$-5.150425000$

$-6.070810000$

$-2.777480000$

$-3.709739000$

$-1.926540000$

$-2.717238000$

$-0.772257000$

$-0.675926000$

$-3.603511000$

$-3.661829000$

$-3.066806000$

$-5.292315000$

0.786580000

$-5.322275000$

$-1.408609000$

$-4.521510000$

$-2.779343000$

1.209745000

0.457853000

3.521013000

$-4.422286000$

$-4.373973000$

2.579095000

$-1.360952000$

$-0.800673000$

$-6.190948000$

$-7.163435000$

$-5.739126000$

$-6.407290000$

3.130250000

$-2.708814000$

$-3.216514000$

$-4.604405000$

$-4.381323000$

$-3.893203000$

$-5.618638000$

1.756049000

1.434947000

$-3.402381000$

$-4.462244000$

4.170729000

5.057724000

3.767014000

4.523789000

3.025439000

2.271928000

3.978299000

3.186269000

$-0.553756000$
$-0.303209000$

$-0.131141000$

$-0.087531000$

$-0.856799000$

0.778062000

0.227291000

0.659307000

$-0.829214000$

$-1.103159000$

$-2.827287000$

$-3.875868000$

$-2.492266000$

$-2.834342000$

0.045873000

$-0.948650000$

$-0.993820000$

$-1.416651000$

$-0.024199000$

0.228833000

$-1.123664000$

0.287047000

4.018153000

4.217968000

4.203903000

4.769513000

0.222674000

$-0.523588000$

$-0.492500000$

0.756292000

1.597673000

$-1.372492000$

$-0.241859000$

$-0.116435000$

$-0.328236000$

0.959054000

$-0.698914000$

0.670273000

1.195526000

0.239718000

$-1.546901000$

$-2.522165000$

0.921112000

$-1.064076000$

$-1.222961000$

$-2.501513000$

$-2.476702000$

$-3.489312000$

$-2.430900000$

$-0.689277000$

$-1.436117000$

$-1.861356000$

2.305999000

2.215269000

3.028872000

2.738329000

$-0.915164000$

$-1.642061000$

$-1.264769000$

$-1.530378000$

$-1.451912000$

$-0.828207000$

$-1.809496000$

$-2.339896000$

1.881947000

2.666919000

2.374139000

1.351185000

$-0.828946000$ 


$\begin{array}{rrrr}\mathrm{C} & -2.866031000 & 0.148968000 & 1.180844000 \\ \mathrm{H} & -3.833674000 & 0.367046000 & 1.644327000 \\ \mathrm{C} & 2.014840000 & 1.484259000 & 1.093905000 \\ \mathrm{H} & 2.712822000 & 2.183223000 & 1.582610000 \\ \mathrm{C} & 0.654140000 & 1.531152000 & 1.571953000 \\ \mathrm{C} & 0.377524000 & 2.430443000 & 2.624463000 \\ \mathrm{C} & -0.403055000 & 0.702227000 & 1.038244000 \\ \mathrm{H} & 1.207273000 & 3.043401000 & 3.002243000 \\ \mathrm{C} & -0.896633000 & 2.582807000 & 3.211469000 \\ \mathrm{C} & -1.720889000 & 0.862577000 & 1.635788000 \\ \mathrm{C} & -1.125389000 & 3.567114000 & 4.339285000 \\ \mathrm{C} & -1.924142000 & 1.790026000 & 2.696873000 \\ \mathrm{H} & -2.936164000 & 1.883880000 & 3.111985000 \\ \mathrm{O} & -0.231618000 & -0.150265000 & 0.068719000 \\ \mathrm{C} & 0.485666000 & -1.989486000 & -1.889458000 \\ \mathrm{C} & 1.282263000 & -1.295478000 & -2.809930000 \\ \mathrm{H} & 0.804501000 & -2.957375000 & -1.485706000 \\ \mathrm{H} & -0.580121000 & -1.754212000 & -1.800451000 \\ \mathrm{C} & 0.787751000 & -0.169038000 & -3.685605000 \\ \mathrm{H} & 2.208991000 & -1.772952000 & -3.154578000 \\ \mathrm{H} & 1.559953000 & 0.608271000 & -3.820508000 \\ \mathrm{H} & 0.557281000 & -0.568873000 & -4.693886000 \\ \mathrm{H} & -0.126168000 & 0.301052000 & -3.284954000 \\ \mathrm{H} & -2.178651000 & 3.565580000 & 4.666002000 \\ \mathrm{H} & -0.501170000 & 3.325120000 & 5.219076000 \\ \mathrm{H} & -0.869904000 & 4.598015000 & 4.032724000 \\ \mathrm{H} & 3.703676000 & -4.906819000 & 2.434796000 \\ \mathrm{H} & 0.351853000 & -5.996459000 & 0.033934000 \\ \mathrm{H} & 1.347946000 & 6.530409000 & -1.606016000 \\ \mathrm{H} & -5.974924000 & 4.590673000 & 0.429689000 \\ \mathrm{H} & 2.734170000 & -0.959465000 & -1.403313000 \\ \mathrm{H} & -1.923396000 & -1.006392000 & -0.140046000\end{array}$

\section{${ }^{\mathrm{Me}-\mathrm{H}+}$ 1-BHE-C}

Zero-point correction

$\begin{array}{lc}\text { Thermal correction to Energy= } & 1.010322 \\ \text { Thermal correction to Enthalpy= } & 1.011266 \\ \text { Thermal correction to Gibbs Free Energy= } & 0.842243 \\ \text { Sum of electronic and zero-point Energies }= & -2521.332498 \\ \text { Sum of electronic and thermal Energies }= & -2521.270054 \\ \text { Sum of electronic and thermal Enthalpies }= & -2521.269110 \\ \text { Sum of electronic and thermal Free Energies }= & -2521.438132 \\ \text { E solvent }=-2521.15806258 & \end{array}$

$\begin{array}{lrrr}\mathrm{N} & 2.567732000 & 0.710305000 & 0.142909000 \\ \mathrm{C} & 4.871248000 & -0.178535000 & 0.050708000 \\ \mathrm{C} & 3.526415000 & 3.310791000 & -0.954315000 \\ \mathrm{C} & 2.446083000 & 3.201788000 & -1.857100000 \\ \mathrm{H} & 2.237839000 & 2.233083000 & -2.328506000 \\ \mathrm{C} & 3.023502000 & 5.700016000 & -0.671783000 \\ \mathrm{C} & 4.457763000 & -1.484827000 & 0.647131000 \\ \mathrm{C} & 1.952698000 & 5.556565000 & -1.572651000 \\ \mathrm{C} & 3.972564000 & 0.901177000 & -0.159653000 \\ \mathrm{C} & 1.657194000 & 4.319225000 & -2.182302000 \\ \mathrm{C} & 4.426406000 & 2.148390000 & -0.670474000 \\ \mathrm{C} & 3.851863000 & -1.552503000 & 1.919570000 \\ \mathrm{H} & 3.631409000 & -0.627036000 & 2.464547000 \\ \mathrm{C} & 3.898686000 & -3.972510000 & 1.835812000 \\ \mathrm{C} & 3.804228000 & 4.564643000 & -0.372282000 \\ \mathrm{H} & 4.643453000 & 4.654959000 & 0.329484000 \\ \mathrm{C} & 3.582969000 & -2.790816000 & 2.534789000 \\ \mathrm{C} & 6.230129000 & 0.006049000 & -0.279767000 \\ \mathrm{H} & 6.926811000 & -0.820535000 & -0.102692000 \\ \mathrm{C} & 4.498854000 & -3.941504000 & 0.560019000 \\ \mathrm{C} & 6.695865000 & 1.227057000 & -0.783132000 \\ \mathrm{H} & 7.755651000 & 1.352685000 & -1.028936000 \\ \mathrm{C} & 4.774833000 & -2.689514000 & -0.020270000 \\ \mathrm{H} & 5.243060000 & -2.642391000 & -1.011388000 \\ \mathrm{C} & 5.800902000 & 2.286667000 & -0.967436000\end{array}$

\begin{tabular}{|c|c|c|c|}
\hline H & 6.150014000 & 000 & -1.378921000 \\
\hline $\mathrm{N}$ & -2.841354000 & -0.758497000 & 0.249584000 \\
\hline $\mathrm{C}$ & -5.208496000 & -0.597157000 & -0.482248000 \\
\hline $\mathrm{C}$ & -2.747092000 & -3.591078000 & -0.482929000 \\
\hline $\mathrm{C}$ & -2.072610000 & -3.670788000 & 0.757618000 \\
\hline $\mathrm{H}$ & -2.418934000 & -3.067612000 & 1.604901000 \\
\hline $\mathrm{C}$ & -1.251219000 & -5.322970000 & -1.384705000 \\
\hline $\mathrm{C}$ & -5.331095000 & 0.868679000 & -0.196351000 \\
\hline $\mathrm{C}$ & -0.601050000 & -5.374542000 & -0.136132000 \\
\hline $\mathrm{C}$ & -4.020062000 & -1.362890000 & -0.297494000 \\
\hline $\mathrm{C}$ & -0.999743000 & -4.562448000 & 0.945048000 \\
\hline $\mathrm{C}$ & -3.953835000 & -2.734487000 & -0.673378000 \\
\hline $\mathrm{C}$ & -6.309533000 & 1.317263000 & 0.716119000 \\
\hline $\mathrm{H}$ & -6.934248000 & 0.581490000 & 1.238313000 \\
\hline $\mathrm{C}$ & -5.668495000 & 3.612425000 & 0.295751000 \\
\hline $\mathrm{C}$ & -2.320505000 & -4.421244000 & -1.544316000 \\
\hline $\mathrm{H}$ & -2.829670000 & -4.355308000 & -2.513628000 \\
\hline $\mathrm{C}$ & -6.491370000 & 2.691530000 & 0.972171000 \\
\hline $\mathrm{C}$ & -6.337488000 & -1.250951000 & -1.015862000 \\
\hline $\mathrm{H}$ & -7.250343000 & -0.665531000 & -1.166901000 \\
\hline $\mathrm{C}$ & -4.693144000 & 3.196519000 & -0.633231000 \\
\hline $\mathrm{C}$ & -6.292562000 & -2.599718000 & -1.391302000 \\
\hline $\mathrm{H}$ & -7.181125000 & -3.082873000 & -1.810825000 \\
\hline $\mathrm{C}$ & -4.527211000 & 1.817770000 & -0.864241000 \\
\hline $\mathrm{H}$ & -3.778561000 & 1.477479000 & -1.590620000 \\
\hline $\mathrm{C}$ & -5.108188000 & -3.325477000 & -1.231706000 \\
\hline $\mathrm{H}$ & -5.071165000 & -4.385807000 & -1.501283000 \\
\hline $\mathrm{Ni}$ & 1.517012000 & -0.541159000 & -0.841103000 \\
\hline $\mathrm{C}$ & -2.816131000 & 0.159654000 & 1.210692000 \\
\hline $\mathrm{H}$ & -3.777330000 & 0.389473000 & 1.681674000 \\
\hline $\mathrm{C}$ & 2.069768000 & 1.492248000 & 1.073156000 \\
\hline $\mathrm{H}$ & 2.766862000 & 2.193857000 & 1.559581000 \\
\hline $\mathrm{C}$ & 0.712379000 & 1.533736000 & 1.560795000 \\
\hline $\mathrm{C}$ & 0.445452000 & 2.438944000 & 2.611182000 \\
\hline $\mathrm{C}$ & -0.347893000 & 0.691582000 & 1.048741000 \\
\hline $\mathrm{H}$ & 1.277680000 & 3.057573000 & 2.973801000 \\
\hline $\mathrm{C}$ & -0.820626000 & 2.590639000 & 3.214655000 \\
\hline $\mathrm{C}$ & -1.660702000 & 0.860762000 & 1.656950000 \\
\hline $\mathrm{C}$ & -1.037372000 & 3.578813000 & 4.341392000 \\
\hline $\mathrm{C}$ & -1.852390000 & 1.793928000 & 2.715229000 \\
\hline $\mathrm{H}$ & -2.860290000 & 1.890607000 & 3.139603000 \\
\hline $\mathrm{O}$ & -0.177425000 & -0.175946000 & 0.094892000 \\
\hline $\mathrm{C}$ & 0.732500000 & -2.217971000 & -1.702143000 \\
\hline $\mathrm{C}$ & 0.750615000 & -1.224573000 & -2.684758000 \\
\hline $\mathrm{H}$ & 1.541297000 & -2.954690000 & -1.642034000 \\
\hline $\mathrm{H}$ & -0.183941000 & -2.458115000 & -1.150571000 \\
\hline $\mathrm{C}$ & -0.439026000 & -0.407906000 & -3.122867000 \\
\hline $\mathrm{H}$ & 1.605789000 & -1.199975000 & -3.373414000 \\
\hline $\mathrm{H}$ & -0.165635000 & 0.649363000 & -3.291703000 \\
\hline $\mathrm{H}$ & -0.808378000 & -0.798858000 & -4.092352000 \\
\hline $\mathrm{H}$ & -1.266550000 & -0.443534000 & -2.397230000 \\
\hline $\mathrm{H}$ & -0.412048000 & 3.332972000 & 5.219377000 \\
\hline $\mathrm{H}$ & -0.774533000 & 4.607005000 & 4.032218000 \\
\hline $\mathrm{H}$ & -2.089375000 & 3.586263000 & 4.672007000 \\
\hline $\mathrm{H}$ & 3.693216000 & -4.943364000 & 2.306632000 \\
\hline $\mathrm{H}$ & 0.234435000 & -6.073357000 & 0.002329000 \\
\hline $\mathrm{H}$ & 1.336580000 & 6.433107000 & -1.814236000 \\
\hline $\mathrm{H}$ & -5.796542000 & 4.685500000 & 0.489984000 \\
\hline $\mathrm{H}$ & 2.759688000 & -0.881306000 & -1.518480000 \\
\hline $\mathrm{H}$ & -1.906477000 & -1.010086000 & -0.124136000 \\
\hline $\mathrm{C}$ & -0.824591000 & -6.230890000 & -2.520678000 \\
\hline $\mathrm{H}$ & -1.326994000 & -7.214780000 & -2.451724000 \\
\hline $\mathrm{H}$ & -1.081337000 & -5.801072000 & -3.504195000 \\
\hline $\mathrm{H}$ & 0.262700000 & -6.420932000 & -2.502180000 \\
\hline $\mathrm{C}$ & -0.290442000 & -4.660100000 & 2.280240000 \\
\hline $\mathrm{H}$ & -0.739815000 & -3.986364000 & 3.029445000 \\
\hline $\mathrm{H}$ & -0.334311000 & -5.688930000 & 2.682813000 \\
\hline $\mathrm{H}$ & 0.778551000 & -4.393779000 & 2.181720000 \\
\hline
\end{tabular}




$\begin{array}{lrrr}\mathrm{C} & 4.872279000 & -5.226928000 & -0.152051000 \\ \mathrm{H} & 5.867887000 & -5.586561000 & 0.172071000 \\ \mathrm{H} & 4.152708000 & -6.036417000 & 0.063672000 \\ \mathrm{H} & 4.916789000 & -5.088965000 & -1.246362000 \\ \mathrm{C} & 3.015638000 & -2.845609000 & 3.940095000 \\ \mathrm{H} & 2.322014000 & -2.008275000 & 4.133975000 \\ \mathrm{H} & 2.473826000 & -3.789375000 & 4.125316000 \\ \mathrm{H} & 3.821831000 & -2.781374000 & 4.696091000 \\ \mathrm{C} & 0.525381000 & 4.210794000 & -3.184982000 \\ \mathrm{H} & 0.320343000 & 3.159331000 & -3.452601000 \\ \mathrm{H} & -0.409234000 & 4.652387000 & -2.792562000 \\ \mathrm{H} & 0.765022000 & 4.748914000 & -4.121556000 \\ \mathrm{C} & -3.866026000 & 4.215863000 & -1.390433000 \\ \mathrm{H} & -2.914385000 & 3.784603000 & -1.747004000 \\ \mathrm{H} & -4.411835000 & 4.587801000 & -2.278601000 \\ \mathrm{H} & -3.632532000 & 5.094324000 & -0.763483000 \\ \mathrm{C} & -7.561907000 & 3.165638000 & 1.934156000 \\ \mathrm{H} & -8.514771000 & 3.355321000 & 1.403809000 \\ \mathrm{H} & -7.769386000 & 2.415244000 & 2.716720000 \\ \mathrm{H} & -7.273576000 & 4.109131000 & 2.429268000 \\ \mathrm{C} & 3.348698000 & 7.046975000 & -0.057155000 \\ \mathrm{H} & 2.451286000 & 7.685075000 & 0.021184000 \\ \mathrm{H} & 3.783520000 & 6.940658000 & 0.952259000 \\ \mathrm{H} & 4.086819000 & 7.597404000 & -0.671728000\end{array}$

Me-H+ $\mathbf{T S}-\mathbf{1}_{2,1-i n s}$
Zero-point correction=

0.946940 (Hartree/Particle)
Thermal correction to Energy= $\quad 1.008056$

Thermal correction to Enthalpy $=\quad 1.009000$

Thermal correction to Gibbs Free Energy= $\quad 0.843469$

Sum of electronic and zero-point Energies $=\quad-2521.331744$

Sum of electronic and thermal Energies $=\quad-2521.270628$

Sum of electronic and thermal Enthalpies $=\quad-2521.269684$

Sum of electronic and thermal Free Energies $=-2521.435215$

E solvent $=-2521.15531299$

$\begin{array}{lrr}\mathrm{Ni} & -1.340296000 & -1.137075000 \\ \mathrm{C} & -1.246310000 & -2.690441000 \\ \mathrm{~N} & -2.561096000 & 0.135287000 \\ \mathrm{C} & -2.245943000 & 1.393177000 \\ \mathrm{C} & -0.970143000 & 2.032672000 \\ \mathrm{C} & 0.203025000 & 1.330100000 \\ \mathrm{O} & 0.201645000 & 0.071086000 \\ \mathrm{C} & -0.068250000 & -2.663660000 \\ \mathrm{C} & 0.229127000 & -3.621472000 \\ \mathrm{H} & 0.906432000 & -4.423629000 \\ \mathrm{H} & -0.687521000 & -4.090289000 \\ \mathrm{H} & 0.740978000 & -3.115874000 \\ \mathrm{H} & 0.781979000 & -2.111730000 \\ \mathrm{H} & -1.298337000 & -2.148372000 \\ \mathrm{H} & -1.975679000 & -3.501122000 \\ \mathrm{H} & -3.033607000 & 2.043174000 \\ \mathrm{C} & -0.889160000 & 3.408965000 \\ \mathrm{H} & -1.805033000 & 3.909731000 \\ \mathrm{C} & 0.294641000 & 4.169052000 \\ \mathrm{C} & 0.308845000 & 5.647538000 \\ \mathrm{H} & -0.315297000 & 6.222398000 \\ \mathrm{H} & -0.087573000 & 5.841596000 \\ \mathrm{H} & 1.330927000 & 6.059139000 \\ \mathrm{C} & 1.440795000 & 3.488736000 \\ \mathrm{H} & 2.392117000 & 4.031647000 \\ \mathrm{C} & 1.435438000 & 2.102421000 \\ \mathrm{C} & 2.684306000 & 1.525309000 \\ \mathrm{~N} & 2.863084000 & 0.262701000 \\ \mathrm{H} & 3.579802000 & 2.155207000 \\ \mathrm{C} & 4.123811000 & -0.366446000 \\ \mathrm{C} & 5.256521000 & -0.086725000 \\ \mathrm{C} & 6.467291000 & -0.740400000 \\ \mathrm{C} & 6.553915000 & -1.663962000 \\ & & \\ \end{array}$

0.150963000

1.508426000

$-0.596024000$

$-0.810251000$

$-0.595760000$

$-0.126457000$

0.207336000

0.747204000

$-0.377580000$

$-0.020284000$

$-0.772136000$

$-1.215656000$

1.166653000

2.461298000

1.390173000

$-1.223310000$

$-0.900114000$

$-1.242344000$

$-0.788635000$

$-1.115921000$

$-0.406829000$

$-2.129176000$

$-1.070347000$

$-0.374313000$

$-0.299318000$

$-0.049336000$

0.316313000

0.689534000

0.292775000

0.948893000

0.130840000

0.438353000

1.487965000
$5.424491000 \quad-1.942297000$ $4.193811000-1.293145000$ $3.051460000-1.582129000$ $2.325591000 \quad-0.549651000$ $1.800841000-4.938985000$ $1.031274000 \quad-2.189073000$ $\begin{array}{ll}1.426391000 & -4.687008000\end{array}$ $1.737418000 \quad-3.245949000$ 2.743120000 5.239373000 4.402743000 4.437483000 5.315060000 6.167633000 6.117937000 3.574484000 1.318459000 0.595833000 $-4.609738000$ $-3.939206000$ $-4.038161000$ $-4.809147000$ $-5.477350000$ $-5.387512000$

$-3.153750000$ $-5.000056000$ $-6.325889000$ $-6.598220000$ $-5.539718000$ $-4.194478000$ $-3.933736000$

$-3.129869000$

$-3.226200000$

$-2.312260000$

$-1.278816000$

$-1.155162000$ $-2.078325000$ $-0.073344000$

$-2.449564000$ $-6.072802000$

$-4.527833000$

$-2.332660000$

$-2.717151000$

$-3.802065000$

$-3.523273000$

$-7.145174000$

$-7.633698000$

$-5.743923000$

$-4.030004000$

$-3.067188000$

$-2.931945000$

$-1.466110000$

$-0.559772000$

0.855811000

0.167207000

$-0.391952000$

5.341214000

6.768223000

4.162051000

3.159714000

2.732832000

7.339578000

7.503681000

5.491431000

1.893845000

0.338233000

3.295747000

$\mathrm{H} \quad 2.581339000$
2.264721000 2.026730000 2.942898000 3.576085000 5.550385000 4.802805000 4.540121000 4.189366000 3.260762000 $-1.061350000$ $-2.169462000$ $-3.308252000$ $-3.314543000$ $-2.229126000$ $-1.104372000$ $-4.516558000$ 4.518724000 5.244028000 2.721715000 2.926234000 1.939364000 0.775073000 0.605627000 1.571116000 4.199777000 $-0.242909000$ $-0.551232000$ $-1.455883000$ $-2.071313000$ $-1.804138000$ $-0.884966000$ $-2.525770000$ $-2.599055000$ $-3.348368000$ $-4.031970000$ $-3.986403000$ $-3.219550000$ $-4.781109000$ $-3.441522000$ $-0.301344000$ 3.481508000 4.039690000 4.591134000 4.996527000 2.084150000 $-0.040834000$ $-1.680533000$ $-2.801766000$ $-2.052506000$ $-2.621227000$ $-4.392606000$ $-3.410487000$ $-4.623211000$ $-4.819907000$ $-4.350634000$ $-5.827816000$ $-4.195040000$ $-0.244006000$ $-5.308997000$ $-4.959226000$ $-4.259913000$ $-0.191352000$ 1.699910000 3.104551000 3.829423000 4.544490000 2.764589000 3.374218000 


$\begin{array}{rrrr}\mathrm{H} & 1.169179000 & 0.610776000 & 6.130205000 \\ \mathrm{H} & -0.395953000 & -0.047314000 & 5.603517000 \\ \mathrm{H} & 3.733564000 & -0.305311000 & -2.152489000 \\ \mathrm{H} & -2.002806000 & -0.306441000 & -3.203603000 \\ \mathrm{H} & 0.247913000 & -2.425051000 & 5.535421000 \\ \mathrm{H} & 0.456168000 & 1.161013000 & 4.596972000 \\ \mathrm{H} & -2.463813000 & -2.060771000 & 0.096699000 \\ \mathrm{C} & -6.126218000 & 5.116781000 & 1.387465000 \\ \mathrm{H} & -7.087808000 & 5.112120000 & 1.935764000 \\ \mathrm{H} & -5.540569000 & 5.970582000 & 1.771999000 \\ \mathrm{H} & -6.356969000 & 5.309457000 & 0.325390000 \\ \mathrm{C} & 7.132701000 & 3.938995000 & -2.277834000 \\ \mathrm{H} & 8.083136000 & 3.646855000 & -2.764170000 \\ \mathrm{H} & 6.719751000 & 4.783439000 & -2.856701000 \\ \mathrm{H} & 7.383044000 & 4.304580000 & -1.266888000 \\ \mathrm{H} & 1.982287000 & -0.283637000 & 0.757662000\end{array}$

\section{$\mathbf{M e}-\mathbf{H}+$ 3- $\beta-\mathbf{C}$}

Zero-point correction $=$

Thermal correction to Energy=

0.949682 (Hartree/Particle)

Thermal correction to Enthalpy=

1.012082

1.013026

Thermal correction to Gibbs Free Energy $=0.843314$

Sum of electronic and zero-point Energies $=\quad-2521.349245$

Sum of electronic and thermal Energies $=\quad-2521.286845$

Sum of electronic and thermal Enthalpies $=\quad-2521.285900$

Sum of electronic and thermal Free Energies $=-2521.455613$

E solvent $=-2521.17816811$

N $\quad-0.325644000 \quad-2.636337000$

C $\quad-2.258100000 \quad 1.471504000$

C $\quad-0.970141000 \quad 2.083532000$

C $\quad 0.198616000 \quad 1.349863000$

O $0.217229000 \quad 0.064036000$

C $\quad-1.740147000 \quad-3.100369000$

$\mathrm{H} \quad-2.469164000 \quad-2.272311000$

$\mathrm{H} \quad-1.938462000 \quad-3.919268000$

$\mathrm{H} \quad-2.198374000 \quad-3.291416000$

C $\quad 0.672363000 \quad-3.205249000$

H $\quad 0.079639000 \quad-2.434422000$

H $\quad-3.045929000 \quad 2.149810000$

C $\quad-0.872940000 \quad 3.474371000$

$\mathrm{H} \quad-1.782366000 \quad 4.002190000$

C $\quad 0.315517000 \quad 4.218209000$

C $\quad 0.343463000$

$\mathrm{H} \quad-0.354049000$

H $\quad 0.045756000$

$\mathrm{H} \quad 1.351371000$

C $\quad 1.450545000$

H 2.402731000

C 1.431342000

1.431342000
2.666711000

$\begin{array}{ll}\mathrm{N} & 2.835275000 \\ \mathrm{H} & 3.562644000\end{array}$

C 4.087518000

C $\quad 5.245161000$

5.245161000
6.445332000

6.498900000

5.345732000

4.123250000

2.954184000

2.213182000

0.887667000

1.608178000

2.636442000

5.264299000

4.465619000

4.535929000

0.684209000

1.575471000
0.032567000 0.670884000 $-0.627965000$

$-0.781329000$

$-0.535898000$

$-0.106558000$

0.136438000

0.629997000

0.184936000

$-0.082766000$

1.614087000

$-0.316509000$

1.674796000

$-1.146787000$

$-0.755560000$

$-1.073712000$

$-0.588128000$

$-0.837500000$

$-0.165919000$

$-1.874274000$

$-0.672081000$

$-0.193228000$

$-0.067313000$

0.049226000

0.417230000

0.722542000

0.459321000

0.982737000

0.218373000

0.521601000

1.514174000

2.236847000

2.002169000

2.865280000

3.541236000

4.634903000

3.977826000

3.092820000

$-0.917458000$

$-2.066018000$

$-3.152828000$
5.410224000 6.225067000

6.140393000

1.183273000

$-4.699700000$

$-3.979521000$

$-4.051938000$

$-4.843192000$

$-5.561194000$

$-5.499631000$

$-5.001759000$

$-6.314142000$

$-6.555292000$

$-5.478070000$

$-4.143502000$

$-3.913059000$

$-3.056435000$

$-3.114439000$

$-2.163840000$

$-1.132160000$

$-1.050888000$

$-2.010808000$

$-6.171789000$

$-4.639654000$

$-3.495804000$

$-7.148302000$

$-7.581529000$

$-5.660674000$

$-3.917428000$

$-0.384037000$

5.462979000

6.761489000

7.336633000

7.441258000

5.385845000

3.199409000

2.473556000

3.797983000

$-1.966596000$

0.084278000

1.043026000

0.232626000

1.558152000

1.956688000

0.442546000

$-0.587873000$

0.948656000

0.391404000

1.298150000

1.630733000

1.811274000

0.217278000

3.712016000

4.334788000

2.889473000

3.273503000

7.187169000

6.805704000

7.376016000

8.166093000

$-2.257480000$

$-1.264092000$

$-2.905021000$

$-2.683137000$

0.024967000

0.242122000

0.965292000
2.678314000 2.893561000

1.981160000

$-1.109999000$

3.501937000

2.302663000

1.319925000

1.513291000

2.720808000

3.726356000

0.445871000

0.022310000

$-0.998339000$

$-1.619849000$

$-1.237220000$

$-0.188876000$

$-1.940010000$

$-3.348283000$

$-4.059055000$

$-3.334633000$

$-1.932046000$

$-1.247916000$

2.881297000

4.278821000

0.381685000

0.495421000

$-1.309689000$

$-2.398718000$

$-3.900014000$

$-3.875664000$

3.384351000

2.133893000

$-0.534922000$

$-2.278842000$

$-2.835185000$

$-3.896933000$

0.290467000

$-0.184470000$

$-0.155312000$

$-2.750228000$

$-4.195420000$

$-3.338043000$

$-2.551977000$

$-0.334756000$

$-0.037749000$

$-0.351325000$

0.179826000

0.910489000

$-4.952543000$

$-5.596991000$

$-5.306882000$

$-5.118368000$

1.344832000

0.931944000

0.631442000

2.286724000

4.063244000

4.927822000

4.391435000

3.790980000

$-5.564432000$

$-6.043391000$

$-6.013869000$

$-5.837052000$

$-1.168228000$

$-0.198571000$

$-1.744679000$

$-0.954468000$

$-3.066369000$

$-1.938859000$

$-0.867323000$

4.438729000

2.779651000

2.953350000

1.949108000

0.796196000

0.658688000

1.643390000

$-0.242132000$

$-0.546116000$

$-1.472403000$

$-2.112566000$

$-1.850351000$

$-0.911246000$

$-2.599039000$

$-2.731644000$

$-3.487211000$

$-4.119334000$

$-4.022963000$

$-3.250414000$

$-0.239035000$

3.553484000

2.070350000

$-0.017159000$

$-1.694422000$

$-2.860415000$

$-2.226714000$

$-4.714015000$

$-3.905751000$

0.024429000

$-0.067429000$

1.723623000

3.033089000

2.561462000

3.407230000

$-2.122051000$

$-3.195148000$

5.330349000

0.023000000

$-1.321395000$

$-0.411980000$

0.740057000

5.212107000

5.454154000

6.172311000

4.649026000

4.240788000

3.408967000

5.155271000

4.393925000 


$\begin{array}{llll}\mathrm{C} & -3.167309000 & 2.070415000 & 4.211826000 \\ \mathrm{H} & -2.651831000 & 2.991631000 & 4.537456000 \\ \mathrm{H} & -3.813627000 & 1.749205000 & 5.051233000 \\ \mathrm{H} & -2.405440000 & 1.285399000 & 4.063507000 \\ \mathrm{C} & -6.293254000 & 5.009023000 & 1.493074000 \\ \mathrm{H} & -7.274312000 & 4.931802000 & 2.000066000 \\ \mathrm{H} & -5.764884000 & 5.868565000 & 1.941703000 \\ \mathrm{H} & -6.492529000 & 5.243712000 & 0.432964000\end{array}$

\section{Me-H+TS-3 ${ }_{\text {coor }}$}

Zero-point correction= 1.000882 (Hartree/Particle)

Thermal correction to Energy $=\quad 1.066852$

Thermal correction to Enthalpy= $\quad 1.067796$

Thermal correction to Gibbs Free Energy $=\quad 0.889711$

Sum of electronic and zero-point Energies $=\quad-2599.869082$

Sum of electronic and thermal Energies $=\quad-2599.803112$

Sum of electronic and thermal Enthalpies $=\quad-2599.802168$

Sum of electronic and thermal Free Energies $=-2599.980253$

E solvent $=-2599.72474821$

\begin{tabular}{|c|c|c|c|}
\hline $\mathrm{Ni}$ & -1.162832000 & 0.346544000 & -1.109700000 \\
\hline $\mathrm{C}$ & -0.038559000 & 0.702241000 & -2.658299000 \\
\hline $\mathrm{N}$ & -2.504267000 & 0.500169000 & 0.343982000 \\
\hline $\mathrm{C}$ & -2.153980000 & 0.346508000 & 1.600563000 \\
\hline $\mathrm{C}$ & -0.848404000 & -0.011001000 & 2.117051000 \\
\hline $\mathrm{C}$ & 0.325393000 & -0.204248000 & 1.295737000 \\
\hline $\mathrm{O}$ & 0.335516000 & -0.085540000 & -0.003497000 \\
\hline $\mathrm{C}$ & -1.334263000 & 1.243443000 & -3.166635000 \\
\hline $\mathrm{H}$ & -2.185641000 & 1.118409000 & -2.378015000 \\
\hline $\mathrm{H}$ & -1.330117000 & 2.334299000 & -3.324848000 \\
\hline $\mathrm{H}$ & -1.752876000 & 0.709330000 & -4.035907000 \\
\hline $\mathrm{C}$ & 1.080 & 1.691554000 & -2.4071 \\
\hline $\mathrm{H}$ & 0.291388000 & -0.255141000 & -3.092518000 \\
\hline $\mathrm{H}$ & -2.919013000 & 0.520365000 & 2.372970000 \\
\hline $\mathrm{C}$ & -0.746957000 & -0.161189000 & 3.516098000 \\
\hline $\mathrm{H}$ & -1.661235000 & -0.020701000 & 4.108408000 \\
\hline $\mathrm{C}$ & 0.4498 & -0.490349000 & 4.191109000 \\
\hline $\mathrm{C}$ & 0.478594000 & -0.646861000 & 5.697060000 \\
\hline $\mathrm{H}$ & -0.129380000 & -1.510 & 6.024255000 \\
\hline $\mathrm{H}$ & 0.072540000 & 0.247090000 & 6.204555000 \\
\hline $\mathrm{H}$ & 1.506265000 & -0.804111000 & 6.0652 \\
\hline $\mathrm{C}$ & 1.588475000 & -0.662361000 & 3.40 \\
\hline $\mathrm{H}$ & 2.544957000 & -0.907156000 & 3.88 \\
\hline $\mathrm{C}$ & 1.566951000 & -0.531542000 & 1.986490000 \\
\hline $\mathrm{C}$ & 2.802565000 & -0.705497000 & 1.303445000 \\
\hline $\mathrm{N}$ & 2.950364000 & -0.694959000 & -0.0193 \\
\hline $\mathrm{H}$ & 3.712635000 & -0.864337000 & 2000 \\
\hline $\mathrm{C}$ & 4.190436000 & -0.730651000 & -0.734177000 \\
\hline $\mathrm{C}$ & 5.317859000 & 0.012291000 & -0.279875000 \\
\hline $\mathrm{C}$ & 6.498234000 & -0.049808000 & -1.049320000 \\
\hline $\mathrm{C}$ & 6.557327000 & -0.782077000 & -2.242077000 \\
\hline $\mathrm{C}$ & 5.433513000 & -1.491424000 & -2.679877000 \\
\hline $\mathrm{C}$ & 4.238088000 & -1.498750000 & -1.930460000 \\
\hline $\mathrm{C}$ & 3.112119000 & -2.362146000 & -2.395699000 \\
\hline $\mathrm{C}$ & 2.549009000 & -3.345033000 & -1.550858000 \\
\hline $\mathrm{C}$ & 1.166859000 & -4.141851000 & -3.373271000 \\
\hline $\mathrm{C}$ & 1.702857000 & -3.169352000 & -4.241284000 \\
\hline $\mathrm{C}$ & 2.675101000 & -2.283627000 & -3.736809000 \\
\hline $\mathrm{C}$ & 5.322323000 & 0.885945000 & 0.936527000 \\
\hline $\mathrm{C}$ & 4.443034000 & 1.985417000 & 1.058482000 \\
\hline $\mathrm{C}$ & 4.499654000 & 2.835700000 & 2.177170000 \\
\hline $\mathrm{C}$ & 5.449467000 & 2.564279000 & 3.184641000 \\
\hline $\mathrm{C}$ & 6.343049000 & 1.482089000 & 3.090283000 \\
\hline $\mathrm{C}$ & 6.265909000 & 0.646315000 & 1.956321000 \\
\hline $\mathrm{C}$ & 1.583516000 & -4.250195000 & -2.030375000 \\
\hline $\mathrm{C}$ & -4.269797000 & -3.718913000 & 2.890665000 \\
\hline $\mathrm{C}$ & -4.059120000 & -3.760225000 & 1.499050000 \\
\hline G & -4.234778000 & -2.576043000 & 0.754568000 \\
\hline
\end{tabular}

\begin{tabular}{|c|c|c|c|}
\hline & & & \\
\hline & 03000 & 11000 & \\
\hline & 77755000 & -2.538514000 & \\
\hline & 9363000 & -0.123720000 & \\
\hline & 00 & 146928000 & 200007 \\
\hline & 457000 & 1000 & -0.386 \\
\hline & 36000 & 2.2 & \\
\hline & 854000 & 1.987686000 & \\
\hline & -3.903655000 & & \\
\hline & -3.338622000 & 000 & 1000 \\
\hline & 3677000 & 000 & 4700 \\
\hline & -2.749971000 & 3000 & -2.61847000 \\
\hline & -1.700220000 & 5.222550000 & -1.77145700 \\
\hline & -1.463017000 & 4.594879000 & -0.53426100 \\
\hline & -2.280934000 & 3.511009000 & -0.15365500 \\
\hline & -5.174162000 & -0.444503000 & 3.25928300 \\
\hline & -4.129737000 & -4.6 & 400 \\
\hline & & & -0 \\
\hline & -7.009945000 & & \\
\hline & -7 & & 2000 \\
\hline & 785000 & 3. & 0000 \\
\hline & 19000 & & 3000 \\
\hline & & & \\
\hline & & & \\
\hline & & & \\
\hline & & & \\
\hline & & & \\
\hline & & & \\
\hline & & & \\
\hline & & & \\
\hline & & & \\
\hline & & & \\
\hline & 3000 & & \\
\hline & 70000 & & \\
\hline & 00 & & -1 \\
\hline & 60000 & & -1 . \\
\hline & -2.485708000 & & -2 \\
\hline & -1.536946000 & & 48000 \\
\hline & -2.250583000 & & -3.553246000 \\
\hline & -3.528255000 & -1. & 84000 \\
\hline & -0.515682000 & & -1.8 \\
\hline & -1.791771000 & & \\
\hline & & & \\
\hline & & & \\
\hline & 7.0 & & \\
\hline & 7.55 & & \\
\hline & 00 & & \\
\hline & 0 & & \\
\hline & & & \\
\hline & 2 & & \\
\hline & 4. & & 00 \\
\hline & 2 & & \\
\hline & -2 & & -4 \\
\hline & -3.6 & & -4.5 \\
\hline & -3. & & -3.76275600 \\
\hline & 72000 & & 4000 \\
\hline & & & 19000 \\
\hline & 59000 & & 6000 \\
\hline & 44000 & & 5000 \\
\hline & 83000 & & 2000 \\
\hline & 503000 & -5 & 63000 \\
\hline & 063000 & & 00000 \\
\hline & -3.174550000 & -4.8 & -0.1 \\
\hline & -4.959235000 & & \\
\hline & -5.981100000 & & 5.2 \\
\hline & & & \\
\hline & & & \\
\hline & & -3.099867000 & -5.6907150 \\
\hline
\end{tabular}




$\begin{array}{crrr}\mathrm{H} & 1.530748000 & -2.133407000 & -6.151834000 \\ \mathrm{H} & 1.747045000 & -3.895110000 & -6.289712000 \\ \mathrm{H} & 0.173043000 & -3.240060000 & -5.792979000 \\ \mathrm{C} & 1.019566000 & -5.334279000 & -1.133383000 \\ \mathrm{H} & -0.084953000 & -5.351862000 & -1.166617000 \\ \mathrm{H} & 1.366732000 & -6.335574000 & -1.450728000 \\ \mathrm{H} & 1.327035000 & -5.193251000 & -0.083199000\end{array}$

\section{Me-H+3-Coor-C}

Zero-point correction $=$ 1.004786 (Hartree/Particle)

$\begin{array}{lc}\text { Thermal correction to Energy }= & 1.070022 \\ \text { Thermal correction to Enthalpy= } & 1.070967 \\ \text { Thermal correction to Gibbs Free Energy }= & 0.896054 \\ \text { Sum of electronic and zero-point Energies }= & -2599.883570 \\ \text { Sum of electronic and thermal Energies }= & -2599.818334 \\ \text { Sum of electronic and thermal Enthalpies }= & -2599.817390 \\ \text { Sum of electronic and thermal Free Energies }= & -2599.992303 \\ \text { E solvent }=-2599.7347476 & \end{array}$

$\mathrm{Ni} \quad-1.188503000 \quad-0.414255000$

C $\quad 0.278483000 \quad-1.244929000$

$\begin{array}{lll}\mathrm{N} & -2.555644000 & 0.438914000\end{array}$

$\begin{array}{lll}\text { C } & -2.278877000 & 0.651361000\end{array}$

$\begin{array}{lll}\text { C } & -0.990502000 & 0.578075000\end{array}$

C $\quad 0.215804000 \quad 0.180571000$

$\begin{array}{lll}\text { O } & 0.245581000 & -0.237951000\end{array}$

C $\quad 0.015037000 \quad-2.271692000$

$\mathrm{H} \quad-0.498376000 \quad-3.172028000$

$\mathrm{H}-0.556210000$

H $\quad 0.990247000$

C 1.136388000

$\mathrm{H} \quad 0.742108000$

$\mathrm{H} \quad-3.107681000$

C $\quad-0.941587000$

$\mathrm{H} \quad-1.886607000$

C $\quad 0.248825000$

C $\quad 0.232480000$

$\mathrm{H} \quad-0.268114000$

H $\quad-0.311946000$

$\mathrm{H} \quad 1.254786000$

C 1.428831000

H 2.384455000

C 1.452467000

C 2.733037000

$\mathrm{N} \quad 2.966926000$

H 3.619174000

C $\quad 4.268546000$

C $\quad 5.232410000$

C $\quad 6.489678000$

C $\quad 6.776529000$

C $\quad 5.812119000$

C $\quad 4.547422000$

C $\quad 3.609353000$

C $\quad 3.010575000$

$\mathrm{H} \quad 3.011238000$

C $\quad 2.041866000$

C 2.433934000

C $\quad 2.626619000$

C $\quad 3.406529000$

C 4.987848000

C $\quad 3.982030000$

C $\quad 3.813204000$

C $\quad 4.656129000$

C $\quad 5.671145000$

C $\quad 5.825570000$

C $\quad 6.590900000$

C 2.770210000

C $\quad 2.233541000$

C 1.665026000
$-1.270158000$

$-2.358338000$

0.069103000

1.331165000

1.983651000

1.303075000

0.070751000

$-3.454021000$

$-3.075097000$

$-4.312071000$

$-3.858463000$

$-2.842736000$

$-1.498259000$

1.992342000

3.342150000

3.829283000

4.099373000

5.558952000

6.181212000

5.710832000

5.951287000

3.431677000

3.969784000

2.067445000

1.511274000

0.297517000

2.128854000

$-0.289634000$

$-0.174579000$

$-0.781996000$

$-1.504193000$

$-1.613583000$

$-0.997413000$

$-1.069933000$

0.089468000

$-3.758245000$

$-1.222313000$

$-3.725932000$

$-2.400396000$

$-2.308271000$

0.512473000

0.072555000

0.678529000

1.751985000

2.212308000

1.580685000

3.341925000

0.167329000

0.028654000

1.292004000
$-4.731565000$

$-5.346337000$
$-4.174313000 \quad-3.614654000$ $-3.332294000 \quad-3.482117000$ $-3.537732000 \quad-2.403375000$

$-4.584324000 \quad-1.478800000$

$-5.413078000-1.646606000$

$-5.221992000$

$-2.240401000$

$-4.905173000$

$-6.250833000$

$-6.663098000$

$-5.737086000$

$-4.377165000$

$-3.959202000$

$-3.488229000$

$-3.519562000$

$-2.776958000$

$-2.001668000$

$-1.966201000$

$-2.701609000$

$-1.193311000$

$-2.819509000$

$-6.216427000$

$-4.009403000$

$-1.480425000$

$-1.728681000$

$-2.655946000$

$-2.877364000$

$-6.972635000$

$-7.709894000$

$-6.063941000$

$-4.133439000$

$-3.349078000$

$-3.339291000$

$-1.802728000$

$-1.422876000$

$-0.374419000$

$-0.757318000$

$-1.851327000$

4.523816000

7.024449000

7.434692000

6.061479000

6.608335000

3.225507000

2.293735000

1.977950000

7.232090000

7.756328000

6.043966000

2.768773000

1.374976000

3.865158000

3.198217000

1.422887000

2.391208000

3.342334000

$-2.696087000$

1.435368000

0.748592000

$-6.132981000$

$-5.585382000$

$-6.600214000$

$-6.952523000$

2.150322000

0.713603000

1.261076000

$-2.675217000$
2.938081000

1.816320000

0.936384000

1.145755000

2.278470000

3.183751000

1.552694000

0.206813000

$-0.205180000$

$-1.017403000$

$-1.401031000$

$-1.022579000$

$-0.250605000$

$-1.406955000$

$-2.735474000$

$-3.124226000$

$-2.148361000$

$-0.812925000$

$-0.459169000$

0.238467000

$-4.550807000$

2.463166000

3.636513000

0.853450000

2.486415000

1.107559000

0.067956000

0.117896000

$-1.328917000$

$-1.979073000$

$-3.482329000$

$-5.218080000$

$-4.611284000$

$-4.956319000$

$-2.434568000$

$-0.210841000$

0.999842000

0.771364000

2.236707000

3.860105000

2.961557000

4.089808000

1.925165000

$-0.505072000$

0.995253000

$-0.402945000$

$-0.707617000$

$-1.977033000$

$-2.143922000$

$-4.573844000$

$-3.894139000$

$-3.214256000$

1.064660000

2.047296000

1.754935000

$-0.772717000$

0.585040000

$-1.280006000$

1.084726000

4.382995000

5.255166000

4.678833000

4.162943000

$-3.119032000$

$-3.745917000$

$-2.077126000$

$-2.459841000$

$-2.997258000$ 


$\begin{array}{rrrr}\mathrm{H} & -3.591458000 & -1.191997000 & -1.860074000 \\ \mathrm{H} & -2.368614000 & -2.169562000 & -2.854888000 \\ \mathrm{H} & -2.675649000 & 0.950175000 & -2.800449000 \\ \mathrm{H} & -1.478185000 & -0.009695000 & -3.837476000 \\ \mathrm{H} & 2.117259000 & -0.720614000 & -0.255309000\end{array}$

\section{Me-H+ ${ }^{+}$SS-3 ${ }_{\text {ins }}$}

Zero-point correction $=\quad 1.003710($ Hartree/Particle $)$

Thermal correction to Energy= $\quad 1.068600$

Thermal correction to Enthalpy $=\quad 1.069544$

Thermal correction to Gibbs Free Energy= 0.894952

Sum of electronic and zero-point Energies $=-2599.870565$

Sum of electronic and thermal Energies $=-2599.805675$

Sum of electronic and thermal Enthalpies $=\quad-2599.804731$

Sum of electronic and thermal Free Energies $=-2599.979323$

E solvent $=-2599.72062233$

\begin{tabular}{|c|c|c|c|}
\hline & & & \\
\hline & 8000 & 4000 & 6000 \\
\hline & 2000 & -0.387252000 & 0000 \\
\hline & 2.222587000 & -0.286498000 & 02000 \\
\hline & 0.910489000 & -0.069695000 & 4000 \\
\hline & -0.286274000 & 65000 & 1000 \\
\hline & -0.281076000 & 0.066607000 & 18000 \\
\hline & -1.285808000 & -0.249349000 & 3000 \\
\hline & -1.522732000 & 0.660595000 & -2.566 \\
\hline & -1.145182000 & & \\
\hline & -2.1 & & \\
\hline & & & 7000 \\
\hline & & & \\
\hline & 3.0 & & \\
\hline & 0.8 & & \\
\hline & 1.7 & & \\
\hline & -0.3 & & \\
\hline & 0 & & \\
\hline & & & \\
\hline & 0 & & \\
\hline & -1.4 & & \\
\hline & & & \\
\hline & -2.5 & & \\
\hline & -1.5 & & \\
\hline & & & \\
\hline & -2.9 & & \\
\hline & -3.6 & & \\
\hline & -4.2 & & -0 \\
\hline & -5.23 & -0.2 & 6000 \\
\hline & -6.43 & & 9000 \\
\hline & -6.61 & & \\
\hline & -5.5 & & \\
\hline & & & \\
\hline & & & \\
\hline & & & \\
\hline & & & \\
\hline $\mathrm{C}$ & & & \\
\hline C & & & \\
\hline & -5.1 & & \\
\hline & -4. & & \\
\hline & -4.0 & & \\
\hline & -4.9 & & 2.6 \\
\hline & & & 2.7 \\
\hline & & & 1.7 \\
\hline $\mathrm{C}$ & -2.0 & & \\
\hline & & & 2.3 \\
\hline & & & 1.1 \\
\hline $\mathrm{C}$ & & & \\
\hline $\mathrm{C}$ & & & 1.12 \\
\hline & & & \\
\hline & 4.865355000 & 3.383399000 & 2.93338 \\
\hline
\end{tabular}

\begin{tabular}{|c|c|c|}
\hline & & \\
\hline . & 000 & \\
\hline 000 & 000 & 000 \\
\hline 0004000 & 9000 & \\
\hline & 000 & 20( \\
\hline & 364000 & \\
\hline & & \\
\hline PO & 228000 & -2 \\
\hline 000 & 9822000 & \\
\hline 186053000 & -5.266275000 & -1.5 \\
\hline 9000 & 00 & 2000 \\
\hline 7000 & 6000 & 000 \\
\hline 1000 & & 000 \\
\hline 000 & 00 & 000 \\
\hline 6000 & 6739000 & 9000 \\
\hline 6.951062000 & & 56000 \\
\hline 7.815228000 & -0.972957000 & 98000 \\
\hline 6.223283000 & -2.806655000 & 47000 \\
\hline & & \\
\hline & & \\
\hline 7000 & & \\
\hline $\mathrm{H}$ & & \\
\hline$T$ & & \\
\hline $\mathrm{H}$ & & \\
\hline $\mathrm{H}$ & & \\
\hline 0 & & \\
\hline & & \\
\hline & & \\
\hline & & \\
\hline $\mathrm{H}$ & & \\
\hline & & \\
\hline & & \\
\hline 00 & & \\
\hline 00 & & \\
\hline 00 & & \\
\hline 7000 & & 7000 \\
\hline 7000 & & 000 \\
\hline 5000 & & 000 \\
\hline 7000 & & 000 \\
\hline 1000 & & 0000 \\
\hline 3000 & -4.49086 & 7000 \\
\hline 3000 & -5.42460 & 0000 \\
\hline & & 2000 \\
\hline & & 000 \\
\hline & & \\
\hline & & \\
\hline & & \\
\hline & & \\
\hline & & \\
\hline & & \\
\hline & & \\
\hline & & \\
\hline & & \\
\hline & & \\
\hline & & \\
\hline & & -4 \\
\hline & & \\
\hline & & 000 \\
\hline & & 2000 \\
\hline 000 & & 000 \\
\hline 1000 & & 000 \\
\hline 2000 & & 5000 \\
\hline 2000 & 5000 & 4.9 \\
\hline 55000 & 938904000 & 4.6 \\
\hline 000 & .415169000 & 3600 \\
\hline ה & -5.814179000 & -4.23676000 \\
\hline 2780260 & -4.727408000 & -4.54578300 \\
\hline 4.033203000 & -6.271034000 & -3.6935870 \\
\hline
\end{tabular}




$\begin{array}{llll}\mathrm{C} & 1.041221000 & -5.301517000 & 0.719551000 \\ \mathrm{H} & 0.665938000 & -4.563356000 & 1.449630000 \\ \mathrm{H} & 0.171856000 & -5.774553000 & 0.229289000 \\ \mathrm{H} & 1.558068000 & -6.095231000 & 1.292777000\end{array}$

\section{$\mathbf{M e - H +}$ 4- $\boldsymbol{\beta}-\mathbf{T}$}

Zero-point correction $=\quad 1.005757($ Hartree/Particle $)$ $\begin{array}{lc}\text { Thermal correction to Energy= } & 1.070598 \\ \text { Thermal correction to } & 1.071542\end{array}$ Thermal correction to Enthalpy= $\quad 1.071542$

Thermal correction to Gibbs Free Energy $=0.897108$ Sum of electronic and zero-point Energies $=\quad-2599.912211$ Sum of electronic and thermal Energies $=\quad-2599.847370$ Sum of electronic and thermal Enthalpies $=\quad-2599.846426$ Sum of electronic and thermal Free Energies $=-2600.020860$ E solvent $=-2599.76034464$

\begin{tabular}{|c|c|c|c|}
\hline $\mathrm{Ni}$ & 1.264941000 & -0.112590000 & -0.919353000 \\
\hline $\mathrm{C}$ & 2.373513000 & -0.138958000 & -2.461765000 \\
\hline $\mathrm{N}$ & 2.481546000 & -0.638585000 & 0.384864000 \\
\hline $\mathrm{C}$ & 2.124122000 & -0.772277000 & 1.645091000 \\
\hline $\mathrm{C}$ & 0.798527000 & -0.673878000 & 2.209563000 \\
\hline $\mathrm{C}$ & -0.364728000 & -0.281166000 & 1.446054000 \\
\hline $\mathrm{O}$ & -0.301051000 & 0.072437000 & 0.193736000 \\
\hline $\mathrm{C}$ & 1.079340000 & 0.237751000 & -3.106996000 \\
\hline $\mathrm{H}$ & 0.636882000 & -0.599181000 & -3.681097000 \\
\hline $\mathrm{C}$ & 1.031863000 & 1.561758000 & -3.916729000 \\
\hline $\mathrm{C}$ & 1.888927000 & 1.409928000 & -5.1 \\
\hline $\mathrm{H}$ & 1.477677000 & 2.353585000 & 5000 \\
\hline $\mathrm{C}$ & -0.415342000 & 1.955463000 & -4.2 \\
\hline $\mathrm{H}$ & 0.261263000 & 0.393131000 & -2.266590000 \\
\hline $\mathrm{H}$ & 3.159939000 & 0.632797000 & -2.432026000 \\
\hline $\mathrm{H}$ & 2.757574000 & -1.148222000 & 1000 \\
\hline $\mathrm{H}$ & 2.923784000 & -1.03 & 5000 \\
\hline $\mathrm{C}$ & 0.654757000 & -1.009178000 & 4000 \\
\hline $\mathrm{H}$ & 1.560937000 & -1.282 & 000 \\
\hline $\mathrm{C}$ & -0.584460000 & -1.028390000 & 4.249751000 \\
\hline $\mathrm{C}$ & -0.668644000 & -1.407553000 & 5000 \\
\hline $\mathrm{H}$ & -0.114045000 & -0.6 & 7000 \\
\hline $\mathrm{H}$ & -0.236212000 & 360000 & 5000 \\
\hline $\mathrm{H}$ & -1.713903000 & -1.424328000 & 6.064698000 \\
\hline $\mathrm{C}$ & -1.716096000 & 325000 & 3.504994000 \\
\hline $\mathrm{H}$ & -2.702691000 & -0.70 & 3.9864 \\
\hline $\mathrm{C}$ & -1.644425000 & -0.30 & 8000 \\
\hline $\mathrm{C}$ & -2.863742000 & 0.050592000 & 1.491329000 \\
\hline $\mathrm{N}$ & -2.950673000 & 0.573324000 & 0.275036000 \\
\hline $\mathrm{H}$ & -3.809759000 & 407000 & 2.027460000 \\
\hline $\mathrm{C}$ & -4.170148000 & 27000 & -0.417499000 \\
\hline $\mathrm{C}$ & -5.211690000 & -0.084378000 & 53000 \\
\hline $\mathrm{C}$ & -6.374232000 & 0.266335000 & -1.216319000 \\
\hline $\mathrm{C}$ & -6.490862000 & 1.507718000 & -1.854 \\
\hline $\mathrm{C}$ & -5.452600000 & 2.441581000 & -1.763263000 \\
\hline $\mathrm{C}$ & -4.280989000 & 2.160375000 & -1.028159000 \\
\hline $\mathrm{C}$ & -3.260046000 & 3.236532000 & -0.862198000 \\
\hline $\mathrm{C}$ & -2.803166000 & 3.615951000 & 0.419094000 \\
\hline $\mathrm{H}$ & -2.109395000 & 6.828594000 & -3.118703000 \\
\hline $\mathrm{C}$ & -1.518872000 & 5.422458000 & -0.551813000 \\
\hline $\mathrm{C}$ & -1.529736000 & 243000 & -3.050621000 \\
\hline $\mathrm{C}$ & -1.956510000 & 5.073371000 & -1.846196000 \\
\hline $\mathrm{C}$ & -2.826286000 & 76000 & -1.986157000 \\
\hline $\mathrm{C}$ & -5.139130000 & -1.452397000 & 0.104349000 \\
\hline $\mathrm{C}$ & -4.127433000 & -2.369037000 & -0.256580000 \\
\hline $\mathrm{C}$ & -4.117340000 & -3.675739000 & 0.264411000 \\
\hline $\mathrm{C}$ & -5.127874000 & -4.047908000 & 1.174715000 \\
\hline $\mathrm{C}$ & -6.151843000 & -3.159203000 & 1.552472000 \\
\hline $\mathrm{C}$ & -6.144237000 & -1.859614000 & 1.006834000 \\
\hline $\mathrm{C}$ & -7.250094000 & -3.594734000 & 2.501472000 \\
\hline $\mathrm{C}$ & -3.062942000 & -4.676047000 & -0.164217000 \\
\hline $\mathrm{C}$ & -1.932793000 & 4.709601000 & 0.590045000 \\
\hline & -1459794000 & 5110409000 & \\
\hline
\end{tabular}

\begin{tabular}{|c|c|c|}
\hline & & \\
\hline & 00 & \\
\hline 7200 & 58673000 & 1000 \\
\hline & .442817000 & 0 \\
\hline & & \\
\hline 000 & & \\
\hline & 60048 & \\
\hline 000 & 173510000 & \\
\hline 000 & -0.035 & 9000 \\
\hline $6350^{\circ}$ & 0000 & 1000 \\
\hline 5.7108 & -2.176116000 & 6000 \\
\hline 4.3298 & -2.027313000 & 000 \\
\hline 3.895476000 & -0.833217000 & 000 \\
\hline 3.4240 & -3.149278000 & 2423000 \\
\hline 3.513501000 & -3.672344000 & -2.233940000 \\
\hline 2.738629000 & -4.772993000 & 2545000 \\
\hline 1.8682 & -5.363 & \\
\hline 1.7721 & & \\
\hline 2.54 & -3.768 & \\
\hline 0.88 & & \\
\hline 2.851 & -5.32 & \\
\hline & 1. & \\
\hline 0 & & \\
\hline 0 & 4. & \\
\hline & & \\
\hline 2.8 & 5 & \\
\hline 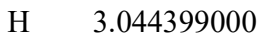 & & \\
\hline 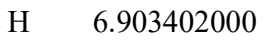 & & \\
\hline$T$ & -1.3 & \\
\hline 0 & & \\
\hline 00 & -3.2 & \\
\hline 00 & -4.6 & \\
\hline 00 & -6.2 & \\
\hline & -5.5 & \\
\hline 00 & -6.226 & \\
\hline 00 & -6.0 & \\
\hline 00 & $-4.8^{\prime}$ & \\
\hline 00 & -6.358 & 52000 \\
\hline 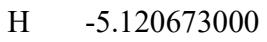 & -5.06 & \\
\hline 00 & 5000 & 000 \\
\hline 4000 & -4.041141000 & 86000 \\
\hline 2000 & -4.356702000 & \\
\hline & 94000 & \\
\hline & 43000 & \\
\hline & -5 & \\
\hline 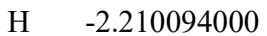 & -4.1 & \\
\hline & -0 . & \\
\hline & & \\
\hline & & \\
\hline & 0 & \\
\hline & 0 & \\
\hline & 0 & \\
\hline & 0 & \\
\hline & 4.8 & 2. \\
\hline 0 & 9000 & 000 \\
\hline & -2.068 & 408000 \\
\hline 000 & 8000 & 000 \\
\hline-0.851 & 2000 & 26000 \\
\hline-0.518 & 94000 & 7000 \\
\hline 5.460 & 3.336209000 & 000 \\
\hline 6.177 & 4.168937000 & 1000 \\
\hline 4.7255 & 3.658914000 & 000 \\
\hline 6.018 & 2.477079000 & 68000 \\
\hline-0.437 & 2.885086000 & -4.8 \\
\hline & 1.166 & \\
\hline & 2.12 & .7500 \\
\hline & 2.3503210 & -5.767814000 \\
\hline 2.934987000 & 1.152622000 & -4.94689100 \\
\hline
\end{tabular}


$\begin{array}{lrrr}\mathrm{H} & 1.485762000 & 0.615174000 & -5.844588000 \\ \mathrm{H} & -2.034313000 & 0.733410000 & -0.185219000\end{array}$

\section{Me-H+TS-1 Transf-1}

Zero-point correction-

0.998653 (Hartree/Particle)

Thermal correction to Enthalpy=

1.064952

Thermal correction to Gibbs Free Energy $=0.887082$ Sum of electronic and zero-point Energies $=\quad-2599.853388$

Sum of electronic and thermal Energies $=\quad-2599.787089$

Sum of electronic and thermal Enthalpies $=-2599.786145$

Sum of electronic and thermal Free Energies $=-2599.964959$

E solvent $=-2599.70380233$

\begin{tabular}{|c|c|c|c|}
\hline & U & & \\
\hline & -2.561284000 & 1027000 & 000 \\
\hline & 120000 & 8000 & 3000 \\
\hline & -4.764393000 & -0.714177000 & 3000 \\
\hline & -4.580256000 & 416623000 & -0.826296000 \\
\hline & -5.940881000 & 297474000 & -1.1 \\
\hline & -6.707024000 & 0.201287000 & -0.770033000 \\
\hline & -6.123835000 & -0.780034000 & 3000 \\
\hline & -6.714881000 & 8861000 & 0.372033000 \\
\hline & -7.759885000 & 0.122713000 & 9000 \\
\hline & -6.400224000 & & 5000 \\
\hline & 914000 & & \\
\hline & & & \\
\hline & & & \\
\hline & & & \\
\hline & -4.6 & & \\
\hline & -3.5 & -3.9 & \\
\hline & & -4.7 & \\
\hline & -2.7 & -3.6 & \\
\hline & -3.1 & & \\
\hline & -2.5 & & \\
\hline & & & \\
\hline & & & \\
\hline & & & \\
\hline & -2.7 & & \\
\hline & -2.2 & & 000 \\
\hline & & & -0 \\
\hline & -3.2 & & -0.2 \\
\hline & -3.1( & & 000 \\
\hline & -2.22 & & \\
\hline & -3.046 & & \\
\hline & -0.9 & & \\
\hline & & & \\
\hline & & & \\
\hline & & & \\
\hline & & & \\
\hline & & & \\
\hline & & & \\
\hline & & & \\
\hline & & & 00 \\
\hline & & & \\
\hline & & & -2.1 \\
\hline & & & -2.5 \\
\hline & & & -2.4 \\
\hline & & & -1.4 \\
\hline & & & 9000 \\
\hline & -0.7 & & -3.7 \\
\hline & & 0.4 & 000 \\
\hline & & & 2000 \\
\hline & & 000 & 000 \\
\hline & -2.4 & 000 & -2 \\
\hline & -2.5507 & -1.217613000 & -3 . \\
\hline & -1.340279000 & -2.548981000 & -2.492948000 \\
\hline & 303000 & -1.845293000 & \\
\hline & -0.484884000 & -2 & $-3.1 / 8005000$ \\
\hline
\end{tabular}

C

C $\quad 3.125668000$

C $\quad 3.054808000$

$\mathrm{H} \quad 3.467712000$

H $\quad 3.371447000$

C $\quad 2.325943000$

C $\quad 2.273799000$

C $\quad 1.907578000$

H 1.294734000

C $\quad 5.037112000$

5.977386000

3.990493000

6.790414000

3.268232000

5.885386000

3.877721000

4.824980000

4.737675000

2.083384000

2.780314000

1.943649000

2.376051000

3.160594000

6.913668000

6.456159000

7.417722000

7.698727000

1.946761000

2.525246000

0.878262000

2.143632000

1.889271000

0.920949000

2.642873000

1.819893000

$-1.537011000$

$-1.012406000$

$-1.837361000$

$-0.812398000$

$-5.490483000$

$-6.268971000$

$-4.873384000$

$-6.008869000$

$-3.497674000$

$-2.595587000$

$-3.650826000$

$-4.356755000$

$-1.978198000$

$-2.696175000$

$-1.597725000$

H $\quad-1.135753000$
$-3.187101000$ 0.344727000 0.229285000 1.319275000 $-0.439170000$ $-0.266156000$ $-0.250890000$ $-0.495209000$ $-0.535352000$ $-1.565795000$ 0.462644000 $-1.557511000$ 0.398091000 $-2.356270000$ 1.167158000 $-0.590096000$ $-0.609410000$ $-2.693226000$ $-3.041053000$ $-3.502599000$ $-2.416623000$ $-3.269194000$ $-4.169038000$ $-4.651737000$ $-4.959709000$ $-5.850781000$ 1.588267000 1.750035000 2.526642000 1.020631000 2.423185000 2.824243000 3.612798000 3.738409000 4.577039000 $-0.542851000$ 4.644348000 4.253920000 4.968026000 5.550626000 3.004101000 3.400764000 2.053951000 3.721779000 $-4.546968000$ $-5.424937000$ $-4.817219000$ $-3.723764000$ $-5.569940000$ $-6.066405000$ $-6.369782000$ $-5.025115000$ $-4.496697000$ $-4.830397000$ $-5.409137000$ $-3.953397000$ $-3.465728000$ $-4.217047000$ $-3.882448000$ $-2.570155000$ 4.827065000 5.379489000 3.988719000 5.514931000 5.596575000 6.335640000 5.009994000 6.167453000
$-1.606527000$ 5.712010000 6.109337000 6.061336000 6.171857000 1.396756000 2.006289000 0.099756000 $-0.577496000$ $-1.532453000$ $-0.315980000$ $-2.251994000$ $-1.039088000$ $-2.978601000$ $-0.852726000$ $-2.007154000$ $-2.565697000$ $-1.761697000$ $-3.080954000$ $-0.693147000$ $-3.915678000$ 0.330416000 $-3.341574000$ $-0.926549000$ $-2.250998000$ $-2.441536000$ 0.655467000 1.695816000 0.527037000 1.801774000 $-0.292431000$ 2.602855000 1.415176000 2.451078000 3.154611000 $-0.444972000$ 1.246559000 0.641437000 2.222096000 0.737644000 3.701332000 4.624660000 3.948392000 3.395352000 $-4.759839000$ $-5.104711000$ $-4.836658000$ $-5.467677000$ 0.216273000 0.031420000 0.349804000 1.173757000 1.560232000 2.473133000 1.009435000 0.928638000 4.577055000 4.342591000 5.392173000 4.961708000 $-4.396379000$ $-4.713020000$ $-5.097815000$ $-4.515503000$ 0.392456000 0.797197000 1.246937000 $-0.036264000$ 


\section{Me-H+ 1 -transf-1}

Zero-point correction $=1.000992($ Hartree/Particle $)$

$\begin{array}{lc}\text { Thermal correction to Energy= } & 1.066485 \\ \text { Thermal correction to Enthalpy= } & 1.067430 \\ \text { Thermal correction to Gibbs Free Energy= } & 0.893793 \\ \text { Sum of electronic and zero-point Energies }= & -2599.862297 \\ \text { Sum of electronic and thermal Energies }= & -2599.796803 \\ \text { Sum of electronic and thermal Enthalpies }= & -2599.795859 \\ \text { Sum of electronic and thermal Free Energies }= & -2599.969496\end{array}$

E solvent $=-2599.71328016$

\begin{tabular}{|c|c|c|c|}
\hline & 1.324829000 & 35546000 & \\
\hline $\mathrm{N}$ & 2.550932000 & -0.560186000 & \\
\hline & 3.990229000 & -0.418939000 & 0.273077000 \\
\hline & 4.622325000 & 0.766704000 & 0. \\
\hline & 4.746800000 & -1.419756000 & -0.399017000 \\
\hline & 6.123853000 & -1.200573000 & \\
\hline & 6.752946000 & -0.030645000 & 77263000 \\
\hline & 6.006245000 & & \\
\hline & & & \\
\hline & & & \\
\hline & 6.701574000 & & 6000 \\
\hline & & & \\
\hline & & & \\
\hline & 3.28 & & \\
\hline & 3.2 & & \\
\hline & 2.74 & & \\
\hline & 2.8 & & \\
\hline & 2.43 & & \\
\hline & 3.53 & & \\
\hline & 4.06 & & \\
\hline & 4.58 & & \\
\hline & 4.32 & & \\
\hline & 4.78 & & \\
\hline & 3.87 & & 000 \\
\hline & 3.27 & & \\
\hline & 2.91 & & -2 \\
\hline 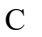 & 3.13 & -4.8 & -0.4 \\
\hline$C$ & 3.58 & & \\
\hline & 3.5 & & \\
\hline$C$ & $2.0^{7}$ & & \\
\hline & & & \\
\hline & & & \\
\hline & & & \\
\hline & & & \\
\hline & & & \\
\hline & & & \\
\hline & & & \\
\hline & & & \\
\hline & & & \\
\hline U & & & \\
\hline & & & \\
\hline & & & \\
\hline & & & \\
\hline & & & \\
\hline$\theta$ & & & \\
\hline$C$ & -1.17 & -0.9 & 000 \\
\hline $\mathrm{H}$ & & & 0000 \\
\hline $\mathrm{H}$ & -1.6 & & 6000 \\
\hline $\mathrm{H}$ & 000 & 000 & 6000 \\
\hline $\mathrm{H}$ & -1.32 & 000 & 1000 \\
\hline $\mathrm{C}$ & $0.8 \mathrm{~s}$ & 000 & 000 \\
\hline $\mathrm{H}$ & -0.18 & & -2 \\
\hline$C$ & & & \\
\hline- & & & \\
\hline $\mathrm{H}$ & & 000 & -0.3 \\
\hline $\mathrm{H}$ & & 2.01 & -1.464921000 \\
\hline & -1.035731000 & -2.331960000 & 5.14534500 \\
\hline
\end{tabular}

$-2.082378000$ $-0.768452000$ $-0.398699000$

$-2.914647000$

$-3.878078000$

$-2.947277000$

$-4.120558000$

$-4.062778000$

$-5.287495000$

$-5.194264000$

$-6.399562000$

$-5.161401000$

$-7.296639000$

$-6.356089000$

$-7.229915000$

$-2.888125000$

$-2.387156000$

$-2.331861000$

$-2.813512000$

$-2.743762000$

$-1.358008000$

$-1.303771000$

$-0.829647000$

$-0.034188000$

$-5.401857000$

$-6.381609000$

$-4.588947000$

$-7.017341000$

$-3.845943000$

$-6.555269000$

$-4.741720000$

$-5.722405000$

$-5.847555000$

$-2.008989000$

$-3.884173000$

$-3.174632000$

$-3.299728000$

$-4.504929000$

$-7.632571000$

$-7.370084000$

$-7.809538000$

$-8.595888000$

$-0.835801000$

$-1.038812000$

0.258748000

$-1.304201000$

$-0.726611000$

0.250599000

$-0.561327000$

$-1.397018000$

3.699339000

2.788918000

4.526483000

3.932192000

2.077846000

1.374987000

1.519327000

2.828086000

4.054029000

3.180804000

4.202554000

4.937750000

2.538974000

3.257704000

2.260882000

1.636786000
$-2.257269000$

$-3.404686000$

$-1.857935000$

$-0.035369000$

$-0.178083000$

0.593310000

0.990898000

2.211111000

0.173220000

2.589021000

0.620894000

3.534850000

$-0.006752000$

1.810259000

2.129800000

3.130397000

3.663204000

3.573500000

3.324096000

3.207396000

4.622150000

4.535810000

5.044803000

5.801671000

$-1.152886000$

$-1.334169000$

$-2.246539000$

$-0.487533000$

$-2.120017000$

$-2.583431000$

$-3.503642000$

$-3.649864000$

$-4.626602000$

0.771961000

$-4.683040000$

$-4.409981000$

$-5.075123000$

$-5.518218000$

$-2.776469000$

$-3.581730000$

$-1.853092000$

$-3.055359000$

5.201308000

6.286640000

5.073890000

4.722246000

5.017663000

4.541950000

6.109714000

4.785083000

5.716335000

6.325063000

6.251260000

5.695362000

2.635205000

3.455046000

1.683285000

2.612999000

$-4.713925000$

$-5.271907000$

$-3.828030000$

$-5.367123000$

$-5.898667000$

$-6.708201000$

$-5.424313000$

$-6.380071000$
5.484560000

5.118696000

5.914442000

1.159987000

1.660185000

$-0.011371000$

$-0.728691000$

$-1.459584000$

$-0.737304000$

$-2.213750000$

$-1.478854000$

$-2.764310000$

$-1.494694000$

$-2.218093000$

$-2.795467000$

$-1.428459000$

$-2.639141000$

$-0.207814000$

$-3.591381000$

0.740059000

$-2.644161000$

$-0.182454000$

$-1.406915000$

$-1.397945000$

$-0.048579000$

0.949078000

$-0.420524000$

1.237967000

$-1.217584000$

1.581358000

0.191864000

1.195610000

1.681651000

$-0.420875000$

$-0.221244000$

$-1.020853000$

0.631946000

$-0.594794000$

2.629587000

3.337166000

3.208406000

2.161114000

$-3.943595000$

$-4.006758000$

$-4.031085000$

$-4.819979000$

1.133372000

1.344685000

1.122807000

1.978617000

1.051310000

1.195006000

1.556079000

$-0.027236000$

4.754514000

4.982572000

4.800779000

5.568243000

$-4.166838000$

$-4.548816000$

$-4.808061000$

$-4.299403000$

0.534838000

0.764407000

1.491939000

0.116187000 
Zero-point correction $=$

Thermal correction to Energy=

Thermal correction to Enthalpy=

0.922673 (Hartree/Particle)

0.983299

0.984244

Thermal correction to Gibbs Free Energy= 0.819064

Sum of electronic and zero-point Energies $=\quad-2482.060957$

Sum of electronic and thermal Energies $=\quad-2482.000331$

Sum of electronic and thermal Enthalpies $=\quad-2481.999387$

Sum of electronic and thermal Free Energies $=-2482.164567$

E solvent $=-2481.87673693$

C

1.253643000

$-1.120209000$

0.334802000

2.534811000

2.242290000

0.955953000

$-0.205861000$

$-0.218127000$

1.721592000

2.446348000

1.860360000

2.234225000

$-0.056373000$

3.030078000

0.850913000

1.755883000

$-0.340771000$

$-0.376823000$

$-1.405520000$

0.234519000

0.022008000

$-1.471522000$

$-2.426790000$

$-1.443444000$

$-2.674902000$

$-2.835683000$

$-3.575305000$

$-4.084079000$

$-5.244408000$

$-6.439514000$

$-6.486839000$

$-5.331701000$

$-4.113135000$

$-2.942129000$

$-2.198976000$

$-0.868541000$

$-1.591770000$

$-2.623681000$

$-5.277439000$

$-4.466660000$

$-4.556791000$

$-5.462927000$

$-6.289993000$

$-6.184012000$

$-1.165071000$

4.734865000

3.991774000

4.049024000

4.845804000

5.586542000

5.542225000

4.987873000

6.294857000

6.522528000

5.436979000

4.107314000

3.890879000

3.008576000

3.049961000

2.080928000
$-2.723040000$

0.226165000

1.505466000

2.083697000

1.301475000

$-0.003144000$

$-3.213091000$

$-2.340027000$

$-3.920001000$

$-3.548367000$

$-2.675506000$

2.221410000

3.490242000

4.055962000

4.202304000

5.714678000

6.097191000

6.168457000

6.085139000

3.446294000

3.954430000

2.024941000

1.370071000

0.058456000

1.982135000

$-0.621011000$

$-0.209250000$

$-0.925691000$

$-2.028785000$

$-2.431137000$

$-1.734410000$

$-2.178260000$

$-1.273836000$

$-3.102273000$

$-4.032991000$

$-3.554411000$

0.906089000

0.889672000

1.915197000

2.971909000

3.013863000

1.970947000

$-1.724956000$

3.097823000

1.900161000

1.037640000

1.347560000

2.549078000

3.435742000

0.407935000

0.014172000

$-0.885506000$

$-1.413712000$

$-1.056637000$

$-0.133168000$

$-1.652553000$

$-3.034775000$

$-3.640937000$
0.095729000

$-0.408522000$

0.619950000

0.614908000

0.292021000

$-0.057770000$

$-0.161766000$

$-0.214654000$

0.150861000

0.619629000

$-1.131020000$

$-1.433708000$

0.898545000

0.350840000

0.611215000

0.094070000

0.162858000

0.055058000

$-0.639263000$

1.124542000

$-0.218994000$

$-0.404291000$

$-0.299928000$

$-0.591419000$

$-0.737248000$

$-0.706495000$

$-0.914127000$

$-0.196351000$

$-0.411339000$

$-1.273177000$

$-1.951061000$

$-1.801068000$

$-2.613427000$

$-3.404590000$

$-3.499386000$

$-2.670894000$

0.803176000

1.959151000

2.919321000

2.695908000

1.557437000

0.615003000

$-4.247949000$

$-3.131480000$

$-3.166315000$

$-2.056529000$

$-0.932870000$

$-0.935351000$

$-2.029357000$

0.224365000

0.586976000

1.634204000

2.340626000

2.023780000

0.958868000

2.843862000

3.145718000

3.966675000
$-4.273202000$
1.050572000 0.986136000 1.963034000 6.201346000 4.686994000 3.477745000 7.135888000 7.545012000 5.608534000 3.852714000 0.287538000

$-5.531066000$

$-6.812215000$

$-7.332224000$

$-7.425719000$

$-5.367153000$

$-3.186158000$

$-2.460430000$

$-3.774743000$

1.931329000

$-0.060713000$

$-1.954250000$

$-3.722379000$

$-2.825458000$

$-3.393604000$

$-4.302790000$

$-7.285696000$

$-8.256753000$

$-6.930668000$

$-7.482943000$

$-1.280614000$

$-1.884724000$

$-0.219940000$

$-1.505672000$

$-0.418199000$

$-0.923343000$

$-0.360150000$

0.609943000

3.169568000

2.423830000

2.633632000

3.811599000

6.361596000

7.348840000

5.859944000

6.549172000

2.153364000

1.153149000

2.795501000

2.573704000

$-0.093039000$

0.172467000

$-0.243191000$

$-1.059138000$

$-0.410366000$

$-2.835958000$ $-1.454403000$ $-0.876762000$ 2.800444000 3.782089000 0.100890000 0.411512000 $-1.174829000$ $-2.094127000$ $-3.650485000$ $-3.295175000$ 3.780560000 1.988127000 $-0.620563000$ $-2.573983000$ $-3.274660000$ $-4.260379000$ $-0.209136000$ 0.054129000 0.201607000 $-3.462348000$ $-0.488380000$ 1.872170000

1.241143000

2.882068000

1.454901000

4.138853000

3.895768000 5.079901000

4.326471000

$-5.514302000$

$-6.005438000$

$-5.694823000$

$-6.027698000$ $-0.756254000$ $-0.650167000$ 0.251550000

$-1.103329000$

1.542847000

0.761396000

2.422864000 


\begin{tabular}{|c|c|c|c|}
\hline C & 4.669287000 & 1.851659000 & -0.768514000 \\
\hline$C$ & 4.033813000 & 1.428026000 & -1.955304000 \\
\hline $\mathrm{H}$ & 3.575773000 & 0.432697000 & -1.995005000 \\
\hline $\mathrm{C}$ & 5.247407000 & 3.975352000 & -1.868944000 \\
\hline $\mathrm{C}$ & 2.769394000 & -1.079234000 & 3.035127000 \\
\hline $\mathrm{C}$ & 4.601885000 & 3.520919000 & -3.034188000 \\
\hline $\mathrm{C}$ & 3.678376000 & 0.364975000 & 1.105558000 \\
\hline $\mathrm{C}$ & 3.999225000 & 2.248084000 & -3.097653000 \\
\hline $\mathrm{C}$ & 4.784688000 & 0.977566000 & 0.443378000 \\
\hline $\mathrm{C}$ & 1.626835000 & -0.362456000 & 3.453811000 \\
\hline $\mathrm{H}$ & 1.520718000 & 0.693768000 & 3.184727000 \\
\hline $\mathrm{C}$ & 0.797177000 & -2.316880000 & 4.624772000 \\
\hline $\mathrm{C}$ & 5.270771000 & 3.129013000 & -0.742796000 \\
\hline $\mathrm{H}$ & 5.757745000 & 3.472242000 & 0.178810000 \\
\hline $\mathrm{C}$ & 0.641071000 & -0.965716000 & 4.260422000 \\
\hline $\mathrm{C}$ & 5.198423000 & -0.619232000 & 2.740990000 \\
\hline $\mathrm{H}$ & 5.349903000 & -1.211070000 & 3.649994000 \\
\hline $\mathrm{C}$ & 5.919469000 & 5.333531000 & -1.832317000 \\
\hline $\mathrm{H}$ & 6.954421000 & 5.275728000 & -2.220785000 \\
\hline $\mathrm{H}$ & 5.979363000 & 5.731381000 & -0.804361000 \\
\hline $\mathrm{H}$ & 5.381568000 & 6.069945000 & -2.454865000 \\
\hline $\mathrm{C}$ & 1.928146000 & -3.062211000 & 4.231331000 \\
\hline $\mathrm{C}$ & 6.291971000 & -0.031362000 & 2.095928000 \\
\hline $\mathrm{H}$ & 7.306114000 & -0.185432000 & 2.479600000 \\
\hline $\mathrm{C}$ & 3.350893000 & 1.767778000 & -4.381219000 \\
\hline $\mathrm{H}$ & 4.112049000 & 1.461603000 & -5.124160000 \\
\hline $\mathrm{H}$ & 2.745585000 & 2.563290000 & -4.851979000 \\
\hline $\mathrm{H}$ & 2.693722000 & 0.898598000 & -4.204010000 \\
\hline $\mathrm{C}$ & 2.903939000 & -2.428821000 & 3.439777000 \\
\hline $\mathrm{H}$ & 3.787405000 & -2.996630000 & 3.121341000 \\
\hline $\mathrm{C}$ & 6.080964000 & 0.761727000 & 0.963153000 \\
\hline $\mathrm{H}$ & 6.931040000 & 1.216052000 & 0.443122000 \\
\hline $\mathrm{C}$ & 2.096006000 & -4.502740000 & 4.672964000 \\
\hline $\mathrm{H}$ & 2.406469000 & -4.559192000 & 5.733739000 \\
\hline $\mathrm{H}$ & 2.864517000 & -5.025243000 & 4.077711000 \\
\hline $\mathrm{H}$ & 1.150881000 & -5.067927000 & 4.584780000 \\
\hline $\mathrm{C}$ & -0.544715000 & -0.162606000 & 4.758202000 \\
\hline $\mathrm{H}$ & -0.323990000 & 0.309603000 & 5.734800000 \\
\hline $\mathrm{H}$ & -1.435294000 & -0.799647000 & 4.901234000 \\
\hline $\mathrm{H}$ & -0.807957000 & 0.648533000 & 4.056366000 \\
\hline $\mathrm{N}$ & -3.026314000 & -0.038982000 & -0.727195000 \\
\hline $\mathrm{C}$ & -4.174538000 & -2.030026000 & -1.554871000 \\
\hline $\mathrm{C}$ & -5.563079000 & 0.901281000 & 0.582622000 \\
\hline $\mathrm{C}$ & -6.497863000 & 1.887214000 & 0.200630000 \\
\hline $\mathrm{H}$ & -7.089559000 & 1.741451000 & -0.711929000 \\
\hline $\mathrm{C}$ & -4.965165000 & 2.251493000 & 2.541401000 \\
\hline $\mathrm{C}$ & -2.949060000 & -2.518304000 & -2.254017000 \\
\hline $\mathrm{C}$ & -5.895034000 & 3.225267000 & 2.124364000 \\
\hline $\mathrm{C}$ & -4.233556000 & -0.799592000 & -0.841672000 \\
\hline $\mathrm{C}$ & -6.676878000 & 3.057376000 & 0.965374000 \\
\hline $\mathrm{C}$ & -5.440969000 & -0.348055000 & -0.234743000 \\
\hline $\mathrm{C}$ & -2.541692000 & -3.860953000 & -2.081117000 \\
\hline $\mathrm{H}$ & -3.085961000 & -4.499052000 & -1.373905000 \\
\hline $\mathrm{C}$ & -0.751739000 & -3.544933000 & -3.678443000 \\
\hline $\mathrm{C}$ & -4.802649000 & 1.094384000 & 1.756251000 \\
\hline $\mathrm{H}$ & -4.090435000 & 0.322206000 & 2.072595000 \\
\hline $\mathrm{C}$ & -1.447085000 & -4.390721000 & -2.790949000 \\
\hline $\mathrm{C}$ & -5.349785000 & -2.806825000 & -1.639050000 \\
\hline $\mathrm{H}$ & -5.316082000 & -3.745438000 & -2.201859000 \\
\hline $\mathrm{C}$ & -4.180088000 & 2.437372000 & 3.824203000 \\
\hline $\mathrm{H}$ & -4.793077000 & 2.174712000 & 4.707510000 \\
\hline $\mathrm{H}$ & -3.280158000 & 1.798660000 & 3.847209000 \\
\hline $\mathrm{H}$ & -3.859948000 & 3.486344000 & 3.954917000 \\
\hline $\mathrm{C}$ & -1.135441000 & -2.203912000 & -3.881249000 \\
\hline $\mathrm{C}$ & -6.550024000 & -2.370334000 & -1.068319000 \\
\hline $\mathrm{H}$ & -7.455051000 & -2.980137000 & -1.158601000 \\
\hline $\mathrm{C}$ & -7.701010000 & 4.099107000 & 0.562210000 \\
\hline $\mathrm{H}$ & -8.652773000 & 3.950527000 & 1.107372000 \\
\hline
\end{tabular}

\begin{tabular}{|c|c|c|c|}
\hline $\mathrm{H}$ & 3258000 & 5.121299000 & 0.793359000 \\
\hline $\mathrm{H}$ & -7.931091000 & 4.049805000 & -0.516087000 \\
\hline $\mathrm{C}$ & -2.231548000 & -1.700080000 & -3.155668000 \\
\hline $\mathrm{H}$ & -2.558689000 & -0.668537000 & -3.330713000 \\
\hline $\mathrm{C}$ & -6.591445000 & -1.150038000 & -0.381799000 \\
\hline $\mathrm{H}$ & -7.520564000 & -0.813381000 & 0.089754000 \\
\hline $\mathrm{C}$ & -0.412218000 & -1.336647000 & -4.892459000 \\
\hline $\mathrm{H}$ & -0.849470000 & -1.455234000 & -5.902459000 \\
\hline $\mathrm{H}$ & -0.477438000 & -0.265783000 & -4.632294000 \\
\hline $\mathrm{H}$ & 0.655216000 & -1.608266000 & -4.969287000 \\
\hline $\mathrm{C}$ & -1.046843000 & -5.843538000 & -2.625179000 \\
\hline $\mathrm{H}$ & -1.592135000 & -6.488226000 & -3.341027000 \\
\hline $\mathrm{H}$ & 0.030658000 & -5.993695000 & -2.812354000 \\
\hline $\mathrm{H}$ & -1.276697000 & -6.216951000 & -1.612042000 \\
\hline $\mathrm{Ni}$ & 1.130274000 & -1.035179000 & 0.205128000 \\
\hline $\mathrm{C}$ & -2.926737000 & 1.286604000 & -0.787067000 \\
\hline $\mathrm{H}$ & -3.845850000 & 1.832393000 & -1.024858000 \\
\hline $\mathrm{C}$ & 1.933086000 & 1.778418000 & 0.517413000 \\
\hline $\mathrm{H}$ & 2.632238000 & 2.566992000 & 0.839879000 \\
\hline $\mathrm{C}$ & 0.647330000 & 2.258699000 & 0.062686000 \\
\hline $\mathrm{C}$ & 0.498478000 & 3.652164000 & -0.090537000 \\
\hline $\mathrm{C}$ & -0.477810000 & 1.387487000 & -0.180552000 \\
\hline $\mathrm{H}$ & 1.376348000 & 4.283175000 & 0.104529000 \\
\hline $\mathrm{C}$ & -0.705835000 & 4.273131000 & -0.492991000 \\
\hline $\mathrm{C}$ & -1.730377000 & 2.023658000 & -0.566562000 \\
\hline $\mathrm{C}$ & -0.789356000 & 5.775959000 & -0.660151000 \\
\hline $\mathrm{C}$ & -1.800150000 & 3.438798000 & -0.719695000 \\
\hline $\mathrm{H}$ & -2.760678000 & 3.877384000 & -1.020252000 \\
\hline $\mathrm{O}$ & -0.431718000 & 0.092551000 & -0.050085000 \\
\hline $\mathrm{C}$ & 2.774575000 & -2.171718000 & -0.528532000 \\
\hline $\mathrm{C}$ & 3.302202000 & -3.451034000 & -1.187368000 \\
\hline $\mathrm{H}$ & 2.664356000 & -1.393568000 & -1.311289000 \\
\hline $\mathrm{H}$ & 3.463767000 & -1.844463000 & 0.262258000 \\
\hline $\mathrm{C}$ & 1.082745000 & -3.197550000 & 0.316039000 \\
\hline $\mathrm{C}$ & -0.124721000 & -2.492718000 & 786000 \\
\hline $\mathrm{C}$ & 4.712641000 & -3.207238000 & -1.765820000 \\
\hline $\mathrm{H}$ & 3.351011000 & -4.280974000 & -0.456973000 \\
\hline $\mathrm{H}$ & 2.627319000 & -3.777188000 & -2.002133000 \\
\hline $\mathrm{H}$ & 5.095173000 & -4.127736000 & -2.241997000 \\
\hline $\mathrm{H}$ & 4.704985000 & -2.409156000 & -2.529841000 \\
\hline $\mathrm{H}$ & 5.426255000 & -2.911570000 & -0.976474000 \\
\hline $\mathrm{H}$ & -0.925828000 & -2.545830000 & -0.184173000 \\
\hline $\mathrm{H}$ & -0.423301000 & -2.251083000 & 1.593382000 \\
\hline $\mathrm{H}$ & 1.676678000 & -3.557899000 & 1.164207000 \\
\hline $\mathrm{H}$ & 1.093621000 & -3.838180000 & -0.569531000 \\
\hline $\mathrm{H}$ & -0.524188000 & 6.302188000 & 0.275277000 \\
\hline $\mathrm{H}$ & -0.094304000 & 6.133817000 & -1.442085000 \\
\hline $\mathrm{H}$ & -1.806260000 & 6.092493000 & -0.946434000 \\
\hline $\mathrm{H}$ & 4.571426000 & 4.172101000 & -3.917981000 \\
\hline $\mathrm{H}$ & 0.101389000 & -3.946834000 & -4.241303000 \\
\hline $\mathrm{H}$ & -6.019986000 & 4.135589000 & 2.725610000 \\
\hline $\mathrm{H}$ & 0.028886000 & -2.796936000 & 5.245478000 \\
\hline $\mathrm{H}$ & -2.121649000 & -0.520777000 & -0.554807000 \\
\hline
\end{tabular}

\section{Me-H+TS-1 BHE-T $_{\text {B }}$}

Zero-point correction $=\quad 0.947829($ Hartree/Particle $)$

Thermal correction to Energy= $\quad 1.009299$

Thermal correction to Enthalpy $=\quad 1.010243$

Thermal correction to Gibbs Free Energy $=\quad 0.843821$

Sum of electronic and zero-point Energies $=\quad-2521.321438$

Sum of electronic and thermal Energies $=\quad-2521.259968$

Sum of electronic and thermal Enthalpies $=\quad-2521.259024$

Sum of electronic and thermal Free Energies $=-2521.425447$

E solvent $=-2521.1476959$

$\begin{array}{lrrr}\mathrm{Ni} & 1.304566000 & -0.661463000 & -0.785990000 \\ \mathrm{C} & 2.821694000 & -1.575012000 & -1.758958000 \\ \mathrm{~N} & 2.344541000 & 0.789408000 & 0.171965000 \\ \mathrm{C} & 1.789739000 & 1.558253000 & 1.080275000\end{array}$




\begin{tabular}{|c|c|c|c|}
\hline C & 0.428062000 & 1.521853000 & 1.564257000 \\
\hline $\mathrm{C}$ & -0.569680000 & 0.634187000 & 1.031426000 \\
\hline $\mathrm{O}$ & -0.344166000 & -0.212477000 & 0.065149000 \\
\hline $\mathrm{C}$ & 1.928327000 & -1.131209000 & -2.750023000 \\
\hline $\mathrm{H}$ & 0.507127000 & -1.808731000 & -1.226164000 \\
\hline $\mathrm{C}$ & 2.147237000 & 0.102029000 & -3.587644000 \\
\hline $\mathrm{H}$ & 1.206862000 & 0.651120000 & -3.772855000 \\
\hline $\mathrm{H}$ & 2.887014000 & 0.788910000 & -3.145044000 \\
\hline $\mathrm{H}$ & 2.525778000 & -0.214036000 & -4.581609000 \\
\hline $\mathrm{H}$ & 1.204263000 & -1.846744000 & -3.160126000 \\
\hline $\mathrm{H}$ & 3.748711000 & -1.022407000 & -1.576894000 \\
\hline $\mathrm{H}$ & 2.788409000 & -2.614807000 & -1.413087000 \\
\hline $\mathrm{H}$ & 2.427013000 & 2.321498000 & 1.558402000 \\
\hline $\mathrm{C}$ & 0.084396000 & 2.405412000 & 2.611025000 \\
\hline $\mathrm{H}$ & 0.867263000 & 3.075231000 & 2.992599000 \\
\hline $\mathrm{C}$ & -1.202783000 & 2.470732000 & 3.185661000 \\
\hline $\mathrm{C}$ & -1.506401000 & 3.436007000 & 4.312602000 \\
\hline $\mathrm{H}$ & -1.282358000 & 4.478631000 & 4.022049000 \\
\hline $\mathrm{H}$ & -0.901596000 & 3.211259000 & 5.210469000 \\
\hline $\mathrm{H}$ & -2.568543000 & 3.389813000 & 4.605494000 \\
\hline $\mathrm{C}$ & -2.175579000 & 1.612736000 & 2.662762000 \\
\hline $\mathrm{H}$ & -3.195391000 & 1.643337000 & 3.067947000 \\
\hline $\mathrm{C}$ & -1.902849000 & 0.704368000 & 1.603678000 \\
\hline $\mathrm{C}$ & -2.983976000 & -0.091177000 & 1.117506000 \\
\hline $\mathrm{N}$ & -2.888272000 & -0.966276000 & 0.122139000 \\
\hline $\mathrm{H}$ & -3.968777000 & 0.022314000 & 1.581499000 \\
\hline $\mathrm{C}$ & -3.944843000 & -1.717561000 & -0.477388000 \\
\hline $\mathrm{C}$ & -3.608702000 & -2.997974000 & -1.003065000 \\
\hline $\mathrm{C}$ & -4.624130000 & -3.746282000 & -1.634475000 \\
\hline $\mathrm{C}$ & -5.933999000 & -3.262425000 & -1.720207000 \\
\hline $\mathrm{C}$ & -6.245089000 & -1.999348000 & -1.198847000 \\
\hline $\mathrm{C}$ & -5.264687000 & -1.191473000 & -0.589264000 \\
\hline $\mathrm{C}$ & -5.669727000 & 0.186090000 & -0.160887000 \\
\hline $\mathrm{C}$ & -5.106907000 & 1.331979000 & -0.765864000 \\
\hline $\mathrm{C}$ & -6.509141000 & 2.752091000 & 0.607102000 \\
\hline $\mathrm{C}$ & -7.098054000 & 1.630328000 & 1.219243000 \\
\hline $\mathrm{C}$ & -6.664659000 & 0.346612000 & 0.824962000 \\
\hline $\mathrm{C}$ & -2.243900000 & -3.593975000 & -0.898105000 \\
\hline $\mathrm{C}$ & -1.625040000 & -4.106162000 & -2.059377000 \\
\hline $\mathrm{C}$ & -0.379588000 & -4.760671000 & -1.993859000 \\
\hline $\mathrm{C}$ & 0.245892000 & -4.885725000 & -0.737758000 \\
\hline $\mathrm{C}$ & -0.345853000 & -4.388941000 & 0.442253000 \\
\hline $\mathrm{C}$ & -1.590177000 & -3.739779000 & 0.346758000 \\
\hline $\mathrm{C}$ & -5.523885000 & 2.623293000 & -0.394680000 \\
\hline $\mathrm{C}$ & 1.316069000 & 5.580529000 & -1.667295000 \\
\hline $\mathrm{C}$ & 0.910232000 & 4.266072000 & -1.968599000 \\
\hline $\mathrm{C}$ & 1.774147000 & 3.200731000 & -1.654400000 \\
\hline $\mathrm{C}$ & 3.043001000 & 3.428073000 & -1.076591000 \\
\hline $\mathrm{C}$ & 3.414754000 & 4.761903000 & -0.787626000 \\
\hline $\mathrm{C}$ & 2.566529000 & 5.846961000 & -1.078302000 \\
\hline $\mathrm{C}$ & 4.033521000 & 2.342226000 & -0.779936000 \\
\hline $\mathrm{C}$ & 5.382676000 & 2.575523000 & -1.137068000 \\
\hline $\mathrm{C}$ & 6.395199000 & 1.664431000 & -0.819082000 \\
\hline $\mathrm{C}$ & 6.082071000 & 0.504123000 & -0.103605000 \\
\hline $\mathrm{C}$ & 4.751279000 & 0.206757000 & 0.268328000 \\
\hline $\mathrm{C}$ & 3.723933000 & 1.116679000 & -0.115137000 \\
\hline $\mathrm{C}$ & 4.510837000 & -1.028122000 & 1.078078000 \\
\hline
\end{tabular}

\subsection{7 $\quad \mathbf{3}^{\mathrm{CF} 3}-\mathrm{H}^{+}$}

\begin{tabular}{|c|c|c|c|}
\hline $\mathrm{C}$ & 5.129715000 & -2.240050000 & 0.694175000 \\
\hline $\mathrm{C}$ & 5.007630000 & -3.408733000 & 1.468287000 \\
\hline $\mathrm{C}$ & 4.247161000 & -3.350907000 & 2.653418000 \\
\hline $\mathrm{C}$ & 3.631211000 & -2.156838000 & 3.077910000 \\
\hline C & 3.762070000 & -1.007496000 & 2.275337000 \\
\hline $\mathrm{H}$ & 4.381075000 & 4.954627000 & -0.305508000 \\
\hline $\mathrm{H}$ & 0.641073000 & 6.416621000 & -1.893471000 \\
\hline $\mathrm{H}$ & 1.451185000 & 2.177489000 & -1.870850000 \\
\hline $\mathrm{H}$ & 5.624800000 & 3.492336000 & -1.684600000 \\
\hline $\mathrm{H}$ & 7.431091000 & 1.870523000 & -1.108525000 \\
\hline $\mathrm{H}$ & 6.876685000 & -0.182781000 & 0.206590000 \\
\hline $\mathrm{H}$ & 5.711027000 & -2.273181000 & -0.236068000 \\
\hline $\mathrm{H}$ & 4.146189000 & -4.254133000 & 3.269968000 \\
\hline $\mathrm{H}$ & 1.213308000 & -5.401466000 & -0.672417000 \\
\hline $\mathrm{H}$ & -2.078840000 & -3.382534000 & 1.261201000 \\
\hline $\mathrm{H}$ & -4.373501000 & -4.737680000 & -2.025721000 \\
\hline $\mathrm{H}$ & -6.712850000 & -3.865417000 & -2.198550000 \\
\hline $\mathrm{H}$ & -7.259475000 & -1.597855000 & -1.292986000 \\
\hline $\mathrm{H}$ & -7.103187000 & -0.542400000 & 1.295739000 \\
\hline $\mathrm{H}$ & -4.350310000 & 1.211643000 & -1.551192000 \\
\hline $\mathrm{H}$ & -2.126451000 & -3.991498000 & -3.028516000 \\
\hline $\mathrm{H}$ & 3.308756000 & -0.071125000 & 2.617080000 \\
\hline $\mathrm{H}$ & -6.834595000 & 3.756457000 & 0.909063000 \\
\hline $\mathrm{H}$ & -1.916445000 & -1.089762000 & -0.234591000 \\
\hline $\mathrm{C}$ & 0.340009000 & -4.567908000 & 1.781555000 \\
\hline $\mathrm{H}$ & -0.272060000 & -4.164432000 & 2.606211000 \\
\hline $\mathrm{H}$ & 0.527814000 & -5.636595000 & 1.994658000 \\
\hline $\mathrm{H}$ & 1.320059000 & -4.055406000 & 1.804239000 \\
\hline $\mathrm{C}$ & 0.252439000 & -5.349857000 & -3.239729000 \\
\hline $\mathrm{H}$ & 1.354342000 & -5.357754000 & -3.173481000 \\
\hline $\mathrm{H}$ & -0.070156000 & -6.398160000 & -3.389627000 \\
\hline $\mathrm{H}$ & -0.034730000 & -4.790702000 & -4.147392000 \\
\hline $\mathrm{C}$ & -4.950782000 & 3.852623000 & -1.070580000 \\
\hline $\mathrm{H}$ & -4.047199000 & 3.611525000 & -1.656024000 \\
\hline $\mathrm{H}$ & -5.685331000 & 4.303796000 & -1.764253000 \\
\hline $\mathrm{H}$ & -4.685190000 & 4.632135000 & -0.333769000 \\
\hline $\mathrm{C}$ & -8.188721000 & 1.792558000 & 2.258658000 \\
\hline $\mathrm{H}$ & -9.190926000 & 1.746606000 & 1.791013000 \\
\hline $\mathrm{H}$ & -8.150104000 & 0.993580000 & 3.019718000 \\
\hline $\mathrm{H}$ & -8.115209000 & 2.764825000 & 2.775537000 \\
\hline $\mathrm{C}$ & 2.997215000 & 7.268926000 & -0.778351000 \\
\hline $\mathrm{H}$ & 3.549853000 & 7.705789000 & -1.632416000 \\
\hline $\mathrm{H}$ & 2.129369000 & 7.923018000 & -0.584764000 \\
\hline $\mathrm{H}$ & 3.665791000 & 7.316134000 & 0.098939000 \\
\hline $\mathrm{C}$ & 5.700158000 & -4.691882000 & 1.053559000 \\
\hline $\mathrm{H}$ & 6.701316000 & -4.771671000 & 1.519201000 \\
\hline $\mathrm{H}$ & 5.127017000 & -5.582307000 & 1.366136000 \\
\hline $\mathrm{H}$ & 5.845174000 & -4.742986000 & -0.039633000 \\
\hline $\mathrm{C}$ & 2.877417000 & -2.100624000 & 4.392428000 \\
\hline $\mathrm{H}$ & 2.126232000 & -1.291513000 & 4.396716000 \\
\hline $\mathrm{H}$ & 2.357722000 & -3.051731000 & 4.603581000 \\
\hline $\mathrm{H}$ & 3.565935000 & -1.913822000 & 5.238908000 \\
\hline $\mathrm{C}$ & -0.424225000 & 4.005534000 & -2.639801000 \\
\hline $\mathrm{H}$ & -0.803017000 & 2.993442000 & -2.411018000 \\
\hline $\mathrm{H}$ & -1.186863000 & 4.738204000 & -2.321440000 \\
\hline $\mathrm{H}$ & -0.341017000 & 4.084709000 & -3.741014000 \\
\hline
\end{tabular}

\section{Thermal correction to Energy= 0.826179 \\ Thermal correction to Gibbs Free Energy $=\quad 0.621609$}

Sum of electronic and zero-point Energies $=\quad-4902.100861$ Sum of electronic and thermal Energies $=\quad-4902.024559$ Sum of electronic and thermal Enthalpies $=-4902.023615$ Sum of electronic and thermal Free Energies $=\quad-4902.228185$ E solvent $=-4902.09603853$

$\mathrm{Ni} \quad 0.902944000 \quad 0.898741000 \quad 1.251108000$ 


\begin{tabular}{|c|c|c|c|}
\hline & & & \\
\hline & & & \\
\hline & 000 & & 3000 \\
\hline & 595000 & 7000 & -1 \\
\hline & -0.189522000 & -0.141026000 & -1.21 \\
\hline & 5000 & 4000 & \\
\hline & 0.668065000 & 999000 & 0000 \\
\hline H & -0.104831000 & 0000 & 15000 \\
\hline & 2.803221000 & 0.8780 & 200 \\
\hline & 2.240259000 & 2.647606000 & 25000 \\
\hline & 1.828146000 & 2.557516000 & -2.121992000 \\
\hline & 0.331000000 & 0.884574000 & 32000 \\
\hline & 0.877892000 & 1.649990000 & 52000 \\
\hline & -0.501833000 & -0.0 & \\
\hline & -0.6 & & \\
\hline & & & \\
\hline & & & 7000 \\
\hline & -1.3 & $-0.6-(x-1)$ & -5 \\
\hline & -1.1 & -0 . & \\
\hline & & & \\
\hline & -1.0 & & \\
\hline & 0 & & \\
\hline & 0 & & \\
\hline & -2.5 & & -1 . \\
\hline & -2.5 & & \\
\hline & -3. & & \\
\hline & -4. & & \\
\hline & -4.0 & & \\
\hline & -2.6 & & \\
\hline & -1.8 & & \\
\hline & 00 & & \\
\hline & 00 & & \\
\hline & 2.4 & & \\
\hline & 1.75 & & \\
\hline & 0.36 & & \\
\hline C & -4.68 & & \\
\hline $\mathrm{C}$ & -4.4 & & 000 \\
\hline C & -5.22 & & \\
\hline & & & \\
\hline & & & \\
\hline & & & \\
\hline & & & \\
\hline & & & \\
\hline & & & \\
\hline & & & \\
\hline & & & \\
\hline & & & \\
\hline & & & \\
\hline & & & \\
\hline & & & \\
\hline $\mathrm{C}$ & & & \\
\hline E & & & \\
\hline C & & & \\
\hline $\mathrm{C}$ & & & \\
\hline $\mathrm{C}$ & & & \\
\hline $\mathrm{C}$ & 00 & & \\
\hline $\mathrm{C}$ & -1. & & 5000 \\
\hline $\mathrm{C}$ & -2.3 & & 000 \\
\hline $\mathrm{C}$ & -1.8 & & 0000 \\
\hline $\mathrm{C}$ & -0.4 & & \\
\hline 11 & & & \\
\hline & & & \\
\hline & & & \\
\hline & & & \\
\hline & & & \\
\hline & & & \\
\hline & & & \\
\hline & -3.398242000 & 4.932563000 & 1.6036570 \\
\hline
\end{tabular}

\begin{tabular}{cc}
$\mathrm{H}$ & -6.775196000 \\
$\mathrm{H}$ & -5.841876000 \\
$\mathrm{H}$ & -5.760695000 \\
$\mathrm{H}$ & -4.619069000 \\
$\mathrm{H}$ & -2.120472000 \\
$\mathrm{H}$ & -0.168019000 \\
$\mathrm{H}$ & -0.205152000 \\
$\mathrm{H}$ & -3.739787000 \\
$\mathrm{H}$ & -0.062159000 \\
$\mathrm{H}$ & 3.541258000 \\
$\mathrm{H}$ & -0.991997000 \\
$\mathrm{C}$ & -1.959334000 \\
$\mathrm{~F}$ & -1.968668000 \\
$\mathrm{~F}$ & -1.169362000 \\
$\mathrm{~F}$ & -3.225241000 \\
$\mathrm{C}$ & -2.726361000 \\
$\mathrm{~F}$ & -2.469279000 \\
$\mathrm{~F}$ & -4.038321000 \\
$\mathrm{~F}$ & -2.533915000 \\
$\mathrm{C}$ & 7.002856000 \\
$\mathrm{~F}$ & 7.954097000 \\
$\mathrm{~F}$ & 6.294431000 \\
$\mathrm{~F}$ & 7.628821000 \\
$\mathrm{C}$ & 4.592706000 \\
$\mathrm{~F}$ & 5.471149000 \\
$\mathrm{~F}$ & 4.555078000 \\
$\mathrm{~F}$ & 3.345481000 \\
$\mathrm{C}$ & 2.479790000 \\
$\mathrm{~F}$ & 3.822204000 \\
$\mathrm{~F}$ & 2.247432000 \\
$\mathrm{~F}$ & 2.039621000 \\
$\mathrm{C}$ & 2.488089000 \\
$\mathrm{~F}$ & 3.656173000 \\
$\mathrm{~F}$ & 2.796310000 \\
$\mathrm{~F}$ & 1.746170000 \\
$\mathrm{C}$ & -5.029901000 \\
$\mathrm{~F}$ & -3.740473000 \\
$\mathrm{~F}$ & -5.299887000 \\
$\mathrm{~F}$ & -5.835426000 \\
$\mathrm{C}$ & -7.395039000 \\
$\mathrm{~F}$ & -8.000981000 \\
$\mathrm{~F}$ & -8.358706000 \\
$\mathrm{~F}$ & -6.758663000 \\
$\mathrm{C}$ & 0.324252000 \\
$\mathrm{H}$ & -0.721927000 \\
& 1.084533000 \\
\hline
\end{tabular}

0.381253000 $-3.728041000$ $-4.136958000$ $-5.755079000$ $-5.770791000$ $-4.948638000$ $-3.676914000$ $-0.489499000$ 4.484307000 $-4.659412000$ $-1.759538000$ 4.712057000 3.422752000 5.435280000 5.183683000 4.890590000 3.936093000 4.803031000 6.097583000 1.263645000 0.300396000 1.271414000 2.467286000 $-1.917351000$ $-2.836596000$ $-2.018839000$ $-2.272212000$ $-5.411315000$ $-5.232324000$ $-6.746241000$ $-4.816974000$ $-3.703826000$ $-4.373677000$ $-2.364883000$ $-3.889973000$ 1.567546000 1.832352000 2.298393000 2.016515000 $-2.208815000$ $-3.360539000$ $-1.286858000$ $-2.469000000$ $-0.170174000$ $-0.491278000$ $-0.961496000$

$-2.198872000$ $-1.214211000$ 1.389256000 2.907413000 3.064469000 $-0.385556000$ 3.757919000 0.757487000 $-1.111875000$ 1.819009000 0.517533000 3.940333000 4.422839000 4.776069000 4.034547000 $-1.067410000$ $-2.010415000$ $-0.729346000$ $-1.666670000$ $-3.579510000$ $-3.687512000$ $-4.745364000$ $-3.479636000$ $-0.473957000$ $-0.936559000$ 0.888288000 $-0.921610000$ $-0.610815000$ $-0.530584000$ $-0.740275000$ $-1.756711000$ 4.154830000 4.298474000 4.096641000 5.276965000 $-0.450872000$ $-0.074728000$ $-1.561061000$ 0.547343000 $-2.843898000$ $-2.449135000$ $-3.093345000$ $-4.022228000$ 2.908985000 2.970281000 2.942402000

\subsection{8 $\mathbf{2}^{\mathbf{C F} 3}-\mathbf{H}^{+}$ CF3-H+ $\mathbf{1 - \beta}-\mathbf{T}$ \\ Zero-point correction=}

Thermal correction to Energy= 0.770633 (Hartree/Particle)

rergy $=0.847710$

Thermal correction to Enthalpy $=\quad 0.848654$

Thermal correction to Gibbs Free Energy= 0.641167

Sum of electronic and zero-point Energies $=\quad-4903.278464$ Sum of electronic and thermal Energies $=\quad-4903.201387$

Sum of electronic and thermal Enthalpies $=\quad-4903.200443$

Sum of electronic and thermal Free Energies $=-4903.407931$

E solvent $=-4903.29516484$

$\begin{array}{lrrr}\mathrm{Ni} & 1.441743000 & 0.561854000 & -0.776134000 \\ \mathrm{C} & 2.769806000 & 0.865971000 & -2.103533000 \\ \mathrm{~N} & 1.526428000 & 2.142034000 & 0.201418000 \\ \mathrm{C} & 0.784301000 & 2.340339000 & 1.274119000 \\ \mathrm{C} & -0.161229000 & 1.437154000 & 1.881260000 \\ \mathrm{C} & -0.520646000 & 0.166344000 & 1.299128000 \\ \mathrm{O} & 0.041909000 & -0.302053000 & 0.222032000 \\ \mathrm{C} & 2.251795000 & -0.454799000 & -2.574636000 \\ \mathrm{H} & 1.453015000 & -0.851707000 & -1.794484000\end{array}$




\begin{tabular}{|c|c|c|c|}
\hline & & & \\
\hline & 19569000 & 92379000 & 6000 \\
\hline & 0.713583000 & .243405000 & -3.987075000 \\
\hline & 2.259535000 & -0.258581000 & 7000 \\
\hline & 001187000 & -1.260434000 & \\
\hline & 31000 & 17000 & 19700 \\
\hline & 27000 & 66000 & -1.6 \\
\hline & 79000 & 349000 & \\
\hline & .784472000 & 00 & \\
\hline & 602000 & 00 & 2000 \\
\hline & .760872000 & 8000 & 3. \\
\hline & -2.367199000 & 12000 & 2500 \\
\hline & -2.772069000 & 142000 & 45000 \\
\hline & 1.612996000 & 50000 & 5.863588000 \\
\hline & 3999000 & 385000 & 5.391518000 \\
\hline & -2.145986000 & 500000 & 203000 \\
\hline & -2.928108000 & -0.7 & \\
\hline & 70000 & & \\
\hline & & & \\
\hline & & & \\
\hline & 20000 & -2.2 & 1. \\
\hline & -2.2 & -3.4 & 94000 \\
\hline & -1 & -4.3 & 000 \\
\hline & -1 & -5.4 & \\
\hline & & 0 & \\
\hline & & -4 & \\
\hline & & & \\
\hline & & -2. & \\
\hline & & -1 . & \\
\hline & & -0 & \\
\hline & & -2.0 & \\
\hline & -5 & & \\
\hline & 6000 & & \\
\hline & 6000 & & \\
\hline & 6000 & & \\
\hline & & 00 & 27000 \\
\hline & 3000 & 00 & 2000 \\
\hline & 74000 & -4.0 & 54000 \\
\hline $\mathrm{C}$ & 35000 & -0.2 & 346000 \\
\hline C & 70000 & 4.1 & 24000 \\
\hline C & 910000 & & \\
\hline & & & \\
\hline & 69000 & & \\
\hline & & & \\
\hline & & & \\
\hline & & & \\
\hline & & & \\
\hline & & & \\
\hline & & & \\
\hline & & & \\
\hline & 2.3 & & 73000 \\
\hline & & & 00 \\
\hline & 5. & & 0 \\
\hline & 6.3 & & 0.8 \\
\hline & 5.9 & & \\
\hline 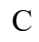 & 68000 & & 24000 \\
\hline $\mathrm{C}$ & 86000 & & 2.014209000 \\
\hline 11 & 6000 & & 07000 \\
\hline $\mathrm{H}$ & 232000 & & 44000 \\
\hline 11 & 16000 & 33000 & 98000 \\
\hline 11 & 60000 & 6.2 & 33000 \\
\hline 11 & 28000 & 6.4 & 020000 \\
\hline & 10000 & 4.5 & 66000 \\
\hline & & 2.1 & \\
\hline & & & \\
\hline & & & -0.7 \\
\hline & 00 & & 1217023000 \\
\hline & -1.274136000 & -6.244589000 & -2.1194040 \\
\hline
\end{tabular}

\begin{tabular}{cc} 
H & -3.748500000 \\
H & -5.267216000 \\
H & -5.755327000 \\
H & -3.819398000 \\
H & 0.376626000 \\
H & 3.167999000 \\
H & -7.369875000 \\
H & -0.858299000 \\
C & 7.521425000 \\
F & 8.206439000 \\
F & 8.388050000 \\
F & 7.100291000 \\
C & 4.356921000 \\
F & 2.995362000 \\
F & 4.893932000 \\
F & 4.723998000 \\
C & 2.888102000 \\
F & 2.118364000 \\
F & 3.166414000 \\
F & 4.064813000 \\
C & 3.081946000 \\
F & 3.330232000 \\
F & 4.283025000 \\
F & 2.418557000 \\
C & -5.514841000 \\
F & -6.388802000 \\
F & -4.260677000 \\
F & -5.787195000 \\
C & -7.737854000 \\
F & -8.882925000 \\
F & -8.031792000 \\
F & -7.319365000 \\
C & -3.007315000 \\
F & -4.160706000 \\
F & -3.342260000 \\
F & -2.419445000 \\
C & -2.204831000 \\
F & -3.495713000 \\
F & -1.452028000 \\
F & -2.096440000 \\
& \\
\hline
\end{tabular}

$-6.522802000$ $-4.800945000$ $-3.999080000$ $-0.857564000$ $-4.661788000$ 2.218449000 0.014808000 $-1.824962000$ 0.103729000 1.017962000 $-0.470224000$ $-0.872331000$ 0.364221000 0.246737000 $-0.832586000$ 1.273021000 $-3.761922000$ $-3.020593000$ $-4.934888000$ $-3.100208000$ $-4.571493000$ $-3.336816000$ $-5.157105000$ $-5.295390000$ 1.144813000 1.951032000 1.665729000 1.222523000 $-2.484332000$ $-1.773432000$ $-3.803216000$ $-2.306016000$ 6.297396000 6.356858000 6.056598000 7.523602000 1.927875000 2.041782000 1.713851000 0.774706000

$-2.296070000$ $-1.313277000$ 1.137415000 $-1.148723000$ $-3.053741000$ 2.511259000 1.164642000 $-0.155680000$ 0.106597000 $-0.632009000$ 0.979015000 $-0.753236000$ 4.051650000 4.124213000 4.398198000 4.996115000 1.653822000 2.501660000 2.277430000 1.476612000 $-3.325522000$ $-3.872927000$ $-3.102699000$ $-4.262510000$ $-0.516191000$ 0.137183000 $-0.336229000$ $-1.845094000$ 2.221844000 2.063265000 2.070393000 3.508035000 $-0.639760000$ $-1.353640000$ 0.661523000 $-0.681827000$ $-3.084003000$ $-3.464216000$ $-4.204082000$ $-2.333586000$

\section{CF3-H+TS-2 coor $_{\text {}}$}

Zero-point correction= $\quad 0.902538$ Thermal correction to Enthalpy= $\quad 0.903482$ Thermal correction to Gibbs Free Energy $=0.689691$ Sum of electronic and zero-point Energies $=\quad-4981.803019$ Sum of electronic and thermal Energies $=\quad-4981.722190$ Sum of electronic and thermal Enthalpies $=\quad-4981.721246$ Sum of electronic and thermal Free Energies $=-4981.935038$ E solvent $=-4981.84421772$

$\begin{array}{rrrr}\mathrm{N} & 1.448682000 & 2.098513000 & -0.367962000 \\ \mathrm{C} & 1.866627000 & 4.430813000 & 0.375900000 \\ \mathrm{C} & 4.298300000 & 1.864995000 & -1.186065000 \\ \mathrm{C} & 4.126014000 & 0.587734000 & -0.617649000 \\ \mathrm{H} & 3.512263000 & 0.469340000 & 0.281156000 \\ \mathrm{C} & 5.710553000 & 0.853196000 & -2.910793000 \\ \mathrm{C} & 0.429694000 & 4.664176000 & 0.710158000 \\ \mathrm{C} & 5.533642000 & -0.416138000 & -2.338343000 \\ \mathrm{C} & 2.350607000 & 3.222700000 & -0.189898000 \\ \mathrm{C} & 4.739414000 & -0.537718000 & -1.188661000 \\ \mathrm{C} & 3.718000000 & 3.096167000 & -0.566868000 \\ \mathrm{C} & -0.577196000 & 4.582092000 & -0.274726000 \\ \mathrm{H} & -0.320497000 & 4.292817000 & -1.297429000 \\ \mathrm{C} & -2.262870000 & 5.305625000 & 1.331827000 \\ \mathrm{C} & 5.100683000 & 1.982189000 & -2.342368000 \\ \mathrm{H} & 5.237272000 & 2.961608000 & -2.812208000 \\ \mathrm{C} & -1.906618000 & 4.909030000 & 0.032067000\end{array}$




\begin{tabular}{|c|c|c|c|}
\hline C & 2.773001000 & 5.490408000 & 0.596238000 \\
\hline 11 & 2.392744000 & 6.425909000 & 1.020830000 \\
\hline $\mathrm{C}$ & -1.266561000 & 5.388733000 & 2.315148000 \\
\hline $\mathrm{C}$ & 4.123056000 & 5.373736000 & 0.246885000 \\
\hline $\mathrm{H}$ & 4.812459000 & 6.205414000 & 0.423933000 \\
\hline $\mathrm{C}$ & 0.070422000 & 5.085603000 & 2.006168000 \\
\hline $\mathrm{H}$ & 0.838964000 & 5.172979000 & 2.781627000 \\
\hline $\mathrm{C}$ & 4.583176000 & 4.190786000 & -0.340675000 \\
\hline $\mathrm{H}$ & 5.639793000 & 4.087716000 & -0.610275000 \\
\hline $\mathrm{N}$ & -1.598664000 & -2.508894000 & 0.091787000 \\
\hline $\mathrm{C}$ & -1.695438000 & -4.226567000 & 1.824198000 \\
\hline $\mathrm{C}$ & -4.579225000 & -2.494049000 & -0.084876000 \\
\hline $\mathrm{C}$ & -5.532970000 & -3.018390000 & -0.983359000 \\
\hline $\mathrm{H}$ & -5.678099000 & -4.101653000 & -1.053707000 \\
\hline $\mathrm{C}$ & -5.183556000 & -0.245000000 & -0.822337000 \\
\hline $\mathrm{C}$ & -0.202254000 & -4.279036000 & 1.880639000 \\
\hline $\mathrm{C}$ & -6.124784000 & -0.769873000 & -1.719515000 \\
\hline $\mathrm{C}$ & -2.382359000 & -3.398045000 & 0.899669000 \\
\hline $\mathrm{C}$ & -6.296740000 & -2.161593000 & -1.792110000 \\
\hline $\mathrm{C}$ & -3.802364000 & -3.410645000 & 0.805876000 \\
\hline $\mathrm{C}$ & 0.483012000 & -3.923858000 & 3.062560000 \\
\hline $\mathrm{H}$ & -0.076879000 & -3.562818000 & 3.930629000 \\
\hline $\mathrm{C}$ & 2.612812000 & -4.516411000 & 2.029671000 \\
\hline $\mathrm{C}$ & -4.419533000 & -1.095852000 & -0.008289000 \\
\hline $\mathrm{H}$ & -3.709711000 & -0.663195000 & 0.704268000 \\
\hline $\mathrm{C}$ & 1.880277000 & -4.031338000 & 3.127370000 \\
\hline $\mathrm{C}$ & -2.449399000 & -5.064439000 & 2.669459000 \\
\hline $\mathrm{H}$ & -1.922338000 & -5.710521000 & 3.379531000 \\
\hline $\mathrm{C}$ & 1.932912000 & -4.884224000 & 0.861242000 \\
\hline $\mathrm{C}$ & -3.846156000 & -5.110952000 & 2.571495000 \\
\hline $\mathrm{H}$ & -4.419650000 & -5.778064000 & 3.222958000 \\
\hline $\mathrm{C}$ & 0.536431000 & -4.755498000 & 0.780113000 \\
\hline $\mathrm{H}$ & 0.019161000 & -5.059073000 & -0.135782000 \\
\hline $\mathrm{C}$ & -4.510150000 & -4.299344000 & 1.642620000 \\
\hline $\mathrm{H}$ & -5.603875000 & -4.310945000 & 1.587577000 \\
\hline $\mathrm{Ni}$ & 0.956594000 & 0.965052000 & 1.040621000 \\
\hline $\mathrm{C}$ & -1.743935000 & -2.305011000 & -1.216354000 \\
\hline $\mathrm{H}$ & -2.436231000 & -2.974273000 & -1.740365000 \\
\hline $\mathrm{C}$ & 1.127191000 & 1.829847000 & -1.620793000 \\
\hline $\mathrm{H}$ & 1.524341000 & 2.516428000 & -2.385430000 \\
\hline $\mathrm{C}$ & 0.312241000 & 0.752978000 & -2.129326000 \\
\hline $\mathrm{C}$ & 0.075721000 & 0.724516000 & -3.520887000 \\
\hline $\mathrm{C}$ & -0.248132000 & -0.285575000 & -1.292707000 \\
\hline $\mathrm{H}$ & 0.527242000 & 1.516585000 & -4.133867000 \\
\hline $\mathrm{C}$ & -0.708903000 & -0.258464000 & -4.166297000 \\
\hline $\mathrm{C}$ & -1.074577000 & -1.289496000 & -1.949290000 \\
\hline $\mathrm{C}$ & -0.918713000 & -0.223292000 & -5.665197000 \\
\hline $\mathrm{C}$ & -1.278384000 & -1.243601000 & -3.359103000 \\
\hline $\mathrm{H}$ & -1.909483000 & -2.016646000 & -3.816656000 \\
\hline $\mathrm{O}$ & -0.047725000 & -0.338347000 & -0.009463000 \\
\hline $\mathrm{C}$ & 2.037061000 & 1.725602000 & 2.415586000 \\
\hline $\mathrm{C}$ & 1.954140000 & 0.371912000 & 3.066942000 \\
\hline $\mathrm{H}$ & 3.030950000 & 2.052538000 & 2.076575000 \\
\hline $\mathrm{H}$ & 1.480015000 & 2.536610000 & 2.914511000 \\
\hline $\mathrm{C}$ & -1.589567000 & 0.428251000 & 2.773550000 \\
\hline $\mathrm{C}$ & -1.494583000 & 1.610254000 & 2.129811000 \\
\hline $\mathrm{C}$ & 1.562504000 & 0.365829000 & 4.560523000 \\
\hline $\mathrm{H}$ & 1.125208000 & -0.233719000 & 2.532982000 \\
\hline $\mathrm{H}$ & 2.862675000 & -0.228555000 & 2.891817000 \\
\hline $\mathrm{H}$ & 1.416153000 & -0.660326000 & 4.934399000 \\
\hline $\mathrm{H}$ & 2.372602000 & 0.830339000 & 5.148214000 \\
\hline $\mathrm{H}$ & 0.641620000 & 0.947606000 & 4.734914000 \\
\hline $\mathrm{H}$ & -1.745296000 & 1.710164000 & 1.068383000 \\
\hline $\mathrm{H}$ & -1.279917000 & 2.535798000 & 2.674154000 \\
\hline $\mathrm{H}$ & -1.424996000 & 0.339189000 & 3.853145000 \\
\hline $\mathrm{H}$ & -1.863277000 & -0.494468000 & 2.248972000 \\
\hline $\mathrm{H}$ & -1.369522000 & 0.733430000 & -5.985978000 \\
\hline $\mathrm{H}$ & 0.038910000 & -0.331042000 & -6.206860000 \\
\hline
\end{tabular}

\begin{tabular}{cr} 
H & -1.584547000 \\
H & 6.004815000 \\
H & 3.701543000 \\
H & -6.720946000 \\
H & -3.301319000 \\
H & -0.890870000 \\
C & -1.628677000 \\
F & -2.828537000 \\
F & -1.701235000 \\
F & -0.689955000 \\
C & -2.950378000 \\
F & -4.183895000 \\
F & -3.042420000 \\
F & -2.643120000 \\
C & -4.971250000 \\
F & -5.659013000 \\
F & -5.340520000 \\
F & -3.639701000 \\
C & -7.259377000 \\
F & -8.250401000 \\
F & -6.604977000 \\
F & -7.830907000 \\
C & 2.633561000 \\
F & 3.427536000 \\
F & 3.444479000 \\
F & 1.791646000 \\
C & 2.676471000 \\
F & 2.206971000 \\
F & 4.009724000 \\
F & 2.506806000 \\
C & 4.476667000 \\
F & 3.218861000 \\
F & 5.359253000 \\
F & 4.528898000 \\
C & 6.609321000 \\
F & 6.213502000 \\
F & 7.895934000 \\
F & 6.605799000 \\
& \\
\hline
\end{tabular}

$-1.036789000$ $-1.296016000$ $-4.602908000$ $-0.104001000$ 5.547099000 $-1.886711000$ 5.710274000 6.332437000 4.557266000 6.498323000 4.906828000 4.594859000 6.127823000 4.007019000 1.249526000 1.948440000 1.699077000 1.552532000 $-2.756018000$ $-1.886598000$ $-3.078104000$ $-3.896177000$ $-3.607638000$ $-4.601658000$ $-2.537340000$ $-3.233707000$ $-5.498715000$ $-5.018475000$ $-5.252042000$ $-6.849150000$ $-1.894687000$ $-2.336626000$ $-2.829844000$ $-1.851493000$ 1.015311000 2.063872000 1.249363000 $-0.101767000$

$-5.997355000$ $-2.783968000$ 2.082690000 $-2.348499000$ 1.573725000 0.532702000 3.749534000 3.849963000 4.488455000 4.337709000 $-1.066536000$ $-0.584852000$ $-1.658930000$ $-2.045196000$ $-0.694197000$ $-1.626904000$ 0.533311000 $-0.842869000$ $-2.803084000$ $-3.124030000$ $-3.956129000$ $-2.331128000$ 4.371277000 4.837688000 4.105586000 5.377313000 $-0.308795000$ $-1.495019000$ $-0.250248000$ $-0.329705000$ $-0.577214000$ $-0.899868000$ $-0.998525000$ 0.788198000 $-4.121671000$ $-4.896138000$ $-3.746961000$ $-4.897391000$

\section{CF3-H+2-Coor-T}

Zero-point correction= Energy= $\quad 0.905304$

Thermal correction to Enthalpy= $\quad 0.906248$ Thermal correction to Gibbs Free Energy $=\quad 0.690797$ Sum of electronic and zero-point Energies $=\quad-4981.820086$ Sum of electronic and thermal Energies $=\quad-4981.739407$ Sum of electronic and thermal Enthalpies $=\quad-4981.738463$ Sum of electronic and thermal Free Energies $=-4981.953914$ E solvent $=-4981.85408369$

$\begin{array}{lrrr}\mathrm{N} & 1.628580000 & 1.873043000 & -0.314659000 \\ \mathrm{C} & 2.255479000 & 4.175537000 & 0.334231000 \\ \mathrm{C} & 4.456282000 & 1.399294000 & -1.225210000 \\ \mathrm{C} & 4.106903000 & 0.113164000 & -0.769063000 \\ \mathrm{H} & 3.438840000 & 0.000080000 & 0.088658000 \\ \mathrm{C} & 5.833600000 & 0.344898000 & -2.957549000 \\ \mathrm{C} & 0.835562000 & 4.519463000 & 0.653096000 \\ \mathrm{C} & 5.484294000 & -0.932415000 & -2.488980000 \\ \mathrm{C} & 2.635052000 & 2.909467000 & -0.187827000 \\ \mathrm{C} & 4.620603000 & -1.036710000 & -1.390754000 \\ \mathrm{C} & 3.985417000 & 2.659553000 & -0.569386000 \\ \mathrm{C} & -0.152090000 & 4.549161000 & -0.352736000 \\ \mathrm{H} & 0.107715000 & 4.286171000 & -1.382379000 \\ \mathrm{C} & -1.822954000 & 5.297474000 & 1.257122000 \\ \mathrm{C} & 5.325855000 & 1.495056000 & -2.337320000 \\ \mathrm{H} & 5.593877000 & 2.478808000 & -2.734900000 \\ \mathrm{C} & -1.465072000 & 4.945138000 & -0.053917000 \\ \mathrm{C} & 3.244281000 & 5.160995000 & 0.533666000\end{array}$




\begin{tabular}{|c|c|c|c|}
\hline & & & \\
\hline & 3928000 & 1000 & 000 \\
\hline & 2785000 & 2244000 & 207406000 \\
\hline & 43664000 & 81182000 & 208000 \\
\hline & 478431000 & & \\
\hline & 000 & 000 & 200 \\
\hline & 1000 & 00 & -0.3 \\
\hline & 5.985819000 & 3.484500000 & -060 \\
\hline & -1.947941000 & & \\
\hline & -2.247311000 & -4.124426000 & \\
\hline & -4.897477000 & -2.07523 & -0.1 \\
\hline & 5.894720000 & -2.432531000 & -1. \\
\hline & -6.167595000 & -3.484676000 & -1.207174000 \\
\hline & -5.218443000 & 0.268592000 & -0.753566000 \\
\hline & -0.762334000 & -4.254791000 & 1.909867000 \\
\hline & -6.203934000 & -0.090948000 & -1.685558000 \\
\hline & -2.829847000 & -3.218840000 & \\
\hline & -6.5 & & \\
\hline & & & \\
\hline & & & \\
\hline & -0 & -3.3 & \\
\hline & 2000 & & \\
\hline & & & \\
\hline & -3.8 & & \\
\hline & & & \\
\hline & 0 & & \\
\hline & -2.6 & & \\
\hline & & & \\
\hline & -4 & & \\
\hline & 0 & & \\
\hline & $-0 .($ & & \\
\hline & -0.4 & & \\
\hline & -5.0 & & \\
\hline & & & \\
\hline & & & \\
\hline & -2.008830000 & & \\
\hline & -2.72 & -2.8 & 000 \\
\hline & 1.327039000 & & -1.56 \\
\hline & 1.830884000 & & \\
\hline & 0.41 & & \\
\hline & 0.256258000 & & \\
\hline & & & \\
\hline & & & \\
\hline & & & \\
\hline & & & \\
\hline & & & \\
\hline & & & \\
\hline & & & \\
\hline & & & \\
\hline & & & \\
\hline & & & \\
\hline & & & \\
\hline & & & \\
\hline & 0 & & \\
\hline & & & \\
\hline & & & 4.07 \\
\hline & & & \\
\hline & & & \\
\hline & & & \\
\hline & 000 & & \\
\hline & 3.37 & & \\
\hline & -1.65 & & \\
\hline & -0.8 & 2.21 & \\
\hline & 0.27 & & \\
\hline & & & \\
\hline & & & \\
\hline & & & -6.14 \\
\hline & -1.457998000 & -1.084021000 & -6.0136970 \\
\hline
\end{tabular}

\begin{tabular}{cr} 
H & 5.875496000 \\
H & 3.118018000 \\
H & -6.706763000 \\
H & -2.849431000 \\
H & -1.218472000 \\
C & -1.217142000 \\
F & -2.363242000 \\
F & -1.409855000 \\
F & -0.232857000 \\
C & -2.477971000 \\
F & -3.752478000 \\
F & -2.389480000 \\
F & -2.273525000 \\
C & -4.858107000 \\
F & -5.232345000 \\
F & -5.436409000 \\
F & -3.499719000 \\
C & -7.550896000 \\
F & -8.447978000 \\
F & -6.926541000 \\
F & -8.235938000 \\
C & 1.942064000 \\
F & 1.454838000 \\
F & 3.286887000 \\
F & 1.685329000 \\
C & 2.152377000 \\
F & 1.710203000 \\
F & 3.484901000 \\
F & 1.986296000 \\
C & 4.191147000 \\
F & 2.853520000 \\
F & 4.865010000 \\
F & 4.374291000 \\
C & 6.804564000 \\
F & 6.659835000 \\
F & 8.091896000 \\
F & 6.620143000 \\
& \\
\hline
\end{tabular}

$-1.829274000$ $-4.674208000$ 0.673149000 5.591057000 $-1.807576000$ 5.538915000 6.253659000 4.354116000 6.205543000 5.058881000 4.928711000 6.265675000 4.102490000 1.722051000 2.506243000 2.209376000 1.858846000 $-1.860344000$ $-0.872830000$ $-2.154427000$ $-2.970816000$ $-3.466450000$ $-4.095492000$ $-3.623029000$ $-2.125741000$ $-5.758431000$ $-5.424043000$ $-5.502900000$ $-7.102258000$ $-2.388236000$ $-2.593969000$ $-3.407089000$ $-2.474726000$ 0.473413000 1.663652000 0.395714000 $-0.520625000$
$-2.976141000$ 2.292522000 $-2.284115000$ 1.491958000 0.555746000 3.702768000 3.813894000 4.370980000 4.360923000 $-1.174186000$ $-0.718267000$ $-1.796236000$ $-2.127056000$ $-0.525148000$ $-1.562677000$ 0.603010000 $-0.364120000$ $-2.889721000$ $-3.138057000$ $-4.066436000$ $-2.506786000$ 4.477621000 5.577701000 4.458456000 4.649311000 $-0.045073000$ $-1.289657000$ 0.013182000 0.092302000 $-0.864215000$ $-1.086437000$ $-1.445996000$ 0.485928000 $-4.115839000$ $-4.760880000$ $-3.683647000$ $-5.026679000$

\section{CF3-H+ + TS- isom $_{\text {iso }}$}

Zero-point correction $=\quad 0.822870($ Hartree/Particle $)$ $\begin{array}{lc}\text { Thermal correction to Energy= } & 0.902144 \\ \text { Thermal correction to Enthalpy= } & 0.903089\end{array}$ Thermal correction to Gibbs Free Energy $=0.691980$ Sum of electronic and zero-point Energies $=\quad-4981.812256$ Sum of electronic and thermal Energies $=-4981.732981$

Sum of electronic and thermal Enthalpies $=\quad-4981.732037$ Sum of electronic and thermal Free Energies $=-4981.943146$ E solvent $=-4981.84315929$

$\begin{array}{lr}\mathrm{N} & 0.810777000 \\ \mathrm{C} & 2.865535000 \\ \mathrm{C} & -0.630097000 \\ \mathrm{C} & -1.687035000 \\ \mathrm{H} & -1.483834000 \\ \mathrm{C} & -2.287009000 \\ \mathrm{C} & 3.641983000 \\ \mathrm{C} & -3.326590000 \\ \mathrm{C} & 1.477969000 \\ \mathrm{C} & -3.015932000 \\ \mathrm{C} & 0.798396000 \\ \mathrm{C} & 3.435176000 \\ \mathrm{H} & 2.713526000 \\ \mathrm{C} & 5.144275000 \\ \mathrm{C} & -0.954192000 \\ \mathrm{H} & -0.154405000 \\ \mathrm{C} & 4.176315000 \\ \mathrm{C} & 3.550572000 \\ \mathrm{H} & 4.615994000 \\ \mathrm{C} & 5.380435000\end{array}$

2.317313000 3.636726000 4.810690000 4.276116000 3.804595000 5.555326000 2.398464000 5.011428000 3.597780000 4.375307000 4.781233000 1.675388000 2.034727000 0.055124000 5.459466000 5.886304000 0.515631000 4.867980000 4.891642000 0.787703000
0.907948000 1.252984000 0.097372000 0.867434000 1.831210000 $-1.549410000$ 1.581388000 $-0.784402000$ 0.934778000 0.430266000 0.534171000 2.774073000 3.513412000 2.142359000 $-1.113394000$ $-1.728153000$ 3.048654000 1.185936000 1.440238000 0.970694000 


\begin{tabular}{|c|c|c|c|}
\hline $\mathrm{C}$ & 2.884719000 & 6.043924000 & 0.820749000 \\
\hline $\mathrm{H}$ & 3.422795000 & 6.996679000 & 0.791235000 \\
\hline $\mathrm{C}$ & 4.641507000 & 1.951147000 & 0.693840000 \\
\hline $\mathrm{H}$ & 4.847764000 & 2.518016000 & -0.220444000 \\
\hline $\mathrm{C}$ & 1.524674000 & 5.993296000 & 0.496601000 \\
\hline $\mathrm{H}$ & 0.994359000 & 6.912022000 & 0.222778000 \\
\hline $\mathrm{N}$ & -0.761891000 & -2.857275000 & -0.326497000 \\
\hline $\mathrm{C}$ & -2.420062000 & -4.313642000 & -1.459176000 \\
\hline $\mathrm{C}$ & 1.394750000 & -3.943402000 & -1.902875000 \\
\hline $\mathrm{C}$ & 2.047114000 & -3.967083000 & -0.653709000 \\
\hline $\mathrm{H}$ & 1.526561000 & -4.346329000 & 0.231359000 \\
\hline $\mathrm{C}$ & 3.444607000 & -3.076004000 & -2.912570000 \\
\hline $\mathrm{C}$ & -3.585095000 & -3.692758000 & -0.755889000 \\
\hline $\mathrm{C}$ & 4.087015000 & -3.088973000 & -1.662713000 \\
\hline $\mathrm{C}$ & -1.076505000 & -3.878378000 & -1.280800000 \\
\hline $\mathrm{C}$ & 3.381773000 & -3.542185000 & -0.539518000 \\
\hline $\mathrm{C}$ & -0.015274000 & -4.407686000 & -2.062648000 \\
\hline $\mathrm{C}$ & -3.801164000 & -2.300015000 & -0.796100000 \\
\hline $\mathrm{H}$ & -3.108400000 & -1.655255000 & -1.347085000 \\
\hline $\mathrm{C}$ & -5.825069000 & -2.536853000 & 0.540778000 \\
\hline $\mathrm{C}$ & 2.113802000 & -3.501229000 & -3.035348000 \\
\hline $\mathrm{H}$ & 1.627301000 & -3.481945000 & -4.015888000 \\
\hline $\mathrm{C}$ & -4.905709000 & -1.729193000 & -0.144000000 \\
\hline $\mathrm{C}$ & -2.662567000 & -5.336204000 & -2.399942000 \\
\hline $\mathrm{H}$ & -3.693218000 & -5.672587000 & -2.554755000 \\
\hline $\mathrm{C}$ & -5.629207000 & -3.927347000 & 0.563927000 \\
\hline $\mathrm{C}$ & -1.624949000 & -5.877022000 & -3.170634000 \\
\hline $\mathrm{H}$ & -1.839907000 & -6.661505000 & -3.903185000 \\
\hline $\mathrm{C}$ & -4.517688000 & -4.502494000 & -0.072338000 \\
\hline $\mathrm{H}$ & -4.365954000 & -5.585745000 & -0.020994000 \\
\hline $\mathrm{C}$ & -0.316637000 & -5.402069000 & -3.015513000 \\
\hline $\mathrm{H}$ & 0.498395000 & -5.825327000 & -3.612031000 \\
\hline $\mathrm{Ni}$ & 0.785830000 & 1.206553000 & -0.820348000 \\
\hline $\mathrm{C}$ & -1.222323000 & -2.796198000 & 0.920492000 \\
\hline $\mathrm{H}$ & -1.799247000 & -3.660198000 & 1.270412000 \\
\hline $\mathrm{C}$ & 0.341703000 & 1.882242000 & 2.051284000 \\
\hline $\mathrm{H}$ & 0.409443000 & 2.551949000 & 2.929249000 \\
\hline $\mathrm{C}$ & -0.282092000 & 0.602630000 & 2.320670000 \\
\hline $\mathrm{C}$ & -0.799821000 & 0.412913000 & 3.620205000 \\
\hline $\mathrm{C}$ & -0.357472000 & -0.477232000 & 1.360022000 \\
\hline $\mathrm{H}$ & -0.705862000 & 1.243518000 & 4.333428000 \\
\hline $\mathrm{C}$ & -1.427918000 & -0.774754000 & 4.055846000 \\
\hline $\mathrm{C}$ & -1.020170000 & -1.698870000 & 1.800733000 \\
\hline $\mathrm{C}$ & -1.958200000 & -0.903169000 & 5.467634000 \\
\hline $\mathrm{C}$ & -1.529725000 & -1.810005000 & 3.125023000 \\
\hline $\mathrm{H}$ & -2.018615000 & -2.748959000 & 3.416141000 \\
\hline $\mathrm{O}$ & 0.133568000 & -0.410571000 & 0.158819000 \\
\hline $\mathrm{C}$ & -0.902897000 & 1.209907000 & -1.829071000 \\
\hline $\mathrm{C}$ & -1.143787000 & 2.128745000 & -3.007155000 \\
\hline $\mathrm{H}$ & -1.046632000 & 0.142490000 & -2.070345000 \\
\hline $\mathrm{H}$ & -1.493619000 & 1.495649000 & -0.938204000 \\
\hline $\mathrm{C}$ & 1.907196000 & 2.167602000 & -2.133807000 \\
\hline $\mathrm{C}$ & 1.784588000 & 0.793142000 & -2.479030000 \\
\hline $\mathrm{C}$ & -2.618763000 & 1.990848000 & -3.470964000 \\
\hline $\mathrm{H}$ & -0.961169000 & 3.180190000 & -2.728270000 \\
\hline $\mathrm{H}$ & -0.481422000 & 1.888692000 & -3.858540000 \\
\hline $\mathrm{H}$ & -2.830049000 & 2.744293000 & -4.248854000 \\
\hline $\mathrm{H}$ & -2.817704000 & 0.989694000 & -3.892396000 \\
\hline $\mathrm{H}$ & -3.321666000 & 2.154528000 & -2.636136000 \\
\hline $\mathrm{H}$ & 1.175277000 & 0.478826000 & -3.334010000 \\
\hline $\mathrm{H}$ & 2.565912000 & 0.079370000 & -2.181974000 \\
\hline $\mathrm{H}$ & 2.790367000 & 2.521278000 & -1.585077000 \\
\hline $\mathrm{H}$ & 1.370606000 & 2.940588000 & -2.693652000 \\
\hline $\mathrm{H}$ & -2.663871000 & -0.087587000 & 5.708200000 \\
\hline $\mathrm{H}$ & -2.486825000 & -1.859637000 & 5.613196000 \\
\hline $\mathrm{H}$ & -1.138849000 & -0.856798000 & 6.208474000 \\
\hline $\mathrm{H}$ & -4.362635000 & 5.084419000 & -1.126051000 \\
\hline $\mathrm{H}$ & -6.682033000 & -2.089765000 & 1.051020000 \\
\hline
\end{tabular}

$\begin{array}{cccc}\text { H } & 5.118607000 & -2.743806000 & -1.565673000 \\ \text { H } & 5.709759000 & -0.855948000 & 2.350224000 \\ \text { H } & -0.175514000 & -2.036409000 & -0.591457000 \\ \text { C } & 6.382572000 & 0.301126000 & -0.053776000 \\ \text { F } & 7.264989000 & -0.585210000 & 0.466869000 \\ \text { F } & 5.739314000 & -0.327974000 & -1.094295000 \\ \text { F } & 7.082064000 & 1.331225000 & -0.596715000 \\ \text { C } & 3.865152000 & -0.280348000 & 4.298581000 \\ \text { F } & 4.922908000 & -1.023842000 & 4.706512000 \\ \text { F } & 3.496520000 & 0.532179000 & 5.329204000 \\ \text { F } & 2.821606000 & -1.138304000 & 4.081231000 \\ \text { C } & 4.167367000 & -2.516337000 & -4.122231000 \\ \text { F } & 3.690281000 & -3.047170000 & -5.279503000 \\ \text { F } & 3.995262000 & -1.161513000 & -4.198473000 \\ \text { F } & 5.500899000 & -2.752343000 & -4.064416000 \\ \text { C } & 4.022624000 & -3.549436000 & 0.832754000 \\ \text { F } & 5.349868000 & -3.265365000 & 0.786464000 \\ \text { F } & 3.433302000 & -2.614054000 & 1.641418000 \\ \text { F } & 3.868475000 & -4.752351000 & 1.445953000 \\ \text { C } & -2.565743000 & 6.197704000 & -2.892527000 \\ \text { F } & -2.139823000 & 5.386605000 & -3.912713000 \\ \text { F } & -3.886768000 & 6.433430000 & -3.085030000 \\ \text { F } & -1.899654000 & 7.375256000 & -3.024358000 \\ \text { C } & -4.145149000 & 3.850037000 & 1.293041000 \\ \text { F } & -3.699373000 & 2.950294000 & 2.217408000 \\ \text { F } & -4.760769000 & 4.855174000 & 1.970357000 \\ \text { F } & -5.095315000 & 3.232073000 & 0.537699000 \\ \text { C } & -5.049302000 & -0.220943000 & -0.160197000 \\ \text { F } & -4.032810000 & 0.361602000 & 0.550024000 \\ \text { F } & -4.968064000 & 0.268231000 & -1.429999000 \\ \text { F } & -6.219225000 & 0.192514000 & 0.377878000 \\ \text { C } & -6.647662000 & -4.822170000 & 1.246558000 \\ \text { F } & -6.059058000 & -5.941076000 & 1.753313000 \\ \text { F } & -7.269588000 & -4.176737000 & 2.268463000 \\ \text { F } & -7.604960000 & -5.225598000 & 0.370150000\end{array}$

\section{CF3-H+2-Coor-C}

Zero-point correction=

Thermal correction to Er

Thermal correction to Enthalpy= $\quad 0.906181$

Thermal correction to Gibbs Free Energy $=0.690291$

Sum of electronic and zero-point Energies $=\quad-4981.819500$

Sum of electronic and thermal Energies $=\quad-4981.738881$

Sum of electronic and thermal Enthalpies $=\quad-4981.737937$

Sum of electronic and thermal Free Energies $=-4981.953828$

E solvent $=-4981.85555858$

$\begin{array}{lrrr}\mathrm{N} & 1.820276000 & 1.830498000 & -0.226381000 \\ \mathrm{C} & 2.434477000 & 4.084609000 & 0.580376000 \\ \mathrm{C} & 4.682873000 & 1.372270000 & -1.020443000 \\ \mathrm{C} & 4.307640000 & 0.075660000 & -0.620801000 \\ \mathrm{H} & 3.589638000 & -0.056993000 & 0.191344000 \\ \mathrm{C} & 6.132708000 & 0.362614000 & -2.718462000 \\ \mathrm{C} & 1.006125000 & 4.442960000 & 0.838220000 \\ \mathrm{C} & 5.759005000 & -0.926379000 & -2.303502000 \\ \mathrm{C} & 2.824179000 & 2.846721000 & -0.001762000 \\ \mathrm{C} & 4.844219000 & -1.058279000 & -1.250367000 \\ \mathrm{C} & 4.190652000 & 2.606309000 & -0.333112000 \\ \mathrm{C} & 0.066597000 & 4.505399000 & -0.212941000 \\ \mathrm{H} & 0.366508000 & 4.240892000 & -1.230921000 \\ \mathrm{C} & -1.651889000 & 5.310878000 & 1.317039000 \\ \mathrm{C} & 5.602812000 & 1.497604000 & -2.087348000 \\ \mathrm{H} & 5.893253000 & 2.491852000 & -2.441165000 \\ \mathrm{C} & -1.243801000 & 4.948517000 & 0.023306000 \\ \mathrm{C} & 3.424453000 & 5.040863000 & 0.889535000 \\ \mathrm{H} & 3.115189000 & 5.995277000 & 1.329559000 \\ \mathrm{C} & -0.725189000 & 5.243805000 & 2.367611000 \\ \mathrm{C} & 4.774246000 & 4.797647000 & 0.609051000 \\ \mathrm{H} & 5.533352000 & 5.545423000 & 0.859708000\end{array}$




\begin{tabular}{|c|c|c|c|}
\hline & 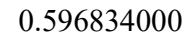 & & \\
\hline & 1.312676000 & & \\
\hline & 000 & & -( \\
\hline & 6.198886000 & 8000 & 3000 \\
\hline & 1.885359000 & 26000 & 0 \\
\hline & 653000 & 4000 & 1 \\
\hline & 4.817585000 & 4000 & 3000 \\
\hline & 5.747058000 & 1000 & -1 \\
\hline & 6.003289000 & -3.446398000 & 46000 \\
\hline & -5.116472000 & 0.324447000 & -1.134217000 \\
\hline & -0.844687000 & 48000 & 3000 \\
\hline & -6.034481000 & & \\
\hline & -2.825095000 & & \\
\hline & -6.346932000 & & \\
\hline & -4.2 & & \\
\hline & & & \\
\hline & 00 & & \\
\hline & 00 & & \\
\hline & 00 & & \\
\hline & 00 & & \\
\hline & 1.1 & & \\
\hline & -3.2 & & \\
\hline & -2.8 & & \\
\hline & & & \\
\hline & 0 & & \\
\hline & -5.2 & & \\
\hline & -0.0 & & \\
\hline & -0.4 & & \\
\hline & -5.0 & & \\
\hline & & & \\
\hline & & & \\
\hline & -1.8 & & 000 \\
\hline & -2.5 & & -1 . \\
\hline & $1.5^{\circ}$ & & \\
\hline & & & \\
\hline & & & \\
\hline & & & \\
\hline & & & \\
\hline & & & \\
\hline & & & \\
\hline & & & \\
\hline & & & \\
\hline & & & \\
\hline & & & \\
\hline & & & \\
\hline & & & \\
\hline & & & \\
\hline & & & \\
\hline & & & \\
\hline $\mathrm{C}$ & & & \\
\hline & & & \\
\hline 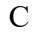 & & & \\
\hline & & & 000 \\
\hline $\mathrm{H}$ & & & \\
\hline $\mathrm{H}$ & -3.7 & & \\
\hline $\mathrm{H}$ & -3.29 & 2000 & 7000 \\
\hline & -2.79 & & 6000 \\
\hline & 3.11 & & \\
\hline & & -0 & \\
\hline & & & \\
\hline & & & \\
\hline & & & \\
\hline & & & 7 \\
\hline & & & \\
\hline & & & \\
\hline & & & \\
\hline & & & -2.797695 \\
\hline & 2.984106000 & -4.725939000 & 2.5809210 \\
\hline
\end{tabular}

$\begin{array}{cr}\text { H } & -1.175067000 \\ \text { C } & -2.195591000 \\ \text { F } & -3.493067000 \\ \text { F } & -2.067221000 \\ \text { F } & -1.948893000 \\ \text { C } & 7.155864000 \\ \text { F } & 6.989475000 \\ \text { F } & 8.421369000 \\ \text { F } & 7.065135000 \\ \text { C } & 4.385108000 \\ \text { F } & 3.057517000 \\ \text { F } & 5.076889000 \\ \text { F } & 4.511134000 \\ \text { C } & -4.755958000 \\ \text { F } & -5.271141000 \\ \text { F } & -5.191569000 \\ \text { F } & -3.392401000 \\ \text { C } & -7.286337000 \\ \text { F } & -8.184916000 \\ \text { F } & -6.587879000 \\ \text { F } & -7.972798000 \\ \text { C } & 2.169074000 \\ \text { F } & 1.798904000 \\ \text { F } & 3.495573000 \\ \text { F } & 2.000667000 \\ \text { C } & 1.687876000 \\ \text { F } & 1.174725000 \\ \text { F } & 3.037520000 \\ \text { F } & 1.375918000 \\ \text { C } & -1.161350000 \\ \text { F } & -2.286835000 \\ \text { F } & -1.426292000 \\ \text { F } & -0.189489000 \\ & \end{array}$

$-1.746759000$ 5.113913000 4.977837000 6.344169000 4.194547000 0.525555000 1.704012000 0.513045000 $-0.481648000$ $-2.418635000$ $-2.608982000$ $-3.429903000$ $-2.532173000$ 1.780120000 2.583516000 2.228656000 1.935649000 $-1.876489000$ $-0.909606000$ $-2.174073000$ $-2.994511000$ $-5.694618000$ $-5.340019000$ $-5.436656000$ $-7.040854000$ $-3.561226000$ $-4.286563000$ $-3.673773000$ $-2.246425000$ 5.520145000 6.274114000 4.342016000 6.152661000

0.519443000 $-1.143904000$ $-0.758918000$ $-1.710470000$ $-2.121616000$ $-3.826529000$ $-4.488579000$ $-3.328102000$ $-4.736448000$ $-0.774545000$ $-1.057360000$ $-1.347949000$ 0.580523000 $-0.923741000$ $-1.881687000$ 0.285986000 $-0.932840000$ $-3.364478000$ $-3.679972000$ $-4.497827000$ $-3.006846000$ 0.142391000 $-1.119849000$ 0.281912000 0.247903000 4.717602000 5.743637000 4.751966000 4.973961000 3.790608000 3.845540000 4.441395000 4.499656000

\section{CF3-H+TS-2 ${ }_{\text {ins }}$}

Zero-point correction $=\quad 0.825447$ (Hartree/Particle $)$

Thermal correction to Energy $=\quad 0.905110$

Thermal correction to Enthalpy $=\quad 0.906054$

Thermal correction to Gibbs Free Energy $=0.696768$

Sum of electronic and zero-point Energies $=\quad-4981.797172$

Sum of electronic and thermal Energies $=\quad-4981.717509$

Sum of electronic and thermal Enthalpies $=\quad-4981.716565$

Sum of electronic and thermal Free Energies $=-4981.925851$

E solvent $=-4981.84480309$

$\begin{array}{lrrr}\mathrm{N} & 1.544744000 & -2.109246000 & -0.002816000 \\ \mathrm{C} & 1.070118000 & -4.487292000 & -0.478592000 \\ \mathrm{C} & 4.496645000 & -2.770138000 & 0.119859000 \\ \mathrm{C} & 4.603644000 & -1.502677000 & -0.490904000 \\ \mathrm{H} & 3.854172000 & -1.171289000 & -1.214526000 \\ \mathrm{C} & 6.567264000 & -2.293834000 & 1.343777000 \\ \mathrm{C} & -0.399988000 & -4.237681000 & -0.443598000 \\ \mathrm{C} & 6.670038000 & -1.040128000 & 0.723549000 \\ \mathrm{C} & 2.022305000 & -3.459938000 & -0.223587000 \\ \mathrm{C} & 5.681365000 & -0.653140000 & -0.193632000 \\ \mathrm{C} & 3.416390000 & -3.757265000 & -0.205325000 \\ \mathrm{C} & -1.010071000 & -3.582195000 & 0.645563000 \\ \mathrm{H} & -0.406949000 & -3.247824000 & 1.492934000 \\ \mathrm{C} & -3.211863000 & -3.825098000 & -0.378356000 \\ \mathrm{C} & 5.493904000 & -3.148204000 & 1.047228000 \\ \mathrm{H} & 5.423049000 & -4.115251000 & 1.555767000 \\ \mathrm{C} & -2.396731000 & -3.377956000 & 0.673164000 \\ \mathrm{C} & 1.526557000 & -5.792731000 & -0.755390000 \\ \mathrm{H} & 0.789277000 & -6.583723000 & -0.930740000 \\ \mathrm{C} & -2.614195000 & -4.497066000 & -1.455944000 \\ \mathrm{C} & 2.893871000 & -6.090156000 & -0.772028000 \\ \mathrm{H} & 3.235333000 & -7.106231000 & -0.994016000 \\ \mathrm{C} & -1.225333000 & -4.706314000 & -1.488526000\end{array}$




\begin{tabular}{|c|c|c|c|}
\hline $\mathrm{H}$ & -0.780483000 & -5.227815000 & -2.342789000 \\
\hline $\mathrm{C}$ & 3.821593000 & -5.082733000 & -0.490079000 \\
\hline $\mathrm{H}$ & 4.893041000 & -5.308378000 & -0.511361000 \\
\hline $\mathrm{N}$ & -1.658509000 & 2.442350000 & 0.317469000 \\
\hline $\mathrm{C}$ & -3.933276000 & 3.287073000 & -0.134310000 \\
\hline $\mathrm{C}$ & -0.483287000 & 4.748476000 & -1.057456000 \\
\hline $\mathrm{C}$ & 0.193211000 & 5.900871000 & -0.607354000 \\
\hline $\mathrm{H}$ & -0.357252000 & 6.686698000 & -0.078949000 \\
\hline $\mathrm{C}$ & 1.625651000 & 3.912700000 & -1.966108000 \\
\hline $\mathrm{C}$ & -4.560160000 & 2.118498000 & 0.557800000 \\
\hline $\mathrm{C}$ & 2.299023000 & 5.053701000 & -1.504481000 \\
\hline $\mathrm{C}$ & -2.529185000 & 3.456976000 & -0.228653000 \\
\hline $\mathrm{C}$ & 1.572578000 & 6.048191000 & -0.830594000 \\
\hline $\mathrm{C}$ & -1.956919000 & 4.596143000 & -0.858574000 \\
\hline $\mathrm{C}$ & -5.399202000 & 1.243682000 & -0.163441000 \\
\hline $\mathrm{H}$ & -5.548424000 & 1.392974000 & -1.236527000 \\
\hline $\mathrm{C}$ & -5.856631000 & -0.030819000 & 1.862028000 \\
\hline $\mathrm{C}$ & 0.247526000 & 3.763828000 & -1.750717000 \\
\hline $\mathrm{H}$ & -0.269679000 & 2.887119000 & -2.152507000 \\
\hline $\mathrm{C}$ & -6.025386000 & 0.167121000 & 0.482568000 \\
\hline $\mathrm{C}$ & -4.763486000 & 4.282413000 & -0.691531000 \\
\hline $\mathrm{H}$ & -5.849653000 & 4.163104000 & -0.617756000 \\
\hline $\mathrm{C}$ & -5.038460000 & 0.848094000 & 2.585993000 \\
\hline $\mathrm{C}$ & -4.217767000 & 5.426950000 & -1.287282000 \\
\hline $\mathrm{H}$ & -4.878198000 & 6.196892000 & -1.699124000 \\
\hline $\mathrm{C}$ & -4.384893000 & 1.909369000 & 1.940052000 \\
\hline $\mathrm{H}$ & -3.768101000 & 2.596870000 & 2.526959000 \\
\hline $\mathrm{C}$ & -2.827975000 & 5.584578000 & -1.362617000 \\
\hline $\mathrm{H}$ & -2.399069000 & 6.463226000 & -1.856128000 \\
\hline $\mathrm{Ni}$ & 0.573843000 & -1.099999000 & -1.398078000 \\
\hline $\mathrm{C}$ & -0.804770000 & 2.664934000 & 1.313601000 \\
\hline $\mathrm{H}$ & -0.899514000 & 3.641813000 & 1.804842000 \\
\hline $\mathrm{C}$ & 1.866633000 & -1.605787000 & 1.169451000 \\
\hline $\mathrm{H}$ & 2.451539000 & -2.246258000 & 1.849376000 \\
\hline $\mathrm{C}$ & 1.519645000 & -0.310174000 & 1.698105000 \\
\hline $\mathrm{C}$ & 2.132903000 & 0.090721000 & 2.901150000 \\
\hline $\mathrm{C}$ & 0.504425000 & 0.517932000 & 1.088667000 \\
\hline $\mathrm{H}$ & 2.877621000 & -0.582842000 & 3.346817000 \\
\hline $\mathrm{C}$ & 1.852388000 & 1.313561000 & 3.554320000 \\
\hline $\mathrm{C}$ & 0.176849000 & 1.757256000 & 1.785668000 \\
\hline $\mathrm{C}$ & 2.579064000 & 1.703564000 & 4.823391000 \\
\hline $\mathrm{C}$ & 0.877839000 & 2.123317000 & 2.974185000 \\
\hline $\mathrm{H}$ & 0.619496000 & 3.078041000 & 3.450830000 \\
\hline $\mathrm{O}$ & -0.101536000 & 0.195210000 & -0.011250000 \\
\hline $\mathrm{C}$ & -0.788141000 & -0.043101000 & -2.594568000 \\
\hline $\mathrm{C}$ & -2.091493000 & -0.794245000 & -2.337280000 \\
\hline $\mathrm{H}$ & -0.614808000 & 0.820159000 & -1.934643000 \\
\hline $\mathrm{H}$ & -0.747318000 & 0.333161000 & -3.626363000 \\
\hline $\mathrm{C}$ & 0.826250000 & -1.200178000 & -3.557712000 \\
\hline $\mathrm{C}$ & 1.494833000 & -2.176838000 & -2.792753000 \\
\hline $\mathrm{C}$ & -3.297592000 & -0.006156000 & -2.903823000 \\
\hline $\mathrm{H}$ & -2.073810000 & -1.789715000 & -2.818518000 \\
\hline $\mathrm{H}$ & -2.233317000 & -0.972537000 & -1.255348000 \\
\hline $\mathrm{H}$ & -4.247211000 & -0.513619000 & -2.661888000 \\
\hline $\mathrm{H}$ & -3.342430000 & 1.017664000 & -2.487970000 \\
\hline $\mathrm{H}$ & -3.229564000 & 0.084366000 & -4.002792000 \\
\hline $\mathrm{H}$ & 1.117241000 & -3.205702000 & -2.788605000 \\
\hline $\mathrm{H}$ & 2.564564000 & -2.067482000 & -2.580337000 \\
\hline $\mathrm{H}$ & 1.365284000 & -0.310092000 & -3.900753000 \\
\hline $\mathrm{H}$ & 0.003199000 & -1.530030000 & -4.200103000 \\
\hline $\mathrm{H}$ & 3.662997000 & 1.826149000 & 4.643705000 \\
\hline $\mathrm{H}$ & 2.197469000 & 2.654697000 & 5.230056000 \\
\hline $\mathrm{H}$ & 2.463114000 & 0.932183000 & 5.606341000 \\
\hline $\mathrm{H}$ & -4.290353000 & -3.644606000 & -0.361403000 \\
\hline $\mathrm{H}$ & 3.371445000 & 5.173715000 & -1.677248000 \\
\hline $\mathrm{H}$ & 7.506536000 & -0.374544000 & 0.951089000 \\
\hline $\mathrm{H}$ & -6.348059000 & -0.866752000 & 2.366830000 \\
\hline $\mathrm{H}$ & -1.585807000 & 1.504476000 & -0.116608000 \\
\hline
\end{tabular}

$\begin{array}{lccc}\text { C } & -3.447843000 & -4.903813000 & -2.653332000 \\ \text { F } & -3.395525000 & -3.933455000 & -3.621663000 \\ \text { F } & -2.989606000 & -6.051318000 & -3.218678000 \\ \text { F } & -4.752447000 & -5.080300000 & -2.329886000 \\ \text { C } & -2.980300000 & -2.557022000 & 1.798633000 \\ \text { F } & -2.875994000 & -1.218906000 & 1.499755000 \\ \text { F } & -4.295700000 & -2.820563000 & 2.005398000 \\ \text { F } & -2.320469000 & -2.751163000 & 2.969697000 \\ \text { C } & 5.737100000 & 0.717942000 & -0.834532000 \\ \text { F } & 6.992031000 & 1.234265000 & -0.826793000 \\ \text { F } & 5.305431000 & 0.681669000 & -2.125804000 \\ \text { F } & 4.932130000 & 1.600175000 & -0.167294000 \\ \text { C } & 7.644266000 & -2.760859000 & 2.304329000 \\ \text { F } & 8.301576000 & -1.712807000 & 2.867740000 \\ \text { F } & 7.113463000 & -3.512130000 & 3.310178000 \\ \text { F } & 8.566531000 & -3.531668000 & 1.667675000 \\ \text { C } & 2.368036000 & 2.795522000 & -2.666719000 \\ \text { F } & 3.627548000 & 3.140226000 & -3.010605000 \\ \text { F } & 2.443567000 & 1.687523000 & -1.858487000 \\ \text { F } & 1.714101000 & 2.393312000 & -3.799373000 \\ \text { C } & 2.286261000 & 7.264824000 & -0.271432000 \\ \text { F } & 3.453899000 & 7.505466000 & -0.919581000 \\ \text { F } & 1.510852000 & 8.378347000 & -0.365595000 \\ \text { F } & 2.576992000 & 7.085525000 & 1.049100000 \\ \text { C } & -6.898086000 & -0.794847000 & -0.298262000 \\ \text { F } & -6.713907000 & -0.666710000 & -1.644144000 \\ \text { F } & -6.612432000 & -2.089865000 & 0.032289000 \\ \text { F } & -8.215623000 & -0.598134000 & -0.043416000 \\ \text { C } & -4.796031000 & 0.606374000 & 4.062188000 \\ \text { F } & -4.518004000 & 1.767495000 & 4.718503000 \\ \text { F } & -5.869954000 & 0.031357000 & 4.659503000 \\ \text { F } & -3.729464000 & -0.224940000 & 4.251523000\end{array}$

\section{CF3-H+2-B-T}

Zero-point correction $=\quad 0.826883($ Hartree/Particle $)$ Thermal correction to Energy $=\quad 0.906508$

Thermal correction to Enthalpy $=\quad 0.907452$

Thermal correction to Gibbs Free Energy= 0.695474

Sum of electronic and zero-point Energies $=\quad-4981.845797$

Sum of electronic and thermal Energies $=\quad-4981.766172$

Sum of electronic and thermal Enthalpies $=\quad-4981.765228$

Sum of electronic and thermal Free Energies $=-4981.977206$

E solvent $=-4981.88392415$

$\begin{array}{lrrr}\mathrm{Ni} & -1.476039000 & 0.727844000 & 0.110479000 \\ \mathrm{O} & 0.160109000 & -0.221140000 & -0.305472000 \\ \mathrm{C} & 0.285195000 & -1.513517000 & -0.218470000 \\ \mathrm{C} & -0.710876000 & -2.367092000 & 0.392567000 \\ \mathrm{C} & -1.882434000 & -1.860064000 & 1.063805000 \\ \mathrm{~N} & -2.319856000 & -0.615298000 & 1.095415000 \\ \mathrm{C} & 1.479345000 & -2.165868000 & -0.733757000 \\ \mathrm{C} & 1.589199000 & -3.586924000 & -0.716770000 \\ \mathrm{C} & -0.516287000 & -3.765820000 & 0.404368000 \\ \mathrm{C} & 0.605690000 & -4.408534000 & -0.164554000 \\ \mathrm{C} & 0.731832000 & -5.916999000 & -0.145902000 \\ \mathrm{C} & 2.592975000 & -1.432631000 & -1.220377000 \\ \mathrm{~N} & 2.679698000 & -0.102793000 & -1.256669000 \\ \mathrm{C} & 3.851719000 & 0.643251000 & -1.603506000 \\ \mathrm{C} & 5.138048000 & 0.223676000 & -1.157925000 \\ \mathrm{C} & 6.260086000 & 0.981204000 & -1.553648000 \\ \mathrm{C} & 6.123458000 & 2.135900000 & -2.332819000 \\ \mathrm{C} & 4.850499000 & 2.555189000 & -2.731454000 \\ \mathrm{C} & 3.694745000 & 1.822139000 & -2.385625000 \\ \mathrm{C} & 5.386020000 & -0.950220000 & -0.260241000 \\ \mathrm{C} & 4.812939000 & -1.041611000 & 1.026292000 \\ \mathrm{C} & 5.140635000 & -2.118918000 & 1.867192000 \\ \mathrm{C} & 6.029395000 & -3.118529000 & 1.442927000 \\ \mathrm{C} & 6.598132000 & -3.031631000 & 0.163637000 \\ \mathrm{C} & 6.284556000 & -1.954490000 & -0.680206000\end{array}$




\begin{tabular}{|c|c|c|c|}
\hline & & & \\
\hline & 000 & 1.484121000 & \\
\hline & 41116000 & 2.019070000 & 025207000 \\
\hline & 21284000 & 397211000 & \\
\hline & 0340000 & 233889000 & \\
\hline & 000 & 41000 & \\
\hline & 503006000 & -0.3 & \\
\hline C & 430545000 & 0000 & \\
\hline C & -4.582960000 & 6000 & 3.79 \\
\hline 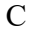 & -5.777201000 & & \\
\hline 4 & -5.841678000 & 3000 & 2. \\
\hline 4 & -4.718157000 & 0000 & 1. \\
\hline 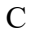 & -2.206717000 & 3000 & 3.337 \\
\hline 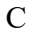 & -2.345806000 & 7000 & 3.68 \\
\hline 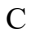 & -1.227701000 & 36000 & 4.00640200 \\
\hline & 0.060050000 & & \\
\hline J & 0.207137000 & & \\
\hline 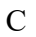 & -0.909354 & & \\
\hline$C$ & -4.880314 & & \\
\hline 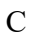 & -4.48 & -1.6 & \\
\hline$c$ & -4.698948 & -2.4 & -1. \\
\hline 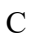 & -5.31150 & -3.7 & \\
\hline$C$ & -5.7 & -4.1 & \\
\hline 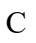 & -5.49 & -3.2 & \\
\hline C & -2.86 & & \\
\hline C & $-1.7^{\prime}$ & & \\
\hline$C$ & -1.28 & & \\
\hline 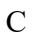 & -1.82 & & \\
\hline $\mathrm{C}$ & -2.29 & & \\
\hline & -2.4 & -2.6 & \\
\hline 1 & -1.2 & & \\
\hline I & 0.67 & & \\
\hline I & 1.69 & & \\
\hline I & -0.07 & & \\
\hline $\mathrm{I}$ & 2.495391000 & & 1 \\
\hline $\mathrm{H}$ & 3.467687000 & & \\
\hline $\mathrm{H}$ & 7.249 & & \\
\hline $\mathrm{H}$ & 7.008257 & & -2.6 \\
\hline 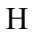 & 4.740148 & & \\
\hline 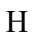 & 6.74 & -1.8 & \\
\hline $\mathrm{H}$ & 4.129930000 & & \\
\hline $\mathrm{H}$ & & & \\
\hline $\mathrm{H}$ & & & \\
\hline $\mathrm{H}$ & & & \\
\hline $\mathrm{H}$ & & & \\
\hline & 07 & & \\
\hline & & & \\
\hline & 45 & & \\
\hline & & & \\
\hline $\mathrm{H}$ & ) & & \\
\hline H & -4 & & -1. \\
\hline 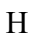 & 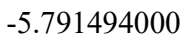 & & \\
\hline & & & -2. \\
\hline & -3 . & & -0.5 \\
\hline & & & \\
\hline $\mathrm{H}$ & & & \\
\hline $\mathrm{H}$ & -0.80 & & 775900 \\
\hline 11 & & & 00 \\
\hline П & -2.439494000 & & -1 . \\
\hline $\mathrm{H}$ & -0.307 & 000 & -0 . \\
\hline 11 & -2.5 & & \\
\hline 11 & -1.67 & 000 & 100 \\
\hline $\mathrm{H}$ & -0.86 & & -0.1 \\
\hline & & & \\
\hline & & & \\
\hline & & & \\
\hline & & & \\
\hline & 4.407580000 & -0.991612000 & 3.82697200 \\
\hline
\end{tabular}

$\begin{array}{lr}\text { F } & 3.220815000 \\ \text { F } & 5.177682000 \\ \text { C } & 7.507857000 \\ \text { F } & 8.472024000 \\ \text { F } & 8.115364000 \\ \text { F } & 6.793824000 \\ \text { C } & 0.671087000 \\ \text { F } & 1.398563000 \\ \text { F } & -0.638418000 \\ \text { F } & 1.040231000 \\ \text { C } & -0.818318000 \\ \text { F } & -1.398994000 \\ \text { F } & -0.322339000 \\ \text { F } & -1.816535000 \\ \text { C } & -4.192194000 \\ \text { F } & -4.408949000 \\ \text { F } & -2.842222000 \\ \text { F } & -4.778734000 \\ \text { C } & -6.407696000 \\ \text { F } & -7.747837000 \\ \text { F } & -6.218272000 \\ \text { F } & -5.945800000 \\ \text { C } & -1.406629000 \\ \text { F } & -2.556815000 \\ \text { F } & -1.461163000 \\ \text { F } & -0.372082000 \\ \text { C } & 1.589371000 \\ \text { F } & 2.463690000 \\ \text { F } & 1.575128000 \\ \text { F } & 2.104399000\end{array}$

$-2.703090000$ $-3.036470000$ $-4.133989000$ $-3.640966000$ $-4.794599000$ $-5.049868000$ 5.729008000 6.400874000 6.043119000 6.206878000 1.105571000 1.640464000 $-0.114758000$ 0.906318000 $-2.100736000$ $-0.779177000$ $-2.306522000$ $-2.815990000$ $-5.434406000$ $-5.259459000$ $-6.305204000$ $-6.024065000$ 4.908592000 5.425327000 5.061311000 5.668856000 0.933424000 1.521188000 $-0.400584000$ 1.090455000

3.130893000 4.066799000 $-0.344516000$ $-1.167301000$ 0.672756000 $-1.061958000$ $-3.232330000$ $-4.159728000$ $-3.422982000$ $-2.005215000$ $-4.605427000$ $-5.706825000$ $-4.931736000$ $-3.683333000$ $-3.327365000$ $-3.574756000$ $-3.418893000$ $-4.319413000$ $-0.219720000$ $-0.066354000$ $-1.245363000$ 0.920447000 4.393116000 3.873728000 5.741394000 3.935972000 3.580152000 4.430415000 3.831507000 2.307823000

\section{CF3-H+TS-2 ${ }_{\text {decoor }}$}

Zero-point correction $=\quad 0.821190($ Hartree/Particle $)$ $\begin{array}{lc}\text { Thermal correction to Energy }= & 0.902213 \\ \text { Thermal correction to Enthalpy }= & 0.903157\end{array}$ Thermal correction to Gibbs Free Energy= $\quad 0.687262$ Sum of electronic and zero-point Energies $=\quad-4981.794113$ Sum of electronic and thermal Energies $=\quad-4981.713090$ Sum of electronic and thermal Enthalpies $=\quad-4981.712146$ Sum of electronic and thermal Free Energies $=-4981.928041$ E solvent $=-4981.83735556$

$\begin{array}{lrrr}\mathrm{N} & 0.832409000 & 2.406565000 & 0.112085000 \\ \mathrm{C} & 2.775421000 & 3.872905000 & -0.415013000 \\ \mathrm{C} & -0.974949000 & 4.800659000 & -0.064609000 \\ \mathrm{C} & -1.540707000 & 5.812565000 & 0.744161000 \\ \mathrm{H} & -0.893235000 & 6.568630000 & 1.200491000 \\ \mathrm{C} & -3.223504000 & 3.894977000 & -0.392763000 \\ \mathrm{C} & 3.777715000 & 2.789258000 & -0.202631000 \\ \mathrm{C} & -3.777347000 & 4.903086000 & 0.408869000 \\ \mathrm{C} & 1.374710000 & 3.707524000 & -0.206848000 \\ \mathrm{C} & -2.923918000 & 5.861367000 & 0.976824000 \\ \mathrm{C} & 0.498988000 & 4.832958000 & -0.334245000 \\ \mathrm{C} & 3.733082000 & 1.924788000 & 0.910783000 \\ \mathrm{H} & 2.936233000 & 2.025457000 & 1.651691000 \\ \mathrm{C} & 5.792487000 & 0.820893000 & 0.200206000 \\ \mathrm{C} & -1.840325000 & 3.840914000 & -0.626365000 \\ \mathrm{H} & -1.436343000 & 3.047728000 & -1.259631000 \\ \mathrm{C} & 4.729574000 & 0.953045000 & 1.104726000 \\ \mathrm{C} & 3.265964000 & 5.143754000 & -0.790815000 \\ \mathrm{H} & 4.346304000 & 5.275733000 & -0.911962000 \\ \mathrm{C} & 5.848962000 & 1.682866000 & -0.907347000 \\ \mathrm{C} & 2.411431000 & 6.235758000 & -0.961921000 \\ \mathrm{H} & 2.811547000 & 7.209876000 & -1.260419000 \\ \mathrm{C} & 4.856472000 & 2.653085000 & -1.107650000 \\ \mathrm{H} & 4.914961000 & 3.303646000 & -1.986275000 \\ \mathrm{C} & 1.042749000 & 6.077241000 & -0.724507000 \\ \mathrm{H} & 0.364255000 & 6.926585000 & -0.856975000 \\ \mathrm{~N} & -1.052095000 & -2.830221000 & 0.146440000\end{array}$




\begin{tabular}{|c|c|c|c|}
\hline & & & \\
\hline & 1.314344000 & 74540000 & \\
\hline & 77160000 & 52269000 & \\
\hline & 34920000 & 3242000 & -1667871000 \\
\hline & 617217000 & 6287000 & \\
\hline & -3.634487000 & 31000 & 0 \\
\hline & 766306000 & 74000 & \\
\hline & -1.151115000 & -4.1 & \\
\hline 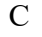 & 368700 & & \\
\hline C & 028897000 & & \\
\hline & -4.804671000 & 3000 & -0 . \\
\hline & -4.79223 & 8000 & 30 \\
\hline & -6.031943000 & -2.362620000 & 29400 \\
\hline & 1.398191000 & -4.182147000 & 1. \\
\hline & 0.513816000 & -4.291685000 & \\
\hline & -5.991237000 & 1000 & 24100 \\
\hline & -2.422777000 & & \\
\hline & -3.37 & & \\
\hline & -4.87 & 6007000 & \\
\hline & 91000 & -6 & \\
\hline & -1.3 & -7 & \\
\hline & -3.6 & -2.6 & \\
\hline & -2.7 & & \\
\hline & -0.0 & & \\
\hline & & & \\
\hline & & & \\
\hline & -1.7 & & \\
\hline & -2.4 & & \\
\hline & & & \\
\hline & & & \\
\hline & -0.6 & & \\
\hline & & & \\
\hline 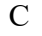 & -0.7 & & \\
\hline & -1.3 & & \\
\hline & -2.27 & & 6000 \\
\hline & -1.6 & & 3900 \\
\hline C & -3.0 & & 83000 \\
\hline $\mathrm{C}$ & -2.3 & -0.6 & 10700 \\
\hline $\mathrm{H}$ & & & \\
\hline $\mathrm{O}$ & -0.1 & & 94200 \\
\hline $\mathrm{C}$ & & & \\
\hline $\mathrm{C}$ & & & \\
\hline $\mathrm{H}$ & & & \\
\hline H & 1.0 & & \\
\hline$c$ & 0 & & 36000 \\
\hline C & -0.4 & & \\
\hline C & & & \\
\hline $\mathrm{H}_{\mathrm{H}}$ & & & \\
\hline & & & \\
\hline$\pi$ & & & \\
\hline & & & 34200 \\
\hline & & & 4 \\
\hline $\mathrm{H}$ & & & 2 \\
\hline 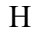 & -0.2 & & \\
\hline $\mathrm{H}$ & & & \\
\hline $\mathrm{H}$ & & & 2900 \\
\hline $\mathrm{H}$ & -3.7 & 4000 & 300000 \\
\hline 11 & -2.3 & 7000 & 400 \\
\hline $\mathrm{H}$ & -3.6 & 000 & 900 \\
\hline 11 & & & 1000 \\
\hline $\mathrm{H}$ & 4.70 & 3000 & 800 \\
\hline$\Pi$ & -4.8 & 4000 & 1600 \\
\hline$H_{2}$ & -6.9 & 0000 & 64600 \\
\hline & & -2.1 & \\
\hline & & & \\
\hline $\mathrm{F}$ & & & \\
\hline & & & \\
\hline & -2.815749000 & 8.100721000 & 1.7687070 \\
\hline
\end{tabular}

$\begin{array}{lr}\text { C } & -4.096366000 \\ \text { F } & -5.416046000 \\ \text { F } & -3.799915000 \\ \text { F } & -3.886829000 \\ \text { C } & -4.926138000 \\ \text { F } & -5.767308000 \\ \text { F } & -3.691064000 \\ \text { F } & -5.364962000 \\ \text { C } & -7.215147000 \\ \text { F } & -8.362456000 \\ \text { F } & -7.275543000 \\ \text { F } & -7.184579000 \\ \text { C } & 2.657373000 \\ \text { F } & 1.681619000 \\ \text { F } & 2.427323000 \\ \text { F } & 3.849285000 \\ \text { C } & 4.911907000 \\ \text { F } & 5.080933000 \\ \text { F } & 6.043043000 \\ \text { F } & 4.813555000 \\ \text { C } & 6.931282000 \\ \text { F } & 7.296410000 \\ \text { F } & 8.039273000 \\ \text { F } & 6.482246000 \\ \text { C } & 4.615052000 \\ \text { F } & 3.411579000 \\ \text { F } & 5.595790000 \\ \text { F } & 4.664620000\end{array}$

2.818528000 3.104992000 2.628856000 1.617919000 $-0.770511000$ 0.193222000 $-0.209981000$ $-1.142676000$ $-3.926075000$ $-3.517505000$ $-5.275866000$ $-3.374794000$ $-3.519626000$ $-2.632944000$ $-4.663051000$ $-3.015616000$ $-3.731346000$ $-2.394939000$ $-4.155396000$ $-4.367086000$ 1.504729000 2.693435000 0.915233000 0.711943000 0.050598000 $-0.607868000$ $-0.890886000$ 0.752862000

$-0.999830000$ $-0.902021000$ $-2.317368000$ $-0.373509000$ $-2.788450000$ $-2.330753000$ $-2.954158000$ $-4.020229000$ 0.426579000 $-0.172114000$ 0.583514000 1.674508000 3.635415000 3.993104000 4.337583000 4.038035000 $-0.885711000$ $-1.151833000$ $-0.269533000$ $-2.081728000$ $-1.952742000$ $-2.502015000$ $-1.439718000$ $-2.974957000$ 2.313499000 2.301683000 2.350877000 3.473979000

\section{CF3-H+TS-2 ${ }_{\text {decoorAr }}$}

Zero-point correction= 0.822804 (Hartree/Particle)

Thermal correction to Energy= $\quad 0.903465$ Thermal correction to Enthalpy $=\quad 0.904409$ Thermal correction to Gibbs Free Energy $=0.691479$ Sum of electronic and zero-point Energies $=\quad-4981.796962$ Sum of electronic and thermal Energies $=\quad-4981.716302$ Sum of electronic and thermal Enthalpies $=\quad-4981.715357$ Sum of electronic and thermal Free Energies $=-4981.928287$ E solvent $=-4981.83711921$

$\begin{array}{lrrr}\mathrm{N} & 1.448295000 & -2.232204000 & 0.129347000 \\ \mathrm{C} & 0.906190000 & -4.351273000 & -0.831988000 \\ \mathrm{C} & 4.447232000 & -3.068685000 & 0.049561000 \\ \mathrm{C} & 4.650934000 & -1.840115000 & -0.613528000 \\ \mathrm{H} & 3.924383000 & -1.503248000 & -1.357531000 \\ \mathrm{C} & 6.550297000 & -2.721245000 & 1.244488000 \\ \mathrm{C} & -0.515640000 & -3.888013000 & -0.863075000 \\ \mathrm{C} & 6.744433000 & -1.499098000 & 0.584033000 \\ \mathrm{C} & 1.921091000 & -3.504487000 & -0.307626000 \\ \mathrm{C} & 5.785866000 & -1.062989000 & -0.345879000 \\ \mathrm{C} & 3.280164000 & -3.937925000 & -0.299337000 \\ \mathrm{C} & -1.142854000 & -3.358476000 & 0.323629000 \\ \mathrm{H} & -0.561823000 & -3.270744000 & 1.247272000 \\ \mathrm{C} & -3.364554000 & -3.835354000 & -0.599003000 \\ \mathrm{C} & 5.412500000 & -3.503502000 & 0.980938000 \\ \mathrm{H} & 5.279289000 & -4.457223000 & 1.502159000 \\ \mathrm{C} & -2.566288000 & -3.389779000 & 0.443537000 \\ \mathrm{C} & 1.232747000 & -5.645681000 & -1.275785000 \\ \mathrm{H} & 0.440237000 & -6.307379000 & -1.640708000 \\ \mathrm{C} & -2.752694000 & -4.287597000 & -1.801941000 \\ \mathrm{C} & 2.558005000 & -6.098903000 & -1.222117000 \\ \mathrm{H} & 2.809315000 & -7.106915000 & -1.566958000 \\ \mathrm{C} & -1.368419000 & -4.320216000 & -1.929529000 \\ \mathrm{H} & -0.919714000 & -4.708574000 & -2.848994000 \\ \mathrm{C} & 3.563635000 & -5.247114000 & -0.750064000 \\ \mathrm{H} & 4.608206000 & -5.576603000 & -0.763941000 \\ \mathrm{~N} & -1.520941000 & 2.492773000 & 0.364281000 \\ \mathrm{C} & -1.890268000 & 4.644476000 & -0.803348000 \\ \mathrm{C} & -4.410106000 & 2.101916000 & 0.652263000\end{array}$




\begin{tabular}{|c|c|c|c|}
\hline & & & \\
\hline & 3000 & 21000 & 1000 \\
\hline & 014084000 & 258868000 & 94000 \\
\hline & 1000 & 397412000 & 1700 \\
\hline & & 50000 & \\
\hline & 7462000 & 12000 & 17 \\
\hline & & 00 & \\
\hline & 000 & 00 & -0.069 \\
\hline & 0.127825000 & & 0.566 \\
\hline & 0.506120000 & & \\
\hline & 2.318665000 & 5.465700000 & \\
\hline & 5.403710000 & 00 & 000 \\
\hline & .697803000 & 1.530359000 & 000 \\
\hline & 1.486810000 & 6.405197000 & \\
\hline & -2.801010000 & 5.589394000 & -1.322126000 \\
\hline & -2.405766000 & 6.479416000 & -1.82 \\
\hline & 1.771869000 & 4.2 & \\
\hline & & & \\
\hline & & & \\
\hline & & 30 & \\
\hline & -0.0 & 3.0 & \\
\hline & & & \\
\hline & & & \\
\hline & -0.2 & -1.6 & -0 . \\
\hline & 0 & & \\
\hline & 0 & 0 & \\
\hline & & 10 & \\
\hline & & & \\
\hline & & & \\
\hline & & & \\
\hline & 0.5 & & \\
\hline & & & \\
\hline & 2.34 & & \\
\hline & 00 & & \\
\hline & 00 & & \\
\hline & 1.326640000 & & \\
\hline & 1.177290000 & & \\
\hline $\mathrm{O}$ & -0.246389 & 00 & \\
\hline & & & \\
\hline & & & \\
\hline & & & \\
\hline & & & \\
\hline & & & \\
\hline & & & \\
\hline & 80000 & 00 & -3.44 \\
\hline & & & \\
\hline & & & \\
\hline & & & \\
\hline & & & \\
\hline & & & \\
\hline & 0 & & \\
\hline & & & \\
\hline & & & \\
\hline & & 00 & \\
\hline & & & \\
\hline & & & \\
\hline & 38000 & -0.6 & \\
\hline & & & 000 \\
\hline & & 00 & \\
\hline & 00 & -0.4 & \\
\hline & 34000 & 5000 & \\
\hline & -1.5 & 00 & \\
\hline & 00 & -3.1 & \\
\hline & 70 & 0 & \\
\hline & & & \\
\hline & & & \\
\hline & & & \\
\hline & 7.262257000 & 0.573778000 & -1.24317400 \\
\hline
\end{tabular}

$\begin{array}{lrrr}\text { F } & 5.311298000 & 0.321135000 & -2.227711000 \\ \text { F } & 5.436145000 & 1.281427000 & -0.257047000 \\ \text { C } & -3.634660000 & -4.662003000 & -2.975378000 \\ \text { F } & -2.982083000 & -5.450085000 & -3.869136000 \\ \text { F } & -4.751412000 & -5.316316000 & -2.567163000 \\ \text { F } & -4.040461000 & -3.539064000 & -3.641080000 \\ \text { C } & -3.157097000 & -2.929643000 & 1.755406000 \\ \text { F } & -2.874862000 & -1.603204000 & 1.964013000 \\ \text { F } & -4.504181000 & -3.077594000 & 1.800674000 \\ \text { F } & -2.621310000 & -3.609581000 & 2.804734000 \\ \text { C } & -6.993128000 & -0.639028000 & -0.011474000 \\ \text { F } & -6.328265000 & -1.627147000 & -0.688055000 \\ \text { F } & -7.855914000 & -1.244499000 & 0.841771000 \\ \text { F } & -7.717453000 & 0.052928000 & -0.930380000 \\ \text { C } & -4.318013000 & 0.502618000 & 4.123395000 \\ \text { F } & -5.020644000 & 1.314416000 & 4.958632000 \\ \text { F } & -4.570947000 & -0.780923000 & 4.487726000 \\ \text { F } & -2.992857000 & 0.747565000 & 4.346562000 \\ \text { C } & 2.638106000 & 3.192462000 & -2.479520000 \\ \text { F } & 3.864655000 & 3.657500000 & -2.807653000 \\ \text { F } & 2.809404000 & 2.121542000 & -1.641168000 \\ \text { F } & 2.051922000 & 2.706613000 & -3.613446000 \\ \text { C } & 2.080415000 & 7.702289000 & -0.252543000 \\ \text { F } & 3.084665000 & 8.142205000 & -1.052966000 \\ \text { F } & 1.142136000 & 8.683017000 & -0.170749000 \\ \text { F } & 2.598952000 & 7.528766000 & 0.996895000\end{array}$

\section{CF3-H+ 1 -Pr-C}

Zero-point correction $=\quad 0.770875($ Hartree $/$ Particle $)$ Thermal correction to Energy $=\quad 0.848122$ Thermal correction to Enthalpy $=\quad 0.849066$ Thermal correction to Gibbs Free Energy $=\quad 0.642066$ Sum of electronic and zero-point Energies $=\quad-4903.272280$ Sum of electronic and thermal Energies $=\quad-4903.195033$ Sum of electronic and thermal Enthalpies $=\quad-4903.194089$ Sum of electronic and thermal Free Energies $=-4903.401089$ E solvent $=-4903.28467233$

$\begin{array}{lrrr}\mathrm{N} & 1.167963000 & -2.311877000 & 0.200520000 \\ \mathrm{C} & 0.503801000 & -4.256724000 & -1.019144000 \\ \mathrm{C} & 4.126934000 & -3.231771000 & -0.130195000 \\ \mathrm{C} & 4.395190000 & -1.963962000 & -0.688584000 \\ \mathrm{H} & 3.689666000 & -1.514377000 & -1.395280000 \\ \mathrm{C} & 6.252440000 & -3.111539000 & 1.072185000 \\ \mathrm{C} & -0.893794000 & -3.722622000 & -0.976266000 \\ \mathrm{C} & 6.508635000 & -1.845101000 & 0.524910000 \\ \mathrm{C} & 1.569986000 & -3.529309000 & -0.421000000 \\ \mathrm{C} & 5.575418000 & -1.279203000 & -0.357385000 \\ \mathrm{C} & 2.909322000 & -4.006748000 & -0.529476000 \\ \mathrm{C} & -1.515393000 & -3.335991000 & 0.267957000 \\ \mathrm{H} & -0.952927000 & -3.425088000 & 1.203981000 \\ \mathrm{C} & -3.740373000 & -3.502070000 & -0.752633000 \\ \mathrm{C} & 5.072210000 & -3.801436000 & 0.749141000 \\ \mathrm{H} & 4.878746000 & -4.783247000 & 1.194034000 \\ \mathrm{C} & -2.941805000 & -3.259776000 & 0.354972000 \\ \mathrm{C} & 0.758960000 & -5.495672000 & -1.634128000 \\ \mathrm{H} & -0.072385000 & -6.069634000 & -2.056865000 \\ \mathrm{C} & -3.129832000 & -3.826537000 & -1.996496000 \\ \mathrm{C} & 2.063127000 & -6.006012000 & -1.682510000 \\ \mathrm{H} & 2.259069000 & -6.971983000 & -2.158555000 \\ \mathrm{C} & -1.747981000 & -3.932040000 & -2.108256000 \\ \mathrm{H} & -1.301465000 & -4.207514000 & -3.068587000 \\ \mathrm{C} & 3.120380000 & -5.257993000 & -1.150176000 \\ \mathrm{H} & 4.148863000 & -5.621971000 & -1.248448000 \\ \mathrm{~N} & -1.244024000 & 2.618114000 & 0.472655000 \\ \mathrm{C} & -1.361812000 & 4.753976000 & -0.768504000 \\ \mathrm{C} & -4.138961000 & 2.492360000 & 0.668528000 \\ \mathrm{C} & -3.862744000 & 2.159119000 & 2.010482000 \\ \mathrm{H} & -3.116685000 & 2.727538000 & 2.574302000\end{array}$




\begin{tabular}{|c|c|c|c|}
\hline $\mathrm{C}$ & -5.821135000 & 0.721877000 & 0.618112000 \\
\hline $\mathrm{C}$ & 0.112932000 & 4.763060000 & -1.013360000 \\
\hline $\mathrm{C}$ & -5.539197000 & 0.392531000 & 1.953022000 \\
\hline $\mathrm{C}$ & -2.019650000 & 3.681829000 & -0.105642000 \\
\hline $\mathrm{C}$ & -4.560914000 & 1.119957000 & 2.645199000 \\
\hline $\mathrm{C}$ & -3.434242000 & 3.616629000 & -0.016897000 \\
\hline $\mathrm{C}$ & 0.906280000 & 5.868662000 & -0.642898000 \\
\hline $\mathrm{H}$ & 0.449251000 & 6.724986000 & -0.135520000 \\
\hline $\mathrm{C}$ & 2.891399000 & 4.792723000 & -1.569908000 \\
\hline $\mathrm{C}$ & -5.134424000 & 1.766536000 & -0.020449000 \\
\hline $\mathrm{H}$ & -5.365090000 & 2.011077000 & -1.062537000 \\
\hline $\mathrm{C}$ & 2.283698000 & 5.878033000 & -0.917992000 \\
\hline $\mathrm{C}$ & -2.155141000 & 5.797849000 & -1.288261000 \\
\hline $\mathrm{H}$ & -1.661737000 & 6.625225000 & -1.809198000 \\
\hline $\mathrm{C}$ & 2.102729000 & 3.697072000 & -1.950506000 \\
\hline $\mathrm{C}$ & -3.552758000 & 5.754808000 & -1.200085000 \\
\hline $\mathrm{H}$ & -4.151920000 & 6.568134000 & -1.621937000 \\
\hline $\mathrm{C}$ & 0.726659000 & 3.682304000 & -1.677669000 \\
\hline $\mathrm{H}$ & 0.120136000 & 2.832197000 & -2.006847000 \\
\hline $\mathrm{C}$ & -4.185363000 & 4.664624000 & -0.588356000 \\
\hline $\mathrm{H}$ & -5.277514000 & 4.631928000 & -0.513755000 \\
\hline $\mathrm{Ni}$ & -0.563502000 & -1.599403000 & -0.457416000 \\
\hline $\mathrm{C}$ & -0.257789000 & 2.795918000 & 1.346209000 \\
\hline $\mathrm{H}$ & -0.123773000 & 3.819863000 & 1.716290000 \\
\hline $\mathrm{C}$ & 1.842127000 & -1.788218000 & 1.197605000 \\
\hline $\mathrm{H}$ & 2.699453000 & -2.337811000 & 1.613173000 \\
\hline $\mathrm{C}$ & 1.592169000 & -0.490157000 & 1.781005000 \\
\hline $\mathrm{C}$ & 2.454037000 & -0.075687000 & 2.819753000 \\
\hline $\mathrm{C}$ & 0.589421000 & 0.425215000 & 1.282357000 \\
\hline $\mathrm{H}$ & 3.195082000 & -0.799981000 & 3.184300000 \\
\hline $\mathrm{C}$ & 2.434719000 & 1.214534000 & 3.394952000 \\
\hline $\mathrm{C}$ & 0.609703000 & 1.776334000 & 1.824707000 \\
\hline $\mathrm{C}$ & 3.382700000 & 1.583100000 & 4.515791000 \\
\hline $\mathrm{C}$ & 1.522684000 & 2.125579000 & 2.862030000 \\
\hline $\mathrm{H}$ & 1.492803000 & 3.150635000 & 3.253759000 \\
\hline $\mathrm{O}$ & -0.312467000 & 0.123508000 & 0.389106000 \\
\hline $\mathrm{C}$ & -1.958574000 & -0.743084000 & -1.492294000 \\
\hline $\mathrm{C}$ & -1.185589000 & -0.213853000 & -2.694600000 \\
\hline $\mathrm{H}$ & -2.737876000 & -1.464411000 & -1.775576000 \\
\hline $\mathrm{H}$ & -2.402056000 & 0.047309000 & -0.860368000 \\
\hline $\mathrm{C}$ & -2.120147000 & 0.531121000 & -3.686845000 \\
\hline $\mathrm{H}$ & -0.369381000 & 0.464439000 & -2.376290000 \\
\hline $\mathrm{H}$ & -0.703732000 & -1.050292000 & -3.239322000 \\
\hline $\mathrm{H}$ & -1.546935000 & 0.896902000 & -4.557194000 \\
\hline $\mathrm{H}$ & -2.912962000 & -0.143307000 & -4.053365000 \\
\hline $\mathrm{H}$ & -2.606237000 & 1.397723000 & -3.203550000 \\
\hline $\mathrm{H}$ & 3.312921000 & 2.654566000 & 4.765928000 \\
\hline $\mathrm{H}$ & 3.159125000 & 1.009286000 & 5.434058000 \\
\hline $\mathrm{H}$ & 4.431481000 & 1.367713000 & 4.242851000 \\
\hline $\mathrm{H}$ & -4.828482000 & -3.436233000 & -0.676218000 \\
\hline $\mathrm{H}$ & -6.069337000 & -0.426159000 & 2.444307000 \\
\hline $\mathrm{H}$ & 7.426278000 & -1.308319000 & 0.778536000 \\
\hline $\mathrm{H}$ & 3.962550000 & 4.806866000 & -1.786574000 \\
\hline $\mathrm{H}$ & -1.371282000 & 1.630165000 & 0.174226000 \\
\hline $\mathrm{C}$ & 7.277691000 & -3.767617000 & 1.977571000 \\
\hline $\mathrm{F}$ & 8.035268000 & -2.842810000 & 2.625679000 \\
\hline $\mathrm{F}$ & 6.675839000 & -4.548937000 & 2.917658000 \\
\hline $\mathrm{F}$ & 8.119562000 & -4.563724000 & 1.266152000 \\
\hline $\mathrm{C}$ & 5.803716000 & 0.103412000 & -0.933528000 \\
\hline $\mathrm{F}$ & 7.092501000 & 0.507433000 & -0.801474000 \\
\hline $\mathrm{F}$ & 5.479890000 & 0.148555000 & -2.252903000 \\
\hline $\mathrm{F}$ & 5.020561000 & 1.026617000 & -0.295052000 \\
\hline $\mathrm{C}$ & -4.006321000 & -3.975199000 & -3.224447000 \\
\hline $\mathrm{F}$ & -3.378795000 & -4.662014000 & -4.213802000 \\
\hline $\mathrm{F}$ & -5.165162000 & -4.616051000 & -2.929398000 \\
\hline $\mathrm{F}$ & -4.336796000 & -2.746311000 & -3.724825000 \\
\hline $\mathrm{C}$ & -3.536351000 & -2.942979000 & 1.709026000 \\
\hline $\mathrm{F}$ & -3.096818000 & -1.723289000 & 2.151236000 \\
\hline
\end{tabular}

$\begin{array}{lrrr}\text { F } & -4.892541000 & -2.910078000 & 1.687396000 \\ \text { F } & -3.150490000 & -3.858367000 & 2.638404000 \\ \text { C } & -6.811404000 & -0.107136000 & -0.174117000 \\ \text { F } & -6.161727000 & -1.077115000 & -0.893545000 \\ \text { F } & -7.707081000 & -0.732657000 & 0.628410000 \\ \text { F } & -7.496761000 & 0.653284000 & -1.067650000 \\ \text { C } & -4.249329000 & 0.818404000 & 4.097438000 \\ \text { F } & -4.717475000 & 1.802507000 & 4.912540000 \\ \text { F } & -4.793020000 & -0.354457000 & 4.505425000 \\ \text { F } & -2.898871000 & 0.747350000 & 4.297178000 \\ \text { C } & 2.699206000 & 2.510056000 & -2.678516000 \\ \text { F } & 4.037732000 & 2.617871000 & -2.834953000 \\ \text { F } & 2.446812000 & 1.348005000 & -1.990516000 \\ \text { F } & 2.139415000 & 2.355213000 & -3.909553000 \\ \text { C } & 3.127458000 & 7.044026000 & -0.438130000 \\ \text { F } & 4.259332000 & 7.182403000 & -1.173602000 \\ \text { F } & 2.435328000 & 8.213297000 & -0.499295000 \\ \text { F } & 3.500892000 & 6.862834000 & 0.861671000\end{array}$

\section{CF3-H+ TS-2}

Zero-point correction $=\quad 0.770275($ Hartree/Particle $)$

Thermal correction to Energy $=\quad 0.846471$

Thermal correction to Enthalpy= $\quad 0.847415$

Thermal correction to Gibbs Free Energy $=\quad 0.644494$

Sum of electronic and zero-point Energies $=\quad-4903.270510$

Sum of electronic and thermal Energies $=\quad-4903.194314$

Sum of electronic and thermal Enthalpies $=\quad-4903.193370$

Sum of electronic and thermal Free Energies $=-4903.396291$

E solvent $=-4903.27821546$

$\begin{array}{lll}2.473570000 & -0.149836000 & -1.045067000\end{array}$

$\begin{array}{lll}3.289600000 & 1.254782000 & -2.816709000\end{array}$

$\begin{array}{lll}5.384777000 & -0.956078000 & -0.438552000\end{array}$

$\begin{array}{lll}6.200083000 & -2.089347000 & -0.637760000\end{array}$

$\begin{array}{lll}6.456400000 & -2.405892000 & -1.654335000\end{array}$

$\begin{array}{lll}5.525702000 & -1.328460000 & 1.972331000\end{array}$

$\begin{array}{lll}1.906258000 & 1.808367000 & -2.968093000\end{array}$

$\begin{array}{lll}6.334857000 & -2.458674000 & 1.768872000\end{array}$

$\begin{array}{llr}3.594759000 & 0.293932000 & -1.810246000\end{array}$

$\begin{array}{lll}6.671911000 & -2.830591000 & 0.459311000\end{array}$

$\begin{array}{lll}4.940166000 & -0.127466000 & -1.601365000\end{array}$

$\begin{array}{lll}0.736405000 & 0.978570000 & -3.040829000\end{array}$

$\begin{array}{lll}0.836074000 & -0.110089000 & -3.035384000\end{array}$

$\begin{array}{rrr}-0.619259000 & 2.904191000 & -3.731977000\end{array}$

$\begin{array}{lll}5.059806000 & -0.579389000 & 0.882527000\end{array}$

$\begin{array}{lll}4.453648000 & 0.313907000 & 1.062545000\end{array}$

$\begin{array}{lll}-0.500314000 & 1.539780000 & -3.456750000\end{array}$

$\begin{array}{lll}4.312445000 & 1.704216000 & -3.674255000\end{array}$

$\begin{array}{lll}4.068515000 & 2.409963000 & -4.475256000\end{array}$

$\begin{array}{lll}0.514085000 & 3.733549000 & -3.604997000\end{array}$

$\begin{array}{lll}5.624912000 & 1.237326000 & -3.524766000\end{array}$

$\begin{array}{lll}6.413139000 & 1.587855000 & -4.198549000\end{array}$

$\begin{array}{lll}1.753309000 & 3.195093000 & -3.241549000\end{array}$

$\begin{array}{lll}2.625613000 & 3.852943000 & -3.165897000\end{array}$

$\begin{array}{lll}5.932174000 & 0.349897000 & -2.486193000\end{array}$

$\begin{array}{lll}6.970542000 & 0.043571000 & -2.319281000\end{array}$

$\begin{array}{lll}-2.704843000 & -0.289836000 & 0.989485000\end{array}$

$\begin{array}{lll}-4.293533000 & 1.444758000 & 1.683064000\end{array}$

$\begin{array}{lll}-4.956520000 & -1.953999000 & -0.062506000\end{array}$

$\begin{array}{lll}-5.645664000 & -3.068255000 & 0.464560000\end{array}$

$\begin{array}{lll}-6.264984000 & -2.952168000 & 1.360320000\end{array}$

$\begin{array}{lll}-4.087245000 & -3.383939000 & -1.838230000\end{array}$

$\begin{array}{lll}-3.214475000 & 2.261195000 & 2.312541000\end{array}$

$\begin{array}{lll}-4.766301000 & -4.489772000 & -1.307343000\end{array}$

$\begin{array}{lll}-4.059681000 & 0.173622000 & 1.090385000\end{array}$

$\begin{array}{lll}-5.546500000 & -4.324862000 & -0.152074000\end{array}$

$\begin{array}{lll}-5.131512000 & -0.616292000 & 0.588343000\end{array}$

$\begin{array}{llll}\mathrm{C} & -3.153275000 & 3.652908000 & 2.072964000 \\ \mathrm{H} & -3.843601000 & 4.115263000 & 1.360607000\end{array}$ 


\begin{tabular}{|c|c|c|c|}
\hline & & & \\
\hline & 41000 & 3000 & \\
\hline & 7940000 & -1.265579000 & 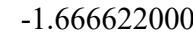 \\
\hline & 90000 & 54000 & \\
\hline & 9134000 & & \\
\hline & 1000 & 00 & 2700 \\
\hline & 000 & 00 & \\
\hline & -6.68 & 1.160440000 & \\
\hline & -7.708 & 421000 & 7000 \\
\hline & -2.3022 & 5000 & 73000 \\
\hline & -2.3 & 6000 & 300 \\
\hline & -6.440 & -0.102215000 & 000 \\
\hline & -7.2700 & -0.698967000 & 72000 \\
\hline & 1.058921000 & 1.172404000 & -0.806701000 \\
\hline & -2.289172000 & -1.513086000 & 1.309855000 \\
\hline & -3.033760000 & -2.166031000 & 1.778709000 \\
\hline & 2.38 & -1.3 & 70000 \\
\hline & 3.21 & & \\
\hline & & & \\
\hline & 1.43 & & \\
\hline & 0.05 & & 0. \\
\hline & 2.38 & & \\
\hline & 0.4 & & \\
\hline & & & \\
\hline & & & \\
\hline & & & \\
\hline & & & \\
\hline & -0 . & & \\
\hline & & & \\
\hline & & & \\
\hline & -0. & & \\
\hline & -0.5 & 2.5 & \\
\hline & & & \\
\hline & & & \\
\hline & & & \\
\hline & & & \\
\hline & & & \\
\hline & 1.39 & 3.0 & 40000 \\
\hline & & & \\
\hline & 6.70 & & \\
\hline & & & \\
\hline & & & \\
\hline & & & \\
\hline & & & \\
\hline & & & \\
\hline & & & \\
\hline & & & \\
\hline & & & \\
\hline & & & \\
\hline & & & \\
\hline & & 0 & 00 \\
\hline & & & \\
\hline & & & 00 \\
\hline & & & -5 . \\
\hline & & & -3 \\
\hline & & & 72000 \\
\hline & & & 10000 \\
\hline & & 309000 & 13000 \\
\hline $\mathrm{F}$ & & 4000 & 55000 \\
\hline $\mathrm{C}$ & & 00 & 33000 \\
\hline & & 77000 & 50000 \\
\hline & & 28000 & 78000 \\
\hline & & 607000 & \\
\hline & & & \\
\hline & & & \\
\hline & & & \\
\hline & & & \\
\hline & -3.227566000 & -3.530743000 & -3.0779740 \\
\hline
\end{tabular}

$\begin{array}{lrrr}\text { F } & -3.518292000 & -2.569994000 & -3.995179000 \\ \text { F } & -1.902920000 & -3.398099000 & -2.763857000 \\ \text { F } & -3.385735000 & -4.740261000 & -3.671822000 \\ \text { C } & -6.233169000 & -5.523234000 & 0.474535000 \\ \text { F } & -7.378944000 & -5.163323000 & 1.112442000 \\ \text { F } & -6.541785000 & -6.465326000 & -0.452229000 \\ \text { F } & -5.421016000 & -6.110517000 & 1.401382000 \\ \text { H } & -0.212813000 & -5.924701000 & 2.067571000 \\ \text { H } & 1.039916000 & -6.089125000 & 0.808078000 \\ \text { H } & 1.482856000 & -5.516307000 & 2.433408000\end{array}$

\section{CF3-H+ $1-\boldsymbol{\beta}-\mathbf{C}$}

Zero-point correction $=\quad 0.770077($ Hartree/Particle) Thermal correction to Energy= $\quad 0.847217$ Thermal correction to Enthalpy $=\quad 0.848161$ Thermal correction to Gibbs Free Energy $=\quad 0.641420$ Sum of electronic and zero-point Energies $=\quad-4903.275860$ Sum of electronic and thermal Energies $=\quad-4903.198721$ Sum of electronic and thermal Enthalpies $=\quad-4903.197776$ Sum of electronic and thermal Free Energies $=-4903.404517$ E solvent $=-4903.29573705$

N 1.2461360000 .235381000

$\begin{array}{llll}\mathrm{C} & 3.392298000 & 3.573006000 & 0.476721000\end{array}$

$\begin{array}{llll}\text { C } & -0.121864000 & 4.625853000 & -0.792375000\end{array}$

$\begin{array}{llll}\text { C } & -1.017335000 & 5.642498000 & -0.400065000\end{array}$

$\begin{array}{llll}\mathrm{H} & -0.666517000 & 6.466112000 & 0.230594000\end{array}$

$\begin{array}{llll}\text { C } & -1.945681000 & 3.553570000 & -2.020505000\end{array}$

$\begin{array}{llll}\text { C } & 4.113939000 & 2.376362000 & 0.902326000\end{array}$

C $\quad-2.835806000 \quad 4.560268000 \quad-1.619298000$

$\begin{array}{llll}\text { C } & 1.990416000 & 3.550476000 & 0.152350000\end{array}$

$\begin{array}{llll}\text { C } & -2.360723000 & 5.605119000 & -0.809337000\end{array}$

$\begin{array}{llll}\text { C } & 1.322812000 & 4.671478000 & -0.414594000\end{array}$

$\begin{array}{llll}\text { C } & 3.890398000 & 1.892121000 & 2.207064000\end{array}$

$\begin{array}{llll}\mathrm{H} & 3.184835000 & 2.405987000 & 2.866688000\end{array}$

$\begin{array}{llll}\text { C } & 5.470262000 & 0.072611000 & 1.839775000\end{array}$

$\begin{array}{lrrr}\text { C } & -0.604135000 & 3.588991000 & -1.614572000\end{array}$

$\begin{array}{llll}\mathrm{H} & 0.081566000 & 2.802889000 & -1.950222000\end{array}$

$\begin{array}{llll}\text { C } & 4.572617000 & 0.757561000 & 2.672903000\end{array}$

$\begin{array}{llll}\text { C } & 4.111681000 & 4.741504000 & 0.046290000\end{array}$

$\begin{array}{llll}\mathrm{H} & 5.192381000 & 4.762237000 & 0.224996000\end{array}$

$\begin{array}{llll}\text { C } & 5.698515000 & 0.552601000 & 0.542235000\end{array}$

$\begin{array}{llll}\mathrm{C} & 3.458247000 & 5.869779000 & -0.468376000\end{array}$

$\begin{array}{llll}\mathrm{H} & 4.028197000 & 6.774136000 & -0.704368000\end{array}$

$\begin{array}{llll}\text { C } & 5.043494000 & 1.707130000 & 0.081916000\end{array}$

$\begin{array}{llll}\text { H } & 5.243373000 & 2.079870000 & -0.928508000\end{array}$

$\begin{array}{llll}\text { C } & 2.077606000 & 5.829991000 & -0.699690000\end{array}$

$\begin{array}{llll}\mathrm{H} & 1.569822000 & 6.692828000 & -1.144808000\end{array}$

$\mathrm{N} \quad-1.348034000 \quad-2.499938000 \quad 0.125816000$

$\begin{array}{llll}\text { C } & -3.109922000 & -4.029683000 & -0.718104000\end{array}$

$\begin{array}{llll}\text { C } & 0.741775000 & -4.200621000 & -0.969813000\end{array}$

$\begin{array}{llll}\text { C } & 1.566714000 & -4.262061000 & -2.115912000\end{array}$

H $\quad 1.129599000 \quad-4.513453000 \quad-3.087581000$

$\begin{array}{llll}\text { C } & 2.706923000 & -3.590354000 & 0.349208000\end{array}$

$\begin{array}{llll}\text { C } & -4.240951000 & -3.152846000 & -0.276115000\end{array}$

$\begin{array}{llll}\text { C } & 3.518044000 & -3.636590000 & -0.793636000\end{array}$

$\begin{array}{llll}\text { C } & -1.735150000 & -3.697038000 & -0.559588000\end{array}$

$\begin{array}{lrrr}\text { C } & -1.735150000 & -3.697038000 & -0.559588000 \\ \text { C } & 2.938587000 & -3.984038000 & -2.025678000\end{array}$

$\begin{array}{llll}\text { C } & -0.709682000 & -4.525164000 & -1.095892000\end{array}$

$\begin{array}{llll}\text { C } & -5.229790000 & -3.676279000 & 0.584553000\end{array}$

$\mathrm{H} \quad-5.138879000 \quad-4.701490000 \quad 0.959105000$

$\begin{array}{llll}\text { C } & -6.474250000 & -1.584412000 & 0.479401000\end{array}$

$\begin{array}{llll}\text { C } & 1.333241000 & -3.873361000 & 0.267619000\end{array}$

$\begin{array}{llll}\mathrm{H} & 0.731716000 & -3.866724000 & 1.181573000\end{array}$

$\begin{array}{lrrr}\mathrm{H} & 0.731716000 & -3.866724000 & 1.181573000 \\ \mathrm{C} & -6.333860000 & -2.895131000 & 0.960749000\end{array}$

C $\quad-3.429261000 \quad-5.235288000 \quad-1.376979000$

$\mathrm{H} \quad-4.484937000 \quad-5.493964000 \quad-1.510345000$

$\begin{array}{llll}\text { C } & -5.498302000 & -1.062560000 & -0.382452000\end{array}$

$\begin{array}{llll}\text { C } & -2.431317000 & -6.066702000 & -1.900356000\end{array}$ 


\begin{tabular}{|c|c|c|c|}
\hline & & & \\
\hline & 7000 & 5693000 & \\
\hline & 1739000 & -1.417897000 & 9000 \\
\hline & 000 & 03472000 & \\
\hline & 300994000 & & \\
\hline & 3120000 & & \\
\hline & & & \\
\hline & -2.641019000 & & \\
\hline & 0.451758000 & & \\
\hline & 5000 & & \\
\hline & -0.4279 & & \\
\hline & 113000 & 000 & \\
\hline & -0.610710000 & & \\
\hline & 147000 & 2.596249000 & 3.683671000 \\
\hline & -2.049936000 & 0.715211000 & 3.764696000 \\
\hline & -1.566811000 & -0.806862000 & \\
\hline & -2.770338000 & & $\$ 775000$ \\
\hline & -2.24 & & \\
\hline & -2.9 & & \\
\hline & & & \\
\hline & & 00 & \\
\hline & & & \\
\hline & & & \\
\hline & 0.6 & & \\
\hline & & & \\
\hline & & & \\
\hline & & & \\
\hline & & & \\
\hline & & & \\
\hline & & & \\
\hline & & & \\
\hline & & & \\
\hline & -3.3 & & \\
\hline & & & \\
\hline & & & \\
\hline & & & 95000 \\
\hline & & & \\
\hline & 00 & & 6625000 \\
\hline & & & \\
\hline & & & \\
\hline & & & \\
\hline & & & \\
\hline & & & \\
\hline & & & \\
\hline & & & 00 \\
\hline & & & \\
\hline & & & \\
\hline & & & \\
\hline & & & \\
\hline & & & \\
\hline & & & 00 \\
\hline & & & \\
\hline & & & 00 \\
\hline & & & \\
\hline & & & \\
\hline & & & 09000 \\
\hline & & & 57000 \\
\hline & & & 06000 \\
\hline$C$ & & & 52000 \\
\hline & & & 303000 \\
\hline & & & 578000 \\
\hline & & 2000 & 667000 \\
\hline & & & 970000 \\
\hline & & & \\
\hline & & & -0 . \\
\hline & & & -2.24207300 \\
\hline & & & \\
\hline & -8.556252000 & -2.858196000 & 1.81148200 \\
\hline
\end{tabular}

$\begin{array}{llll}\text { F } & -7.513028000 & -4.795613000 & 1.767883000 \\ \text { F } & -6.927135000 & -3.264971000 & 3.225901000\end{array}$

\section{CF3-H+ TS-1 BHE-C $_{\text {B }}$}

Zero-point correction $=\quad 0.767472($ Hartree/Particle $)$

Thermal correction to Energy $=\quad 0.844278$

Thermal correction to Enthalpy= $\quad 0.845222$

Thermal correction to Gibbs Free Energy $=0.638986$

Sum of electronic and zero-point Energies $=\quad-4903.259042$

Sum of electronic and thermal Energies $=\quad-4903.182236$

Sum of electronic and thermal Enthalpies $=\quad-4903.181292$

Sum of electronic and thermal Free Energies $=-4903.387528$

E solvent $=-4903.27156218$

$\begin{array}{llll}\text { C } & 2.494473000 & 4.230050000 & 0.121454000\end{array}$

$\begin{array}{llll}\text { C } & -1.338890000 & 4.680764000 & -0.031754000\end{array}$

C $\quad-1.860964000 \quad 3.675293000 \quad-0.868882000$

H $\quad-1.177062000 \quad 3.054353000 \quad-1.455794000$

$\begin{array}{llll}\text { C } & -3.628729000 & 5.270945000 & 0.596120000\end{array}$

$\begin{array}{llll}\text { C } & 3.559217000 & 3.189060000 & 0.205933000\end{array}$

$\begin{array}{llll}\text { C } & -4.140092000 & 4.260903000 & -0.235481000\end{array}$

$\begin{array}{llll}\text { C } & 1.108418000 & 3.920657000 & 0.183848000\end{array}$

$\begin{array}{llll}\text { C } & -3.245141000 & 3.465904000 & -0.965399000\end{array}$

C $\quad 0.131018000 \quad 4.948366000 \quad 0.031480000$

$\begin{array}{llll}\text { C } & 3.602698000 & 2.228996000 & 1.237078000\end{array}$

$\begin{array}{llll}\mathrm{H} & 2.850024000 & 2.240155000 & 2.030192000\end{array}$

$\begin{array}{llll}\text { C } & 5.628937000 & 1.247937000 & 0.291815000\end{array}$

$\begin{array}{llll}\text { C } & -2.244617000 & 5.480258000 & 0.699532000\end{array}$

$\begin{array}{llll}\mathrm{H} & -1.867358000 & 6.269925000 & 1.357581000\end{array}$

$\begin{array}{llll}\text { C } & 4.631386000 & 1.273510000 & 1.277248000\end{array}$

$\begin{array}{llll}\text { C } & 2.882451000 & 5.575477000 & -0.054015000\end{array}$

$\mathrm{H} \quad 3.951099000 \quad 5.815232000 \quad-0.073285000$

$\begin{array}{llll}\text { C } & 5.599037000 & 2.210086000 & -0.729051000\end{array}$

$\begin{array}{llll}\text { C } & 1.932820000 & 6.596778000 & -0.169959000\end{array}$

$\begin{array}{llll}\mathrm{H} & 2.253794000 & 7.635165000 & -0.300359000\end{array}$

C $\quad 4.580485000 \quad 3.173804000 \quad-0.770088000$

$\begin{array}{llll}\mathrm{H} & 4.564652000 & 3.910315000 & -1.580368000\end{array}$

$\begin{array}{llll}\text { C } & 0.570715000 & 6.280053000 & -0.136196000\end{array}$

$\mathrm{H} \quad-0.177826000 \quad 7.067041000 \quad-0.277757000$

$\mathrm{N} \quad-0.658715000 \quad-2.805047000 \quad-0.023696000$

$\begin{array}{llll}\text { C } & -1.879813000 & -4.562397000 & -1.290624000\end{array}$

C $\quad 1.850591000 \quad-4.124036000 \quad-0.448419000$

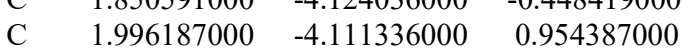

H $\quad 1.176382000 \quad-4.459509000 \quad 1.591133000$

$\begin{array}{llll}\text { C } & 4.123820000 & -3.261154000 & -0.650190000\end{array}$

C $\quad-3.218915000 \quad-3.937588000 \quad-1.028829000$

$\begin{array}{llll}\text { C } & 4.264648000 & -3.254309000 & 0.746836000\end{array}$

$\begin{array}{llll}\text { C } & -0.669030000 & -4.028483000 & -0.773087000\end{array}$

$\begin{array}{llll}\text { C } & 3.197347000 & -3.689902000 & 1.544331000\end{array}$

$\begin{array}{llll}\text { C } & 0.586675000 & -4.624598000 & -1.067591000\end{array}$

$\begin{array}{llll}\text { C } & -3.986043000 & -4.328328000 & 0.084912000\end{array}$

H $\quad-3.615151000 \quad-5.110520000 \quad 0.757618000$

$\begin{array}{llll}\text { C } & -5.737010000 & -2.747822000 & -0.526921000\end{array}$

$\begin{array}{llll}\text { C } & 2.933544000 & -3.705696000 & -1.249206000\end{array}$

$\mathrm{H} \quad 2.843807000 \quad-3.717957000 \quad-2.340540000$

$\begin{array}{llll}\text { C } & -5.226623000 & -3.719694000 & 0.344108000\end{array}$

\begin{tabular}{llll}
$\mathrm{C}$ & -5.226623000 & -3.719694000 & 0.344108000 \\
\hline
\end{tabular}

H $\quad-2.730919000 \quad-6.114600000 \quad-2.526895000$

$\begin{array}{llll}\text { C } & -4.993285000 & -2.386147000 & -1.662912000\end{array}$

$\begin{array}{llll}\text { C } & -0.568596000 & -6.278942000 & -2.444892000\end{array}$

$\mathrm{H} \quad-0.532167000 \quad-7.156723000 \quad-3.097971000$

$\begin{array}{llll}\mathrm{C} & -3.739002000 & -2.966482000 & -1.908685000\end{array}$

$\mathrm{H} \quad-3.163194000 \quad-2.660674000 \quad-2.787577000$

C $\quad 0.615954000 \quad-5.744602000 \quad-1.920943000$

H $\quad 1.580085000 \quad-6.215515000 \quad-2.140894000$

$\mathrm{Ni} \quad 1.067687000 \quad 1.164432000 \quad-0.876541000$

$\begin{array}{llll}\text { C } & -1.423801000 & -2.484926000 & 1.014612000\end{array}$

$\mathrm{H} \quad-2.073363000 \quad-3.270738000 \quad 1.412790000$ 


\begin{tabular}{|c|c|c|c|}
\hline & & & \\
\hline & & 75000 & 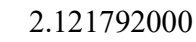 \\
\hline & -0.748459000 & 181000 & \\
\hline & -1.560953000 & 5000 & \\
\hline & -0.652122000 & -0.087701000 & 00 \\
\hline & 1.606059000 & 77000 & 26000 \\
\hline & 401000 & & 1000 \\
\hline & 4000 & & 400 \\
\hline & 0000 & & \\
\hline & 5000 & & \\
\hline & 5000 & 00 & 6700 \\
\hline & 0.07 & 00 & 2000 \\
\hline & & & \\
\hline & & & \\
\hline I & & & \\
\hline & 0.9 & & \\
\hline & -0.1 & & \\
\hline & & & -3 \\
\hline & -0.5 & & 000 \\
\hline & & & \\
\hline & -0.9 & & 2000 \\
\hline & -2.5 & & 5000 \\
\hline & & & \\
\hline & -3.8 & & 9000 \\
\hline & 6.41 & & \\
\hline & & & 000 \\
\hline & -5 . & & 1000 \\
\hline & -6.7 & & 3000 \\
\hline & & & 400( \\
\hline & & & \\
\hline & & & \\
\hline & & & \\
\hline & 6.0 & & \\
\hline & & & \\
\hline & & & \\
\hline & & & \\
\hline & & & \\
\hline & & & \\
\hline & & & \\
\hline & & & 7000 \\
\hline & & & 7000 \\
\hline & & & 3000 \\
\hline & & & 000 \\
\hline & & & 000 \\
\hline & 000 & & 0000 \\
\hline & 000 & 000 & 14000 \\
\hline & -6.3 & -0. & 000 \\
\hline & & -0.7 & \\
\hline & & & -3 \\
\hline & & & \\
\hline & & & 8000 \\
\hline & & & \\
\hline & & & 2.7 \\
\hline & & & \\
\hline$F$ & & & \\
\hline 1 & & & \\
\hline$\Gamma$ & 5.28 & -3.3 & -2.729267000 \\
\hline $\mathrm{C}$ & & -3.7 & \\
\hline $\mathrm{F}$ & 2.22 & & 3.646 \\
\hline $\mathrm{F}$ & 3.36 & -5.0 & 3.488437000 \\
\hline$\Gamma$ & 4.425939000 & 6000 & \\
\hline & & & \\
\hline
\end{tabular}

\section{CF3-H+ 1 -BHE-C}

Zero-point correction= Thermal correction to Energy=

Thermal correction to Enthalpy= 0.768042 (Hartree/Particle) 0.845541 0.846485

Thermal correction to Gibbs Free Energy=
Sum of electronic and zero-point Energies= Sum of electronic and thermal Energies= Sum of electronic and thermal Enthalpies= $-4903.261610$ $-4903.184112$ $-4903.183168$

Sum of electronic and thermal Free Energies $=-4903.390949$ E solvent $=-4903.27755965$

$1.950006000 \quad 4.528507000$

$-1.904517000 \quad 4.604232000$

$-2.328953000 \quad 3.578043000$

$-1.596023000 \quad 3.069436000$

$-4.229469000 \quad 4.909185000$

$3.094170000 \quad 3.572010000$

$-4.642587000 \quad 3.877073000$

$0.602012000 \quad 4.092255000$

$-3.681726000 \quad 3.215884000$

$-0.469777000$

5.021248000

3.322975000

2.738114000

2.670552000

5.266306000

$-2.874154000$

$-2.570683000$

4.406657000

2.211263000

3.253062000

5.047529000

1.166820000

H $\quad 1.386202000$

3.982932000

3.821314000

$-0.159407000$

$-0.981634000$

2.801360000

1.747906000

5.270061000

6.075228000

1.843279000

5.900202000

6.234080000

2.585988000

6.829040000

7.892061000

3.499815000

4.143251000

6.388003000

7.101332000

$-0.228684000$

$-2.841064000$

$-1.199672000$

2.425881000

$-4.772242000$

2.518406000

$-3.874537000$

$-3.790264000$

1.726188000

4.582148000

$-4.216371000$

$-2.743241000$

$-2.605975000$

$-4.288293000$

4.667958000

$-2.661140000$

$-0.072724000$

3.634596000

1.250535000

$-3.374540000$

$-2.954088000$

$-4.083292000$

$-3.198507000$

$-4.544161000$

$-4.750558000$

$-5.487745000$

$-5.249570000$

$-3.356789000$

3.478331000

3.431288000

$-4.681011000$

$-0.970238000$

$-1.832291000$

$-4.498633000$

0.331377000

0.486574000

$-3.184165000$

$-2.606792000$

$-3.357690000$

$-3.424998000$

$-4.271653000$

$-5.927917000$

$-6.464435000$

$-2.922587000$

$-6.376381000$

$-7.271382000$

$-3.376579000$

$-3.019041000$

$-5.688543000$

1.432199000

2.449305000

$-6.055917000$

1.357213000

$-2.598828000$

$-1.040732000$

$-3.456625000$

$-1.582403000$

$-0.377828000$

2.400681000

3.241549000

1.116261000

$-0.882565000$

$-1.700571000$

1.095815000

0.324018000

0.020231000

$-0.066078000$

$-0.934838000$

$-1.569255000$

0.628750000

0.078623000

$-0.227658000$

0.114001000

$-1.008419000$

$-0.019722000$

1.189978000

2.066289000

0.092541000

0.712447000

1.390168000

1.196758000

$-0.171986000$

$-0.229771000$

$-1.012394000$

$-0.262175000$

$-0.404047000$

$-1.015399000$

$-1.886432000$

$-0.193317000$

$-0.316353000$

0.026073000

$-1.199470000$

$-0.275665000$

1.129957000

1.754441000

$-0.440936000$

$-0.998726000$

0.956758000

$-0.677110000$

1.738170000

$-0.909362000$

0.086108000

0.779916000

$-0.608414000$

$-1.058285000$

$-2.150857000$

0.288265000

$-1.972597000$

$-2.382801000$

$-1.713732000$

$-2.237944000$

$-2.848882000$

$-1.905201000$

$-2.763178000$

$-1.709830000$

$-1.883595000$

$-0.948764000$

1.049730000

1.461489000

1.402039000

2.068679000

1.818430000

2.970907000

1.116392000

3.485639000

$-1.881601000$

$-0.116125000$

2.049242000

$-2.314120000 \quad-0.068638000$

3.479929000

1.647192000 


\begin{tabular}{|c|c|c|c|}
\hline & 0 & $-\mathrm{c}-\mathrm{c}-\mathrm{c}$ & \\
\hline & -2.071196000 & 796000 & 809000 \\
\hline & -2.556251000 & -2.184958000 & 126059000 \\
\hline & 0.153981000 & -0.180539000 & 0059445000 \\
\hline & 1.894405000 & 0.122287000 & 20е6е \\
\hline & 0.758085000 & 0.539288000 & -2.90763700 \\
\hline 11 & 2.861086000 & 0.612101000 & -2.3705870 \\
\hline $\mathrm{H}$ & 1.925013000 & -0.856555000 & -1.713550000 \\
\hline $\mathrm{C}$ & -0.503918000 & -0.259124000 & \\
\hline $\mathrm{H}$ & 0.868473000 & 1.397533000 & -3.584226000 \\
\hline $\mathrm{H}$ & -1.409059000 & 0.365048000 & -3.009515000 \\
\hline $\mathrm{H}$ & -0.506139000 & -0.665814000 & -4.13292900 \\
\hline $\mathrm{H}$ & -0.584232000 & -1.100070000 & -2.394458000 \\
\hline & -2.596881000 & 0.187324000 & 5.619693000 \\
\hline & -3.951157000 & 0.784230000 & 4.631731000 \\
\hline & -3.724104000 & -0.969405000 & 4.866899000 \\
\hline & 6.091452000 & 1.032191000 & 0.088495000 \\
\hline$\pi$ & 5.521908000 & -2.1 & 1.432948000 \\
\hline $\mathrm{H}$ & -5.698306000 & & -0.29 \\
\hline$H$ & -6.2653 & -2.9 & \\
\hline $\mathrm{H}$ & 790000 & 2.4 & -1.7327 \\
\hline $\mathrm{C}$ & 5.88 & & -2.26 \\
\hline & 6.1250 & & -2.8 \\
\hline $\mathrm{F}$ & 7.07 & & \\
\hline $\mathrm{F}$ & 5.22 & & \\
\hline $\mathrm{C}$ & 4.57 & & 2.3 \\
\hline & 3.58 & -0.0 & 2.4 \\
\hline $\mathrm{F}$ & 5.76 & 0.2 & 2.3 \\
\hline $\mathrm{F}$ & 4.50 & 1.6 & 3.5 \\
\hline $\mathrm{C}$ & 4.0 & 2.0 & 30400 \\
\hline & 0 & 2.0 & -2.1 \\
\hline $\mathrm{F}$ & 00 & 2.0 & -3.0 \\
\hline $\mathrm{F}$ & -3.7 & 0.8 & -1.268449000 \\
\hline & 00 & 5.5 & 376000 \\
\hline & -6.4 & 5.5 & $1.02307100 \mathrm{C}$ \\
\hline & 00 & & 2.76 \\
\hline $\mathrm{F}$ & -4.9 & 6.8 & 1.7326 \\
\hline $\mathrm{C}$ & -5.13 & -1.994511000 & -2.733167000 \\
\hline $\mathrm{F}$ & -6.00 & -1.1 & -2.146559000 \\
\hline $\mathrm{F}$ & -4.19 & -1.25 & -3.388530000 \\
\hline $\mathrm{F}$ & -5.818 & -2.700701000 & -3.670614000 \\
\hline $\mathrm{C}$ & -5.416882000 & -4.695922000 & 1.542475000 \\
\hline $\mathrm{F}$ & -6.734578000 & -4.388604000 & 1.497456000 \\
\hline $\mathrm{F}$ & -5.294 & -6.030625000 & 1.762413000 \\
\hline $\mathrm{F}$ & -4.88 & -4.0 & 358000 \\
\hline $\mathrm{C}$ & & & -1.316029000 \\
\hline $\mathrm{F}$ & 5.1 & & 302000 \\
\hline $\mathrm{F}$ & & & \\
\hline $\mathrm{F}$ & & & \\
\hline $\mathrm{C}$ & & & \\
\hline $\mathrm{F}$ & & & \\
\hline$x^{2}$ & 3.726 & & 3.764244000 \\
\hline 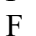 & 4.770 & -2.4 & 3.711864000 \\
\hline 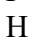 & 0.301610000 & -2.000198000 & -0.28201500 \\
\hline
\end{tabular}

\begin{tabular}{|c|c|c|c|}
\hline \multicolumn{4}{|c|}{ CF3-H+TS-1 2,1-ins } \\
\hline \multicolumn{2}{|c|}{ Zero-point correction $=$} & \multicolumn{2}{|c|}{0.767011 (Hartree/Particle) } \\
\hline \multicolumn{3}{|c|}{ Thermal correction to Energy= } & 0.843935 \\
\hline \multicolumn{3}{|c|}{ Thermal correction to Enthalpy= } & 0.844880 \\
\hline \multicolumn{3}{|c|}{ Thermal correction to Gibbs Free Energy= } & 0.639505 \\
\hline \multicolumn{3}{|c|}{ Sum of electronic and zero-point Energies $=$} & -4903.2598 \\
\hline \multicolumn{3}{|c|}{ Sum of electronic and thermal Energies= } & -4903.18297 \\
\hline \multicolumn{4}{|c|}{ Sum of electronic and thermal Enthalpies $=$} \\
\hline \multicolumn{4}{|c|}{$\begin{array}{l}\text { Sum of electronic and thermal Free Energies }=-4903.387402 \\
\text { E solvent }=-4903.27276693\end{array}$} \\
\hline $\mathrm{Ni}$ & -0.164246000 & -1.275050000 & -1.178 \\
\hline $\mathrm{C}$ & -0.080275000 & -0.761771000 & -3.183524000 \\
\hline $\mathrm{N}$ & 0.556282000 & -2.556630000 & 0.062516000 \\
\hline
\end{tabular}

\begin{tabular}{|c|c|c|}
\hline & & \\
\hline & 000 & \\
\hline 000 & 7000 & \\
\hline & & \\
\hline & & \\
\hline & -0.2 & \\
\hline 000 & & \\
\hline 267000 & -1.255310000 & \\
\hline 841000 & -0.075518000 & -1 . \\
\hline-0.776666000 & 000 & -2 \\
\hline 0.948770000 & 0000 & -3.2 \\
\hline-0.346569000 & 7000 & -3 \\
\hline 9000 & -3.008 & \\
\hline 936000 & -0.7291 & \\
\hline 2.383555000 & -1.636997000 & $3.3 \mathrm{~s}-\mathrm{r}-\mathrm{c}$ \\
\hline 2.184728000 & 0.508036000 & \\
\hline 3.00 & & \\
\hline 4.0 & & \\
\hline 9000 & & \\
\hline & & \\
\hline & & \\
\hline & & \\
\hline 0.8 & & \\
\hline & 2 & \\
\hline & & \\
\hline & 3.7 & \\
\hline & & \\
\hline & & \\
\hline & & \\
\hline & & \\
\hline-0 . & & 000 \\
\hline-0.1 & & \\
\hline 12 & & \\
\hline & & \\
\hline & & \\
\hline 2 & & \\
\hline 2.2 & & \\
\hline-3.4 & 3.3721 & \\
\hline-4.4 & & \\
\hline 38000 & & \\
\hline-5.3 & & \\
\hline-4 & & \\
\hline & & \\
\hline 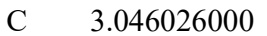 & & \\
\hline 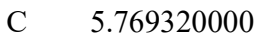 & & \\
\hline & & \\
\hline & & \\
\hline & & \\
\hline & & \\
\hline & & \\
\hline & & -0.3 \\
\hline & & \\
\hline & & \\
\hline & & 5000 \\
\hline & & 70000 \\
\hline & & 9000 \\
\hline & & 8000 \\
\hline 4000 & & 0000 \\
\hline 60000 & & 1000 \\
\hline 074000 & -2.9 & 6000 \\
\hline 860 & 6000 & 5000 \\
\hline 0000 & 5000 & 6000 \\
\hline 200 & 3000 & 400 \\
\hline & -2.4 & 200 \\
\hline & -2.372 & 1 \\
\hline & & -0.7 \\
\hline & & -1.23 \\
\hline & & -1.137772 \\
\hline
\end{tabular}




$\begin{array}{crrr}\text { H } & -2.500029000 & -4.731825000 & -2.487433000 \\ \mathrm{H} & -5.545625000 & -2.505454000 & -0.364891000 \\ \mathrm{H} & -6.026384000 & 0.872887000 & 1.988853000 \\ \mathrm{H} & -2.596270000 & 3.465327000 & 2.426580000 \\ \mathrm{H} & -4.053798000 & 5.763033000 & -0.658158000 \\ \mathrm{H} & -2.494494000 & 7.489889000 & -1.576057000 \\ \mathrm{H} & -0.038375000 & 7.038864000 & -1.650446000 \\ \mathrm{H} & 1.943596000 & 6.536455000 & 0.185726000 \\ \mathrm{H} & 0.936019000 & 3.035756000 & -2.157077000 \\ \mathrm{H} & -4.539614000 & 3.077003000 & -1.428356000 \\ \mathrm{H} & -1.708205000 & -3.226577000 & 1.495451000 \\ \mathrm{H} & 5.080145000 & 4.147792000 & -1.604827000 \\ \mathrm{H} & -0.234083000 & -2.385305000 & -2.122732000 \\ \mathrm{H} & -0.856862000 & 1.963950000 & -0.089121000 \\ \mathrm{C} & -5.014296000 & -3.766921000 & -2.748658000 \\ \mathrm{~F} & -4.901385000 & -4.990993000 & -3.328901000 \\ \mathrm{~F} & -6.317866000 & -3.563674000 & -2.436869000 \\ \mathrm{~F} & -4.673205000 & -2.843076000 & -3.702265000 \\ \mathrm{C} & -4.063740000 & -2.024537000 & 1.917704000 \\ \mathrm{~F} & -3.799641000 & -2.722781000 & 3.052262000 \\ \mathrm{~F} & -3.352685000 & -0.853102000 & 2.003523000 \\ \mathrm{~F} & -5.384191000 & -1.700503000 & 1.920734000 \\ \mathrm{C} & 6.278805000 & -4.612275000 & 2.138961000 \\ \mathrm{~F} & 6.057431000 & -4.034261000 & 3.356500000 \\ \mathrm{~F} & 6.066801000 & -5.947871000 & 2.282935000 \\ \mathrm{~F} & 7.587646000 & -4.422463000 & 1.832176000 \\ \mathrm{C} & 5.274795000 & -1.016083000 & -1.261542000 \\ \mathrm{~F} & 6.620426000 & -0.877484000 & -1.363947000 \\ \mathrm{~F} & 4.750114000 & -0.976607000 & -2.516318000 \\ \mathrm{~F} & 4.804759000 & 0.082640000 & -0.592016000 \\ \mathrm{C} & 4.642534000 & 6.331024000 & 0.000557000 \\ \mathrm{~F} & 5.838332000 & 6.266383000 & -0.636823000 \\ \mathrm{~F} & 4.230335000 & 7.627363000 & -0.000020000 \\ \mathrm{~F} & 4.848029000 & 5.973374000 & 1.301049000 \\ \mathrm{C} & 3.429251000 & 2.304702000 & -2.841091000 \\ \mathrm{~F} & 2.772601000 & 1.163910000 & -2.442352000 \\ \mathrm{~F} & 3.065591000 & 2.524967000 & -4.133192000 \\ \mathrm{~F} & 4.757155000 & 2.051294000 & -2.816512000 \\ \mathrm{C} & -6.400069000 & 1.230360000 & -0.696349000 \\ \mathrm{~F} & -7.510759000 & 0.860309000 & -0.014213000 \\ \mathrm{~F} & -6.778529000 & 2.023361000 & -1.732047000 \\ \mathrm{~F} & -5.862175000 & 0.089897000 & -1.242518000 \\ \mathrm{C} & -4.227937000 & 1.834165000 & 3.831761000 \\ \mathrm{~F} & -2.929839000 & 1.686127000 & 4.230348000 \\ \mathrm{~F} & -4.757768000 & 2.847570000 & 4.568812000 \\ \mathrm{~F} & -4.895107000 & 0.697237000 & 4.152402000\end{array}$

\section{CF3-H+3- $\boldsymbol{\beta}-\mathbf{C}$}

Zero-point correction $=0.770114$ (Hartree/Particle) Thermal correction to Energy= $\quad 0.847394$ Thermal correction to Enthalpy= $\quad 0.848338$

Thermal correction to Gibbs Free Energy= $\quad 0.640729$ Sum of electronic and zero-point Energies $=\quad-4903.277118$ Sum of electronic and thermal Energies $=\quad-4903.199838$ Sum of electronic and thermal Enthalpies $=\quad-4903.198894$ Sum of electronic and thermal Free Energies $=-4903.406504$ E solvent $=-4903.29635779$

$\begin{array}{lrrc}\mathrm{Ni} & 1.355902000 & 1.053488000 & -0.321151000 \\ \mathrm{C} & 0.776584000 & 2.520182000 & 0.789661000 \\ \mathrm{~N} & 2.317145000 & -0.172340000 & -1.480973000 \\ \mathrm{C} & 1.947865000 & -1.427010000 & -1.615686000 \\ \mathrm{C} & 0.798286000 & -2.053025000 & -1.004771000 \\ \mathrm{C} & -0.164793000 & -1.344349000 & -0.193733000 \\ \mathrm{O} & -0.075069000 & -0.078440000 & 0.123734000 \\ \mathrm{C} & 2.116110000 & 2.977353000 & 0.325780000 \\ \mathrm{H} & 2.592320000 & 2.221023000 & -0.460529000 \\ \mathrm{H} & 2.100463000 & 3.899889000 & -0.280173000 \\ \mathrm{H} & 2.896565000 & 3.010226000 & 1.103352000\end{array}$

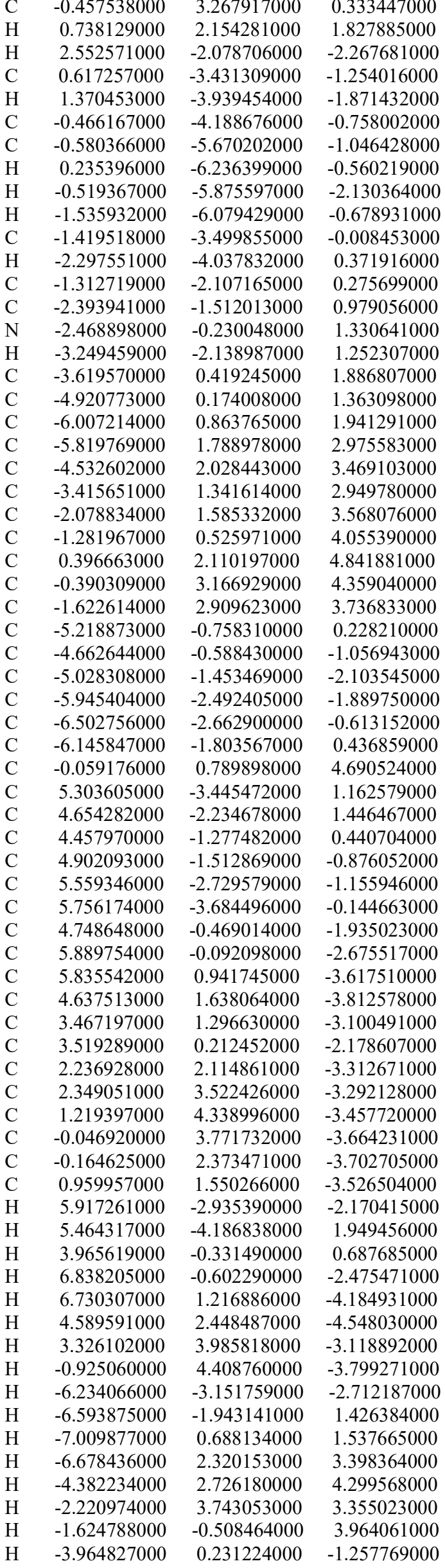




$\begin{array}{lrrr}\text { H } & 0.840774000 & 0.466319000 & -3.591249000 \\ \text { H } & 1.358929000 & 2.310457000 & 5.321262000 \\ \text { H } & -0.620538000 & 4.157655000 & 0.975876000 \\ \text { H } & -0.370873000 & 3.618325000 & -0.709431000 \\ \text { H } & -1.366406000 & 2.644848000 & 0.410148000 \\ \text { H } & -1.613334000 & 0.317171000 & 1.109098000 \\ \text { C } & 0.070290000 & 4.601657000 & 4.528595000 \\ \text { F } & 1.423880000 & 4.683951000 & 4.623665000 \\ \text { F } & -0.319310000 & 5.369323000 & 3.466290000 \\ \text { F } & -0.459004000 & 5.162992000 & 5.645286000 \\ \text { C } & 0.753531000 & -0.351079000 & 5.273910000 \\ \text { F } & 0.466421000 & -1.532244000 & 4.659314000 \\ \text { F } & 2.087311000 & -0.120530000 & 5.141469000 \\ \text { F } & 0.496210000 & -0.507666000 & 6.599201000 \\ \text { C } & -7.445121000 & -3.821016000 & -0.345102000 \\ \text { F } & -6.751740000 & -4.909175000 & 0.099436000 \\ \text { F } & -8.359669000 & -3.506395000 & 0.610832000 \\ \text { F } & -8.113817000 & -4.192810000 & -1.465644000 \\ \text { C } & -4.393166000 & -1.263018000 & -3.467206000 \\ \text { F } & -4.998492000 & -2.012836000 & -4.422266000 \\ \text { F } & -4.447594000 & 0.038913000 & -3.858692000 \\ \text { F } & -3.075704000 & -1.622012000 & -3.436863000 \\ \text { C } & 1.374974000 & 5.835707000 & -3.289650000 \\ \text { F } & 2.515001000 & 6.290226000 & -3.871232000 \\ \text { F } & 0.328061000 & 6.523045000 & -3.807446000 \\ \text { F } & 1.453450000 & 6.149536000 & -1.956116000 \\ \text { C } & -1.527948000 & 1.730565000 & -3.832119000 \\ \text { F } & -1.472802000 & 0.563181000 & -4.520887000 \\ \text { F } & -2.025468000 & 1.435253000 & -2.580696000 \\ \text { F } & -2.426797000 & 2.543336000 & -4.435857000 \\ \text { C } & 4.095881000 & -1.951222000 & 2.824372000 \\ \text { F } & 4.587726000 & -2.791336000 & 3.766578000 \\ \text { F } & 4.363747000 & -0.673074000 & 3.214607000 \\ \text { F } & 2.733293000 & -2.085868000 & 2.822564000 \\ \text { C } & 6.396146000 & -5.014402000 & -0.491953000 \\ \text { F } & 5.460364000 & -5.890802000 & -0.961602000 \\ \text { F } & 7.338871000 & -4.874990000 & -1.462727000 \\ \text { F } & 6.984613000 & -5.587803000 & 0.588814000\end{array}$

\section{CF3-H+TS-3 ${ }_{\text {coor }}$}

Zero-point correction=

$\begin{array}{lc}\text { Thermal correction to Energy= } & 0.902273 \\ \text { Thermal correction to Enthalpy= } & 0.903217 \\ \text { Thermal correction to Gibbs Free Energy= } & 0.689274 \\ \text { Sum of electronic and zero-point Energies }= & -4981.799176 \\ \text { Sum of electronic and thermal Energies }= & -4981.718386 \\ \text { Sum of electronic and thermal Enthalpies }= & -4981.717442 \\ \text { Sum of electronic and thermal Free Energies }= & -4981.931385 \\ \text { E solvent }=-4981.84172045 & \end{array}$

0.821483 (Hartree/Particle) 0.902273 $-4981.799176$ $-4981.718386$ E solvent $=-4981.84172045$

$\begin{array}{lrr}\mathrm{Ni} & 1.285765000 & 0.972481000 \\ \mathrm{C} & 0.429535000 & 1.453128000 \\ \mathrm{~N} & 2.252673000 & 1.041374000 \\ \mathrm{C} & 1.816057000 & 0.359754000 \\ \mathrm{C} & 0.689132000 & -0.545824000 \\ \mathrm{C} & -0.271655000 & -0.705172000 \\ \mathrm{O} & -0.185988000 & -0.103430000 \\ \mathrm{C} & 1.544362000 & 2.450020000 \\ \mathrm{H} & 2.232607000 & 2.313316000 \\ \mathrm{H} & 1.214277000 & 3.498261000 \\ \mathrm{H} & 2.265883000 & 2.323029000 \\ \mathrm{C} & -0.988368000 & 1.973520000 \\ \mathrm{H} & 0.556623000 & 0.624576000 \\ \mathrm{H} & 2.334767000 & 0.494847000 \\ \mathrm{C} & 0.530905000 & -1.277888000 \\ \mathrm{H} & 1.301983000 & -1.166062000 \\ \mathrm{C} & -0.533954000 & -2.174112000 \\ \mathrm{C} & -0.621555000 & -2.936107000 \\ \mathrm{H} & -1.294875000 & -3.805478000\end{array}$

0.811404000 2.493928000 $-0.926863000$ $-1.963045000$ $-2.016023000$ $-0.949083000$ 0.206139000 2.488854000 1.557377000 2.407197000 3.313577000 2.393697000 3.205255000 $-2.924326000$ $-3.212154000$ $-3.985409000$ $-3.454730000$ $-4.760097000$ $-4.676868000$
$-3.599526000$

$-4.926457000$

$-5.937724000$

$-5.650463000$

$-4.339710000$

$-3.301357000$

$-1.942098000$

$-1.338200000$

0.573993000

$-0.020491000$

$-1.270893000$

$-5.322317000$

$-4.783236000$

$-5.236237000$

$-6.223674000$

$-6.765439000$

$-6.321944000$

$-0.094205000$

5.071513000

5.253194000

5.100621000

4.767383000

4.629344000

4.760586000

4.657495000

5.830381000

5.822144000

4.626081000

3.426583000

3.447041000

2.194155000

2.276976000

1.146993000

$-0.096107000$

$-0.186865000$

0.940779000

4.424405000

5.178227000

5.249099000

6.754980000

6.737804000

4.601289000

3.229530000

$-0.975042000$

$-6.576878000$

$-6.757512000$

$-6.960878000$

$-6.451854000$

$-4.114573000$

$-1.724522000$

$-1.858856000$

$-4.029093000$

0.841171000

1.538870000

$-1.242744000$

$-1.139088000$

$-1.726746000$

3.280585000

2.505254000

$-3.299660000$

$-2.292902000$

$-5.074482000$

$-295967000$

$-5.573808000$

$-2.964350000$

$-2.450120000$

$-2.599961000$

$-1.583249000 \quad-1.217529000$

$-1.752179000 \quad-0.292809000$

$\begin{array}{ll}-1.195512000 & 0.917976000\end{array}$

$-2.375409000 \quad-0.572027000$

$-1.203218000 \quad 1.839852000$

$-0.914409000 \quad 1.416689000$

$-0.875647000 \quad 2.400732000$

$-1.080942000 \quad 3.756094000$

$-1.369700000 \quad 4.155264000$

$-1.457955000 \quad 3.206898000$

$-1.890521000 \quad 3.651022000$

$-3.035834000 \quad 3.090618000$

$-2.804818000 \quad 4.583381000$

$-1.662096000 \quad 5.143070000$

$-1.210006000 \quad 4.689299000$

$-0.654128000-0.004517000$

$0.405441000-0.763497000$

$0.635613000 \quad-2.074849000$

$-0.178813000-2.647081000$

$-1.231525000-1.893660000$

$-1.466892000 \quad-0.583695000$

$-3.489123000 \quad 3.556916000$

$-3.033634000 \quad-2.526578000$

$-2.592433000 \quad-1.207233000$

$-1.233246000 \quad-0.879379000$

$-0.289595000-1.870476000$

$-0.734212000 \quad-3.201410000$

$-2.095756000 \quad-3.521889000$

$1.179330000-1.560748000$

$1.948657000-1.706141000$

$3.330700000-1.485764000$

$3.961087000-1.130668000$

$3.234425000-0.958824000$

$1.820850000-1.158479000$

$4.000790000-0.610536000$

$\begin{array}{ll}5.057974000 & 0.324657000\end{array}$

$5.819649000 \quad 0.657427000$

$5.550285000 \quad 0.064449000$

$\begin{array}{ll}4.508799000 & -0.870381000\end{array}$

$3.741713000-1.206864000$

$-0.009685000 \quad-3.997813000$

$-4.092371000 \quad-2.775139000$

$\begin{array}{ll}-0.908760000 & 0.155185000\end{array}$

$1.443646000 \quad-2.005984000$

$3.916503000-1.614626000$

$5.050427000-1.019629000$

$5.274128000 \quad 0.818884000$

$6.146722000 \quad 0.322760000$

$0.013467000 \quad-3.663220000$

$-2.287370000 \quad-0.003751000$

$\begin{array}{ll}-0.643125000 & 2.087108000\end{array}$

$-1.026129000 \quad 4.499743000$

$\begin{array}{ll}-1.574913000 & 5.207295000\end{array}$

$-0.322315000 \quad 5.141908000$

$\begin{array}{ll}-3.603615000 & 2.313390000\end{array}$

$1.071186000-0.330906000$

$2.961389000 \quad-1.963958000$

$-3.164288000 \quad 4.950838000$

$2.563846000 \quad 3.299459000$

$2.630660000 \quad 1.520085000$

$1.155473000 \quad 2.339546000$

$\begin{array}{ll}-0.274199000 & 2.331589000\end{array}$

$-1.090244000 \quad 1.580166000$

$-0.073252000 \quad 3.382503000$ 


$\begin{array}{cccc}\text { H } & 4.191752000 & 0.191938000 & 1.937874000 \\ \text { H } & 1.644269000 & -1.614285000 & 2.006923000 \\ \text { H } & 2.785156000 & -1.371081000 & 0.559440000 \\ \text { C } & 1.246595000 & 6.846411000 & 1.765791000 \\ \text { F } & 0.297060000 & 7.808310000 & 1.656074000 \\ \text { F } & 1.073861000 & 6.238693000 & 2.982998000 \\ \text { F } & 2.463606000 & 7.449287000 & 1.789368000 \\ \text { C } & -1.527932000 & 4.134348000 & -1.461709000 \\ \text { F } & -2.414882000 & 5.155875000 & -1.419079000 \\ \text { F } & -1.415696000 & 3.716850000 & -2.748151000 \\ \text { F } & -2.077830000 & 3.089006000 & -0.749719000 \\ \text { C } & 0.738975000 & -0.859081000 & 6.178209000 \\ \text { F } & 1.546593000 & -1.639133000 & 6.935275000 \\ \text { F } & 1.544602000 & 0.072416000 & 5.560315000 \\ \text { F } & -0.092708000 & -0.173680000 & 7.001681000 \\ \text { C } & 0.548788000 & -4.687384000 & 2.887641000 \\ \text { F } & 1.495912000 & -5.260341000 & 3.668390000 \\ \text { F } & -0.373775000 & -5.634360000 & 2.569998000 \\ \text { F } & 1.151936000 & -4.310292000 & 1.717513000 \\ \text { C } & -7.789410000 & -2.162556000 & -2.514542000 \\ \text { F } & -7.177788000 & -3.250690000 & -3.065200000 \\ \text { F } & -8.669612000 & -2.621856000 & -1.584679000 \\ \text { F } & -8.494194000 & -1.548130000 & -3.498098000 \\ \text { C } & -4.621906000 & 1.770508000 & -2.870826000 \\ \text { F } & -5.262679000 & 1.976179000 & -4.049049000 \\ \text { F } & -4.651113000 & 2.935727000 & -2.168585000 \\ \text { F } & -3.313248000 & 1.504015000 & -3.155022000 \\ \text { C } & 5.670288000 & -3.570182000 & -0.125532000 \\ \text { F } & 5.022734000 & -3.310134000 & 1.046897000 \\ \text { F } & 5.400457000 & -4.854900000 & -0.474352000 \\ \text { F } & 7.003982000 & -3.479323000 & 0.125063000 \\ \text { C } & 4.504501000 & -2.532212000 & -4.948506000 \\ \text { F } & 5.148508000 & -1.732955000 & -5.837544000 \\ \text { F } & 4.880941000 & -3.812830000 & -5.173951000 \\ \text { F } & 3.161877000 & -2.444653000 & -5.244940000 \\ \text { H } & -1.643133000 & -0.652048000 & 1.141279000\end{array}$

\section{CF3-H+3-Coor-C}

Zero-point correction= $\begin{array}{lc}\text { Thermal correction to Energy }= & 0.904170 \\ \text { Thermal correction to Enthalpy= } & 0.905114 \\ \text { Thermal correction to Gibbs Free Energy= } & 0.693571 \\ \text { Sum of electronic and zero-point Energies= } & -4981.819141 \\ \text { Sum of electronic and thermal Energies }= & -4981.738647 \\ \text { Sum of electronic and thermal Enthalpies }= & -4981.737703 \\ \text { Sum of electronic and thermal Free Energies }= & -4981.949247\end{array}$ E solvent $=-4981.85175762$

$\begin{array}{lrrr}\mathrm{Ni} & 0.994160000 & 0.669403000 & 1.347405000 \\ \mathrm{C} & -0.482058000 & 0.491641000 & 2.640260000 \\ \mathrm{~N} & 2.027030000 & 1.587914000 & -0.210333000 \\ \mathrm{C} & 1.703219000 & 1.427820000 & -1.472844000 \\ \mathrm{C} & 0.678390000 & 0.581722000 & -2.045064000 \\ \mathrm{C} & -0.209792000 & -0.264047000 & -1.272983000 \\ \mathrm{O} & -0.190022000 & -0.337577000 & 0.019950000 \\ \mathrm{C} & -0.316415000 & 0.536880000 & 4.147150000 \\ \mathrm{H} & 0.275744000 & -0.301825000 & 4.550060000 \\ \mathrm{H} & 0.114211000 & 1.491928000 & 4.493660000 \\ \mathrm{H} & -1.322034000 & 0.463546000 & 4.612689000 \\ \mathrm{C} & -1.107952000 & 1.729853000 & 2.013988000 \\ \mathrm{H} & -0.932522000 & -0.458312000 & 2.307625000 \\ \mathrm{H} & 2.279993000 & 2.000859000 & -2.220173000 \\ \mathrm{C} & 0.580011000 & 0.592954000 & -3.452508000 \\ \mathrm{H} & 1.272710000 & 1.241855000 & -4.006164000 \\ \mathrm{C} & -0.345119000 & -0.180402000 & -4.190240000 \\ \mathrm{C} & -0.381929000 & -0.119261000 & -5.702240000 \\ \mathrm{H} & 0.566168000 & -0.479536000 & -6.142275000 \\ \mathrm{H} & -0.535337000 & 0.915243000 & -6.059472000 \\ \mathrm{H} & -1.196864000 & -0.740011000 & -6.109863000\end{array}$

$-1.953844000$

$-2.148978000$

$\mathrm{N} \quad-2.226961000$

$-2.907483000$

$-3.306149000$

$-4.658612000$

$-5.677406000$

$-5.376747000$

$-4.041806000$

$-2.987570000$

$-1.578120000$

$-0.717280000$

1.031441000

0.182094000

$-1.118255000$

$-5.050867000$

$-4.557889000$

$-4.973197000$

$-5.893318000$

$-6.392996000$

$-5.978598000$

0.571814000

5.379309000

4.580462000

4.263895000

4.740334000

5.543693000

5.858670000

4.468770000

5.570943000

5.416353000

4.136610000

3.004667000

3.171872000

1.664645000

1.374410000

0.152135000

$-0.786894000$

$-0.496637000$

0.712229000

5.913939000

5.623660000

3.644544000

6.568827000

6.286936000

3.992924000

2.105426000

$-1.732838000$

$-6.228989000$

$-6.382225000$

$-6.720997000$

$-6.184790000$

$-3.798378000$

$-1.778431000$

$-1.064025000$

$-3.863504000$

0.926926000

2.037593000

$-2.060331000$

$-1.249208000$

$-0.462865000$

2.175436000

2.478140000

2.781043000

1.596204000

H $\quad 3.336872000$
$-0.989140000$

$-1.601258000$

$-1.053677000$

$-1.867814000$

2.076197000

$-2.367083000$

$-2.741989000$

$-2.431867000$

$-3.135531000$

$-4.073084000$

$-4.328885000$

$-3.684523000$

$-4.035578000$

$-4.638948000$

$-4.835068000$

$-4.224386000$

$-3.837053000$

$-1.335919000$

$-0.027737000$

1.018887000

0.782188000

$-0.516114000$

$-1.568739000$

$-5.046041000$

$-1.490130000$

$-1.578504000$

$-0.429419000$

0.836230000

0.920389000

$-0.231295000$

2.062870000

2.885117000

4.047348000

4.428768000

3.648352000

2.440080000

4.188915000

4.497591000

5.098882000

5.428119000

5.136043000

4.515032000

1.893743000

$-2.385102000$

$-0.517378000$

2.577971000

4.660444000

5.363706000

4.264461000

5.905223000

1.600979000

$-2.574692000$

$-2.896774000$

$-4.594239000$

$-5.063557000$

$-3.381438000$

$-4.825514000$

0.178703000

4.312922000

$-5.151492000$

1.520666000

2.527532000

2.194785000

$-0.566551000$

0.773772000

$-1.127433000$

$-1.194420000$

1.272411000
$-3.455820000$

$-3.983951000$

$-2.032370000$

$-1.395260000$

$-0.081399000$

$-2.009611000$

0.587329000

0.266860000

0.943012000

1.939157000

2.277023000

1.598611000

1.951199000

1.010538000

2.697523000

3.633091000

3.269367000

$-0.671604000$

$-0.486966000$

$-1.322471000$

$-2.355729000$

$-2.541272000$

$-1.707896000$

1.387854000

$-2.754128000$

$-1.605177000$

$-0.866348000$

$-1.254120000$

$-2.412968000$

$-3.151636000$

$-0.443730000$

$-0.115693000$

0.646314000

1.065392000

0.749906000

0.020588000

1.128735000

2.472737000

2.818708000

1.830126000

0.486725000

0.138572000

$-2.750655000$

$-3.332239000$

0.027911000

$-0.446787000$

0.900194000

1.618021000

3.253574000

2.101940000

$-2.997629000$

$-1.865074000$

0.712069000

2.462353000

3.051855000

4.014379000

$-0.010449000$

0.333139000

$-0.914395000$

2.985943000

1.497050000

2.761882000

1.216625000

2.399765000

2.720742000

1.676391000

3.083648000

2.256814000 


$\begin{array}{cccc}\text { H } & 2.133038000 & 1.209881000 & 3.663413000 \\ \text { H } & -1.455895000 & -1.628519000 & 0.455802000 \\ \text { C } & -4.371987000 & 2.395227000 & -1.123638000 \\ \text { F } & -5.149878000 & 3.373956000 & -1.641040000 \\ \text { F } & -4.177964000 & 2.662142000 & 0.201677000 \\ \text { F } & -3.147592000 & 2.476139000 & -1.725451000 \\ \text { C } & -7.339637000 & -0.808377000 & -3.690649000 \\ \text { F } & -8.029207000 & 0.297873000 & -4.067102000 \\ \text { F } & -6.647995000 & -1.252793000 & -4.779262000 \\ \text { F } & -8.236391000 & -1.775666000 & -3.359354000 \\ \text { C } & 1.439886000 & -5.800499000 & 0.398571000 \\ \text { F } & 1.166061000 & -5.435038000 & -0.884039000 \\ \text { F } & 2.761751000 & -5.580483000 & 0.626602000 \\ \text { F } & 1.226725000 & -7.140618000 & 0.493741000 \\ \text { C } & 0.688290000 & -3.920466000 & 5.027595000 \\ \text { F } & -0.316382000 & -3.924469000 & 5.940655000 \\ \text { F } & 1.634800000 & -4.801849000 & 5.428275000 \\ \text { F } & 1.261067000 & -2.669577000 & 5.072160000 \\ \text { C } & -0.190480000 & 5.292183000 & 4.280073000 \\ \text { F } & -1.085275000 & 6.292119000 & 4.468749000 \\ \text { F } & -0.752679000 & 4.145073000 & 4.789970000 \\ \text { F } & 0.911257000 & 5.556957000 & 5.028240000 \\ \text { C } & -1.471725000 & 5.559951000 & -0.593943000 \\ \text { F } & -1.334389000 & 6.879979000 & -0.887739000 \\ \text { F } & -1.277408000 & 4.861951000 & -1.748051000 \\ \text { F } & -2.761035000 & 5.364612000 & -0.200762000 \\ \text { C } & 6.768070000 & -0.121789000 & -4.360682000 \\ \text { F } & 6.646070000 & 1.092765000 & -4.965442000 \\ \text { F } & 8.072747000 & -0.270654000 & -4.006171000 \\ \text { F } & 6.483653000 & -1.078648000 & -5.284950000 \\ \text { C } & 3.997657000 & -2.900243000 & -1.157452000 \\ \text { F } & 2.672876000 & -2.986561000 & -1.491113000 \\ \text { F } & 4.628995000 & -3.960728000 & -1.712206000 \\ \text { F } & 4.065177000 & -3.030412000 & 0.201994000\end{array}$

\section{CF3-H+ ${ }^{-}$TS-3 $3_{\text {ins }}$}

Zero-point correction $=\quad 0.823481($ Hartree/Particle $)$

Thermal correction to Energy= $\quad 0.903611$

Thermal correction to Enthalpy= $\quad 0.904555$

Thermal correction to Gibbs Free Energy $=\quad 0.689968$

Sum of electronic and zero-point Energies $=\quad-4981.800707$

Sum of electronic and thermal Energies $=\quad-4981.720577$

Sum of electronic and thermal Enthalpies $=\quad-4981.719633$

Sum of electronic and thermal Free Energies $=-4981.934220$

E solvent $=-4981.84035989$

$\begin{array}{lrrr}\mathrm{Ni} & 0.560553000 & 1.244506000 & 1.169467000 \\ \mathrm{C} & -0.984444000 & 0.749817000 & 2.575523000 \\ \mathrm{~N} & 1.305605000 & 2.189000000 & -0.339726000 \\ \mathrm{C} & 1.154019000 & 1.807547000 & -1.591880000 \\ \mathrm{C} & 0.472569000 & 0.640231000 & -2.098802000 \\ \mathrm{C} & -0.132964000 & -0.360791000 & -1.247531000 \\ \mathrm{O} & -0.138317000 & -0.276355000 & 0.048358000 \\ \mathrm{C} & -1.187350000 & -0.627630000 & 3.194484000 \\ \mathrm{H} & -0.595144000 & -1.407664000 & 2.685858000 \\ \mathrm{H} & -0.908172000 & -0.642834000 & 4.263798000 \\ \mathrm{H} & -2.256756000 & -0.912452000 & 3.135659000 \\ \mathrm{C} & -1.598731000 & 1.900450000 & 3.360664000 \\ \mathrm{H} & -1.407811000 & 0.761415000 & 1.550249000 \\ \mathrm{H} & 1.593379000 & 2.458008000 & -2.366518000 \\ \mathrm{C} & 0.424696000 & 0.487858000 & -3.500738000 \\ \mathrm{H} & 0.903164000 & 1.262405000 & -4.115895000 \\ \mathrm{C} & -0.199062000 & -0.598762000 & -4.154913000 \\ \mathrm{C} & -0.218024000 & -0.691234000 & -5.665723000 \\ \mathrm{H} & 0.806566000 & -0.733470000 & -6.078412000 \\ \mathrm{H} & -0.715808000 & 0.186832000 & -6.116030000 \\ \mathrm{H} & -0.753894000 & -1.592394000 & -6.006710000 \\ \mathrm{C} & -0.782311000 & -1.570334000 & -3.342012000 \\ \mathrm{H} & -1.272514000 & -2.436283000 & -3.806030000\end{array}$

C -2.073000000

C $\quad-3.481656000$

C -4.132408000

$-3.424821000$

$-2.040290000$

$-1.341100000$

0.149270000

0.961816000

2.968772000

2.162702000

0.762493000

$-4.303925000$

$-4.216077000$

$-5.029802000$

$-5.948687000$

$-6.045083000$

$-5.233051000$

2.360544000

5.678046000

4.832417000

4.105469000

4.212889000

5.063048000

5.788389000

3.512663000

4.290718000

3.716889000

2.340109000

1.518958000

2.117355000

0.051006000

$-0.411110000$

$-1.778451000$

$-2.708901000$

$-2.255121000$

$-0.893199000$

5.147755000

6.240714000

3.460558000

5.368141000

4.337538000

1.872810000

0.303231000

$-3.770895000$

$-6.582513000$

$-5.321034000$

$-5.219061000$

$-3.955059000$

$-1.478576000$

0.146361000

0.501689000

$-3.516355000$

$-0.564478000$

4.057922000

$-2.702841000$

$-1.321750000$

$-1.335666000$

1.609286000

1.066453000

1.238736000

2.644873000

0.557593000

1.619141000

$\mathrm{H} \quad-0.813209000$
$-1.494752000$ $-2.549878000$

$-2.676697000$

$-3.332670000$

$-3.664758000$

$-3.796598000$

$-4.779957000$

$-5.571740000$

$-5.409347000$

$-4.469737000$

$-4.381582000$

$-4.824176000$

$-4.310856000$

$-3.857387000$

$-3.911474000$

$-2.909145000$

$-1.505967000$

$-0.679147000$

$-1.234930000$

$-2.631579000$

$-3.463858000$

$-4.803810000$

$-0.078070000$

$-0.234184000$

0.852657000

2.127016000

2.276599000

1.185329000

3.331458000

4.488200000

5.662449000

5.704957000

4.578461000

3.378164000

4.738634000

5.316297000

5.574432000

5.281373000

4.720797000

4.443615000

3.252853000

$-0.927882000$

0.706310000

4.443379000

6.545502000

6.634658000

5.570066000

5.493418000

$-0.588977000$

$-4.551265000$

$-4.883972000$

$-6.314552000$

$-6.036953000$

$-3.585614000$

$-5.214864000$

$-1.052871000$

4.027610000

$-4.277991000$

1.817028000

1.888242000

2.885210000

2.142488000

0.962256000

3.122099000

2.143962000

1.059970000

0.014804000

$-1.927743000$
$-1.918687000$

$-1.198577000$

0.124978000

$-1.750239000$

0.883203000

0.741339000

1.517393000

2.430542000

2.574761000

1.792086000

1.894341000

0.831769000

2.112809000

3.169008000

3.073647000

$-0.134991000$

$-0.032868000$

$-0.820986000$

$-1.724183000$

$-1.827486000$

$-1.040197000$

0.949443000

$-2.015852000$

$-0.906394000$

$-0.397712000$

$-0.991435000$

$-2.108611000$

$-2.613369000$

$-0.446444000$

$-0.210898000$

0.285613000

0.531487000

0.306775000

$-0.164199000$

0.532189000

1.734413000

1.929504000

0.922200000

$-0.282652000$

$-0.474612000$

$-2.597698000$

$-2.410025000$

0.474148000

$-0.403049000$

0.468027000

0.873950000

2.525043000

1.071236000

$-2.337424000$

$-1.134939000$

1.429064000

3.035149000

3.274775000

3.917736000

$-0.081727000$

0.675940000

$-1.430278000$

2.194461000

3.310360000

4.430863000

2.942222000

2.550421000

3.161562000

2.878539000

2.187002000

4.125865000

3.079947000

0.603429000 


$\begin{array}{lrrr}\text { C } & -4.933797000 & 0.821948000 & -0.641189000 \\ \text { F } & -5.426373000 & 1.491798000 & -1.709365000 \\ \text { F } & -5.610940000 & 1.235159000 & 0.463473000 \\ \text { F } & -3.627282000 & 1.204448000 & -0.468979000 \\ \text { C } & -6.978871000 & -3.251884000 & -2.850142000 \\ \text { F } & -8.043193000 & -2.450051000 & -3.107010000 \\ \text { F } & -6.327914000 & -3.461679000 & -4.030002000 \\ \text { F } & -7.447239000 & -4.456495000 & -2.426305000 \\ \text { C } & 3.195691000 & -5.389432000 & -0.172485000 \\ \text { F } & 2.712005000 & -5.015643000 & -1.391336000 \\ \text { F } & 4.493832000 & -5.001637000 & -0.096531000 \\ \text { F } & 3.167476000 & -6.749956000 & -0.132060000 \\ \text { C } & 2.807953000 & -3.232167000 & 4.387599000 \\ \text { F } & 2.026754000 & -3.354476000 & 5.493630000 \\ \text { F } & 4.017620000 & -3.778336000 & 4.658398000 \\ \text { F } & 3.008983000 & -1.888603000 & 4.187705000 \\ \text { C } & -2.246855000 & 6.103336000 & 3.269800000 \\ \text { F } & -3.460511000 & 6.702926000 & 3.183677000 \\ \text { F } & -2.358400000 & 5.084485000 & 4.177977000 \\ \text { F } & -1.365501000 & 7.000616000 & 3.786705000 \\ \text { C } & -3.231611000 & 4.498788000 & -1.419418000 \\ \text { F } & -3.351414000 & 5.615851000 & -2.187099000 \\ \text { F } & -2.823912000 & 3.488644000 & -2.240998000 \\ \text { F } & -4.473622000 & 4.188460000 & -0.959108000 \\ \text { C } & 6.725659000 & 1.399616000 & -3.786157000 \\ \text { F } & 6.170531000 & 2.234563000 & -4.709841000 \\ \text { F } & 7.893855000 & 1.966896000 & -3.380972000 \\ \text { F } & 7.028355000 & 0.230345000 & -4.409139000 \\ \text { C } & 4.641485000 & -1.600626000 & -0.289114000 \\ \text { F } & 3.496637000 & -2.189580000 & -0.760970000 \\ \text { F } & 5.671210000 & -2.436165000 & -0.563566000 \\ \text { F } & 4.508604000 & -1.529199000 & 1.068275000\end{array}$

\section{$\mathrm{CF}^{-\mathrm{H}+} \boldsymbol{4}-\boldsymbol{\beta}-\mathbf{T}$}

Zero-point correction $=\quad 0.825326($ Hartree/Particle $)$ Thermal correction to Energy= $\quad 0.905379$ Thermal correction to Enthalpy= $\quad 0.906323$

Thermal correction to Gibbs Free Energy $=\quad 0.690379$ Sum of electronic and zero-point Energies $=-4981.844522$ Sum of electronic and thermal Energies $=\quad-4981.764469$ Sum of electronic and thermal Enthalpies $=\quad-4981.763525$ Sum of electronic and thermal Free Energies $=-4981.979469$ E solvent $=-4981.88530171$

$\begin{array}{lrrr}\mathrm{Ni} & 0.349612000 & -1.159982000 & -0.966919000 \\ \mathrm{C} & 0.626403000 & -2.118678000 & -2.588503000 \\ \mathrm{~N} & 0.634585000 & -2.492862000 & 0.303478000 \\ \mathrm{C} & 0.532027000 & -2.252707000 & 1.595963000 \\ \mathrm{C} & 0.083708000 & -1.040858000 & 2.238228000 \\ \mathrm{C} & -0.215373000 & 0.174317000 & 1.516159000 \\ \mathrm{O} & -0.043600000 & 0.294998000 & 0.231996000 \\ \mathrm{C} & 0.390413000 & -0.751428000 & -3.142439000 \\ \mathrm{H} & -0.553769000 & -0.689154000 & -3.718914000 \\ \mathrm{C} & 1.558651000 & -0.034384000 & -3.866585000 \\ \mathrm{C} & 1.847738000 & -0.738508000 & -5.206577000 \\ \mathrm{H} & 2.453651000 & -0.114584000 & -3.218886000 \\ \mathrm{C} & 1.244401000 & 1.458555000 & -4.073845000 \\ \mathrm{H} & 0.115066000 & -0.019937000 & -2.248639000 \\ \mathrm{H} & 1.640674000 & -2.535903000 & -2.691402000 \\ \mathrm{H} & -0.164402000 & -2.865502000 & -2.746460000 \\ \mathrm{H} & 0.772218000 & -3.087816000 & 2.273891000 \\ \mathrm{C} & -0.098853000 & -1.078554000 & 3.637957000 \\ \mathrm{H} & 0.154980000 & -2.009399000 & 4.163303000 \\ \mathrm{C} & -0.606843000 & 0.001749000 & 4.394132000 \\ \mathrm{C} & -0.792023000 & -0.116503000 & 5.891967000 \\ \mathrm{H} & 0.174070000 & -0.279876000 & 6.403837000 \\ \mathrm{H} & -1.447592000 & -0.967976000 & 6.149564000 \\ \mathrm{H} & -1.244846000 & 0.795639000 & 6.314322000 \\ \mathrm{C} & -0.930877000 & 1.165588000 & 3.694753000\end{array}$
-1.338600000
-0.736468000 $-1.055693000$ $-0.791401000$ $-1.529280000$

$-1.142429000$ $-2.510682000$ $-2.820905000$ $-1.810778000$ $-0.470462000$ $-0.105575000$ 1.336193000 1.947957000 4.090869000 3.493378000 2.129978000 $-3.621055000$

$-3.693839000$

$-4.770056000$

$-5.788984000$

$-5.721664000$

$-4.652253000$

3.310931000

5.639656000

4.719187000

3.651381000

3.484790000

4.416071000

5.476629000

2.398706000

2.748338000

1.784092000

0.443754000

0.041437000

1.033172000

$-1.409882000$

$-2.111749000$

$-3.481079000$

$-4.178882000$

$-3.492995000$

$-2.125505000$

4.312310000

6.471766000

2.942066000

3.801285000

2.076339000

$-0.320772000$

$-1.581974000$

$-5.239783000$

$-6.627234000$

$-4.621745000$

$-3.872656000$

$-2.070220000$

0.319382000

1.678446000

1.375488000

$-2.917215000$

$-1.624853000$

5.150962000

2.103269000

0.371925000

1.024223000

2.693899000

2.104079000

0.970478000

$-0.305683000$

$-4.837346000$

2.026088000

1.290716000

2.534928000

2.855514000

3.318604000

4.118622000

4.486166000

5.756138000

6.608834000

6.205313000

4.960531000

4.576028000

4.145187000

3.908685000

4.355306000

4.685433000

3.548086000

2.280496000

1.419763000

1.804768000

3.066605000

3.936344000

3.811372000

$-1.565651000$

$-1.198197000$

$-2.044532000$

$-3.284846000$

$-3.654051000$

$-2.797871000$

$-4.226860000$

$-5.553411000$

$-6.468997000$

$-6.074606000$

$-4.767366000$

$-3.843961000$

$-4.417074000$

$-4.603925000$

$-4.300669000$

$-3.816735000$

$-3.656064000$

$-3.954325000$

$-4.613756000$

$-0.906190000$

$-1.738144000$

$-5.850225000$

$-7.489779000$

$-6.792332000$

$-4.976289000$

$-3.568051000$

1.133994000

4.919842000

6.048782000

7.586876000

6.874163000

5.025665000

4.104107000

1.970409000

$-3.853820000$

3.645264000

1.981444000

1.592805000

1.962363000

$-0.252214000$

$-1.802926000$

$-0.688361000$

2.108190000

0.101692000

$-0.507782000$
4.241105000

2.288988000

1.683272000

0.420259000

2.288041000

$-0.177814000$

$-0.274220000$

$-0.804739000$

$-1.267775000$

$-1.213921000$

$-0.658150000$

$-0.599658000$

0.595900000

$-0.540707000$

$-1.729785000$

$-1.762562000$

0.073402000

$-0.538422000$

$-0.274720000$

0.609186000

1.221827000

0.953558000

0.620567000

1.129846000

0.137505000

$-0.194645000$

0.450210000

1.444100000

1.783336000

0.041120000

$-0.296687000$

$-0.732964000$

$-0.825303000$

$-0.476513000$

$-0.050174000$

$-0.557219000$

$-1.766231000$

$-1.855483000$

$-0.739860000$

0.474072000

0.566128000

1.960913000

1.389693000

$-0.969214000$

$-0.239858000$

$-0.999406000$

$-1.142380000$

$-2.650041000$

$-0.816205000$

0.814752000

1.434077000

$-0.891457000$

$-1.685658000$

$-1.571880000$

$-2.699918000$

1.526527000

$-1.244508000$

1.532581000

$-0.517845000$

$-4.525776000$

$-4.740914000$

$-3.113058000$

$-5.722468000$

$-5.062720000$

$-5.878576000$

$-0.113780000$

$-1.017553000$

$-1.044333000$ 


$\begin{array}{lrrr}\text { F } & -5.717876000 & -0.764095000 & -0.451794000 \\ \text { F } & -5.211080000 & 0.283809000 & -2.312634000 \\ \text { C } & -6.776693000 & 3.466808000 & 2.236095000 \\ \text { F } & -6.940489000 & 4.816739000 & 2.277093000 \\ \text { F } & -7.978738000 & 2.902954000 & 1.954627000 \\ \text { F } & -6.417417000 & 3.062818000 & 3.488104000 \\ \text { C } & 4.310478000 & 4.409662000 & -3.005781000 \\ \text { F } & 5.614068000 & 4.687161000 & -2.753618000 \\ \text { F } & 4.271971000 & 3.207631000 & -3.657929000 \\ \text { F } & 3.834140000 & 5.348329000 & -3.866367000 \\ \text { C } & 3.912319000 & 3.282112000 & 1.906299000 \\ \text { F } & 3.362603000 & 3.886626000 & 2.997318000 \\ \text { F } & 3.672640000 & 1.941084000 & 2.038032000 \\ \text { F } & 5.253875000 & 3.462754000 & 1.955897000 \\ \text { C } & 4.887790000 & 0.078562000 & -0.652904000 \\ \text { F } & 5.401139000 & -0.163731000 & -1.890106000 \\ \text { F } & 3.678304000 & 0.699088000 & -0.852277000 \\ \text { F } & 5.706931000 & 0.970277000 & -0.035409000 \\ \text { C } & 6.414356000 & -3.177025000 & 2.913229000 \\ \text { F } & 7.649108000 & -2.637854000 & 2.739533000 \\ \text { F } & 5.937219000 & -2.727799000 & 4.109321000 \\ \text { F } & 6.553370000 & -4.526770000 & 3.014769000 \\ \text { C } & -4.195804000 & -4.531009000 & -3.172038000 \\ \text { F } & -3.441812000 & -4.083484000 & -4.218930000 \\ \text { F } & -4.423968000 & -5.855558000 & -3.382312000 \\ \text { F } & -5.392892000 & -3.891388000 & -3.218893000 \\ \text { C } & -4.200725000 & -3.095229000 & 1.688269000 \\ \text { F } & -3.995878000 & -1.744907000 & 1.797635000 \\ \text { F } & -5.540068000 & -3.297201000 & 1.642564000 \\ \text { F } & -3.729020000 & -3.652824000 & 2.840294000\end{array}$

CF3-H+TS-1 Transf-1 $^{2}$

Zero-point correction $=0.818977($ Hartree/Particle $)$

Thermal correction to Energy=

Thermal correction to Enthalpy= $\quad 0.901099$

Thermal correction to Gibbs Free Energy $=0.683695$

Sum of electronic and zero-point Energies $=\quad-4981.782686$

Sum of electronic and thermal Energies $=\quad-4981.701508$

Sum of electronic and thermal Enthalpies $=\quad-4981.700564$

Sum of electronic and thermal Free Energies $=-4981.917968$

E solvent $=-4981.82136464$

$\begin{array}{lrrr}\mathrm{Ni} & 1.202981000 & 0.731224000 & 1.193881000 \\ \mathrm{~N} & 2.490730000 & 0.739651000 & -0.304172000 \\ \mathrm{C} & 3.922303000 & 0.850404000 & -0.117682000 \\ \mathrm{C} & 4.783949000 & -0.185604000 & -0.592461000 \\ \mathrm{C} & 4.474137000 & 1.972864000 & 0.560843000 \\ \mathrm{C} & 5.859869000 & 2.004483000 & 0.823168000 \\ \mathrm{C} & 6.703939000 & 0.970040000 & 0.404950000 \\ \mathrm{C} & 6.167465000 & -0.101573000 & -0.314267000 \\ \mathrm{H} & 6.818908000 & -0.913556000 & -0.654764000 \\ \mathrm{H} & 7.776381000 & 1.009387000 & 0.620634000 \\ \mathrm{H} & 6.275732000 & 2.880369000 & 1.332991000 \\ \mathrm{C} & 4.329608000 & -1.370119000 & -1.388977000 \\ \mathrm{C} & 3.680894000 & 3.177906000 & 0.943765000 \\ \mathrm{C} & 4.986612000 & -1.668415000 & -2.603005000 \\ \mathrm{H} & 5.778441000 & -1.005679000 & -2.968067000 \\ \mathrm{C} & 4.632804000 & -2.801480000 & -3.353102000 \\ \mathrm{C} & 5.302524000 & -3.038998000 & -4.692473000 \\ \mathrm{C} & 3.624755000 & -3.667001000 & -2.903374000 \\ \mathrm{H} & 3.354379000 & -4.552913000 & -3.483363000 \\ \mathrm{C} & 2.971318000 & -3.378518000 & -1.695731000 \\ \mathrm{C} & 1.929705000 & -4.326727000 & -1.144746000 \\ \mathrm{C} & 3.313253000 & -2.240580000 & -0.948945000 \\ \mathrm{H} & 2.789500000 & -2.032030000 & -0.012928000 \\ \mathrm{C} & 3.761267000 & 3.685740000 & 2.258126000 \\ \mathrm{H} & 4.336783000 & 3.143196000 & 3.015381000 \\ \mathrm{C} & 3.108574000 & 4.879836000 & 2.605463000 \\ \mathrm{C} & 3.138303000 & 5.345310000 & 4.047311000\end{array}$

$\mathrm{O}$

(1)

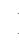

F

F

C

H

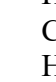

$\stackrel{\mathrm{H}}{\mathrm{H}}$

$\mathrm{H}$

$\stackrel{\mathrm{H}}{\mathrm{H}}$

$\stackrel{\mathrm{C}}{\mathrm{H}}$

$\mathrm{C}$

$\mathrm{H}$

800623000

\begin{tabular}{lr} 
C $\quad-0.675116000$ \\
\hline
\end{tabular}

$\mathrm{H} \quad-1.658085000$

H $\quad-0.529000000$

H $\quad 0.098849000$

C $\quad-2.752269000$

$\mathrm{H} \quad-3.665999000$

$\mathrm{N} \quad-2.861655000$

C $\quad-4.107584000$

C $\quad-4.203634000$

C $\quad-5.204871000$

C $\quad-5.411361000$

C -6.405678000

$\mathrm{H} \quad-5.489862000$

H $\quad-7.250720000$

C -6.509487000

H $\quad-7.445705000$

C $\quad-3.096997000$

C -2.552527000

C $\quad-2.650861000$

$\begin{array}{ll}\mathrm{C} & -2.650861000 \\ \mathrm{H} & -2.898895000\end{array}$

$\mathrm{H} \quad-3.082249000$

C -1.564508000

C -1.688439000
5.600051000 6.541653000 5.105020000 5.845169000 3.901974000 3.551374000 0.619170000 0.751672000 0.300784000 0.261451000 0.526271000 $-0.107342000$ $-0.470207000$ $-0.773178000$ $-0.465790000$ $-0.055280000$ $-0.021907000$

1.246421000 2.331890000 $-5.103800000$ $-3.629599000$ $-5.142044000$ $-4.333578000$ $-2.266696000$ $-2.713510000$ 5.747492000 5.318419000 7.161711000 6.670508000 4.673723000 5.107720000 1.282885000 3.108694000 2.571872000 0.526942000 1.231603000 0.866129000 0.722773000 $-0.562917000$ $-1.189028000$ $-0.625336000$ $-1.928100000$ $-1.182787000$ $-1.983768000$ $-2.545245000$ $-0.110780000$ $-0.474042000$ 0.904106000 $-0.759839000$ $-0.825299000$

$-1.025233000$ $-0.943936000$ $-1.103016000$ $-2.111407000$ $-0.240025000$ $-2.240972000$ $-0.437752000$ $-3.024381000$ 0.228961000

$-1.420696000$ $-1.543565000$ $-3.073937000$ $-3.153033000$ $-3.982961000$ $-2.465185000$ $-3.959261000$ $-4.107601000$ $-4.952813000$
1.644288000 1.910356000 0.332563000 $-0.713728000$ $-0.014857000$ $-1.049985000$ $-1.550905000$ $-2.338942000$ $-2.039421000$ $-3.440887000$ $-4.056802000$ $-4.097291000$ $-3.283840000$ $-3.746414000$ $-1.859036000$ $-1.182010000$ 0.114363000 2.021719000 1.947656000 $-0.154788000$ $-0.593572000$ $-2.099286000$ $-5.087427000$ $-5.660242000$ $-4.651327000$ $-1.945500000$ $-0.816122000$ $-0.414591000$ 4.159039000 4.786317000 4.622131000 2.862739000 2.121942000 1.212205000 3.038119000 3.714572000 3.970309000 2.208906000 3.138867000 2.697248000 3.618893000 2.535951000 1.961339000 3.323536000 1.647512000 $-5.608133000$ $-5.950677000$ $-6.021431000$ $-6.056656000$ $-1.154957000$ $-1.727238000$ 0.167117000 0.868219000 1.861041000 0.585240000 2.577184000 1.299271000 3.338383000 1.097539000 2.291038000 2.844857000 2.147008000 3.443502000 1.163289000 4.222629000 0.158035000 3.743211000 1.478100000 


$\begin{array}{lrrr}\text { C } & -1.130722000 & -5.014425000 & 2.767043000 \\ \text { H } & -0.370105000 & -5.762226000 & 3.005983000 \\ \text { C } & -5.131998000 & 0.901950000 & -0.376715000 \\ \text { C } & -6.115922000 & 1.056728000 & -1.377738000 \\ \text { C } & -4.122483000 & 1.877597000 & -0.268671000 \\ \text { H } & -6.914569000 & 0.314309000 & -1.477894000 \\ \text { H } & -3.364975000 & 1.795612000 & 0.517463000 \\ \text { C } & -6.078393000 & 2.155807000 & -2.249710000 \\ \text { C } & -4.085796000 & 2.971003000 & -1.147851000 \\ \text { C } & -5.060755000 & 3.118972000 & -2.143464000 \\ \text { H } & -5.034224000 & 3.975052000 & -2.822728000 \\ \text { C } & -0.970744000 & -4.111686000 & 5.136688000 \\ \text { F } & -0.332482000 & -2.921206000 & 5.384659000 \\ \text { F } & -1.935844000 & -4.239419000 & 6.084209000 \\ \text { F } & -0.067895000 & -5.105234000 & 5.311446000 \\ \text { C } & -1.316456000 & -6.002775000 & 0.448906000 \\ \text { F } & -1.467988000 & -5.534430000 & -0.821075000 \\ \text { F } & -0.028298000 & -6.411397000 & 0.596104000 \\ \text { F } & -2.109475000 & -7.100959000 & 0.573726000 \\ \text { C } & -7.102501000 & 2.266723000 & -3.363916000 \\ \text { F } & -6.648398000 & 1.657205000 & -4.496550000 \\ \text { F } & -8.275446000 & 1.669202000 & -3.023546000 \\ \text { F } & -7.361103000 & 3.562480000 & -3.673950000 \\ \text { C } & -2.962306000 & 3.972250000 & -0.984058000 \\ \text { F } & -2.972243000 & 4.926688000 & -1.941879000 \\ \text { F } & -3.014698000 & 4.583533000 & 0.229500000 \\ \text { F } & -1.749551000 & 3.328672000 & -1.045655000 \\ \text { H } & -1.975242000 & -0.790709000 & 0.685197000\end{array}$

\section{CF3-H+1-transf-1}

Zero-point correction $=\quad 0.820699$ (Hartree/Particle)

Thermal correction to Energy= $\quad 0.901408$

Thermal correction to Enthalpy= $\quad 0.902352$

Thermal correction to Gibbs Free Energy $=\quad 0.688414$

Sum of electronic and zero-point Energies $=\quad-4981.789940$

Sum of electronic and thermal Energies $=\quad-4981.709231$

Sum of electronic and thermal Enthalpies $=\quad-4981.708287$

Sum of electronic and thermal Free Energies $=-4981.922225$

E solvent $=-4981.83108181$

$\begin{array}{lrrr}\mathrm{Ni} & -0.788668000 & 1.178486000 & -1.120089000 \\ \mathrm{~N} & -1.570870000 & 2.019467000 & 0.582270000 \\ \mathrm{C} & -2.738305000 & 2.870228000 & 0.541030000 \\ \mathrm{C} & -4.001548000 & 2.376299000 & 0.985418000 \\ \mathrm{C} & -2.658096000 & 4.161988000 & -0.049161000 \\ \mathrm{C} & -3.833992000 & 4.917893000 & -0.226874000 \\ \mathrm{C} & -5.081012000 & 4.425025000 & 0.174871000 \\ \mathrm{C} & -5.154434000 & 3.168081000 & 0.782510000 \\ \mathrm{H} & -6.119673000 & 2.777505000 & 1.122881000 \\ \mathrm{H} & -5.985764000 & 5.024352000 & 0.031902000 \\ \mathrm{H} & -3.751552000 & 5.918099000 & -0.666397000 \\ \mathrm{C} & -4.218387000 & 1.031356000 & 1.613793000 \\ \mathrm{C} & -1.366583000 & 4.789263000 & -0.459227000 \\ \mathrm{C} & -3.838115000 & 0.753742000 & 2.942149000 \\ \mathrm{H} & -3.365852000 & 1.530478000 & 3.551139000 \\ \mathrm{C} & -4.112565000 & -0.495469000 & 3.524061000 \\ \mathrm{C} & -4.798065000 & -1.483686000 & 2.803080000 \\ \mathrm{H} & -5.013038000 & -2.454438000 & 3.255420000 \\ \mathrm{C} & -5.216920000 & -1.200595000 & 1.494831000 \\ \mathrm{C} & -4.932467000 & 0.042266000 & 0.906479000 \\ \mathrm{H} & -5.270838000 & 0.247010000 & -0.114620000 \\ \mathrm{C} & -1.152302000 & 5.149955000 & -1.804870000 \\ \mathrm{H} & -1.916500000 & 4.921021000 & -2.555153000 \\ \mathrm{C} & 0.038876000 & 5.787500000 & -2.192330000 \\ \mathrm{C} & 1.020219000 & 6.104206000 & -1.240975000 \\ \mathrm{H} & 1.944204000 & 6.603328000 & -1.543410000 \\ \mathrm{C} & 0.801651000 & 5.766092000 & 0.104892000 \\ \mathrm{C} & -0.376836000 & 5.107756000 & 0.492989000 \\ \mathrm{H} & -0.531878000 & 4.855309000 & 1.546421000\end{array}$

$-1.510773000$

$-0.081933000$

0.293513000

$-0.159991000$

1.201560000

1.744362000

2.458381000

1.401970000

0.454707000

0.137944000

$-1.420228000$

1.057194000

0.897984000

0.762886000

1.727401000

1.645967000

0.426727000

2.536849000

1.993928000

1.042739000

$-1.561553000$

$-0.869081000$

$-2.493855000$

$-1.671734000$

$-2.546846000$

$-3.343862000$

1.528788000

2.268157000

1.940565000

0.621314000

2.044629000

2.798907000

1.799723000

2.500954000

1.731365000

3.922720000

2.408749000

4.550403000

1.820871000

5.644575000

3.805954000

4.316784000

0.239413000

$-0.486471000$

$-0.474272000$

0.045592000

0.061299000

$-1.889662000$

$-1.877205000$

$-2.595196000$

$-3.688574000$

4.785839000

5.776905000

4.669509000

5.881257000

3.937308000

6.622009000

5.507523000

6.488348000

7.140381000

1.032813000

5.291858000

4.141953000

6.309532000

5.141792000

7.708774000

8.138658000
.708509000

2.239969000

0.721891000

0.658129000

1.384485000

$-0.285925000$

$-1.203730000$

$-1.954536000$

$-1.217003000$

0.248367000

$-0.302817000$

2.229954000

2.249257000

1.728572000

3.277237000

1.767602000

0.537530000

2.374430000

0.892687000

$-0.124510000$

$-0.052591000$

$-0.115095000$

$-0.963992000$

0.017146000

0.440829000

$-0.712721000$

0.699164000

$-0.293450000$

$-1.073588000$

0.678244000

$-0.486278000$

$-2.184812000$

$-2.839634000$

$-2.373963000$

$-3.277922000$

$-4.089455000$

$-3.334579000$

$-4.955964000$

$-4.248959000$

$-5.592057000$

$-4.293043000$

$-5.047003000$

$-5.737491000$

$-4.073273000$

$-3.912362000$

$-4.259176000$

$-3.765404000$

$-4.434247000$

$-3.913651000$

$-4.259270000$

$-4.078639000$

$-4.057508000$

$-2.436241000$

$-2.981296000$

$-1.034095000$

$-4.068099000$

$-0.587632000$

$-2.140588000$

$-0.199587000$

$-0.745503000$

$-0.093650000$

$-1.772713000$

1.297657000

1.650648000

1.996979000

1.709400000

$-2.761362000$

$-1.905236000$
1.759416000

2.633201000

2.088483000

3.444738000

4.132702000

3.979708000

3.086241000

3.449385000

1.700352000

1.154128000

$-0.105603000$

$-1.893744000$

$-1.207340000$

$-2.485809000$

$-0.988786000$

$-0.485837000$

$-3.025442000$

$-3.238320000$

$-3.580886000$

$-2.215037000$

$-3.737477000$

$-2.606006000$

$-2.622475000$

$-1.580814000$

$-3.543332000$

$-0.766465000$

$-1.690532000$

5.456726000

5.702173000

5.784756000

6.057763000

0.885871000

1.337278000

$-0.410387000$

$-1.275071000$

$-2.153154000$

$-1.272004000$

$-3.034836000$

$-2.144046000$

$-3.705050000$

$-2.156870000$

$-3.022218000$

$-3.700813000$

$-2.137829000$

$-3.339281000$

$-0.936450000$

$-4.284316000$

0.000674000

$-3.327734000$

$-0.932682000$

$-2.123585000$

$-2.111395000$

$-0.443109000$

0.400126000

$-0.529203000$

0.487616000

$-1.209120000$

1.143791000

0.225816000

1.067723000

1.654592000

$-0.776123000$

0.147423000

0.808255000

0.700735000

$-1.141796000$

2.002455000

2.964932000 


$\begin{array}{lrrr}\text { F } & 7.257518000 & -3.891408000 & 2.616534000 \\ \text { F } & 8.783434000 & -3.112559000 & 1.247584000 \\ \text { C } & -2.671659000 & -3.745403000 & -4.617294000 \\ \text { F } & -3.226838000 & -4.920668000 & -5.010593000 \\ \text { F } & -3.685534000 & -2.850624000 & -4.454574000 \\ \text { F } & -1.881058000 & -3.298546000 & -5.631322000 \\ \text { C } & -2.605468000 & -4.367635000 & 0.389051000 \\ \text { F } & -2.776194000 & -3.119480000 & 0.938624000 \\ \text { F } & -3.831244000 & -4.923295000 & 0.256280000 \\ \text { F } & -1.902110000 & -5.099729000 & 1.294653000 \\ \text { C } & -5.999319000 & -2.216361000 & 0.688065000 \\ \text { F } & -6.102239000 & -3.410340000 & 1.320411000 \\ \text { F } & -7.257180000 & -1.769053000 & 0.427563000 \\ \text { F } & -5.402804000 & -2.434827000 & -0.526858000 \\ \text { C } & -3.605655000 & -0.767452000 & 4.924103000 \\ \text { F } & -4.178007000 & -1.863379000 & 5.477568000 \\ \text { F } & -2.248718000 & -0.975550000 & 4.913398000 \\ \text { F } & -3.828903000 & 0.292106000 & 5.749404000 \\ \text { C } & 0.271623000 & 6.056302000 & -3.664556000 \\ \text { F } & 1.305259000 & 6.907018000 & -3.875747000 \\ \text { F } & 0.560464000 & 4.888452000 & -4.323936000 \\ \text { F } & -0.836353000 & 6.576521000 & -4.25566000 \\ \text { C } & 1.818953000 & 6.143792000 & 1.163193000 \\ \text { F } & 1.464772000 & 7.290841000 & 1.801284000 \\ \text { F } & 1.917835000 & 5.168733000 & 2.115571000 \\ \text { F } & 3.054814000 & 6.330624000 & 0.631323000\end{array}$

\section{CF3-H+ 1 -Transf-2}

Zero-point correction=

Thermal correction to Energy

0.742934 (Hartree/Particle)

Thermal correction to Enthalpy=

0.818600

Thermal correction to Gibbs Free Energy $=\quad 0.615243$

Sum of electronic and zero-point Energies $=\quad-4863.989183$

Sum of electronic and thermal Energies $=\quad-4863.913518$

Sum of electronic and thermal Enthalpies $=\quad-4863.912574$

Sum of electronic and thermal Free Energies $=-4864.116874$

E solvent $=-4863.9954785$

$\begin{array}{lrrr}\mathrm{N} & 2.383765000 & -0.316679000 & -1.237376000 \\ \mathrm{C} & 3.441490000 & 0.896107000 & -3.105883000 \\ \mathrm{C} & 5.052368000 & -1.441075000 & -0.479773000 \\ \mathrm{C} & 4.642853000 & -1.032456000 & 0.806556000 \\ \mathrm{H} & 4.117987000 & -0.080680000 & 0.936166000 \\ \mathrm{C} & 6.029697000 & -3.448118000 & 0.521069000 \\ \mathrm{C} & 2.166513000 & 1.596895000 & -3.441951000 \\ \mathrm{C} & 5.615198000 & -3.037304000 & 1.797011000 \\ \mathrm{C} & 3.560162000 & -0.007923000 & -2.010705000 \\ \mathrm{C} & 4.922196000 & -1.824147000 & 1.930621000 \\ \mathrm{C} & 4.825541000 & -0.578685000 & -1.681020000 \\ \mathrm{C} & 0.918004000 & 0.938689000 & -3.519820000 \\ \mathrm{H} & 0.856290000 & -0.142890000 & -3.380210000 \\ \mathrm{C} & -0.205254000 & 3.041276000 & -4.055964000 \\ \mathrm{C} & 5.753238000 & -2.659065000 & -0.607488000 \\ \mathrm{H} & 6.085848000 & -2.995614000 & -1.595203000 \\ \mathrm{C} & -0.249156000 & 1.656357000 & -3.830248000 \\ \mathrm{C} & 4.585303000 & 1.171693000 & -3.886070000 \\ \mathrm{H} & 4.486354000 & 1.838284000 & -4.749575000 \\ \mathrm{C} & 1.030480000 & 3.699950000 & -3.983884000 \\ \mathrm{C} & 5.821462000 & 0.585085000 & -3.594779000 \\ \mathrm{H} & 6.695867000 & 0.806713000 & -4.214901000 \\ \mathrm{C} & 2.203806000 & 2.986111000 & -3.692246000 \\ \mathrm{H} & 3.156374000 & 3.522880000 & -3.628425000 \\ \mathrm{C} & 5.937999000 & -0.271312000 & -2.494136000 \\ \mathrm{H} & 6.913756000 & -0.692200000 & -2.228729000 \\ \mathrm{~N} & -2.553466000 & -0.020845000 & 1.238877000 \\ \mathrm{C} & -3.709153000 & 1.858371000 & 2.308597000 \\ \mathrm{C} & -5.179306000 & -1.079227000 & 0.239813000 \\ \mathrm{H} & -4.582171000 & -1.191266000 & -1.033332000 \\ & -3.952281000 & -0.385385000 & -1.424499000\end{array}$

C -0.134286000

C -6.013798000

H $\quad-6.494305000$

C $\quad-0.376615000$

C $\quad-4.910965000$

H $\quad-4.868079000$

C -1.041087000

C -6.145534000

H $\quad-7.068139000$

C $\quad-2.178025000$

$\mathrm{H} \quad-2.870838000$

C -6.197253000

$\mathrm{H} \quad-7.157985000$

$\mathrm{Ni} \quad 1.308581000$

\begin{tabular}{lr}
$\mathrm{C}$ & -2.366939000 \\
\hline
\end{tabular}

$\mathrm{H} \quad-3.192052000$

C 2.093765000

H 2.747969000

C $\quad 0.967615000$

0.889591000

$-0.081769000$

1.704777000

$-0.166209000$

$-1.207369000$

$-0.164488000$

$-1.205320000$

$-2.066940000$

$-0.095244000$

$-1.122183000$

0.005601000

0.637650000

$-1.116687000$

$-5.833344000$

5.840919000

0.743484000

2.494305000

0.596975000

1.909962000

$-0.302007000$

0.471437000

1.860229000

2.636924000

$-1.727627000$

4.410588000

5.063149000

4.538039000

3.081224000

6.718724000

7.349987000

5.812222000

7.636799000

$-7.078497000$

$-8.067140000$

$-7.649324000$

$-6.307429000$

$-4.146656000$

$-2.811260000$

$-4.668652000$

$-4.265141000$

$-0.743688000$
$-3.257125000$

2.414999000

$-3.365569000$

0.654573000

$-2.328294000$

0.146773000

1.617314000

0.550643000

3.570463000

$-2.122945000$

$-2.046390000$

2.192686000

2.543526000

3.457237000

4.369220000

2.046442000

2.585169000

3.799294000

4.436503000

0.856315000

0.475659000

1.069831000

$-1.339345000$

$-1.934472000$

$-1.590620000$

$-2.333170000$

$-2.132349000$

$-3.538247000$

$-1.318691000$

$-4.128969000$

$-4.221697000$

$-2.017145000$

$-5.731263000$

$-3.439316000$

$-3.928066000$

$-0.011052000$

$-6.106652000$

$-6.208287000$

$-6.080470000$

3.597211000

$-4.240045000$

$-3.646099000$

4.018336000

2.269525000

2.677422000

3.148069000

3.010236000

2.580349000

3.895186000

3.429187000

0.515245000

$-1.375961000$

$-1.982565000$

$-0.029178000$

$-1.669165000$

$-4.786398000$

$-5.187075000$

$-5.753585000$

$-4.740642000$

$-4.396701000$

$-3.939684000$

$-5.121362000$

$-5.247865000$

$-2.428933000$

$-2.679227000$

$-3.425994000$

$-1.265649000$

5.843691000
$-0.098767000$

2.855464000

$-1.365123000$

1.552465000

$-1.825147000$

1.087881000

3.570757000

3.717220000

4.041297000

0.696222000

1.677327000

4.152457000

2.587266000

3.189012000

3.326070000

2.157058000

2.395258000

2.735126000

2.176713000

1.421452000

1.059353000

$-0.409695000$

1.267618000

1.672953000

$-1.089244000$

$-1.573502000$

$-0.363984000$

$-0.254050000$

0.201127000

$-0.693664000$

0.388091000

0.802228000

0.500110000

0.893307000

1.366232000

0.186250000

0.896901000

$-0.481738000$

1.176349000

$-4.291080000$

$-1.992832000$

2.676264000

4.516013000

$-0.715624000$

0.343938000

$-0.158323000$

$-0.194515000$

1.429816000

$-0.967388000$

0.620844000

0.905440000

3.284009000

4.306633000

3.441331000

3.415631000

0.339072000

1.471780000

0.011051000

$-0.663865000$

0.446978000

1.261247000

$-0.547910000$

1.184757000

$-3.179986000$

$-3.035914000$

$-3.937639000$

$-3.875539000$

3.130043000 


$\begin{array}{cc}\text { F } & -0.130430000 \\ \text { F } & 0.088805000 \\ \text { F } & -1.875282000 \\ \text { C } & 0.643664000 \\ \text { F } & 1.273119000 \\ \text { F } & 0.077094000 \\ \text { F } & 1.603480000 \\ \text { C } & 1.109155000 \\ \text { F } & 2.200044000 \\ \text { F } & 0.007533000 \\ \text { F } & 1.219783000 \\ \text { C } & -1.580817000 \\ \text { F } & -2.118719000 \\ F & -2.486742000 \\ \text { F } & -1.459372000\end{array}$

6.375448000 6.026821000 6.556234000 1.305771000 1.948286000 0.174675000 0.917409000 5.207666000 5.600142000 5.737285000 5.765784000 0.939646000 0.950811000 1.531620000 $-0.360583000$
4.217787000 2.062988000 2.888677000 4.836836000 5.851271000 5.335335000 3.942729000 $-4.099833000$ $-4.806876000$ $-4.684841000$ $-2.850611000$ $-3.826140000$ $-2.553914000$ $-4.639603000$ $-4.190605000$

\section{${ }^{\text {CF3-H+ }}+{ }^{-T S}-2_{\text {ins- }}$}

Zero-point correction $=$ $\begin{array}{lc}\text { Thermal correction to Energy= } & 0.904461 \\ \text { Thermal correction to Enthalpy= } & 0.905405 \\ \text { Thermal correction to Gibbs Free Energy= } & 0.692182 \\ \text { Sum of electronic and zero-point Energies }= & -4981.795944 \\ \text { Sum of electronic and thermal Energies }= & -4981.715943 \\ \text { Sum of electronic and thermal Enthalpies }= & -4981.714999 \\ \text { Sum of electronic and thermal Free Energies }= & -4981.928222\end{array}$ E solvent $=-4981.8283591$

\begin{tabular}{|c|c|c|c|}
\hline $\mathrm{N}$ & 2.181921000 & -0.177184000 & -1.385728000 \\
\hline $\mathrm{C}$ & 3.274570000 & 1.289083000 & -3.054971000 \\
\hline C & 4.725608000 & -1.733745000 & -1.128461000 \\
\hline & 4.493201000 & -1.591666000 & 0.253677000 \\
\hline $\mathrm{H}$ & 4.155075000 & -0.629989000 & 56000 \\
\hline C & 5.433464000 & -4.038975000 & -0.719114000 \\
\hline $\mathrm{C}$ & 2.059443000 & 2.144695000 & 000 \\
\hline C & 5.195528000 & -3.889985000 & 5000 \\
\hline C & 910000 & 0.174917000 & 000 \\
\hline $\mathrm{C}$ & 4.726241000 & -2.656873000 & 000 \\
\hline 0 & 4.550105000 & -0.584275000 & 000 \\
\hline $\mathrm{C}$ & 0.760926000 & 1.611021000 & -3.356 \\
\hline $\mathrm{H}$ & 0.6099 & 7000 & 000 \\
\hline $\mathrm{C}$ & 4000 & 00 & 000 \\
\hline $\mathrm{C}$ & 5.204848000 & 037000 & 3000 \\
\hline $\mathrm{H}$ & 5.4014 & 95000 & 2000 \\
\hline$C$ & -0.345210000 & 2.460518000 & 3000 \\
\hline $\mathrm{C}$ & 4.403783000 & 1.606290000 & 000 \\
\hline $\mathrm{H}$ & 4.335442000 & 2.4 & 000 \\
\hline $\mathrm{C}$ & 1.098608000 & 574000 & -3.3 \\
\hline $\mathrm{C}$ & 5.581056000 & 5000 & 2000 \\
\hline $\mathrm{H}$ & 6.442854000 & 697000 & 4000 \\
\hline C & 2.209927000 & 384000 & 000 \\
\hline $\mathrm{H}$ & 3.204037000 & 3.98 & $-3 .($ \\
\hline $\mathrm{C}$ & 5.649590000 & 851000 & 8000 \\
\hline $\mathrm{H}$ & 6.576427000 & 483000 & -2.790 \\
\hline $\mathrm{N}$ & -2.635485000 & 104000 & 0000 \\
\hline $\mathrm{C}$ & -3.380524000 & 1.334680000 & 68000 \\
\hline $\mathrm{C}$ & -5.475592000 & 17616000 & 5000 \\
\hline $\mathrm{C}$ & -6.470181000 & -1.494596000 & 9000 \\
\hline $\mathrm{H}$ & -6.870717000 & -1.640545000 & 1.599 \\
\hline $\mathrm{C}$ & -5.440169000 & -1.158031000 & -1.986656000 \\
\hline $\mathrm{C}$ & -2.001611000 & 1.426425000 & 3.622829000 \\
\hline $\mathrm{C}$ & -6.426102000 & -2.129596000 & -1.758557000 \\
\hline C & -3.709141000 & 0.481246000 & 1.966827000 \\
\hline $\mathrm{C}$ & -6.941203000 & -2.291614000 & -0.463 \\
\hline $\mathrm{C}$ & -5.050879000 & 0.349113000 & 1.512860000 \\
\hline $\mathrm{C}$ & -1.422549000 & 2.690620000 & 3.878970000 \\
\hline $\mathrm{H}$ & -1.961212000 & 3.603162000 & 3.604808000 \\
\hline $\mathrm{C}$ & 0.552957000 & 1.627036000 & 4.836884000 \\
\hline $\mathrm{C}$ & -4.965038000 & -0.352384000 & -0.937419000 \\
\hline & 2000 & 0.415044000 & -1.148047000 \\
\hline
\end{tabular}

$-0.157456000$ $-4.412548000$ $-4.167907000$ $-0.015638000$ $-5.738220000$ $-6.530567000$ $-1.281124000$ $-1.717487000$ $-6.050245000$ $-7.083940000$ 1.208055000 $-2.624019000$ $-3.496149000$ 1.737115000 2.275540000 0.582084000 0.388894000 $-0.396037000$ 1.149220000 $-0.717103000$ $-1.562765000$ $-0.842222000$ $-1.676849000$ $-2.561652000$ $-0.297755000$ 3.020440000 3.783984000 2.985229000 3.509362000 1.228457000 $-0.025403000$ 5.247987000 3.769383000 3.319547000 5.805021000 5.305918000 5.763343000 $-0.539006000$ $-0.657961000$ 1.560624000 1.535130000 $-0.759738000$ $-0.044205000$ $-1.810646000$ 5.387153000 1.534921000 $-6.790280000$ $-1.051677000$ $-1.760549000$ 1.299101000 0.269081000 1.395772000 2.447956000 $-1.733270000$ $-1.727675000$ $-2.264453000$ $-2.587153000$ $-4.838482000$ $-5.586672000$ $-4.712117000$ $-3.589388000$ $-8.048633000$ $-8.062924000$ $-7.901192000$ $-9.266715000$ 0.490015000 1.430416000 $-0.410579000$

2.785258000 2.078788000 2.724393000 0.370853000 1.957195000 2.532085000 0.268789000 $-0.721428000$ 1.090726000 1.004131000

1.132151000

$-1.482399000$

$-2.087650000$ $-1.395392000$ $-2.009527000$ $-2.038209000$ $-3.405861000$ $-1.330231000$ $-3.913904000$ $-4.156664000$ $-2.085653000$ $-5.631518000$ $-3.472613000$ $-4.011687000$ $-0.068422000$ 1.998161000 3.049462000 1.053408000 1.857098000 3.230474000 2.567860000 2.594838000 4.028883000 3.198208000 3.347759000 1.634625000 2.471901000 2.355502000 2.609764000 3.840203000 3.600976000 $-5.818172000$ $-6.216740000$ $-6.035504000$ $-4.717178000$ 1.702496000 $-2.750505000$ 4.514340000 0.301135000 5.890663000 6.582147000 6.266651000 6.282549000 1.865157000 0.611277000 1.770400000 2.625309000 $-0.994109000$ $-1.596334000$ 0.320396000 $-1.544723000$ $-3.294622000$ $-4.281666000$ $-3.876196000$ $-2.690737000$ 4.137863000 4.384840000 5.150386000
4.478322000 3.664874000 4.514962000 4.584319000 3.233240000 3.723041000 3.984274000 3.824821000 2.178528000 1.827678000 $-0.169558000$ 0.928163000 1.199248000 $-1.610559000$ $-2.351323000$ $-1.027094000$ $-1.313370000$ $-0.238214000$ $-1.922241000$ $-0.852996000$ 0.201644000 $-1.170381000$ $-0.107060000$ 0.255861000 0.066319000 0.472086000 1.284380000 1.055731000 $-0.503181000$ 0.384004000 0.332840000 1.473994000 0.769999000 2.276121000 2.059271000 2.017551000 0.504543000 1.279482000 $-0.563036000$ $-0.463926000$ 1.364024000 $-2.256317000$ $-0.677160000$ $-0.831779000$ 1.343829000 5.312335000 $-2.580852000$ $-3.649533000$ 1.069017000 $-3.271436000$ $-3.818158000$ $-1.957881000$ $-3.882791000$ $-3.583347000$ $-4.098999000$ $-2.308903000$ $-4.306813000$ $-3.368272000$ $-4.326481000$ $-3.698600000$ $-3.416538000$ $-0.200416000$ $-1.133796000$ 1.023425000 $-0.217424000$ 4.692349000 3.718253000 4.641056000 


\section{CF3-H+ TS-1}

Zero-point correction= Thermal correction to Energy= Thermal correction to Enthalpy= 0.767869 (Hartree/Particle)

Thermal correction to Gibbs Free Energy $=0.643057$ Sum of electronic and zero-point Energies $=-4903.253390$ Sum of electronic and thermal Energies $=\quad-4903.177758$ Sum of electronic and thermal Enthalpies $=\quad-4903.176814$ Sum of electronic and thermal Free Energies $=-4903.378201$ E solvent $=-4903.27006202$

\begin{tabular}{|c|c|c|c|}
\hline $\mathrm{Ni}$ & 1.441272000 & 0.299832000 & -0.921037000 \\
\hline $\mathrm{C}$ & 3.096698000 & 0.529218000 & -2.052432000 \\
\hline $\mathrm{N}$ & 1.697535000 & 1.890789000 & 0.289818000 \\
\hline $\mathrm{C}$ & 0.945814000 & 2.088631000 & 1.351045000 \\
\hline $\mathrm{C}$ & -0.143723000 & 1.273946000 & 129000 \\
\hline $\mathrm{C}$ & -0.616528000 & 0.110678000 & 3000 \\
\hline $\mathrm{O}$ & -0.094878000 & -0.329095000 & 0.025818000 \\
\hline $\mathrm{C}$ & 2.051359000 & 0.387321000 & -2.982399000 \\
\hline $\mathrm{H}$ & 1.249224000 & -0.966250000 & 5668000 \\
\hline $\mathrm{C}$ & 1.385581000 & 1.536622000 & -3.692173000 \\
\hline $\mathrm{H}$ & 0.286579000 & 1.436687000 & -3.7 \\
\hline $\mathrm{H}$ & 1.642 & 8000 & -3.2 \\
\hline $\mathrm{H}$ & 1.733596000 & 1.538432000 & 7000 \\
\hline $\mathrm{H}$ & 1.927114000 & -0.590182000 & -3.4 \\
\hline $\mathrm{H}$ & 3.512372000 & 1.522805000 & -1.854095000 \\
\hline $\mathrm{H}$ & 3.736125000 & -0.332069000 & 2401000 \\
\hline $\mathrm{H}$ & 1.1508 & 7000 & 000 \\
\hline $\mathrm{C}$ & -0.774120000 & 1.668 & 000 \\
\hline $\mathrm{H}$ & -0.391783000 & 2.562 & 1000 \\
\hline $\mathrm{C}$ & -1.858969000 & 0.977876000 & 4000 \\
\hline $\mathrm{C}$ & -2.508611000 & 1.455 & 8000 \\
\hline $\mathrm{H}$ & -1.8695 & 2.18 & 7000 \\
\hline $\mathrm{H}$ & -2.709 & 0.61 & \\
\hline $\mathrm{H}$ & -3.47596 & 1.952416000 & 59000 \\
\hline $\mathrm{C}$ & -2.322782000 & -0.155374000 & 2.934425000 \\
\hline $\mathrm{H}$ & -3.168772000 & -0.720950000 & 3.346958000 \\
\hline $\mathrm{C}$ & -1.744970000 & -0.604312000 & 1.715830000 \\
\hline $\mathrm{C}$ & -2.305032000 & -1.757960000 & 23000 \\
\hline $\mathrm{N}$ & -1.857006000 & -2.280574000 & -0.043144000 \\
\hline $\mathrm{H}$ & -3.153002000 & -2.260710000 & 1.572522000 \\
\hline $\mathrm{C}$ & -2.382339000 & -3.408781000 & -0.746816000 \\
\hline $\mathrm{C}$ & -1.443984000 & -4.291607000 & -1.352975000 \\
\hline $\mathrm{C}$ & -1.930655000 & -5.395849000 & -2.080475000 \\
\hline $\mathrm{C}$ & -3.305189000 & -5.640688000 & -2.182388000 \\
\hline $\mathrm{C}$ & -4.215967000 & -4.762835000 & -1.580732000 \\
\hline $\mathrm{C}$ & -3.783142000 & -3.622104000 & -0.872610000 \\
\hline $\mathrm{C}$ & -4.829504000 & -2.683988000 & -0.355650000 \\
\hline $\mathrm{C}$ & -4.882317000 & -1.336315000 & -0.771646000 \\
\hline $\mathrm{C}$ & -6.929008000 & -0.994118000 & 0.511353000 \\
\hline $\mathrm{C}$ & -6.878819000 & -2.332218000 & 0.929653000 \\
\hline $\mathrm{C}$ & -5.840417000 & -3.173690000 & 0.498475000 \\
\hline $\mathrm{C}$ & 0.030195000 & -4.092814000 & -1.227214000 \\
\hline $\mathrm{C}$ & 0.840509000 & -4.089548000 & -2.384716000 \\
\hline $\mathrm{C}$ & 2.229557000 & -3.925300000 & -2.280334000 \\
\hline
\end{tabular}

$\mathrm{C}$
$\mathrm{C}$
$\mathrm{C}$
$\mathrm{C}$
$\mathrm{C}$
$\mathrm{C}$
$\mathrm{C}$
$\mathrm{C}$
$\mathrm{C}$
$\mathrm{C}$
$\mathrm{C}$
$\mathrm{C}$

$2.838892000 \quad-3.756289000$ $2.038690000-3.772586000$ $0.648584000-3.945211000$ $-5.927092000 \quad-0.503251000$ $-1.972231000$ $-1.532028000$ $-0.199343000$ 0.734118000 0.276906000 $-1.055715000$ 2.177780000 3.142741000 4.509784000 4.944221000 4.023430000 2.640183000 4.537763000 5.492895000 5.970670000 5.548910000 4.633880000 4.122612000 0.962663000 $-3.011735000$

0.112801000 2.804285000 5.234584000 6.008701000 5.845059000 5.914024000 3.919759000 0.051463000 $-1.211844000$

$-3.668666000$ $-5.292603000$ $-5.821650000$ $-4.127487000$ 0.381501000 3.406746000 $-7.750615000$

$-1.011765000$ 6.847373000 7.754353000 7.512476000 6.076210000

$-1.488628000$ $-2.830257000$ $-0.867627000$ $-1.172276000$ $-2.476978000$ $-3.732486000$ $-2.045350000$

$-2.544951000$

$-5.944577000$

$-7.198359000$

$-5.173214000$

$-5.450829000$

$-7.919770000$

$-9.103183000$

$-8.141472000$

$-7.504029000$

3.081517000

3.354271000

4.273371000

5.305336000

4.014221000

3.634013000

4.538499000

5.834929000

6.211952000

4.200731000

5.183243000

4.964847000

3.756698000

2.743972000

2.957753000

1.480899000

0.707310000

$-0.494174000$

$-0.916437000$

$-0.124940000$

1.054445000

6.552070000

5.598444000

2.618959000

6.126909000

5.739747000

3.589703000

1.027021000

$-1.855329000$

$-3.609310000$

$-3.991365000$

$-6.084945000$

$-6.511259000$

$-4.929497000$

$-4.219502000$

$-0.939555000$

$-4.208935000$

1.652054000

$-0.346550000$

$-1.787754000$

$-1.382452000$

$-0.664840000$

$-2.310625000$

$-2.060770000$

7.629477000

7.710737000

8.091089000

8.478367000

2.991083000

3.469535000

2.561804000

1.873281000

0.950761000

1.469618000

1.708327000

1.100488000

$-2.861972000$

$-2.211544000$

$-4.190591000$

$-2.697471000$

$-3.852773000$

$-2.544315000$

$-4.473442000$

2.458844000

$-4.403889000$

$-3.513052000$

2.644760000

$-2.200143000$

2.059959000
$-1.024811000$

0.125777000

0.030437000

$-0.340208000$

$-1.152046000$

$-1.475173000$

$-1.251916000$

$-0.705059000$

$-0.371872000$

$-0.596395000$

$-0.498018000$

$-0.823379000$

$-0.623494000$

$-0.065268000$

0.276664000

0.027465000

0.890001000

0.201488000

0.750405000

2.018444000

2.730393000

2.169051000

0.090555000

$-1.317841000$

$-1.510442000$

$-1.264133000$

$-0.892761000$

0.132624000

$-0.784888000$

2.441098000

$-0.944861000$

0.946226000

$-2.536270000$

$-2.737471000$

$-1.691200000$

0.822726000

$-1.458162000$

$-3.371158000$

2.740244000

0.828933000

$-0.410244000$

$-0.103532000$

$-0.815387000$

0.623963000

$-1.023296000$

$-0.273119000$

$-0.071879000$

0.848142000

$-1.288618000$

$-2.065970000$

$-2.216598000$

$-3.291433000$

$-1.273723000$

$-0.770929000$

$-0.745666000$

0.063057000

$-2.029278000$

1.898116000

1.764315000

1.711234000

3.187050000

$-3.531045000$

$-3.851646000$

$-3.362439000$

$-4.603947000$

1.487662000

1.839911000

2.454027000 


$\begin{array}{rrr}3.979729000 & -3.755266000 & 1.509745000 \\ 4.218188000 & -0.528159000 & 4.130316000 \\ 2.947576000 & -0.112324000 & 4.411010000 \\ 4.259505000 & -1.876495000 & 4.299641000 \\ 5.035655000 & 0.025746000 & 5.066304000\end{array}$

\section{References}

[1] Lindoy, L. F.; Meehan, G. V.; Svenstrup, N. Mono- and Diformylation of 4-Substituted Phenols: A New Application of the Duff Reaction. Synthesis 1998, 1998, 1029-1032.

[2] Zuideveld, M. A.; Wehrmann, P.; Röhr, C.; Mecking, S. Remote Substituents Controlling Catalytic Polymerization by Very Active and Robust Neutral Nickel(II) Complexes. Angew. Chem. Int. Ed. 2004, 43, 869-873.

[3] Göttker-Schnetmann, I.; Mecking, S. A Practical Synthesis of [(tmeda)Ni( $\left.\left.\mathrm{CH}_{3}\right)_{2}\right]$, Isotopically Labeled [(tmeda)Ni $\left({ }^{13} \mathrm{CH}_{3}\right)_{2}$ ], and Neutral Chelated-Nickel Methyl Complexes. Organometallics 2020, 39, 3433-3440.

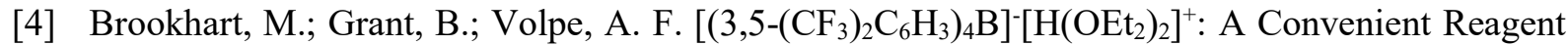
for Generation and Stabilization of Cationic, Highly Electrophilic Organometallic Complexes. Organometallics 1992, 11, 3920-3922.

[5] Dolomanov, O. V.; Bourhis, L. J.; Gildea, R. J.; Howard, J. A. K.; Puschmann, H. OLEX2: A Complete Structure Solution, Refinement and Analysis Program. J. Appl. Crystallogr. 2009, 42, 339-341.

[6] Sheldrick, G. SHELXT - Integrated space-group and Crystal-structure determination. Acta Cryst C 2015, 71, 3-8.

[7] Sheldrick, G. M. Crystal structure refinement with SHELXL. Acta Cryst C 2015, 71, 3-8.

[8] Faruggia, L. ORTEP-3 V2.02 for Windows, University of Glasgow: Scotland 2008.

[9] Sheldrick, G. M. Crystal structure refinement with SHELXL. Acta Cryst. C 2015, 71, 3-8.

[10] Göttker-Schnetmann, I.; Wehrmann, P.; Röhr, C.; Mecking, S. Substituent Effects in ( $\left.\kappa^{2}-\mathrm{N}, \mathrm{O}\right)-$ Salicylaldiminato Nickel(II)-Methyl Pyridine Polymerization Catalysts: Terphenyls Controlling Polyethylene Microstructures. Organometallics 2007, 26, 2348-2362.

[11] Perdew, J. P. Density-functional Approximation for the Correlation Energy of the Inhomogeneous Electron Gas. Phys. Rev. B 1986, 33, 8822-8824.

[12] Perdew, J. P. Erratum: Density-functional Approximation for the Correlation Energy of the Inhomogeneous Electron Gas. Phys. Rev. B 1986, 34, 7406.

[13] Becke, A. D. Density-functional Exchange-energy Approximation with Correct Asymptotic Behavior. Phys. Rev. A 1988, 38, 3098-3100.

[14] Frisch, M. J.; Trucks, G. W.; Schlegel, H. B.; Scuseria, G. E.; Robb, M. A.; Cheeseman, J. R.; Scalmani, G.; Barone, V.; Mennucci, B.; Petersson, G. A.; Nakatsuji, H.; Caricato, M.; Li, X.; Hratchian, H. P.; Izmaylov, A. F.; Bloino, J.; Zheng, G.; Sonnenberg, J. L; Hada, M.; Ehara, M.; Toyota, K.; Fukuda, R.; Hasegawa, J.; Ishida, M.; Nakajima, T.; Honda, Y.; Kitao, O.; Nakai, H.; Vreven, T.; Montgomery, J. A.; Peralta, J. E.; Ogliaro, F.; Bearpark, M.; Heyd, J. J.; Brothers, E.; Kudin, K. N.; Staroverov, V. N.; Kobayashi, R.; Normand, J.; Raghavachari, K.; Rendell, A.; Burant, J. C.; Iyengar, S. S.; Tomasi, J.; Cossi, M;. Rega, N.; Millam, J. M.; Klene, M.; Knox, J. E.; Cross, J. B.; Bakken, V.; Adamo, C.; Jaramillo, J.; Gomperts, R.; Stratmann, R. E.; Yazyev, O.; Austin, A. J.; Cammi, R.; Pomelli, C.; Ochterski, J. W. R.; Martin, L.; Morokuma, K.; Zakrzewski, V. G.; Voth, G. A.; Salvador, P.; Dannenberg, J. J.; Dapprich, S.; Daniels, A. D.; Farkas, Ö.; 
Foresman, J. B.; Ortiz, J. V.; Cioslowski, J.; Fox D. J. Gaussian 09 Revision A.1, Gaussian, Inc., Wallingford, CT, 2009.

[15] Weigend, F.; Ahlrichs, R. Balanced Basis Sets of Split Valence, Triple Zeta Valence and Quadruple Zeta Valence Quality for H to Rn: Design and Assessment of Accuracy. PCCP 2005, 7, 3297 3305.

[16] Häussermann, U.; Dolg, M.; Stoll, H.; Preuss, H.; Schwerdtfeger, P.; Pitzer, R. M. Accuracy of energy-adjusted quasirelativistic ab initio pseudopotentials. Mol. Phys. 1993, 78, 1211-1224.

[17] Küchle, W.; Dolg, M.; Stoll, H.; Preuss, H. Energy-adjusted pseudopotentials for the actinides. Parameter sets and test calculations for thorium and thorium monoxide. J. Chem. Phys. 1994, 100, $7535-7542$.

[18] Leininger, T.; Nicklass, A.; Stoll, H.; Dolg, M.; Schwerdtfeger, P. The accuracy of the pseudopotential approximation. II. A comparison of various core sizes for indium pseudopotentials in calculations for spectroscopic constants of $\mathrm{InH}, \mathrm{InF}$, and InCl. J. Chem. Phys. 1996, 105, 10521059.

[19] Tomasi, J.; Persico, M. Molecular Interactions in Solution: An Overview of Methods Based on Continuous Distributions of the Solvent. Chem. Rev. 1994, 94, 2027-2094.

[20] Barone, V.; Cossi, M. Quantum Calculation of Molecular Energies and Energy Gradients in Solution by a Conductor Solvent Model. J. Phys. Chem. A 1998, 102, 1995-2001. 